\title{
INITIAL SLUDGE BATCH 4 TANK 40 DECANT VARIABILITY STUDY WITH FRIT 510
}

\author{
F.C. Raszewski \\ T.B. Edwards \\ D.K. Peeler \\ D.R. Best \\ I.A. Reamer \\ R.J. Workman
}

May 2008

Process Science and Engineering Section Savannah River National Laboratory Aiken, SC 29808

Prepared for the U.S. Department of Energy Under Contract Number DEAC09-96SR18500

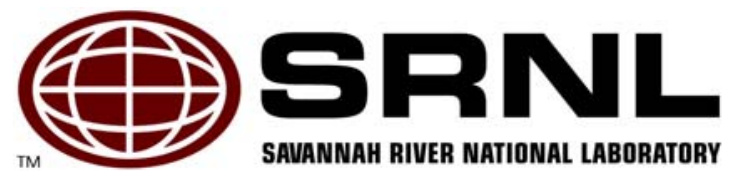


WSRC-STI-2008-00149

Revision 0

\section{DISCLAIMER}

This report was prepared by Washington Savannah River Company (WSRC) for the United States Department of Energy under Contract No. DE-AC09-96SR18500 and is an account of work performed under that contract. Neither the United States Department of Energy, nor WSRC, nor any of their employees makes any warranty, expressed or implied, or assumes any legal liability or responsibility for the accuracy, completeness, or usefulness, of any information, apparatus, or product or process disclosed herein or represents that its use will not infringe privately owned rights. Reference herein to any specific commercial product, process, or service by trademark, name, manufacturer or otherwise does not necessarily constitute or imply endorsement, recommendation, or favoring of same by WSRC or by the United States Government or any agency thereof. The views and opinions of the authors expressed herein do not necessarily state or reflect those of the United States Government or any agency thereof.

\section{Printed in the United States of America}

Prepared For U.S. Department of Energy

The Savannah River National Laboratory is operated for the U.S. Department of Energy by Washington Savannah River Company. 
Keywords: SB4, Frit 510, decant, variability, durability

Retention: permanent

\section{INITIAL SLUDGE BATCH 4 TANK 40 DECANT VARIABILITY STUDY WITH FRIT 510}

F.C. Raszewski

T.B. Edwards

D.K. Peeler

D.R. Best

I.A. Reamer

R.J. Workman

May 2008

Process Science and Engineering Section Savannah River National Laboratory Aiken, SC 29808

Prepared for the U.S. Department of Energy Under Contract Number DEAC09-96SR18500

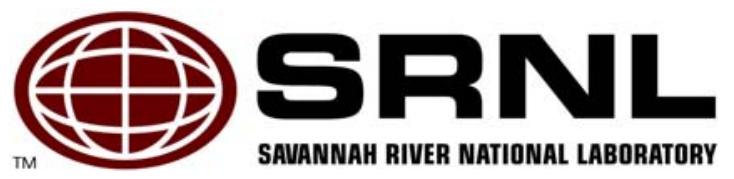




\section{REVIEWS AND APPROVALS}

AUTHORS:

F.C. Raszewski, Process Science and Engineering Section

Date

T.B. Edwards, Statistical Consulting Section

Date

D.K. Peeler, Process Science and Engineering Section

Date

D.R. Best, Process Science and Engineering Section

Date

I.A. Reamer, Process Science and Engineering Section

Date

R.J. Workman, Process Science and Engineering Section

Date

\section{TECHNICAL REVIEWER:}

K.M. Fox, Process Science and Engineering Section

Date

\section{APPROVERS:}

J.C. Griffin, Manager, E\&CPT Research Programs

Date

C.C. Herman, Manager, Process Engineering Technology Group

Date

J.E. Occhipinti, Manager, Process Cognizant Engineering

Date

Waste Solidification Engineering 
WSRC-STI-2008-00149

Revision 0

\section{EXECUTIVE SUMMARY}

Sludge Batch 4 (SB4) is currently being processed in the Defense Waste Processing Facility (DWPF) using Frit 510. The slurry pumps in Tank 40 are experiencing in-leakage of bearing water, which is causing the sludge slurry feed in Tank 40 to become dilute at a rapid rate. Currently, the DWPF is removing this dilution water by performing caustic boiling during the Sludge Receipt and Adjustment Tank (SRAT) cycle. In order to alleviate prolonged SRAT cycle times that may eventually impact canister production rates, decant scenarios of 100, 150, and 200 kilogallons of supernate were proposed for Tank 40 during the DWPF March outage. Based on the results of the preliminary assessment issued by the Savannah River National Laboratory (SRNL), the Liquid Waste Organization (LWO) issued a Technical Task Request (TTR) for SRNL to (i) perform a more detailed evaluation using updated SB4 compositional information and (ii) assess the viability of Frit 510 and determine any potential impacts on the SB4 system. As defined in the TTR, LWO requested that SRNL validate the sludge-only SB4 flowsheet and the coupled operations flowsheet using the 100K gallon decant volume as well as the addition of $3 \mathrm{wt} \%$ sodium on a calcined oxide basis.

Approximately 12 historical glasses were identified during a search of the $\mathrm{ComPro}^{\mathrm{TM}}$ database that are located within at least one of the five glass regions defined by the proposed SB4 flowsheet options. While these glasses meet the requirements of a variability study there was some concern that the compositional coverage did not adequately bound all cases. Therefore, SRNL recommended that a supplemental experimental variability study be performed to support the various SB4 flowsheet options that may be implemented for future SB4 operations in DWPF. Eighteen glasses were selected based on nominal sludge projections representing the current as well as the proposed flowsheets over a WL interval of interest to DWPF $(32-42 \%)$. The intent of the experimental portion of the variability study is to demonstrate that the glasses of the Frit 510-modified SB4 compositional region (Cases \#1-5) are both acceptable relative to the Environmental Assessment (EA) reference glass and predictable by the current DWPF process control models for durability.

Frit 510 is a viable option for the processing of SB4 after a Tank 40 decant and the addition of products from the Actinide Removal Process (ARP). The addition of ARP did not have any negative impacts on the acceptability and predictability of the variability study glasses. The results of the variability study indicate that all of the study glasses (both quenched and centerline canister cooled (ccc)) have normalized releases for boron that are well below the reference EA glass $(16.695 \mathrm{~g} / \mathrm{L})$. The durabilities of all of the study glasses are predictable using the current Product Composition Control System (PCCS) durability models with the exception of SB4VAR24ccc (Case \#2 at 41\%). PCCS is not applicable to non-homogeneous glasses (i.e. glasses containing crystals such as acmite and nepheline), thus SB4VAR24ccc should not be predictable as it contains nepheline.

The presence of nepheline has been confirmed in both SB4VAR13ccc and SB4VAR24ccc by X-ray diffraction (XRD). These two glasses are the first results which indicate that the current nepheline discriminator value of 0.62 is not conservative. The nepheline discriminator was implemented into PCCS for SB4 based on the fact that all of the historical glasses evaluated with nepheline values of 0.62 or greater did not contain nepheline via XRD analysis. Although these two glasses do cause some concern over the use of the 0.62 nepheline value for future DWPF glass systems, the impact to the current SB4 system is of little concern. More specifically, the formation of nepheline was observed in glasses targeting 41 or $42 \%$ WL. Current processing of the Frit 510-SB4 system in DWPF has nominally targeted $34 \% \mathrm{WL}$. For the SB4 variability study glasses targeting these lower WLs, nepheline formation was not observed and the minimal difference in PCT response between quenched and ccc versions supported its absence. 


\section{TABLE OF CONTENTS}

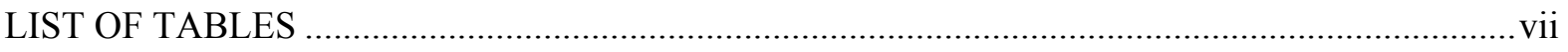

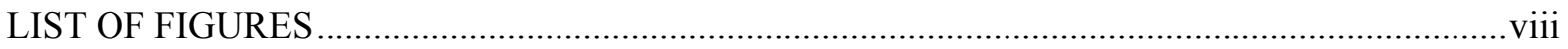

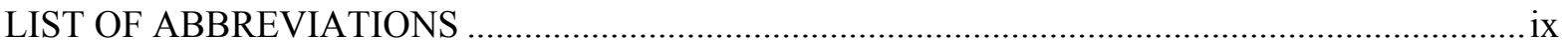

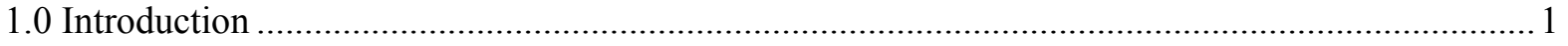

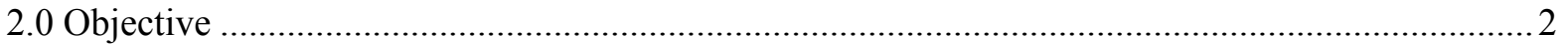

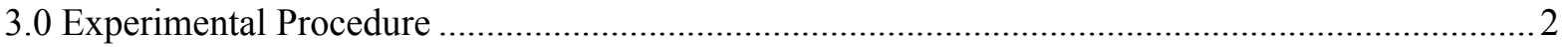

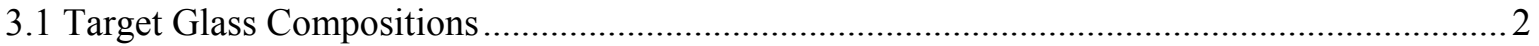

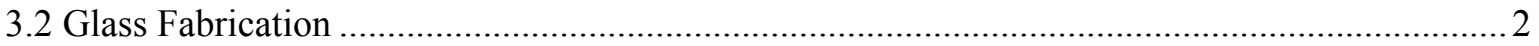

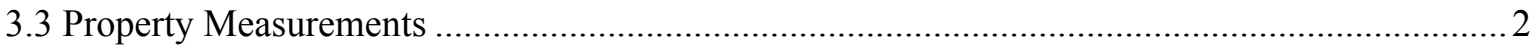

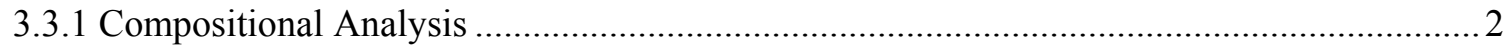

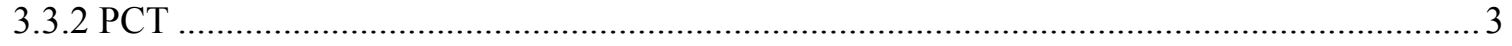

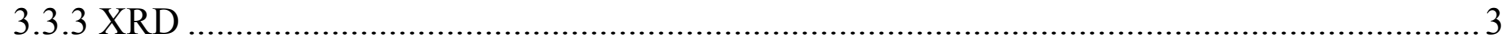

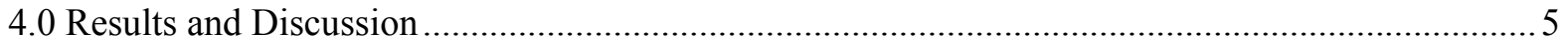

4.1 Statistical Review of Chemical Composition Measurements ................................................... 5

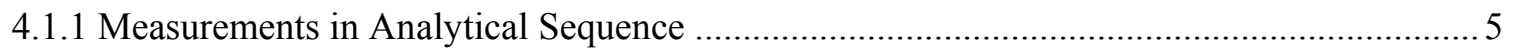

4.1.2 Batch 1 and Uranium Standard Results................................................................................. 5

4.1.3 Composition Measurements by Glass Identifier ........................................................... 6

4.1.4 Measured versus Targeted Compositions ......................................................................... 7

4.1.5 MAR Assessment of the SB4/Decant Variability Study (VS) Glasses ................................ 7

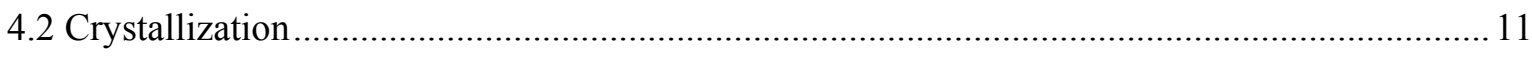

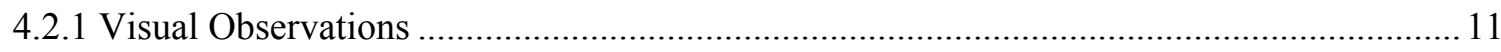

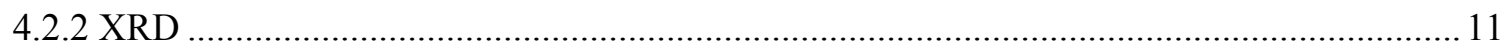

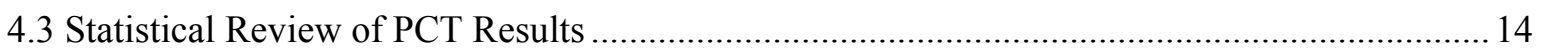

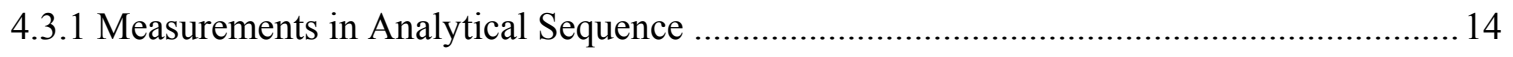

4.3.2 Results for the Samples of the Multi-Element Solution Standard ..................................... 15

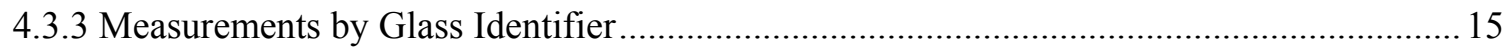

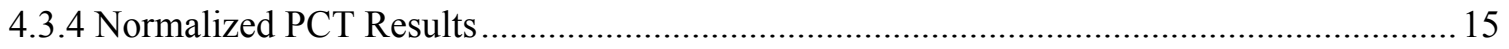

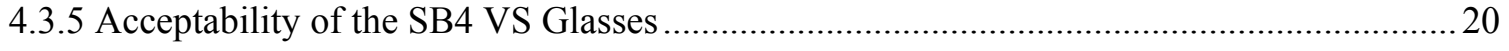

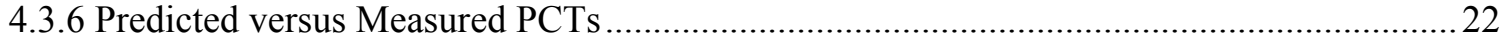

4.4 A Review of the Nepheline Assessments for DWPF's SB4 Process Batches ........................... 23

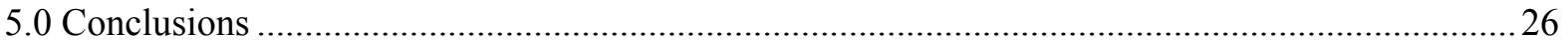

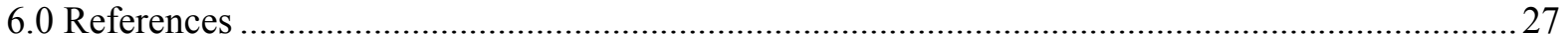




\section{LIST OF TABLES}

Table 1. Target Compositions of the SB4 Tank 40 Decant Variability Study Glasses......

Table 2. Results of MAR Assessment of SB4 VS Glasses for Measured, Bias-Corrected, and Targeted Compositional Views

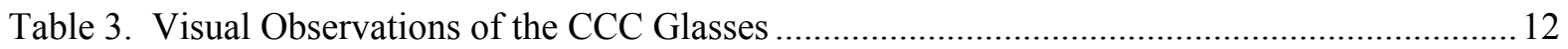

Table 4. Results from Samples of the Multi-Element Solution Standard ......................................... 15

Table 5. Normalized PCTs by Glass ID/Compositional View ....................................................... 17

Table 6. Nepheline Discriminator Values Recorded by DWPF during the Processing of SB4 ..........23

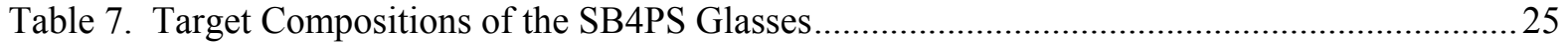

Table 8. Measured Compositions of the SB4PS Glasses ................................................................2

Table 9. Normalized* PCT Responses of the Frit 510 - SB4 Supplemental Glasses (quenched and ccc) 


\section{LIST OF FIGURES}

Figure 1. XRD pattern of the ccc version of the SB4VAR11 glass (Case \#1 at 34\% WL)................ 13

Figure 2. XRD pattern of the ccc version of the SB4VAR13 glass (Case \#1 at 42\% WL)................ 13

Figure 3. XRD pattern of the ccc version of the SB4VAR24 glass (Case \#2 at 41\% WL)............... 14

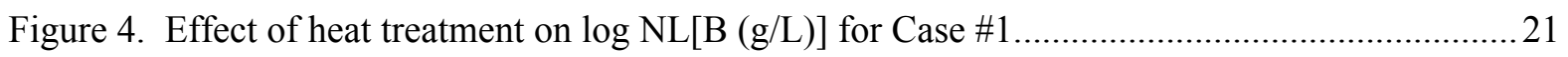

Figure 5. Log NL[B (g/L) versus B Del Gp model with a 95\% confidence interval for individual

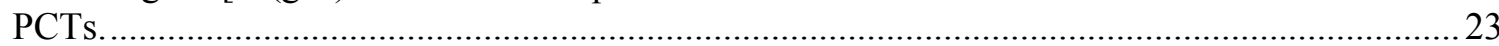




\section{LIST OF ABBREVIATIONS}

\begin{tabular}{|c|c|}
\hline $\mathrm{AD}$ & Analytical Development \\
\hline ANOVA & Analysis of Variance \\
\hline ARM & Approved Reference Material \\
\hline ARP & Actinide Removal Process \\
\hline B Del Gp & $\Delta \mathrm{Gp}$ value for boron \\
\hline $\mathrm{bc}$ & Bias-Corrected \\
\hline $\operatorname{ccc}$ & Centerline Canister Cooling \\
\hline $\mathrm{CPC}$ & Chemical Processing Cell \\
\hline DWPF & Defense Waste Processing Facility \\
\hline EA & Environmental Assessment \\
\hline ICP-AES & Inductively Coupled Plasma-Atomic Emission Spectroscopy \\
\hline ID & Identification \\
\hline LM & Lithium Metaborate \\
\hline LWO & Liquid Waste Organization \\
\hline MAR & Measure Acceptability Region \\
\hline $\mathrm{NL}[\mathrm{B}]$ & Normalized Boron Release \\
\hline $\mathrm{NL}[\mathrm{Li}]$ & Normalized Lithium Release \\
\hline $\mathrm{NL}[\mathrm{Na}]$ & Normalized Sodium Release \\
\hline $\mathrm{NL}[\mathrm{Si}]$ & Normalized Silicon Release \\
\hline PCCS & Product Composition Control System \\
\hline PCT & Product Consistency Test \\
\hline $\mathrm{PF}$ & Sodium Peroxide Fusion \\
\hline ppm & Parts per million \\
\hline PSAL & Process Science Analytical Laboratory \\
\hline SB4 & Sludge Batch 4 \\
\hline SME & Slurry Mix Evaporator \\
\hline SRAT & Sludge Receipt and Adjustment Tank \\
\hline SRNL & Savannah River National Laboratory \\
\hline $\mathrm{T}_{\mathrm{L}}$ Pred & Liquidus Temperature Prediction \\
\hline TTR & Technical Task Request \\
\hline $\mathrm{U}_{\text {std }}$ & Uranium Standard \\
\hline Visc Pred & Viscosity Prediction \\
\hline VS & Variability Study \\
\hline WL & Waste Loading \\
\hline XRD & $\mathrm{X}$-ray Diffraction \\
\hline
\end{tabular}


WSRC-STI-2008-00149

Revision 0

\subsection{Introduction}

Sludge Batch 4 (SB4) is currently being processed in the Defense Waste Processing Facility (DWPF) using Frit 510. The slurry pumps in Tank 40 are experiencing in-leakage of bearing water, which is causing the sludge slurry feed in Tank 40 to become dilute at a rapid rate. Currently, the DWPF is removing this dilution water by performing caustic boiling during the Sludge Receipt and Adjustment Tank (SRAT) cycle. In order to alleviate prolonged SRAT cycle times that may eventually impact canister production rates, decant scenarios of varying amounts of supernate have been proposed for Tank 40. The Savannah River National Laboratory (SRNL) has issued a preliminary assessment evaluating the possible downstream impacts of three (100, 150, and 200 kilogallon) decant scenarios on DWPF glass formulation and the Chemical Processing Cell (CPC). ${ }^{1}$ Based on the results of the preliminary assessment issued by the Savannah River National Laboratory (SRNL), the Liquid Waste Organization (LWO) issued a Technical Task Request (TTR) for SRNL to (i) perform a more detailed evaluation using updated SB4 compositional information and (ii) assess the viability of Frit 510 and determine any potential impacts on the SB4 system. ${ }^{2}$ As defined in the TTR, LWO requested that SRNL validate the sludge-only SB4 flowsheet and the coupled operations flowsheet using the 100K gallon decant volume as well as the addition of $3 \mathrm{wt} \%$ sodium on a calcined oxide basis.

The following five nominal SB4 sludge compositions were identified to support this study ${ }^{\mathrm{a}, 3}$ :

Case \#1: SB4 Nominal

Case \#2: SB4 after the 100K gallon decant

Case \#3: SB4 after the $100 \mathrm{~K}$ gallon decant with a $3 \mathrm{wt} \% \mathrm{Na}_{2} \mathrm{O}$ addition

Case \#4: SB4 after the 100K gallon decant and with addition of Actinide Removal Process (ARP) $\mathrm{J}$

Case \#5: SB4 after the 100K gallon decant with the addition of $3 \mathrm{wt} \% \mathrm{Na}_{2} \mathrm{O}$ and ARP $\mathrm{J}$

These potential SB4 flowsheet modifications (i.e., $100 \mathrm{~K}$ gallon decant, potential addition of $\mathrm{Na}_{2} \mathrm{O}$, and/or transitioning to a coupled operations flowsheet) resulted in compositional shifts in the SB4 system as described in WSRC-STI-2008-00017. ${ }^{3}$ Nominal sludge compositions (i.e. without any variation in the concentration of individual components) for each case were coupled with the nominal Frit 510 composition over a waste loading (WL) interval of $25-50 \%$ in order to define the glass regions of interest. The projected operating windows determined in the assessment suggested that Frit 510 remains a viable option for processing SB4 following the Tank 40 decant based on either a sludge-only or coupled operations flowsheet. In addition, the $\mathrm{Na}_{2} \mathrm{O}$ addition was also determined to be viable if melt rate was reduced due to the decrease in $\mathrm{Na}_{2} \mathrm{O}$ content after the decant. With respect to the SB4 variability study, approximately 12 historical glasses were identified during a search of the ComPro $^{\mathrm{TM}}$ database that are located within at least one of the five glass regions defined by the proposed SB4 flowsheet options. ${ }^{4}$ While these glasses meet the requirements of a variability study there was some concern that the compositional coverage did not adequately bound all cases. Therefore, SRNL recommended that a supplemental, experimental variability study be performed to support the various SB4 flowsheet options that may be implemented for future SB4 operations in DWPF. Eighteen glasses were selected based on nominal sludge projections representing the current as well as the proposed flowsheets over a WL interval of interest to DWPF (32-42\%). The glass selection strategy used to define these glass compositions is described in detail in WSRC-STI-2008-

\footnotetext{
${ }^{a}$ Compositional projections of SB4 with the $100 \mathrm{~K}$ gallon decant and SB4 with the $100 \mathrm{~K}$ gallon decant and a $3 \mathrm{wt}^{\mathrm{a}} \mathrm{Na} \mathrm{O}_{2} \mathrm{O}$ addition were provided by LWO, while SRNL subsequently added the ARP/MCU stream to these compositions to assess the scenarios. Compositional information for ARP additions was obtained from X-CLC-S-00113, Rev. 0, Actinide Removal Process Material Balance Calculation with Low Curie Salt Feed, S.G. Subosits, 9/24/2004. Details of these compositions used in this assessment are provided in WSRC-STI-2008-00017.
} 
WSRC-STI-2008-00149

Revision 0

00017. ${ }^{3}$ These eighteen glasses were fabricated and characterized using chemical composition analysis, X-ray Diffraction (XRD) and the Product Consistency Test (PCT).

\subsection{Objective}

The intent of the experimental portion of the variability study is to demonstrate that the glasses of the Frit 510-modified SB4 compositional region (Cases \#1-5) are both acceptable relative to the Environmental Assessment (EA) reference glass and predictable by the current process control models for durability. ${ }^{5-7}$

\subsection{Experimental Procedure}

\subsection{Target Glass Compositions}

Target glass compositions of the eighteen SB4 Tank 40 decant variability study glasses are presented in Table 1. The glass identification (ID) represents both the case number (see Section 1.0) and the waste loading. For the glass SB4VAR11, "SB4VAR" refers to the SB4 Variability Study, "1" refers to Case \#1 (SB4 Nominal) and "1" refers to the first or lowest WL (34\%), whereas SB4VAR54 represents Case \#5 (SB4 after the $100 \mathrm{~K}$ gallon decant with the addition of $3 \mathrm{wt} \% \mathrm{Na}_{2} \mathrm{O}$ and $\mathrm{ARP} J$ ) at the highest WL (41\%), and so on.

\subsection{Glass Fabrication}

Each variability study glass was prepared from the proper proportions of reagent-grade metal oxides, carbonates, $\mathrm{H}_{3} \mathrm{BO}_{3}$, and salts in $150 \mathrm{~g}$ batches. ${ }^{8}$ The raw materials were thoroughly mixed and placed into a $95 \%$ platinum / $5 \%$ gold, $250 \mathrm{ml}$ crucible. Batched materials were placed into a hightemperature furnace at the target melt temperature of $1150^{\circ} \mathrm{C} .{ }^{9}$ The crucible was removed from the furnace after an isothermal hold at $1150^{\circ} \mathrm{C}$ for 1 hour. The molten glass was quenched by pouring the liquid onto a clean, stainless steel plate. The glass pour patty was used as a sampling stock for the various property measurements (i.e., chemical composition, durability testing and XRD).

Approximately $25 \mathrm{~g}$ of each glass was heat-treated to simulate cooling along the centerline of a DWPF-type canister to gauge the effects of thermal history on the product performance. ${ }^{7}$ This cooling schedule is referred to as the centerline canister cooling (ccc) curve.

\subsection{Property Measurements}

\subsubsection{Compositional Analysis}

To confirm that the as-fabricated glasses met the target compositions, a representative sample from each glass was submitted to the SRNL Process Science Analytical Laboratory (PSAL) for chemical analysis under the auspices of an analytical plan (SRNL-SCS-2008-00003). ${ }^{10}$ Two dissolution techniques were utilized by PSAL, sodium peroxide fusion (PF) and lithium-metaborate (LM). Samples prepared by LM were used to measure silver (Ag), aluminum (Al), barium (Ba), calcium $(\mathrm{Ca})$, cadmium $(\mathrm{Cd})$, cerium $(\mathrm{Ce})$, cobalt $(\mathrm{Co})$, chromium $(\mathrm{Cr})$, copper $(\mathrm{Cu})$, iron $(\mathrm{Fe})$, potassium $(\mathrm{K})$, lanthanum $(\mathrm{La})$, magnesium $(\mathrm{Mg})$, manganese $(\mathrm{Mn})$, sodium $(\mathrm{Na})$, niobium $(\mathrm{Nb})$, nickel $(\mathrm{Ni})$, phosphorous $(\mathrm{P})$, lead $(\mathrm{Pb})$, sulfur $(\mathrm{S})$, strontium $(\mathrm{Sr})$, thorium $(\mathrm{Th})$, titanium $(\mathrm{Ti})$, uranium $(\mathrm{U})$, zinc $(\mathrm{Zn})$, and zirconium $(\mathrm{Zr})$ concentrations. Samples prepared by PF were used to measure boron (B), lithium ( $\mathrm{Li}$ ) and silicon ( $\mathrm{Si}$ ) concentrations. Each glass was prepared in duplicate for each cation 
dissolution technique (PF and LM). It should be noted that several of the elements in this list (e.g., $\mathrm{Ag}, \mathrm{Cd}, \mathrm{Co}, \mathrm{Nb}$, and $\mathrm{Th}$ ) are not present in these glasses or are present at very small concentrations. For each study glass, measurements were obtained from samples prepared in duplicate by each of these dissolution methods. All of the prepared samples were analyzed (twice for each element of interest) by Inductively Coupled Plasma-Atomic Emission Spectroscopy (ICP-AES) with the instrumentation being re-calibrated between the duplicate analyses. The analytical plan was developed in such a way as to provide the opportunity to evaluate potential sources of bias and error. Glass standards were also intermittently measured to assess the performance of the ICP-AES instrument over the course of these analyses.

\subsubsection{PCT}

A 7-day PCT was performed in triplicate on each quenched and ccc glass to assess chemical durability using Method A of the PCT procedure (ASTM C1285-97). ${ }^{11}$ Also included in the experimental test matrix was the EA glass, the Approved Reference Material (ARM) glass, and blanks from the sample cleaning batch. Samples were ground, washed, and prepared according to the standard procedure. ${ }^{11,12}$ The resulting solutions were sampled (filtered and acidified) and analyzed by PSAL under the auspices of an analytical plan (SRNL-SCS-2008-00050). ${ }^{13}$ Samples of a multielement, standard solution were also included in the analytical plan (as a check on the accuracy of the ICP-AES). Normalized release rates were calculated based on target, measured, and bias-corrected (bc) compositions using the average of the logs of the leachate concentrations.

\subsubsection{XRD}

Representative samples of quenched and ccc glasses were submitted to SRNL Analytical Development (AD) for XRD analysis. Samples were analyzed under conditions providing a detection limit of approximately $0.5 \mathrm{vol} \%$, i.e. no crystals can be detected if the amount in the sample is less than $\sim 0.5 \mathrm{vol} \%$. 
Table 1. Target Compositions of the SB4 Tank 40 Decant Variability Study Glasses

\begin{tabular}{|c|c|c|c|c|c|c|c|c|c|c|c|c|c|c|c|c|c|c|}
\hline \multirow[b]{2}{*}{ Glass ID } & \multicolumn{3}{|c|}{ Case \#1 } & \multicolumn{4}{|c|}{ Case \#2 } & \multicolumn{3}{|c|}{ Case \#3 } & \multicolumn{4}{|c|}{ Case \#4 } & \multicolumn{4}{|c|}{ Case \#5 } \\
\hline & $\begin{array}{l}\vec{z} \\
\text { 妾 } \\
\text { के }\end{array}$ & 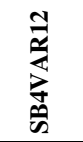 & $\begin{array}{l}\frac{m}{\underline{z}} \\
\frac{1}{d} \\
\text { के }\end{array}$ & 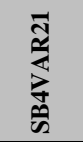 & 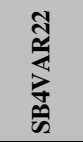 & 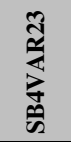 & 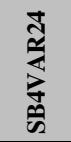 & 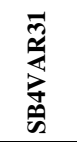 & 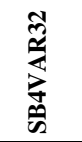 & 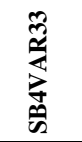 & 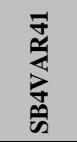 & 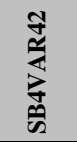 & 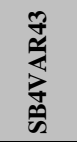 & 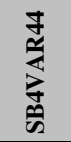 & 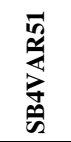 & 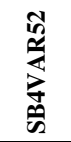 & 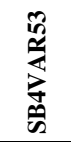 & 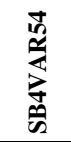 \\
\hline Frit & 510 & 510 & 510 & 510 & 510 & 510 & 510 & 510 & 510 & 510 & 510 & 510 & 510 & 510 & 510 & 510 & 510 & 510 \\
\hline WL & 34 & 38 & 42 & 32 & 35 & 38 & 41 & 32 & 36 & 40 & 32 & 35 & 38 & 41 & 32 & 35 & 38 & 41 \\
\hline$\overline{\mathbf{A l}_{2} \mathbf{O}_{3}}$ & 8.46 & 9.46 & 10.46 & 8.31 & 9.09 & 9.86 & 10.64 & 8.00 & 9.01 & 10.01 & 8.00 & 8.75 & 9.50 & 10.25 & 7.71 & 8.43 & 9.15 & 9.88 \\
\hline$\overline{\mathrm{B}_{2} \mathrm{O}_{3}}$ & 9.24 & 8.68 & 8.12 & 9.52 & 9.10 & 8.68 & 8.26 & 9.52 & 8.96 & 8.40 & 9.52 & 9.10 & 8.68 & 8.26 & 9.52 & 9.10 & 8.68 & 8.26 \\
\hline$\overline{\mathrm{BaO}}$ & 0.03 & 0.03 & 0.03 & 0.02 & 0.03 & 0.03 & 0.03 & 0.02 & 0.03 & 0.03 & 0.02 & 0.03 & 0.03 & 0.03 & 0.02 & 0.03 & 0.03 & 0.03 \\
\hline $\begin{array}{l}\mathrm{CaO} \\
\end{array}$ & 0.93 & 1.04 & 1.15 & 0.91 & 1.00 & 1.09 & 1.17 & 0.88 & 0.99 & 1.10 & 0.88 & 0.97 & 1.05 & 1.13 & 0.85 & 0.93 & 1.01 & 1.09 \\
\hline $\mathrm{Ce}_{2} \mathrm{O}_{3}$ & 0.02 & 0.02 & 0.03 & 0.02 & 0.02 & 0.03 & 0.03 & 0.02 & 0.02 & 0.03 & 0.02 & 0.03 & 0.03 & 0.03 & 0.02 & 0.02 & 0.03 & 0.03 \\
\hline $\mathrm{Cr}_{2} \mathbf{O}_{3}$ & 0.05 & 0.06 & 0.07 & 0.05 & 0.06 & 0.06 & 0.07 & 0.05 & 0.06 & 0.06 & 0.05 & 0.06 & 0.06 & 0.07 & 0.05 & 0.05 & 0.06 & 0.06 \\
\hline $\mathrm{CuO}$ & 0.02 & 0.02 & 0.02 & 0.02 & 0.02 & 0.02 & 0.02 & 0.02 & 0.02 & 0.02 & 0.02 & 0.02 & 0.02 & 0.02 & 0.02 & 0.02 & 0.02 & 0.02 \\
\hline $\mathrm{Fe}_{2} \mathrm{O}_{3}$ & 9.62 & 10.75 & 11.88 & 9.47 & 10.36 & 11.25 & 12.14 & 9.13 & 10.27 & 11.41 & 9.20 & 10.06 & 10.92 & 11.79 & 8.87 & 9.70 & 10.53 & 11.37 \\
\hline$\overline{\mathrm{K}_{2} \mathrm{O}}$ & 0.12 & 0.13 & 0.15 & 0.12 & 0.13 & 0.14 & 0.15 & 0.11 & 0.13 & 0.14 & 0.11 & 0.12 & 0.13 & 0.15 & 0.11 & 0.12 & 0.13 & 0.14 \\
\hline $\mathrm{La}_{2} \mathrm{O}_{3}$ & 0.02 & 0.02 & 0.02 & 0.02 & 0.02 & 0.02 & 0.02 & 0.02 & 0.02 & 0.02 & 0.02 & 0.02 & 0.02 & 0.02 & 0.02 & 0.02 & 0.02 & 0.02 \\
\hline $\mathrm{Li}_{2} \mathrm{O}$ & 5.28 & 4.96 & 4.64 & 5.44 & 5.20 & 4.96 & 4.72 & 5.44 & 5.12 & 4.80 & 5.44 & 5.20 & 4.96 & 4.72 & 5.44 & 5.20 & 4.96 & 4.72 \\
\hline MgO & 0.91 & 1.02 & 1.13 & 0.90 & 0.98 & 1.07 & 1.15 & 0.87 & 0.97 & 1.08 & 0.86 & 0.94 & 1.02 & 1.10 & 0.83 & 0.90 & 0.98 & 1.06 \\
\hline MnO & 1.93 & 2.16 & 2.39 & 1.90 & 2.08 & 2.26 & 2.44 & 1.83 & 2.06 & 2.29 & 1.88 & 2.06 & 2.23 & 2.41 & 1.82 & 1.99 & 2.16 & 2.33 \\
\hline$\overline{\mathrm{Na}_{2} \mathrm{O}}$ & 12.26 & 12.76 & 13.26 & 10.98 & 11.26 & 11.54 & 11.82 & 11.93 & 12.42 & 12.92 & 11.34 & 11.66 & 11.97 & 12.28 & 12.25 & 12.65 & 13.04 & 13.44 \\
\hline $\mathrm{NiO}$ & 0.54 & 0.60 & 0.66 & 0.53 & 0.58 & 0.63 & 0.68 & 0.51 & 0.57 & 0.63 & 0.52 & 0.57 & 0.62 & 0.67 & 0.50 & 0.55 & 0.60 & 0.65 \\
\hline$\overline{\text { PbO }}$ & 0.02 & 0.02 & 0.03 & 0.02 & 0.02 & 0.02 & 0.03 & 0.02 & 0.02 & 0.03 & 0.02 & 0.02 & 0.03 & 0.03 & 0.02 & 0.02 & 0.02 & 0.03 \\
\hline $\mathrm{SO}_{4}$ & 0.45 & 0.50 & 0.55 & 0.32 & 0.35 & 0.38 & 0.41 & 0.32 & 0.36 & 0.40 & 0.35 & 0.38 & 0.41 & 0.45 & 0.35 & 0.38 & 0.41 & 0.45 \\
\hline $\mathrm{SiO}_{2}$ & 47.10 & 44.41 & 41.72 & 48.49 & 46.47 & 44.46 & 42.44 & 48.46 & 45.77 & 43.07 & 48.46 & 46.44 & 44.42 & 42.40 & 48.43 & 46.40 & 44.38 & 42.36 \\
\hline $\mathrm{TiO}_{2}$ & 0.01 & 0.01 & 0.02 & 0.01 & 0.01 & 0.02 & 0.02 & 0.01 & 0.01 & 0.02 & 0.43 & 0.47 & 0.51 & 0.55 & 0.43 & 0.47 & 0.51 & 0.55 \\
\hline$\overline{\mathbf{U}_{3} \mathbf{O}_{8}}$ & 2.94 & 3.28 & 3.63 & 2.89 & 3.16 & 3.43 & 3.70 & 2.79 & 3.13 & 3.48 & 2.80 & 3.06 & 3.32 & 3.59 & 2.70 & 2.95 & 3.21 & 3.46 \\
\hline ZnO & 0.03 & 0.03 & 0.03 & 0.03 & 0.03 & 0.03 & 0.03 & 0.02 & 0.03 & 0.03 & 0.03 & 0.03 & 0.03 & 0.03 & 0.02 & 0.03 & 0.03 & 0.03 \\
\hline$\overline{\mathrm{ZrO}_{2}}$ & 0.03 & 0.03 & 0.03 & 0.03 & 0.03 & 0.03 & 0.03 & 0.03 & 0.03 & 0.03 & 0.03 & 0.03 & 0.03 & 0.04 & 0.03 & 0.03 & 0.03 & 0.04 \\
\hline
\end{tabular}


WSRC-STI-2008-00149

Revision 0

\subsection{Results and Discussion}

\subsection{Statistical Review of Chemical Composition Measurements}

Table A1 in Appendix A provides the elemental concentration measurements derived from the samples prepared using LM, and Table A3 in Appendix A provides the measurements derived from the samples prepared using PF. Measurements for standards (Batch 1 and a uranium standard, $U_{\text {std }}$ ) that were included in the PSAL analytical plan along with the study glasses are also provided in these two tables.

The elemental concentrations were converted to oxide concentrations by multiplying the values for each element by the gravimetric factor for the corresponding oxide (Table A2 and Table A4). During this process, an elemental concentration that was determined to be below the detection limit of the analytical procedures used by the PSAL was reduced to half of that detection limit as the oxide concentration was determined.

In the following sections, the analytical sequences of the measurements are explored, the measurements of the standards are investigated and used for bias correction, the measurements for each glass are reviewed, the average chemical compositions (measured and bias-corrected) for each glass are determined, and comparisons are made between the measurements and the targeted compositions for the glasses.

\subsubsection{Measurements in Analytical Sequence}

Exhibit A1 in Appendix A provides plots of the measurements generated by the PSAL for samples prepared using the LM method. The plots are in analytical sequence with different symbols and colors being used to represent each of the study and standard glasses. Similar plots are provided in Exhibit A2 in Appendix A for the samples prepared using the PF method. These plots include all of the measurement data from Tables A1 and A3. While looking for obvious patterns in these plots is difficult, a relatively small value for $\mathrm{NiO}(0.078 \mathrm{wt} \%)$ for one of the uranium standard glasses $\left(\mathrm{U}_{\text {std }} \mathrm{LM} 2-122\right)$ does stand out. Otherwise, a review of these plots indicates no significant patterns or trends in the analytical process over the course of these measurements. Better opportunities for such reviews are provided in the discussions that follow.

\subsubsection{Batch 1 and Uranium Standard Results}

In this section, the PSAL measurements of the chemical compositions of the Batch 1 and uranium standard $\left(\mathrm{U}_{\text {std }}\right)$ glasses are reviewed. These measurements are investigated across the ICP analytical blocks, and the results are used to bias correct the measurements for the study glasses.

Exhibit A3 in Appendix A provides statistical analyses of the Batch 1 and $\mathrm{U}_{\text {std }}$ results generated by the LM prep method by analytical set and calibration block/sub-block for each oxide of interest. The results include analysis of variance (ANOVA) investigations looking for statistically significant differences between the means of these groups for each of the oxides for each of the standards. The results from the statistical tests for the Batch 1 standard may be summarized as follows: the $\mathrm{Al}_{2} \mathrm{O}_{3}$, $\mathrm{CaO}, \mathrm{Ce}_{2} \mathrm{O}_{3}$ (a detection limit effect), $\mathrm{Cr}_{2} \mathrm{O}_{3}, \mathrm{CuO}, \mathrm{Fe}_{2} \mathrm{O}_{3}, \mathrm{~K}_{2} \mathrm{O}, \mathrm{MgO}, \mathrm{Na}_{2} \mathrm{O}, \mathrm{NiO}, \mathrm{TiO}_{2}$, and $\mathrm{ZrO}_{2}$ measurements indicate a significant ICP calibration effect on the block averages at the 5\% significance level. For the $\mathrm{U}_{\text {std }}$, the $\mathrm{Al}_{2} \mathrm{O}_{3}, \mathrm{CaO}, \mathrm{Ce}_{2} \mathrm{O}_{3}$ (a detection limit effect), $\mathrm{Cr}_{2} \mathrm{O}_{3}, \mathrm{Fe}_{2} \mathrm{O}_{3}, \mathrm{~K}_{2} \mathrm{O}$, $\mathrm{MgO}, \mathrm{Na}_{2} \mathrm{O}, \mathrm{SiO}_{2}, \mathrm{TiO}_{2}$, and $\mathrm{U}_{3} \mathrm{O}_{8}$ measurements indicate a significant ICP calibration effect on the 
block averages at the 5\% significance level. The reference values for the oxide concentrations of the standards are given in the header for each set of measurements in the exhibit.

Exhibit A4 in Appendix A provides a similar set of analyses for the measurements derived from samples prepared via the PF method. The results from the statistical tests for the Batch 1 standard may be summarized as follows: the $\mathrm{Li}_{2} \mathrm{O}$ measurements indicate significant ICP calibration effects on the block averages at the $5 \%$ significance level. For the $\mathrm{U}_{\text {std }}$, the $\mathrm{Li}_{2} \mathrm{O}$ measurements indicate significant ICP calibration effects on the block averages at the 5\% significance level. The reference values for the oxide concentrations of the standards are given in the header for each set of measurements in the exhibit.

Some of these results provide incentive for adjusting the measurements by the effects of the ICP calibration. Therefore, the oxide measurements of the study glasses are bias corrected for the effect of the ICP calibration on each of the analytical blocks and sub-blocks. The basis for the bias correction is presented as part of Exhibits $\mathrm{A} 3$ and $\mathrm{A} 4$ - the average measurement for Batch 1 for each ICP block/sub-block for $\mathrm{Al}_{2} \mathrm{O}_{3}, \mathrm{~B}_{2} \mathrm{O}_{3}, \mathrm{BaO}, \mathrm{CaO}, \mathrm{Cr}_{2} \mathrm{O}_{3}, \mathrm{CuO}, \mathrm{Fe}_{2} \mathrm{O}_{3}, \mathrm{Li}_{2} \mathrm{O}, \mathrm{MgO}, \mathrm{MnO}, \mathrm{Na}_{2} \mathrm{O}, \mathrm{NiO}$, $\mathrm{SiO}_{2}$, and $\mathrm{TiO}_{2}$ and the average measurement for $\mathrm{U}_{\text {std }}$ for each ICP set/block for $\mathrm{U}_{3} \mathrm{O}_{8}$. Thus, the Batch 1 results serve as the basis for bias correcting all of the selected oxides except uranium. The $\mathrm{U}_{\text {std }}$ results were used to bias correct for uranium. The Batch 1 results were used to conduct the bias correction as long as the reference value for the oxide concentration in the Batch 1 glass was greater than or equal to $0.1 \mathrm{wt} \%$. Thus, applying this approach and based upon the information in the exhibits, the Batch 1 results were used to bias correct the $\mathrm{Al}_{2} \mathrm{O}_{3}, \mathrm{~B}_{2} \mathrm{O}_{3}, \mathrm{BaO}, \mathrm{CaO}, \mathrm{Cr}_{2} \mathrm{O}_{3}, \mathrm{CuO}, \mathrm{Fe}_{2} \mathrm{O}_{3}$, $\mathrm{K}_{2} \mathrm{O}, \mathrm{Li}_{2} \mathrm{O}, \mathrm{MgO}, \mathrm{MnO}, \mathrm{Na}_{2} \mathrm{O}, \mathrm{NiO}, \mathrm{SiO}_{2}$, and $\mathrm{TiO}_{2}$ measurements. No bias correction was conducted for $\mathrm{Ce}_{2} \mathrm{O}_{3}, \mathrm{La}_{2} \mathrm{O}_{3}, \mathrm{PbO}, \mathrm{SO}_{4}, \mathrm{ThO}_{2}, \mathrm{ZnO}$, or $\mathrm{ZrO}_{2}$.

The bias correction was conducted as follows. For each oxide, let $\overline{\mathrm{a}}_{\mathrm{ij}}$ be the average measurement for the $\mathrm{i}^{\text {th }}$ oxide at analytical block $\mathrm{j}$ for Batch 1 (or $\mathrm{U}_{\text {std }}$ for uranium), and let $\mathrm{t}_{\mathrm{i}}$ be the reference value for the $\mathrm{i}^{\text {th }}$ oxide for Batch 1 (or for $\mathrm{U}_{\text {std }}$ if uranium). (The averages and reference values are provided in Exhibits A3 and A4.) Let $\bar{c}_{i j k}$ be the average measurement for the $i^{\text {th }}$ oxide at analytical block $j$ for the $\mathrm{k}^{\text {th }}$ glass. The bias adjustment was conducted as follows

$$
\bar{c}_{i j k} \bullet\left(1-\frac{\bar{a}_{i j}-t_{i}}{\bar{a}_{i j}}\right)=\bar{c}_{i j k} \bullet \frac{t_{i}}{\bar{a}_{i j}}
$$

Bias-corrected measurements are indicated by a "bc" suffix, and such adjustments were performed for all of the oxides of this study except for $\mathrm{Ce}_{2} \mathrm{O}_{3}, \mathrm{La}_{2} \mathrm{O}_{3}, \mathrm{PbO}, \mathrm{SO}_{4}, \mathrm{ThO}_{2}, \mathrm{ZnO}$, and $\mathrm{ZrO}_{2}$. Both measured and measured "bc" values are included in the discussion that follows. Since no bias correction was performed on $\mathrm{Ce}_{2} \mathrm{O}_{3}, \mathrm{La}_{2} \mathrm{O}_{3}, \mathrm{PbO}, \mathrm{SO}_{4}, \mathrm{ThO}_{2}, \mathrm{ZnO}$, and $\mathrm{ZrO}_{2}$, the original values are included for completeness (e.g., to allow a sum of oxides to be computed for the bias-corrected results).

\subsubsection{Composition Measurements by Glass Identifier}

Exhibits A5 and A6 in Appendix A provide plots of the oxide concentration measurements by Glass ID (including Batch 1 and $\mathrm{U}_{\text {std }}$ ) by analytical solution ID for the LM and PF preparation methods, respectively, for each set of analyses. Different symbols and colors are used to represent the different glasses. These plots show the individual measurements across the duplicates of each preparation 
WSRC-STI-2008-00149

Revision 0

method and the two ICP calibrations within each analytical set. A review of the plots presented in these exhibits reveals the repeatability of the four individual, oxide values for each glass. $\mathrm{The} \mathrm{Al}_{2} \mathrm{O}_{3}$ results in set 1 for SB4VAR23 show differences that appear to be due to a dissolution effect for this glass. Although $\mathrm{Al}_{2} \mathrm{O}_{3}$ differences are observed, all of the data were used to compute measured and measured bias corrected compositional views. A considerable amount of scatter is observed in the $\mathrm{Fe}_{2} \mathrm{O}_{3}$ results for both sets of analyses. These issues will not have any impact on the conclusions of this report.

\subsubsection{Measured versus Targeted Compositions}

The four measurements for each oxide of each glass (over both preparation methods) were averaged to determine a representative chemical composition for each glass. These determinations were conducted both for the measured and for the bias-corrected data. A sum of oxides was also computed for each glass based upon both the measured and bias-corrected values. Exhibit A7 in Appendix A provides plots showing results for each glass for each oxide to help highlight the comparisons among the measured, bias-corrected, and targeted values.

Some observations from the plots of Exhibit A7 are offered: In general, there were some difficulties in reaching the targeted concentrations for $\mathrm{Fe}_{2} \mathrm{O}_{3}$ (measured values generally lower than targeted) and $\mathrm{SiO}_{2}$ (measured values generally higher than targeted). In addition, the $\mathrm{SO}_{4}$ concentration for SB4VAR21 appears to be low (53\% below its targeted value). Table A4 in Appendix A provides a summary of the average compositions as well as the targeted compositions and some associated differences and relative differences. Notice that the targeted sums of oxides for the standard glasses are not $100 \%$ due to incomplete coverage of the oxides in the Batch 1 and $\mathrm{U}_{\text {std }}$ glasses. All of the oxide sums (both measured and bias-corrected) for the study glasses fall within the interval of 95 to $105 \mathrm{wt} \%$. Entries in Table A4 show the relative differences between the measured or bias-corrected values and the targeted values. These differences are shaded when they are greater than or equal to $5 \%$. Overall, the comparisons between the measured and targeted compositions suggest only minor difficulties in reaching the targeted compositions for some of the oxides for some of the glasses.

\subsubsection{MAR Assessment of the SB4/Decant Variability Study (VS) Glasses}

Another assessment that can be made for these SB4 VS glasses is how well they satisfy the Measurement Acceptability Region (MAR) criteria of DWPF's Product Composition Control System (PCCS). All of the glasses that were selected for this study satisfied these criteria. That is, the targeted composition for each of these glasses fell within the MAR of PCCS as described in WSRCSTI-2008-00017. ${ }^{3}$ In this section, the results of the MAR assessment of the measured and measured bias-corrected compositions for these glasses are presented along with the results for the targeted compositions.

Table 2 provides the percent waste loading ( $\% \mathrm{WL})$, the frit, the glass identifier with compositional view, the B Del Gp Value ( $\Delta \mathrm{Gp}$ value for boron), the normalized leachate for boron in grams/Liter $(\mathrm{NL}[\mathrm{B}(\mathrm{g} / \mathrm{L})])$, the liquidus temperature prediction in degrees Celsius $\left(\mathrm{T}_{\mathrm{L}}\right.$ Pred $\left.\left({ }^{\circ} \mathrm{C}\right)\right)$, the viscosity prediction in Poise (Visc Pred (P)), the sum of oxides (in wt\%), the $\mathrm{Al}_{2} \mathrm{O}_{3}$ concentration (in wt\%), the $\mathrm{Na}_{2} \mathrm{SO}_{4}$ concentration (in $\mathrm{wt} \%$ ), and the total alkali concentration $\left(\mathrm{R}_{2} \mathrm{O}\right)$ as a $\mathrm{wt} \%$, the nepheline value, and the overall MAR assessment. ${ }^{\mathrm{b}}$ With the exception of SB4VAR54 (target and measured bc), all of the MAR results for these glass compositional views indicate that they would pass the PCCS

\footnotetext{
${ }^{\mathrm{b}}$ Although the PCCS limit of $0.88 \mathrm{wt} \% \mathrm{Na}_{2} \mathrm{SO}_{4}$ was not used during the MAR assessment, both the projected and measured $\mathrm{Na}_{2} \mathrm{SO}_{4}$ concentrations for all SB4 cases meet this criterion.
} 
MAR and be both processable and acceptable by the DWPF. As for the SB4VAR54 glass, it was noted during the glass selection process that the targeted composition failed the low viscosity criterion. ${ }^{3}$ The measured composition has a slightly higher predicted viscosity (27.2 Poise) as compared to both the target and measured bias corrected compositional views of 24.4 and 24.6 Poise respectively, which both fail the low viscosity constraint. 
Table 2. Results of MAR Assessment of SB4 VS Glasses for Measured, Bias-Corrected, and Targeted Compositional Views

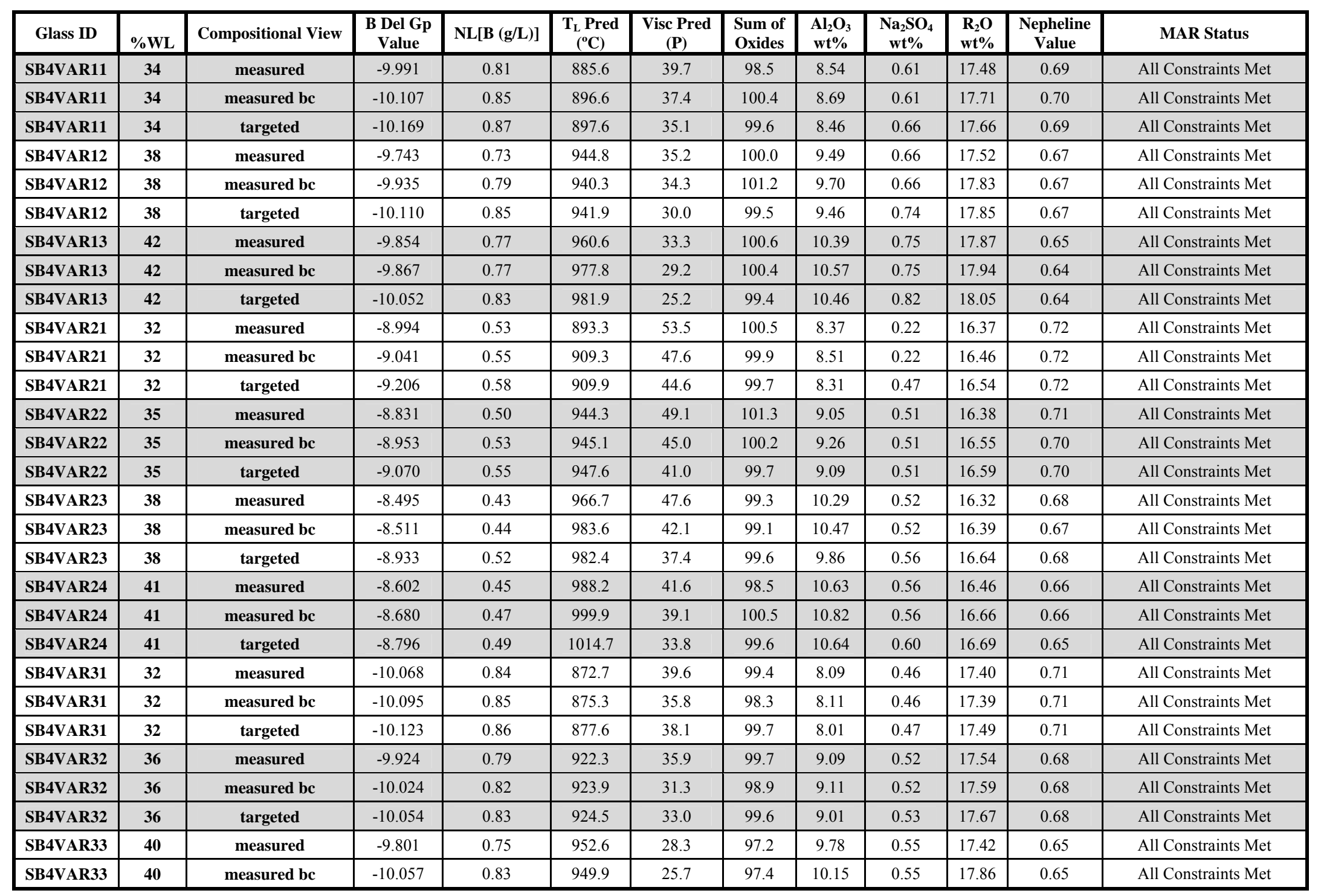


Table 2 cont. Results of MAR Assessment of SB4 VS Glasses for Measured, Bias-Corrected, and Targeted Compositional Views

\begin{tabular}{|c|c|c|c|c|c|c|c|c|c|c|c|c|}
\hline Glass ID & $\% \mathrm{WL}$ & Compositional View & $\begin{array}{c}\text { B Del Gp } \\
\text { Value }\end{array}$ & $\mathrm{NL}[\mathrm{B}(\mathrm{g} / \mathrm{L})]$ & $\begin{array}{c}T_{L} \text { Pred } \\
\left({ }^{\circ} \mathrm{C}\right)\end{array}$ & $\begin{array}{c}\text { Visc Pred } \\
\text { (P) }\end{array}$ & $\begin{array}{l}\text { Sum of } \\
\text { Oxides }\end{array}$ & $\begin{array}{c}\mathrm{Al}_{2} \mathbf{O}_{3} \\
\mathrm{wt} \%\end{array}$ & $\begin{array}{c}\mathrm{Na}_{2} \mathrm{SO}_{4} \\
\mathrm{wt} \%\end{array}$ & $\begin{array}{l}\mathrm{R}_{2} \mathrm{O} \\
\mathrm{wt} \%\end{array}$ & \begin{tabular}{|c|c|}
$\begin{array}{c}\text { Nepheline } \\
\text { Value }\end{array}$ \\
\end{tabular} & MAR Status w/o Na2SO 4 \\
\hline SB4VAR33 & 40 & targeted & -9.986 & 0.81 & 967.1 & 28.0 & 99.6 & 10.01 & 0.59 & 17.86 & 0.65 & All Constraints Met \\
\hline SB4VAR41 & 32 & measured & -9.468 & 0.65 & 889.0 & 48.2 & 102.1 & 8.14 & 0.49 & 16.96 & 0.72 & All Constraints Met \\
\hline SB4VAR41 & 32 & targeted & -9.546 & 0.67 & 894.8 & 41.6 & 99.7 & 8.00 & 0.51 & 16.90 & 0.71 & All Constraints Met \\
\hline SB4VAR42 & 35 & measured & -9.094 & 0.56 & 932.5 & 43.9 & 100.0 & 8.68 & 0.52 & 16.59 & 0.70 & All Constraints Met \\
\hline SB4VAR42 & 35 & measured bc & -9.217 & 0.59 & 933.0 & 40.1 & 99.0 & 8.88 & 0.52 & 16.76 & 0.69 & All Constraints Met \\
\hline SB4VAR42 & 35 & targeted & -9.441 & 0.64 & 931.3 & 37.8 & 99.6 & 8.75 & 0.56 & 16.98 & 0.69 & All Constraints Met \\
\hline SB4VAR43 & 38 & measured & -8.936 & 0.52 & 969.1 & 40.3 & 100.4 & 9.41 & 0.59 & 16.65 & 0.68 & All Constraints Met \\
\hline SB4VAR43 & 38 & measured bc & -9.119 & 0.56 & 964.3 & 39.3 & 101.6 & 9.63 & 0.59 & 16.95 & 0.68 & All Constraints Met \\
\hline SB4VAR43 & 38 & targeted & -9.336 & 0.62 & 965.1 & 34.1 & 99.6 & 9.50 & 0.61 & 17.07 & 0.67 & All Constraints Met \\
\hline SB4VAR44 & 41 & measured & -9.033 & 0.54 & 970.0 & 38.6 & 98.5 & 10.26 & 0.61 & 16.90 & 0.66 & All Constraints Met \\
\hline SB4VAR44 & 41 & measured bc & -9.110 & 0.56 & 981.9 & 36.4 & 100.5 & 10.44 & 0.61 & 17.11 & 0.66 & All Constraints Met \\
\hline SB4VAR44 & 41 & targeted & -9.231 & 0.59 & 996.1 & 30.4 & 99.6 & 10.25 & 0.66 & 17.15 & 0.65 & All Constraints Met \\
\hline SB4VAR51 & 32 & measured bc & -10.432 & 0.97 & 856.6 & 37.2 & 100.2 & 7.96 & 0.49 & 17.91 & 0.71 & All Constraints Met \\
\hline SB4VAR51 & 32 & targeted & -10.417 & 0.97 & 864.4 & 35.8 & 99.7 & 7.71 & 0.51 & 17.80 & 0.71 & All Constraints Met \\
\hline SB4VAR52 & 35 & measured & -10.152 & 0.87 & 893.1 & 36.7 & 99.2 & 8.58 & 0.53 & 17.74 & 0.69 & All Constraints Met \\
\hline SB4VAR52 & 35 & measured bc & -10.261 & 0.91 & 904.3 & 34.6 & 101.1 & 8.73 & 0.53 & 17.97 & 0.69 & All Constraints Met \\
\hline SB4VAR52 & 35 & targeted & -10.393 & 0.96 & 899.2 & 31.8 & 99.6 & 8.43 & 0.56 & 17.96 & 0.69 & All Constraints Met \\
\hline SB4VAR53 & 38 & measured & -10.392 & 0.96 & 907.9 & 33.4 & 100.4 & 9.17 & 0.57 & 18.18 & 0.67 & All Constraints Met \\
\hline SB4VAR53 & 38 & measured bc & -10.414 & 0.97 & 924.3 & 29.4 & 100.1 & 9.33 & 0.57 & 18.25 & 0.67 & All Constraints Met \\
\hline SB4VAR53 & 38 & targeted & -10.371 & 0.95 & 931.3 & 28.0 & 99.6 & 9.16 & 0.61 & 18.13 & 0.67 & All Constraints Met \\
\hline SB4VAR54 & 41 & measured & -10.124 & 0.86 & 956.7 & 27.2 & 99.4 & 9.86 & 0.64 & 18.05 & 0.65 & All Constraints Met \\
\hline SB4VAR54 & 41 & measured bc & -10.238 & 0.90 & 957.3 & 24.6 & 98.5 & 10.09 & 0.64 & 18.23 & 0.64 & lvisc \\
\hline SB4VAR54 & 41 & targeted & -10.347 & 0.94 & 960.8 & 24.4 & 99.6 & 9.88 & 0.66 & 18.30 & 0.64 & lvisc \\
\hline
\end{tabular}


WSRC-STI-2008-00149

Revision 0

\subsection{Crystallization}

\subsubsection{Visual Observations}

Prior to discussing the visual observations, a brief explanation of the terms used to describe the asfabricated (quenched) and ccc glasses is necessary. "Surface" refers to the top of the sample that has not touched the steel plate during quenching or the walls of the crucible during the ccc treatment. The term "bulk" refers to the cross-section of the glass sample. "Homogeneous" indicates that there is no crystallization evident on the surface or in the bulk of the glass. Other terms such as "haze", "clusters", and/or "silver/metallic patches" imply that the surface or bulk of the glass contains crystals or some other characteristic feature. "Black and shiny" implies that crystallization is not apparent to the un-aided eye.

The surface and bulk of each as-fabricated (quenched) glass were "black and shiny," which indicates that the glasses are free of crystallization and homogeneous.

Crystallization is much more prevalent in the ccc glasses given that the kinetics for crystallization are more favorable during the slow cooling of the ccc treatment. Only three of the ccc glasses were determined to be homogeneous based on visual observations as shown in Table 3, which is not surprising as these glasses had a target WL of only $32 \%$. The remainder of the ccc glasses did contain some degree of crystallization on the surface, characterized by "haze" and "silver/metallic patches." Historically, metallic-like features on the surface of DWPF glasses are due to the precipitation of spinels during the slower cooling process. Crystallization was not present in the bulk of any glasses, except SB4VAR24 (Case \#2 at 41\% WL), which contained patches of silver/metallic crystals on the surface that extended $3 \mathrm{~mm}$ into the bulk.

\subsubsection{XRD}

Each of the quenched glasses is amorphous (within the detection limit of the instrument), which corresponds to the visual observations (Section 4.2.1). Two of the ccc samples contain crystalline material, while all others are amorphous (within the detection limit of the instrument). Both nepheline and trevorite were detected in samples SB4VAR13ccc (Case \#1 at 42\% WL) and SB4VAR24ccc (Case \#2 at $41 \% \mathrm{WL}$ ). A representative pattern ${ }^{c}$ of an amorphous sample is shown in Figure 1, while the XRD patterns of the glasses containing nepheline and trevorite are shown in Figure 2 and Figure 3. The presence of nepheline was not expected in SB4VAR13ccc or SB4VAR24ccc. The MAR assessments suggest that the nepheline discriminator values of these glasses are well above the 0.62 value currently being used to delineate compositions that are prone to nepheline formation from those that are not. These data are the initial results that suggest that the current nepheline discriminator and the use of the 0.62 values may not be conservative for specific compositional regions. As nepheline is present in these two glasses, a negative impact on the PCT response is anticipated.

\footnotetext{
${ }^{\mathrm{c}}$ The remainder of the XRD patterns (quenched and ccc) can be viewed in the laboratory notebook for the SB4 variability study on pages 116-118 (WSRC-NB-2006-00168).
} 
Table 3. Visual Observations of the CCC Glasses

\begin{tabular}{|c|c|c|c|c|c|}
\hline \multirow{2}{*}{ Glass ID } & \multirow{2}{*}{ Frit } & \multirow{2}{*}{ WL } & \multicolumn{2}{|l|}{ Visual Observations } & \multirow{2}{*}{ XRD } \\
\hline & & & Surface & Bulk & \\
\hline SB4VAR11 & \multirow{18}{*}{510} & 34 & Light haze & Clean & Amorphous \\
\hline SB4VAR12 & & 38 & Light/shiny haze, three silver/metallic patches of crystals & Clean & Amorphous \\
\hline SB4VAR13 & & 42 & Haze, large patches of silver/metallic crystals & Clean & Nepheline and Trevorite \\
\hline SB4VAR21 & & 32 & Very light crystals & Clean & Amorphous \\
\hline SB4VAR22 & & 35 & Silver haze with crystals & Clean & Amorphous \\
\hline SB4VAR23 & & 38 & Silver haze, several patches of silver/metallic patches & Clean & Amorphous \\
\hline SB4VAR24 & & 41 & Crystals, large patches of silver/metallic crystals & Crystal patches extend $1 / 8$ " into bulk & Nepheline and Trevorite \\
\hline SB4VAR31 & & 32 & Black and shiny & Clean & Amorphous \\
\hline SB4VAR32 & & 36 & Shiny, silver metallic haze & Clean & Amorphous \\
\hline SB4VAR33 & & 40 & Haze, several silver/metallic patches of crystals & Clean & Amorphous \\
\hline SB4VAR41 & & 32 & Black and shiny & Clean & Amorphous \\
\hline SB4VAR42 & & 35 & Silver haze, few patches of silver crystals & Clean & Amorphous \\
\hline SB4VAR43 & & 38 & Silver haze, few patches of silver crystals & Clean & Amorphous \\
\hline SB4VAR44 & & 41 & Silver haze, few patches of silver crystals & Clean & Amorphous \\
\hline SB4VAR51 & & 32 & Black and shiny & Clean & Amorphous \\
\hline SB4VAR52 & & 35 & Silver haze & Clean & Amorphous \\
\hline SB4VAR53 & & 38 & Silver haze, few patches of silver crystals & Clean & Amorphous \\
\hline SB4VAR54 & & 41 & Large patches of silver crystals & Clean & Amorphous \\
\hline
\end{tabular}




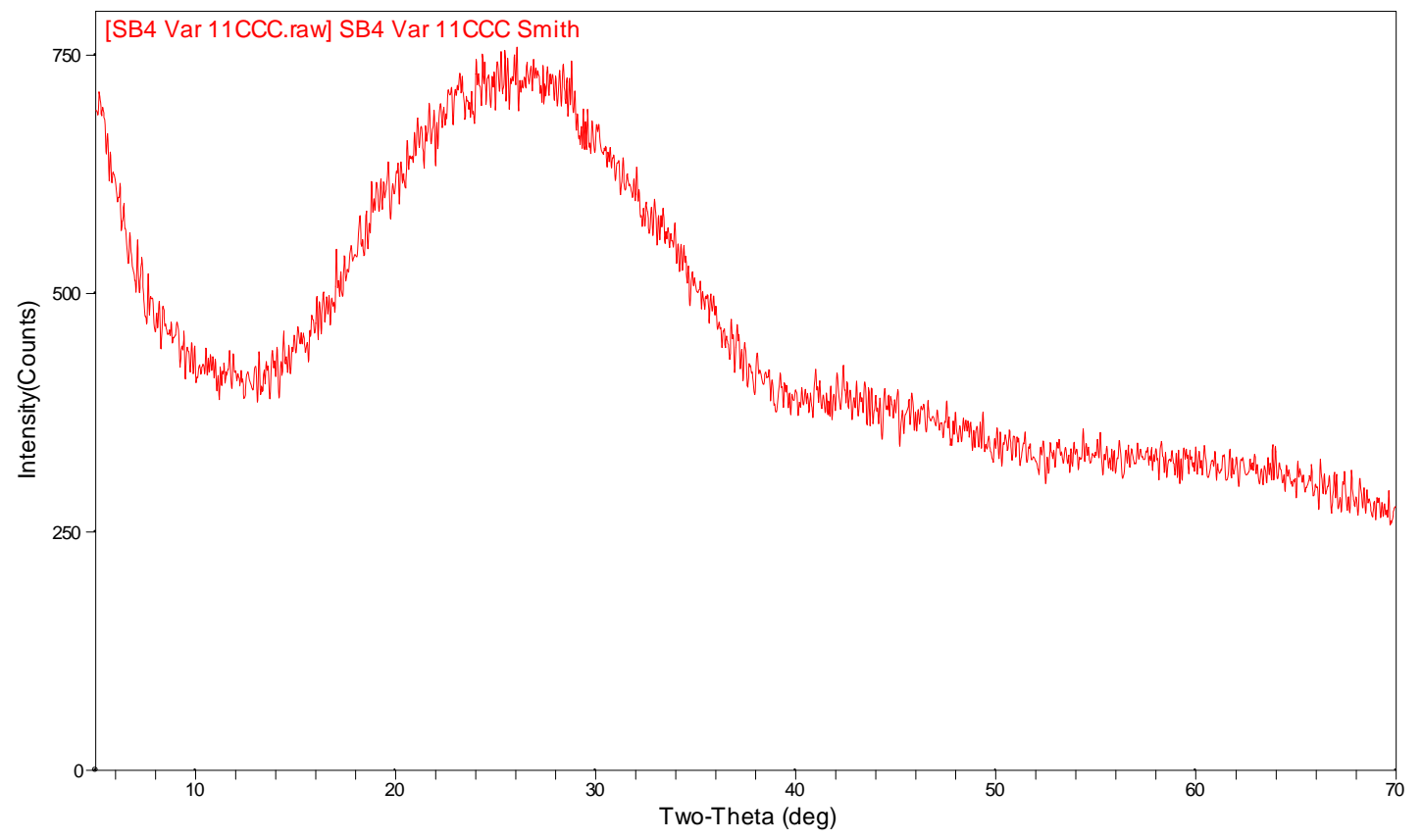

Figure 1. XRD pattern of the ccc version of the SB4VAR11 glass (Case \#1 at 34\% WL).

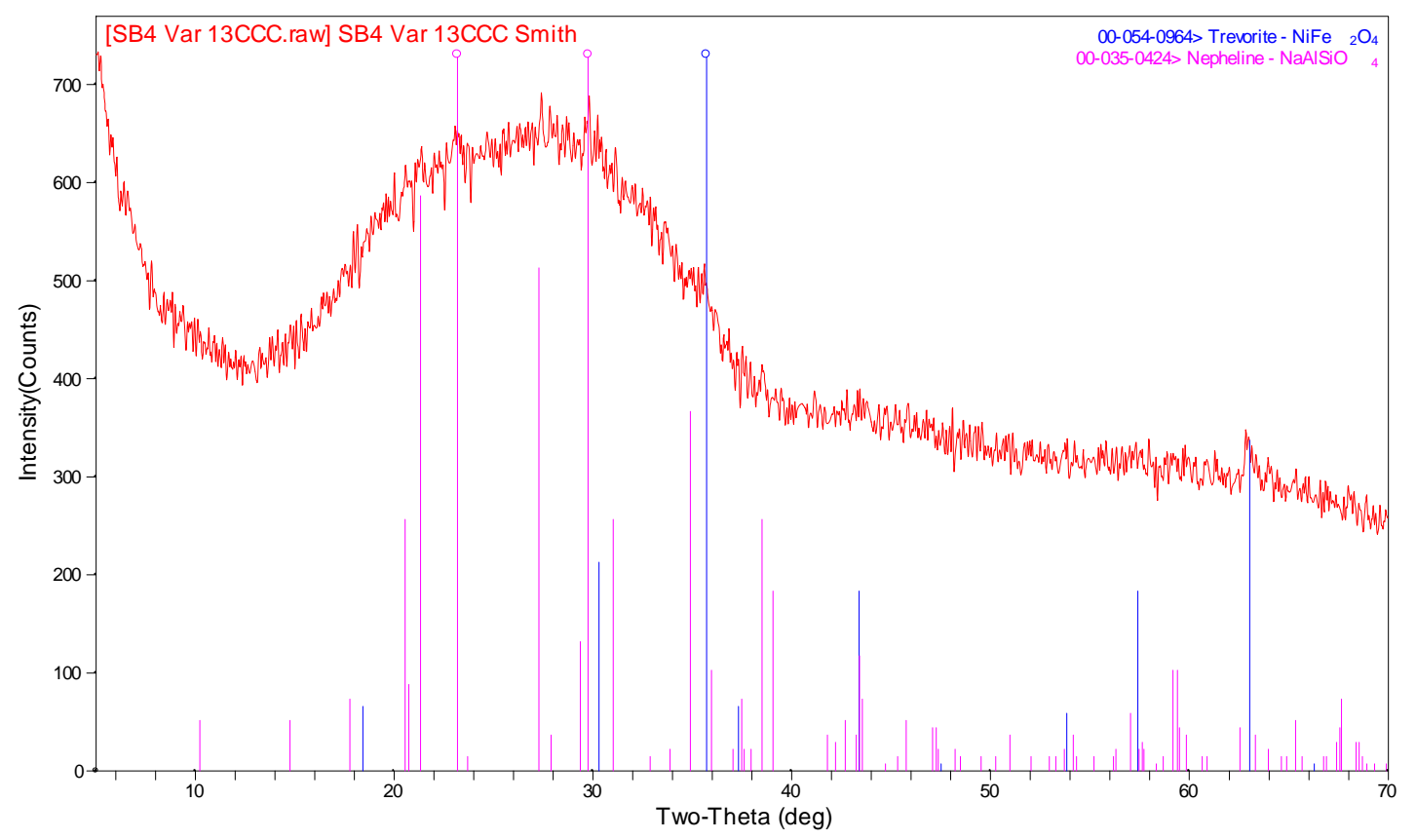

Figure 2. XRD pattern of the ccc version of the SB4VAR13 glass (Case \#1 at $42 \% \mathrm{WL}$ ). 


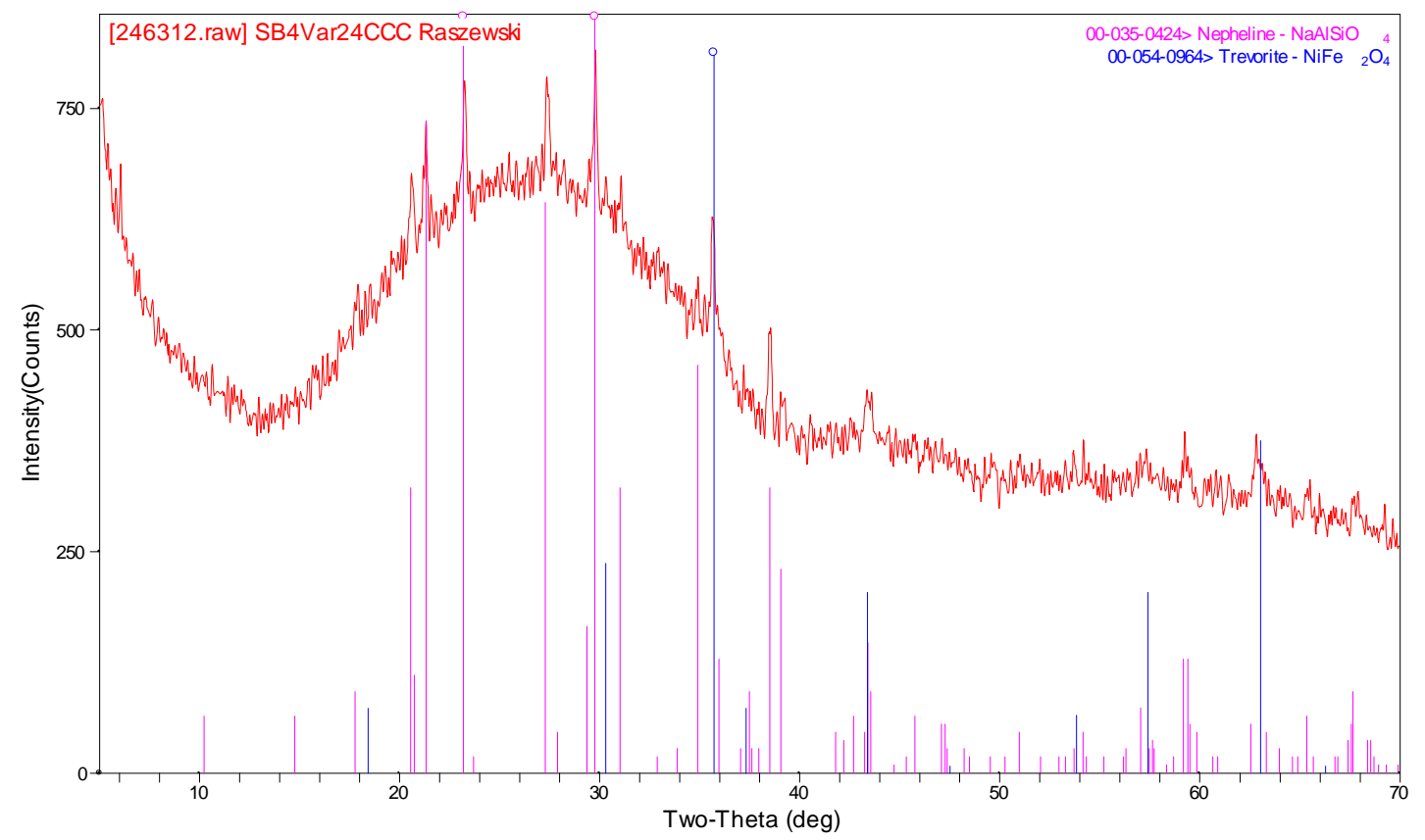

Figure 3. XRD pattern of the ccc version of the SB4VAR24 glass (Case \#2 at $41 \% \mathrm{WL}$ ).

\subsection{Statistical Review of PCT Results}

Table B1 in Appendix B provides the elemental leachate concentration measurements determined by the PSAL for the solution samples generated by the PCTs. One of the quality control checkpoints for the PCT procedure is solution-weight loss over the course of the 7-day test. None of these PCT results indicated a solution-weight loss problem. Any measurement in Table B1 below the detection limit of the analytical procedure (indicated by a "<") was replaced by $1 / 2$ of the detection limit in subsequent analyses. In addition to adjustments for detection limits, the values were adjusted for the dilution factors: the values for the study glasses, the blanks, and the ARM glass in Table B1 were multiplied by 1.6667 to determine the values in parts per million (ppm) and the values for EA were multiplied by 16.6667. Table B2 in Appendix B provides the resulting measurements.

In the following sections, the analytical sequence of the measurements is explored, the measurements of the standards are investigated and used to assess the overall accuracy of the ICP measurement process, the measurements for each glass are reviewed, plots are provided that explore the effects of heat treatment on the PCTs for these glasses, the PCTs are normalized using the compositions (targeted, measured, and bias-corrected) presented in Table A4, and the normalized PCTs are compared to durability predictions for these compositions generated from the current DWPF models. $^{14}$

\subsubsection{Measurements in Analytical Sequence}

Exhibit B1 in Appendix B provides plots of the leachate (ppm) concentrations in analytical sequence as generated by the PSAL for all of the data and for the data from only the study glasses. A different 
WSRC-STI-2008-00149

Revision 0

color and symbol are used for each study glass or standard. No issues are observed in these plots.

\subsubsection{Results for the Samples of the Multi-Element Solution Standard}

Exhibit B2 in Appendix B provides analyses of the PSAL measurements of the samples of the multielement solution standard by analytical set and ICP calibration block. An ANOVA investigating for statistically significant differences among the block averages for these samples for each element of interest is included in these exhibits. There was no indication of a statistically significant (at a 5\% level) difference among the averages of these measurements for any of the elements of interest. Averaging the measured concentrations for each set of triplicates helps to minimize the impact of any potential ICP-AES effects.

Table 4 summarizes the average measurements and the reference values for the 4 primary elements of interest. The results indicate consistent and accurate measurements from the PSAL processes used to conduct these analyses.

Table 4. Results from Samples of the Multi-Element Solution Standard

\begin{tabular}{|c|c|c|c|c|}
\hline Analytical & Avg B & Avg Li & Avg Na & Avg Si \\
\hline Set/Block & (ppm) & (ppm) & (ppm) & (ppm) \\
\hline $\mathbf{q} / \mathbf{1}$ & 20.2 & 10.0 & 81.2 & 49.4 \\
\hline $\mathbf{q} / \mathbf{2}$ & 20.3 & 9.8 & 80.1 & 50.6 \\
\hline $\mathbf{q} / \mathbf{3}$ & 19.4 & 9.9 & 80.4 & 50.4 \\
\hline $\mathbf{r} / \mathbf{1}$ & 20.1 & 9.8 & 79.3 & 50.0 \\
\hline $\mathbf{r} / \mathbf{2}$ & 20.4 & 9.8 & 80.1 & 52.0 \\
\hline $\mathbf{r} / \mathbf{3}$ & 20.8 & 10.0 & 80.7 & 51.5 \\
\hline Grand Average & 20.2 & 9.9 & 80.3 & 50.7 \\
\hline Reference Value & 20 & 10 & 81 & 50 \\
\hline \% difference & $1.03 \%$ & $-1.22 \%$ & $-0.85 \%$ & $1.30 \%$ \\
\hline
\end{tabular}

\subsubsection{Measurements by Glass Identifier}

Exhibit B3 in Appendix B provides plots (grouped by analytical set) of the leachate concentrations for each type of submitted sample: the study glasses by heat treatment and the standards (EA, ARM, the multi-element solution standard, and blanks). Exhibit B4 in Appendix B provide plots of the leachate concentrations for the PCT results of just the study glasses by heat treatment. These plots allow for the assessment of the repeatability of the measurements, which suggests some scatter in the triplicate values for some analytes for some of the glasses. Also, note that some differences between the values for the two heat treatments for some glasses are evident. More will be said regarding comparisons between the heat treatments in the discussions that follow.

\subsubsection{Normalized PCT Results}

PCT leachate concentrations are typically normalized using the cation composition (expressed as a weight percent) in the glass to obtain a grams per liter $(\mathrm{g} / \mathrm{L})$ leachate concentration. The normalization of the PCTs is usually conducted using the measured compositions of the glasses. This is the preferred normalization process for the PCTs. For completeness, the targeted cation and the bias-corrected cation compositions were also used to conduct this normalization. 
As is the usual convention, the common logarithm of the normalized PCT (normalized leachate, NL) for each element of interest was determined and used for comparison. To accomplish this computation, one must

1.) Determine the common logarithm of the elemental parts per million (ppm) leachate concentration for each of the triplicates and each of the elements of interest (these values are provided in Table B2 of Appendix B),

2.) Average the common logarithms over the triplicates for each element of interest, and then

Normalizing Using Measured Composition (preferred method)

3a.) Subtract a quantity equal to 1 plus the common logarithm of the average cation measured concentration (expressed as a weight percent of the glass) from the average computed in step 2 .

Or Normalizing Using Target Composition

3b.) Subtract a quantity equal to 1 plus the common logarithm of the target cation concentration (expressed as a weight percent of the glass) from the average computed in step 2 .

Or Normalizing Using Measured Bias-Corrected Composition

3c.) Subtract a quantity equal to 1 plus the common logarithm of the measured biascorrected cation concentration (expressed as a weight percent of the glass) from the average computed in step 2.

Exhibit B5 in Appendix B provides scatter plots for these results and offers an opportunity to investigate the consistency in the leaching across the elements for the glasses of this study. All combinations of the normalizations of the PCTs (i.e., those generated using the targeted, measured, and bias-corrected compositional views) and both heat treatments are represented in the series of scatter plots. Consistency in the leaching across the elements is typically demonstrated by a high degree of linear correlation among the values for pairs of these elements. The smallest correlation in this plot is that for $\mathrm{Li}$ and $\mathrm{Si}$, with a value of $\sim 95 \%$.

Table 5 summarizes the normalized PCTs for the glasses of this study, which are listed by glass identifier. The following sections discuss the two primary objectives of the variability study: (1) acceptability of the glasses with respect to the EA glass and (2) applicability of the current PCCS durability models for the measured PCT responses. 
Table 5. Normalized PCTs by Glass ID/Compositional View

\begin{tabular}{|c|c|c|c|c|c|c|c|c|c|c|c|c|c|}
\hline $\begin{array}{c}\text { Glass } \\
\text { ID }\end{array}$ & $\begin{array}{l}\text { WL } \\
(\%)\end{array}$ & $\begin{array}{c}\text { Sludge } \\
\text { Case }\end{array}$ & $\begin{array}{c}\text { Heat } \\
\text { Treatment }\end{array}$ & $\begin{array}{c}\text { Compositional } \\
\text { View }\end{array}$ & $\begin{array}{c}\text { Nepheline } \\
\text { Assessment }\end{array}$ & $\begin{array}{c}\log N L[B \\
(g / L)]\end{array}$ & $\begin{array}{c}\log N L[\mathrm{Li} \\
(\mathrm{g} / \mathrm{L})]\end{array}$ & $\begin{array}{c}\log N L[N a \\
(g / L)]\end{array}$ & $\begin{array}{c}\log N \mathrm{~L} \\
{[\mathrm{Si}(\mathrm{g} / \mathrm{L})]}\end{array}$ & $\begin{array}{l}\text { NL[B } \\
\text { (g/L)] }\end{array}$ & $\begin{array}{l}\mathrm{NL}[\mathrm{Li} \\
(\mathrm{g} / \mathrm{L})]\end{array}$ & $\begin{array}{c}\text { NL[Na } \\
(g / L)]\end{array}$ & $\begin{array}{l}\text { NL[Si } \\
(\mathrm{g} / \mathrm{L})]\end{array}$ \\
\hline ARM & $\cdot$ & & & reference & 0.753 & -0.276 & -0.208 & -0.270 & -0.537 & 0.53 & 0.62 & 0.54 & 0.29 \\
\hline ARM & . & & & reference & 0.753 & -0.324 & -0.249 & -0.300 & -0.565 & 0.47 & 0.56 & 0.50 & 0.27 \\
\hline EA & . & & & reference & 0.704 & 1.243 & 0.973 & 1.116 & 0.597 & 17.52 & 9.40 & 13.05 & 3.96 \\
\hline EA & 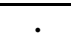 & & & reference & 0.704 & 1.241 & 0.959 & 1.108 & 0.597 & 17.41 & 9.10 & 12.83 & 3.95 \\
\hline SB4VAR11 & 34 & Case 1 & ccc & measured & 0.694 & -0.157 & -0.143 & -0.199 & -0.344 & 0.70 & 0.72 & 0.63 & 0.45 \\
\hline SB4VAR11 & 34 & Case 1 & CCC & measured bc & 0.695 & -0.166 & -0.164 & -0.199 & -0.350 & 0.68 & 0.69 & 0.63 & 0.45 \\
\hline SB4VAR11 & 34 & Case 1 & CCC & targeted & 0.694 & -0.164 & -0.159 & -0.199 & -0.344 & 0.69 & 0.69 & 0.63 & 0.45 \\
\hline SB4VAR11 & 34 & Case 1 & quenched & measured & 0.694 & -0.144 & -0.140 & -0.175 & -0.346 & 0.72 & 0.72 & 0.67 & 0.45 \\
\hline SB4VAR11 & 34 & Case 1 & quenched & targeted & 0.694 & -0.152 & -0.156 & -0.175 & -0.346 & 0.70 & 0.70 & 0.67 & 0.45 \\
\hline SB4VAR12 & 38 & Case 1 & CCC & measured & 0.673 & -0.128 & -0.126 & -0.124 & -0.342 & 0.74 & 0.75 & 0.75 & 0.45 \\
\hline SB4VAR12 & 38 & Case 1 & ccc & measured bc & 0.673 & -0.138 & -0.147 & -0.126 & -0.347 & 0.73 & 0.71 & 0.75 & 0.45 \\
\hline SB4VAR12 & 38 & Case 1 & ccc & targeted & 0.667 & -0.132 & -0.135 & -0.132 & -0.334 & 0.74 & 0.73 & 0.74 & 0.46 \\
\hline SB4VAR12 & 38 & Case 1 & quenched & measured & 0.673 & -0.101 & -0.116 & -0.120 & -0.325 & 0.79 & 0.77 & 0.76 & 0.47 \\
\hline SB4VAR12 & 38 & Case 1 & quenched & measured bc & 0.673 & -0.110 & -0.137 & -0.123 & -0.330 & 0.78 & 0.73 & 0.75 & 0.47 \\
\hline SB4VAR12 & 38 & Case 1 & quenched & targeted & 0.667 & -0.105 & -0.125 & -0.129 & -0.316 & 0.79 & 0.75 & 0.74 & 0.48 \\
\hline SB4VAR13 & 42 & Case 1 & сcC & measured & 0.651 & 0.175 & 0.132 & 0.042 & -0.244 & 1.49 & 1.36 & 1.10 & 0.57 \\
\hline SB4VAR13 & 42 & Case 1 & ccC & measured bc & 0.644 & 0.188 & 0.123 & 0.042 & -0.233 & 1.54 & 1.33 & 1.10 & 0.58 \\
\hline SB4VAR13 & 42 & Case 1 & quenched & measured bc & 0.644 & -0.081 & -0.106 & -0.071 & -0.339 & 0.83 & 0.78 & 0.85 & 0.46 \\
\hline SB4VAR13 & 42 & Case 1 & quenched & targeted & 0.638 & -0.080 & -0.099 & -0.076 & -0.327 & 0.83 & 0.80 & 0.84 & 0.47 \\
\hline SB4VAR21 & 32 & Case 2 & CCC & measured & 0.723 & -0.200 & -0.152 & -0.218 & -0.365 & 0.63 & 0.70 & 0.61 & 0.43 \\
\hline SB4VAR21 & 32 & Case 2 & ccc & measured bc & 0.717 & -0.187 & -0.161 & -0.217 & -0.355 & 0.65 & 0.69 & 0.61 & 0.44 \\
\hline SB4VAR21 & 32 & Case 2 & CCC & targeted & 0.715 & -0.190 & -0.151 & -0.225 & -0.351 & 0.65 & 0.71 & 0.60 & 0.45 \\
\hline SB4VAR21 & 32 & Case 2 & quenched & measured & 0.723 & -0.198 & -0.142 & -0.208 & -0.366 & 0.63 & 0.72 & 0.62 & 0.43 \\
\hline SB4VAR21 & 32 & Case 2 & quenched & measured bc & 0.717 & -0.184 & -0.150 & -0.207 & -0.355 & 0.65 & 0.71 & 0.62 & 0.44 \\
\hline SB4VAR21 & 32 & Case 2 & quenched & targeted & 0.715 & -0.188 & -0.141 & -0.215 & -0.351 & 0.65 & 0.72 & 0.61 & 0.45 \\
\hline SB4VAR22 & 35 & Case 2 & CCC & measured & 0.706 & -0.186 & -0.136 & -0.184 & -0.365 & 0.65 & 0.73 & 0.65 & 0.43 \\
\hline SB4VAR22 & 35 & Case 2 & ccc & measured bc & 0.698 & -0.173 & -0.145 & -0.187 & -0.354 & 0.67 & 0.72 & 0.65 & 0.44 \\
\hline SB4VAR22 & 35 & Case 2 & CCC & targeted & 0.696 & -0.173 & -0.139 & -0.191 & -0.347 & 0.67 & 0.73 & 0.64 & 0.45 \\
\hline SB4VAR22 & 35 & Case 2 & quenched & measured & 0.706 & -0.170 & -0.125 & -0.192 & -0.360 & 0.68 & 0.75 & 0.64 & 0.44 \\
\hline SB4VAR22 & 35 & Case 2 & quenched & measured bc & 0.698 & -0.157 & -0.134 & -0.195 & -0.350 & 0.70 & 0.73 & 0.64 & 0.45 \\
\hline SB4VAR22 & 35 & Case 2 & quenched & targeted & 0.696 & -0.157 & -0.128 & -0.200 & -0.343 & 0.70 & 0.75 & 0.63 & 0.45 \\
\hline SB4VAR23 & 38 & Case 2 & ccc & measured & 0.676 & -0.140 & -0.104 & -0.163 & -0.347 & 0.73 & 0.79 & 0.69 & 0.45 \\
\hline SB4VAR23 & 38 & Case 2 & CCC & measured bc & 0.669 & -0.126 & -0.112 & -0.162 & -0.337 & 0.75 & 0.77 & 0.69 & 0.46 \\
\hline SB4VAR23 & 38 & Case 2 & CCC & targeted & 0.675 & -0.138 & -0.117 & -0.170 & -0.340 & 0.73 & 0.76 & 0.68 & 0.46 \\
\hline SB4VAR23 & 38 & Case 2 & quenched & measured & 0.676 & -0.156 & -0.112 & -0.168 & -0.360 & 0.70 & 0.77 & 0.68 & 0.44 \\
\hline SB4VAR23 & 38 & Case 2 & quenched & measured bc & 0.669 & -0.142 & -0.121 & -0.168 & -0.350 & 0.72 & 0.76 & 0.68 & 0.45 \\
\hline
\end{tabular}


Table 4 cont. Normalized PCTs by Glass ID/Compositional View

\begin{tabular}{|c|c|c|c|c|c|c|c|c|c|c|c|c|c|}
\hline $\begin{array}{l}\text { Glass } \\
\text { ID }\end{array}$ & $\begin{array}{l}\text { WL } \\
(\%)\end{array}$ & $\begin{array}{c}\text { Sludge } \\
\text { Case }\end{array}$ & $\begin{array}{c}\text { Heat } \\
\text { Treatment }\end{array}$ & $\begin{array}{c}\text { Compositional } \\
\text { View }\end{array}$ & $\begin{array}{c}\text { Nepheline } \\
\text { Assessment }\end{array}$ & $\begin{array}{c}\log N L[B \\
(\mathrm{g} / \mathrm{L})]\end{array}$ & $\begin{array}{l}\log N L[L i \\
(g / L)]\end{array}$ & $\begin{array}{c}\log \mathrm{NL}[\mathrm{Na} \\
(\mathrm{g} / \mathrm{L})]\end{array}$ & $\begin{array}{c}\log \mathrm{NL} \\
{[\mathrm{Si}(\mathrm{g} / \mathrm{L})]}\end{array}$ & $\begin{array}{l}\text { NL[B } \\
(\mathrm{g} / \mathrm{L})]\end{array}$ & $\begin{array}{l}\text { NL[Li } \\
(\mathrm{g} / \mathrm{L})]\end{array}$ & $\begin{array}{l}\text { NL[Na } \\
\text { (g/L)] }\end{array}$ & $\begin{array}{l}\mathrm{NL}[\mathrm{Si} \\
(\mathrm{g} / \mathrm{L})]\end{array}$ \\
\hline SB4VAR24 & 41 & Case 2 & cCC & measured & 0.659 & 0.257 & 0.230 & 0.054 & -0.223 & 1.81 & 1.70 & 1.13 & 0.60 \\
\hline SB4VAR24 & 41 & Case 2 & CCC & measured bc & 0.660 & 0.247 & 0.209 & 0.055 & -0.228 & 1.77 & 1.62 & 1.13 & 0.59 \\
\hline SB4VAR24 & 41 & Case 2 & cCC & targeted & 0.654 & 0.251 & 0.218 & 0.049 & -0.217 & 1.78 & 1.65 & 1.12 & 0.61 \\
\hline SB4VAR24 & 41 & Case 2 & quenched & measured & 0.659 & -0.109 & -0.090 & -0.140 & -0.341 & 0.78 & 0.81 & 0.72 & 0.46 \\
\hline SB4VAR24 & 41 & Case 2 & quenched & measured bc & 0.660 & -0.118 & -0.111 & -0.140 & -0.346 & 0.76 & 0.77 & 0.72 & 0.45 \\
\hline SB4VAR24 & 41 & Case 2 & quenched & targeted & 0.654 & -0.115 & -0.102 & -0.146 & -0.335 & 0.77 & 0.79 & 0.71 & 0.46 \\
\hline SB4VAR31 & 32 & Case 3 & cCC & measured & 0.708 & -0.175 & -0.153 & -0.196 & -0.343 & 0.67 & 0.70 & 0.64 & 0.45 \\
\hline SB4VAR31 & 32 & Case 3 & cCC & measured bc & 0.706 & -0.170 & -0.168 & -0.188 & -0.334 & 0.68 & 0.68 & 0.65 & 0.46 \\
\hline SB4VAR31 & 32 & Case 3 & CCC & targeted & 0.709 & -0.170 & -0.156 & -0.198 & -0.343 & 0.68 & 0.70 & 0.63 & 0.45 \\
\hline SB4VAR31 & 32 & Case 3 & quenched & measured & 0.708 & -0.143 & -0.138 & -0.180 & -0.328 & 0.72 & 0.73 & 0.66 & 0.47 \\
\hline SB4VAR31 & 32 & $\begin{array}{l}\text { Case } 3 \\
\end{array}$ & quenched & measured bc & 0.706 & -0.137 & -0.153 & -0.172 & -0.319 & 0.73 & 0.70 & 0.67 & 0.48 \\
\hline SB4VAR31 & 32 & $\begin{array}{l}\text { Case } 3 \\
\end{array}$ & quenched & targeted & 0.709 & -0.137 & -0.141 & -0.182 & -0.328 & 0.73 & 0.72 & 0.66 & 0.47 \\
\hline SB4VAR32 & 36 & Case 3 & CCC & measured & 0.682 & -0.134 & -0.123 & -0.153 & -0.330 & 0.73 & 0.75 & 0.70 & 0.47 \\
\hline SB4VAR32 & 36 & Case 3 & CCC & measured bc & 0.680 & -0.136 & -0.145 & -0.145 & -0.321 & 0.73 & 0.72 & 0.72 & 0.48 \\
\hline SB4VAR32 & 36 & Case 3 & CCC & targeted & 0.681 & -0.132 & -0.132 & -0.154 & -0.327 & 0.74 & 0.74 & 0.70 & 0.47 \\
\hline SB4VAR32 & 36 & Case 3 & quenched & measured & 0.682 & -0.137 & -0.136 & -0.162 & -0.333 & 0.73 & 0.73 & 0.69 & 0.46 \\
\hline SB4VAR32 & 36 & Case 3 & quenched & measured bc & 0.680 & -0.140 & -0.158 & -0.154 & -0.324 & 0.73 & 0.69 & 0.70 & 0.47 \\
\hline SB4VAR32 & 36 & Case 3 & quenched & targeted & 0.681 & -0.135 & -0.144 & -0.163 & -0.330 & 0.73 & 0.72 & 0.69 & 0.47 \\
\hline SB4VAR33 & 40 & Case 3 & CCC & measured & 0.655 & -0.088 & -0.101 & -0.071 & -0.304 & 0.82 & 0.79 & 0.85 & 0.50 \\
\hline SB4VAR33 & 40 & Case 3 & CCC & measured bc & 0.646 & -0.083 & -0.116 & -0.080 & -0.299 & 0.83 & 0.77 & 0.83 & 0.50 \\
\hline SB4VAR33 & 40 & $\begin{array}{l}\text { Case } 3 \\
\end{array}$ & CCC & targeted & 0.653 & -0.084 & -0.103 & -0.086 & -0.312 & 0.83 & 0.79 & 0.82 & 0.49 \\
\hline SB4VAR33 & 40 & Case 3 & quenched & measured & 0.655 & -0.108 & -0.109 & -0.083 & -0.314 & 0.78 & 0.78 & 0.83 & 0.49 \\
\hline SB4VAR33 & 40 & $\begin{array}{l}\text { Case } 3 \\
\end{array}$ & quenched & measured bc & 0.646 & -0.103 & -0.125 & -0.091 & -0.309 & 0.79 & 0.75 & 0.81 & 0.49 \\
\hline SB4VAR33 & 40 & Case 3 & quenched & targeted & 0.653 & -0.104 & -0.111 & -0.097 & -0.322 & 0.79 & 0.77 & 0.80 & 0.48 \\
\hline SB4VAR41 & 32 & Case 4 & CCC & measured & 0.721 & -0.170 & -0.119 & -0.196 & -0.338 & 0.68 & 0.76 & 0.64 & 0.46 \\
\hline SB4VAR41 & 32 & Case 4 & CCC & measured bc & 0.714 & -0.157 & -0.128 & -0.199 & -0.328 & 0.70 & 0.75 & 0.63 & 0.47 \\
\hline SB4VAR41 & 32 & Case 4 & CCC & targeted & 0.715 & -0.159 & -0.118 & -0.195 & -0.320 & 0.69 & 0.76 & 0.64 & 0.48 \\
\hline SB4VAR41 & 32 & Case 4 & quenched & measured & 0.721 & -0.191 & -0.139 & -0.197 & -0.364 & 0.64 & 0.73 & 0.63 & 0.43 \\
\hline SB4VAR41 & 32 & Case 4 & quenched & measured bc & 0.714 & -0.178 & -0.148 & -0.200 & -0.354 & 0.66 & 0.71 & 0.63 & 0.44 \\
\hline SB4VAR41 & 32 & Case 4 & quenched & targeted & 0.715 & -0.181 & -0.138 & -0.196 & -0.346 & 0.66 & 0.73 & 0.64 & 0.45 \\
\hline SB4VAR42 & 35 & Case 4 & cCC & measured & 0.703 & -0.162 & -0.114 & -0.167 & -0.342 & 0.69 & 0.77 & 0.68 & 0.45 \\
\hline SB4VAR42 & 35 & Case 4 & CCC & measured bc & 0.695 & -0.148 & -0.123 & -0.170 & -0.332 & 0.71 & 0.75 & 0.68 & 0.47 \\
\hline SB4VAR42 & 35 & $\begin{array}{l}\text { Case } 4 \\
\end{array}$ & CCC & targeted & 0.695 & -0.159 & -0.124 & -0.178 & -0.334 & 0.69 & 0.75 & 0.66 & 0.46 \\
\hline SB4VAR42 & 35 & $\begin{array}{l}\text { Case } 4 \\
\end{array}$ & quenched & measured & 0.703 & -0.161 & -0.122 & -0.166 & -0.348 & 0.69 & 0.75 & 0.68 & 0.45 \\
\hline SB4VAR42 & 35 & Case 4 & quenched & measured bc & 0.695 & -0.148 & -0.131 & -0.169 & -0.337 & 0.71 & 0.74 & 0.68 & 0.46 \\
\hline SB4VAR42 & 35 & Case 4 & quenched & targeted & 0.695 & -0.159 & -0.132 & -0.177 & -0.339 & 0.69 & 0.74 & 0.67 & 0.46 \\
\hline SB4VAR43 & 38 & Case 4 & CCC & measured & 0.684 & -0.160 & -0.124 & -0.165 & -0.356 & 0.69 & 0.75 & 0.68 & 0.44 \\
\hline SB4VAR43 & 38 & Case 4 & CCC & measured bc & 0.684 & -0.169 & -0.145 & -0.168 & -0.361 & 0.68 & 0.72 & 0.68 & 0.44 \\
\hline SB4VAR43 & 38 & Case 4 & CCC & targeted & 0.674 & -0.162 & -0.133 & -0.178 & -0.345 & 0.69 & 0.74 & 0.66 & 0.45 \\
\hline SB4VAR43 & 38 & Case 4 & quenched & measured & 0.684 & -0.144 & -0.113 & -0.139 & -0.340 & 0.72 & 0.77 & 0.73 & 0.46 \\
\hline SB4VAR43 & 38 & Case 4 & quenched & measured bc & 0.684 & -0.154 & -0.134 & -0.142 & -0.345 & 0.70 & 0.73 & 0.72 & 0.45 \\
\hline
\end{tabular}


Table 4 cont. Normalized PCTs by Glass ID/Compositional View

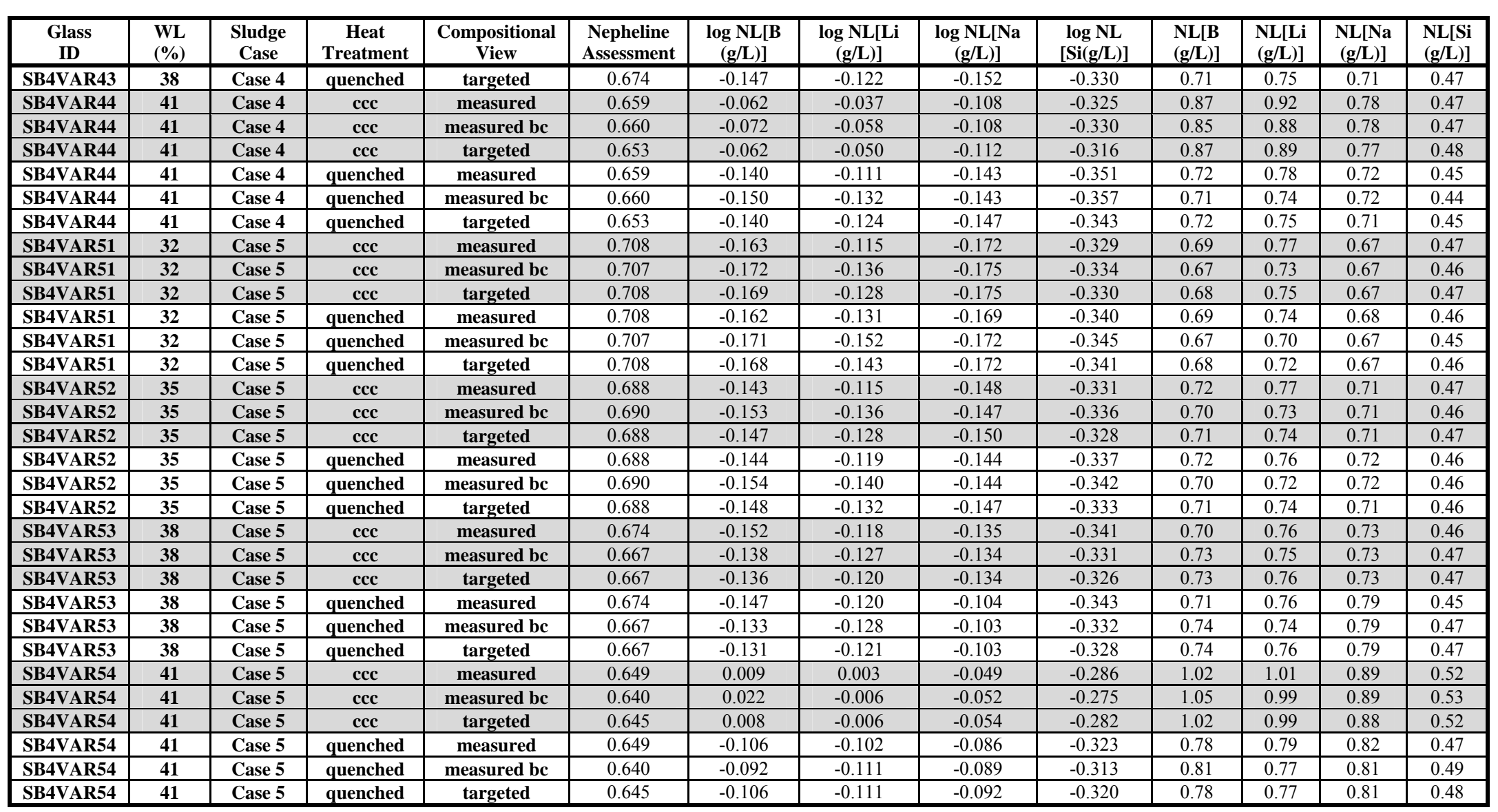


WSRC-STI-2008-00149

Revision 0

\subsubsection{Acceptability of the SB4 VS Glasses}

The NL [B] for each quenched and ccc glass is well below that of the reference EA glass (16.695 $\mathrm{g} / \mathrm{L}){ }^{12}$ For the quenched glasses, the NL [B] (based on measured bc compositions) ranges from 0.65 $\mathrm{g} / \mathrm{L}$ (SB4VAR21) to $0.83 \mathrm{~g} / \mathrm{L}$ (SB4VAR13). Within each SB4 post-decant option, the general trend observed for the quenched glasses is a gradual decrease in durability with increasing WL. For example, in Case \#1 (SB4 nominal pre-decant) the NL [B] for glasses targeting 34, 38, and 42\% WL are $0.70,0.78$, and $0.83 \mathrm{~g} / \mathrm{L}$, respectively and in Case \#2 (SB4 after the $100 \mathrm{~K}$ decant), the NL [B] for glasses targeting $32,35,38,41 \% \mathrm{WL}$ are $0.65,0.70,0.72$, and $0.76 \mathrm{~g} / \mathrm{L}$, respectively. Similar trends are also observed for Cases \#3, \#4, and \#5. Regardless of the SB4 option or targeted WL, the durabilities of the quenched Frit 510-based glasses are very acceptable.

With respect to the ccc versions of the variability study glasses, the NL [B] (based on measured bc compositions) range from $0.65 \mathrm{~g} / \mathrm{L}$ (SB4VAR21ccc) to $1.77 \mathrm{~g} / \mathrm{L}$ (SB4VAR24ccc). As in the quenched glasses, the PCT responses for all of the ccc glasses are acceptable relative to the EA reference glass $(16.695 \mathrm{~g} / \mathrm{L})$. A gradual decrease in durability with increasing WL (within a specific option) is observed. For example, the NL [B] of the Case \#1 ccc glasses are $0.68,0.73$, and $1.54 \mathrm{~g} / \mathrm{L}$ for the 34, 38, and 41\% WL targets and for Case \#2, the NL [B] of the 32, 35, 38, and 41\% WL glasses are $0.65,0.67,0.75$, and $1.77 \mathrm{~g} / \mathrm{L}$. Again, all of the NL [B] values are very acceptable relative to EA and the durability trend as a function of WL is consistent for both quenched and cce glasses.

One primary difference is observed for two of the ccc glasses, specifically SB4VAR13ccc (Case \#1 at $42 \% \mathrm{WL}$ ) and SB4VAR24cce (Case \#2 at 41\% WL). Although the PCT responses are acceptable, there is a considerable increase (a factor of approximately 2) in the PCT response as the WL is raised to the maximum level considered in this study. Consider Figure 4 which shows the log NL [B] for the Case \#1 (Frit 510-SB4 nominal (pre-decant)) glasses as a function of WL, compositional view, and thermal treatment (quenched and $\mathrm{ccc}$ ). There is very little difference (no practical significance) between the quenched and ccc glasses targeting 34 and 38\% WL; however, a significant and potentially practical difference exists between the quenched and ccc versions of the glass at $42 \% \mathrm{WL}$. More specifically, the NL [B] of the SB4VAR12ccc (Case \#1 at 38\% WL) is $0.73 \mathrm{~g} / \mathrm{L}$, while SB4VAR13ccc (Case \#1 at 42\% WL) has a NL [B] of $1.54 \mathrm{~g} / \mathrm{L}$. This same trend is observed for Case \#2 (post $100 \mathrm{~K}$ decant) as the NL [B] of the ccc glasses at 38 and $41 \% \mathrm{WL}$ are 0.75 and $1.77 \mathrm{~g} / \mathrm{L}$, respectively. The magnitude of the decrease in durability for these two glasses is inconsistent with the general differences of the lower WL glasses within their respective options. Such a trend in NL [B] would suggest that nepheline formation may be responsible for the lower durabilities of these glasses. In fact, nepheline formation was observed in both SB4VAR13ccc and SB4VAR24ccc (refer to Section 4.2.2). While the increased NL [B] of SB4VAR44ccc (Case \#4 at 41\% WL) and SB4VAR54ccc (Case \#5 at 41\%) would suggest the presence of nepheline, none was detected with XRD. Due to the detection limit of the instrument, these results indicate that the PCT response of a sample may be more sensitive to the presence of nepheline than XRD.

Although not an issue in terms of acceptability, the fact that nepheline was detected in two of the study glasses are the first results which indicate that the current nepheline discriminator value of 0.62 is not conservative. The nepheline discriminator was implemented into PCCS for SB4 based on the fact that all of the historical glasses evaluated with nepheline values of 0.62 or greater did not contain nepheline via XRD analysis. ${ }^{15}$ During that assessment, glasses were identified with nepheline discriminator values less than 0.62 that were not characterized by the formation of nepheline upon slow cooling. These results suggest that there was either a compositional or thermal (kinetic) effect not fully captured in the discriminator but at that point the 0.62 value was deemed to be conservative. The current results (XRD patterns and PCT response for SB4VAR13 and SB4VAR24) suggest that 
the discriminator is not conservative within this glass compositional region. These results have altered concurrent experimental studies that continue to focus on both compositional and heat treatment effects for nepheline formation in an attempt to gain a more fundamental understanding of its formation and impacts to DWPF type glasses.

Although these two glasses do cause some concern over the continued use of the 0.62 nepheline value to predict nepheline in ccc glasses for future DWPF glass systems, the impact on the current DWPF operating window for the SB4 system is of little concern. Nepheline was only observed in glasses targeting 41 or $42 \%$ WL. Current processing of the Frit $510-$ SB4 system in DWPF has nominally targeted $34 \% \mathrm{WL}$. For those SB4 variability study glasses targeting these lower WLs, nepheline formation was not observed and the minimal difference in PCT response between quenched and ccc versions supported its absence. It should also be noted that the glasses targeting the higher WLs in the two "post decanted" systems (Case \#3 and Case \#4) did not formally show nepheline formation via XRD results, but a comparison of the quenched and ccc PCT response do suggest crystallization may be present below the detection limit of the $\mathrm{X}$-ray diffractometer.

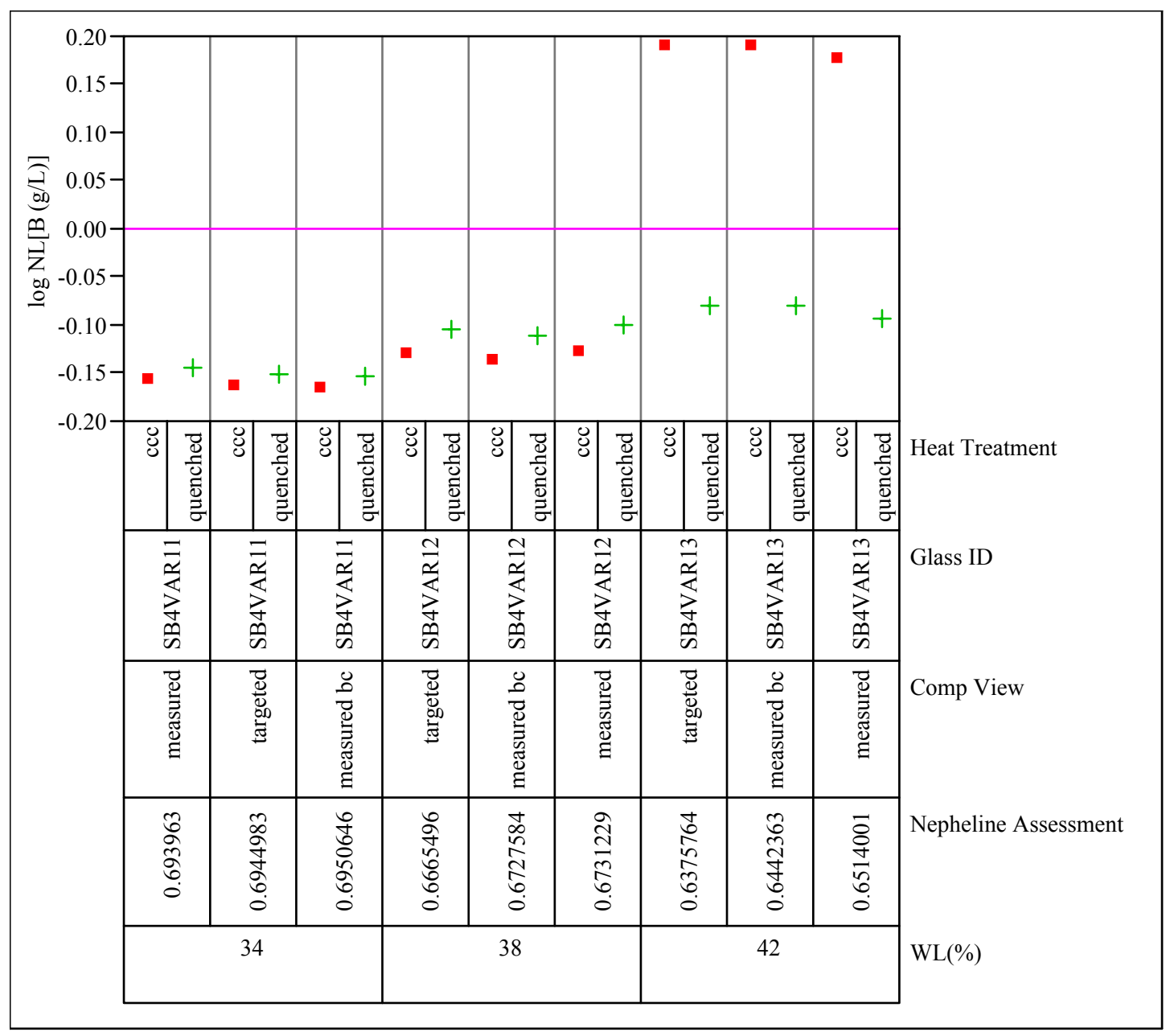

Figure 4. Effect of heat treatment on $\log$ NL[B (g/L)] for Case \#1. 
WSRC-STI-2008-00149

Revision 0

\subsubsection{Predicted versus Measured PCTs}

As discussed in Section 4.3.5 and shown in Table 5, the durabilities of the SB4/Decant VS glasses are all very acceptable when compared to the durability of EA, which meets one objective of the variability study. The second objective is to evaluate the applicability of the current durability model to the compositional region of interest. The term applicability refers to the ability of the current durability model to predict the PCT response of the study glasses. Note that the current durability model is only valid for homogeneous glasses and is not expected to predict the PCT response of glasses containing crystallization that would significantly alter the response (i.e., nepheline). Nonhomogeneous glasses (based on XRD analysis) in this study include SB4VAR13ccc (Case \#1 at 42\% WL) and SB4VAR24ccc (Case \#2 at 41\% WL).

Exhibit B8 in Appendix B provides plots of the DWPF models that relate the logarithm of the normalized PCT (for each element of interest) to a linear function of a free energy of hydration term $\left(\Delta \mathrm{G}_{\mathrm{p}}, \mathrm{kcal} / 100 \mathrm{~g}\right.$ glass $)$ derived for all of the glass compositional views and heat treatments. ${ }^{14}$ Prediction limits (at a 95\% confidence) for an individual PCT result are also plotted along with the linear fit. The EA and ARM results are also indicated on these plots. Exhibit B9 in Appendix B provides a version of these plots for the quenched glasses only and Exhibit B10 in Appendix B provides a version for ccc glasses only. While all of these glasses show acceptable PCT responses, some points fall above the confidence limits for the ccc versions of the study glasses.

Figure 5 provides a closer look at the PCT response that falls above the confidence interval, which is the ccc version of glass SB4VAR24. The PCT response for the quenched version of this glass is also included on the plot along with the EA and ARM results. The points lying above the $95 \%$ confidence interval are associated with the three compositional views (target, measured and measured $\mathrm{bc}$ ) of SB4VAR24ccc. As previously mentioned, SB4VAR24ccc contains nepheline, which is the cause for the higher PCT response and the inability of the $\Delta \mathrm{G}_{\mathrm{P}}$ model to predict the NL [B]. Implementation of the nepheline discriminator in DWPF's PCCS is the primary mechanism to avoid the production of a glass that precipitates nepheline upon slow cooling, thus creating a potentially unacceptable glass.

Although the PCT response for the SB4VAR24ccc glass is acceptable, there is currently no tool or model within the DWPF's process control system that would have prevented this glass (as well as SB4VAR13) from being processed. As previously mentioned, targeted WLs for the Frit 510-SB4 system have been approximately $34 \%$, so a comfortable processing margin exists for the operating window since nepheline formed only in glasses targeting a WL of either 41 or $42 \% \mathrm{WL}$. These data do identify the need for a better understanding of the current nepheline discriminator over DWPF compositions of interest. 


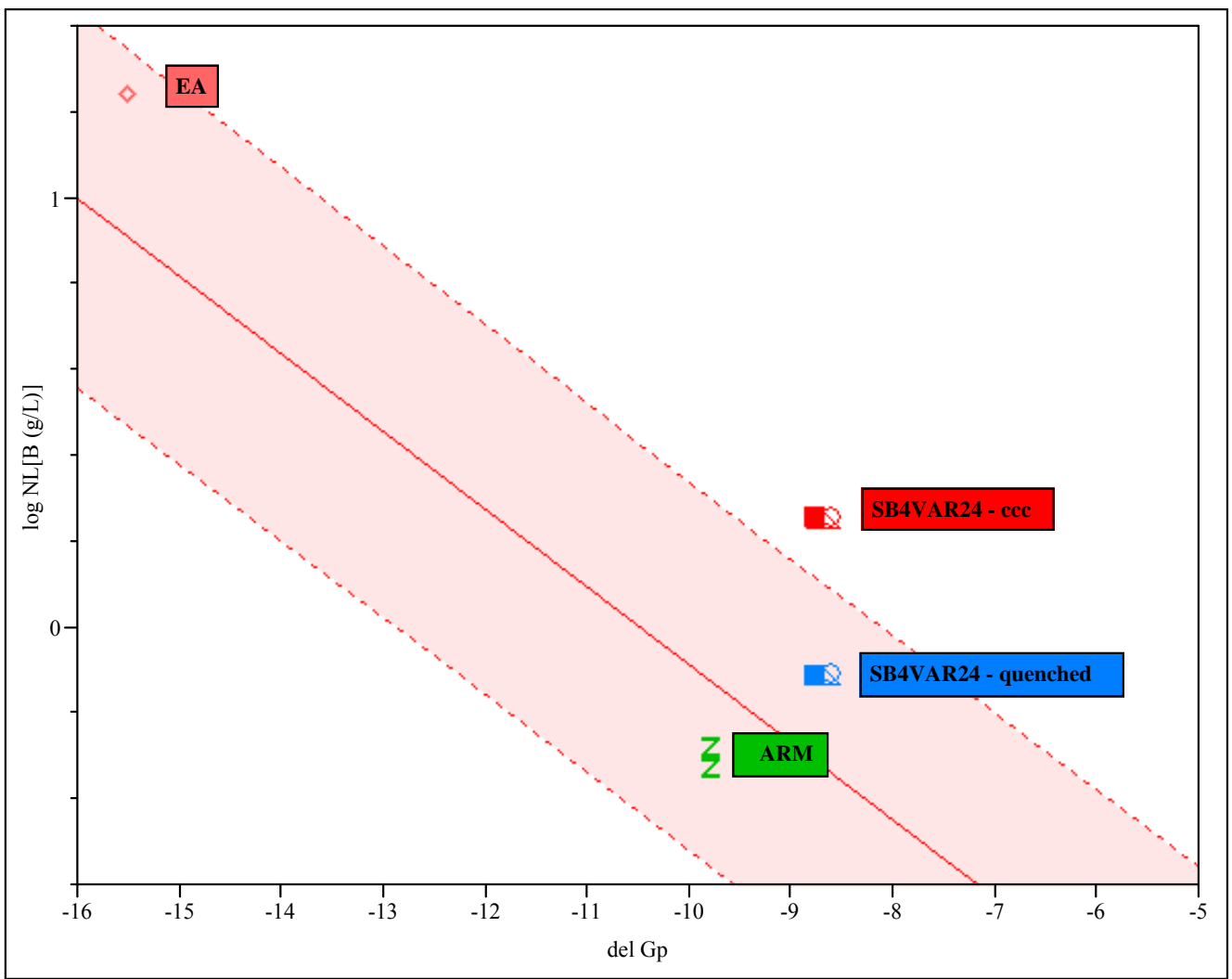

Figure 5. Log NL[B (g/L) versus B Del Gp model with a 95\% confidence interval for individual PCTs.

\subsection{A Review of the Nepheline Assessments for DWPF's SB4 Process Batches}

In order to evaluate the potential impacts of the two non-conservative data points from this study on DWPF operations with SB4 (pre-Tank 40 decant), values of the nepheline discriminator for the SB4 SME batches 402 through 436 that have been processed by the DWPF were assessed. These values were obtained from the Sample_Date Folder maintained by the DWPF Chem Group on the WG09 server and are shown in Table 6. The smallest nepheline discriminator value the DWPF has recorded as part of the SB4 processing to date is 0.704 , which is well above the 0.62 nominal limit for this constraint as well as being above the 0.654 value of the SB4VAR24 glass of this study.

Table 6. Nepheline Discriminator Values Recorded by DWPF during the Processing of SB4

\begin{tabular}{|c|c|c|c|c|c|c|c|c|c|c|c|c|}
\hline SME Batch & $\mathbf{4 0 2}$ & $\mathbf{4 0 3}$ & $\mathbf{4 0 4}$ & $\mathbf{4 0 5}$ & $\mathbf{4 0 6}$ & $\mathbf{4 0 7}$ & $\mathbf{4 0 8}$ & $\mathbf{4 0 9}$ & $\mathbf{4 1 0}$ & $\mathbf{4 1 1}$ & $\mathbf{4 1 2}$ & $\mathbf{4 1 3}$ \\
\hline Nepheline Value & 0.719 & 0.743 & 0.727 & 0.742 & 0.726 & 0.735 & 0.723 & 0.722 & 0.725 & 0.719 & 0.717 & 0.723 \\
\hline SME Batch & $\mathbf{4 1 4}$ & $\mathbf{4 1 5}$ & $\mathbf{4 1 6}$ & $\mathbf{4 1 7}$ & $\mathbf{4 1 8}$ & $\mathbf{4 1 9}$ & $\mathbf{4 2 0}$ & $\mathbf{4 2 1}$ & $\mathbf{4 2 2}$ & $\mathbf{4 2 3}$ & $\mathbf{4 2 4}$ & $\mathbf{4 2 5}$ \\
\hline Nepheline Value & 0.721 & 0.752 & 0.729 & 0.728 & 0.730 & 0.712 & 0.720 & 0.732 & 0.731 & 0.724 & 0.711 & 0.707 \\
\hline SME Batch & $\mathbf{4 2 6}$ & $\mathbf{4 2 7}$ & $\mathbf{4 2 8}$ & $\mathbf{4 2 9}$ & $\mathbf{4 3 0}$ & $\mathbf{4 3 1}$ & $\mathbf{4 3 2}$ & $\mathbf{4 3 3}$ & $\mathbf{4 3 4}$ & $\mathbf{4 3 5}$ & $\mathbf{4 3 6}$ & \\
\hline Nepheline Value & 0.723 & 0.717 & 0.704 & 0.722 & 0.722 & 0.709 & 0.719 & 0.721 & 0.722 & 0.727 & 0.735 & \\
\hline
\end{tabular}


The nepheline values predicted by the actual DWPF SME analytical data in combination with the data of the study glasses provide no indication that the production glasses within the Frit 510-SB4 are not acceptable. To support this statement, SRNL fabricated five supplemental glasses based on the specific SB4 SME batch information from WG09. Specifically, elemental compositional information from SME batches 434 and $435^{\mathrm{d}}$ were obtained from WG09, converted to oxides, and normalized. The SME data suggested that the WLs for these two Frit 510-SB4 nominal (pre-Tank 40 decant) glasses were approximately 33 and $32 \%$, respectively. In addition to these two specific glasses three other glasses were made using the nominal Case \#1 variability study composition coupled with Frit 510 at WLs of $\sim 32,33$, and $34 \% \mathrm{WL}^{\mathrm{e}}$ The target and measured compositions of these five supplemental glasses are shown in Table 7 and Table 8 . $^{\mathrm{f}}$ The nomenclature for these pre-decant glasses can be summarized as follows:

1.) SB4PS1: Nominal glass targeting the measured composition of SME batch 434 (normalized)

2.) SB4PS2: Nominal glass targeting the measured composition of SME batch 435 (normalized)

3.) SB4PS3: Nominal glass targeting a Frit 510 - SB4 Case \#1 glass at $32 \% \mathrm{WL}$

4.) SB4PS4: Nominal glass targeting a Frit 510 - SB4 Case \#1 glass at 33\% WL

5.) SB4PS5: Nominal glass targeting a Frit $510-\mathrm{SB} 4$ Case \#1 glass at $34 \% \mathrm{WL}$

Table 9 summarizes the PCT results (normalized to the target compositions) of both the quenched and ccc versions of these five supplemental glasses. The results indicate that all of the glasses are acceptable and predictable ${ }^{\mathrm{g}}$ regardless of thermal history (quenched or $\mathrm{ccc}$ ). The NL [B] ranges from $0.41 \mathrm{~g} / \mathrm{L}$ (SB4PS1ccc) to $0.52 \mathrm{~g} / \mathrm{L}$ (SB4PS3 quenched and SB4PS4 ccc). When comparing the quenched glasses to their ccc counterparts, there is very little (if any) difference between the PCT response, which suggests that the ccc glasses are void of nepheline. These results have also been confirmed by XRD. ${ }^{\mathrm{h}, \mathrm{i}}$ The PCT responses of these supplemental glasses are comparable to the lower WL glasses of the initial 18 variability study glasses. More specifically, the PCT responses of the quenched and ccc SB4VAR11 (Case \#1, pre-Tank 40 decant glass at 34\% WL) are 0.70 and $0.68 \mathrm{NL}$ [B], respectively. Again, these data coupled with nepheline values predicted by the actual SME analytical data and the measured PCT response from the study glasses indicate that the production glasses are void of nepheline and are very acceptable regardless of the thermal history. Even though the PCT responses for the higher WL post-Tank 40 decant study glasses are acceptable and no nepheline was observed via XRD, it is recommended that DWPF continue to target lower WLs during processing of SB4.

\footnotetext{
${ }^{\mathrm{d}}$ It should be noted that SB4 pour stream samples were also pulled during processing of these two SME batches. The first pour stream sample was pulled on 3/12/08 during the processing SME 434. The second pour stream sample was pulled on 3/18/08 during the processing SME 435. The two pour stream samples have been transported to SRNL, but have not been characterized.

${ }^{\mathrm{e}}$ The $\mathrm{Al}_{2} \mathrm{O}_{3}, \mathrm{Fe}_{2} \mathrm{O}_{3}, \mathrm{Na}_{2} \mathrm{O}$ and $\mathrm{U}_{3} \mathrm{O}_{8}$ content of each $\mathrm{SME}$ batch were compared. All of the variability study glasses and supplemental glasses have compositions that are within the bounds of the SME batch compositions, with the exception of 2 glasses (SB4VAR24 and SB4VAR54). Both of these glasses targeted higher WLs (41\%), so they are not expected to fall within the bounds of the SME batches. A comparison of the data is shown in Appendix C, Table C1.

${ }^{\mathrm{f}}$ No analytical plan was used as guidance for the chemical composition measurements, so there are no measured bc values.

The measured compositions in Table 8 are an average of the values obtained by the LM and PF methods.

${ }^{\mathrm{g}}$ For more information see page 135 of WSRC-NB-2006-00168.

${ }^{\mathrm{h}}$ The XRD patterns of the supplemental glasses (ccc) can be viewed in the laboratory notebook for the SB4 variability study on page 132 (WSRC-NB-2006-00168).

i During the testing of the supplemental glasses, replicates of SB4VAR13ccc (Case \#1 at 42\% WL) and SB4VAR24ccc (Case \#2 at $41 \% \mathrm{WL}$ ) were fabricated and tested to confirm reproducibility. While the composition and PCT results correspond to the previous values, the XRD patterns do not indicate the presence of nepheline (see pages 132 and 134 of WSRC-SB-2006-00168 for the PCT and XRD results). We have recognized the issues and will integrate these results into future nepheline testing.
} 
Table 7. Target Compositions of the SB4PS Glasses

\begin{tabular}{|c|c|c|c|c|c|}
\hline Glass ID & $\begin{array}{l}\bar{n} \\
\tilde{f} \\
\dot{\infty}\end{array}$ & $\begin{array}{l}\text { బे } \\
\text { \&े } \\
\text { के }\end{array}$ & $\begin{array}{l}\mathscr{n} \\
\tilde{f} \\
\tilde{\omega}\end{array}$ & $\begin{array}{l}\text { W } \\
\hat{f} \\
\text { के }\end{array}$ & $\begin{array}{l}\text { L } \\
\text { \&े } \\
\text { की }\end{array}$ \\
\hline Frit & \multirow{2}{*}{ SME 434} & \multirow{2}{*}{ SME 435} & 510 & 510 & 510 \\
\hline WL & & & 31.7 & 33.2 & 34 \\
\hline $\mathrm{Al}_{2} \mathbf{O}_{3}$ & 7.61 & 7.37 & 7.92 & 8.29 & 8.49 \\
\hline $\mathbf{B}_{2} \mathbf{O}_{3}$ & 8.53 & 8.77 & 9.56 & 9.35 & 9.24 \\
\hline $\mathrm{CaO}$ & 0.84 & 0.79 & 0.87 & 0.91 & 0.93 \\
\hline $\mathrm{Cr}_{2} \mathrm{O}_{3}$ & 0.05 & 0.04 & 0.05 & 0.05 & 0.05 \\
\hline $\mathrm{CuO}$ & 0.01 & 0.00 & 0.02 & 0.02 & 0.02 \\
\hline $\mathrm{Fe}_{2} \mathrm{O}_{3}$ & 8.07 & 8.03 & 9.00 & 9.42 & 9.65 \\
\hline $\mathbf{K}_{2} \mathbf{O}$ & 0.08 & 0.02 & 0.11 & 0.12 & 0.12 \\
\hline $\mathbf{L i}_{2} \mathbf{O}$ & 5.19 & 5.31 & 5.46 & 5.34 & 5.28 \\
\hline MgO & 0.79 & 0.85 & 0.85 & 0.89 & 0.92 \\
\hline $\mathrm{MnO}$ & 1.43 & 1.57 & 1.81 & 1.89 & 1.94 \\
\hline $\mathrm{Na}_{2} \mathrm{O}$ & 12.12 & 11.98 & 11.99 & 12.18 & 12.28 \\
\hline $\mathrm{NiO}$ & 0.45 & 0.46 & 0.50 & 0.52 & 0.54 \\
\hline $\mathrm{SO}_{4}$ & 0.44 & 0.42 & 0.42 & 0.44 & 0.45 \\
\hline $\mathrm{SiO}_{2}$ & 51.37 & 51.48 & 48.66 & 47.65 & 47.11 \\
\hline $\mathrm{TiO}_{2}$ & 0.09 & 0.07 & 0.01 & 0.01 & 0.01 \\
\hline $\mathrm{U}_{3} \mathbf{O}_{8}$ & 2.73 & 2.68 & 2.75 & 2.88 & 2.94 \\
\hline $\mathrm{ZrO}_{2}$ & 0.19 & 0.15 & 0.03 & 0.03 & 0.03 \\
\hline
\end{tabular}

Table 8. Measured Compositions of the SB4PS Glasses

\begin{tabular}{|c|c|c|c|c|c|}
\hline Glass ID & $\begin{array}{l}\bar{n} \\
\text { \&े } \\
\text { कै }\end{array}$ & $\begin{array}{l}\text { W } \\
\text { से } \\
\text { कै }\end{array}$ & $\begin{array}{l}\mathscr{n} \\
\tilde{f} \\
\tilde{\omega}\end{array}$ & $\begin{array}{l}\text { W } \\
\text { \&े } \\
\text { के }\end{array}$ & $\begin{array}{l}\text { L } \\
\text { है } \\
\text { \% } \\
\text { की }\end{array}$ \\
\hline Frit & \multirow{2}{*}{ SME 434} & \multirow{2}{*}{ SME 435} & 510 & 510 & 510 \\
\hline$\overline{W L}$ & & & 31.7 & 33.2 & 34 \\
\hline $\mathrm{Al}_{2} \mathrm{O}_{3}$ & 7.69 & 7.41 & 7.89 & 8.61 & 8.51 \\
\hline $\mathbf{B}_{2} \mathbf{O}_{3}$ & 8.87 & 9.29 & 9.71 & 9.60 & 9.42 \\
\hline $\mathrm{CaO}$ & 0.82 & 0.77 & 0.85 & 0.91 & 0.89 \\
\hline $\mathrm{Cr}_{2} \mathrm{O}_{3}$ & 0.07 & 0.05 & 0.05 & 0.06 & 0.06 \\
\hline $\mathrm{CuO}$ & 0.02 & 0.01 & 0.02 & 0.03 & 0.03 \\
\hline $\mathbf{F e}_{2} \mathbf{O}_{3}$ & 7.80 & 7.91 & 8.66 & 9.47 & 9.33 \\
\hline$\overline{K_{2}} \mathbf{O}$ & 0.08 & 0.02 & 0.12 & 0.12 & 0.11 \\
\hline $\mathbf{L i}_{2} \mathbf{O}$ & 5.19 & 5.46 & 5.49 & 5.43 & 5.27 \\
\hline MgO & 0.78 & 0.83 & 0.83 & 0.91 & 0.91 \\
\hline MnO & 1.43 & 1.58 & 1.80 & 1.96 & 1.94 \\
\hline $\mathrm{Na}_{2} \mathrm{O}$ & 12.47 & 12.30 & 12.40 & 13.00 & 12.39 \\
\hline $\mathrm{NiO}$ & 0.41 & 0.43 & 0.45 & 0.49 & 0.50 \\
\hline $\mathrm{SO}_{4}$ & 0.39 & 0.39 & 0.39 & 0.40 & 0.41 \\
\hline $\mathrm{SiO}_{2}$ & 51.25 & 51.93 & 48.72 & 47.94 & 46.87 \\
\hline $\mathrm{TiO}_{2}$ & 0.09 & 0.07 & 0.02 & 0.02 & 0.02 \\
\hline $\mathrm{U}_{3} \mathrm{O}_{8}$ & 2.73 & 2.67 & 2.68 & 2.94 & 2.92 \\
\hline$\overline{\mathrm{ZrO}_{2}}$ & 0.00 & 0.14 & 0.02 & 0.02 & 0.02 \\
\hline
\end{tabular}


WSRC-STI-2008-00149

Revision 0

Table 9. Normalized* PCT Responses of the Frit 510 - SB4 Supplemental Glasses (quenched and (CC)

\begin{tabular}{|l|c|c|c|c|}
\hline \multirow{2}{*}{ Sample } & \multicolumn{4}{|c|}{ NL (g/L) } \\
\cline { 2 - 5 } & $\mathbf{B}$ & $\mathbf{L i}$ & $\mathbf{N a}$ & $\mathbf{S i}$ \\
\hline SB4PS-1 & 0.44 & 0.43 & 0.62 & 0.46 \\
\hline SB4PS-1-ccc & 0.41 & 0.41 & 0.61 & 0.45 \\
\hline SB4PS-2 & 0.44 & 0.43 & 0.63 & 0.46 \\
\hline SB4PS-2-ccc & 0.42 & 0.42 & 0.61 & 0.45 \\
\hline SB4PS-3 & 0.52 & 0.45 & 0.69 & 0.48 \\
\hline SB4PS-3-ccc & 0.49 & 0.44 & 0.66 & 0.48 \\
\hline SB4PS-4 & 0.49 & 0.43 & 0.66 & 0.47 \\
\hline SB4PS-4-ccc & 0.52 & 0.45 & 0.70 & 0.50 \\
\hline SB4PS-5 & 0.50 & 0.44 & 0.74 & 0.48 \\
\hline SB4PS-5-ccc & 0.50 & 0.44 & 0.71 & 0.49 \\
\hline
\end{tabular}

*Based on measured compositions

\subsection{Conclusions}

Frit 510 is a viable option for the processing of SB4 after a Tank 40 decant and the addition of products from the Actinide Removal Process (ARP). The addition of ARP did not have any negative impacts on the acceptability and predictability of the variability study glasses.

The durabilities of all of the study glasses (both quenched and ccc) are well below that of the NL [B] reference EA glass $(16.695 \mathrm{~g} / \mathrm{L})$. For the quenched glasses, the NL [B] (based on measured bc compositions) ranges from $0.65 \mathrm{~g} / \mathrm{L}$ (SB4VAR21) to $0.83 \mathrm{~g} / \mathrm{L}$ (SB4VAR13). With respect to the ccc versions of the variability study glasses, the NL [B] (based on measured bc compositions) range from $0.65 \mathrm{~g} / \mathrm{L}$ (SB4VAR21ccc) to $1.77 \mathrm{~g} / \mathrm{L}$ (SB4VAR24ccc). A gradual decrease in durability is observed with increasing WL for the quenched glasses. Although a similar trend is observed for the ccc glasses, the primary distinction is the magnitude of the difference at higher WLs. The durabilities of all of the study glasses are predictable using the current Product Composition Control System (PCCS) durability models with the exception of SB4VAR24ccc (Case \#2 at 41\%). PCCS is not applicable to non-homogeneous glasses (i.e. glasses containing crystals such as acmite and nepheline), thus SB4VAR24ccc should not be predictable as it contains nepheline.

The presence of nepheline has been confirmed in both SB4VAR13ccc and SB4VAR24ccc by X-ray diffraction (XRD). These two glasses are the first results which indicate that the current nepheline discriminator value of 0.62 is not conservative. The nepheline discriminator was implemented into PCCS for SB4 based on the fact that all of the historical glasses evaluated with nepheline values of 0.62 or greater did not contain nepheline via XRD analysis. Although these two glasses do cause some concern over the use of the 0.62 nepheline value for future DWPF glass systems, the impact to the current SB4 system is of little concern. Specifically, the formation of nepheline was observed in glasses targeting 41 or $42 \%$ WL. Current processing of the Frit 510-SB4 system in DWPF has nominally targeted 34\% WL. For the SB4 variability study glasses targeting these lower WLs, nepheline formation was not observed and the minimal difference in PCT response between quenched 
WSRC-STI-2008-00149

Revision 0

and ccc versions supported its absence.

\subsection{References}

1. M.E. Stone, F.C. Raszewski, B.R. Pickenheim, T.B. Edwards, and D.K. Peeler, "Impacts of a Tank 40 Supernate Decant to DWPF Glass Formulation and CPC Processing: A Preliminary Assessment for SB4," Savannah River National Laboratory, Aiken, SC, Report No. SRNLPSE-2007-00257, 2007.

2. B.A. Davis, "SB4 Flowsheet and Variability Studies for Tank 40H 100,000 Gallon Decant Technical Task Request," Liquid Waste Organization, Aiken, SC, Report No. HLW-DWPFTTR-2008-0009, Rev. 0, 2007.

3. F.C. Raszewski, T.B. Edwards, and D.K. Peeler, "The Impact of a Tank 40H Decant on the Projected Operating Windows for SB4 and Glass Selection Strategy in Support of the Variability Study," Savannah River National Laboratory, Aiken, SC, Report No. WSRC-STI2008-00017, 2008.

4. A.S. Taylor, T.B. Edwards, J.C. George, T.K. Snyder, and D.K. Peeler, "The SRNL Composition - Properties (ComPro ${ }^{\mathrm{TM}}$ ) Database," Westinghouse Savannah River Company, Aiken, SC, Report No. WSRC-RP-2004-00704, Revision 0, 2004.

5. $\quad$ K.M. Fox, T.B. Edwards, D.K. Peeler, D.R. Best, I.A. Reamer, and R.J. Workman, "High Level Waste (HLW) Sludge Batch 4 (SB4) Variability Study," Washington Savannah River Company, Aiken, SC, Report No. WSRC-STI-2006-00204, Revision 0, 2006.

6. K.M. Fox, T.B. Edwards, D.K. Peeler, D.R. Best, I.A. Reamer, and R.J. Workman, "High Level Waste (HLW) Sludge Batch 4 (SB4) with Frit 418: Results of a Phase II Variability Study," Washington Savannah River Company, Aiken, SC, Report No. WSRC-STI-200600329, Revision 0, 2006.

7. S.L. Marra and C.M. Jantzen, "Characterization of Projected DWPF Glass Heat Treated to Simulate Canister Centerline Cooling," Westinghouse Savannah River Company, Aiken, SC, Report No. WSRC-TR-92-142, Rev. 1, 1993.

8. "Glass Batching," Savannah River National Laboratory, Aiken, SC, Report No. ITS-0001, Rev. 1, 2007.

9. "Glass Melting," Savannah River National Laboratory, Aiken, SC, Report No. ITS-0003, Rev. 2, 2007.

10. T.B. Edwards, "Analytical Plans for Measuring the Chemical Compositions of Glasses from an EM-20 Study and Glasses from Two DWPF Studies," Savannah River National Laboratory, Aiken, SC, Report No. SRNL-SCS-2008-00003, 2008.

11. "Standard Test Methods for Determining Chemical Durability of Nuclear, Hazardous, and Mixed Waste Glasses and Multiphase Glass Ceramics: The Product Consistency Test (PCT)," ASTM International, West Conshohocken, PA, Report No. ASTM C 1285-02, 2002. 
12. C.M. Jantzen, N.E. Bibler, D.C. Beam, C.L. Crawford, and M.A. Pickett, "Characterization of the Defense Waste Processing Facility (DWPF) Environmental Assessment (EA) Glass Standard Reference Material," Westinghouse Savannah River Company, Aiken, SC, Report No. WSRC-TR-92-346, Rev. 1, 1993.

13. T.B. Edwards, "Analytical Plans for Measuring the PCT Solutions of Glasses from an EM-20 Study and Glasses from Two DWPF Studies," Savannah River National Laboratory, Aiken, SC, Report No. SRNL-SCS-2008-00005, 2008.

14. C.M. Jantzen, J.B. Picket, K.G. Brown, T.B. Edwards, and D.C. Beam, "Process/Product Models for the Defense Waste Processing Facility (DWPF): Part I. Predicting Glass Durability from Composition Using a Thermodynamic Hydration Energy Reaction Model (THERMO)," Westinghouse Savannah River Company, Aiken, SC, Report No. WSRC-TR93-672, Rev. 1, 1995.

15. T.B. Edwards, D.K. Peeler, and K.M. Fox, "The Nepheline Discriminator: Justification and DWPF PCCS Implementation Details," Washington Savannah River Company, Aiken, SC, Report No. WSRC-STI-2006-00014, Revision 0, 2006. 


\section{APPENDIX A}

TABLES AND EXHIBITS SUPPORTING THE ANALYSIS OF THE CHEMICAL COMPOSITION MEASUREMENTS OF THE SB4/DECANT VARIABILITY STUDY GLASSES 
WSRC-STI-2008-00149

Revision 0

This page intentionally left blank. 
Table A1. Measured Elemental Concentrations (wt\%) for Samples Prepared Using Lithium Metaborate (part 1)

\begin{tabular}{|c|c|c|c|c|c|c|c|c|c|c|c|c|c|c|c|c|c|c|c|}
\hline Set & Glass ID & Block & $\begin{array}{c}\text { Sub- } \\
\text { Block }\end{array}$ & Sequence & Lab ID & $\begin{array}{c}\begin{array}{c}\mathrm{Ag} \\
\text { (wt\%) }\end{array} \\
\end{array}$ & $\begin{array}{c}\mathrm{Al} \\
\text { (wt\%) }\end{array}$ & $\begin{array}{c}\begin{array}{c}\mathrm{Ba} \\
\text { (wt\%) }\end{array} \\
\text { (a) }\end{array}$ & $\begin{array}{c}\mathrm{Ca} \\
\text { (wt\%) }\end{array}$ & $\begin{array}{c}\begin{array}{c}\mathrm{Cd} \\
\text { (wt\%) }\end{array} \\
(\mathrm{w})\end{array}$ & $\begin{array}{c}\mathrm{Ce} \\
\text { (wt\%) }\end{array}$ & $\begin{array}{c}\begin{array}{c}\mathrm{Co} \\
\text { (wt\%) }\end{array} \\
\end{array}$ & $\begin{array}{c}\mathrm{Cr} \\
\text { (wt\%) }\end{array}$ & $\begin{array}{c}\begin{array}{c}\mathrm{Cu} \\
\text { (wt\%) }\end{array} \\
\text { (a) }\end{array}$ & $\begin{array}{c}\mathrm{Fe} \\
\text { (wt\%) }\end{array}$ & $\begin{array}{c}\mathrm{K} \\
\text { (wt\%) }\end{array}$ & $\begin{array}{c}\begin{array}{c}\mathrm{La} \\
\text { (wt\%) }\end{array} \\
\end{array}$ & $\begin{array}{c}\begin{array}{c}\mathrm{Mg} \\
\text { (wt\%) }\end{array} \\
\end{array}$ & $\begin{array}{c}\mathrm{Mn} \\
\text { (wt\%) }\end{array}$ \\
\hline$\frac{1}{1}$ & Batch 1 & 1 & 1 & 1 & BCHLM1-111 & $<0.100$ & 2.55 & 0.124 & 0.834 & $<0.010$ & 0.019 & $<0.010$ & 0.071 & 0.297 & 9.14 & 3.09 & $<0.100$ & 0.821 & 1.38 \\
\hline 1 & U std & 1 & $\frac{1}{1}$ & $\frac{1}{2}$ & UstdLM1-111 & $<0.100$ & 2.07 & $<0.010$ & 0.896 & $<0.010$ & $<0.010$ & $<0.010$ & 0.158 & $<0.010$ & 9.24 & 2.87 & $<0.100$ & 0.705 & 2.2 \\
\hline 1 & SB4VAR51 & 1 & 1 & 3 & M04LM11 & $<0.100$ & 4.05 & 0.017 & 0.569 & $<0.010$ & 0.017 & $<0.010$ & 0.035 & 0.021 & 6.08 & 0.094 & $<0.100$ & 0.486 & 1.44 \\
\hline 1 & SB4VAR42 & 1 & 1 & 4 & M05LM11 & $<0.100$ & 4.6 & 0.02 & 0.654 & $<0.010$ & 0.019 & $<0.010$ & 0.038 & 0.022 & 7.01 & 0.111 & $<0.100$ & 0.558 & 1.65 \\
\hline$\frac{1}{1}$ & SB4VAR43 & 1 & $\frac{1}{1}$ & 5 & M06LM21 & $<0.100$ & 5.1 & 0.025 & 0.724 & $<0.010$ & 0.021 & $<0.010$ & 0.035 & 0.024 & 7.85 & 0.118 & $<0.100$ & 0.609 & 1.86 \\
\hline 1 & SB4VAR41 & 1 & 1 & 6 & M01LM21 & $<0.100$ & 4.38 & 0.018 & 0.615 & $<0.010$ & 0.017 & $<0.010$ & 0.037 & 0.019 & 6.54 & 0.107 & $<0.100$ & 0.501 & 1.55 \\
\hline 1 & SB4VAR54 & 1 & 1 & 7 & M03LM11 & $<0.100$ & 5.28 & 0.023 & 0.736 & $<0.010$ & 0.02 & $<0.010$ & 0.041 & 0.025 & 8.01 & 0.119 & $<0.100$ & 0.628 & 1.88 \\
\hline 1 & SB4VAR51 & 1 & 1 & 8 & M04LM21 & $<0.100$ & 4.22 & 0.019 & 0.588 & $<0.010$ & 0.016 & $<0.010$ & 0.035 & 0.023 & 6.22 & 0.103 & $<0.100$ & 0.485 & 1.47 \\
\hline 1 & SB4VAR54 & 1 & 1 & 9 & M03LM21 & $<0.100$ & 5.22 & 0.023 & 0.737 & $<0.010$ & 0.02 & $<0.010$ & 0.041 & 0.024 & 7.81 & 0.121 & $<0.100$ & 0.635 & 1.84 \\
\hline 1 & Batch 1 & 1 & 1 & 10 & BCHLM1-112 & $<0.100$ & 2.58 & 0.128 & 0.838 & $<0.010$ & 0.018 & $<0.010$ & 0.073 & 0.298 & 9.09 & 3.06 & $<0.100$ & 0.844 & 1.37 \\
\hline 1 & U std & 1 & 1 & 11 & UstdLM1-112 & $<0.100$ & 2.09 & $<0.010$ & 0.905 & $<0.010$ & $<0.010$ & $<0.010$ & 0.163 & $<0.010$ & 9.27 & 2.93 & $<0.100$ & 0.729 & 2.2 \\
\hline$\frac{1}{1}$ & SB4VAR41 & $\frac{1}{1}$ & $\frac{1}{1}$ & $\frac{11}{12}$ & M01LM11 & $<0.100$ & $\begin{array}{ll}2.03 \\
4.31\end{array}$ & 0.019 & 0.016 & $<0.010$ & 0.018 & $\begin{array}{l}<0.010 \\
\end{array}$ & $\begin{array}{l}0.038 \\
0.038\end{array}$ & 0.02 & 6.21 & 0.108 & $<0.100$ & 0.517 & $\frac{2.2}{1.48}$ \\
\hline 1 & SB4VAR22 & 1 & 1 & 13 & M12LM11 & $<0.100$ & 4.79 & 0.023 & 0.681 & $<0.010$ & 0.015 & $<0.010$ & 0.045 & 0.022 & 6.89 & 0.106 & $<0.100$ & 0.615 & 1.57 \\
\hline 1 & SB4VAR43 & 1 & 1 & 14 & M06LM11 & $<0.100$ & 4.99 & 0.026 & 0.712 & $<0.010$ & 0.021 & $<0.010$ & 0.036 & 0.025 & 7.29 & 0.17 & $<0.100$ & 0.622 & 1.71 \\
\hline$\frac{1}{1}$ & SB4VAR12 & $\frac{1}{1}$ & $\frac{1}{1}$ & $\frac{147}{15}$ & M15LM11 & $<0.100$ & 5.08 & 0.02 & 0.703 & $<0.010$ & 0.016 & $<0.010$ & 0.041 & 0.023 & 7.33 & 0.127 & $<0.100$ & 0.028 & 1.68 \\
\hline 1 & SB4VAR22 & 1 & 1 & 16 & M12LM21 & $<0.100$ & 4.75 & 0.023 & 0.674 & $<0.010$ & 0.014 & $<0.010$ & 0.044 & 0.022 & 6.74 & 0.109 & $<0.100$ & 0.605 & 1.58 \\
\hline 1 & SB4VAR12 & 1 & 1 & 17 & M15LM21 & $<0.100$ & 5.03 & 0.021 & 0.703 & $<0.010$ & 0.016 & $<0.010$ & 0.042 & 0.022 & 7.22 & 0.114 & $<0.100$ & 0.618 & 1.65 \\
\hline 1 & SB4VAR42 & 1 & 1 & 18 & M05LM21 & $<0.100$ & 4.55 & 0.021 & 0.668 & $<0.010$ & 0.019 & $<0.010$ & 0.038 & 0.022 & 6.81 & 0.109 & $<0.100$ & 0.569 & 1.6 \\
\hline 1 & Batch 1 & 1 & 1 & 19 & BCHLM1-113 & $<0.100$ & 2.6 & 0.129 & 0.864 & $<0.010$ & 0.019 & $<0.010$ & 0.074 & $\begin{array}{l}0.022 \\
0.306\end{array}$ & 9.36 & 2.97 & $<0.100$ & $\begin{array}{l}0.0052 \\
0.852\end{array}$ & 1.41 \\
\hline 1 & U std & 1 & 1 & 20 & UstdLM1-113 & $<0.100$ & 2.11 & $<0.010$ & 0.909 & $<0.010$ & $<0.010$ & $<0.010$ & 0.164 & $<0.010$ & 9.63 & 2.85 & $<0.100$ & 0.728 & 2.29 \\
\hline 1 & Batch 1 & 1 & 2 & 1 & BCHLM1-121 & $<0.100$ & 2.49 & 0.127 & 0.833 & $<0.010$ & 0.02 & $<0.010$ & 0.072 & 0.302 & 8.76 & 3.02 & $<0.100$ & 0.829 & 1.28 \\
\hline 1 & U std & 1 & 2 & $\frac{1}{2}$ & UstdLM1-121 & $<0.100$ & 2.04 & $<0.010$ & 0.883 & $<0.010$ & $<0.010$ & $<0.010$ & 0.159 & $<0.010$ & 9.53 & 2.83 & $<0.100$ & 0.708 & 2.23 \\
\hline 1 & SB4VAR22 & $\frac{1}{1}$ & 2 & 3 & M12LM22 & $<0.100$ & 4.85 & 0.023 & $\begin{array}{l}0.0083 \\
\end{array}$ & $<0.010$ & 0.016 & $<0.010$ & 0.043 & 0.021 & 7.26 & $\begin{array}{l}2.05 \\
0.109\end{array}$ & $<0.100$ & 0.579 & 1.66 \\
\hline 1 & SB4VAR42 & 1 & 2 & 4 & M05LM12 & $<0.100$ & 4.64 & 0.021 & 0.656 & $<0.010$ & 0.02 & $<0.010$ & 0.038 & 0.022 & 6.99 & 0.11 & $<0.100$ & 0.554 & 1.6 \\
\hline 1 & SB4VAR51 & 1 & 2 & 5 & M04LM22 & $<0.100$ & 4.14 & 0.02 & 0.585 & $<0.010$ & 0.018 & $<0.010$ & 0.035 & 0.022 & 6.05 & 0.1 & $<0.100$ & 0.475 & 1.4 \\
\hline 1 & SB4VAR54 & $\frac{1}{1}$ & $\frac{2}{2}$ & 6 & M03LM22 & $<0.100$ & 5.18 & $\begin{array}{l}0.023 \\
\end{array}$ & 0.736 & $<0.010$ & 0.022 & $<0.010$ & 0.041 & $\begin{array}{l}0.023 \\
0.023\end{array}$ & 7.82 & 0.119 & $<0.100$ & 0.415 & 1.77 \\
\hline 1 & SB4VAR51 & 1 & 2 & 7 & M04LM12 & $<0.100$ & 4.07 & 0.018 & 0.574 & $<0.010$ & 0.018 & $<0.010$ & 0.035 & 0.021 & 6.03 & 0.094 & $<0.100$ & 0.475 & 1.4 \\
\hline 1 & SB4VAR12 & 1 & 2 & 8 & M15LM22 & $<0.100$ & 4.95 & 0.021 & 0.686 & $<0.010$ & 0.017 & $<0.010$ & 0.042 & 0.021 & 7.56 & 0.109 & $<0.100$ & 0.6 & 1.69 \\
\hline 1 & SB4VAR43 & 1 & 2 & 9 & M06LM12 & $<0.100$ & 4.88 & 0.027 & 0.698 & $<0.010$ & 0.022 & $<0.010$ & 0.035 & 0.024 & 7.91 & 0.166 & $<0.100$ & 0.596 & 1.83 \\
\hline 1 & Batch 1 & 1 & 2 & 10 & BCHLM1-122 & $<0.100$ & 2.46 & 0.126 & 0.845 & $<0.010$ & 0.02 & $<0.010$ & 0.072 & 0.299 & 9.77 & 2.89 & $<0.100$ & 0.827 & 1.43 \\
\hline 1 & U std & 1 & 2 & 11 & UstdLM1-122 & $<0.100$ & 2.04 & $<0.010$ & 0.886 & $<0.010$ & $<0.010$ & $<0.010$ & 0.16 & $<0.010$ & 9.93 & 2.83 & $<0.100$ & 0.704 & 2.34 \\
\hline 1 & SB4VAR41 & 1 & 2 & 12 & M01LM22 & $<0.100$ & 4.16 & 0.019 & 0.608 & $<0.010$ & 0.019 & $<0.010$ & 0.037 & 0.018 & 6.42 & 0.105 & $<0.100$ & 0.488 & 1.49 \\
\hline 1 & SB4VAR22 & 1 & 2 & 13 & M12LM12 & $<0.100$ & 4.77 & 0.023 & 0.668 & $<0.010$ & 0.016 & $<0.010$ & 0.044 & 0.021 & 7.64 & 0.102 & $<0.100$ & 0.586 & 1.71 \\
\hline$\frac{1}{1}$ & SB4VAR42 & $\frac{1}{1}$ & $\frac{2}{2}$ & $\frac{5}{14}$ & M05LM22 & $<0.100$ & 4.58 & 0.021 & 0.65 & $<0.010$ & 0.02 & $<0.010$ & 0.038 & $\begin{array}{l}0.021 \\
0.021\end{array}$ & 7.47 & 0.105 & $<0.100$ & 0.555 & 1.73 \\
\hline 1 & SB4VAR12 & 1 & 2 & 15 & M15LM12 & $<0.100$ & 5.02 & 0.021 & 0.709 & $<0.010$ & 0.017 & $<0.010$ & 0.041 & 0.022 & 8.33 & 0.125 & $<0.100$ & 0.576 & 1.89 \\
\hline 1 & SB4VAR43 & 1 & 2 & 16 & M06LM22 & $<0.100$ & 4.96 & 0.026 & 0.697 & $<0.010$ & 0.022 & $<0.010$ & 0.035 & 0.023 & 8.38 & 0.112 & $<0.100$ & 0.602 & 1.96 \\
\hline$\frac{1}{1}$ & SB4VAR41 & $\frac{1}{1}$ & $\frac{2}{2}$ & 17 & M01LM12 & $<0.100$ & $\begin{array}{l}4.30 \\
4.38\end{array}$ & 0.020 & $\begin{array}{l}0.0304 \\
0.604\end{array}$ & $<0.010$ & 0.019 & $<0.010$ & $\begin{array}{l}0.035 \\
0.038\end{array}$ & $\begin{array}{l}0.025 \\
0.019\end{array}$ & 6.71 & 0.103 & $<0.100$ & $\begin{array}{l}0.002 \\
0.509\end{array}$ & 1.56 \\
\hline 1 & SB4VAR54 & 1 & 2 & 18 & M03LM12 & $<0.100$ & 5.2 & 0.023 & 0.733 & $<0.010$ & 0.022 & $<0.010$ & 0.041 & 0.024 & 7.95 & 0.117 & $<0.100$ & 0.618 & 1.84 \\
\hline 1 & Batch 1 & 1 & 2 & 19 & BCHLM1-123 & $<0.100$ & 2.47 & 0.123 & 0.842 & $<0.010$ & 0.019 & $<0.010$ & 0.071 & 0.295 & 8.88 & 2.94 & $<0.100$ & 0.817 & 1.3 \\
\hline$\frac{1}{1}$ & $\frac{\mathrm{Dathi}}{\mathrm{U} \text { std }}$ & $\frac{1}{1}$ & $\frac{2}{2}$ & $\frac{15}{20}$ & $\begin{array}{l}\text { DUnLIII-120 } \\
\text { UstdLM1-123 }\end{array}$ & $<0.100$ & $\begin{array}{l}2 . .47 \\
.04\end{array}$ & $\frac{0.123}{<0.010}$ & 0.0481 & $<0.010$ & $\frac{0.017}{<0.010}$ & $<<0.010$ & 0.158 & $\frac{0.253}{<0.010}$ & 0.15 & 2.83 & $<0.100$ & $\begin{array}{l}0.017 \\
0.699 \\
\end{array}$ & 2.15 \\
\hline$\frac{1}{1}$ & Batch 1 & $\frac{1}{2}$ & 1 & 1 & BCHLM1-211 & $<0.100$ & 2.52 & 0.126 & 0.853 & $<0.010$ & 0.02 & $<0.010$ & 0.072 & 0.3 & 8.72 & $\begin{array}{ll}2.05 \\
.01\end{array}$ & $<0.100$ & 0.814 & 1.32 \\
\hline 1 & U std & 2 & 1 & $\frac{1}{2}$ & UstdLM1-211 & $<0.100$ & 2.08 & $<0.010$ & 0.908 & $<0.010$ & $<0.010$ & $<0.010$ & 0.16 & $<0.010$ & 8.99 & 2.93 & $<0.100$ & 0.707 & 2.15 \\
\hline 1 & SB4VAR11 & 2 & 1 & 3 & $\begin{array}{l}\text { M02LM21 } \\
\end{array}$ & $<0.100$ & 4.5 & 0.02 & 0.632 & $<0.010$ & 0.017 & $<0.010$ & 0.04 & 0.02 & 6.4 & 0.1 & $<0.100$ & 0.54 & 1.46 \\
\hline 1 & SB4VAR53 & $\frac{2}{2}$ & $\frac{1}{1}$ & 4 & M11LM11 & $<0.100$ & $\begin{array}{l}4.54 \\
4.84\end{array}$ & 0.023 & $\begin{array}{l}0.032 \\
0.708\end{array}$ & $<0.010$ & 0.02 & $<0.010$ & 0.041 & 0.022 & 6.95 & 0.156 & $<0.100$ & 0.567 & 1.46 \\
\hline$\frac{1}{1}$ & SB4VAR44 & $\frac{2}{2}$ & $\frac{1}{1}$ & 5 & M08LM11 & $<0.100$ & 5.44 & 0.026 & 0.773 & $<0.010$ & 0.022 & $<0.010$ & $\begin{array}{l}0.0412 \\
0.042\end{array}$ & 0.025 & 7.66 & 0.13 & $<0.100$ & 0.634 & 1.85 \\
\hline 1 & SB4VAR52 & 2 & 1 & 6 & M09LM11 & $<0.100$ & 4.61 & 0.021 & 0.643 & $<0.010$ & 0.019 & $<0.010$ & 0.039 & 0.02 & 6.58 & 0.098 & $<0.100$ & 0.549 & 1.56 \\
\hline 1 & SB4VAR13 & 2 & $\frac{1}{1}$ & 7 & M14LM21 & $<0.100$ & 5.46 & 0.026 & 0.769 & $<0.010$ & 0.02 & $<0.010$ & 0.042 & 0.025 & 7.8 & 0.113 & $<0.100$ & 0.667 & 1.81 \\
\hline 1 & SB4VAR23 & 2 & $\frac{1}{1}$ & 8 & M10LM11 & $<0.100$ & 5.66 & 0.024 & 0.734 & $<0.010$ & 0.017 & $<0.010$ & 0.039 & 0.025 & 7.23 & 0.114 & $<0.100$ & 0.63 & 1.69 \\
\hline 1 & SB4VAR23 & 2 & 1 & 9 & M10LM21 & $<0.100$ & 5.12 & 0.023 & 0.726 & $<0.010$ & 0.017 & $<0.010$ & 0.037 & 0.023 & 7.29 & 0.122 & $<0.100$ & 0.604 & 1.71 \\
\hline 1 & SB4VAR24 & 2 & 1 & 10 & M07LM11 & $<0.100$ & 5.62 & 0.025 & 0.812 & $<0.010$ & 0.021 & $<0.010$ & 0.04 & 0.025 & 7.68 & 0.15 & $<0.100$ & 0.642 & 1.84 \\
\hline 1 & Batch 1 & $\frac{2}{2}$ & $\frac{1}{1}$ & 11 & BCHLM1-212 & $<0.100$ & $\begin{array}{l}0.02 \\
2.53\end{array}$ & 0.026 & $\begin{array}{l}0.038 \\
0.838\end{array}$ & $<0.010$ & 0.019 & $<0.010$ & $\begin{array}{l}0.072 \\
0.072\end{array}$ & $\begin{array}{l}0.0299 \\
0.299\end{array}$ & 8.32 & $\begin{array}{l}0.15 \\
3.02 \\
\end{array}$ & $<0.100$ & 0.01 & 1.04 \\
\hline 1 & U std & 2 & $\frac{1}{1}$ & 12 & UstdLM1-212 & $<0.100$ & 2.08 & $<0.010$ & 0.0907 & $<0.010$ & $<0.010$ & $<0.010$ & 0.159 & $<0.010$ & 8.51 & 2.89 & $<0.100$ & 0.694 & 2.05 \\
\hline
\end{tabular}


Table A1. Measured Elemental Concentrations (wt\%) for Samples Prepared Using Lithium Metaborate (part 1)

\begin{tabular}{|c|c|c|c|c|c|c|c|c|c|c|c|c|c|c|c|c|c|c|c|}
\hline Set & Glass ID & Block & $\begin{array}{c}\text { Sub- } \\
\text { Block }\end{array}$ & Sequence & Lab ID & $\begin{array}{c}\begin{array}{c}\mathrm{Ag} \\
\text { (wt\%) }\end{array} \\
\end{array}$ & $\begin{array}{c}\mathrm{Al} \\
\text { (wt\%) }\end{array}$ & $\begin{array}{c}\begin{array}{c}\mathrm{Ba} \\
\text { (wt\%) }\end{array} \\
\text { (a) }\end{array}$ & $\begin{array}{c}\mathrm{Ca} \\
\text { (wt\%) }\end{array}$ & $\begin{array}{c}\mathrm{Cd} \\
\text { (wt\%) }\end{array}$ & $\begin{array}{c}\mathrm{Ce} \\
(\mathrm{wt} \%)\end{array}$ & $\begin{array}{c}\begin{array}{c}\mathrm{Co} \\
\text { (wt\%) }\end{array} \\
\end{array}$ & $\begin{array}{c}\mathrm{Cr} \\
\text { (wt\%) }\end{array}$ & $\begin{array}{c}\begin{array}{c}\mathrm{Cu} \\
\text { (wt\%) }\end{array} \\
\text { (a) }\end{array}$ & $\begin{array}{c}\mathrm{Fe} \\
\text { (wt\%) }\end{array}$ & $\begin{array}{c}\mathrm{K} \\
\text { (wt\%) }\end{array}$ & $\begin{array}{c}\begin{array}{c}\mathrm{La} \\
\text { (wt\%) }\end{array} \\
\end{array}$ & $\begin{array}{c}\mathrm{Mg} \\
\text { (wt\%) }\end{array}$ & $\begin{array}{c}\mathrm{Mn} \\
\text { (wt\%) }\end{array}$ \\
\hline$\frac{1}{1}$ & SB4VAR13 & $\frac{1}{2}$ & 1 & 13 & M14LM11 & $<0.100$ & 5.49 & 0.026 & 0.777 & $<0.010$ & 0.021 & $<0.010$ & 0.042 & 0.024 & 7.59 & 0.116 & $<0.100$ & 0.674 & 1.76 \\
\hline 1 & SB4VAR11 & 2 & $\frac{1}{1}$ & 14 & M02LM11 & $<0.100$ & 4.5 & 0.019 & 0.632 & $<0.010$ & 0.016 & $<0.010$ & 0.038 & 0.019 & 6.06 & 0.111 & $<0.100$ & 0.518 & 1.4 \\
\hline 1 & SB4VAR21 & 2 & 1 & 15 & M13LM21 & $<0.100$ & 4.42 & 0.021 & 0.633 & $<0.010$ & 0.015 & $<0.010$ & 0.038 & 0.02 & 5.86 & 0.099 & $<0.100$ & 0.522 & 1.36 \\
\hline 1 & SB4VAR53 & 2 & 1 & 16 & M11LM21 & $<0.100$ & 4.79 & 0.023 & 0.712 & $<0.010$ & 0.02 & $<0.010$ & 0.04 & 0.024 & 6.65 & 0.117 & $<0.100$ & 0.567 & 1.59 \\
\hline$\frac{1}{1}$ & SB4VAR52 & 2 & $\frac{1}{1}$ & 17 & M09LM21 & $<0.100$ & 4.43 & 0.021 & 0.64 & $<0.010$ & 0.019 & $<0.010$ & 0.039 & 0.02 & 6.29 & 0.095 & $<0.100$ & 0.536 & 1.49 \\
\hline 1 & SB4VAR44 & 2 & 1 & 18 & M08LM21 & $<0.100$ & 5.28 & 0.026 & 0.765 & $<0.010$ & 0.022 & $<0.010$ & 0.048 & 0.024 & 7.05 & 0.117 & $<0.100$ & 0.638 & 1.7 \\
\hline 1 & SB4VAR24 & 2 & 1 & 19 & M07LM21 & $<0.100$ & 5.56 & 0.025 & 0.807 & $<0.010$ & 0.021 & $<0.010$ & 0.042 & 0.025 & 7.74 & 0.18 & $<0.100$ & 0.663 & 1.84 \\
\hline 1 & SB4VAR21 & 2 & 1 & 20 & M13LM11 & $<0.100$ & 4.38 & 0.021 & 0.624 & $<0.010$ & 0.015 & $<0.010$ & 0.038 & 0.02 & 6.1 & 0.098 & $<0.100$ & 0.526 & 1.41 \\
\hline 1 & Batch 1 & 2 & 1 & 21 & BCHLM1-213 & $<0.100$ & 2.51 & 0.125 & 0.854 & $<0.010$ & 0.019 & $<0.010$ & 0.072 & 0.303 & 8.45 & 2.96 & $<0.100$ & 0.812 & 1.28 \\
\hline 1 & U std & 2 & 1 & 22 & UstdLM1-213 & $<0.100$ & 2.06 & $<0.010$ & 0.911 & $<0.010$ & $<0.010$ & $<0.010$ & 0.157 & $<0.010$ & 8.7 & 2.84 & $<0.100$ & 0.689 & 2.08 \\
\hline 1 & Batch 1 & 2 & 2 & 1 & BCHLM1-221 & $<0.100$ & 2.51 & 0.128 & 0.847 & $<0.010$ & 0.019 & $<0.010$ & 0.073 & 0.299 & 8.82 & 2.98 & $<0.100$ & 0.815 & 1.34 \\
\hline$\frac{1}{1}$ & U std & $\frac{2}{2}$ & $\frac{2}{2}$ & $\frac{1}{2}$ & UstdLM1-221 & $<0.100$ & 2.06 & $<0.010$ & $\begin{array}{l}0.047 \\
0.924\end{array}$ & $<0.010$ & $<0.010$ & $\begin{array}{l}<0.010 \\
\end{array}$ & $\begin{array}{l}0.158 \\
0.158\end{array}$ & $<0.010$ & $\begin{array}{l}0.02 \\
8.96\end{array}$ & 2.84 & $<0.100$ & 0.098 & $\frac{1.04}{2.14}$ \\
\hline 1 & SB4VAR24 & 2 & 2 & 3 & M07LM22 & $<0.100$ & 5.68 & 0.027 & 0.807 & $<0.010$ & 0.021 & $<0.010$ & 0.043 & 0.024 & 7.84 & 0.18 & $<0.100$ & 0.666 & 1.87 \\
\hline 1 & SB4VAR13 & 2 & 2 & 4 & M14LM12 & $<0.100$ & 5.51 & 0.028 & 0.789 & $<0.010$ & 0.02 & $<0.010$ & 0.042 & 0.024 & 7.81 & 0.118 & $<0.100$ & 0.676 & 1.8 \\
\hline$\frac{1}{1}$ & SB4VAR24 & $\frac{2}{2}$ & $\frac{2}{2}$ & $\frac{4}{5}$ & M07LM12 & $<0.100$ & 5.64 & 0.026 & 0.822 & $<0.010$ & 0.021 & $<0.010$ & 0.041 & 0.025 & 7.91 & 0.151 & $<0.100$ & 0.639 & 1.89 \\
\hline 1 & SB4VAR23 & 2 & 2 & 6 & M10LM12 & $<0.100$ & 5.81 & 0.025 & 0.752 & $<0.010$ & 0.017 & $<0.010$ & 0.038 & 0.025 & 7.39 & 0.117 & $<0.100$ & 0.628 & 1.72 \\
\hline 1 & SB4VAR52 & 2 & 2 & 7 & M09LM12 & $<0.100$ & 4.62 & 0.023 & 0.642 & $<0.010$ & 0.019 & $<0.010$ & 0.04 & 0.02 & 6.72 & 0.096 & $<0.100$ & 0.549 & 1.59 \\
\hline 1 & SB4VAR21 & 2 & 2 & 8 & M13LM22 & $<0.100$ & 4.42 & 0.023 & 0.617 & $<0.010$ & 0.014 & $<0.010$ & 0.038 & 0.02 & 6.28 & 0.095 & $<0.100$ & 0.528 & 1.45 \\
\hline 1 & $\begin{array}{l}\text { SB4VAR23 } \\
\end{array}$ & 2 & 2 & 9 & M10LM22 & $<0.100$ & 5.2 & 0.024 & 0.739 & $<0.010$ & 0.017 & $<0.010$ & 0.037 & 0.023 & 7.3 & 0.035 & $<0.100$ & 0.602 & 1.71 \\
\hline 1 & SB4VAR11 & 2 & 2 & 10 & M02LM12 & $<0.100$ & 4.53 & 0.021 & 0.631 & $<0.010$ & 0.016 & $<0.010$ & 0.038 & 0.019 & 6.35 & 0.11 & $<0.100$ & 0.518 & 1.46 \\
\hline 1 & Batch 1 & 2 & 2 & 11 & BCHLM1-222 & $<0.100$ & 2.65 & 0.128 & 0.846 & $<0.010$ & 0.019 & $<0.010$ & 0.073 & 0.301 & 8.77 & 3.01 & $<0.100$ & 0.823 & 1.34 \\
\hline 1 & U std & 2 & 2 & 12 & UstdLM1-222 & $<0.100$ & 2.1 & $<0.010$ & 0.89 & $<0.010$ & $<0.010$ & $<0.010$ & 0.16 & $<0.010$ & 8.85 & 2.86 & $<0.100$ & 0.701 & 2.12 \\
\hline 1 & SB4VAR53 & 2 & 2 & 13 & M11LM22 & $<0.100$ & 4.87 & 0.025 & 0.702 & $<0.010$ & 0.02 & $<0.010$ & 0.041 & 0.023 & 6.66 & 0.115 & $<0.100$ & 0.568 & 1.59 \\
\hline 1 & SB4VAR44 & 2 & 2 & 14 & M08LM12 & $<0.100$ & 5.59 & 0.027 & 0.774 & $<0.010$ & 0.022 & $<0.010$ & 0.042 & 0.025 & 7.26 & 0.129 & $<0.100$ & 0.635 & 1.77 \\
\hline 1 & SB4VAR52 & 2 & 2 & 15 & M09LM22 & $<0.100$ & 4.5 & 0.023 & 0.634 & $<0.010$ & 0.019 & $<0.010$ & 0.039 & 0.02 & 6.26 & 0.093 & $<0.100$ & 0.54 & 1.48 \\
\hline 1 & SB4VAR11 & $\frac{2}{2}$ & $\frac{2}{2}$ & 16 & M02LM22 & $<0.100$ & $\begin{array}{l}4.5 \\
4.54\end{array}$ & 0.022 & $\begin{array}{l}0.034 \\
0.628\end{array}$ & $<0.010$ & 0.016 & $<0.010$ & 0.04 & 0.02 & 6.43 & 0.098 & $<0.100$ & 0.541 & $\begin{array}{l}1.40 \\
1.48\end{array}$ \\
\hline 1 & SB4VAR44 & 2 & 2 & 17 & M08LM22 & $<0.100$ & 5.4 & 0.028 & 0.763 & $<0.010$ & 0.022 & $<0.010$ & 0.049 & 0.024 & 7.6 & 0.116 & $<0.100$ & 0.65 & 1.84 \\
\hline 1 & SB4VAR21 & 2 & 2 & 18 & M13LM12 & $<0.100$ & 4.49 & 0.023 & 0.629 & $<0.010$ & 0.014 & $<0.010$ & 0.038 & 0.02 & 6.3 & 0.096 & $<0.100$ & 0.531 & 1.46 \\
\hline 1 & SB4VAR13 & 2 & 2 & 19 & M14LM22 & $<0.100$ & 5.54 & 0.027 & 0.77 & $<0.010$ & 0.02 & $<0.010$ & 0.042 & 0.024 & 7.85 & 0.115 & $<0.100$ & 0.663 & 1.82 \\
\hline$\frac{1}{1}$ & SB4VAR53 & $\frac{2}{2}$ & $\frac{z}{2}$ & 20 & M11LM12 & $<0.100$ & $\begin{array}{l}0.04 \\
4.91\end{array}$ & 0.024 & 0.712 & $<0.010$ & $\begin{array}{l}0.02 \\
0.019\end{array}$ & $<0.010$ & 0.04 & $\begin{array}{l}0.024 \\
0.022\end{array}$ & 6.95 & 0.159 & $<0.100$ & 0.0568 & 1.02 \\
\hline 1 & Batch 1 & 2 & 2 & 21 & BCHLM1-223 & $<0.100$ & 2.5 & 0.127 & 0.859 & $<0.010$ & 0.019 & $<0.010$ & 0.072 & 0.301 & 8.21 & 2.88 & $<0.100$ & 0.813 & 1.25 \\
\hline 1 & U std & 2 & 2 & 22 & UstdLM1-223 & $<0.100$ & 2.12 & $<0.010$ & 0.895 & $<0.010$ & $<0.010$ & $<0.010$ & 0.156 & $<0.010$ & 8.96 & 2.88 & $<0.100$ & 0.694 & 2.14 \\
\hline 2 & Batch 1 & 1 & 1 & 1 & BCHLM2-111 & & 2.49 & 0.126 & 0.834 & $<0.010$ & 0.011 & $<0.010$ & 0.073 & 0.295 & 9.33 & 2.49 & $<0.100$ & 0.819 & 1.42 \\
\hline$\frac{2}{2}$ & U std & $\frac{1}{1}$ & $\frac{1}{1}$ & $\frac{1}{2}$ & UstdLM2-111 & & 2.03 & $<0.010$ & $\begin{array}{l}0.0342 \\
0.892\end{array}$ & $<0.010$ & 0.001 & $<0.010$ & 0.162 & $<0.010$ & 9.5 & 2.22 & $<0.100$ & 0.71 & $\frac{1.42}{2.27}$ \\
\hline 2 & SB5-01 & 1 & 1 & 3 & N02LM11 & & 5.73 & 0.031 & 2.41 & 0.014 & 0.084 & $<0.010$ & 0.077 & $<0.010$ & 6.26 & 0.014 & $<0.100$ & 0.263 & 1.51 \\
\hline 2 & SB5-05 & 1 & 1 & 4 & N07LM11 & & 5.12 & 0.035 & 2.5 & 0.013 & 0.052 & $<0.010$ & 0.087 & $<0.010$ & 6.87 & 0.013 & $<0.100$ & 0.296 & 1.66 \\
\hline$\frac{2}{2}$ & SB5-04 & $\frac{1}{1}$ & $\frac{1}{1}$ & $\frac{4}{5}$ & N03LM21 & & $\begin{array}{l}5.82 \\
5.89\end{array}$ & 0.033 & $\begin{array}{c}.563 \\
0.463\end{array}$ & 0.016 & 0.057 & $<0.010$ & 0.077 & 0.01 & 6.15 & 0.015 & $<0.100$ & $\begin{array}{l}0.273 \\
0.273\end{array}$ & 1.5 \\
\hline 2 & SB5-04 & 1 & 1 & 6 & N03LM11 & & 5.9 & 0.033 & 0.47 & 0.016 & 0.058 & $<0.010$ & 0.078 & 0.011 & 6.09 & 0.012 & $<0.100$ & 0.276 & 1.5 \\
\hline 2 & SB5-12 & 1 & 1 & 7 & N13LM21 & & 4.21 & 0.038 & 0.568 & 0.017 & 0.126 & $<0.010$ & 0.109 & 0.011 & 6.85 & 0.016 & $<0.100$ & 0.326 & 1.71 \\
\hline$\frac{2}{2}$ & $\frac{S D J 12}{S B 5-02}$ & $\frac{1}{1}$ & $\frac{1}{1}$ & 8 & $\begin{array}{l}\text { N12LM21 } \\
\text { N121 }\end{array}$ & & $\begin{array}{l}4.21 \\
5.76\end{array}$ & $\begin{array}{l}.0030 \\
0.032\end{array}$ & $\begin{array}{l}0.300 \\
1.47\end{array}$ & 0.014 & $\begin{array}{l}0.126 \\
0.083\end{array}$ & $<0.010$ & 0.08 & $<0.010$ & 0.05 & 0.010 & $<0.100$ & 0.0271 & $\frac{1.71}{1.43}$ \\
\hline 2 & SB5-11 & $\frac{1}{1}$ & $\frac{1}{1}$ & 9 & N14LM11 & & 4.18 & 0.037 & 1.57 & 0.018 & 0.126 & $<0.010$ & 0.098 & 0.011 & 6.54 & 0.016 & $<0.100$ & 0.316 & 1.63 \\
\hline 2 & SB5-07 & 1 & 1 & 10 & N15LM21 & & 5.07 & 0.035 & 1.5 & 0.015 & 0.083 & $<0.010$ & 0.086 & 0.01 & 6.51 & 0.018 & $<0.100$ & 0.295 & 1.6 \\
\hline 2 & Batch 1 & 1 & 1 & 11 & BCHLM2-112 & & 2.44 & 0.126 & 0.839 & $<0.010$ & 0.011 & $<0.010$ & 0.074 & 0.298 & 9.18 & 2.51 & $<0.100$ & 0.824 & 1.4 \\
\hline 2 & U std & 1 & 1 & 12 & UstdLM1-112 & & 2.04 & $<0.010$ & 0.89 & $<0.010$ & 0.066 & $<0.010$ & 0.162 & $<0.010$ & 9.47 & 2.22 & $<0.100$ & 0.705 & 2.28 \\
\hline$\frac{2}{2}$ & SB5-02 & $\frac{1}{1}$ & $\frac{1}{1}$ & 13 & N12LM11 & & 5.75 & 0.032 & 1.43 & 0.014 & 0.084 & $<0.010$ & 0.083 & $<0.010$ & $\frac{3.47}{6}$ & 0.011 & $<0.100$ & 0.271 & 1.47 \\
\hline 2 & SB5-11 & 1 & 1 & 14 & N14LM21 & & 4.13 & 0.038 & 1.56 & 0.018 & 0.126 & $<0.010$ & 0.096 & 0.011 & 6.96 & 0.017 & $<0.100$ & 0.322 & 1.75 \\
\hline 2 & SB4VAR33 & 1 & 1 & 15 & N05LM21 & & 5.18 & 0.023 & 0.747 & $<0.010$ & 0.019 & $<0.010$ & 0.038 & 0.024 & 7.44 & 0.126 & $<0.100$ & 0.619 & 1.76 \\
\hline 2 & SB5-05 & 1 & $\frac{1}{1}$ & 16 & N07LM21 & & 5.11 & 0.034 & 2.55 & 0.012 & 0.051 & $<0.010$ & 0.085 & $<0.010$ & 6.42 & 0.017 & $<0.100$ & 0.289 & 1.56 \\
\hline 2 & SB5-12 & 1 & 1 & 17 & N13LM11 & & 4.18 & 0.038 & 0.576 & 0.017 & 0.125 & $<0.010$ & 0.109 & 0.01 & 6.82 & 0.016 & $<0.100$ & 0.325 & 1.71 \\
\hline 2 & SB4VAR33 & 1 & 1 & 18 & N05LM11 & & 5.17 & 0.023 & 0.745 & $<0.010$ & 0.02 & $<0.010$ & 0.038 & 0.023 & 7.58 & 0.117 & $<0.100$ & 0.635 & 1.79 \\
\hline 2 & SB5-01 & 1 & $\frac{1}{1}$ & 19 & N02LM21 & & 5.96 & 0.032 & 2.56 & 0.014 & $\begin{array}{l}0.02 \\
0.087\end{array}$ & $<0.010$ & 0.078 & $<0.010$ & 6.32 & 0.013 & $<0.100$ & 0.0351 & 1.53 \\
\hline$\frac{2}{2}$ & SB5-07 & $\frac{1}{1}$ & $\frac{1}{1}$ & 20 & N15LM11 & & 5.03 & 0.035 & 1.54 & 0.016 & 0.085 & $<0.010$ & 0.089 & 0.014 & 6.75 & 0.014 & $<0.100$ & 0.305 & 1.65 \\
\hline
\end{tabular}


Table A1. Measured Elemental Concentrations (wt\%) for Samples Prepared Using Lithium Metaborate (part 1)

\begin{tabular}{|c|c|c|c|c|c|c|c|c|c|c|c|c|c|c|c|c|c|c|c|}
\hline Set & Glass ID & Block & $\begin{array}{c}\text { Sub- } \\
\text { Block }\end{array}$ & Sequence & Lab ID & $\begin{array}{c}\begin{array}{c}\mathrm{Ag} \\
(\mathrm{wt} \%)\end{array} \\
\end{array}$ & $\begin{array}{c}\begin{array}{c}\mathrm{Al} \\
(\mathrm{wt} \%)\end{array} \\
\end{array}$ & $\begin{array}{c}\begin{array}{c}\mathrm{Ba} \\
(\mathrm{wt} \%)\end{array} \\
\end{array}$ & $\begin{array}{c}\begin{array}{c}\mathrm{Ca} \\
\text { (wt\%) }\end{array} \\
\end{array}$ & $\begin{array}{c}\begin{array}{c}\mathrm{Cd} \\
\text { (wt\%) }\end{array} \\
\end{array}$ & $\begin{array}{c}\begin{array}{c}\mathrm{Ce} \\
(\mathrm{wt} \%)\end{array} \\
\end{array}$ & $\begin{array}{c}\begin{array}{c}\mathrm{Co} \\
\text { (wt\%) }\end{array} \\
\end{array}$ & $\begin{array}{c}\mathrm{Cr} \\
\text { (wt\%) }\end{array}$ & $\begin{array}{c}\begin{array}{c}\mathrm{Cu} \\
(\mathrm{wt} \%)\end{array} \\
\end{array}$ & $\begin{array}{c}\begin{array}{c}\mathrm{Fe} \\
\text { (wt\%) }\end{array} \\
\end{array}$ & $\begin{array}{c}\mathrm{K} \\
\text { (wt\%) }\end{array}$ & $\begin{array}{c}\begin{array}{c}\mathrm{La} \\
(\text { wt\%) }\end{array} \\
\end{array}$ & $\begin{array}{c}\mathrm{Mg} \\
\text { (wt\%) }\end{array}$ & $\begin{array}{c}\begin{array}{c}\mathrm{Mn} \\
\text { (wt\%) }\end{array} \\
\end{array}$ \\
\hline 2 & Batch 1 & 1 & 1 & 21 & BCHLM2-113 & & 2.53 & 0.126 & 0.85 & $<0.010$ & 0.011 & $<0.010$ & 0.073 & 0.3 & 9.28 & 2.51 & $<0.100$ & 0.827 & 1.41 \\
\hline 2 & U std & 1 & 1 & 22 & UstdLM2-113 & & 2.04 & $<0.010$ & 0.896 & $<0.010$ & 0.073 & $<0.010$ & 0.161 & $<0.010$ & 9.5 & 2.22 & $<0.100$ & 0.702 & 2.28 \\
\hline 2 & Batch 1 & 1 & 2 & 1 & BCHLM2-121 & & $\begin{array}{l}2.49 \\
\end{array}$ & 0.126 & $\begin{array}{ll}0.84 \\
\end{array}$ & $<0.010$ & $\begin{array}{ll}0.01 \\
\end{array}$ & $<0.010$ & 0.073 & 0.3 & $\begin{array}{l}8.63 \\
\end{array}$ & 2.49 & $<0.100$ & 0.828 & 1.3 \\
\hline 2 & U std & 1 & 2 & 2 & UstdLM2-121 & & 2.03 & $<0.010$ & 0.892 & $<0.010$ & 0 & $<0.010$ & 0.161 & $<0.010$ & 8.72 & 2.21 & $<0.100$ & 0.707 & 2.08 \\
\hline 2 & SB5-12 & 1 & 2 & 3 & $\begin{array}{l}\text { N13LM22 } \\
\end{array}$ & & 4.13 & 0.037 & 0.568 & 0.015 & 0.125 & $<0.010$ & 0.108 & 0.011 & 6.48 & 0.016 & $<0.100$ & 0.326 & 1.61 \\
\hline 2 & SB5-05 & 1 & 2 & 4 & N07LM22 & & 5.02 & 0.032 & 2.46 & 0.009 & 0.05 & $<0.010$ & 0.083 & $<0.010$ & 6.47 & 0.017 & $<0.100$ & 0.286 & 1.55 \\
\hline 2 & SB4VAR33 & 1 & 2 & 5 & N05LM22 & & 5.19 & 0.021 & 0.747 & $<0.010$ & 0.019 & $<0.010$ & 0.037 & 0.023 & 7.43 & 0.127 & $<0.100$ & 0.62 & 1.74 \\
\hline 2 & $\begin{array}{l}\text { SB4VAR33 } \\
\end{array}$ & 1 & 2 & 6 & N05LM12 & & 5.17 & 0.022 & 0.734 & $<0.010$ & 0.019 & $<0.010$ & 0.038 & 0.022 & 7.57 & 0.114 & $<0.100$ & 0.629 & 1.76 \\
\hline 2 & $\begin{array}{l}\text { SB5-01 } \\
\end{array}$ & 1 & 2 & 7 & N02LM12 & & 5.83 & 0.029 & 2.33 & 0.011 & 0.082 & $<0.010$ & 0.074 & $<0.010$ & 5.8 & 0.013 & $<0.100$ & 0.257 & 1.39 \\
\hline 2 & SB5-04 & 1 & 2 & 8 & N03LM12 & & 5.83 & 0.032 & 0.464 & $\begin{array}{ll}0.014 \\
\end{array}$ & 0.057 & $<0.010$ & 0.077 & 0.011 & 5.95 & 0.012 & $<0.100$ & 0.273 & 1.44 \\
\hline 2 & SB5-01 & 1 & 2 & 9 & N02LM22 & & 5.87 & 0.03 & 2.42 & 0.012 & 0.085 & $<0.010$ & 0.077 & $<0.010$ & 5.87 & 0.012 & $<0.100$ & 0.267 & 1.41 \\
\hline 2 & SB5-07 & 1 & 2 & 10 & N15LM12 & & 4.9 & 0.033 & 1.48 & 0.013 & 0.084 & $<0.010$ & 0.086 & 0.014 & 6.5 & 0.014 & $<0.100$ & 0.299 & 1.57 \\
\hline 2 & Batch 1 & 1 & 2 & 11 & BCHLM2-122 & & 2.44 & 0.123 & 0.832 & $<0.010$ & 0.01 & $<0.010$ & 0.071 & 0.298 & 8.95 & 2.47 & $<0.100$ & 0.817 & 1.34 \\
\hline 2 & U std & 1 & 2 & 12 & $\begin{array}{l}\text { UstdLM2-122 } \\
\end{array}$ & & 2.07 & $<0.010$ & 0.882 & $<0.010$ & 0.028 & $<0.010$ & 0.157 & $<0.010$ & 9.5 & 2.18 & $<0.100$ & 0.688 & 2.26 \\
\hline 2 & SB5-04 & 1 & 2 & 13 & N03LM22 & & 5.76 & 0.031 & 0.469 & 0.013 & 0.056 & $<0.010$ & 0.075 & 0.01 & 6.19 & 0.012 & $<0.100$ & 0.264 & 1.5 \\
\hline 2 & SB5-11 & 1 & 2 & 14 & N14LM22 & & 4.17 & 0.037 & 1.52 & 0.016 & 0.125 & $<0.010$ & 0.094 & 0.011 & 6.83 & 0.017 & $<0.100$ & 0.317 & 1.68 \\
\hline 2 & $\begin{array}{l}\text { SB5-05 } \\
\end{array}$ & 1 & 2 & 15 & N07LM12 & & 5.03 & 0.034 & $\begin{array}{l}2.43 \\
\end{array}$ & 0.01 & 0.052 & $<0.010$ & 0.087 & $<0.010$ & $\begin{array}{l}6.64 \\
\end{array}$ & 0.013 & $<0.100$ & 0.299 & 1.59 \\
\hline 2 & SB5-02 & 1 & 2 & 16 & N12LM12 & & 5.7 & 0.031 & 1.38 & 0.012 & 0.082 & $<0.010$ & 0.083 & $<0.010$ & 5.89 & 0.011 & $<0.100$ & 0.274 & 1.42 \\
\hline 2 & SB5-11 & 1 & 2 & 17 & N14LM12 & & 4.09 & 0.037 & 1.47 & 0.016 & 0.126 & $<0.010$ & 0.099 & 0.011 & 6.65 & 0.016 & $<0.100$ & 0.325 & 1.63 \\
\hline 2 & SB5-02 & 1 & 2 & 18 & N12LM22 & & 5.66 & 0.031 & 1.37 & 0.012 & 0.083 & $<0.010$ & 0.081 & $<0.010$ & 5.73 & 0.012 & $<0.100$ & 0.276 & 1.38 \\
\hline 2 & SB5-12 & 1 & 2 & 19 & N13LM12 & & 4.11 & 0.037 & 0.569 & 0.015 & 0.125 & $<0.010$ & 0.109 & 0.01 & 7.43 & 0.016 & $<0.100$ & 0.329 & 1.83 \\
\hline 2 & SB5-07 & 1 & 2 & 20 & N15LM22 & & 5.01 & 0.034 & 1.46 & 0.013 & 0.082 & $<0.010$ & 0.086 & 0.01 & 7.13 & 0.017 & $<0.100$ & 0.297 & 1.72 \\
\hline 2 & Batch 1 & 1 & 2 & 21 & BCHLM2-123 & & 2.53 & 0.126 & 0.832 & $<0.010$ & 0.01 & $<0.010$ & 0.073 & 0.295 & 9.61 & 2.45 & $<0.100$ & 0.832 & 1.44 \\
\hline 2 & U std & 1 & 2 & 22 & UstdLM2-123 & & 2.04 & $<0.010$ & 0.877 & $<0.010$ & 0 & $<0.010$ & 0.164 & $<0.010$ & 9.81 & 2.16 & $<0.100$ & 0.724 & 2.3 \\
\hline 2 & Batch 1 & 2 & 1 & 1 & BCHLM2-211 & & 2.49 & 0.129 & 0.873 & $<0.010$ & 0.013 & $<0.010$ & 0.075 & 0.311 & $\begin{array}{l}8.99 \\
\end{array}$ & 2.6 & $<0.100$ & 0.826 & 1.35 \\
\hline 2 & U std & 2 & 1 & 2 & UstdLM2-211 & & 2.04 & $<0.010$ & 0.927 & $<0.010$ & 0.002 & $<0.010$ & 0.163 & $<0.010$ & 9.32 & 2.31 & $<0.100$ & 0.704 & 2.21 \\
\hline 2 & $\begin{array}{l}\text { SB4VAR32 } \\
\end{array}$ & 2 & 1 & 3 & $\begin{array}{l}\text { N11LM21 } \\
\end{array}$ & & 4.66 & 0.022 & 0.722 & $<0.010$ & 0.018 & $<0.010$ & 0.041 & 0.023 & 6.82 & 0.119 & $<0.100$ & 0.563 & 1.58 \\
\hline 2 & $\begin{array}{l}\text { SB4VAR31 } \\
\end{array}$ & 2 & 1 & 4 & N06LM11 & & $\begin{array}{lll}4.14 \\
\end{array}$ & 0.021 & $\begin{array}{ll}0.628 \\
\end{array}$ & $<0.010$ & 0.018 & $<0.010$ & 0.038 & 0.02 & $\begin{array}{ll}6.01 \\
\end{array}$ & 0.091 & $<0.100$ & 0.53 & 1.39 \\
\hline 2 & SB5-03 & 2 & 1 & 5 & N09LM11 & & 5.72 & 0.033 & 1.38 & 0.014 & 0.089 & $<0.010$ & 0.08 & 0.012 & 6.05 & 0.01 & $<0.100$ & 0.271 & 1.47 \\
\hline 2 & SB5-09 & 2 & 1 & 6 & N04LM11 & & 4.17 & 0.036 & 2.43 & 0.015 & 0.084 & $<0.010$ & 0.09 & 0.011 & 7.38 & 0.021 & $<0.100$ & 0.315 & 1.75 \\
\hline 2 & SB5-03 & 2 & 1 & 7 & N09LM21 & & 5.79 & 0.034 & 1.39 & 0.014 & 0.091 & $<0.010$ & 0.082 & 0.013 & 6.37 & 0.008 & $<0.100$ & 0.278 & 1.55 \\
\hline 2 & SB4VAR32 & 2 & 1 & 8 & N11LM11 & & 4.75 & 0.022 & 0.711 & $<0.010$ & 0.018 & $<0.010$ & 0.047 & 0.022 & 7.6 & 0.116 & $<0.100$ & 0.569 & 1.74 \\
\hline 2 & SB5-10 & 2 & 1 & 9 & N081LM11 & & 4.27 & 0.038 & 1.57 & 0.018 & 0.126 & $<0.010$ & 0.084 & 0.011 & 7.55 & 0.019 & $<0.100$ & 0.316 & 1.85 \\
\hline 2 & Batch 1 & 2 & 1 & 10 & BCHLM2-212 & & 2.52 & 0.127 & 0.867 & $<0.010$ & 0.013 & $<0.010$ & 0.074 & 0.305 & 9.48 & 2.6 & $<0.100$ & 0.818 & 1.42 \\
\hline 2 & U std & 2 & 1 & 11 & $\begin{array}{l}\text { UstdLM2-212 } \\
\end{array}$ & & 2.05 & $<0.010$ & 0.944 & $<0.010$ & 0.002 & $<0.010$ & 0.166 & $<0.010$ & 9.23 & 2.36 & $<0.100$ & 0.719 & 2.19 \\
\hline 2 & SB5-08 & 2 & 1 & 12 & N10LM21 & & 4.83 & 0.037 & 0.533 & 0.014 & 0.056 & $<0.010$ & 0.088 & 0.01 & 6.6 & 0.012 & $<0.100$ & 0.299 & 1.58 \\
\hline 2 & SB5-06 & 2 & 1 & 13 & N01LM11 & & 4.99 & 0.037 & 1.42 & 0.015 & 0.094 & $<0.010$ & 0.083 & 0.011 & 6.88 & 0.013 & $<0.100$ & 0.305 & 1.65 \\
\hline 2 & $\begin{array}{l}\text { SB4VAR31 } \\
\end{array}$ & 2 & 1 & 14 & N06LM21 & & 4.22 & 0.021 & 0.636 & $<0.010$ & 0.018 & $<0.010$ & 0.038 & 0.02 & 6.42 & 0.106 & $<0.100$ & 0.514 & 1.49 \\
\hline 2 & SB5-10 & 2 & 1 & 15 & N08LM21 & & $\begin{array}{lll}4.17 \\
\end{array}$ & 0.039 & 1.46 & 0.018 & 0.128 & $<0.010$ & 0.085 & 0.011 & 7 & 0.018 & $<0.100$ & 0.32 & 1.72 \\
\hline 2 & SB5-08 & 2 & 1 & 16 & N10LM11 & & 5.03 & 0.038 & 0.564 & 0.016 & 0.056 & $<0.010$ & 0.093 & 0.012 & 6.72 & 0.078 & $<0.100$ & 0.31 & 1.61 \\
\hline 2 & SB5-09 & 2 & 1 & 17 & N04LM21 & & 4.16 & 0.038 & 2.3 & 0.016 & 0.088 & $<0.010$ & 0.094 & 0.011 & 7.16 & 0.016 & $<0.100$ & 0.329 & 1.68 \\
\hline 2 & $\begin{array}{l}\text { SB5-06 } \\
\end{array}$ & 2 & 1 & 18 & $\begin{array}{l}\text { N01LM21 } \\
\end{array}$ & & 5.21 & 0.033 & 1.54 & 0.013 & 0.088 & $<0.010$ & 0.075 & 0.01 & 6.75 & 0.013 & $<0.100$ & 0.278 & 1.52 \\
\hline 2 & Batch 1 & 2 & 1 & 19 & BCHLM2-213 & & 2.54 & 0.129 & 0.881 & $<0.010$ & 0.013 & $<0.010$ & 0.075 & 0.313 & 9.36 & 2.63 & $<0.100$ & 0.824 & 1.41 \\
\hline 2 & U std & 2 & 1 & 20 & UstdLM2-213 & & 2.05 & $<0.010$ & 0.933 & $<0.010$ & 0.002 & $<0.010$ & 0.165 & $<0.010$ & 9.92 & 2.33 & $<0.100$ & 0.71 & 2.35 \\
\hline 2 & Batch 1 & 2 & 2 & 1 & BCHLM2-221 & & 2.62 & 0.127 & 0.853 & $<0.010$ & 0.01 & $<0.010$ & 0.073 & 0.302 & $\begin{array}{l}8.77 \\
\end{array}$ & 2.53 & $<0.100$ & 0.836 & 1.31 \\
\hline 2 & U std & 2 & 2 & 2 & UstdLM2-221 & & 2.21 & $<0.010$ & 0.906 & $<0.010$ & $<0.010$ & $<0.010$ & 0.163 & $<0.010$ & 9.38 & 2.23 & $<0.100$ & 0.721 & 2.22 \\
\hline 2 & $\begin{array}{l}\text { SB4VAR31 } \\
\end{array}$ & 2 & 2 & 3 & N06LM22 & & 4.4 & 0.019 & 0.622 & $<0.010$ & 0.015 & $<0.010$ & 0.035 & 0.016 & 6.11 & 0.104 & $<0.100$ & 0.517 & 1.41 \\
\hline 2 & $\begin{array}{l}\text { SB4VAR32 } \\
\end{array}$ & 2 & 2 & 4 & N11LM22 & & 4.91 & 0.02 & 0.692 & $<0.010$ & 0.016 & $<0.010$ & 0.039 & 0.018 & 7.18 & 0.11 & $<0.100$ & 0.578 & 1.64 \\
\hline 2 & SB5-10 & 2 & 2 & 5 & N08LM12 & & 4.39 & 0.036 & 1.6 & 0.015 & 0.121 & $<0.010$ & 0.081 & $<0.010$ & 7.16 & 0.018 & $<0.100$ & 0.314 & 1.74 \\
\hline 2 & SB5-06 & 2 & 2 & 6 & N01LM22 & & 5.21 & 0.033 & 1.56 & 0.011 & 0.089 & $<0.010$ & 0.078 & $<0.010$ & 6.74 & 0.012 & $<0.100$ & 0.297 & 1.6 \\
\hline 2 & $\begin{array}{l}\text { SB4VAR31 } \\
\end{array}$ & 2 & 2 & 7 & N06LM12 & & 4.37 & 0.019 & 0.609 & $<0.010$ & 0.016 & $<0.010$ & 0.036 & 0.016 & 6.15 & 0.087 & $<0.100$ & 0.538 & 1.41 \\
\hline 2 & SB5-09 & 2 & 2 & 8 & N04LM12 & & 4.43 & 0.034 & 2.59 & 0.012 & 0.081 & $<0.010$ & 0.089 & $<0.010$ & 7.28 & 0.021 & $<0.100$ & 0.32 & 1.71 \\
\hline
\end{tabular}


Table A1. Measured Elemental Concentrations (wt\%) for Samples Prepared Using Lithium Metaborate (part 1)

\begin{tabular}{|c|c|c|c|c|c|c|c|c|c|c|c|c|c|c|c|c|c|c|c|}
\hline Set & Glass ID & Block & $\begin{array}{c}\text { Sub- } \\
\text { Block }\end{array}$ & Sequence & Lab ID & $\begin{array}{c}\mathrm{Ag} \\
\text { (wt\%) }\end{array}$ & $\begin{array}{c}\begin{array}{c}\mathrm{Al} \\
(\mathrm{wt} \%)\end{array} \\
\end{array}$ & $\begin{array}{c}\mathrm{Ba} \\
\text { (wt\%) }\end{array}$ & $\begin{array}{c}\begin{array}{c}\mathrm{Ca} \\
\text { (wt\%) }\end{array} \\
\end{array}$ & $\begin{array}{c}\begin{array}{c}\mathrm{Cd} \\
\text { (wt\%) }\end{array} \\
\end{array}$ & $\begin{array}{c}\begin{array}{c}\mathrm{Ce} \\
(\mathrm{wt} \%)\end{array} \\
\end{array}$ & $\begin{array}{c}\begin{array}{c}\mathrm{Co} \\
\text { (wt\%) }\end{array} \\
\end{array}$ & $\begin{array}{c}\begin{array}{c}\mathrm{Cr} \\
\text { (wt\%) }\end{array} \\
\end{array}$ & $\begin{array}{c}\begin{array}{c}\mathrm{Cu} \\
(\mathrm{w} t \%)\end{array} \\
\end{array}$ & $\begin{array}{c}\begin{array}{c}\mathrm{Fe} \\
(\mathrm{wt} \%)\end{array} \\
\end{array}$ & $\begin{array}{c}\mathrm{K} \\
\text { (wt\%) }\end{array}$ & $\begin{array}{c}\begin{array}{c}\mathrm{La} \\
(\mathrm{wt} \%)\end{array} \\
\end{array}$ & $\begin{array}{c}\begin{array}{c}\mathrm{Mg} \\
\text { (wt\%) }\end{array} \\
\end{array}$ & $\begin{array}{c}\begin{array}{c}\mathrm{Mn} \\
\text { (wt\%) }\end{array} \\
\end{array}$ \\
\hline 2 & SB5-08 & 2 & 2 & 9 & N10LM12 & & 5.27 & 0.037 & 0.541 & 0.014 & 0.052 & $<0.010$ & 0.092 & $<0.010$ & 6.73 & 0.073 & $<0.100$ & 0.314 & 1.59 \\
\hline 2 & Batch 1 & 2 & 2 & 10 & BCHLM2-222 & & 2.66 & 0.128 & 0.859 & $<0.010$ & 0.01 & $<0.010$ & 0.073 & 0.304 & 8.91 & 2.53 & $<0.100$ & 0.843 & 1.31 \\
\hline 2 & U std & 2 & 2 & 11 & UstdLM2-222 & & 2.2 & $<0.010$ & 0.928 & $<0.010$ & $<0.010$ & $<0.010$ & 0.164 & $<0.010$ & 9.39 & 2.26 & $<0.100$ & 0.731 & 2.2 \\
\hline 2 & SB5-03 & 2 & 2 & 12 & N09LM12 & & 6.14 & 0.032 & 1.55 & 0.012 & 0.086 & $<0.010$ & 0.081 & $\begin{array}{l}<0.010 \\
\end{array}$ & 5.85 & 0.008 & $<0.100$ & 0.282 & 1.41 \\
\hline 2 & SB5-10 & 2 & 2 & 13 & N08LM22 & & 4.41 & 0.037 & 1.62 & 0.016 & 0.122 & $<0.010$ & 0.085 & $<0.010$ & 6.99 & 0.015 & $<0.100$ & 0.33 & 1.71 \\
\hline 2 & SB5-09 & 2 & 2 & 14 & N04LM22 & & 4.34 & 0.038 & 2.58 & 0.014 & 0.085 & $<0.010$ & 0.096 & $<0.010$ & 6.63 & 0.014 & $<0.100$ & 0.348 & 1.57 \\
\hline 2 & SB5-03 & 2 & 2 & 15 & N09LM22 & & 5.96 & 0.034 & $\begin{array}{ll}1.51 \\
\end{array}$ & 0.013 & $\begin{array}{l}0.089 \\
\end{array}$ & $<0.010$ & 0.085 & $\begin{array}{l}<0.010 \\
\end{array}$ & 5.59 & 0.007 & $<0.100$ & 0.298 & 1.36 \\
\hline 2 & $\begin{array}{l}\text { SB5-08 } \\
\end{array}$ & 2 & 2 & 16 & N10LM22 & & 5.01 & 0.037 & 0.513 & 0.012 & 0.054 & $<0.010$ & 0.089 & $<0.010$ & 6.59 & 0.01 & $<0.100$ & 0.314 & 1.48 \\
\hline 2 & $\begin{array}{l}\text { SB5-06 } \\
\end{array}$ & 2 & 2 & 17 & N01LM12 & & 5.19 & 0.036 & 1.56 & 0.012 & 0.092 & $<0.010$ & 0.083 & $<0.010$ & 6.51 & 0.012 & $<0.100$ & 0.315 & 1.56 \\
\hline 2 & SB4VAR32 & 2 & 2 & 18 & N11LM12 & & 4.92 & 0.021 & 0.677 & $<0.010$ & 0.016 & $<0.010$ & 0.046 & 0.018 & 6.72 & 0.106 & $<0.100$ & 0.6 & 1.55 \\
\hline 2 & Batch 1 & 2 & 2 & 19 & BCHLM2-223 & & $\begin{array}{l}2.62 \\
\end{array}$ & 0.134 & 0.833 & $<0.010$ & $\begin{array}{ll}0.011 \\
\end{array}$ & $<0.010$ & 0.077 & 0.304 & $\begin{array}{l}8.91 \\
\end{array}$ & 2.48 & $<0.100$ & 0.888 & 1.34 \\
\hline 2 & U std & 2 & 2 & 20 & UstdLM2-223 & & 2.2 & $<0.010$ & 0.881 & $<0.010$ & $<0.010$ & $<0.010$ & 0.173 & $<0.010$ & 9.16 & 2.17 & $<0.100$ & 0.767 & 2.18 \\
\hline
\end{tabular}


Table A2. Measured Elemental Concentrations (wt\%) for Samples Prepared Using Lithium Metaborate (part 2)

\begin{tabular}{|c|c|c|c|c|c|c|c|c|c|c|c|c|c|c|c|c|c|c|}
\hline Set & Glass ID & Block & Sub-Block & Sequence & Lab ID & Na (wt\%) & $\mathrm{Nb}(\mathrm{wt} \%)$ & $\mathrm{Ni}$ (wt\%) & \begin{tabular}{|l|}
$\mathrm{P}$ (wt\%) \\
\end{tabular} & $\mathrm{Pb}(\mathrm{wt} \%)$ & S (wt\%) & Si (wt\%) & Sr (wt\%) & Th (wt\%) & Ti (wt\%) & $\mathrm{U}$ (wt\%) & Zn (wt\%) & $\mathrm{Zr}(\mathrm{wt} \%)$ \\
\hline 1 & Batch 1 & 1 & 1 & 1 & BCHLM1-111 & 6.68 & 0.035 & 0.549 & \begin{tabular}{|l|}
$<0.100$ \\
\end{tabular} & $<0.020$ & \begin{tabular}{|l|}
$<0.100$ \\
\end{tabular} & & $<0.010$ & $<0.100$ & 0.389 & \begin{tabular}{|l|}
$<0.100$ \\
\end{tabular} & $<<0.010$ & 0.062 \\
\hline 1 & U std & 1 & 1 & 2 & UstdLM1-111 & 8.71 & 0.052 & 0.789 & \begin{tabular}{|c|}
$<0.100$ \\
\end{tabular} & $<0.020$ & \begin{tabular}{|c|}
$<0.100$ \\
\end{tabular} & & $<0.010$ & $<0.100$ & 0.565 & 1.97 & $<0.010$ & $<0.010$ \\
\hline 1 & \begin{tabular}{|l|} 
SB4VAR51 \\
\end{tabular} & 1 & 1 & 3 & M04LM11 & 8.83 & 0.025 & 0.355 & \begin{tabular}{|l|}
$<0.100$ \\
\end{tabular} & 0.02 & \begin{tabular}{|l|}
0.109 \\
\end{tabular} & & $<0.010$ & $<0.100$ & 0.238 & 2.21 & 0.013 & 0.016 \\
\hline 1 & \begin{tabular}{|l|} 
SB4VAR42 \\
\end{tabular} & 1 & 1 & 4 & M05LM11 & 8.51 & 0.028 & 0.408 & \begin{tabular}{|l|}
$<0.100$ \\
\end{tabular} & 0.023 & 0.116 & & \begin{tabular}{|c|}
$<010$ \\
\end{tabular} & $<0.100$ & 0.267 & 2.52 & 0.017 & 0.019 \\
\hline 1 & \begin{tabular}{|l|} 
SB4VAR43 \\
\end{tabular} & 1 & 1 & 5 & M06LM21 & 8.82 & 0.032 & 0.445 & \begin{tabular}{|l|}
$<0.100$ \\
\end{tabular} & 0.024 & 0.135 & & $<0.010$ & $<0.100$ & 0.314 & 2.81 & 0.019 & 0.02 \\
\hline 1 & \begin{tabular}{|l|} 
SB4VAR41 \\
\end{tabular} & 1 & 1 & 6 & M01LM21 & 8.64 & 0.025 & 0.363 & \begin{tabular}{|c|}
$<0.100$ \\
\end{tabular} & 0.02 & 0.11 & & $<<0.010$ & $<0.100$ & 0.238 & 2.38 & 0.014 & 0.019 \\
\hline 1 & \begin{tabular}{|l|} 
SB4VAR54 \\
\end{tabular} & 1 & 1 & 7 & M03LM11 & 9.93 & 0.033 & 0.46 & \begin{tabular}{|c|}
$<0.100$ \\
\end{tabular} & 0.025 & 0.144 & & $<0.010$ & $<0.100$ & 0.32 & 2.9 & 0.023 & 0.024 \\
\hline 1 & \begin{tabular}{|l|} 
SB4VAR51 \\
\end{tabular} & 1 & 1 & 8 & M04LM21 & 9.23 & 0.025 & 0.352 & \begin{tabular}{|l|}
$<0.100$ \\
\end{tabular} & 0.02 & 0.113 & & $<0.010$ & $<0.100$ & 0.242 & 2.29 & 0.014 & 0.019 \\
\hline 1 & \begin{tabular}{|l|} 
SB4VAR54 \\
\end{tabular} & 1 & 1 & 9 & M03LM21 & 9.88 & 0.033 & 0.46 & \begin{tabular}{|l|}
$<0.100$ \\
\end{tabular} & 0.025 & \begin{tabular}{|l|}
0.147 \\
\end{tabular} & & $<<0.010$ & $<0.100$ & 0.32 & 2.88 & 0.022 & 0.024 \\
\hline 1 & Batch 1 & 1 & 1 & 10 & BCHLM1-112 & 6.85 & 0.034 & 0.564 & \begin{tabular}{|l|}
$<0.100$ \\
\end{tabular} & $<0.020$ & \begin{tabular}{|c|}
$<0.100$ \\
\end{tabular} & & $<0.010$ & $<0.100$ & 0.395 & \begin{tabular}{|l|}
$<0.100$ \\
\end{tabular} & $<0.010$ & 0.064 \\
\hline 1 & U std & 1 & 1 & 11 & UstdLM1-112 & 8.64 & 0.052 & 0.818 & \begin{tabular}{|l|}
$<0.100$ \\
\end{tabular} & $<0.020$ & \begin{tabular}{|c|}
$<0.100$ \\
\end{tabular} & & $<0.010$ & $<0.100$ & 0.578 & 2 & $<0.010$ & $<0.010$ \\
\hline 1 & SB4VAR41 & 1 & 1 & 12 & M01LM11 & 8.55 & 0.026 & 0.375 & \begin{tabular}{|l|}
$<0.100$ \\
\end{tabular} & 0.021 & 0.112 & & $<0.010$ & $<0.100$ & 0.243 & 2.35 & 0.015 & 0.019 \\
\hline 1 & \begin{tabular}{|l|} 
SB4VAR22 \\
\end{tabular} & 1 & 1 & 13 & M12LM11 & 8.29 & $<0.020$ & 0.436 & \begin{tabular}{|l|}
$<0.100$ \\
\end{tabular} & 0.023 & 0.117 & & $<0.010$ & $<0.100$ & 0.015 & 2.6 & 0.018 & 0.016 \\
\hline 1 & \begin{tabular}{|l|} 
SB4VAR43 \\
\end{tabular} & 1 & 1 & 14 & M06LM11 & 8.78 & 0.033 & 0.462 & \begin{tabular}{|l|}
$<0.100$ \\
\end{tabular} & 0.026 & 0.14 & & $<0.010$ & $<0.100$ & 0.312 & 2.73 & 0.021 & 0.021 \\
\hline 1 & SB4VAR12 & 1 & 1 & 15 & M15LM11 & 9.54 & $<0.020$ & 0.425 & \begin{tabular}{|l|}
$<0.100$ \\
\end{tabular} & 0.02 & 0.15 & & $<0.010$ & $<0.100$ & 0.012 & 2.73 & 0.017 & 0.02 \\
\hline 1 & SB4VAR22 & 1 & 1 & 16 & M12LM21 & 8.27 & $<0.020$ & 0.428 & \begin{tabular}{|l|}
$<0.100$ \\
\end{tabular} & 0.022 & 0.114 & & $<0.010$ & $<0.100$ & 0.015 & 2.58 & 0.017 & 0.016 \\
\hline 1 & SB4VAR12 & 1 & 1 & 17 & M15LM21 & 9.44 & $<0.020$ & 0.441 & \begin{tabular}{|l|}
$<0.100$ \\
\end{tabular} & 0.021 & 0.152 & & $<0.010$ & $<0.100$ & 0.013 & 2.73 & 0.018 & 0.021 \\
\hline 1 & SB4VAR42 & 1 & 1 & 18 & M05LM21 & 8.36 & 0.029 & 0.419 & \begin{tabular}{|l|}
$<0.100$ \\
\end{tabular} & 0.023 & 0.122 & & $<0.010$ & $<0.100$ & 0.274 & 2.48 & 0.019 & 0.02 \\
\hline 1 & Batch 1 & 1 & 1 & 19 & BCHLM1-113 & 6.84 & 0.035 & 0.57 & \begin{tabular}{|l|}
$<0.100$ \\
\end{tabular} & $<0.020$ & \begin{tabular}{|c|}
$<0.100$ \\
\end{tabular} & & $<0.010$ & $<0.100$ & 0.4 & \begin{tabular}{|l|}
$<0.100$ \\
\end{tabular} & $<0.010$ & 0.065 \\
\hline 1 & U std & 1 & 1 & 20 & UstdLM1-113 & 8.63 & 0.052 & 0.812 & \begin{tabular}{|l|}
$<0.100$ \\
\end{tabular} & $<0.020$ & \begin{tabular}{|c|}
$<0.100$ \\
\end{tabular} & & $<0.010$ & $<0.100$ & 0.574 & 2.01 & $<0.010$ & $<0.010$ \\
\hline 1 & Batch 1 & 1 & 2 & 1 & BCHLM1-121 & 6.56 & 0.039 & 0.557 & \begin{tabular}{|l|}
$<0.100$ \\
\end{tabular} & $<0.020$ & \begin{tabular}{|l|}
$<0.100$ \\
\end{tabular} & & $<0.010$ & $<0.100$ & 0.388 & \begin{tabular}{|l|}
$<0.100$ \\
\end{tabular} & $<0.010$ & 0.063 \\
\hline 1 & U std & 1 & 2 & 2 & UstdLM1-121 & 8.53 & 0.056 & 0.793 & \begin{tabular}{|l|}
$<0.100$ \\
\end{tabular} & $<0.020$ & \begin{tabular}{|c|}
$<0.100$ \\
\end{tabular} & & $<0.010$ & $<0.100$ & 0.552 & 1.93 & $<0.010$ & $<0.010$ \\
\hline \begin{tabular}{|l|}
1 \\
\end{tabular} & \begin{tabular}{|l|} 
SB4VAR22 \\
\end{tabular} & 1 & 2 & 3 & M12LM22 & 8.31 & $<0.020$ & 0.411 & \begin{tabular}{|l|}
$<0.100$ \\
\end{tabular} & 0.022 & \begin{tabular}{|l|}
0.114 \\
\end{tabular} & & $<0.010$ & $<0.100$ & 0.017 & 2.58 & 0.016 & 0.016 \\
\hline \begin{tabular}{|l|l|}
1 & \\
\end{tabular} & SB4VAR42 & 1 & 2 & 4 & M05LM12 & 8.63 & 0.032 & 0.401 & \begin{tabular}{|l|}
$<0.100$ \\
\end{tabular} & 0.023 & 0.115 & & $<<0.010$ & $<0.100$ & 0.265 & 2.46 & 0.017 & 0.019 \\
\hline 1 & \begin{tabular}{|l|} 
SB4VAR51 \\
\end{tabular} & 1 & 2 & 5 & M04LM22 & 9.11 & 0.029 & 0.342 & \begin{tabular}{|l|}
$<0.100$ \\
\end{tabular} & $<0.020$ & 0.111 & & $<0.010$ & $<0.100$ & 0.234 & 2.18 & 0.014 & 0.019 \\
\hline \begin{tabular}{|l|l|}
1 \\
\end{tabular} & SB4VAR54 & 1 & 2 & 6 & M03LM22 & 9.88 & 0.036 & 0.448 & \begin{tabular}{|l|}
$<0.100$ \\
\end{tabular} & 0.025 & 0.14 & & $<0.010$ & $<0.100$ & 0.31 & 2.76 & 0.021 & 0.024 \\
\hline \begin{tabular}{|l|}
1 \\
\end{tabular} & \begin{tabular}{|l|} 
SB4VAR51 \\
\end{tabular} & 1 & 2 & 7 & M04LM12 & 8.93 & 0.029 & 0.347 & \begin{tabular}{|l|}
$<0.100$ \\
\end{tabular} & 0.02 & 0.107 & & $<0.010$ & $<0.100$ & 0.234 & 2.14 & 0.013 & 0.017 \\
\hline \begin{tabular}{l|l}
1 \\
\end{tabular} & SB4VAR12 & 1 & 2 & 8 & M15LM22 & 9.08 & $<0.020$ & 0.426 & \begin{tabular}{|l|}
$<0.100$ \\
\end{tabular} & 0.02 & 0.148 & & $<0.010$ & $<0.100$ & 0.015 & 2.56 & 0.017 & 0.021 \\
\hline \begin{tabular}{|l|l|}
1 \\
\end{tabular} & \begin{tabular}{|l|} 
SB4VAR43 \\
\end{tabular} & 1 & 2 & 9 & M06LM12 & 8.44 & 0.036 & 0.443 & \begin{tabular}{|l|}
$<0.100$ \\
\end{tabular} & 0.024 & 0.13 & & $<0.010$ & $<0.100$ & 0.306 & 2.63 & 0.021 & 0.021 \\
\hline \begin{tabular}{|l|}
1 \\
\end{tabular} & Batch 1 & 1 & 2 & 10 & BCHLM1-122 & 6.35 & 0.038 & 0.551 & \begin{tabular}{|l|}
$<0.100$ \\
\end{tabular} & $<0.020$ & \begin{tabular}{|l|}
$<0.100$ \\
\end{tabular} & & $<0.010$ & $<0.100$ & 0.385 & \begin{tabular}{|l|}
$<0.100$ \\
\end{tabular} & $<0.010$ & 0.064 \\
\hline \begin{tabular}{|l|}
1 \\
\end{tabular} & U std & 1 & 2 & 11 & UstdLM1-122 & 8.42 & 0.055 & 0.792 & \begin{tabular}{|l|}
$<0.100$ \\
\end{tabular} & $<0.020$ & \begin{tabular}{|c|}
$<0.100$ \\
\end{tabular} & & $<0.010$ & $<0.100$ & 0.557 & 1.92 & $<0.010$ & $<0.010$ \\
\hline \begin{tabular}{|l|}
1 \\
\end{tabular} & \begin{tabular}{|l|} 
SB4VAR41 \\
\end{tabular} & 1 & 2 & 12 & M01LM22 & 8.08 & 0.029 & 0.351 & \begin{tabular}{|l|}
$<0.100$ \\
\end{tabular} & 0.02 & 0.109 & & $<0.010$ & $<0.100$ & 0.231 & 2.21 & 0.014 & 0.019 \\
\hline \begin{tabular}{|l|}
1 \\
\end{tabular} & \begin{tabular}{|l|} 
SB4VAR22 \\
\end{tabular} & 1 & 2 & 13 & M12LM12 & 8 & $<0.020$ & 0.418 & \begin{tabular}{|l|}
$<0.100$ \\
\end{tabular} & 0.022 & 0.114 & & $<0.010$ & $<0.100$ & 0.017 & 2.56 & 0.017 & 0.016 \\
\hline \begin{tabular}{|l|}
1 \\
\end{tabular} & SB4VAR42 & 1 & 2 & 14 & M05LM22 & 8.25 & 0.032 & 0.404 & \begin{tabular}{|l|}
$<0.100$ \\
\end{tabular} & 0.023 & 0.115 & & $<0.010$ & $<0.100$ & 0.267 & 2.46 & 0.019 & 0.02 \\
\hline \begin{tabular}{l|l}
1 \\
\end{tabular} & SB4VAR12 & 1 & 2 & 15 & M15LM12 & 9.07 & $<0.020$ & 0.406 & \begin{tabular}{|c|}
$<0.100$ \\
\end{tabular} & $<0.020$ & 0.143 & & $\begin{array}{l}<0.010 \\
\end{array}$ & $<0.100$ & 0.015 & 2.69 & 0.017 & 0.02 \\
\hline \begin{tabular}{|l|}
1 \\
\end{tabular} & SB4VAR43 & 1 & 2 & 16 & M06LM22 & 8.44 & 0.036 & 0.437 & \begin{tabular}{|l|}
$<0.100$ \\
\end{tabular} & 0.024 & 0.129 & & $<0.010$ & $<0.100$ & 0.305 & 2.71 & 0.019 & 0.02 \\
\hline \begin{tabular}{|l|l}
1 & \\
\end{tabular} & SB4VAR41 & 1 & 2 & 17 & M01LM12 & 8.51 & 0.029 & 0.366 & \begin{tabular}{|l|}
$<0.100$ \\
\end{tabular} & 0.02 & 0.11 & & $<0.010$ & $<0.100$ & 0.238 & 2.36 & 0.015 & 0.019 \\
\hline \begin{tabular}{|l|}
1 \\
\end{tabular} & SB4VAR54 & 1 & 2 & 18 & M03LM12 & 9.73 & 0.037 & 0.449 & \begin{tabular}{|l|}
$<0.100$ \\
\end{tabular} & 0.025 & 0.143 & & $<0.010$ & $<0.100$ & 0.313 & 2.82 & 0.023 & 0.024 \\
\hline \begin{tabular}{|l|l}
1 \\
\end{tabular} & Batch 1 & 1 & 2 & 19 & BCHLM1-123 & 6.53 & 0.037 & 0.543 & \begin{tabular}{|l|}
$<0.100$ \\
\end{tabular} & $<0.020$ & \begin{tabular}{|c|}
$<0.100$ \\
\end{tabular} & & $<0.010$ & $<0.100$ & 0.384 & \begin{tabular}{|l|}
$<0.100$ \\
\end{tabular} & $<0.010$ & 0.063 \\
\hline \begin{tabular}{|l|}
1 \\
\end{tabular} & U std & 1 & 2 & 20 & UstdLM1-123 & 8.43 & 0.055 & 0.782 & \begin{tabular}{|l|}
$<0.100$ \\
\end{tabular} & $<0.020$ & \begin{tabular}{|c|}
$<0.100$ \\
\end{tabular} & & $<0.010$ & $<0.100$ & 0.552 & 1.91 & $<0.010$ & $<0.010$ \\
\hline 1 & Batch 1 & 2 & 1 & 1 & BCHLM1-211 & 6.6 & 0.036 & 0.547 & \begin{tabular}{|l|}
$<0.100$ \\
\end{tabular} & $<0.020$ & \begin{tabular}{|l|}
$<0.100$ \\
\end{tabular} & & $<0.010$ & $<0.100$ & 0.387 & \begin{tabular}{|l|}
$<0.100$ \\
\end{tabular} & $<0.010$ & 0.064 \\
\hline 1 & U std & 2 & 1 & 2 & UstdLM1-211 & 8.67 & 0.053 & 0.788 & \begin{tabular}{|c|}
$<0.100$ \\
\end{tabular} & $<0.020$ & \begin{tabular}{|c|}
$<0.100$ \\
\end{tabular} & & \begin{tabular}{|l|}
$<0.010$ \\
\end{tabular} & $<0.100$ & 0.558 & 1.96 & $<0.010$ & $<0.010$ \\
\hline \begin{tabular}{|l|}
1 \\
\end{tabular} & \begin{tabular}{|l|} 
SB4VAR11 \\
\end{tabular} & 2 & 1 & 3 & M02LM21 & 9.16 & $<0.020$ & 0.388 & \begin{tabular}{|l|}
$<0.100$ \\
\end{tabular} & 0.022 & 0.14 & & $<0.010$ & $<0.100$ & 0.011 & 2.4 & 0.019 & 0.02 \\
\hline 4 & \begin{tabular}{|l|} 
SB4VAR53 \\
\end{tabular} & 2 & 1 & 4 & M11 & 9.8 & 0.031 & 0.428 & \begin{tabular}{|l|}
$<0.100$ \\
\end{tabular} & 0.025 & 0.13 & & \begin{tabular}{|l|}
$<0.010$ \\
\end{tabular} & $<0.100$ & 0.286 & 2.58 & 0.019 & 0.022 \\
\hline \begin{tabular}{|l|}
1 \\
\end{tabular} & SB4VAR44 & 2 & 1 & 5 & M08LM11 & 9.06 & 0.032 & 0.438 & \begin{tabular}{|l|}
$<0.100$ \\
\end{tabular} & 0.028 & 0.139 & & $<0.010$ & $<0.100$ & 0.3 & 2.9 & 0.024 & 0.023 \\
\hline \begin{tabular}{|l|l}
1 & \\
\end{tabular} & SB4VAR52 & 2 & 1 & 6 & M09LM11 & 9.49 & 0.03 & 0.404 & \begin{tabular}{|c|}
$<0.100$ \\
\end{tabular} & 0.022 & 0.125 & & $<0.010$ & $<0.100$ & 0.276 & 2.47 & 0.019 & 0.02 \\
\hline 1 & SB4VAR13 & 2 & 1 & $\overline{7}$ & M14LM21 & 9.68 & $<0.020$ & 0.465 & $<0.100$ & 0.028 & 0.17 & & $<0.010$ & $<0.100$ & 0.014 & 2.95 & 0.022 & 0.024 \\
\hline
\end{tabular}


Table A2. Measured Elemental Concentrations (wt\%) for Samples Prepared Using Lithium Metaborate (part 2)

\begin{tabular}{|c|c|c|c|c|c|c|c|c|c|c|c|c|c|c|c|c|c|c|}
\hline Set & Glass ID & Block & Sub-Block & Sequence & Lab ID & Na (wt\%) & $\mathrm{Nb}(\mathrm{wt} \%)$ & $\mathrm{Ni}$ (wt\%) & $\mathrm{P}$ (wt\%) & $\mathrm{Pb}$ (wt\%) & S (wt\%) & Si (wt\%) & Sr (wt\%) & Th (wt\%) & Ti (wt\%) & $\mathrm{U}$ (wt\%) & Zn (wt\%) & $\mathrm{Zr}(\mathrm{wt} \%)$ \\
\hline \begin{tabular}{|l|}
1 \\
\end{tabular} & \begin{tabular}{|l|} 
SB4VAR23 \\
\end{tabular} & 2 & 1 & 8 & $\begin{array}{l}\text { M10LM11 } \\
\end{array}$ & 8.32 & $<0.020$ & 0.438 & $<<0.100$ & 0.025 & 0.121 & & $<0.010$ & \begin{tabular}{|l|}
$<0.100$ \\
\end{tabular} & 0.014 & 2.75 & 0.021 & 0.022 \\
\hline 1 & SB4VAR23 & 2 & 1 & 9 & M10LM21 & 8.27 & $<0.020$ & 0.418 & $<0.100$ & 0.024 & 0.116 & & \begin{tabular}{|l|}
$<0.010$ \\
\end{tabular} & $<0.100$ & 0.014 & 2.7 & 0.02 & 0.02 \\
\hline 1 & \begin{tabular}{|l|} 
SB4VAR24 \\
\end{tabular} & 2 & 1 & 10 & M07LM11 & 8.6 & $<0.020$ & 0.419 & $<0.100$ & 0.027 & 0.126 & & $<0.010$ & $<0.100$ & 0.013 & 2.96 & 0.021 & 0.024 \\
\hline \begin{tabular}{|l|}
1 \\
\end{tabular} & Batch 1 & 2 & 1 & 11 & BCHLM1-212 & 6.63 & 0.034 & 0.544 & $<0.100$ & $<0.020$ & $<0.100$ & & \begin{tabular}{|c|}
$<010$ \\
\end{tabular} & $<0.100$ & 0.384 & $<0.100$ & $<0.010$ & 0.064 \\
\hline \begin{tabular}{|l|}
1 \\
\end{tabular} & U std & 2 & 1 & 12 & UstdLM1-212 & 8.54 & 0.052 & 0.782 & $<0.100$ & $<0.020$ & $<0.100$ & & $<0.010$ & $<0.100$ & 0.558 & 1.93 & $<0.010$ & $<0.010$ \\
\hline \begin{tabular}{|l|l}
1 \\
\end{tabular} & SB4VAR13 & 2 & 1 & 13 & $\begin{array}{l}\text { M14LM11 } \\
\end{array}$ & 9.57 & $<0.020$ & 0.466 & $<0.100$ & 0.029 & 0.17 & & $<0.010$ & $<0.100$ & 0.014 & 2.93 & 0.022 & 0.024 \\
\hline 1 & SB4VAR11 & 2 & 1 & 14 & M02LM11 & 8.97 & $<0.020$ & 0.369 & $<0.100$ & 0.021 & 0.139 & & $<0.010$ & $<0.100$ & 0.01 & 2.37 & 0.016 & 0.019 \\
\hline \begin{tabular}{|l|}
1 \\
\end{tabular} & \begin{tabular}{|l|} 
SB4VAR21 \\
\end{tabular} & 2 & 1 & 15 & M13LM21 & 7.93 & $<0.020$ & 0.373 & $<0.100$ & 0.021 & $<0.100$ & & $<0.010$ & $<0.100$ & 0.012 & 2.32 & 0.016 & 0.019 \\
\hline \begin{tabular}{|l|}
1 \\
\end{tabular} & \begin{tabular}{|l|} 
SB4VAR53 \\
\end{tabular} & 2 & 1 & 16 & M11LM21 & 9.33 & 0.031 & 0.416 & $<0.100$ & 0.026 & $\begin{array}{ll}0.127 \\
\end{array}$ & & $<<0.010$ & $<0.100$ & 0.286 & 2.56 & 0.02 & 0.022 \\
\hline \begin{tabular}{|l|}
1 \\
\end{tabular} & \begin{tabular}{|l|} 
SB4VAR52 \\
\end{tabular} & 2 & 1 & 17 & M09LM21 & 8.99 & 0.03 & 0.394 & $<0.100$ & 0.023 & 0.114 & & $<0.010$ & $<0.100$ & 0.273 & 2.37 & 0.018 & 0.022 \\
\hline 1 & \begin{tabular}{|l|} 
SB4VAR44 \\
\end{tabular} & 2 & 1 & 18 & M08LM21 & 8.71 & 0.033 & 0.452 & $<0.100$ & 0.029 & 0.134 & & $<0.010$ & $<0.100$ & 0.303 & 2.8 & 0.024 & 0.025 \\
\hline \begin{tabular}{|l|}
1 \\
\end{tabular} & \begin{tabular}{|l|} 
SB4VAR24 \\
\end{tabular} & 2 & 1 & 19 & M07LM21 & 8.45 & $<0.020$ & 0.444 & $<0.100$ & 0.029 & 0.129 & & $<0.010$ & $<0.100$ & 0.014 & 2.97 & 0.022 & 0.024 \\
\hline \begin{tabular}{|l|}
1 \\
\end{tabular} & \begin{tabular}{|l|} 
SB4VAR21 \\
\end{tabular} & 2 & 1 & 20 & M13LM11 & 7.86 & $<0.020$ & 0.377 & $<0.100$ & 0.022 & $<0.100$ & & $<0.010$ & $<0.100$ & 0.012 & 2.31 & 0.017 & 0.02 \\
\hline 1 & Batch 1 & 2 & 1 & 21 & BCHLM1-213 & 6.51 & 0.034 & 0.542 & $<0.100$ & $<0.020$ & $<0.100$ & & $<0.010$ & $<0.100$ & 0.383 & $<0.100$ & $<0.010$ & 0.064 \\
\hline 1 & U std & 2 & 1 & 22 & UstdLM1-213 & 8.42 & 0.051 & 0.777 & $<0.100$ & $<0.020$ & $<0.100$ & & $<0.010$ & $<0.100$ & 0.556 & 1.92 & $<0.010$ & $<0.010$ \\
\hline \begin{tabular}{|l|}
1 \\
\end{tabular} & Batch 1 & 2 & 2 & 1 & BCHLM1-221 & 6.72 & 0.038 & 0.549 & $<0.100$ & $<0.020$ & $<0.100$ & & $<0.010$ & $<0.100$ & 0.386 & $<0.100$ & $<0.010$ & 0.063 \\
\hline \begin{tabular}{|l|}
1 \\
\end{tabular} & U std & 2 & 2 & 2 & UstdLM1-221 & 8.61 & 0.055 & 0.779 & $<0.100$ & $<0.020$ & $<0.100$ & & $<0.010$ & $<0.100$ & 0.561 & 1.97 & $<0.010$ & $<0.010$ \\
\hline 1 & \begin{tabular}{|l|} 
SB4VAR24 \\
\end{tabular} & 2 & 2 & 3 & M07LM22 & 8.85 & $<0.020$ & 0.448 & $<0.100$ & 0.029 & 0.127 & & $<0.010$ & $<0.100$ & 0.014 & 3.05 & 0.022 & 0.023 \\
\hline \begin{tabular}{|l|}
1 \\
\end{tabular} & SB4VAR13 & 2 & 2 & 4 & M14LM12 & 9.73 & $<0.020$ & 0.468 & $<0.100$ & 0.028 & 0.17 & & $<0.010$ & $<0.100$ & 0.014 & 2.97 & 0.022 & 0.023 \\
\hline 1 & \begin{tabular}{|l|} 
SB4VAR24 \\
\end{tabular} & 2 & 2 & 5 & M07LM12 & 8.72 & $<0.020$ & 0.418 & $<0.100$ & 0.027 & 0.124 & & $<0.010$ & $<0.100$ & 0.013 & 3.03 & 0.021 & 0.023 \\
\hline \begin{tabular}{|l|}
1 \\
\end{tabular} & \begin{tabular}{|l|} 
SB4VAR23 \\
\end{tabular} & 2 & 2 & 6 & M10LM12 & 8.64 & $<0.020$ & 0.437 & $<0.100$ & 0.024 & 0.117 & & $<0.010$ & $<0.100$ & 0.014 & 2.82 & 0.021 & 0.021 \\
\hline 1 & \begin{tabular}{|l|} 
SB4VAR52 \\
\end{tabular} & 2 & 2 & 7 & M09LM12 & 9.51 & 0.033 & 0.403 & $<0.100$ & 0.023 & $\begin{array}{ll}0.119 \\
\end{array}$ & & $<0.010$ & $<0.100$ & 0.276 & 2.49 & 0.018 & 0.019 \\
\hline \begin{tabular}{|l|}
1 \\
\end{tabular} & \begin{tabular}{|l|} 
SB4VAR21 \\
\end{tabular} & 2 & 2 & 8 & M13LM22 & 8.02 & $<0.020$ & 0.379 & $<0.100$ & 0.022 & $<0.100$ & & $<0.010$ & $<0.100$ & 0.012 & 2.35 & 0.016 & 0.018 \\
\hline \begin{tabular}{|l|}
1 \\
\end{tabular} & \begin{tabular}{|l|} 
SB4VAR23 \\
\end{tabular} & 2 & 2 & 9 & M10LM22 & 8.48 & $<0.020$ & 0.416 & $<0.100$ & 0.023 & $\begin{array}{ll}0.114 \\
\end{array}$ & & $<0.010$ & $<0.100$ & 0.013 & 2.78 & 0.02 & 0.019 \\
\hline 1 & SB4VAR11 & 2 & 2 & 10 & M02 & 9.04 & $<0.020$ & 0.369 & $<0.100$ & 0.021 & 0.137 & & $<0.010$ & $<0.100$ & 0.01 & 2.41 & 0.016 & 0.018 \\
\hline \begin{tabular}{|l|l}
1 \\
\end{tabular} & Batch 1 & 2 & 2 & 11 & BCHLM1-222 & 7.02 & 0.037 & 0.548 & $<0.100$ & $<0.020$ & $<0.100$ & & $<0.010$ & $<0.100$ & 0.386 & $<0.100$ & $<0.010$ & 0.063 \\
\hline \begin{tabular}{|l|}
1 \\
\end{tabular} & U std & 2 & 2 & 12 & UstdLM1-222 & 8.8 & 0.055 & 0.794 & $<0.100$ & $<0.020$ & $<0.100$ & & $<0.010$ & $<0.100$ & 0.561 & 1.97 & $<0.010$ & $<0.010$ \\
\hline \begin{tabular}{|l|l}
1 \\
\end{tabular} & SB4VAR53 & 2 & 2 & 13 & M11LM22 & 9.6 & 0.034 & 0.418 & $<0.100$ & 0.025 & 0.127 & & $<0.010$ & $<0.100$ & 0.283 & 2.63 & 0.02 & 0.021 \\
\hline 1 & SB4VAR44 & 2 & 2 & 14 & M08LM12 & 9.35 & 0.035 & 0.44 & $<0.100$ & 0.028 & 0.138 & & $<0.010$ & $<0.100$ & 0.298 & 2.97 & 0.023 & 0.022 \\
\hline \begin{tabular}{|l|}
1 \\
\end{tabular} & \begin{tabular}{|l|} 
SB4VAR52 \\
\end{tabular} & 2 & 2 & 15 & M09LM22 & 9.32 & 0.033 & 0.402 & $<0.100$ & 0.023 & 0.116 & & $<0.010$ & $<0.100$ & 0.277 & 2.4 & 0.018 & 0.021 \\
\hline 1 & \begin{tabular}{|l|} 
SB4VAR11 \\
\end{tabular} & 2 & 2 & 16 & M02LM22 & 9.23 & $<0.020$ & 0.391 & $<0.100$ & 0.022 & 0.135 & & $<0.010$ & $<0.100$ & 0.01 & 2.41 & 0.019 & 0.019 \\
\hline \begin{tabular}{|l|}
1 \\
\end{tabular} & SB4VAR44 & 2 & 2 & 17 & M08LM22 & 9.01 & 0.036 & 0.463 & $<0.100$ & 0.029 & 0.137 & & $<0.010$ & $<0.100$ & 0.305 & 2.89 & 0.024 & 0.024 \\
\hline 1 & \begin{tabular}{|l|} 
SB4VAR21 \\
\end{tabular} & 2 & 2 & 18 & M13LM12 & 8.25 & $<0.020$ & 0.38 & $<0.100$ & 0.021 & $<0.100$ & & $<0.010$ & $<0.100$ & 0.012 & 2.4 & 0.017 & 0.018 \\
\hline \begin{tabular}{|l|}
1 \\
\end{tabular} & \begin{tabular}{|l|} 
SB4VAR13 \\
\end{tabular} & 2 & 2 & 19 & M14LM22 & 9.91 & $<0.020$ & 0.461 & $<0.100$ & 0.028 & 0.164 & & $<0.010$ & $<0.100$ & 0.013 & 2.98 & 0.022 & 0.023 \\
\hline \begin{tabular}{|l|l}
1 \\
\end{tabular} & \begin{tabular}{|l|} 
SB4VAR53 \\
\end{tabular} & 2 & 2 & 20 & M11LM12 & 10.04 & 0.033 & 0.428 & $<0.100$ & 0.025 & 0.127 & & $\begin{array}{l}<0.010 \\
\end{array}$ & $<0.100$ & 0.284 & 2.61 & 0.019 & 0.021 \\
\hline 1 & Batch 1 & 2 & 2 & 21 & BCHLM1-223 & 6.64 & 0.037 & 0.544 & $<0.100$ & $<0.020$ & $<0.100$ & & $<0.010$ & $<0.100$ & 0.384 & $<0.100$ & $<0.010$ & 0.063 \\
\hline \begin{tabular}{|l|l}
1 \\
\end{tabular} & U std & 2 & 2 & 22 & UstdLM1-223 & 8.89 & 0.054 & 0.771 & $<0.100$ & $<0.020$ & $<0.100$ & & $<0.010$ & $<0.100$ & 0.545 & 1.99 & $<0.010$ & $<0.010$ \\
\hline \begin{tabular}{|l}
2 \\
\end{tabular} & Batch 1 & 1 & 1 & 1 & BCHLM2-111 & 6.59 & 0.03 & 0.551 & $<0.100$ & $<0.020$ & $<0.100$ & 23.9 & $<0.010$ & $<0.100$ & 0.387 & $<0.100$ & $<0.010$ & 0.062 \\
\hline \begin{tabular}{|l|}
2 \\
\end{tabular} & U std & 1 & 1 & 2 & UstdLM2-111 & 8.36 & 0.048 & 0.793 & $<0.100$ & $<0.020$ & $<0.100$ & 21.2 & $<0.010$ & $<0.100$ & 0.563 & 1.93 & $<0.010$ & $<0.010$ \\
\hline 2 & SB5-01 & 1 & 1 & 3 & N02LM11 & 9.83 & $<0.010$ & 0.705 & $<0.100$ & $<0.020$ & $<0.100$ & 21.3 & 0.096 & $<0.100$ & $<0.010$ & 2.26 & $<0.010$ & 0.065 \\
\hline \begin{tabular}{|l|}
2 \\
\end{tabular} & SB5-05 & 1 & 1 & 4 & $\begin{array}{l}\text { N07LM11 } \\
\end{array}$ & 10.1 & $<0.010$ & 0.802 & $<0.100$ & $<0.020$ & $<0.100$ & 21.3 & 0.108 & $<0.100$ & 0.01 & 2.59 & $<0.010$ & 0.073 \\
\hline 2 & SB5-04 & 1 & 1 & 5 & $\begin{array}{l}\text { N03LM21 } \\
\end{array}$ & 10.8 & $<0.010$ & 0.724 & $<0.100$ & $<0.020$ & $<0.100$ & 20.7 & 0.089 & $<0.100$ & $<0.010$ & 2.34 & $<0.010$ & 0.068 \\
\hline \begin{tabular}{|l|}
2 \\
\end{tabular} & SB5-04 & 1 & 1 & 6 & $\begin{array}{l}\text { N03LM11 } \\
\end{array}$ & 10.8 & $<0.010$ & 0.729 & $<0.100$ & $<0.020$ & $<0.100$ & 20.8 & 0.09 & $<0.100$ & 0.01 & 2.34 & $<0.010$ & 0.068 \\
\hline \begin{tabular}{|l|}
2 \\
\end{tabular} & SB5-12 & 1 & 1 & 7 & N13LM21 & 10.5 & $<0.010$ & 0.882 & $<0.100$ & $<0.020$ & 0.102 & 20.9 & 0.109 & $<0.100$ & 0.011 & 2.74 & $<0.010$ & 0.08 \\
\hline \begin{tabular}{|l}
2 \\
\end{tabular} & SB5-02 & 1 & 1 & 8 & $\begin{array}{l}\text { N12LM21 } \\
\end{array}$ & 9.96 & $<0.010$ & 0.72 & $<0.100$ & $<0.020$ & $<0.100$ & 21.2 & 0.09 & $<0.100$ & 0.011 & 2.26 & $<0.010$ & 0.067 \\
\hline \begin{tabular}{|l|}
2 \\
\end{tabular} & SB5-11 & 1 & 1 & 9 & N14LM11 & 9.95 & $<0.010$ & 0.864 & $<0.100$ & $<0.020$ & 0.102 & 20.3 & 0.112 & $<0.100$ & 0.011 & 2.67 & $<0.010$ & 0.082 \\
\hline \begin{tabular}{|l}
2 \\
\end{tabular} & SB5-07 & 1 & 1 & 10 & N15LM21 & 10.1 & $<0.010$ & 0.778 & $<0.100$ & $<0.020$ & $<0.100$ & 20.9 & 0.108 & $<0.100$ & 0.01 & 2.58 & $<0.010$ & 0.071 \\
\hline
\end{tabular}


Table A2. Measured Elemental Concentrations (wt\%) for Samples Prepared Using Lithium Metaborate (part 2)

\begin{tabular}{|c|c|c|c|c|c|c|c|c|c|c|c|c|c|c|c|c|c|c|}
\hline Set & Glass ID & Block & Sub-Block & Sequence & Lab ID & Na (wt\%) & $\mathrm{Nb}(\mathrm{wt} \%)$ & Ni (wt\%) & $\mathrm{P}(\mathrm{wt} \%)$ & $\overline{\mathrm{Pb}(\mathrm{wt} \%)}$ & S (wt\%) & Si (wt\%) & Sr (wt\%) & Th (wt\%) & Ti (wt\%) & $\mathrm{U}$ (wt\%) & Zn (wt\%) & $\mathrm{Zr}$ (wt\%) \\
\hline \begin{tabular}{|l|}
2 \\
\end{tabular} & Batch 1 & 1 & 1 & 11 & BCHLM2-112 & 6.37 & 0.029 & 0.551 & \begin{tabular}{|c|c|}
$<0.100$ \\
\end{tabular} & $<0.020$ & $<0.100$ & 23.6 & $<0.010$ & $<0.100$ & 0.386 & $<0.100$ & $<0.010$ & 0.063 \\
\hline \begin{tabular}{|l|}
2 \\
\end{tabular} & U std & 1 & 1 & 12 & UstdLM1-112 & 8.42 & 0.047 & 0.795 & $<0.100$ & $<0.020$ & $<0.100$ & 21.4 & $<0.010$ & $<0.100$ & 0.564 & 1.93 & $<0.010$ & $<0.010$ \\
\hline \begin{tabular}{|l|}
2 \\
\end{tabular} & SB5-02 & 1 & 1 & 13 & N12LM11 & 9.83 & $<0.010$ & 0.732 & $<0.100$ & $<0.020$ & $<0.100$ & 21.4 & 0.091 & $<0.100$ & 0.011 & 2.29 & $<0.010$ & 0.065 \\
\hline \begin{tabular}{|l|}
2 \\
\end{tabular} & SB5-11 & 1 & 1 & 14 & N14LM21 & 9.67 & $<0.010$ & 0.884 & $<0.100$ & $<0.020$ & 0.104 & 20.8 & 0.113 & $<0.100$ & 0.011 & 2.69 & $<0.010$ & 0.082 \\
\hline \begin{tabular}{|l|}
2 \\
\end{tabular} & \begin{tabular}{|l|} 
SB4VAR33 \\
\end{tabular} & 1 & 1 & 15 & $\begin{array}{l}\text { N05LM21 } \\
\end{array}$ & 9.26 & $<0.010$ & 0.432 & $<0.100$ & 0.026 & 0.127 & 19.8 & $<0.010$ & $<0.100$ & 0.013 & 2.79 & $<0.010$ & 0.02 \\
\hline \begin{tabular}{|l|}
2 \\
\end{tabular} & SB5-05 & 1 & 1 & 16 & N07LM21 & 10.1 & $<0.010$ & 0.777 & $<0.100$ & $<0.020$ & $<0.100$ & 20.8 & 0.11 & $<0.100$ & 0.011 & 2.55 & $<0.010$ & 0.071 \\
\hline \begin{tabular}{|l|}
2 \\
\end{tabular} & SB5-12 & 1 & 1 & 17 & N13LM11 & 10.2 & $<0.010$ & 0.879 & $<0.100$ & $<0.020$ & 0.1 & 20.8 & 0.111 & $<0.100$ & 0.011 & 2.71 & $<0.010$ & 0.079 \\
\hline \begin{tabular}{|l|}
2 \\
\end{tabular} & SB4VAR33 & 1 & 1 & 18 & N05LM11 & 9.26 & $<0.010$ & 0.447 & $<0.100$ & 0.026 & 0.124 & 19.8 & $<0.010$ & $<0.100$ & 0.014 & 2.81 & $<0.010$ & 0.021 \\
\hline \begin{tabular}{|l|}
2 \\
\end{tabular} & SB5-01 & 1 & 1 & 19 & N02LM21 & 10.19 & $<0.010$ & 0.739 & $<0.100$ & $<0.020$ & $<0.100$ & 21.9 & 0.099 & $<0.100$ & $<0.010$ & 2.38 & $<0.010$ & 0.062 \\
\hline \begin{tabular}{|l|}
2 \\
\end{tabular} & SB5-07 & 1 & 1 & 20 & $\begin{array}{l}\text { N15LM11 } \\
\end{array}$ & 9.9 & $<0.010$ & 0.816 & \begin{tabular}{|c|}
$<0.100$ \\
\end{tabular} & $<0.020$ & 0.102 & 21 & 0.107 & $<0.100$ & 0.01 & 2.56 & $<0.010$ & 0.072 \\
\hline \begin{tabular}{|l|}
2 \\
\end{tabular} & Batch 1 & 1 & 1 & 21 & BCHLM2-113 & 6.69 & 0.029 & 0.553 & $<0.100$ & $<0.020$ & $<0.100$ & 24.1 & $<0.010$ & $<0.100$ & 0.388 & $<0.100$ & $<0.010$ & 0.064 \\
\hline \begin{tabular}{|l|}
2 \\
\end{tabular} & U std & 1 & 1 & 22 & UstdLM2-113 & 8.37 & 0.047 & 0.794 & \begin{tabular}{|c|}
$<0.100$ \\
\end{tabular} & $<0.020$ & $<0.100$ & 21.3 & $<0.010$ & $<0.100$ & 0.558 & 1.94 & $<0.010$ & $<0.010$ \\
\hline \begin{tabular}{|l|}
2 \\
\end{tabular} & Batch 1 & 1 & 2 & 1 & BCHLM2-121 & 6.6 & 0.029 & 0.551 & $<0.100$ & $<0.020$ & $<0.100$ & 23.2 & $<0.010$ & $<0.100$ & 0.389 & $<0.100$ & $<0.010$ & 0.062 \\
\hline \begin{tabular}{|l|}
2 \\
\end{tabular} & U std & 1 & 2 & 2 & UstdLM2-121 & 8.56 & 0.047 & 0.79 & $<0.100$ & $<0.020$ & $<0.100$ & 21.5 & $<0.010$ & $<0.100$ & 0.564 & 1.94 & $<0.010$ & $<0.010$ \\
\hline \begin{tabular}{|l|}
2 \\
\end{tabular} & SB5-12 & 1 & 2 & 3 & N13LM22 & 10.3 & $<0.010$ & 0.878 & $<0.100$ & $<0.020$ & $<0.100$ & 21.2 & 0.108 & $<0.100$ & $<0.010$ & 2.69 & $<0.010$ & 0.078 \\
\hline \begin{tabular}{|l|}
2 \\
\end{tabular} & SB5-05 & 1 & 2 & 4 & N07LM22 & 10 & $<0.010$ & 0.772 & $<0.100$ & $<0.020$ & $<0.100$ & 20.6 & 0.109 & $<0.100$ & $<0.010$ & 2.54 & $<0.010$ & 0.07 \\
\hline \begin{tabular}{|l|}
2 \\
\end{tabular} & SB4VAR33 & 1 & 2 & 5 & N05LM22 & 9.29 & $<0.010$ & 0.429 & $<0.100$ & 0.024 & 0.122 & 19.7 & $<0.010$ & $<0.100$ & 0.012 & 2.8 & $<0.010$ & 0.019 \\
\hline \begin{tabular}{|l|}
2 \\
\end{tabular} & \begin{tabular}{|l|} 
SB4VAR33 \\
\end{tabular} & 1 & 2 & 6 & N05LM12 & 9.26 & $<0.010$ & 0.444 & $<0.100$ & 0.025 & 0.123 & 19.7 & $<0.010$ & $<0.100$ & 0.013 & 2.8 & $<0.010$ & 0.02 \\
\hline \begin{tabular}{|l|}
2 \\
\end{tabular} & SB5-01 & 1 & 2 & 7 & $\begin{array}{l}\text { N02LM12 } \\
\end{array}$ & 10.1 & $<0.010$ & 0.69 & $<0.100$ & $<0.020$ & $<0.100$ & 20.9 & 0.095 & $<0.100$ & $<0.010$ & 2.29 & $<0.010$ & 0.063 \\
\hline \begin{tabular}{|l|}
2 \\
\end{tabular} & SB5-04 & 1 & 2 & 8 & N03LM12 & 10.8 & $<0.010$ & 0.721 & $<0.100$ & $<0.020$ & $<0.100$ & 20.4 & 0.088 & $<0.100$ & $<0.010$ & 2.33 & $<0.010$ & 0.067 \\
\hline \begin{tabular}{|l|}
2 \\
\end{tabular} & SB5-01 & 1 & 2 & 9 & N02LM22 & 10.1 & $<0.010$ & 0.727 & $<0.100$ & $<0.020$ & $<0.100$ & 21 & 0.096 & $<0.100$ & $<0.010$ & 2.3 & $<0.010$ & 0.061 \\
\hline \begin{tabular}{|l|}
2 \\
\end{tabular} & $\begin{array}{l}\text { SB5-07 } \\
\text { SB }\end{array}$ & 1 & 2 & 10 & N15LM12 & 9.75 & $<0.010$ & 0.8 & $<0.100$ & $<0.020$ & $<0.100$ & 20.4 & 0.105 & $<0.100$ & $<0.010$ & 2.52 & $<0.010$ & 0.07 \\
\hline \begin{tabular}{|l|}
2 \\
\end{tabular} & Batch 1 & 1 & 2 & 11 & BCHLM2-122 & 6.42 & 0.027 & 0.545 & $<0.100$ & $<0.020$ & $<0.100$ & 23.2 & $<0.010$ & $<0.100$ & 0.384 & $<0.100$ & $<0.010$ & 0.062 \\
\hline \begin{tabular}{|l|}
2 \\
\end{tabular} & U std & 1 & 2 & 12 & UstdLM2-122 & 8.56 & 0.045 & 0.078 & $<0.100$ & $<0.020$ & $<0.100$ & 21.5 & $<0.010$ & $<0.100$ & 0.552 & 1.98 & $<0.010$ & $<0.010$ \\
\hline \begin{tabular}{|l|}
2 \\
\end{tabular} & SB5-04 & 1 & 2 & 13 & N03LM22 & 10.6 & $<0.010$ & 0.705 & $<0.100$ & $<0.020$ & $<0.100$ & 20.5 & 0.089 & $<0.100$ & $<0.010$ & 2.33 & $<0.010$ & 0.065 \\
\hline \begin{tabular}{|l|}
2 \\
\end{tabular} & SB5-11 & 1 & 2 & 14 & N14LM22 & 9.81 & $<0.010$ & 0.871 & $<0.100$ & $<0.020$ & $<0.100$ & 20.7 & 0.112 & $<0.100$ & 0.01 & 2.73 & $<0.010$ & 0.081 \\
\hline \begin{tabular}{|l|}
2 \\
\end{tabular} & SB5-05 & 1 & 2 & 15 & N07LM12 & 9.97 & $<0.010$ & 0.811 & $<0.100$ & $<0.020$ & $<0.100$ & 20.8 & 0.106 & $<0.100$ & $<0.010$ & 2.56 & $<0.010$ & 0.073 \\
\hline \begin{tabular}{|l|}
2 \\
\end{tabular} & SB5-02 & 1 & 2 & 16 & N12 & 9.8 & $<0.010$ & 0.738 & $<0.100$ & $<0.020$ & $<0.100$ & 21.2 & 0.089 & $<0.100$ & $<0.010$ & 2.28 & $<0.010$ & 0.066 \\
\hline \begin{tabular}{|l|}
2 \\
\end{tabular} & SB5-11 & 1 & 2 & 17 & N14LM12 & 9.69 & $<0.010$ & 0.886 & $<0.100$ & $<0.020$ & 0.1 & 20.8 & 0.111 & $<0.100$ & $<0.010$ & 2.66 & $<0.010$ & 0.082 \\
\hline \begin{tabular}{|l|}
2 \\
\end{tabular} & SB5-02 & 1 & 2 & 18 & N12 & 9.77 & $<0.010$ & 0.735 & $<0.100$ & $<0.020$ & $<0.100$ & 20.8 & 0.09 & $<0.100$ & $<0.010$ & 2.24 & $<0.010$ & 0.067 \\
\hline \begin{tabular}{|l|}
2 \\
\end{tabular} & SB5-12 & 1 & 2 & 19 & N13 & 9.96 & $<0.010$ & 0.89 & $<0.100$ & $<0.020$ & $<0.100$ & 21.1 & 0.109 & $<0.100$ & $<0.010$ & 2.72 & $<0.010$ & 0.079 \\
\hline \begin{tabular}{|l|}
2 \\
\end{tabular} & SB5-07 & 1 & 2 & 20 & N15LM22 & 9.83 & $<0.010$ & 0.782 & $<0.100$ & $<0.020$ & $<0.100$ & 21.4 & 0.106 & $<0.100$ & $<0.010$ & 2.57 & $<0.010$ & 0.071 \\
\hline \begin{tabular}{|l|}
2 \\
\end{tabular} & Batch 1 & 1 & 2 & 21 & BCHLM2-12 & 6.62 & 0.028 & 0.557 & $<0.100$ & $<0.020$ & $<0.100$ & 24.5 & & & 0.387 & $<0.100$ & $<0.010$ & 0.063 \\
\hline \begin{tabular}{|l|}
2 \\
\end{tabular} & U std & 1 & 2 & 22 & UstdLM2-123 & 8.42 & 0.047 & 0.804 & $<0.100$ & $<0.020$ & $<0.100$ & 21.5 & $<0.010$ & $<0.100$ & 0.562 & 1.95 & $<0.010$ & $<0.010$ \\
\hline \begin{tabular}{|l|}
2 \\
\end{tabular} & Batch 1 & 2 & 1 & 1 & BCHLM2-211 & 6.62 & 0.032 & 0.557 & $<0.100$ & $<0.020$ & $<0.100$ & 23.5 & $<0.010$ & $<0.100$ & 0.397 & $<0.100$ & $<0.010$ & 0.065 \\
\hline \begin{tabular}{|l|}
2 \\
\end{tabular} & U std & 2 & 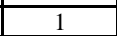 & 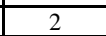 & UstdLM2-211 & 855 & 0.051 & 0.799 & $<0.100$ & $<0.020$ & $<0.100$ & 21.2 & $<0.010$ & $<0.100$ & 0.575 & 1.94 & $<0.010$ & $<0.010$ \\
\hline \begin{tabular}{|l|}
2 \\
\end{tabular} & SB4VAR32 & 2 & 1 & 3 & N11LM21 & 8.97 & $<0.010$ & 0.399 & $<0.100$ & $<0.020$ & \begin{tabular}{|l|}
0.116 \\
\end{tabular} & 21 & $<0.010$ & $<0.100$ & 0.013 & 2.51 & $<0.010$ & 0.019 \\
\hline \begin{tabular}{|l|}
2 \\
\end{tabular} & \begin{tabular}{|l|} 
SB4VAR31 \\
\end{tabular} & 2 & 1 & 4 & N06LM11 & 8.56 & $<0.010$ & 0.38 & $<0.100$ & $<0.020$ & 0.104 & 22 & $<0.010$ & $<0.100$ & 0.015 & 2.21 & $<0.010$ & 0.018 \\
\hline \begin{tabular}{|l|}
2 \\
\end{tabular} & SB5-03 & 2 & 1 & 5 & N09LM11 & 9.9 & $<0.010$ & 0.726 & $<0.100$ & $<0.020$ & $\begin{array}{l}<0.100 \\
\end{array}$ & 20.8 & 0.099 & $<0.100$ & 0.01 & 2.29 & $<0.010$ & 0.069 \\
\hline \begin{tabular}{|l|}
2 \\
\end{tabular} & SB5-09 & 2 & 1 & 6 & N04LM11 & 9.82 & $<0.010$ & 0.836 & $<0.100$ & $<0.020$ & 0.104 & 21 & 0.123 & $<0.100$ & 0.012 & 2.69 & $<0.010$ & 0.085 \\
\hline \begin{tabular}{|l|}
2 \\
\end{tabular} & SB5-03 & 2 & 1 & 7 & N09L & 9.93 & $<0.010$ & 0.753 & $<0.100$ & $<0.020$ & $<0.100$ & 21.3 & 0.097 & $<0.100$ & 0.011 & 2.34 & $<0.010$ & 0.071 \\
\hline \begin{tabular}{|l|l}
2 \\
\end{tabular} & SB4VAR32 & 2 & 1 & 8 & N11LM11 & 8.93 & $<0.010$ & 0.408 & $<0.100$ & $<0.020$ & 0.117 & 21.9 & $<0.010$ & $<0.100$ & 0.013 & 2.57 & $<0.010$ & 0.019 \\
\hline \begin{tabular}{|l|}
2 \\
\end{tabular} & SB5-10 & 2 & 1 & 9 & N08lLM11 & 9.87 & $<0.010$ & 0.849 & $<0.100$ & $<0.020$ & 0.11 & 22.2 & 0.122 & $<0.100$ & 0.013 & 2.75 & $<0.010$ & 0.08 \\
\hline \begin{tabular}{|l|}
2 \\
\end{tabular} & Batch 1 & 2 & 1 & 10 & BCHL & 6.59 & 0.031 & 0.553 & $<0.100$ & $<0.020$ & $<0.100$ & 24.1 & $<0.010$ & $<0.100$ & 0.393 & $<0.100$ & $<0.010$ & 0.065 \\
\hline \begin{tabular}{|l|}
2 \\
\end{tabular} & U std & 2 & 1 & 11 & UstdLM2-212 & 8.58 & 0.051 & 0.812 & $<0.100$ & $<0.020$ & $<0.100$ & 21.1 & $<0.010$ & $<0.100$ & 0.585 & 1.94 & $<0.010$ & $<0.010$ \\
\hline \begin{tabular}{|l}
2 \\
\end{tabular} & SB5-08 & 2 & 1 & 12 & N10LM21 & 9.96 & $<0.010$ & 0.808 & \begin{tabular}{|c|}
$<0.100$ \\
\end{tabular} & $<0.020$ & $<0.100$ & 21.2 & 0.104 & $<0.100$ & 0.012 & 2.49 & $<0.010$ & 0.072 \\
\hline$=$ & SB5-06 & 2 & 1 & 13 & N01LM11 & 9.91 & $<0.010$ & 0.819 & $<0.100$ & $<0.020$ & 0.102 & 21.2 & 0.111 & $<0.100$ & 0.011 & 2.55 & $<0.010$ & 0.076 \\
\hline
\end{tabular}


Table A2. Measured Elemental Concentrations (wt\%) for Samples Prepared Using Lithium Metaborate (part 2)

\begin{tabular}{|c|c|c|c|c|c|c|c|c|c|c|c|c|c|c|c|c|c|c|}
\hline Set] & Glass ID & Block & Sub-Block & Sequence & Lab ID & $\mathrm{Na}(\mathrm{wt} \%)$ & $\mathrm{Nb}(\mathrm{wt} \%)$ & Ni (wt\%) & \begin{tabular}{|l|}
$\mathrm{P}$ (wt\%) \\
\end{tabular} & $\mathrm{Pb}(\mathrm{wt} \%)$ & S (wt\%) & Si (wt\%) & Sr (wt\%) & Th (wt\%) & \begin{tabular}{|l|} 
Ti (wt\%) \\
\end{tabular} & $\mathrm{U}$ (wt\%) & Zn (wt\%) & $\mathrm{Zr}(\mathrm{wt} \%)$ \\
\hline \begin{tabular}{|l|}
2 \\
\end{tabular} & \begin{tabular}{|l|} 
SB4VAR31 \\
\end{tabular} & 2 & 1 & 14 & N06LM21 & 8.69 & $<0.010$ & 0.362 & \begin{tabular}{|c|}
$<0.100$ \\
\end{tabular} & $<0.020$ & 0.103 & 22.8 & $<0.010$ & $<0.100$ & 0.015 & 2.26 & $<0.010$ & 0.017 \\
\hline 2 & SB5-10 & 2 & 1 & 15 & N08LM21 & 9.8 & $<0.010$ & 0.86 & \begin{tabular}{|c|}
$<0.100$ \\
\end{tabular} & $<0.020$ & 0.107 & 21.4 & 0.121 & $<0.100$ & 0.012 & 2.69 & $<0.010$ & 0.081 \\
\hline \begin{tabular}{|l|}
2 \\
\end{tabular} & SB5-08 & 2 & 1 & 16 & N10LM11 & 10.4 & $<0.010$ & 0.877 & \begin{tabular}{|c|}
$<0.100$ \\
\end{tabular} & $<0.020$ & 0.105 & 20.7 & 0.11 & $<0.100$ & 0.012 & 2.56 & $<0.010$ & 0.075 \\
\hline \begin{tabular}{|l|}
2 \\
\end{tabular} & $\begin{array}{l}\text { SB5-09 } \\
\end{array}$ & 2 & 1 & 17 & N04LM21 & 9.8 & $<0.010$ & 0.89 & \begin{tabular}{|l|}
$<0.100$ \\
\end{tabular} & $<0.020$ & 0.107 & 20.7 & 0.118 & $<0.100$ & 0.012 & 2.68 & $<0.010$ & 0.089 \\
\hline \begin{tabular}{|l|}
2 \\
\end{tabular} & SB5-06 & 2 & 1 & 18 & N01LM21 & 10.2 & $<0.010$ & 0.74 & \begin{tabular}{|l|}
$<0.100$ \\
\end{tabular} & $<0.020$ & \begin{tabular}{|c|}
$<0.100$ \\
\end{tabular} & 21.7 & 0.106 & $<0.100$ & 0.01 & 2.65 & $<0.010$ & 0.071 \\
\hline 2 & Batch 1 & 2 & 1 & 19 & BCHLM2-213 & 6.66 & 0.031 & 0.558 & \begin{tabular}{|c|}
$<0.100$ \\
\end{tabular} & $<0.020$ & \begin{tabular}{|c|}
$<0.100$ \\
\end{tabular} & 24.2 & $<0.010$ & $<0.100$ & 0.399 & \begin{tabular}{|l|}
$<0.100$ \\
\end{tabular} & $<0.010$ & 0.066 \\
\hline 2 & U std & 2 & 1 & 20 & UstdLM2-213 & 8.49 & 0.051 & 0.81 & \begin{tabular}{|l|}
$<0.100$ \\
\end{tabular} & $<0.020$ & \begin{tabular}{|c|}
$<0.100$ \\
\end{tabular} & 21.7 & $<0.010$ & $<0.100$ & 0.578 & 1.96 & $<0.010$ & $<0.010$ \\
\hline \begin{tabular}{|l|}
2 \\
\end{tabular} & Batch 1 & 2 & 2 & 1 & BCHLM2-221 & 6.91 & 0.028 & 0.557 & \begin{tabular}{|c|}
$<0.100$ \\
\end{tabular} & $<0.020$ & $<0.100$ & 23.7 & $<0.010$ & $<0.100$ & 0.394 & $<0.100$ & $<0.010$ & 0.062 \\
\hline \begin{tabular}{|l|}
2 \\
\end{tabular} & U std & 2 & 2 & 2 & \begin{tabular}{|l|} 
UstdLM2-221 \\
\end{tabular} & 9.13 & 0.047 & 0.805 & \begin{tabular}{|l|}
$<0.100$ \\
\end{tabular} & $<0.020$ & \begin{tabular}{|l|}
$<0.100$ \\
\end{tabular} & 22 & $<0.010$ & $<0.100$ & 0.575 & 2.11 & $<0.010$ & $<0.010$ \\
\hline \begin{tabular}{|l|}
2 \\
\end{tabular} & \begin{tabular}{|l|} 
SB4VAR31 \\
\end{tabular} & 2 & 2 & 3 & N06LM22 & 9.02 & $<0.010$ & 0.357 & \begin{tabular}{|c|}
$<0.100$ \\
\end{tabular} & $<0.020$ & 0.104 & 22.9 & $<0.010$ & $<0.100$ & 0.012 & 2.41 & $<0.010$ & 0.014 \\
\hline 2 & SB4VAR32 & 2 & 2 & 4 & N11LM22 & 9.24 & $<0.010$ & 0.405 & \begin{tabular}{|l|}
$<0.100$ \\
\end{tabular} & $<0.020$ & 0.115 & 21.9 & $<0.010$ & $<0.100$ & 0.01 & 2.71 & $<0.010$ & 0.016 \\
\hline \begin{tabular}{|l|}
2 \\
\end{tabular} & SB5-10 & 2 & 2 & 5 & N08L & 10.1 & $<0.010$ & 0.839 & \begin{tabular}{|l|}
$<0.100$ \\
\end{tabular} & $<0.020$ & 0.107 & 22.1 & 0.118 & $<0.100$ & $<0.010$ & 2.88 & $<0.010$ & 0.077 \\
\hline 2 & $\begin{array}{l}\text { SB5-06 } \\
\end{array}$ & 2 & 2 & 6 & N01L & 0.2 & $<0.010$ & 0.789 & \begin{tabular}{|l|}
$<0.100$ \\
\end{tabular} & $<0.020$ & \begin{tabular}{|c|}
$<0.100$ \\
\end{tabular} & 21.7 & 0.107 & $<0.100$ & $<0.010$ & 2.69 & $<0.010$ & 0.072 \\
\hline \begin{tabular}{|l|}
2 \\
\end{tabular} & SB4VAR31 & 2 & 2 & 7 & N06LM12 & 8.97 & $<0.010$ & 0.38 & \begin{tabular}{|l|}
$<0.100$ \\
\end{tabular} & $<0.020$ & 0.104 & 22.9 & $<0.010$ & $<0.100$ & 0.012 & 2.38 & $<0.010$ & 0.016 \\
\hline 2 & SB5-09 & 2 & 2 & 8 & N04LM12 & 10.4 & $<0.010$ & 0.843 & \begin{tabular}{|l|}
$<0.100$ \\
\end{tabular} & $<0.020$ & 0.107 & 21.6 & 0.12 & $<0.100$ & $<0.010$ & 2.87 & $<0.010$ & 0.083 \\
\hline \begin{tabular}{|l|l}
2 \\
\end{tabular} & SB5-08 & 2 & 2 & 9 & $\begin{array}{l}\text { N10LM12 } \\
\end{array}$ & 10.9 & $<0.010$ & 0.881 & \begin{tabular}{|l|}
$<0.100$ \\
\end{tabular} & $<0.020$ & 0.101 & 21.3 & 0.105 & $<0.100$ & $<0.010$ & 2.71 & $<0.010$ & 0.073 \\
\hline \begin{tabular}{|l|}
2 \\
\end{tabular} & Batch 1 & 2 & 2 & 10 & BCHLM2-222 & 7.1 & 0.027 & 0.562 & \begin{tabular}{|l|}
$<0.100$ \\
\end{tabular} & $<0.020$ & \begin{tabular}{|c|}
$<0.100$ \\
\end{tabular} & 24.2 & $<0.010$ & $<0.100$ & 0.396 & \begin{tabular}{|l|}
$<0.100$ \\
\end{tabular} & $<0.010$ & 0.063 \\
\hline 2 & U std & 2 & 2 & 11 & UstdLM2-222 & 9.17 & 0.047 & 0.812 & \begin{tabular}{|l|}
$<0.100$ \\
\end{tabular} & $<0.020$ & \begin{tabular}{|c|}
$<0.100$ \\
\end{tabular} & 22.1 & $<0.010$ & $<0.100$ & 0.579 & 2.08 & $<0.010$ & $<0.010$ \\
\hline \begin{tabular}{|l|l|}
2 \\
\end{tabular} & SB5-03 & 2 & 2 & 12 & $\begin{array}{l}\text { N09LM12 } \\
\end{array}$ & 10.7 & $<0.010$ & 0.747 & \begin{tabular}{|l|}
$<0.100$ \\
\end{tabular} & $<0.020$ & \begin{tabular}{|c|}
$<0.100$ \\
\end{tabular} & 21.5 & 0.093 & $<0.100$ & $<0.010$ & 2.46 & $<0.010$ & 0.068 \\
\hline 2 & SB5-10 & 2 & 2 & 13 & N08LM22 & 10.4 & $<0.010$ & 0.877 & \begin{tabular}{|l|}
$<0.100$ \\
\end{tabular} & $<0.020$ & 0.104 & 22.1 & 0.111 & $<0.100$ & $<0.010$ & 2.86 & $<0.010$ & 0.078 \\
\hline \begin{tabular}{|l|}
2 \\
\end{tabular} & SB5-09 & 2 & 2 & 1 & N04LM22 & 10.3 & $<0.010$ & 0.926 & \begin{tabular}{|c|}
$<0.100$ \\
\end{tabular} & $<0.020$ & 0.108 & 20.7 & 0112 & $<0.100$ & $<0.010$ & 2.82 & $<0.010$ & 0.088 \\
\hline \begin{tabular}{|l|}
2 \\
\end{tabular} & SB5-03 & 2 & 2 & 15 & N09LM22 & 10.4 & $<0.010$ & 0.796 & \begin{tabular}{|l|}
$<0.100$ \\
\end{tabular} & $<0.020$ & \begin{tabular}{|c|}
$<0.100$ \\
\end{tabular} & 20.8 & 0.092 & $<0.100$ & $<0.010$ & 2.42 & $<0.010$ & 0.071 \\
\hline \begin{tabular}{|l|}
2 \\
\end{tabular} & SB5-08 & 2 & 2 & 16 & N10LM22 & 10.4 & $<0.010$ & 0.838 & \begin{tabular}{|c|}
$<0.100$ \\
\end{tabular} & $<0.020$ & \begin{tabular}{|l|}
$<0.100$ \\
\end{tabular} & 21.3 & 0.098 & $<0.100$ & $<0.010$ & 2.57 & $<0.010$ & 0.071 \\
\hline \begin{tabular}{|l|l|}
2 \\
\end{tabular} & SB5-06 & 2 & 2 & 17 & N01LM12 & 10.4 & $<0.010$ & 0.84 & \begin{tabular}{|l|}
$<0.100$ \\
\end{tabular} & $<0.020$ & 0.103 & 21.4 & 0.106 & $<0.100$ & $<0.010$ & 2.65 & $<0.010$ & 0.075 \\
\hline 2 & SB4VAR32 & 2 & 2 & 18 & N11LM12 & 9.61 & $<0.010$ & 0.419 & \begin{tabular}{|l|}
$<0.100$ \\
\end{tabular} & $<0.020$ & 0.118 & 21.4 & $<0.010$ & $<0.100$ & 0.01 & 2.66 & $<0.010$ & 0.017 \\
\hline 2 & Batch 1 & 2 & 2 & 19 & BCHLM2-223 & 6.93 & 0.028 & 0.586 & $<0.100$ & $<0.020$ & $<0.100$ & 24.1 & $<0.010$ & $<0.100$ & 0.407 & $<0.100$ & $<0.010$ & 0.064 \\
\hline 2 & U std & 2 & 2 & 20 & UstdLM2-223 & 9.21 & 0.048 & 0.851 & \begin{tabular}{|l|}
$<0.100$ \\
\end{tabular} & $<0.020$ & $<0.100$ & 22 & $<0.010$ & $<0.100$ & 0.592 & 2.09 & $<0.010$ & $<0.010$ \\
\hline
\end{tabular}


Table A3. Measured Elemental Concentrations (wt\%) for Samples Prepared Using Peroxide Fusion

\begin{tabular}{|c|c|c|c|c|c|c|c|c|}
\hline Set & Glass ID & Block & Sub-Block & Sequence & Lab ID & B (wt\%) & Li (wt\%) & Si (wt\%) \\
\hline 1 & Batch 1 & 1 & 1 & 1 & BCHPF1-111 & 2.57 & 2.03 & 24.1 \\
\hline 1 & U std & 1 & 1 & 2 & UstdPF1-111 & 2.86 & 1.37 & 21.3 \\
\hline 1 & SB4VAR54 & 1 & 1 & 3 & M03PF11 & 2.55 & 2.17 & 19.6 \\
\hline 1 & SB4VAR41 & 1 & 1 & 4 & M01PF21 & 2.84 & 2.51 & 22.9 \\
\hline 1 & SB4VAR21 & 1 & 1 & 5 & M13PF11 & 2.95 & 2.52 & 22.9 \\
\hline 1 & SB4VAR54 & 1 & 1 & 6 & M03PF21 & 2.5 & 2.12 & 20 \\
\hline 1 & SB4VAR53 & 1 & 1 & 7 & M11PF21 & 2.69 & 2.26 & 20.8 \\
\hline 1 & SB4VAR22 & 1 & 1 & 8 & M12PF21 & 2.75 & 2.35 & 21.7 \\
\hline 1 & SB4VAR42 & 1 & 1 & 9 & M05PF21 & 2.83 & 2.4 & 22.3 \\
\hline 1 & SB4VAR23 & 1 & 1 & 10 & M10PF21 & 2.65 & 2.23 & 20.8 \\
\hline 1 & Batch 1 & 1 & 1 & 11 & BCHPF1-112 & 2.33 & 1.97 & 23.1 \\
\hline 1 & U std & 1 & 1 & 12 & UstdPF1-112 & 2.73 & 1.36 & 20.5 \\
\hline 1 & SB4VAR13 & 1 & 1 & 13 & M14PF21 & 2.54 & 2.14 & 19.9 \\
\hline 1 & SB4VAR13 & 1 & 1 & 14 & M14PF11 & 2.55 & 2.11 & 20.3 \\
\hline 1 & SB4VAR42 & 1 & 1 & 15 & M05PF11 & 2.7 & 2.28 & 21.1 \\
\hline 1 & SB4VAR23 & 1 & 1 & 16 & M10PF11 & 2.58 & 2.19 & 20.5 \\
\hline 1 & SB4VAR53 & 1 & 1 & 17 & M11PF11 & 2.7 & 2.29 & 21.2 \\
\hline 1 & SB4VAR22 & 1 & 1 & 18 & M12PF11 & 2.9 & 2.41 & 22.7 \\
\hline 1 & SB4VAR21 & 1 & 1 & 19 & M13PF21 & 3.08 & 2.55 & 23.8 \\
\hline 1 & SB4VAR41 & 1 & 1 & 20 & M01PF11 & 3.14 & 2.55 & 24.2 \\
\hline 1 & Batch 1 & 1 & 1 & 21 & BCHPF1-113 & 2.52 & 2.04 & 24.6 \\
\hline 1 & U std & 1 & 1 & 22 & UstdPF1-113 & 2.96 & 1.41 & 22.3 \\
\hline 1 & Batch 1 & 1 & 2 & 1 & BCHPF1-121 & 2.53 & 2.02 & 23.9 \\
\hline 1 & U std & 1 & 2 & 2 & UstdPF1-121 & 2.86 & 1.37 & 20.8 \\
\hline 1 & SB4VAR54 & 1 & 2 & 3 & M03PF22 & 2.58 & 2.13 & 20.1 \\
\hline 1 & SB4VAR42 & 1 & 2 & 4 & M05PF12 & 2.9 & 2.35 & 22.2 \\
\hline 1 & SB4VAR41 & 1 & 2 & 5 & M01PF12 & 3.05 & 2.5 & 23.4 \\
\hline 1 & SB4VAR54 & 1 & 2 & 6 & M03PF12 & 2.62 & 2.17 & 20.2 \\
\hline 1 & SB4VAR21 & 1 & 2 & 7 & M13PF22 & 3.01 & 2.52 & 23.5 \\
\hline 1 & SB4VAR23 & 1 & 2 & 8 & M10PF12 & 2.74 & 2.25 & 21.4 \\
\hline 1 & SB4VAR21 & 1 & 2 & 9 & M13PF12 & 3.06 & 2.54 & 23.5 \\
\hline 1 & SB4VAR42 & 1 & 2 & 10 & M05PF22 & 2.94 & 2.42 & 23 \\
\hline 1 & Batch 1 & 1 & 2 & 11 & BCHPF1-122 & 2.48 & 2 & 23.9 \\
\hline 1 & U std & 1 & 2 & 12 & UstdPF1-122 & 2.86 & 1.38 & 21.3 \\
\hline 1 & SB4VAR23 & 1 & 2 & 13 & M10PF22 & 2.86 & 2.27 & 21.8 \\
\hline 1 & SB4VAR53 & 1 & 2 & 14 & M11PF12 & 2.9 & 2.33 & 22.3 \\
\hline 1 & SB4VAR41 & 1 & 2 & 15 & M01PF22 & 3.09 & 2.57 & 24 \\
\hline 1 & SB4VAR13 & 1 & 2 & 16 & M14PF22 & 2.63 & 2.17 & 20.8 \\
\hline 1 & SB4VAR53 & 1 & 2 & 17 & M11PF22 & 2.89 & 2.31 & 21.6 \\
\hline 1 & SB4VAR22 & 1 & 2 & 18 & M12PF12 & 3.06 & 2.44 & 23.5 \\
\hline 1 & SB4VAR13 & 1 & 2 & 19 & M14PF12 & 2.71 & 2.18 & 21.1 \\
\hline 1 & SB4VAR22 & 1 & 2 & 20 & M12PF22 & 2.96 & 2.41 & 22.5 \\
\hline 1 & Batch 1 & 1 & 2 & 21 & BCHPF1-123 & 2.52 & 2.04 & 24.7 \\
\hline 1 & U std & 1 & 2 & 22 & UstdPF1-123 & 2.95 & 1.4 & 21.8 \\
\hline 1 & Batch 1 & 2 & 1 & 1 & BCHPF1-211 & 2.43 & 1.97 & 23.3 \\
\hline 1 & U std & 2 & 1 & 2 & UstdPF1-211 & 2.75 & 1.35 & 20.9 \\
\hline 1 & SB4VAR12 & 2 & 1 & 3 & M15PF21 & 2.7 & 2.26 & 21.4 \\
\hline 1 & SB4VAR52 & 2 & 1 & 4 & M09PF11 & 2.79 & 2.34 & 22 \\
\hline 1 & SB4VAR43 & 2 & 1 & 5 & M06PF21 & 2.69 & 2.25 & 21.4 \\
\hline 1 & SB4VAR51 & 2 & 1 & 6 & M04PF11 & 2.89 & 2.45 & 23 \\
\hline 1 & SB4VAR52 & 2 & 1 & 7 & M09PF21 & 2.8 & 2.35 & 21.9 \\
\hline 1 & SB4VAR44 & 2 & 1 & 8 & M08PF21 & 2.56 & 2.14 & 20.4 \\
\hline 1 & SB4VAR24 & 2 & 1 & 9 & M07PF21 & 2.58 & 2.14 & 20.5 \\
\hline 1 & Batch 1 & 2 & 1 & 10 & BCHPF1-212 & 2.31 & 1.96 & 23.2 \\
\hline 1 & U std & 2 & 1 & 11 & UstdPF1-212 & 2.75 & 1.36 & 20.8 \\
\hline 1 & SB4VAR44 & 2 & 1 & 12 & M08PF11 & 2.54 & 2.11 & 19.9 \\
\hline 1 & SB4VAR24 & 2 & 1 & 13 & M07PF11 & 2.54 & 2.14 & 20.3 \\
\hline 1 & SB4VAR11 & 2 & 1 & 14 & M02PF11 & 2.81 & 2.34 & 22 \\
\hline 1 & SB4VAR11 & 2 & 1 & 15 & M02PF21 & 2.81 & 2.4 & 22.4 \\
\hline 1 & SB4VAR51 & 2 & 1 & 16 & M04PF21 & 2.92 & 2.47 & 22.5 \\
\hline 1 & SB4VAR12 & 2 & 1 & 17 & M15PF11 & 2.71 & 2.26 & 21.6 \\
\hline 1 & SB4VAR43 & 2 & 1 & 18 & M06PF11 & 2.63 & 2.25 & 21.3 \\
\hline 1 & Batch 1 & 2 & 1 & 19 & BCHPF1-213 & 2.35 & 1.97 & 23.6 \\
\hline 1 & U std & 2 & 1 & 20 & UstdPF1-213 & 2.73 & 1.35 & 21 \\
\hline 1 & Batch 1 & 2 & 2 & 1 & BCHPF1-221 & 2.46 & 1.97 & 23.3 \\
\hline 1 & U std & 2 & 2 & 2 & UstdPF1-221 & 2.79 & 1.35 & 20.4 \\
\hline 1 & SB4VAR43 & 2 & 2 & 3 & M06PF22 & 2.69 & 2.26 & 21 \\
\hline 1 & SB4VAR43 & 2 & 2 & 4 & M06PF12 & 2.71 & 2.26 & 21.4 \\
\hline
\end{tabular}


Table A3. Measured Elemental Concentrations (wt\%) for Samples Prepared Using Peroxide Fusion

\begin{tabular}{|c|c|c|c|c|c|c|c|c|}
\hline Set & Glass ID & Block & Sub-Block & Sequence & Lab ID & B (wt\%) & $\mathrm{Li}(\mathrm{wt} \%)$ & Si (wt\%) \\
\hline 1 & SB4VAR52 & 2 & 2 & 5 & M09PF12 & 2.82 & 2.34 & 21.9 \\
\hline 1 & SB4VAR51 & 2 & 2 & 6 & M04PF22 & 2.95 & 2.46 & 22.3 \\
\hline 1 & SB4VAR44 & 2 & 2 & 7 & M08PF22 & 2.59 & 2.14 & 20.4 \\
\hline 1 & SB4VAR52 & 2 & 2 & 8 & M09PF22 & 2.79 & 2.35 & 21.6 \\
\hline 1 & SB4VAR24 & 2 & 2 & 9 & M07PF22 & 2.52 & 2.14 & 20 \\
\hline 1 & Batch 1 & 2 & 2 & 10 & BCHPF1-222 & 2.29 & 1.94 & 22.7 \\
\hline 1 & U std & 2 & 2 & 11 & UstdPF1-222 & 2.74 & 1.35 & 20.5 \\
\hline 1 & SB4VAR11 & 2 & 2 & 12 & M02PF12 & 2.87 & 2.34 & 22 \\
\hline 1 & SB4VAR44 & 2 & 2 & 13 & M08PF12 & 2.57 & 2.12 & 20.2 \\
\hline 1 & SB4VAR12 & 2 & 2 & 14 & M15PF12 & 2.67 & 2.27 & 21.1 \\
\hline 1 & SB4VAR24 & 2 & 2 & 15 & M07PF12 & 2.48 & 2.11 & 19.8 \\
\hline 1 & SB4VAR12 & 2 & 2 & 16 & M15PF22 & 2.61 & 2.24 & 20.6 \\
\hline 1 & SB4VAR11 & 2 & 2 & 17 & M02PF22 & 2.79 & 2.37 & 21.8 \\
\hline 1 & SB4VAR51 & 2 & 2 & 18 & M04PF12 & 2.9 & 2.44 & 22.5 \\
\hline 1 & Batch 1 & 2 & 2 & 19 & BCHPF1-223 & 2.34 & 1.95 & 23.1 \\
\hline 1 & U std & 2 & 2 & 20 & UstdPF1-223 & 2.7 & 1.35 & 20.3 \\
\hline 2 & Batch 1 & 1 & 1 & 1 & BCHPF2-111 & 2.45 & 1.97 & \\
\hline 2 & U std & 1 & 1 & 2 & UstdPF2-111 & 2.8 & 1.35 & \\
\hline 2 & SB5-04 & 1 & 1 & 3 & N03PF11 & 3.13 & 1.46 & \\
\hline 2 & SB5-09 & 1 & 1 & 4 & N04PF21 & 2.02 & 1.98 & \\
\hline 2 & SB5-08 & 1 & 1 & 5 & N10PF21 & 3.12 & 1.42 & \\
\hline 2 & SB5-09 & 1 & 1 & 6 & N04PF11 & 1.99 & 1.98 & \\
\hline 2 & SB5-04 & 1 & 1 & 7 & N03PF21 & 3.26 & 1.43 & \\
\hline 2 & SB5-08 & 1 & 1 & 8 & N10PF11 & 3.14 & 1.42 & \\
\hline 2 & SB5-03 & 1 & 1 & 9 & N09PF11 & 2.56 & 1.6 & \\
\hline 2 & Batch 1 & 1 & 1 & 10 & BCHPF2-112 & 2.3 & 1.91 & \\
\hline 2 & U std & 1 & 1 & 11 & UstdPF2-112 & 2.78 & 1.35 & \\
\hline 2 & SB4VAR32 & 1 & 1 & 12 & N11PF11 & 2.86 & 2.32 & \\
\hline 2 & SB5-12 & 1 & 1 & 13 & N13PF21 & 3.15 & 1.45 & \\
\hline 2 & SB5-11 & 1 & 1 & 14 & N14PF11 & 2.68 & 1.68 & \\
\hline 2 & SB5-12 & 1 & 1 & 15 & N13PF11 & 3.11 & 1.43 & \\
\hline 2 & SB5-03 & 1 & 1 & 16 & N09PF21 & 2.65 & 1.68 & \\
\hline 2 & SB4VAR32 & 1 & 1 & 17 & N11PF21 & 2.7 & 2.32 & \\
\hline 2 & SB5-11 & 1 & 1 & 18 & N14PF21 & 2.62 & 1.69 & \\
\hline 2 & Batch 1 & 1 & 1 & 19 & BCHPF2-113 & 2.36 & 1.95 & \\
\hline 2 & U std & 1 & 1 & 20 & UstdPF2-113 & 2.71 & 1.34 & \\
\hline 2 & Batch 1 & 1 & 2 & 1 & BCHPF1-121 & 2.5 & 1.98 & \\
\hline 2 & U std & 1 & 2 & 2 & UstdPF1-121 & 2.85 & 1.36 & \\
\hline 2 & SB5-12 & 1 & 2 & 3 & N13PF22 & 3.16 & 1.45 & \\
\hline 2 & SB5-11 & 1 & 2 & 4 & N14PF12 & 2.72 & 1.7 & \\
\hline 2 & SB5-03 & 1 & 2 & 5 & N09PF22 & 2.76 & 1.72 & \\
\hline 2 & SB5-11 & 1 & 2 & 6 & N14PF22 & 2.74 & 1.7 & \\
\hline 2 & SB5-03 & 1 & 2 & 7 & N09PF12 & 2.6 & 1.62 & \\
\hline 2 & SB5-08 & 1 & 2 & 8 & N10PF12 & 3.18 & 1.45 & \\
\hline 2 & SB4VAR32 & 1 & 2 & 9 & N11PF12 & 2.84 & 2.32 & \\
\hline 2 & Batch 1 & 1 & 2 & 10 & BCHPF1-122 & 2.44 & 1.97 & \\
\hline 2 & U std & 1 & 2 & 11 & UstdPF1-122 & 2.83 & 1.36 & \\
\hline 2 & SB5-08 & 1 & 2 & 12 & N10PF22 & 3.17 & 1.44 & \\
\hline 2 & SB4VAR32 & 1 & 2 & 13 & N11PF22 & 2.79 & 2.36 & \\
\hline 2 & SB5-04 & 1 & 2 & 14 & N03PF12 & 3.19 & 1.45 & \\
\hline 2 & SB5-04 & 1 & 2 & 15 & N03PF22 & 3.22 & 1.45 & \\
\hline 2 & SB5-09 & 1 & 2 & 16 & N04PF22 & 1.98 & 2 & \\
\hline 2 & SB5-12 & 1 & 2 & 17 & N13PF12 & 3.06 & 1.45 & \\
\hline 2 & SB5-09 & 1 & 2 & 18 & N04PF12 & 2 & 2 & \\
\hline 2 & Batch 1 & 1 & 2 & 19 & BCHPF2-123 & 2.37 & 1.94 & \\
\hline 2 & U std & 1 & 2 & 20 & UstdPF1-123 & 2.79 & 1.36 & \\
\hline 2 & Batch 1 & 2 & 1 & 1 & BCHPF2-211 & 2.5 & 1.98 & \\
\hline 2 & U std & 2 & 1 & 2 & UstdPF2-211 & 2.84 & 1.37 & \\
\hline 2 & SB5-06 & 2 & 1 & 3 & N01PF21 & 2.19 & 2 & \\
\hline 2 & SB5-05 & 2 & 1 & 4 & N07PF11 & 2.01 & 2.02 & \\
\hline 2 & SB4VAR33 & 2 & 1 & 5 & N05PF11 & 2.67 & 2.23 & \\
\hline 2 & SB5-10 & 2 & 1 & 6 & N08PF21 & 2.17 & 2 & \\
\hline 2 & SB5-02 & 2 & 1 & 7 & N12PF11 & 2.15 & 2.01 & \\
\hline 2 & SB5-07 & 2 & 1 & 8 & N15PF21 & 2.62 & 1.7 & \\
\hline 2 & SB5-01 & 2 & 1 & 9 & N02PF11 & 1.89 & 1.96 & \\
\hline 2 & SB5-02 & 2 & 1 & 10 & N12PF21 & 2.12 & 2 & \\
\hline 2 & Batch 1 & 2 & 1 & 11 & BCHPF2-212 & 2.35 & 1.97 & \\
\hline 2 & U std & 2 & 1 & 12 & UstdPF2-212 & 2.77 & 1.37 & \\
\hline 2 & SB4VAR31 & 2 & 1 & 13 & N06PF21 & 2.96 & 2.48 & \\
\hline
\end{tabular}


Revision 0

Table A3. Measured Elemental Concentrations (wt\%) for Samples Prepared Using Peroxide Fusion

\begin{tabular}{|c|c|c|c|c|c|c|c|c|}
\hline Set & Glass ID & Block & Sub-Block & Sequence & Lab ID & $\mathrm{B}(\mathrm{wt} \%)$ & $\mathrm{Li}(\mathrm{wt} \%)$ & $\mathrm{Si}(\mathrm{wt} \%)$ \\
\hline 2 & SB5-05 & 2 & 1 & 14 & N07PF21 & 1.97 & 1.99 & \\
\hline 2 & SB4VAR33 & 2 & 1 & 15 & N05PF21 & 2.57 & 2.2 & \\
\hline 2 & SB5-01 & 2 & 1 & 16 & N02PF21 & 1.9 & 1.96 & \\
\hline 2 & SB5-10 & 2 & 1 & 17 & N08PF11 & 2.05 & 2 & \\
\hline 2 & SB4VAR31 & 2 & 1 & 18 & N06PF11 & 2.91 & 2.5 & \\
\hline 2 & SB5-06 & 2 & 1 & 19 & N01PF11 & 2.16 & 1.97 & \\
\hline 2 & SB5-07 & 2 & 1 & 20 & N15PF11 & 2.68 & 1.71 & \\
\hline 2 & Batch 1 & 2 & 1 & 21 & BCHPF2-213 & 2.37 & 1.99 & \\
\hline 2 & U std & 2 & 1 & 22 & UstdPF2-213 & 2.72 & 1.36 & \\
\hline 2 & Batch 1 & 2 & 2 & 1 & BCHPF2-221 & 2.5 & 1.98 & \\
\hline 2 & U std & 2 & 2 & 2 & UstdPF2-221 & 2.83 & 1.38 & \\
\hline 2 & SB5-06 & 2 & 2 & 3 & N01PF12 & 2.27 & 2.01 & \\
\hline 2 & SB4VAR33 & 2 & 2 & 4 & N05PF22 & 2.66 & 2.23 & \\
\hline 2 & SB5-01 & 2 & 2 & 5 & N02PF22 & 1.93 & 1.95 & \\
\hline 2 & SB5-10 & 2 & 2 & 6 & N08PF22 & 2.19 & 2.02 & \\
\hline 2 & SB5-05 & 2 & 2 & 7 & N07PF22 & 2 & 2.02 & \\
\hline 2 & SB4VAR31 & 2 & 2 & 8 & N06PF22 & 3.03 & 2.48 & \\
\hline 2 & SB5-07 & 2 & 2 & 9 & N15PF12 & 2.78 & 1.73 & \\
\hline 2 & SB4VAR33 & 2 & 2 & 10 & N05PF12 & 2.64 & 2.23 & \\
\hline 2 & Batch 1 & 2 & 2 & 11 & BCHPF2-222 & 2.43 & 1.98 & \\
\hline 2 & U std & 2 & 2 & 12 & UstdPF2-222 & 2.79 & 1.38 & \\
\hline 2 & SB5-05 & 2 & 2 & 13 & N07PF12 & 2.09 & 2.04 & \\
\hline 2 & SB5-01 & 2 & 2 & 14 & N02PF12 & 1.99 & 1.98 & \\
\hline 2 & SB5-06 & 2 & 2 & 15 & N01PF22 & 2.18 & 2.01 & \\
\hline 2 & SB5-10 & 2 & 2 & 16 & N08PF12 & 2.14 & 2.03 & \\
\hline 2 & SB5-02 & 2 & 2 & 17 & N12PF22 & 2.18 & 2.03 & \\
\hline 2 & SB5-07 & 2 & 2 & 18 & N15PF22 & 2.65 & 1.72 & \\
\hline 2 & SB5-02 & 2 & 2 & 19 & N12PF12 & 2.26 & 2.06 & \\
\hline 2 & SB4VAR31 & 2 & 2 & 20 & N06PF12 & 3.08 & 2.58 & \\
\hline 2 & Batch 1 & 2 & 2 & 21 & BCHPF2-223 & 2.52 & 2.02 & \\
\hline 2 & U std & 2 & 2 & 22 & UstdPF2-223 & 2.88 & 1.4 & \\
\hline
\end{tabular}


WSRC-STI-2008-00149

Revision 0

Table A4. Average Measured and Bias-Corrected Chemical Compositions Versus Targeted Compositions by Oxide by SB4-Decant Variability Study Glass

\begin{tabular}{|c|c|c|c|c|c|c|c|c|c|}
\hline & & & & Measured & & & & & \% Diff \\
\hline & & & Measured & $\begin{array}{c}\text { Bias- } \\
\text { Corrected (BC) }\end{array}$ & Targeted & $\begin{array}{c}\text { Diff } \\
\text { of }\end{array}$ & $\begin{array}{c}\text { Diff } \\
\text { of }\end{array}$ & $\begin{array}{c}\% \text { Diff } \\
\text { of }\end{array}$ & $\begin{array}{c}\text { of } \\
\text { Meas. }\end{array}$ \\
\hline Set & Glass ID & Oxide & (wt\%) & (wt\%) & (wt\%) & Measured & Meas BC & Measured & $\mathrm{BC}$ \\
\hline 1 & Batch 1 & $\mathrm{Al}_{2} \mathrm{O}_{3}$ & 4.7820 & 4.8770 & 4.8770 & -0.0950 & 0.0000 & $-1.9 \%$ & $0.0 \%$ \\
\hline 1 & Batch 1 & $\mathrm{~B}_{2} \mathrm{O}_{3}$ & 7.8163 & 7.7770 & 7.7770 & 0.0393 & 0.0000 & $0.5 \%$ & $0.0 \%$ \\
\hline 1 & Batch 1 & $\mathrm{BaO}$ & 0.1411 & 0.1510 & 0.1510 & -0.0099 & 0.0000 & $-6.5 \%$ & $0.0 \%$ \\
\hline 1 & Batch 1 & $\mathrm{CaO}$ & 1.1838 & 1.2200 & 1.2200 & -0.0362 & 0.0000 & $-3.0 \%$ & $0.0 \%$ \\
\hline 1 & Batch 1 & $\mathrm{Ce}_{2} \mathrm{O}_{3}$ & 0.0224 & 0.0224 & 0.0000 & 0.0224 & 0.0224 & & \\
\hline 1 & Batch 1 & $\mathrm{Cr}_{2} \mathrm{O}_{3}$ & 0.1056 & 0.1070 & 0.1070 & -0.0014 & 0.0000 & $-1.3 \%$ & $0.0 \%$ \\
\hline 1 & Batch 1 & $\mathrm{CuO}$ & 0.3755 & 0.3990 & 0.3990 & -0.0235 & 0.0000 & $-5.9 \%$ & $0.0 \%$ \\
\hline 1 & Batch 1 & $\mathrm{Fe}_{2} \mathrm{O}_{3}$ & 12.6636 & 12.8390 & 12.8390 & -0.1754 & 0.0000 & $-1.4 \%$ & $0.0 \%$ \\
\hline 1 & Batch 1 & $\mathrm{~K}_{2} \mathrm{O}$ & 3.5967 & 3.3270 & 3.3270 & 0.2697 & 0.0000 & $8.1 \%$ & $0.0 \%$ \\
\hline 1 & Batch 1 & $\mathrm{La}_{2} \mathrm{O}_{3}$ & 0.0586 & 0.0586 & 0.0000 & 0.0586 & 0.0586 & & \\
\hline 1 & Batch 1 & $\mathrm{Li}_{2} \mathrm{O}$ & 4.2807 & 4.4290 & 4.4290 & -0.1483 & 0.0000 & $-3.3 \%$ & $0.0 \%$ \\
\hline 1 & Batch 1 & $\mathrm{MgO}$ & 1.3649 & 1.4190 & 1.4190 & -0.0541 & 0.0000 & $-3.8 \%$ & $0.0 \%$ \\
\hline 1 & Batch 1 & $\mathrm{MnO}$ & 1.7173 & 1.7260 & 1.7260 & -0.0087 & 0.0000 & $-0.5 \%$ & $0.0 \%$ \\
\hline 1 & Batch 1 & $\mathrm{Na}_{2} \mathrm{O}$ & 8.9788 & 9.0030 & 9.0030 & -0.0242 & 0.0000 & $-0.3 \%$ & $0.0 \%$ \\
\hline 1 & Batch 1 & $\mathrm{NiO}$ & 0.7007 & 0.7510 & 0.7510 & -0.0503 & 0.0000 & $-6.7 \%$ & $0.0 \%$ \\
\hline 1 & Batch 1 & $\mathrm{PbO}$ & 0.0108 & 0.0108 & 0.0000 & 0.0108 & 0.0108 & & \\
\hline 1 & Batch 1 & $\mathrm{SiO}_{2}$ & 50.5410 & 50.2200 & 50.2200 & 0.3210 & 0.0000 & $0.6 \%$ & $0.0 \%$ \\
\hline 1 & Batch 1 & $\mathrm{SO}_{4}$ & 0.1498 & 0.1498 & 0.0000 & 0.1498 & 0.1498 & & \\
\hline 1 & Batch 1 & $\mathrm{TiO}_{2}$ & 0.6465 & 0.6770 & 0.6770 & -0.0305 & 0.0000 & $-4.5 \%$ & $0.0 \%$ \\
\hline 1 & Batch 1 & $\mathrm{U}_{3} \mathrm{O}_{8}$ & 0.0590 & 0.0615 & 0.0000 & 0.0590 & 0.0615 & & \\
\hline 1 & Batch 1 & $\mathrm{ZnO}$ & 0.0062 & 0.0062 & 0.0000 & 0.0062 & 0.0062 & & \\
\hline 1 & Batch 1 & $\mathrm{ZrO}_{2}$ & 0.0858 & 0.0858 & 0.0980 & -0.0122 & -0.0122 & $-12.5 \%$ & $-12.5 \%$ \\
\hline 1 & Batch 1 & Sum & 99.2872 & 99.3172 & 99.0200 & 0.2672 & 0.2972 & $0.3 \%$ & $0.3 \%$ \\
\hline 1 & SB4VAR11 & $\mathrm{Al}_{2} \mathrm{O}_{3}$ & 8.5358 & 8.6855 & 8.4639 & 0.0719 & 0.2216 & $0.8 \%$ & $2.6 \%$ \\
\hline 1 & SB4VAR11 & $\mathrm{B}_{2} \mathrm{O}_{3}$ & 9.0801 & 9.2797 & 9.2400 & -0.1599 & 0.0397 & $-1.7 \%$ & $0.4 \%$ \\
\hline 1 & SB4VAR11 & $\mathrm{BaO}$ & 0.0229 & 0.0244 & 0.0252 & -0.0023 & -0.0008 & $-9.2 \%$ & $-3.1 \%$ \\
\hline 1 & SB4VAR11 & $\mathrm{CaO}$ & 0.8825 & 0.9058 & 0.9283 & -0.0458 & -0.0225 & $-4.9 \%$ & $-2.4 \%$ \\
\hline 1 & SB4VAR11 & $\mathrm{Ce}_{2} \mathrm{O}_{3}$ & 0.0190 & 0.0190 & 0.0221 & -0.0031 & -0.0031 & $-13.9 \%$ & $-13.9 \%$ \\
\hline 1 & SB4VAR11 & $\mathrm{Cr}_{2} \mathrm{O}_{3}$ & 0.0570 & 0.0577 & 0.0527 & 0.0043 & 0.0050 & $8.2 \%$ & $9.5 \%$ \\
\hline 1 & SB4VAR11 & $\mathrm{CuO}$ & 0.0244 & 0.0259 & 0.0195 & 0.0049 & 0.0064 & $25.2 \%$ & $32.8 \%$ \\
\hline 1 & SB4VAR11 & $\mathrm{Fe}_{2} \mathrm{O}_{3}$ & 9.0214 & 9.4768 & 9.6198 & -0.5984 & -0.1430 & $-6.2 \%$ & $-1.5 \%$ \\
\hline 1 & SB4VAR11 & $\mathrm{K}_{2} \mathrm{O}$ & 0.1262 & 0.1171 & 0.1190 & 0.0071 & -0.0020 & $6.0 \%$ & $-1.6 \%$ \\
\hline 1 & SB4VAR11 & $\mathrm{La}_{2} \mathrm{O}_{3}$ & 0.0586 & 0.0586 & 0.0166 & 0.0420 & 0.0420 & $253.3 \%$ & $253.3 \%$ \\
\hline 1 & SB4VAR11 & $\mathrm{Li}_{2} \mathrm{O}$ & 5.0862 & 5.3385 & 5.2800 & -0.1938 & 0.0585 & $-3.7 \%$ & $1.1 \%$ \\
\hline 1 & SB4VAR11 & $\mathrm{MgO}$ & 0.8777 & 0.9221 & 0.9129 & -0.0352 & 0.0092 & $-3.9 \%$ & $1.0 \%$ \\
\hline 1 & SB4VAR11 & $\mathrm{MnO}$ & 1.8722 & 1.9275 & 1.9320 & -0.0598 & -0.0045 & $-3.1 \%$ & $-0.2 \%$ \\
\hline 1 & SB4VAR11 & $\mathrm{Na}_{2} \mathrm{O}$ & 12.2668 & 12.2547 & 12.2570 & 0.0098 & -0.0023 & $0.1 \%$ & $0.0 \%$ \\
\hline 1 & SB4VAR11 & $\mathrm{NiO}$ & 0.4826 & 0.5220 & 0.5353 & -0.0527 & -0.0133 & $-9.8 \%$ & $-2.5 \%$ \\
\hline 1 & SB4VAR11 & $\mathrm{PbO}$ & 0.0232 & 0.0232 & 0.0211 & 0.0021 & 0.0021 & $9.8 \%$ & $9.8 \%$ \\
\hline 1 & SB4VAR11 & $\mathrm{SiO}_{2}$ & 47.1716 & 47.7308 & 47.1049 & 0.0667 & 0.6259 & $0.1 \%$ & $1.3 \%$ \\
\hline 1 & SB4VAR11 & $\mathrm{SO}_{4}$ & 0.4127 & 0.4127 & 0.4483 & -0.0356 & -0.0356 & $-7.9 \%$ & $-7.9 \%$ \\
\hline 1 & SB4VAR11 & $\mathrm{TiO}_{2}$ & 0.0171 & 0.0180 & 0.0130 & 0.0041 & 0.0050 & $31.5 \%$ & $38.6 \%$ \\
\hline 1 & SB4VAR11 & $\mathrm{U}_{3} \mathrm{O}_{8}$ & 2.8271 & 2.9482 & 2.9352 & -0.1081 & 0.0130 & $-3.7 \%$ & $0.4 \%$ \\
\hline 1 & SB4VAR11 & $\mathrm{ZnO}$ & 0.0218 & 0.0218 & 0.0263 & -0.0045 & -0.0045 & $-17.2 \%$ & $-17.2 \%$ \\
\hline 1 & SB4VAR11 & $\mathrm{ZrO}_{2}$ & 0.0257 & 0.0257 & 0.0268 & -0.0011 & -0.0011 & $-4.2 \%$ & $-4.2 \%$ \\
\hline 1 & SB4VAR11 & Sum & 98.9126 & 100.7958 & 100.0000 & -1.0873 & 0.7958 & $-1.1 \%$ & $0.8 \%$ \\
\hline 1 & SB4VAR12 & $\mathrm{Al}_{2} \mathrm{O}_{3}$ & 9.4853 & 9.6987 & 9.4596 & 0.0257 & 0.2391 & $0.3 \%$ & $2.5 \%$ \\
\hline 1 & SB4VAR12 & $\mathrm{B}_{2} \mathrm{O}_{3}$ & 8.6052 & 8.7944 & 8.6800 & -0.0748 & 0.1144 & $-0.9 \%$ & $1.3 \%$ \\
\hline 1 & SB4VAR12 & $\mathrm{BaO}$ & 0.0232 & 0.0248 & 0.0281 & -0.0049 & -0.0033 & $-17.6 \%$ & $-11.6 \%$ \\
\hline 1 & SB4VAR12 & $\mathrm{CaO}$ & 0.9798 & 1.0138 & 1.0375 & -0.0577 & -0.0237 & $-5.6 \%$ & $-2.3 \%$ \\
\hline 1 & SB4VAR12 & $\mathrm{Ce}_{2} \mathrm{O}_{3}$ & 0.0193 & 0.0193 & 0.0247 & -0.0054 & -0.0054 & $-21.8 \%$ & $-21.8 \%$ \\
\hline 1 & SB4VAR12 & $\mathrm{Cr}_{2} \mathrm{O}_{3}$ & 0.0607 & 0.0615 & 0.0589 & 0.0018 & 0.0026 & $3.0 \%$ & $4.5 \%$ \\
\hline 1 & SB4VAR12 & $\mathrm{CuO}$ & 0.0275 & 0.0293 & 0.0218 & 0.0057 & 0.0075 & $26.3 \%$ & $34.4 \%$ \\
\hline 1 & SB4VAR12 & $\mathrm{Fe}_{2} \mathrm{O}_{3}$ & 10.8800 & 10.6604 & 10.7516 & 0.1284 & -0.0912 & $1.2 \%$ & $-0.8 \%$ \\
\hline 1 & SB4VAR12 & $\mathrm{K}_{2} \mathrm{O}$ & 0.1430 & 0.1319 & 0.1330 & 0.0100 & -0.0011 & $7.5 \%$ & $-0.8 \%$ \\
\hline 1 & SB4VAR12 & $\mathrm{La}_{2} \mathrm{O}_{3}$ & 0.0586 & 0.0586 & 0.0185 & 0.0401 & 0.0401 & $217.0 \%$ & $217.0 \%$ \\
\hline 1 & SB4VAR12 & $\mathrm{Li}_{2} \mathrm{O}$ & 4.8602 & 5.1013 & 4.9600 & -0.0998 & 0.1413 & $-2.0 \%$ & $2.8 \%$ \\
\hline 1 & SB4VAR12 & $\mathrm{MgO}$ & 0.9950 & 1.0236 & 1.0203 & -0.0253 & 0.0033 & $-2.5 \%$ & $0.3 \%$ \\
\hline
\end{tabular}


WSRC-STI-2008-00149

Revision 0

Table A4. Average Measured and Bias-Corrected Chemical Compositions Versus Targeted Compositions by Oxide by SB4-Decant Variability Study Glass

\begin{tabular}{|c|c|c|c|c|c|c|c|c|c|}
\hline & & & & Measured & & & & & \% Diff \\
\hline & & & Measured & $\begin{array}{c}\text { Bias- } \\
\text { Corrected (BC) }\end{array}$ & Targeted & $\begin{array}{c}\text { Diff } \\
\text { of }\end{array}$ & $\begin{array}{c}\text { Diff } \\
\text { of }\end{array}$ & $\begin{array}{c}\% \text { Diff } \\
\text { of }\end{array}$ & $\begin{array}{c}\text { of } \\
\text { Meas. }\end{array}$ \\
\hline Set & Glass ID & Oxide & $(\mathrm{wt} \%)$ & $(\mathrm{wt} \%)$ & $(\mathrm{wt} \%)$ & Measured & Meas BC & Measured & $\mathrm{BC}$ \\
\hline 1 & SB4VAR12 & $\mathrm{MnO}$ & 2.2305 & 2.1919 & 2.1593 & 0.0712 & 0.0326 & $3.3 \%$ & $1.5 \%$ \\
\hline 1 & SB4VAR12 & $\mathrm{Na}_{2} \mathrm{O}$ & 12.5128 & 12.5957 & 12.7578 & -0.2450 & -0.1621 & $-1.9 \%$ & $-1.3 \%$ \\
\hline 1 & SB4VAR12 & $\mathrm{NiO}$ & 0.5402 & 0.5737 & 0.5983 & -0.0581 & -0.0246 & $-9.7 \%$ & $-4.1 \%$ \\
\hline 1 & SB4VAR12 & $\mathrm{PbO}$ & 0.0191 & 0.0191 & 0.0236 & -0.0045 & -0.0045 & $-19.0 \%$ & $-19.0 \%$ \\
\hline 1 & SB4VAR12 & $\mathrm{SiO}_{2}$ & 45.2997 & 45.8339 & 44.4114 & 0.8883 & 1.4225 & $2.0 \%$ & $3.2 \%$ \\
\hline 1 & SB4VAR12 & $\mathrm{SO}_{4}$ & 0.4441 & 0.4441 & 0.5011 & -0.0569 & -0.0569 & $-11.4 \%$ & $-11.4 \%$ \\
\hline 1 & SB4VAR12 & $\mathrm{TiO}_{2}$ & 0.0229 & 0.0239 & 0.0146 & 0.0083 & 0.0093 & $57.1 \%$ & $63.6 \%$ \\
\hline 1 & SB4VAR12 & $\mathrm{U}_{3} \mathrm{O}_{8}$ & 3.1573 & 3.2923 & 3.2805 & -0.1232 & 0.0118 & $-3.8 \%$ & $0.4 \%$ \\
\hline 1 & SB4VAR12 & $\mathrm{ZnO}$ & 0.0215 & 0.0215 & 0.0294 & -0.0079 & -0.0079 & $-27.0 \%$ & $-27.0 \%$ \\
\hline 1 & SB4VAR12 & $\mathrm{ZrO}_{2}$ & 0.0277 & 0.0277 & 0.0300 & -0.0023 & -0.0023 & $-7.7 \%$ & $-7.7 \%$ \\
\hline 1 & SB4VAR12 & Sum & 100.4137 & 101.6415 & 100.0000 & 0.4137 & 1.6415 & $0.4 \%$ & $1.6 \%$ \\
\hline 1 & SB4VAR13 & $\mathrm{Al}_{2} \mathrm{O}_{3}$ & 10.3923 & 10.5745 & 10.4554 & -0.0631 & 0.1191 & $-0.6 \%$ & $1.1 \%$ \\
\hline 1 & SB4VAR13 & $\mathrm{B}_{2} \mathrm{O}_{3}$ & 8.3959 & 8.1375 & 8.1200 & 0.2759 & 0.0175 & $3.4 \%$ & $0.2 \%$ \\
\hline 1 & SB4VAR13 & $\mathrm{BaO}$ & 0.0299 & 0.0319 & 0.0311 & -0.0012 & 0.0008 & $-4.0 \%$ & $2.5 \%$ \\
\hline 1 & SB4VAR13 & $\mathrm{CaO}$ & 1.0861 & 1.1148 & 1.1467 & -0.0606 & -0.0319 & $-5.3 \%$ & $-2.8 \%$ \\
\hline 1 & SB4VAR13 & $\mathrm{Ce}_{2} \mathrm{O}_{3}$ & 0.0237 & 0.0237 & 0.0273 & -0.0036 & -0.0036 & $-13.1 \%$ & $-13.1 \%$ \\
\hline 1 & SB4VAR13 & $\mathrm{Cr}_{2} \mathrm{O}_{3}$ & 0.0614 & 0.0621 & 0.0651 & -0.0037 & -0.0030 & $-5.7 \%$ & $-4.6 \%$ \\
\hline 1 & SB4VAR13 & $\mathrm{CuO}$ & 0.0304 & 0.0322 & 0.0241 & 0.0063 & 0.0081 & $26.0 \%$ & $33.6 \%$ \\
\hline 1 & SB4VAR13 & $\mathrm{Fe}_{2} \mathrm{O}_{3}$ & 11.0980 & 11.6585 & 11.8833 & -0.7853 & -0.2248 & $-6.6 \%$ & $-1.9 \%$ \\
\hline 1 & SB4VAR13 & $\mathrm{K}_{2} \mathrm{O}$ & 0.1391 & 0.1291 & 0.1470 & -0.0079 & -0.0179 & $-5.4 \%$ & $-12.2 \%$ \\
\hline 1 & SB4VAR13 & $\mathrm{La}_{2} \mathrm{O}_{3}$ & 0.0586 & 0.0586 & 0.0205 & 0.0381 & 0.0381 & $186.0 \%$ & $186.0 \%$ \\
\hline 1 & SB4VAR13 & $\mathrm{Li}_{2} \mathrm{O}$ & 4.6287 & 4.7217 & 4.6400 & -0.0113 & 0.0817 & $-0.2 \%$ & $1.8 \%$ \\
\hline 1 & SB4VAR13 & $\mathrm{MgO}$ & 1.1111 & 1.1673 & 1.1277 & -0.0166 & 0.0396 & $-1.5 \%$ & $3.5 \%$ \\
\hline 1 & SB4VAR13 & $\mathrm{MnO}$ & 2.3209 & 2.3896 & 2.3866 & -0.0657 & 0.0030 & $-2.8 \%$ & $0.1 \%$ \\
\hline 1 & SB4VAR13 & $\mathrm{Na}_{2} \mathrm{O}$ & 13.1059 & 13.0917 & 13.2587 & -0.1528 & -0.1670 & $-1.2 \%$ & $-1.3 \%$ \\
\hline 1 & SB4VAR13 & $\mathrm{NiO}$ & 0.5917 & 0.6400 & 0.6612 & -0.0695 & -0.0212 & $-10.5 \%$ & $-3.2 \%$ \\
\hline 1 & SB4VAR13 & $\mathrm{PbO}$ & 0.0304 & 0.0304 & 0.0261 & 0.0043 & 0.0043 & $16.6 \%$ & $16.6 \%$ \\
\hline 1 & SB4VAR13 & $\mathrm{SiO}_{2}$ & 43.9091 & 42.8560 & 41.7179 & 2.1912 & 1.1381 & $5.3 \%$ & $2.7 \%$ \\
\hline 1 & SB4VAR13 & $\mathrm{SO}_{4}$ & 0.5048 & 0.5048 & 0.5538 & -0.0490 & -0.0490 & $-8.8 \%$ & $-8.8 \%$ \\
\hline 1 & SB4VAR13 & $\mathrm{TiO}_{2}$ & 0.0229 & 0.0242 & 0.0161 & 0.0068 & 0.0081 & $42.5 \%$ & $50.2 \%$ \\
\hline 1 & SB4VAR13 & $\mathrm{U}_{3} \mathrm{O}_{8}$ & 3.4875 & 3.6368 & 3.6258 & -0.1383 & 0.0110 & $-3.8 \%$ & $0.3 \%$ \\
\hline 1 & SB4VAR13 & $\mathrm{ZnO}$ & 0.0274 & 0.0274 & 0.0325 & -0.0051 & -0.0051 & $-15.7 \%$ & $-15.7 \%$ \\
\hline 1 & SB4VAR13 & $\mathrm{ZrO}_{2}$ & 0.0317 & 0.0317 & 0.0331 & -0.0014 & -0.0014 & $-4.1 \%$ & $-4.1 \%$ \\
\hline 1 & SB4VAR13 & Sum & 101.0877 & 100.9447 & 100.0001 & 1.0877 & 0.9447 & $1.1 \%$ & $0.9 \%$ \\
\hline 1 & SB4VAR21 & $\mathrm{Al}_{2} \mathrm{O}_{3}$ & 8.3658 & 8.5123 & 8.3067 & 0.0591 & 0.2056 & $0.7 \%$ & $2.5 \%$ \\
\hline 1 & SB4VAR21 & $\mathrm{B}_{2} \mathrm{O}_{3}$ & 9.7402 & 9.4419 & 9.5200 & 0.2202 & -0.0781 & $2.3 \%$ & $-0.8 \%$ \\
\hline 1 & SB4VAR21 & $\mathrm{BaO}$ & 0.0246 & 0.0262 & 0.0248 & -0.0002 & 0.0014 & $-1.0 \%$ & $5.7 \%$ \\
\hline 1 & SB4VAR21 & $\mathrm{CaO}$ & 0.8755 & 0.8987 & 0.9141 & -0.0386 & -0.0154 & $-4.2 \%$ & $-1.7 \%$ \\
\hline 1 & SB4VAR21 & $\mathrm{Ce}_{2} \mathrm{O}_{3}$ & 0.0170 & 0.0170 & 0.0218 & -0.0048 & -0.0048 & $-21.9 \%$ & $-21.9 \%$ \\
\hline 1 & SB4VAR21 & $\mathrm{Cr}_{2} \mathrm{O}_{3}$ & 0.0555 & 0.0562 & 0.0519 & 0.0036 & 0.0043 & $7.0 \%$ & $8.3 \%$ \\
\hline 1 & SB4VAR21 & $\mathrm{CuO}$ & 0.0250 & 0.0266 & 0.0192 & 0.0058 & 0.0074 & $30.4 \%$ & $38.3 \%$ \\
\hline 1 & SB4VAR21 & $\mathrm{Fe}_{2} \mathrm{O}_{3}$ & 8.7712 & 9.2133 & 9.4727 & -0.7015 & -0.2594 & $-7.4 \%$ & $-2.7 \%$ \\
\hline 1 & SB4VAR21 & $\mathrm{K}_{2} \mathrm{O}$ & 0.1168 & 0.1084 & 0.1172 & -0.0004 & -0.0088 & $-0.3 \%$ & $-7.5 \%$ \\
\hline 1 & SB4VAR21 & $\mathrm{La}_{2} \mathrm{O}_{3}$ & 0.0586 & 0.0586 & 0.0163 & 0.0423 & 0.0423 & $259.8 \%$ & $259.8 \%$ \\
\hline 1 & SB4VAR21 & $\mathrm{Li}_{2} \mathrm{O}$ & 5.4522 & 5.5619 & 5.4400 & 0.0122 & 0.1219 & $0.2 \%$ & $2.2 \%$ \\
\hline 1 & SB4VAR21 & $\mathrm{MgO}$ & 0.8735 & 0.9177 & 0.8989 & -0.0254 & 0.0188 & $-2.8 \%$ & $2.1 \%$ \\
\hline 1 & SB4VAR21 & $\mathrm{MnO}$ & 1.8335 & 1.8875 & 1.9024 & -0.0689 & -0.0149 & $-3.6 \%$ & $-0.8 \%$ \\
\hline 1 & SB4VAR21 & $\mathrm{Na}_{2} \mathrm{O}$ & 10.8042 & 10.7917 & 10.9820 & -0.1778 & -0.1903 & $-1.6 \%$ & $-1.7 \%$ \\
\hline 1 & SB4VAR21 & $\mathrm{NiO}$ & 0.4801 & 0.5192 & 0.5271 & -0.0470 & -0.0079 & $-8.9 \%$ & $-1.5 \%$ \\
\hline 1 & SB4VAR21 & $\mathrm{PbO}$ & 0.0232 & 0.0232 & 0.0208 & 0.0024 & 0.0024 & $11.3 \%$ & $11.3 \%$ \\
\hline 1 & SB4VAR21 & $\mathrm{SiO}_{2}$ & 50.1131 & 48.9153 & 48.4911 & 1.6220 & 0.4242 & $3.3 \%$ & $0.9 \%$ \\
\hline 1 & SB4VAR21 & $\mathrm{SO}_{4}$ & 0.1498 & 0.1498 & 0.3175 & -0.1677 & -0.1677 & $-52.8 \%$ & $-52.8 \%$ \\
\hline 1 & SB4VAR21 & $\mathrm{TiO}_{2}$ & 0.0200 & 0.0211 & 0.0128 & 0.0072 & 0.0083 & $56.4 \%$ & $64.9 \%$ \\
\hline 1 & SB4VAR21 & $\mathrm{U}_{3} \mathrm{O}_{8}$ & 2.7652 & 2.8834 & 2.8902 & -0.1250 & -0.0068 & $-4.3 \%$ & $-0.2 \%$ \\
\hline 1 & SB4VAR21 & $\mathrm{ZnO}$ & 0.0205 & 0.0205 & 0.0259 & -0.0054 & -0.0054 & $-20.7 \%$ & $-20.7 \%$ \\
\hline 1 & SB4VAR21 & $\mathrm{ZrO}_{2}$ & 0.0253 & 0.0253 & 0.0264 & -0.0011 & -0.0011 & $-4.1 \%$ & $-4.1 \%$ \\
\hline 1 & SB4VAR21 & Sum & 100.6110 & 100.0758 & 99.9998 & 0.6112 & 0.0760 & $0.6 \%$ & $0.1 \%$ \\
\hline 1 & SB4VAR22 & $\mathrm{Al}_{2} \mathrm{O}_{3}$ & 9.0507 & 9.2565 & 9.0855 & -0.0348 & 0.1710 & $-0.4 \%$ & $1.9 \%$ \\
\hline
\end{tabular}


WSRC-STI-2008-00149

Revision 0

Table A4. Average Measured and Bias-Corrected Chemical Compositions Versus Targeted Compositions by Oxide by SB4-Decant Variability Study Glass

\begin{tabular}{|c|c|c|c|c|c|c|c|c|c|}
\hline & & & & Measured & & & & & \% Diff \\
\hline & & & Measured & $\begin{array}{c}\text { Bias- } \\
\text { Corrected (BC) }\end{array}$ & Targeted & $\begin{array}{c}\text { Diff } \\
\text { of }\end{array}$ & $\begin{array}{c}\text { Diff } \\
\text { of }\end{array}$ & $\begin{array}{c}\% \text { Diff } \\
\text { of }\end{array}$ & $\begin{array}{c}\text { of } \\
\text { Meas. }\end{array}$ \\
\hline Set & Glass ID & Oxide & $(\mathrm{wt} \%)$ & $(\mathrm{wt} \%)$ & $(\mathrm{wt} \%)$ & Measured & Meas BC & Measured & $\mathrm{BC}$ \\
\hline 1 & SB4VAR22 & $\mathrm{B}_{2} \mathrm{O}_{3}$ & 9.3941 & 9.1045 & 9.1000 & 0.2941 & 0.0045 & $3.2 \%$ & $0.0 \%$ \\
\hline 1 & SB4VAR22 & $\mathrm{BaO}$ & 0.0257 & 0.0275 & 0.0271 & -0.0014 & 0.0004 & $-5.2 \%$ & $1.6 \%$ \\
\hline 1 & SB4VAR22 & $\mathrm{CaO}$ & 0.9466 & 0.9794 & 0.9998 & -0.0532 & -0.0204 & $-5.3 \%$ & $-2.0 \%$ \\
\hline 1 & SB4VAR22 & $\mathrm{Ce}_{2} \mathrm{O}_{3}$ & 0.0179 & 0.0179 & 0.0238 & -0.0059 & -0.0059 & $-24.9 \%$ & $-24.9 \%$ \\
\hline 1 & SB4VAR22 & $\mathrm{Cr}_{2} \mathrm{O}_{3}$ & 0.0643 & 0.0652 & 0.0568 & 0.0075 & 0.0084 & $13.2 \%$ & $14.9 \%$ \\
\hline 1 & SB4VAR22 & $\mathrm{CuO}$ & 0.0269 & 0.0286 & 0.0210 & 0.0059 & 0.0076 & $28.2 \%$ & $36.4 \%$ \\
\hline 1 & SB4VAR22 & $\mathrm{Fe}_{2} \mathrm{O}_{3}$ & 10.1973 & 9.9915 & 10.3607 & -0.1634 & -0.3692 & $-1.6 \%$ & $-3.6 \%$ \\
\hline 1 & SB4VAR22 & $\mathrm{K}_{2} \mathrm{O}$ & 0.1283 & 0.1183 & 0.1282 & 0.0001 & -0.0099 & $0.1 \%$ & $-7.7 \%$ \\
\hline 1 & SB4VAR22 & $\mathrm{La}_{2} \mathrm{O}_{3}$ & 0.0586 & 0.0586 & 0.0178 & 0.0408 & 0.0408 & $229.4 \%$ & $229.4 \%$ \\
\hline 1 & SB4VAR22 & $\mathrm{Li}_{2} \mathrm{O}$ & 5.1723 & 5.2763 & 5.2000 & -0.0277 & 0.0763 & $-0.5 \%$ & $1.5 \%$ \\
\hline 1 & SB4VAR22 & $\mathrm{MgO}$ & 0.9888 & 1.0172 & 0.9832 & 0.0056 & 0.0340 & $0.6 \%$ & $3.5 \%$ \\
\hline 1 & SB4VAR22 & $\mathrm{MnO}$ & 2.1047 & 2.0681 & 2.0808 & 0.0239 & -0.0127 & $1.1 \%$ & $-0.6 \%$ \\
\hline 1 & SB4VAR22 & $\mathrm{Na}_{2} \mathrm{O}$ & 11.0772 & 11.1544 & 11.2616 & -0.1844 & -0.1072 & $-1.6 \%$ & $-1.0 \%$ \\
\hline 1 & SB4VAR22 & $\mathrm{NiO}$ & 0.5386 & 0.5720 & 0.5765 & -0.0379 & -0.0045 & $-6.6 \%$ & $-0.8 \%$ \\
\hline 1 & SB4VAR22 & $\mathrm{PbO}$ & 0.0240 & 0.0240 & 0.0228 & 0.0012 & 0.0012 & $5.1 \%$ & $5.1 \%$ \\
\hline 1 & SB4VAR22 & $\mathrm{SiO}_{2}$ & 48.3482 & 47.1892 & 46.4746 & 1.8736 & 0.7146 & $4.0 \%$ & $1.5 \%$ \\
\hline 1 & SB4VAR22 & $\mathrm{SO}_{4}$ & 0.3438 & 0.3438 & 0.3473 & -0.0035 & -0.0035 & $-1.0 \%$ & $-1.0 \%$ \\
\hline 1 & SB4VAR22 & $\mathrm{TiO}_{2}$ & 0.0267 & 0.0278 & 0.0140 & 0.0127 & 0.0138 & $90.6 \%$ & $98.5 \%$ \\
\hline 1 & SB4VAR22 & $\mathrm{U}_{3} \mathrm{O}_{8}$ & 3.0423 & 3.1734 & 3.1612 & -0.1189 & 0.0122 & $-3.8 \%$ & $0.4 \%$ \\
\hline 1 & SB4VAR22 & $\mathrm{ZnO}$ & 0.0212 & 0.0212 & 0.0283 & -0.0071 & -0.0071 & $-25.2 \%$ & $-25.2 \%$ \\
\hline 1 & SB4VAR22 & $\mathrm{ZrO}_{2}$ & 0.0216 & 0.0216 & 0.0289 & -0.0073 & -0.0073 & $-25.2 \%$ & $-25.2 \%$ \\
\hline 1 & SB4VAR22 & Sum & 101.6196 & 100.5370 & 99.9999 & 1.6197 & 0.5371 & $1.6 \%$ & $0.5 \%$ \\
\hline 1 & SB4VAR23 & $\mathrm{Al}_{2} \mathrm{O}_{3}$ & 10.2931 & 10.4731 & 9.8642 & 0.4289 & 0.6089 & $4.3 \%$ & $6.2 \%$ \\
\hline 1 & SB4VAR23 & $\mathrm{B}_{2} \mathrm{O}_{3}$ & 8.7179 & 8.4490 & 8.6800 & 0.0379 & -0.2310 & $0.4 \%$ & $-2.7 \%$ \\
\hline 1 & SB4VAR23 & $\mathrm{BaO}$ & 0.0268 & 0.0286 & 0.0294 & -0.0026 & -0.0008 & $-8.9 \%$ & $-2.7 \%$ \\
\hline 1 & SB4VAR23 & $\mathrm{CaO}$ & 1.0323 & 1.0595 & 1.0855 & -0.0532 & -0.0260 & $-4.9 \%$ & $-2.4 \%$ \\
\hline 1 & SB4VAR23 & $\mathrm{Ce}_{2} \mathrm{O}_{3}$ & 0.0199 & 0.0199 & 0.0258 & -0.0059 & -0.0059 & $-22.9 \%$ & $-22.9 \%$ \\
\hline 1 & SB4VAR23 & $\mathrm{Cr}_{2} \mathrm{O}_{3}$ & 0.0552 & 0.0558 & 0.0617 & -0.0065 & -0.0059 & $-10.6 \%$ & $-9.5 \%$ \\
\hline 1 & SB4VAR23 & $\mathrm{CuO}$ & 0.0300 & 0.0319 & 0.0228 & 0.0072 & 0.0091 & $31.8 \%$ & $39.8 \%$ \\
\hline 1 & SB4VAR23 & $\mathrm{Fe}_{2} \mathrm{O}_{3}$ & 10.4404 & 10.9679 & 11.2488 & -0.8084 & -0.2809 & $-7.2 \%$ & $-2.5 \%$ \\
\hline 1 & SB4VAR23 & $\mathrm{K}_{2} \mathrm{O}$ & 0.1436 & 0.1333 & 0.1392 & 0.0045 & -0.0059 & $3.2 \%$ & $-4.2 \%$ \\
\hline 1 & SB4VAR23 & $\mathrm{La}_{2} \mathrm{O}_{3}$ & 0.0586 & 0.0586 & 0.0193 & 0.0393 & 0.0393 & $203.8 \%$ & $203.8 \%$ \\
\hline 1 & SB4VAR23 & $\mathrm{Li}_{2} \mathrm{O}$ & 4.8117 & 4.9084 & 4.9600 & -0.1483 & -0.0516 & $-3.0 \%$ & $-1.0 \%$ \\
\hline 1 & SB4VAR23 & $\mathrm{MgO}$ & 1.0215 & 1.0732 & 1.0675 & -0.0460 & 0.0057 & $-4.3 \%$ & $0.5 \%$ \\
\hline 1 & SB4VAR23 & $\mathrm{MnO}$ & 2.2047 & 2.2700 & 2.2591 & -0.0544 & 0.0109 & $-2.4 \%$ & $0.5 \%$ \\
\hline 1 & SB4VAR23 & $\mathrm{Na}_{2} \mathrm{O}$ & 11.3603 & 11.3469 & 11.5412 & -0.1809 & -0.1943 & $-1.6 \%$ & $-1.7 \%$ \\
\hline 1 & SB4VAR23 & $\mathrm{NiO}$ & 0.5437 & 0.5880 & 0.6259 & -0.0822 & -0.0379 & $-13.1 \%$ & $-6.1 \%$ \\
\hline 1 & SB4VAR23 & $\mathrm{PbO}$ & 0.0259 & 0.0259 & 0.0247 & 0.0012 & 0.0012 & $4.7 \%$ & $4.7 \%$ \\
\hline 1 & SB4VAR23 & $\mathrm{SiO}_{2}$ & 45.1927 & 44.1084 & 44.4582 & 0.7345 & -0.3498 & $1.7 \%$ & $-0.8 \%$ \\
\hline 1 & SB4VAR23 & $\mathrm{SO}_{4}$ & 0.3505 & 0.3505 & 0.3770 & -0.0265 & -0.0265 & $-7.0 \%$ & $-7.0 \%$ \\
\hline 1 & SB4VAR23 & $\mathrm{TiO}_{2}$ & 0.0229 & 0.0242 & 0.0152 & 0.0077 & 0.0090 & $50.9 \%$ & $59.1 \%$ \\
\hline 1 & SB4VAR23 & $\mathrm{U}_{3} \mathrm{O}_{8}$ & 3.2575 & 3.3968 & 3.4322 & -0.1747 & -0.0354 & $-5.1 \%$ & $-1.0 \%$ \\
\hline 1 & SB4VAR23 & $\mathrm{ZnO}$ & 0.0255 & 0.0255 & 0.0307 & -0.0052 & -0.0052 & $-16.9 \%$ & $-16.9 \%$ \\
\hline 1 & SB4VAR23 & $\mathrm{ZrO}_{2}$ & 0.0277 & 0.0277 & 0.0314 & -0.0037 & -0.0037 & $-11.8 \%$ & $-11.8 \%$ \\
\hline 1 & SB4VAR23 & Sum & 99.6625 & 99.4231 & 99.9999 & -0.3374 & -0.5767 & $-0.3 \%$ & $-0.6 \%$ \\
\hline 1 & SB4VAR24 & $\mathrm{Al}_{2} \mathrm{O}_{3}$ & 10.6284 & 10.8147 & 10.6430 & -0.0146 & 0.1717 & $-0.1 \%$ & $1.6 \%$ \\
\hline 1 & SB4VAR24 & $\mathrm{B}_{2} \mathrm{O}_{3}$ & 8.1463 & 8.3254 & 8.2600 & -0.1137 & 0.0654 & $-1.4 \%$ & $0.8 \%$ \\
\hline 1 & SB4VAR24 & $\mathrm{BaO}$ & 0.0287 & 0.0307 & 0.0317 & -0.0030 & -0.0010 & $-9.3 \%$ & $-3.2 \%$ \\
\hline 1 & SB4VAR24 & $\mathrm{CaO}$ & 1.1362 & 1.1661 & 1.1712 & -0.0350 & -0.0051 & $-3.0 \%$ & $-0.4 \%$ \\
\hline 1 & SB4VAR24 & $\mathrm{Ce}_{2} \mathrm{O}_{3}$ & 0.0246 & 0.0246 & 0.0279 & -0.0033 & -0.0033 & $-11.8 \%$ & $-11.8 \%$ \\
\hline 1 & SB4VAR24 & $\mathrm{Cr}_{2} \mathrm{O}_{3}$ & 0.0607 & 0.0614 & 0.0665 & -0.0058 & -0.0051 & $-8.8 \%$ & $-7.7 \%$ \\
\hline 1 & SB4VAR24 & $\mathrm{CuO}$ & 0.0310 & 0.0329 & 0.0246 & 0.0064 & 0.0083 & $25.9 \%$ & $33.6 \%$ \\
\hline 1 & SB4VAR24 & $\mathrm{Fe}_{2} \mathrm{O}_{3}$ & 11.1409 & 11.7035 & 12.1369 & -0.9960 & -0.4334 & $-8.2 \%$ & $-3.6 \%$ \\
\hline 1 & SB4VAR24 & $\mathrm{K}_{2} \mathrm{O}$ & 0.1991 & 0.1847 & 0.1502 & 0.0489 & 0.0345 & $32.5 \%$ & $23.0 \%$ \\
\hline 1 & SB4VAR24 & $\mathrm{La}_{2} \mathrm{O}_{3}$ & 0.0586 & 0.0586 & 0.0209 & 0.0377 & 0.0377 & $180.6 \%$ & $180.6 \%$ \\
\hline 1 & SB4VAR24 & $\mathrm{Li}_{2} \mathrm{O}$ & 4.5911 & 4.8188 & 4.7200 & -0.1289 & 0.0988 & $-2.7 \%$ & $2.1 \%$ \\
\hline 1 & SB4VAR24 & $\mathrm{MgO}$ & 1.0820 & 1.1368 & 1.1518 & -0.0698 & -0.0150 & $-6.1 \%$ & $-1.3 \%$ \\
\hline 1 & SB4VAR24 & $\mathrm{MnO}$ & 2.4016 & 2.4726 & 2.4375 & -0.0359 & 0.0351 & $-1.5 \%$ & $1.4 \%$ \\
\hline
\end{tabular}


WSRC-STI-2008-00149

Revision 0

Table A4. Average Measured and Bias-Corrected Chemical Compositions Versus Targeted Compositions by Oxide by SB4-Decant Variability Study Glass

\begin{tabular}{|c|c|c|c|c|c|c|c|c|c|}
\hline & & & & Measured & & & & & \% Diff \\
\hline & & & Measured & $\begin{array}{c}\text { Bias- } \\
\text { Corrected (BC) }\end{array}$ & Targeted & $\begin{array}{c}\text { Diff } \\
\text { of }\end{array}$ & $\begin{array}{c}\text { Diff } \\
\text { of }\end{array}$ & $\begin{array}{c}\% \text { Diff } \\
\text { of }\end{array}$ & $\begin{array}{c}\text { of } \\
\text { Meas. }\end{array}$ \\
\hline Set & Glass ID & Oxide & $(\mathrm{wt} \%)$ & $(\mathrm{wt} \%)$ & $(w t \%)$ & Measured & Meas BC & Measured & $\mathrm{BC}$ \\
\hline 1 & SB4VAR24 & $\mathrm{Na}_{2} \mathrm{O}$ & 11.6669 & 11.6534 & 11.8207 & -0.1538 & -0.1673 & $-1.3 \%$ & $-1.4 \%$ \\
\hline 1 & SB4VAR24 & $\mathrm{NiO}$ & 0.5500 & 0.5949 & 0.6754 & -0.1254 & -0.0805 & $-18.6 \%$ & $-11.9 \%$ \\
\hline 1 & SB4VAR24 & $\mathrm{PbO}$ & 0.0302 & 0.0302 & 0.0267 & 0.0035 & 0.0035 & $13.0 \%$ & $13.0 \%$ \\
\hline 1 & SB4VAR24 & $\mathrm{SiO}_{2}$ & 43.1069 & 43.6162 & 42.4417 & 0.6652 & 1.1745 & $1.6 \%$ & $2.8 \%$ \\
\hline 1 & SB4VAR24 & $\mathrm{SO}_{4}$ & 0.3790 & 0.3790 & 0.4068 & -0.0278 & -0.0278 & $-6.8 \%$ & $-6.8 \%$ \\
\hline 1 & SB4VAR24 & $\mathrm{TiO}_{2}$ & 0.0225 & 0.0237 & 0.0164 & 0.0061 & 0.0073 & $37.3 \%$ & $44.7 \%$ \\
\hline 1 & SB4VAR24 & $\mathrm{U}_{3} \mathrm{O}_{8}$ & 3.5405 & 3.6919 & 3.7031 & -0.1626 & -0.0112 & $-4.4 \%$ & $-0.3 \%$ \\
\hline 1 & SB4VAR24 & $\mathrm{ZnO}$ & 0.0268 & 0.0268 & 0.0332 & -0.0064 & -0.0064 & $-19.4 \%$ & $-19.4 \%$ \\
\hline 1 & SB4VAR24 & $\mathrm{ZrO}_{2}$ & 0.0317 & 0.0317 & 0.0338 & -0.0021 & -0.0021 & $-6.1 \%$ & $-6.1 \%$ \\
\hline 1 & SB4VAR24 & Sum & 98.8839 & 100.8786 & 100.0000 & -1.1161 & 0.8786 & $-1.1 \%$ & $0.9 \%$ \\
\hline 1 & SB4VAR41 & $\mathrm{Al}_{2} \mathrm{O}_{3}$ & 8.1390 & 8.3219 & 7.9963 & 0.1427 & 0.3256 & $1.8 \%$ & $4.1 \%$ \\
\hline 1 & SB4VAR41 & $\mathrm{B}_{2} \mathrm{O}_{3}$ & 9.7563 & 9.4568 & 9.5200 & 0.2363 & -0.0632 & $2.5 \%$ & $-0.7 \%$ \\
\hline 1 & SB4VAR41 & $\mathrm{BaO}$ & 0.0212 & 0.0227 & 0.0249 & -0.0037 & -0.0022 & $-14.8 \%$ & $-8.7 \%$ \\
\hline 1 & SB4VAR41 & $\mathrm{CaO}$ & 0.8546 & 0.8842 & 0.8848 & -0.0302 & -0.0006 & $-3.4 \%$ & $-0.1 \%$ \\
\hline 1 & SB4VAR41 & $\mathrm{Ce}_{2} \mathrm{O}_{3}$ & 0.0214 & 0.0214 & 0.0233 & -0.0019 & -0.0019 & $-8.2 \%$ & $-8.2 \%$ \\
\hline 1 & SB4VAR41 & $\mathrm{Cr}_{2} \mathrm{O}_{3}$ & 0.0548 & 0.0556 & 0.0510 & 0.0038 & 0.0046 & $7.5 \%$ & $9.0 \%$ \\
\hline 1 & SB4VAR41 & $\mathrm{CuO}$ & 0.0238 & 0.0253 & 0.0190 & 0.0048 & 0.0063 & $25.2 \%$ & $33.2 \%$ \\
\hline 1 & SB4VAR41 & $\mathrm{Fe}_{2} \mathrm{O}_{3}$ & 9.2502 & 9.0625 & 9.1990 & 0.0512 & -0.1365 & $0.6 \%$ & $-1.5 \%$ \\
\hline 1 & SB4VAR41 & $\mathrm{K}_{2} \mathrm{O}$ & 0.1274 & 0.1175 & 0.1133 & 0.0141 & 0.0042 & $12.5 \%$ & $3.7 \%$ \\
\hline 1 & SB4VAR41 & $\mathrm{La}_{2} \mathrm{O}_{3}$ & 0.0586 & 0.0586 & 0.0165 & 0.0421 & 0.0421 & $255.4 \%$ & $255.4 \%$ \\
\hline 1 & SB4VAR41 & $\mathrm{Li}_{2} \mathrm{O}$ & 5.4522 & 5.5619 & 5.4400 & 0.0122 & 0.1219 & $0.2 \%$ & $2.2 \%$ \\
\hline 1 & SB4VAR41 & $\mathrm{MgO}$ & 0.8354 & 0.8595 & 0.8564 & -0.0210 & 0.0031 & $-2.5 \%$ & $0.4 \%$ \\
\hline 1 & SB4VAR41 & $\mathrm{MnO}$ & 1.9626 & 1.9275 & 1.8810 & 0.0816 & 0.0465 & $4.3 \%$ & $2.5 \%$ \\
\hline 1 & SB4VAR41 & $\mathrm{Na}_{2} \mathrm{O}$ & 11.3839 & 11.4605 & 11.3434 & 0.0405 & 0.1171 & $0.4 \%$ & $1.0 \%$ \\
\hline 1 & SB4VAR41 & $\mathrm{NiO}$ & 0.4629 & 0.4916 & 0.5227 & -0.0598 & -0.0311 & $-11.4 \%$ & $-6.0 \%$ \\
\hline 1 & SB4VAR41 & $\mathrm{PbO}$ & 0.0218 & 0.0218 & 0.0217 & 0.0001 & 0.0001 & $0.5 \%$ & $0.5 \%$ \\
\hline 1 & SB4VAR41 & $\mathrm{SiO}_{2}$ & 50.5410 & 49.3329 & 48.4580 & 2.0830 & 0.8749 & $4.3 \%$ & $1.8 \%$ \\
\hline 1 & SB4VAR41 & $\mathrm{SO}_{4}$ & 0.3303 & 0.3303 & 0.3475 & -0.0172 & -0.0172 & $-5.0 \%$ & $-5.0 \%$ \\
\hline 1 & SB4VAR41 & $\mathrm{TiO}_{2}$ & 0.3962 & 0.4121 & 0.4279 & -0.0318 & -0.0158 & $-7.4 \%$ & $-3.7 \%$ \\
\hline 1 & SB4VAR41 & $\mathrm{U}_{3} \mathrm{O}_{8}$ & 2.7416 & 2.8590 & 2.7991 & -0.0575 & 0.0599 & $-2.1 \%$ & $2.1 \%$ \\
\hline 1 & SB4VAR41 & $\mathrm{ZnO}$ & 0.0180 & 0.0180 & 0.0258 & -0.0078 & -0.0078 & $-30.0 \%$ & $-30.0 \%$ \\
\hline 1 & SB4VAR41 & $\mathrm{ZrO}_{2}$ & 0.0257 & 0.0257 & 0.0283 & -0.0026 & -0.0026 & $-9.3 \%$ & $-9.3 \%$ \\
\hline 1 & SB4VAR41 & Sum & 102.4788 & 101.3274 & 99.9999 & 2.4789 & 1.3275 & $2.5 \%$ & $1.3 \%$ \\
\hline 1 & SB4VAR42 & $\mathrm{Al}_{2} \mathrm{O}_{3}$ & 8.6775 & 8.8748 & 8.7460 & -0.0685 & 0.1288 & $-0.8 \%$ & $1.5 \%$ \\
\hline 1 & SB4VAR42 & $\mathrm{B}_{2} \mathrm{O}_{3}$ & 9.1526 & 8.8707 & 9.1000 & 0.0526 & -0.2293 & $0.6 \%$ & $-2.5 \%$ \\
\hline 1 & SB4VAR42 & $\mathrm{BaO}$ & 0.0232 & 0.0248 & 0.0273 & -0.0041 & -0.0025 & $-15.1 \%$ & $-9.0 \%$ \\
\hline 1 & SB4VAR42 & $\mathrm{CaO}$ & 0.9193 & 0.9512 & 0.9678 & -0.0485 & -0.0166 & $-5.0 \%$ & $-1.7 \%$ \\
\hline 1 & SB4VAR42 & $\mathrm{Ce}_{2} \mathrm{O}_{3}$ & 0.0228 & 0.0228 & 0.0255 & -0.0026 & -0.0026 & $-10.4 \%$ & $-10.4 \%$ \\
\hline 1 & SB4VAR42 & $\mathrm{Cr}_{2} \mathrm{O}_{3}$ & 0.0555 & 0.0563 & 0.0558 & -0.0003 & 0.0005 & $-0.5 \%$ & $1.0 \%$ \\
\hline 1 & SB4VAR42 & $\mathrm{CuO}$ & 0.0272 & 0.0290 & 0.0208 & 0.0064 & 0.0082 & $30.9 \%$ & $39.3 \%$ \\
\hline 1 & SB4VAR42 & $\mathrm{Fe}_{2} \mathrm{O}_{3}$ & 10.1080 & 9.9032 & 10.0615 & 0.0465 & -0.1583 & $0.5 \%$ & $-1.6 \%$ \\
\hline 1 & SB4VAR42 & $\mathrm{K}_{2} \mathrm{O}$ & 0.1310 & 0.1208 & 0.1239 & 0.0071 & -0.0031 & $5.7 \%$ & $-2.5 \%$ \\
\hline 1 & SB4VAR42 & $\mathrm{La}_{2} \mathrm{O}_{3}$ & 0.0586 & 0.0586 & 0.0181 & 0.0405 & 0.0405 & $224.0 \%$ & $224.0 \%$ \\
\hline 1 & SB4VAR42 & $\mathrm{Li}_{2} \mathrm{O}$ & 5.0862 & 5.1885 & 5.2000 & -0.1138 & -0.0115 & $-2.2 \%$ & $-0.2 \%$ \\
\hline 1 & SB4VAR42 & $\mathrm{MgO}$ & 0.9270 & 0.9538 & 0.9367 & -0.0097 & 0.0171 & $-1.0 \%$ & $1.8 \%$ \\
\hline 1 & SB4VAR42 & $\mathrm{MnO}$ & 2.1240 & 2.0863 & 2.0573 & 0.0667 & 0.0290 & $3.2 \%$ & $1.4 \%$ \\
\hline 1 & SB4VAR42 & $\mathrm{Na}_{2} \mathrm{O}$ & 11.3738 & 11.4551 & 11.6568 & -0.2831 & -0.2017 & $-2.4 \%$ & $-1.7 \%$ \\
\hline 1 & SB4VAR42 & $\mathrm{NiO}$ & 0.5192 & 0.5514 & 0.5717 & -0.0525 & -0.0203 & $-9.2 \%$ & $-3.6 \%$ \\
\hline 1 & SB4VAR42 & $\mathrm{PbO}$ & 0.0248 & 0.0248 & 0.0237 & 0.0011 & 0.0011 & $4.5 \%$ & $4.5 \%$ \\
\hline 1 & SB4VAR42 & $\mathrm{SiO}_{2}$ & 47.3855 & 46.2490 & 46.4385 & 0.9470 & -0.1895 & $2.0 \%$ & $-0.4 \%$ \\
\hline 1 & SB4VAR42 & $\mathrm{SO}_{4}$ & 0.3505 & 0.3505 & 0.3801 & -0.0296 & -0.0296 & $-7.8 \%$ & $-7.8 \%$ \\
\hline 1 & SB4VAR42 & $\mathrm{TiO}_{2}$ & 0.4474 & 0.4655 & 0.4680 & -0.0206 & -0.0025 & $-4.4 \%$ & $-0.5 \%$ \\
\hline 1 & SB4VAR42 & $\mathrm{U}_{3} \mathrm{O}_{8}$ & 2.9244 & 3.0501 & 3.0615 & -0.1371 & -0.0114 & $-4.5 \%$ & $-0.4 \%$ \\
\hline 1 & SB4VAR42 & $\mathrm{ZnO}$ & 0.0224 & 0.0224 & 0.0282 & -0.0058 & -0.0058 & $-20.5 \%$ & $-20.5 \%$ \\
\hline 1 & SB4VAR42 & $\mathrm{ZrO}_{2}$ & 0.0263 & 0.0263 & 0.0309 & -0.0046 & -0.0046 & $-14.8 \%$ & $-14.8 \%$ \\
\hline 1 & SB4VAR42 & Sum & 100.3873 & 99.3361 & 100.0001 & 0.3872 & -0.6640 & $0.4 \%$ & $-0.7 \%$ \\
\hline 1 & SB4VAR43 & $\mathrm{Al}_{2} \mathrm{O}_{3}$ & 9.4144 & 9.6252 & 9.4956 & -0.0812 & 0.1296 & $-0.9 \%$ & $1.4 \%$ \\
\hline 1 & SB4VAR43 & $\mathrm{B}_{2} \mathrm{O}_{3}$ & 8.6293 & 8.8191 & 8.6800 & -0.0507 & 0.1391 & $-0.6 \%$ & $1.6 \%$ \\
\hline
\end{tabular}


WSRC-STI-2008-00149

Revision 0

Table A4. Average Measured and Bias-Corrected Chemical Compositions Versus Targeted Compositions by Oxide by SB4-Decant Variability Study Glass

\begin{tabular}{|c|c|c|c|c|c|c|c|c|c|}
\hline & & & & Measured & & & & & \% Diff \\
\hline & & & Measured & $\begin{array}{c}\text { Bias- } \\
\text { Corrected (BC) }\end{array}$ & Targeted & $\begin{array}{c}\text { Diff } \\
\text { of }\end{array}$ & $\begin{array}{c}\text { Diff } \\
\text { of }\end{array}$ & $\begin{array}{c}\% \text { Diff } \\
\text { of }\end{array}$ & $\begin{array}{c}\text { of } \\
\text { Meas. }\end{array}$ \\
\hline Set & Glass ID & Oxide & (wt\%) & (wt\%) & $(\mathrm{wt} \%)$ & Measured & Meas BC & Measured & $\mathrm{BC}$ \\
\hline 1 & SB4VAR43 & $\mathrm{BaO}$ & 0.0290 & 0.0311 & 0.0296 & -0.0006 & 0.0015 & $-1.9 \%$ & $5.1 \%$ \\
\hline 1 & SB4VAR43 & $\mathrm{CaO}$ & 0.9903 & 1.0246 & 1.0507 & -0.0604 & -0.0261 & $-5.8 \%$ & $-2.5 \%$ \\
\hline 1 & SB4VAR43 & $\mathrm{Ce}_{2} \mathrm{O}_{3}$ & 0.0252 & 0.0252 & 0.0277 & -0.0025 & -0.0025 & $-9.0 \%$ & $-9.0 \%$ \\
\hline 1 & SB4VAR43 & $\mathrm{Cr}_{2} \mathrm{O}_{3}$ & 0.0515 & 0.0523 & 0.0606 & -0.0091 & -0.0083 & $-15.0 \%$ & $-13.8 \%$ \\
\hline 1 & SB4VAR43 & $\mathrm{CuO}$ & 0.0300 & 0.0320 & 0.0225 & 0.0075 & 0.0095 & $33.5 \%$ & $42.1 \%$ \\
\hline 1 & SB4VAR43 & $\mathrm{Fe}_{2} \mathrm{O}_{3}$ & 11.2339 & 11.0068 & 10.9239 & 0.3100 & 0.0829 & $2.8 \%$ & $0.8 \%$ \\
\hline 1 & SB4VAR43 & $\mathrm{K}_{2} \mathrm{O}$ & 0.1705 & 0.1572 & 0.1345 & 0.0359 & 0.0227 & $26.7 \%$ & $16.8 \%$ \\
\hline 1 & SB4VAR43 & $\mathrm{La}_{2} \mathrm{O}_{3}$ & 0.0586 & 0.0586 & 0.0196 & 0.0390 & 0.0390 & $199.2 \%$ & $199.2 \%$ \\
\hline 1 & SB4VAR43 & $\mathrm{Li}_{2} \mathrm{O}$ & 4.8548 & 5.0957 & 4.9600 & -0.1052 & 0.1357 & $-2.1 \%$ & $2.7 \%$ \\
\hline 1 & SB4VAR43 & $\mathrm{MgO}$ & 1.0070 & 1.0361 & 1.0170 & -0.0100 & 0.0191 & $-1.0 \%$ & $1.9 \%$ \\
\hline 1 & SB4VAR43 & $\mathrm{MnO}$ & 2.3758 & 2.3344 & 2.2337 & 0.1421 & 0.1007 & $6.4 \%$ & $4.5 \%$ \\
\hline 1 & SB4VAR43 & $\mathrm{Na}_{2} \mathrm{O}$ & 11.6198 & 11.6971 & 11.9703 & -0.3505 & -0.2732 & $-2.9 \%$ & $-2.3 \%$ \\
\hline 1 & SB4VAR43 & $\mathrm{NiO}$ & 0.5685 & 0.6038 & 0.6207 & -0.0522 & -0.0169 & $-8.4 \%$ & $-2.7 \%$ \\
\hline 1 & SB4VAR43 & $\mathrm{PbO}$ & 0.0264 & 0.0264 & 0.0258 & 0.0006 & 0.0006 & $2.3 \%$ & $2.3 \%$ \\
\hline 1 & SB4VAR43 & $\mathrm{SiO}_{2}$ & 45.5136 & 46.0542 & 44.4189 & 1.0947 & 1.6353 & $2.5 \%$ & $3.7 \%$ \\
\hline 1 & SB4VAR43 & $\mathrm{SO}_{4}$ & 0.4000 & 0.4000 & 0.4127 & -0.0127 & -0.0127 & $-3.1 \%$ & $-3.1 \%$ \\
\hline 1 & SB4VAR43 & $\mathrm{TiO}_{2}$ & 0.5158 & 0.5366 & 0.5081 & 0.0077 & 0.0285 & $1.5 \%$ & $5.6 \%$ \\
\hline 1 & SB4VAR43 & $\mathrm{U}_{3} \mathrm{O}_{8}$ & 3.2074 & 3.3446 & 3.3239 & -0.1165 & 0.0207 & $-3.5 \%$ & $0.6 \%$ \\
\hline 1 & SB4VAR43 & $\mathrm{ZnO}$ & 0.0249 & 0.0249 & 0.0307 & -0.0058 & -0.0058 & $-18.9 \%$ & $-18.9 \%$ \\
\hline 1 & SB4VAR43 & $\mathrm{ZrO}_{2}$ & 0.0277 & 0.0277 & 0.0336 & -0.0059 & -0.0059 & $-17.6 \%$ & $-17.6 \%$ \\
\hline 1 & SB4VAR43 & Sum & 100.7744 & 102.0135 & 100.0001 & 0.7744 & 2.0134 & $0.8 \%$ & $2.0 \%$ \\
\hline 1 & SB4VAR44 & $\mathrm{Al}_{2} \mathrm{O}_{3}$ & 10.2553 & 10.4345 & 10.2453 & 0.0100 & 0.1892 & $0.1 \%$ & $1.8 \%$ \\
\hline 1 & SB4VAR44 & $\mathrm{B}_{2} \mathrm{O}_{3}$ & 8.2590 & 8.4406 & 8.2600 & -0.0010 & 0.1806 & $0.0 \%$ & $2.2 \%$ \\
\hline 1 & SB4VAR44 & $\mathrm{BaO}$ & 0.0299 & 0.0319 & 0.0319 & -0.0020 & 0.0000 & $-6.4 \%$ & $-0.1 \%$ \\
\hline 1 & SB4VAR44 & $\mathrm{CaO}$ & 1.0756 & 1.1040 & 1.1337 & -0.0581 & -0.0297 & $-5.1 \%$ & $-2.6 \%$ \\
\hline 1 & SB4VAR44 & $\mathrm{Ce}_{2} \mathrm{O}_{3}$ & 0.0258 & 0.0258 & 0.0298 & -0.0041 & -0.0041 & $-13.7 \%$ & $-13.7 \%$ \\
\hline 1 & SB4VAR44 & $\mathrm{Cr}_{2} \mathrm{O}_{3}$ & 0.0661 & 0.0669 & 0.0654 & 0.0007 & 0.0015 & $1.1 \%$ & $2.3 \%$ \\
\hline 1 & SB4VAR44 & $\mathrm{CuO}$ & 0.0307 & 0.0325 & 0.0243 & 0.0064 & 0.0082 & $26.2 \%$ & $33.9 \%$ \\
\hline 1 & SB4VAR44 & $\mathrm{Fe}_{2} \mathrm{O}_{3}$ & 10.5691 & 11.1031 & 11.7863 & -1.2172 & -0.6832 & $-10.3 \%$ & $-5.8 \%$ \\
\hline 1 & SB4VAR44 & $\mathrm{K}_{2} \mathrm{O}$ & 0.1482 & 0.1375 & 0.1451 & 0.0030 & -0.0077 & $2.1 \%$ & $-5.3 \%$ \\
\hline 1 & SB4VAR44 & $\mathrm{La}_{2} \mathrm{O}_{3}$ & 0.0586 & 0.0586 & 0.0212 & 0.0374 & 0.0374 & $176.6 \%$ & $176.6 \%$ \\
\hline 1 & SB4VAR44 & $\mathrm{Li}_{2} \mathrm{O}$ & 4.5803 & 4.8076 & 4.7200 & -0.1397 & 0.0876 & $-3.0 \%$ & $1.9 \%$ \\
\hline 1 & SB4VAR44 & $\mathrm{MgO}$ & 1.0601 & 1.1137 & 1.0972 & -0.0371 & 0.0165 & $-3.4 \%$ & $1.5 \%$ \\
\hline 1 & SB4VAR44 & $\mathrm{MnO}$ & 2.3112 & 2.3796 & 2.4100 & -0.0988 & -0.0304 & $-4.1 \%$ & $-1.3 \%$ \\
\hline 1 & SB4VAR44 & $\mathrm{Na}_{2} \mathrm{O}$ & 12.1758 & 12.1614 & 12.2837 & -0.1079 & -0.1223 & $-0.9 \%$ & $-1.0 \%$ \\
\hline 1 & SB4VAR44 & $\mathrm{NiO}$ & 0.5704 & 0.6169 & 0.6697 & -0.0993 & -0.0528 & $-14.8 \%$ & $-7.9 \%$ \\
\hline 1 & SB4VAR44 & $\mathrm{PbO}$ & 0.0307 & 0.0307 & 0.0278 & 0.0029 & 0.0029 & $10.4 \%$ & $10.4 \%$ \\
\hline 1 & SB4VAR44 & $\mathrm{SiO}_{2}$ & 43.2673 & 43.7836 & 42.3993 & 0.8680 & 1.3843 & $2.0 \%$ & $3.3 \%$ \\
\hline 1 & SB4VAR44 & $\mathrm{SO}_{4}$ & 0.4104 & 0.4104 & 0.4453 & -0.0348 & -0.0348 & $-7.8 \%$ & $-7.8 \%$ \\
\hline 1 & SB4VAR44 & $\mathrm{TiO}_{2}$ & 0.5029 & 0.5302 & 0.5482 & -0.0453 & -0.0180 & $-8.3 \%$ & $-3.3 \%$ \\
\hline 1 & SB4VAR44 & $\mathrm{U}_{3} \mathrm{O}_{8}$ & 3.4079 & 3.5535 & 3.5863 & -0.1784 & -0.0328 & $-5.0 \%$ & $-0.9 \%$ \\
\hline 1 & SB4VAR44 & $\mathrm{ZnO}$ & 0.0296 & 0.0296 & 0.0331 & -0.0035 & -0.0035 & $-10.7 \%$ & $-10.7 \%$ \\
\hline 1 & SB4VAR44 & $\mathrm{ZrO}_{2}$ & 0.0317 & 0.0317 & 0.0362 & -0.0045 & -0.0045 & $-12.3 \%$ & $-12.3 \%$ \\
\hline 1 & SB4VAR44 & Sum & 98.8966 & 100.8844 & 99.9998 & -1.1032 & 0.8846 & $-1.1 \%$ & $0.9 \%$ \\
\hline 1 & SB4VAR51 & $\mathrm{Al}_{2} \mathrm{O}_{3}$ & 7.7847 & 7.9605 & 7.7091 & 0.0756 & 0.2514 & $1.0 \%$ & $3.3 \%$ \\
\hline 1 & SB4VAR51 & $\mathrm{B}_{2} \mathrm{O}_{3}$ & 9.3860 & 9.5924 & 9.5200 & -0.1340 & 0.0724 & $-1.4 \%$ & $0.8 \%$ \\
\hline 1 & SB4VAR51 & $\mathrm{BaO}$ & 0.0207 & 0.0221 & 0.0241 & -0.0034 & -0.0020 & $-14.3 \%$ & $-8.1 \%$ \\
\hline 1 & SB4VAR51 & $\mathrm{CaO}$ & 0.8101 & 0.8383 & 0.8532 & -0.0431 & -0.0149 & $-5.0 \%$ & $-1.7 \%$ \\
\hline 1 & SB4VAR51 & $\mathrm{Ce}_{2} \mathrm{O}_{3}$ & 0.0202 & 0.0202 & 0.0225 & -0.0023 & -0.0023 & $-10.3 \%$ & $-10.3 \%$ \\
\hline 1 & SB4VAR51 & $\mathrm{Cr}_{2} \mathrm{O}_{3}$ & 0.0512 & 0.0519 & 0.0492 & 0.0020 & 0.0027 & $4.0 \%$ & $5.5 \%$ \\
\hline 1 & SB4VAR51 & $\mathrm{CuO}$ & 0.0272 & 0.0290 & 0.0183 & 0.0089 & 0.0107 & $48.8 \%$ & $58.3 \%$ \\
\hline 1 & SB4VAR51 & $\mathrm{Fe}_{2} \mathrm{O}_{3}$ & 8.7140 & 8.5366 & 8.8716 & -0.1576 & -0.3350 & $-1.8 \%$ & $-3.8 \%$ \\
\hline 1 & SB4VAR51 & $\mathrm{K}_{2} \mathrm{O}$ & 0.1177 & 0.1086 & 0.1092 & 0.0085 & -0.0006 & $7.8 \%$ & $-0.6 \%$ \\
\hline 1 & SB4VAR51 & $\mathrm{La}_{2} \mathrm{O}_{3}$ & 0.0586 & 0.0586 & 0.0160 & 0.0426 & 0.0426 & $266.5 \%$ & $266.5 \%$ \\
\hline 1 & SB4VAR51 & $\mathrm{Li}_{2} \mathrm{O}$ & 5.2854 & 5.5476 & 5.4400 & -0.1546 & 0.1076 & $-2.8 \%$ & $2.0 \%$ \\
\hline 1 & SB4VAR51 & $\mathrm{MgO}$ & 0.7964 & 0.8194 & 0.8253 & -0.0289 & -0.0059 & $-3.5 \%$ & $-0.7 \%$ \\
\hline 1 & SB4VAR51 & $\mathrm{MnO}$ & 1.8432 & 1.8094 & 1.8152 & 0.0280 & -0.0058 & $1.5 \%$ & $-0.3 \%$ \\
\hline 1 & SB4VAR51 & $\mathrm{Na}_{2} \mathrm{O}$ & 12.1657 & 12.2525 & 12.2471 & -0.0814 & 0.0054 & $-0.7 \%$ & $0.0 \%$ \\
\hline
\end{tabular}


WSRC-STI-2008-00149

Revision 0

Table A4. Average Measured and Bias-Corrected Chemical Compositions Versus Targeted Compositions by Oxide by SB4-Decant Variability Study Glass

\begin{tabular}{|c|c|c|c|c|c|c|c|c|c|}
\hline & & & & Measured & & & & & \% Diff \\
\hline & & & Measured & $\begin{array}{c}\text { Bias- } \\
\text { Corrected (BC) }\end{array}$ & Targeted & $\begin{array}{c}\text { Diff } \\
\text { of }\end{array}$ & $\begin{array}{c}\text { Diff } \\
\text { of }\end{array}$ & $\begin{array}{c}\% \text { Diff } \\
\text { of }\end{array}$ & $\begin{array}{c}\text { of } \\
\text { Meas. }\end{array}$ \\
\hline Set & Glass ID & Oxide & (wt\%) & (wt\%) & $(\mathrm{wt} \%)$ & Measured & Meas BC & Measured & $\mathrm{BC}$ \\
\hline 1 & SB4VAR51 & $\mathrm{NiO}$ & 0.4441 & 0.4717 & 0.5045 & -0.0604 & -0.0328 & $-12.0 \%$ & $-6.5 \%$ \\
\hline 1 & SB4VAR51 & $\mathrm{PbO}$ & 0.0189 & 0.0189 & 0.0210 & -0.0021 & -0.0021 & $-10.2 \%$ & $-10.2 \%$ \\
\hline 1 & SB4VAR51 & $\mathrm{SiO}_{2}$ & 48.2947 & 48.8669 & 48.4272 & -0.1325 & 0.4397 & $-0.3 \%$ & $0.9 \%$ \\
\hline 1 & SB4VAR51 & $\mathrm{SO}_{4}$ & 0.3295 & 0.3295 & 0.3475 & -0.0180 & -0.0180 & $-5.2 \%$ & $-5.2 \%$ \\
\hline 1 & SB4VAR51 & $\mathrm{TiO}_{2}$ & 0.3953 & 0.4112 & 0.4275 & -0.0322 & -0.0163 & $-7.5 \%$ & $-3.8 \%$ \\
\hline 1 & SB4VAR51 & $\mathrm{U}_{3} \mathrm{O}_{8}$ & 2.6001 & 2.7113 & 2.6992 & -0.0991 & 0.0121 & $-3.7 \%$ & $0.4 \%$ \\
\hline 1 & SB4VAR51 & $\mathrm{ZnO}$ & 0.0168 & 0.0168 & 0.0249 & -0.0081 & -0.0081 & $-32.5 \%$ & $-32.5 \%$ \\
\hline 1 & SB4VAR51 & $\mathrm{ZrO}_{2}$ & 0.0240 & 0.0240 & 0.0274 & -0.0034 & -0.0034 & $-12.5 \%$ & $-12.5 \%$ \\
\hline 1 & SB4VAR51 & Sum & 99.2046 & 100.4973 & 100.0001 & -0.7954 & 0.4972 & $-0.8 \%$ & $0.5 \%$ \\
\hline 1 & SB4VAR52 & $\mathrm{Al}_{2} \mathrm{O}_{3}$ & 8.5783 & 8.7287 & 8.4319 & 0.1464 & 0.2968 & $1.7 \%$ & $3.5 \%$ \\
\hline 1 & SB4VAR52 & $\mathrm{B}_{2} \mathrm{O}_{3}$ & 9.0157 & 9.2139 & 9.1000 & -0.0843 & 0.1139 & $-0.9 \%$ & $1.3 \%$ \\
\hline 1 & SB4VAR52 & $\mathrm{BaO}$ & 0.0246 & 0.0262 & 0.0263 & -0.0017 & -0.0001 & $-6.6 \%$ & $-0.3 \%$ \\
\hline 1 & SB4VAR52 & $\mathrm{CaO}$ & 0.8951 & 0.9188 & 0.9332 & -0.0381 & -0.0144 & $-4.1 \%$ & $-1.5 \%$ \\
\hline 1 & SB4VAR52 & $\mathrm{Ce}_{2} \mathrm{O}_{3}$ & 0.0223 & 0.0223 & 0.0246 & -0.0024 & -0.0024 & $-9.7 \%$ & $-9.7 \%$ \\
\hline 1 & SB4VAR52 & $\mathrm{Cr}_{2} \mathrm{O}_{3}$ & 0.0574 & 0.0581 & 0.0539 & 0.0035 & 0.0042 & $6.4 \%$ & $7.7 \%$ \\
\hline 1 & SB4VAR52 & $\mathrm{CuO}$ & 0.0250 & 0.0266 & 0.0200 & 0.0050 & 0.0066 & $25.2 \%$ & $32.8 \%$ \\
\hline 1 & SB4VAR52 & $\mathrm{Fe}_{2} \mathrm{O}_{3}$ & 9.2394 & 9.7063 & 9.7033 & -0.4639 & 0.0030 & $-4.8 \%$ & $0.0 \%$ \\
\hline 1 & SB4VAR52 & $\mathrm{K}_{2} \mathrm{O}$ & 0.1150 & 0.1067 & 0.1195 & -0.0044 & -0.0127 & $-3.7 \%$ & $-10.6 \%$ \\
\hline 1 & SB4VAR52 & $\mathrm{La}_{2} \mathrm{O}_{3}$ & 0.0586 & 0.0586 & 0.0175 & 0.0411 & 0.0411 & $235.1 \%$ & $235.1 \%$ \\
\hline 1 & SB4VAR52 & $\mathrm{Li}_{2} \mathrm{O}$ & 5.0486 & 5.2990 & 5.2000 & -0.1514 & 0.0990 & $-2.9 \%$ & $1.9 \%$ \\
\hline 1 & SB4VAR52 & $\mathrm{MgO}$ & 0.9013 & 0.9469 & 0.9027 & -0.0014 & 0.0442 & $-0.2 \%$ & $4.9 \%$ \\
\hline 1 & SB4VAR52 & $\mathrm{MnO}$ & 1.9755 & 2.0341 & 1.9854 & -0.0099 & 0.0487 & $-0.5 \%$ & $2.5 \%$ \\
\hline 1 & SB4VAR52 & $\mathrm{Na}_{2} \mathrm{O}$ & 12.5735 & 12.5600 & 12.6453 & -0.0718 & -0.0853 & $-0.6 \%$ & $-0.7 \%$ \\
\hline 1 & SB4VAR52 & $\mathrm{NiO}$ & 0.5100 & 0.5515 & 0.5518 & -0.0418 & -0.0003 & $-7.6 \%$ & $0.0 \%$ \\
\hline 1 & SB4VAR52 & $\mathrm{PbO}$ & 0.0245 & 0.0245 & 0.0230 & 0.0015 & 0.0015 & $6.5 \%$ & $6.5 \%$ \\
\hline 1 & SB4VAR52 & $\mathrm{SiO}_{2}$ & 46.7437 & 47.2986 & 46.4048 & 0.3389 & 0.8938 & $0.7 \%$ & $1.9 \%$ \\
\hline 1 & SB4VAR52 & $\mathrm{SO}_{4}$ & 0.3550 & 0.3550 & 0.3801 & -0.0251 & -0.0251 & $-6.6 \%$ & $-6.6 \%$ \\
\hline 1 & SB4VAR52 & $\mathrm{TiO}_{2}$ & 0.4595 & 0.4844 & 0.4675 & -0.0080 & 0.0169 & $-1.7 \%$ & $3.6 \%$ \\
\hline 1 & SB4VAR52 & $\mathrm{U}_{3} \mathrm{O}_{8}$ & 2.8684 & 2.9913 & 2.9522 & -0.0838 & 0.0391 & $-2.8 \%$ & $1.3 \%$ \\
\hline 1 & SB4VAR52 & $\mathrm{ZnO}$ & 0.0227 & 0.0227 & 0.0273 & -0.0046 & -0.0046 & $-16.8 \%$ & $-16.8 \%$ \\
\hline 1 & SB4VAR52 & $\mathrm{ZrO}_{2}$ & 0.0277 & 0.0277 & 0.0300 & -0.0023 & -0.0023 & $-7.7 \%$ & $-7.7 \%$ \\
\hline 1 & SB4VAR52 & Sum & 99.5419 & 101.4620 & 100.0003 & -0.4584 & 1.4617 & $-0.5 \%$ & $1.5 \%$ \\
\hline 1 & SB4VAR53 & $\mathrm{Al}_{2} \mathrm{O}_{3}$ & 9.1688 & 9.3294 & 9.1546 & 0.0142 & 0.1748 & $0.2 \%$ & $1.9 \%$ \\
\hline 1 & SB4VAR53 & $\mathrm{B}_{2} \mathrm{O}_{3}$ & 8.9996 & 8.7219 & 8.6800 & 0.3196 & 0.0419 & $3.7 \%$ & $0.5 \%$ \\
\hline 1 & SB4VAR53 & $\mathrm{BaO}$ & 0.0265 & 0.0283 & 0.0286 & -0.0021 & -0.0003 & $-7.3 \%$ & $-1.0 \%$ \\
\hline 1 & SB4VAR53 & $\mathrm{CaO}$ & 0.9913 & 1.0175 & 1.0132 & -0.0219 & 0.0043 & $-2.2 \%$ & $0.4 \%$ \\
\hline 1 & SB4VAR53 & $\mathrm{Ce}_{2} \mathrm{O}_{3}$ & 0.0231 & 0.0231 & 0.0268 & -0.0036 & -0.0036 & $-13.5 \%$ & $-13.5 \%$ \\
\hline 1 & SB4VAR53 & $\mathrm{Cr}_{2} \mathrm{O}_{3}$ & 0.0592 & 0.0599 & 0.0585 & 0.0007 & 0.0014 & $1.2 \%$ & $2.4 \%$ \\
\hline 1 & SB4VAR53 & $\mathrm{CuO}$ & 0.0285 & 0.0302 & 0.0217 & 0.0068 & 0.0085 & $31.2 \%$ & $39.2 \%$ \\
\hline 1 & SB4VAR53 & $\mathrm{Fe}_{2} \mathrm{O}_{3}$ & 9.7255 & 10.2172 & 10.5350 & -0.8095 & -0.3178 & $-7.7 \%$ & $-3.0 \%$ \\
\hline 1 & SB4VAR53 & $\mathrm{K}_{2} \mathrm{O}$ & 0.1647 & 0.1529 & 0.1297 & 0.0350 & 0.0232 & $27.0 \%$ & $17.9 \%$ \\
\hline 1 & SB4VAR53 & $\mathrm{La}_{2} \mathrm{O}_{3}$ & 0.0586 & 0.0586 & 0.0190 & 0.0396 & 0.0396 & $208.6 \%$ & $208.6 \%$ \\
\hline 1 & SB4VAR53 & $\mathrm{Li}_{2} \mathrm{O}$ & 4.9463 & 5.0457 & 4.9600 & -0.0137 & 0.0857 & $-0.3 \%$ & $1.7 \%$ \\
\hline 1 & SB4VAR53 & $\mathrm{MgO}$ & 0.9411 & 0.9887 & 0.9801 & -0.0390 & 0.0086 & $-4.0 \%$ & $0.9 \%$ \\
\hline 1 & SB4VAR53 & $\mathrm{MnO}$ & 2.0917 & 2.1538 & 2.1556 & -0.0639 & -0.0018 & $-3.0 \%$ & $-0.1 \%$ \\
\hline 1 & SB4VAR53 & $\mathrm{Na}_{2} \mathrm{O}$ & 13.0655 & 13.0507 & 13.0435 & 0.0220 & 0.0072 & $0.2 \%$ & $0.1 \%$ \\
\hline 1 & SB4VAR53 & $\mathrm{NiO}$ & 0.5376 & 0.5815 & 0.5991 & -0.0615 & -0.0176 & $-10.3 \%$ & $-2.9 \%$ \\
\hline 1 & SB4VAR53 & $\mathrm{PbO}$ & 0.0272 & 0.0272 & 0.0249 & 0.0023 & 0.0023 & $9.2 \%$ & $9.2 \%$ \\
\hline 1 & SB4VAR53 & $\mathrm{SiO}_{2}$ & 45.9415 & 44.8393 & 44.3823 & 1.5592 & 0.4570 & $3.5 \%$ & $1.0 \%$ \\
\hline 1 & SB4VAR53 & $\mathrm{SO}_{4}$ & 0.3827 & 0.3827 & 0.4127 & -0.0300 & -0.0300 & $-7.3 \%$ & $-7.3 \%$ \\
\hline 1 & SB4VAR53 & $\mathrm{TiO}_{2}$ & 0.4750 & 0.5007 & 0.5076 & -0.0326 & -0.0069 & $-6.4 \%$ & $-1.4 \%$ \\
\hline 1 & SB4VAR53 & $\mathrm{U}_{3} \mathrm{O}_{8}$ & 3.0600 & 3.1909 & 3.2053 & -0.1453 & -0.0144 & $-4.5 \%$ & $-0.4 \%$ \\
\hline 1 & SB4VAR53 & $\mathrm{ZnO}$ & 0.0243 & 0.0243 & 0.0296 & -0.0053 & -0.0053 & $-18.0 \%$ & $-18.0 \%$ \\
\hline 1 & SB4VAR53 & $\mathrm{ZrO}_{2}$ & 0.0290 & 0.0290 & 0.0325 & -0.0035 & -0.0035 & $-10.6 \%$ & $-10.6 \%$ \\
\hline 1 & SB4VAR53 & Sum & 100.7679 & 100.4536 & 100.0002 & 0.7677 & 0.4534 & $0.8 \%$ & $0.5 \%$ \\
\hline 1 & SB4VAR54 & $\mathrm{Al}_{2} \mathrm{O}_{3}$ & 9.8632 & 10.0854 & 9.8773 & -0.0141 & 0.2081 & $-0.1 \%$ & $2.1 \%$ \\
\hline 1 & SB4VAR54 & $\mathrm{B}_{2} \mathrm{O}_{3}$ & 8.2510 & 7.9977 & 8.2600 & -0.0090 & -0.2623 & $-0.1 \%$ & $-3.2 \%$ \\
\hline 1 & SB4VAR54 & $\mathrm{BaO}$ & 0.0257 & 0.0275 & 0.0308 & -0.0051 & -0.0033 & $-16.6 \%$ & $-10.6 \%$ \\
\hline
\end{tabular}


WSRC-STI-2008-00149

Revision 0

Table A4. Average Measured and Bias-Corrected Chemical Compositions Versus Targeted Compositions by Oxide by SB4-Decant Variability Study Glass

\begin{tabular}{|c|c|c|c|c|c|c|c|c|c|}
\hline & & & & Measured & & & & & \% Diff \\
\hline & & & Measured & $\begin{array}{c}\text { Bias- } \\
\text { Corrected (BC) }\end{array}$ & Targeted & $\begin{array}{c}\text { Diff } \\
\text { of }\end{array}$ & $\begin{array}{c}\text { Diff } \\
\text { of }\end{array}$ & $\begin{array}{c}\% \text { Diff } \\
\text { of }\end{array}$ & $\begin{array}{c}\text { of } \\
\text { Meas. }\end{array}$ \\
\hline Set & Glass ID & Oxide & $(\mathrm{wt} \%)$ & $(\mathrm{wt} \%)$ & $(\mathrm{wt} \%)$ & Measured & Meas BC & Measured & $\mathrm{BC}$ \\
\hline 1 & SB4VAR54 & $\mathrm{CaO}$ & 1.0291 & 1.0649 & 1.0931 & -0.0640 & -0.0282 & $-5.9 \%$ & $-2.6 \%$ \\
\hline 1 & SB4VAR54 & $\mathrm{Ce}_{2} \mathrm{O}_{3}$ & 0.0246 & 0.0246 & 0.0289 & -0.0043 & -0.0043 & $-14.8 \%$ & $-14.8 \%$ \\
\hline 1 & SB4VAR54 & $\mathrm{Cr}_{2} \mathrm{O}_{3}$ & 0.0599 & 0.0608 & 0.0631 & -0.0032 & -0.0023 & $-5.0 \%$ & $-3.7 \%$ \\
\hline 1 & SB4VAR54 & $\mathrm{CuO}$ & 0.0300 & 0.0320 & 0.0235 & 0.0065 & 0.0085 & $27.8 \%$ & $36.0 \%$ \\
\hline 1 & SB4VAR54 & $\mathrm{Fe}_{2} \mathrm{O}_{3}$ & 11.2911 & 11.0614 & 11.3667 & -0.0756 & -0.3053 & $-0.7 \%$ & $-2.7 \%$ \\
\hline 1 & SB4VAR54 & $\mathrm{K}_{2} \mathrm{O}$ & 0.1433 & 0.1322 & 0.1399 & 0.0034 & -0.0077 & $2.4 \%$ & $-5.5 \%$ \\
\hline 1 & SB4VAR54 & $\mathrm{La}_{2} \mathrm{O}_{3}$ & 0.0586 & 0.0586 & 0.0205 & 0.0381 & 0.0381 & $186.0 \%$ & $186.0 \%$ \\
\hline 1 & SB4VAR54 & $\mathrm{Li}_{2} \mathrm{O}$ & 4.6234 & 4.7163 & 4.7200 & -0.0966 & -0.0037 & $-2.0 \%$ & $-0.1 \%$ \\
\hline 1 & SB4VAR54 & $\mathrm{MgO}$ & 1.0348 & 1.0646 & 1.0574 & -0.0226 & 0.0072 & $-2.1 \%$ & $0.7 \%$ \\
\hline 1 & SB4VAR54 & $\mathrm{MnO}$ & 2.3661 & 2.3230 & 2.3258 & 0.0403 & -0.0028 & $1.7 \%$ & $-0.1 \%$ \\
\hline 1 & SB4VAR54 & $\mathrm{Na}_{2} \mathrm{O}$ & 13.2845 & 13.3779 & 13.4416 & -0.1571 & -0.0637 & $-1.2 \%$ & $-0.5 \%$ \\
\hline 1 & SB4VAR54 & $\mathrm{NiO}$ & 0.5780 & 0.6139 & 0.6464 & -0.0684 & -0.0325 & $-10.6 \%$ & $-5.0 \%$ \\
\hline 1 & SB4VAR54 & $\mathrm{PbO}$ & 0.0269 & 0.0269 & 0.0269 & 0.0000 & 0.0000 & $0.1 \%$ & $0.1 \%$ \\
\hline 1 & SB4VAR54 & $\mathrm{SiO}_{2}$ & 42.7325 & 41.7100 & 42.3599 & 0.3726 & -0.6499 & $0.9 \%$ & $-1.5 \%$ \\
\hline 1 & SB4VAR54 & $\mathrm{SO}_{4}$ & 0.4299 & 0.4299 & 0.4453 & -0.0153 & -0.0153 & $-3.4 \%$ & $-3.4 \%$ \\
\hline 1 & SB4VAR54 & $\mathrm{TiO}_{2}$ & 0.5267 & 0.5479 & 0.5477 & -0.0210 & 0.0002 & $-3.8 \%$ & $0.0 \%$ \\
\hline 1 & SB4VAR54 & $\mathrm{U}_{3} \mathrm{O}_{8}$ & 3.3489 & 3.4923 & 3.4583 & -0.1094 & 0.0340 & $-3.2 \%$ & $1.0 \%$ \\
\hline 1 & SB4VAR54 & $\mathrm{ZnO}$ & 0.0277 & 0.0277 & 0.0319 & -0.0042 & -0.0042 & $-13.2 \%$ & $-13.2 \%$ \\
\hline 1 & SB4VAR54 & $\mathrm{ZrO}_{2}$ & 0.0324 & 0.0324 & 0.0351 & -0.0027 & -0.0027 & $-7.6 \%$ & $-7.6 \%$ \\
\hline 1 & SB4VAR54 & Sum & 99.7885 & 98.9079 & 100.0001 & -0.2116 & -1.0921 & $-0.2 \%$ & $-1.1 \%$ \\
\hline 1 & U std & $\mathrm{Al}_{2} \mathrm{O}_{3}$ & 3.9191 & 3.9973 & 4.1000 & -0.1809 & -0.1027 & $-4.4 \%$ & $-2.5 \%$ \\
\hline 1 & U std & $\mathrm{B}_{2} \mathrm{O}_{3}$ & 9.0372 & 8.9927 & 9.2090 & -0.1718 & -0.2163 & $-1.9 \%$ & $-2.3 \%$ \\
\hline 1 & U std & $\mathrm{BaO}$ & 0.0056 & 0.0060 & 0.0000 & 0.0056 & 0.0060 & & \\
\hline 1 & U std & $\mathrm{CaO}$ & 1.2587 & 1.2971 & 1.3010 & -0.0423 & -0.0039 & $-3.3 \%$ & $-0.3 \%$ \\
\hline 1 & U std & $\mathrm{Ce}_{2} \mathrm{O}_{3}$ & 0.0059 & 0.0059 & 0.0000 & 0.0059 & 0.0059 & & \\
\hline 1 & U std & $\mathrm{Cr}_{2} \mathrm{O}_{3}$ & 0.2329 & 0.2360 & 0.0000 & 0.2329 & 0.2360 & & \\
\hline 1 & U std & $\mathrm{CuO}$ & 0.0063 & 0.0067 & 0.0000 & 0.0063 & 0.0067 & & \\
\hline 1 & U std & $\mathrm{Fe}_{2} \mathrm{O}_{3}$ & 13.0722 & 13.2536 & 13.1960 & -0.1238 & 0.0576 & $-0.9 \%$ & $0.4 \%$ \\
\hline 1 & U std & $\mathrm{K}_{2} \mathrm{O}$ & 3.4512 & 3.1926 & 2.9990 & 0.4522 & 0.1936 & $15.1 \%$ & $6.5 \%$ \\
\hline 1 & U std & $\mathrm{La}_{2} \mathrm{O}_{3}$ & 0.0586 & 0.0586 & 0.0000 & 0.0586 & 0.0586 & & \\
\hline 1 & U std & $\mathrm{Li}_{2} \mathrm{O}$ & 2.9423 & 3.0444 & 3.0570 & -0.1147 & -0.0126 & $-3.8 \%$ & $-0.4 \%$ \\
\hline 1 & U std & $\mathrm{MgO}$ & 1.1685 & 1.2148 & 1.2100 & -0.0415 & 0.0048 & $-3.4 \%$ & $0.4 \%$ \\
\hline 1 & U std & $\mathrm{MnO}$ & 2.8073 & 2.8218 & 2.8920 & -0.0847 & -0.0702 & $-2.9 \%$ & $-2.4 \%$ \\
\hline 1 & U std & $\mathrm{Na}_{2} \mathrm{O}$ & 11.6029 & 11.6360 & 11.7950 & -0.1921 & -0.1590 & $-1.6 \%$ & $-1.3 \%$ \\
\hline 1 & U std & $\mathrm{NiO}$ & 1.0050 & 1.0771 & 1.1200 & -0.1150 & -0.0429 & $-10.3 \%$ & $-3.8 \%$ \\
\hline 1 & U std & $\mathrm{PbO}$ & 0.0108 & 0.0108 & 0.0000 & 0.0108 & 0.0108 & & \\
\hline 1 & U std & $\mathrm{SiO}_{2}$ & 44.9075 & 44.6236 & 45.3530 & -0.4455 & -0.7294 & $-1.0 \%$ & $-1.6 \%$ \\
\hline 1 & U std & $\mathrm{SO}_{4}$ & 0.1498 & 0.1498 & 0.0000 & 0.1498 & 0.1498 & & \\
\hline 1 & U std & $\mathrm{TiO}_{2}$ & 0.9337 & 0.9777 & 1.0490 & -0.1153 & -0.0713 & $-11.0 \%$ & $-6.8 \%$ \\
\hline 1 & U std & $\mathrm{U}_{3} \mathrm{O}_{8}$ & 2.3073 & 2.4060 & 2.4060 & -0.0987 & 0.0000 & $-4.1 \%$ & $0.0 \%$ \\
\hline 1 & U std & $\mathrm{ZnO}$ & 0.0062 & 0.0062 & 0.0000 & 0.0062 & 0.0062 & & \\
\hline 1 & U std & $\mathrm{ZrO}_{2}$ & 0.0068 & 0.0068 & 0.0000 & 0.0068 & 0.0068 & & \\
\hline 1 & U std & Sum & 98.8956 & 99.0212 & 99.6870 & -0.7914 & -0.6658 & $-0.8 \%$ & $-0.7 \%$ \\
\hline 2 & Batch 1 & $\mathrm{Al}_{2} \mathrm{O}_{3}$ & 4.7820 & 4.8770 & 4.8770 & -0.0950 & 0.0000 & $-1.9 \%$ & $0.0 \%$ \\
\hline 2 & Batch 1 & $\mathrm{~B}_{2} \mathrm{O}_{3}$ & 7.8056 & 7.7770 & 7.7770 & 0.0286 & 0.0000 & $0.4 \%$ & $0.0 \%$ \\
\hline 2 & Batch 1 & $\mathrm{BaO}$ & 0.1421 & 0.1510 & 0.1510 & -0.0089 & 0.0000 & $-5.9 \%$ & $0.0 \%$ \\
\hline 2 & Batch 1 & $\mathrm{CaO}$ & 1.1885 & 1.2200 & 1.2200 & -0.0315 & 0.0000 & $-2.6 \%$ & $0.0 \%$ \\
\hline 2 & Batch 1 & $\mathrm{Ce}_{2} \mathrm{O}_{3}$ & 0.0130 & 0.0130 & 0.0000 & 0.0130 & 0.0130 & & \\
\hline 2 & Batch 1 & $\mathrm{Cr}_{2} \mathrm{O}_{3}$ & 0.1077 & 0.1070 & 0.1070 & 0.0007 & 0.0000 & $0.6 \%$ & $0.0 \%$ \\
\hline 2 & Batch 1 & $\mathrm{CuO}$ & 0.3781 & 0.3990 & 0.3990 & -0.0209 & 0.0000 & $-5.2 \%$ & $0.0 \%$ \\
\hline 2 & Batch 1 & $\mathrm{Fe}_{2} \mathrm{O}_{3}$ & 13.0341 & 12.8393 & 12.8390 & 0.1951 & 0.0003 & $1.5 \%$ & $0.0 \%$ \\
\hline 2 & Batch 1 & $\mathrm{~K}_{2} \mathrm{O}$ & 3.0406 & 3.3270 & 3.3270 & -0.2864 & 0.0000 & $-8.6 \%$ & $0.0 \%$ \\
\hline 2 & Batch 1 & $\mathrm{La}_{2} \mathrm{O}_{3}$ & 0.0586 & 0.0586 & 0.0000 & 0.0586 & 0.0586 & & \\
\hline 2 & Batch 1 & $\mathrm{Li}_{2} \mathrm{O}$ & 4.2412 & 4.4290 & 4.4290 & -0.1878 & 0.0000 & $-4.2 \%$ & $0.0 \%$ \\
\hline 2 & Batch 1 & $\mathrm{MgO}$ & 1.3794 & 1.4190 & 1.4190 & -0.0396 & 0.0000 & $-2.8 \%$ & $0.0 \%$ \\
\hline 2 & Batch 1 & $\mathrm{MnO}$ & 1.7700 & 1.7260 & 1.7260 & 0.0440 & 0.0000 & $2.6 \%$ & $0.0 \%$ \\
\hline 2 & Batch 1 & $\mathrm{Na}_{2} \mathrm{O}$ & 8.9979 & 9.0030 & 9.0030 & -0.0051 & 0.0000 & $-0.1 \%$ & $0.0 \%$ \\
\hline 2 & Batch 1 & $\mathrm{NiO}$ & 0.7085 & 0.7510 & 0.7510 & -0.0425 & 0.0000 & $-5.7 \%$ & $0.0 \%$ \\
\hline
\end{tabular}


WSRC-STI-2008-00149

Revision 0

Table A4. Average Measured and Bias-Corrected Chemical Compositions Versus Targeted Compositions by Oxide by SB4-Decant Variability Study Glass

\begin{tabular}{|c|c|c|c|c|c|c|c|c|c|}
\hline & & & & Measured & & & & & \% Diff \\
\hline & & & Measured & $\begin{array}{c}\text { Bias- } \\
\text { Corrected (BC) }\end{array}$ & Targeted & $\begin{array}{c}\text { Diff } \\
\text { of }\end{array}$ & $\begin{array}{c}\text { Diff } \\
\text { of }\end{array}$ & $\begin{array}{c}\% \text { Diff } \\
\text { of }\end{array}$ & $\begin{array}{c}\text { of } \\
\text { Meas. }\end{array}$ \\
\hline Set & Glass ID & Oxide & $(\mathrm{wt} \%)$ & $(\mathrm{wt} \%)$ & $(\mathrm{wt} \%)$ & Measured & Meas BC & Measured & $\mathrm{BC}$ \\
\hline 2 & Batch 1 & $\mathrm{PbO}$ & 0.0108 & 0.0108 & 0.0000 & 0.0108 & 0.0108 & & \\
\hline 2 & Batch 1 & $\mathrm{SiO}_{2}$ & 51.0401 & 50.2200 & 50.2200 & 0.8201 & 0.0000 & $1.6 \%$ & $0.0 \%$ \\
\hline 2 & Batch 1 & $\mathrm{SO}_{4}$ & 0.1498 & 0.1498 & 0.0000 & 0.1498 & 0.1498 & & \\
\hline 2 & Batch 1 & $\mathrm{TiO}_{2}$ & 0.6543 & 0.6770 & 0.6770 & -0.0227 & 0.0000 & $-3.4 \%$ & $0.0 \%$ \\
\hline 2 & Batch 1 & $\mathrm{U}_{3} \mathrm{O}_{8}$ & 0.0590 & 0.0607 & 0.0000 & 0.0590 & 0.0607 & & \\
\hline 2 & Batch 1 & $\mathrm{ZnO}$ & 0.0062 & 0.0062 & 0.0000 & 0.0062 & 0.0062 & & \\
\hline 2 & Batch 1 & $\mathrm{ZrO}_{2}$ & 0.0857 & 0.0857 & 0.0980 & -0.0123 & -0.0123 & $-12.6 \%$ & $-12.6 \%$ \\
\hline 2 & Batch 1 & Sum & 99.6532 & 99.3071 & 99.0200 & 0.6332 & 0.2871 & $0.6 \%$ & $0.3 \%$ \\
\hline 2 & SB4VAR31 & $\mathrm{Al}_{2} \mathrm{O}_{3}$ & 8.0918 & 8.1107 & 8.0047 & 0.0871 & 0.1060 & $1.1 \%$ & $1.3 \%$ \\
\hline 2 & SB4VAR31 & $\mathrm{B}_{2} \mathrm{O}_{3}$ & 9.6436 & 9.5258 & 9.5200 & 0.1236 & 0.0058 & $1.3 \%$ & $0.1 \%$ \\
\hline 2 & SB4VAR31 & $\mathrm{BaO}$ & 0.0223 & 0.0234 & 0.0239 & -0.0016 & -0.0005 & $-6.6 \%$ & $-2.0 \%$ \\
\hline 2 & SB4VAR31 & $\mathrm{CaO}$ & 0.8728 & 0.8838 & 0.8809 & -0.0081 & 0.0029 & $-0.9 \%$ & $0.3 \%$ \\
\hline 2 & SB4VAR31 & $\mathrm{Ce}_{2} \mathrm{O}_{3}$ & 0.0196 & 0.0196 & 0.0210 & -0.0013 & -0.0013 & $-6.4 \%$ & $-6.4 \%$ \\
\hline 2 & SB4VAR31 & $\mathrm{Cr}_{2} \mathrm{O}_{3}$ & 0.0537 & 0.0528 & 0.0500 & 0.0037 & 0.0028 & $7.4 \%$ & $5.6 \%$ \\
\hline 2 & SB4VAR31 & $\mathrm{CuO}$ & 0.0225 & 0.0234 & 0.0185 & 0.0040 & 0.0049 & $21.8 \%$ & $26.5 \%$ \\
\hline 2 & SB4VAR31 & $\mathrm{Fe}_{2} \mathrm{O}_{3}$ & 8.8248 & 8.7410 & 9.1282 & -0.3034 & -0.3872 & $-3.3 \%$ & $-4.2 \%$ \\
\hline 2 & SB4VAR31 & $\mathrm{K}_{2} \mathrm{O}$ & 0.1168 & 0.1260 & 0.1130 & 0.0039 & 0.0130 & $3.4 \%$ & $11.5 \%$ \\
\hline 2 & SB4VAR31 & $\mathrm{La}_{2} \mathrm{O}_{3}$ & 0.0586 & 0.0586 & 0.0157 & 0.0429 & 0.0429 & $273.5 \%$ & $273.5 \%$ \\
\hline 2 & SB4VAR31 & $\mathrm{Li}_{2} \mathrm{O}$ & 5.4038 & 5.5956 & 5.4400 & -0.0362 & 0.1556 & $-0.7 \%$ & $2.9 \%$ \\
\hline 2 & SB4VAR31 & $\mathrm{MgO}$ & 0.8702 & 0.8876 & 0.8663 & 0.0039 & 0.0213 & $0.4 \%$ & $2.5 \%$ \\
\hline 2 & SB4VAR31 & $\mathrm{MnO}$ & 1.8400 & 1.8137 & 1.8333 & 0.0067 & -0.0196 & $0.4 \%$ & $-1.1 \%$ \\
\hline 2 & SB4VAR31 & $\mathrm{Na}_{2} \mathrm{O}$ & 11.8759 & 11.6629 & 11.9326 & -0.0567 & -0.2697 & $-0.5 \%$ & $-2.3 \%$ \\
\hline 2 & SB4VAR31 & $\mathrm{NiO}$ & 0.4705 & 0.4940 & 0.5079 & -0.0374 & -0.0139 & $-7.4 \%$ & $-2.7 \%$ \\
\hline 2 & SB4VAR31 & $\mathrm{PbO}$ & 0.0108 & 0.0108 & 0.0201 & -0.0093 & -0.0093 & $-46.4 \%$ & $-46.4 \%$ \\
\hline 2 & SB4VAR31 & $\mathrm{SiO}_{2}$ & 48.4551 & 47.4604 & 48.4587 & -0.0036 & -0.9983 & $0.0 \%$ & $-2.1 \%$ \\
\hline 2 & SB4VAR31 & $\mathrm{SO}_{4}$ & 0.3108 & 0.3108 & 0.3175 & -0.0067 & -0.0067 & $-2.1 \%$ & $-2.1 \%$ \\
\hline 2 & SB4VAR31 & $\mathrm{TiO}_{2}$ & 0.0225 & 0.0230 & 0.0124 & 0.0101 & 0.0106 & $81.6 \%$ & $85.4 \%$ \\
\hline 2 & SB4VAR31 & $\mathrm{U}_{3} \mathrm{O}_{8}$ & 2.7298 & 2.7575 & 2.7852 & -0.0554 & -0.0277 & $-2.0 \%$ & $-1.0 \%$ \\
\hline 2 & SB4VAR31 & $\mathrm{ZnO}$ & 0.0062 & 0.0062 & 0.0250 & -0.0188 & -0.0188 & $-75.1 \%$ & $-75.1 \%$ \\
\hline 2 & SB4VAR31 & $\mathrm{ZrO}_{2}$ & 0.0220 & 0.0220 & 0.0254 & -0.0034 & -0.0034 & $-13.6 \%$ & $-13.6 \%$ \\
\hline 2 & SB4VAR31 & Sum & 99.7442 & 98.6098 & 100.0002 & -0.2560 & -1.3904 & $-0.3 \%$ & $-1.4 \%$ \\
\hline 2 & SB4VAR32 & $\mathrm{Al}_{2} \mathrm{O}_{3}$ & 9.0885 & 9.1102 & 9.0053 & 0.0832 & 0.1049 & $0.9 \%$ & $1.2 \%$ \\
\hline 2 & SB4VAR32 & $\mathrm{B}_{2} \mathrm{O}_{3}$ & 9.0077 & 9.0534 & 8.9600 & 0.0477 & 0.0934 & $0.5 \%$ & $1.0 \%$ \\
\hline 2 & SB4VAR32 & $\mathrm{BaO}$ & 0.0237 & 0.0249 & 0.0269 & -0.0032 & -0.0020 & $-11.8 \%$ & $-7.5 \%$ \\
\hline 2 & SB4VAR32 & $\mathrm{CaO}$ & 0.9801 & 0.9925 & 0.9910 & -0.0109 & 0.0015 & $-1.1 \%$ & $0.1 \%$ \\
\hline 2 & SB4VAR32 & $\mathrm{Ce}_{2} \mathrm{O}_{3}$ & 0.0199 & 0.0199 & 0.0236 & -0.0037 & -0.0037 & $-15.6 \%$ & $-15.6 \%$ \\
\hline 2 & SB4VAR32 & $\mathrm{Cr}_{2} \mathrm{O}_{3}$ & 0.0632 & 0.0621 & 0.0563 & 0.0069 & 0.0058 & $12.3 \%$ & $10.3 \%$ \\
\hline 2 & SB4VAR32 & $\mathrm{CuO}$ & 0.0253 & 0.0263 & 0.0208 & 0.0045 & 0.0055 & $21.9 \%$ & $26.6 \%$ \\
\hline 2 & SB4VAR32 & $\mathrm{Fe}_{2} \mathrm{O}_{3}$ & 10.1223 & 10.0235 & 10.2693 & -0.1470 & -0.2458 & $-1.4 \%$ & $-2.4 \%$ \\
\hline 2 & SB4VAR32 & $\mathrm{K}_{2} \mathrm{O}$ & 0.1358 & 0.1464 & 0.1271 & 0.0087 & 0.0193 & $6.9 \%$ & $15.2 \%$ \\
\hline 2 & SB4VAR32 & $\mathrm{La}_{2} \mathrm{O}_{3}$ & 0.0586 & 0.0586 & 0.0177 & 0.0409 & 0.0409 & $231.3 \%$ & $231.3 \%$ \\
\hline 2 & SB4VAR32 & $\mathrm{Li}_{2} \mathrm{O}$ & 5.0163 & 5.2831 & 5.1200 & -0.1037 & 0.1631 & $-2.0 \%$ & $3.2 \%$ \\
\hline 2 & SB4VAR32 & $\mathrm{MgO}$ & 0.9577 & 0.9765 & 0.9746 & -0.0169 & 0.0019 & $-1.7 \%$ & $0.2 \%$ \\
\hline 2 & SB4VAR32 & $\mathrm{MnO}$ & 2.1014 & 2.0710 & 2.0624 & 0.0390 & 0.0086 & $1.9 \%$ & $0.4 \%$ \\
\hline 2 & SB4VAR32 & $\mathrm{Na}_{2} \mathrm{O}$ & 12.3848 & 12.1611 & 12.4241 & -0.0393 & -0.2630 & $-0.3 \%$ & $-2.1 \%$ \\
\hline 2 & SB4VAR32 & $\mathrm{NiO}$ & 0.5189 & 0.5447 & 0.5714 & -0.0525 & -0.0267 & $-9.2 \%$ & $-4.7 \%$ \\
\hline 2 & SB4VAR32 & $\mathrm{PbO}$ & 0.0108 & 0.0108 & 0.0226 & -0.0118 & -0.0118 & $-52.3 \%$ & $-52.3 \%$ \\
\hline 2 & SB4VAR32 & $\mathrm{SiO}_{2}$ & 46.1019 & 45.1559 & 45.7660 & 0.3359 & -0.6101 & $0.7 \%$ & $-1.3 \%$ \\
\hline 2 & SB4VAR32 & $\mathrm{SO}_{4}$ & 0.3490 & 0.3490 & 0.3572 & -0.0082 & -0.0082 & $-2.3 \%$ & $-2.3 \%$ \\
\hline 2 & SB4VAR32 & $\mathrm{TiO}_{2}$ & 0.0192 & 0.0196 & 0.0139 & 0.0053 & 0.0057 & $38.0 \%$ & $40.9 \%$ \\
\hline 2 & SB4VAR32 & $\mathrm{U}_{3} \mathrm{O}_{8}$ & 3.0807 & 3.1127 & 3.1333 & -0.0526 & -0.0206 & $-1.7 \%$ & $-0.7 \%$ \\
\hline 2 & SB4VAR32 & $\mathrm{ZnO}$ & 0.0062 & 0.0062 & 0.0281 & -0.0219 & -0.0219 & $-77.9 \%$ & $-77.9 \%$ \\
\hline 2 & SB4VAR32 & $\mathrm{ZrO}_{2}$ & 0.0240 & 0.0240 & 0.0286 & -0.0046 & -0.0046 & $-16.2 \%$ & $-16.2 \%$ \\
\hline 2 & SB4VAR32 & Sum & 100.0960 & 99.2324 & 100.0002 & 0.0958 & -0.7677 & $0.1 \%$ & $-0.8 \%$ \\
\hline 2 & SB4VAR33 & $\mathrm{Al}_{2} \mathrm{O}_{3}$ & 9.7829 & 10.1544 & 10.0058 & -0.2229 & 0.1486 & $-2.2 \%$ & $1.5 \%$ \\
\hline 2 & SB4VAR33 & $\mathrm{B}_{2} \mathrm{O}_{3}$ & 8.4844 & 8.3827 & 8.4000 & 0.0844 & -0.0173 & $1.0 \%$ & $-0.2 \%$ \\
\hline 2 & SB4VAR33 & $\mathrm{BaO}$ & 0.0248 & 0.0268 & 0.0298 & -0.0050 & -0.0030 & $-16.6 \%$ & $-10.2 \%$ \\
\hline 2 & SB4VAR33 & $\mathrm{CaO}$ & 1.0400 & 1.0823 & 1.1011 & -0.0611 & -0.0188 & $-5.6 \%$ & $-1.7 \%$ \\
\hline
\end{tabular}


Table A4. Average Measured and Bias-Corrected Chemical Compositions Versus Targeted Compositions by Oxide by SB4-Decant Variability Study Glass

\begin{tabular}{|c|c|c|c|c|c|c|c|c|c|}
\hline & & & & "Measured & & & & & \% Diff \\
\hline & & & Measured & $\begin{array}{c}\text { Bias- } \\
\text { Corrected (BC) }\end{array}$ & Targeted & $\begin{array}{c}\text { Diff } \\
\text { of }\end{array}$ & $\begin{array}{c}\text { Diff } \\
\text { of }\end{array}$ & $\begin{array}{c}\text { \% Diff } \\
\text { of }\end{array}$ & $\begin{array}{c}\text { of } \\
\text { Meas. }\end{array}$ \\
\hline Set & Glass ID & Oxide & $(\mathrm{wt} \%)$ & (wt\%) & $(\mathrm{wt} \%)$ & Measured & Meas BC & Measured & $\mathrm{BC}$ \\
\hline 2 & SB4VAR33 & $\mathrm{Ce}_{2} \mathrm{O}_{3}$ & 0.0225 & 0.0225 & 0.0262 & -0.0037 & -0.0037 & $-13.9 \%$ & $-13.9 \%$ \\
\hline 2 & SB4VAR33 & $\mathrm{Cr}_{2} \mathrm{O}_{3}$ & 0.0552 & 0.0555 & 0.0626 & -0.0074 & -0.0071 & $-11.9 \%$ & $-11.4 \%$ \\
\hline 2 & SB4VAR33 & $\mathrm{CuO}$ & 0.0288 & 0.0308 & 0.0231 & 0.0057 & 0.0077 & $24.6 \%$ & $33.5 \%$ \\
\hline 2 & SB4VAR33 & $\mathrm{Fe}_{2} \mathrm{O}_{3}$ & 10.7299 & 10.5166 & 11.4103 & -0.6804 & -0.8937 & $-6.0 \%$ & $-7.8 \%$ \\
\hline 2 & SB4VAR33 & $\mathrm{K}_{2} \mathrm{O}$ & 0.1458 & 0.1619 & 0.1412 & 0.0046 & 0.0207 & $3.2 \%$ & $14.7 \%$ \\
\hline 2 & SB4VAR33 & $\mathrm{La}_{2} \mathrm{O}_{3}$ & 0.0586 & 0.0586 & 0.0196 & 0.0390 & 0.0390 & $199.2 \%$ & $199.2 \%$ \\
\hline 2 & SB4VAR33 & $\mathrm{Li}_{2} \mathrm{O}$ & 4.7848 & 4.9548 & 4.8000 & -0.0152 & 0.1548 & $-0.3 \%$ & $3.2 \%$ \\
\hline 2 & SB4VAR33 & $\mathrm{MgO}$ & 1.0377 & 1.0769 & 1.0828 & -0.0451 & -0.0059 & $-4.2 \%$ & $-0.5 \%$ \\
\hline 2 & SB4VAR33 & $\mathrm{MnO}$ & 2.2757 & 2.1969 & 2.2916 & -0.0159 & -0.0947 & $-0.7 \%$ & $-4.1 \%$ \\
\hline 2 & SB4VAR33 & $\mathrm{Na}_{2} \mathrm{O}$ & 12.4926 & 12.7415 & 12.9157 & -0.4231 & -0.1742 & $-3.3 \%$ & $-1.3 \%$ \\
\hline 2 & SB4VAR33 & $\mathrm{NiO}$ & 0.5574 & 0.5966 & 0.6349 & -0.0775 & -0.0383 & $-12.2 \%$ & $-6.0 \%$ \\
\hline 2 & SB4VAR33 & $\mathrm{PbO}$ & 0.0272 & 0.0272 & 0.0251 & 0.0021 & 0.0021 & $8.4 \%$ & $8.4 \%$ \\
\hline 2 & SB4VAR33 & $\mathrm{SiO}_{2}$ & 42.2512 & 41.7624 & 43.0734 & -0.8222 & -1.3110 & $-1.9 \%$ & $-3.0 \%$ \\
\hline 2 & SB4VAR33 & $\mathrm{SO}_{4}$ & 0.3715 & 0.3715 & 0.3969 & -0.0254 & -0.0254 & $-6.4 \%$ & $-6.4 \%$ \\
\hline 2 & SB4VAR33 & $\mathrm{TiO}_{2}$ & 0.0217 & 0.0228 & 0.0154 & 0.0063 & 0.0074 & $40.8 \%$ & $47.7 \%$ \\
\hline 2 & SB4VAR33 & $\mathrm{U}_{3} \mathrm{O}_{8}$ & 3.3018 & 3.4637 & 3.4814 & -0.1796 & -0.0177 & $-5.2 \%$ & $-0.5 \%$ \\
\hline 2 & SB4VAR33 & $\mathrm{ZnO}$ & 0.0062 & 0.0062 & 0.0312 & -0.0250 & -0.0250 & $-80.1 \%$ & $-80.1 \%$ \\
\hline 2 & SB4VAR33 & $\mathrm{ZrO}_{2}$ & 0.0270 & 0.0270 & 0.0318 & -0.0048 & -0.0048 & $-15.0 \%$ & $-15.0 \%$ \\
\hline 2 & SB4VAR33 & Sum & 97.5277 & 97.7396 & 99.9999 & -2.4722 & -2.2603 & $-2.5 \%$ & $-2.3 \%$ \\
\hline 2 & U std & $\mathrm{Al}_{2} \mathrm{O}_{3}$ & 3.9365 & 4.0138 & 4.1000 & -0.1635 & -0.0862 & $-4.0 \%$ & $-2.1 \%$ \\
\hline 2 & U std & $\mathrm{B}_{2} \mathrm{O}_{3}$ & 9.0130 & 8.9811 & 9.2090 & -0.1960 & -0.2279 & $-2.1 \%$ & $-2.5 \%$ \\
\hline 2 & U std & $\mathrm{BaO}$ & 0.0056 & 0.0059 & 0.0000 & 0.0056 & 0.0059 & & \\
\hline 2 & U std & $\mathrm{CaO}$ & 1.2649 & 1.2983 & 1.3010 & -0.0361 & -0.0027 & $-2.8 \%$ & $-0.2 \%$ \\
\hline 2 & U std & $\mathrm{Ce}_{2} \mathrm{O}_{3}$ & 0.0184 & 0.0184 & 0.0000 & 0.0184 & 0.0184 & & \\
\hline 2 & U std & $\mathrm{Cr}_{2} \mathrm{O}_{3}$ & 0.2388 & 0.2374 & 0.0000 & 0.2388 & 0.2374 & & \\
\hline 2 & U std & $\mathrm{CuO}$ & 0.0063 & 0.0066 & 0.0000 & 0.0063 & 0.0066 & & \\
\hline 2 & U std & $\mathrm{Fe}_{2} \mathrm{O}_{3}$ & 13.4511 & 13.2525 & 13.1960 & 0.2551 & 0.0565 & $1.9 \%$ & $0.4 \%$ \\
\hline 2 & U std & $\mathrm{K}_{2} \mathrm{O}$ & 2.6973 & 2.9511 & 2.9990 & -0.3017 & -0.0479 & $-10.1 \%$ & $-1.6 \%$ \\
\hline 2 & U std & $\mathrm{La}_{2} \mathrm{O}_{3}$ & 0.0586 & 0.0586 & 0.0000 & 0.0586 & 0.0586 & & \\
\hline 2 & U std & $\mathrm{Li}_{2} \mathrm{O}$ & 2.9387 & 3.0688 & 3.0570 & -0.1183 & 0.0118 & $-3.9 \%$ & $0.4 \%$ \\
\hline 2 & U std & $\mathrm{MgO}$ & 1.1868 & 1.2208 & 1.2100 & -0.0232 & 0.0108 & $-1.9 \%$ & $0.9 \%$ \\
\hline 2 & U std & $\mathrm{MnO}$ & 2.8858 & 2.8149 & 2.8920 & -0.0062 & -0.0771 & $-0.2 \%$ & $-2.7 \%$ \\
\hline 2 & U std & $\mathrm{Na}_{2} \mathrm{O}$ & 11.6624 & 11.6666 & 11.7950 & -0.1326 & -0.1284 & $-1.1 \%$ & $-1.1 \%$ \\
\hline 2 & U std & $\mathrm{NiO}$ & 0.9483 & 1.0044 & 1.1200 & -0.1717 & -0.1156 & $-15.3 \%$ & $-10.3 \%$ \\
\hline 2 & U std & $\mathrm{PbO}$ & 0.0108 & 0.0108 & 0.0000 & 0.0108 & 0.0108 & & \\
\hline 2 & U std & $\mathrm{SiO}_{2}$ & 46.0841 & 45.3438 & 45.3530 & 0.7311 & -0.0092 & $1.6 \%$ & $0.0 \%$ \\
\hline 2 & U std & $\mathrm{SO}_{4}$ & 0.1498 & 0.1498 & 0.0000 & 0.1498 & 0.1498 & & \\
\hline 2 & U std & $\mathrm{TiO}_{2}$ & 0.9517 & 0.9847 & 1.0490 & -0.0973 & -0.0643 & $-9.3 \%$ & $-6.1 \%$ \\
\hline 2 & U std & $\mathrm{U}_{3} \mathrm{O}_{8}$ & 2.3378 & 2.4060 & 2.4060 & -0.0682 & 0.0000 & $-2.8 \%$ & $0.0 \%$ \\
\hline 2 & U std & $\mathrm{ZnO}$ & 0.0062 & 0.0062 & 0.0000 & 0.0062 & 0.0062 & & \\
\hline 2 & U std & $\mathrm{ZrO}_{2}$ & 0.0068 & 0.0068 & 0.0000 & 0.0068 & 0.0068 & & \\
\hline 2 & U std & Sum & 99.8598 & 99.5075 & 99.6870 & 0.1728 & -0.1795 & $0.2 \%$ & $-0.2 \%$ \\
\hline
\end{tabular}




\section{Exhibit A1. Oxide Measurements in Analytical Sequence for Samples Prepared Using the LM Method}
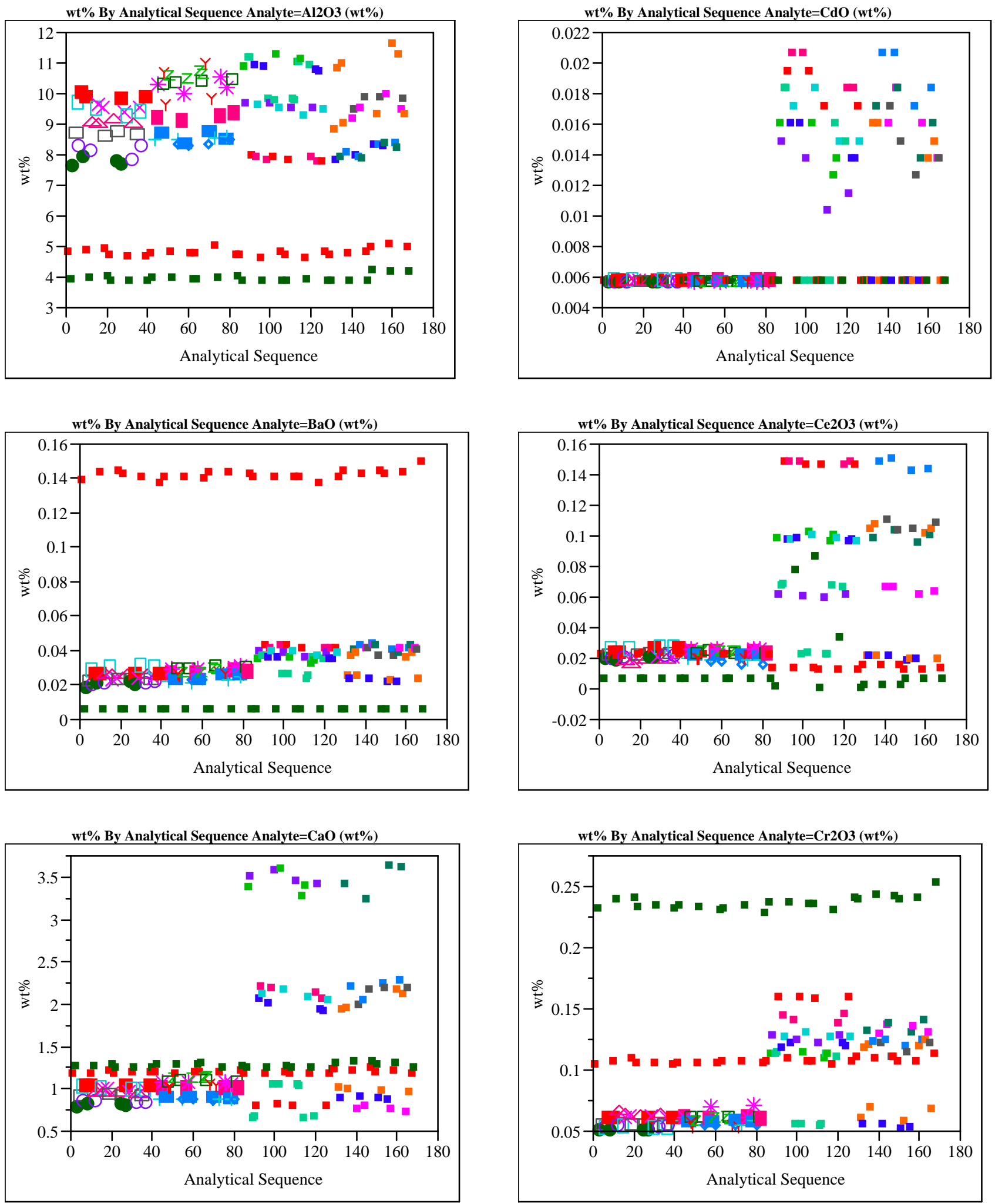


\section{Exhibit A1. Oxide Measurements in Analytical Sequence for Samples Prepared Using the LM Method}
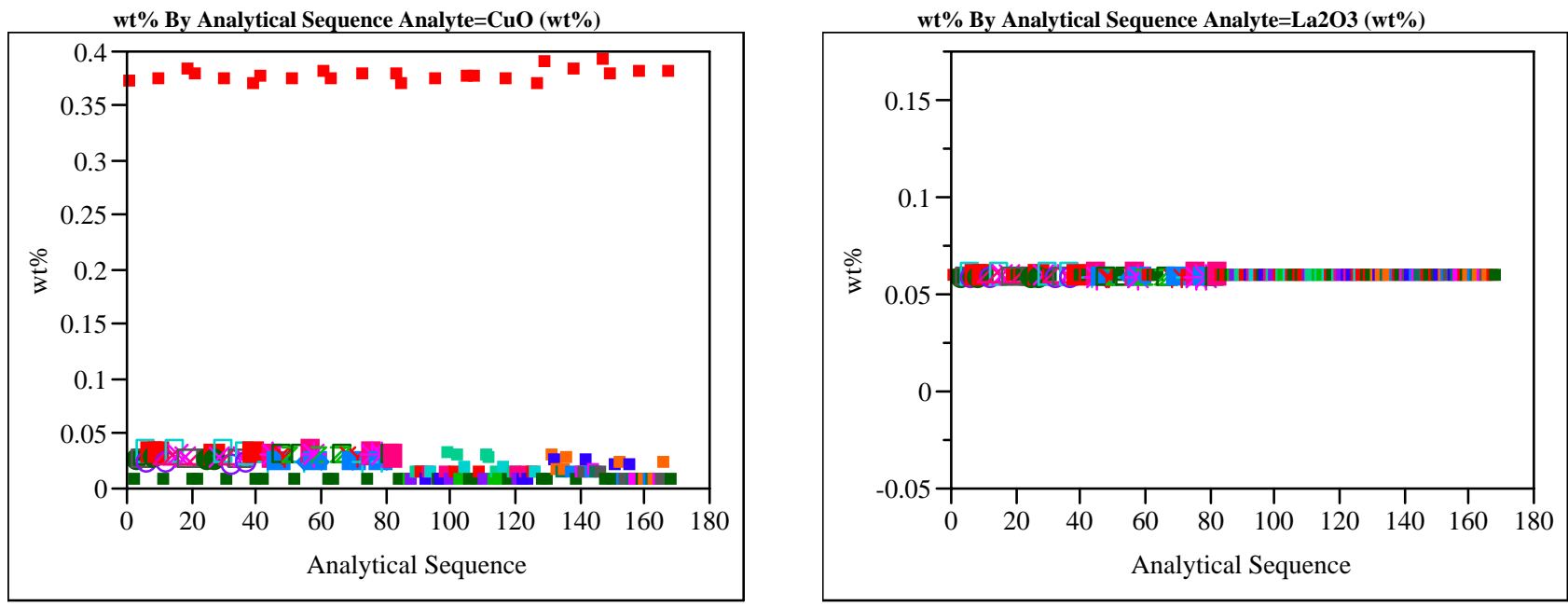

wt \% By Analytical Sequence Analyte=Fe2O3 (wt\%)
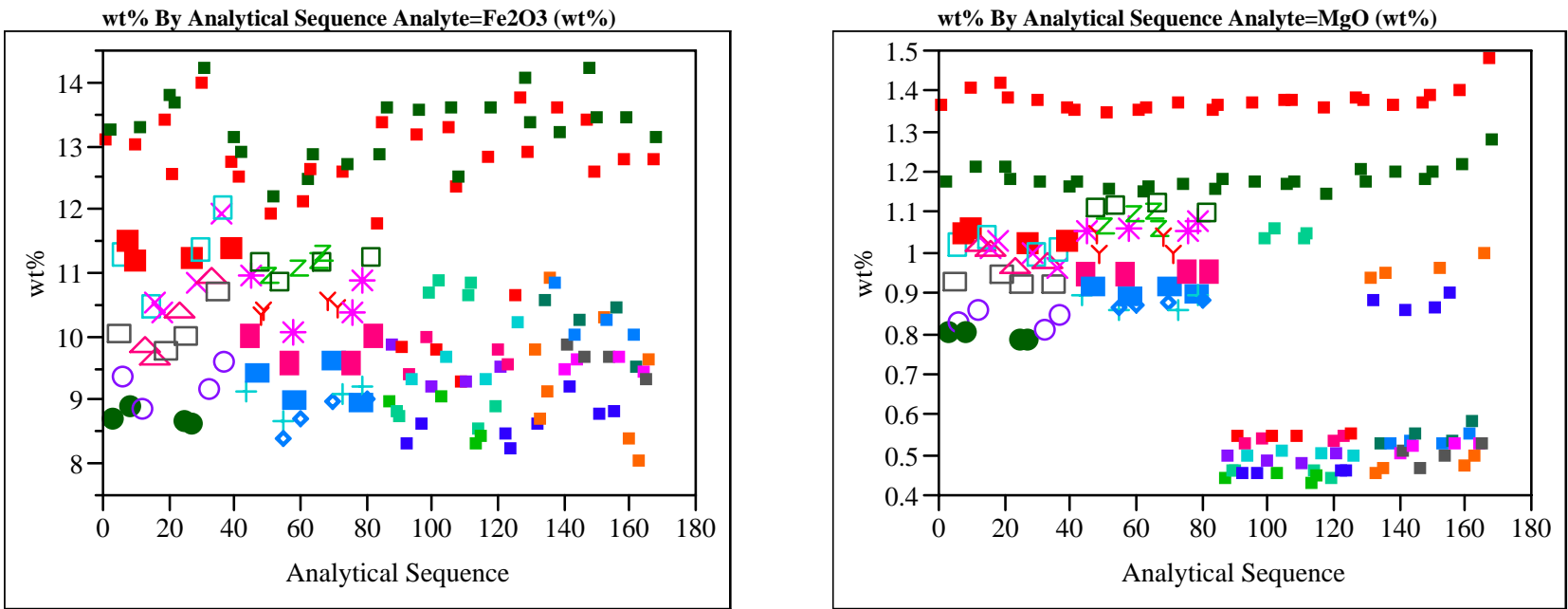

wt \% By Analytical Sequence Analyte=K2O (wt\%)
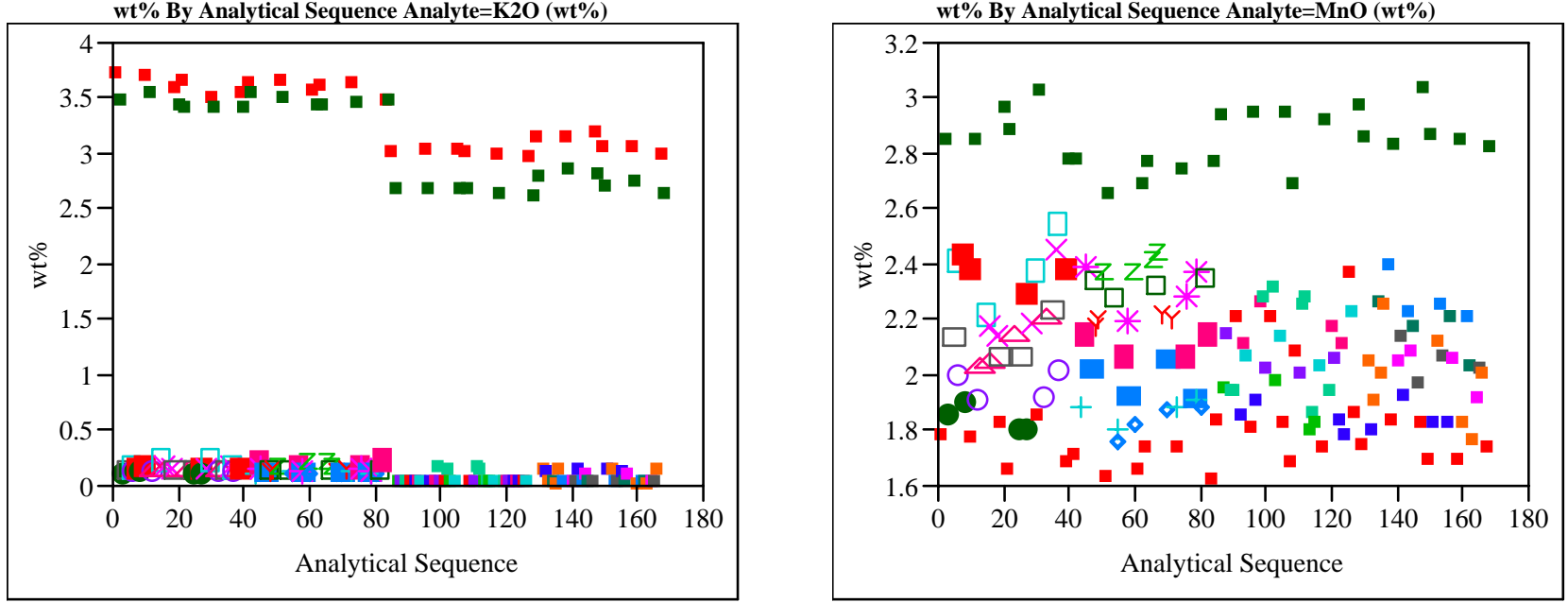


\section{Exhibit A1. Oxide Measurements in Analytical Sequence for Samples Prepared Using the LM Method}
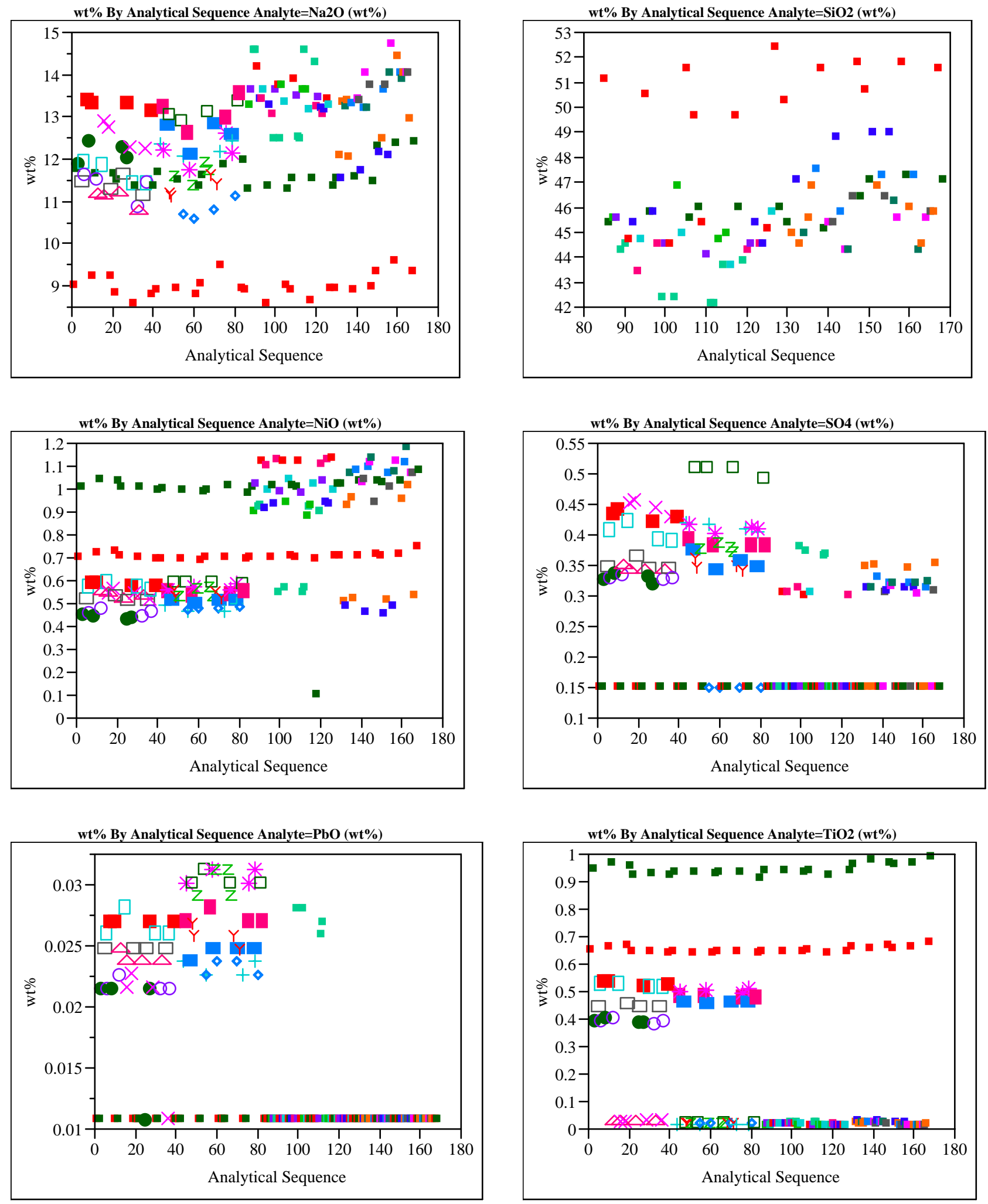
Exhibit A1. Oxide Measurements in Analytical Sequence for Samples Prepared Using the LM Method
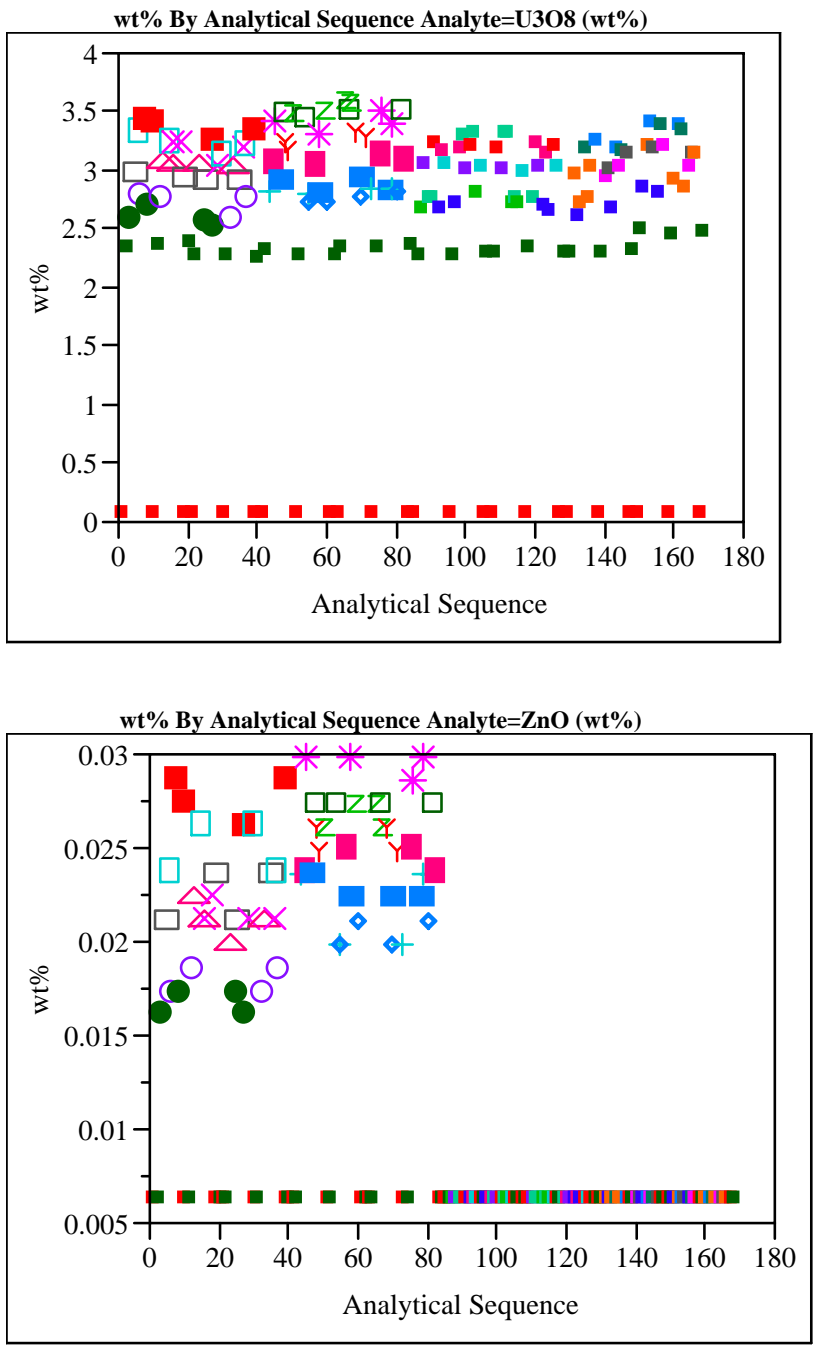

wt \% By Analytical Sequence Analyte $=\mathrm{ZrO} 2$ (wt \%)

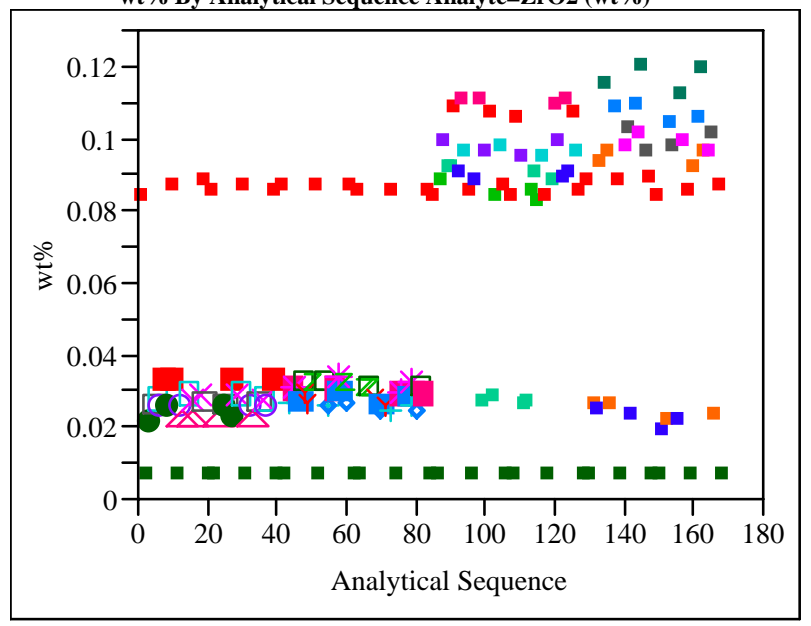




\section{Exhibit A2. Oxide Measurements in Analytical Sequence for Samples Prepared}

\section{Using the PF Method}

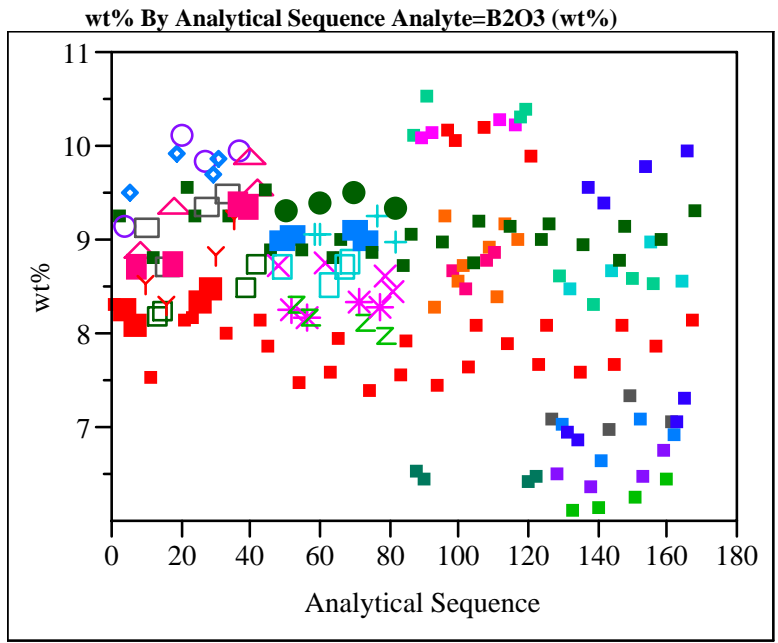

wt \% By Analytical Sequence Analyte=Li2O (wt\%)

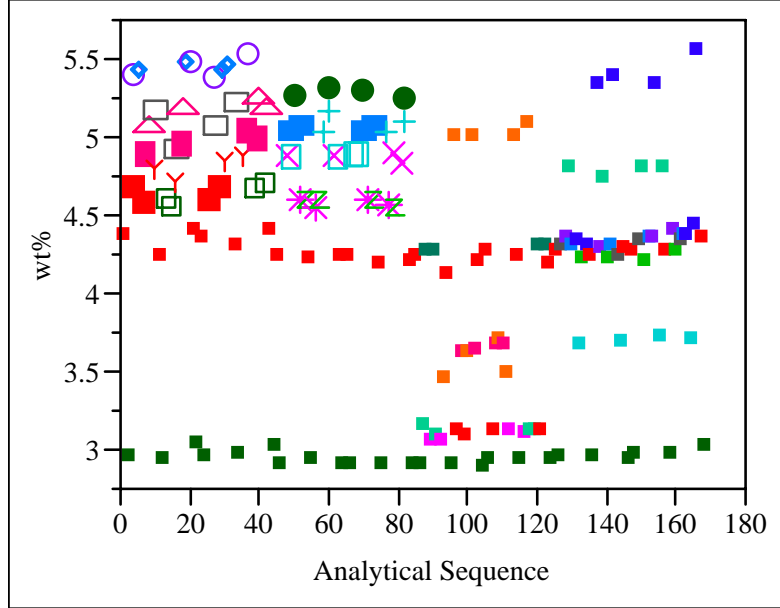

wt \% By Analytical Sequence Analyte=SiO2 (wt\%)

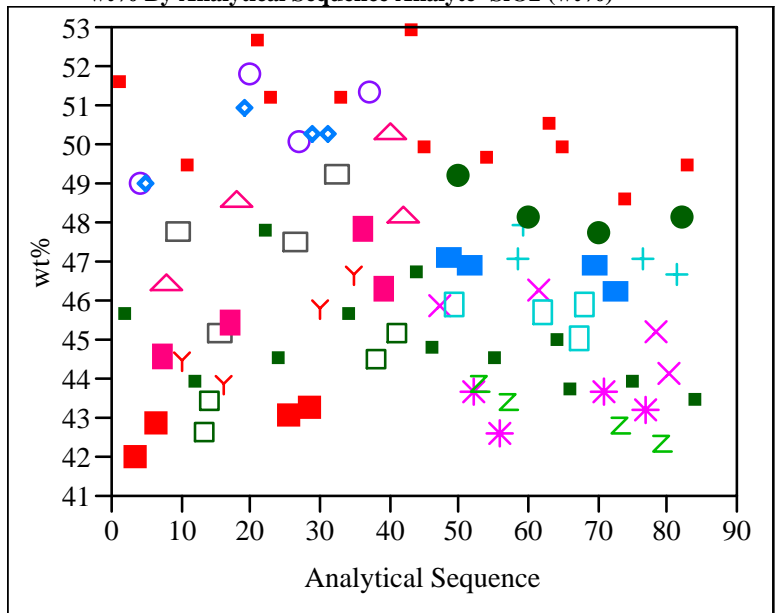




\section{Exhibit A3. PSAL Measurements by Analytical Block for Samples of the Standard Glasses Prepared Using the LM Method}

Oneway Analysis of $w$ t\% By Set/Block/Sub-Block Glass ID=Batch 1, 3 (wt\%)

Reference Value $=4.877$ wt $\%$

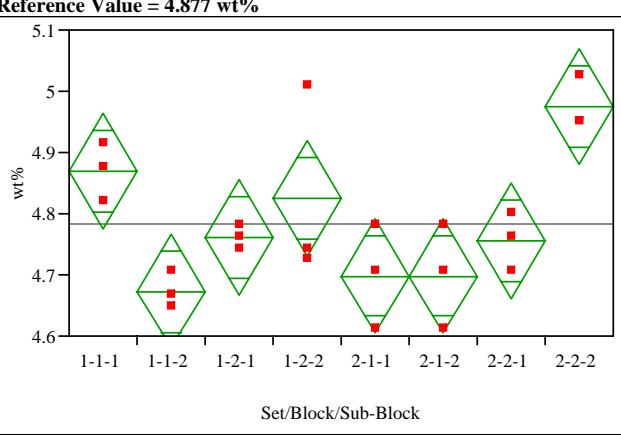

Oneway Anova

Summary of Fit

$\begin{array}{lr}\text { Rsquare } & 0.700547 \\ \text { Adj Rsquare } & 0.569536 \\ \text { Root Mean Square Error } & 0.076849 \\ \text { Mean of Response } & 4.78201 \\ \text { Observations (or Sum Wgts) } & 24\end{array}$

Analysis of Variance
Source

Source DF Sum of Squares Mean Square F Ratio Prob $>$ F

$\begin{array}{llll}\text { Error } & 16 & 0.09449156 & 0.005906 \\ \text { C. Total } & 23 & 0.31554708 & \end{array}$

Means for Oneway Anova

Level Number Mean Std Error Lower 95\% Upper 95\%

$\begin{array}{lllllll}1-1-1 & & 3 & 4.86861 & 0.04437 & 4.7746 & 4.9627 \\ 1-1-2 & & 3 & 4.67336 & 0.04437 & 4593 & 4.7674\end{array}$

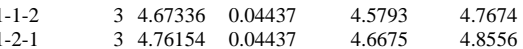

$\begin{array}{lllllll}1-2-2 & & 3 & 4.82452 & 0.04437 & 4.7305 & 4.9186 \\ 2-1-1 & & 3 & 4.69856 & 0.04437 & 4.6045 & 4.7926\end{array}$

$\begin{array}{llllll}2-1-2 & 3 & 4.69856 & 0.04437 & 4.6045 & 4.7926\end{array}$

$\begin{array}{llllll}2-2-1 & 3 & 4.75524 & 0.04437 & 4.6612 & 4.8493 \\ 2-2-2 & 3 & 4.97568 & 0.04437 & 4.8816 & 5.0697\end{array}$

Std Error uses a pooled estimate of error variance
Oneway Analysis of wt\% By Set/Block/Sub-Block Glass ID=Batch 1, Analyte $=\mathbf{B a O}(\mathrm{wt} \%)$

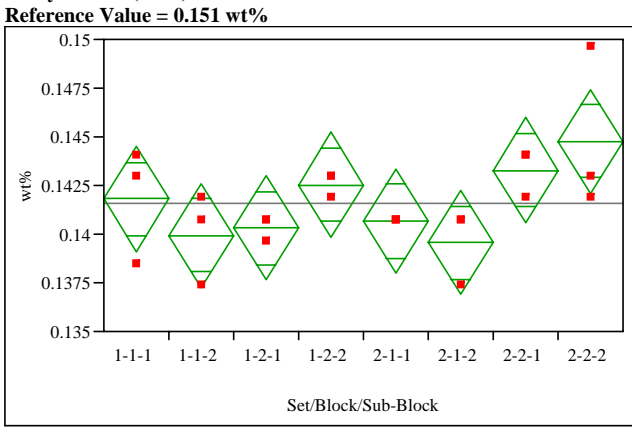

Oneway Anova
Summary of Fit

$\begin{array}{lr} & \\ \text { Rsquare } & 0.477273 \\ \text { Adj Rsquare } & 0.24858 \\ \text { Root Mean Square Error } & 0.002186 \\ \text { Mean of Response } & 0.141609 \\ \text { Observations (or Sum Wgts) } & 24\end{array}$

Analysis of Varian

Source $\quad$ DF Sum of Squares Mean Square F Ratio Prob $>$ F

$\begin{array}{lrrrrr}\text { Set/Block/Sub-Block } & 7 & 0.00006981 & 9.9726 \mathrm{e}-6 & 2.0870 & 0.1057\end{array}$

E. Total

$\begin{array}{ll}16 & 0.00007646 \\ 23 & 0.00014626\end{array}$

Means for Oneway Anov

Level Number Mean Std Error Lower 95\% Upper 95\%

$\begin{array}{lllllll}1-1-1 & 3 & 0.141796 & 0.00126 & 0.13912 & 0.1444 \\ 1-12 & 3 & 0.13935 & 0.00126 & 0.1326 & 0.1268\end{array}$

$\begin{array}{lllllll}1-1-2 & & 3 & 0.1413955 & 0.00126 & 0.139126 & 0.1426 \\ 1-2-1 & & 3 & 0.140307 & 0.00126 & 0.13763 & 0.14298\end{array}$

$\begin{array}{lllllll}1-2-2 & & 3 & 0.142540 & 0.00126 & 0.13986 & 0.14522 \\ 1 & & 3 & 0.14679 & 0.00126 & 0.1380 & 0.1435\end{array}$

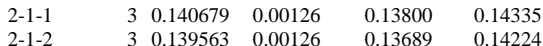

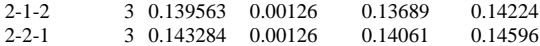

$\begin{array}{llllll}2-2-2 & 3 & 0.144773 & 0.00126 & 0.14210 & 0.14745\end{array}$

Std Error uses a pooled estimate of error variance
Oneway Analysis of wt\% By Set/Block/Sub-Block Glass ID=Batch 1, Analyte $=\mathrm{CaO}(\mathrm{wt} \%)$

Reference Value $=1.220 \mathrm{wt} \%$

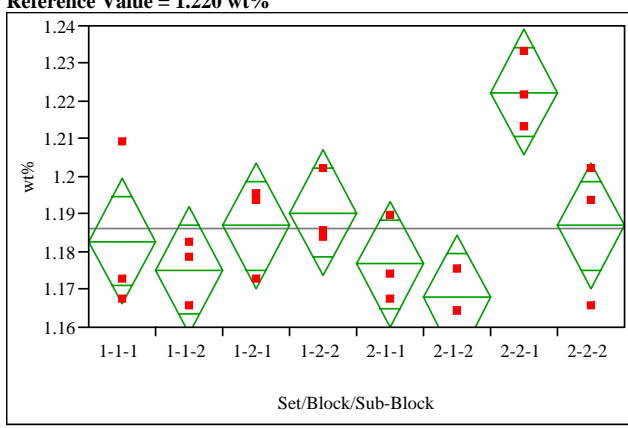

Oneway Anova

$\begin{array}{ll}\text { Rsquare } & 0.655368 \\ \text { Adj Rsquare } & 0.504591\end{array}$

Root Mean Square Error $\quad 0.013638$

$\begin{array}{lr}\text { Mean of Response } & 1.186172 \\ \text { Observations (or Sum Wgts) } & 24\end{array}$

Analysis of Variance

Source DF Sum of Squares Mean Square F Ratio Prob > F

$\begin{array}{llllll}\text { Set/Block/Sub-Block } & 7 & 0.00565891 & 0.000808 & 4.3466 & 0.0071\end{array}$

$\begin{array}{llll}\text { Crror } & 16 & 0.00297580 & 0.000186 \\ \text { C. Total } & 23 & 0.00863470 & \end{array}$

Means for Oneway Anova

Level Number Mean Std Error Lower 95\% Upper 95\%

$\begin{array}{llllll}1-1-1 & 3 & 1.18279 & 0.00787 & 1.1661 & 1.1995\end{array}$

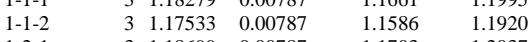

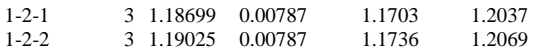

$\begin{array}{lllllll}2-1-1 & & 3 & 1.17673 & 0.00787 & 1.1600 & 1.1934 \\ 2-1-2 & & 3 & 1.16787 & 0.0787 & 1.1512 & 1.1846\end{array}$

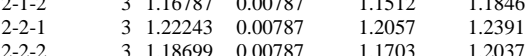

Std Error uses a pooled estimate of error variance 


\section{Exhibit A3. PSAL Measurements by Analytical Block for Samples of the}

\section{Standard Glasses Prepared Using the LM Method}

Oneway Analysis of wt\% By Set/Block/Sub-Block Glass ID=Batch 1, nalyte $=\mathrm{CdO}(\mathrm{wt} \%)$

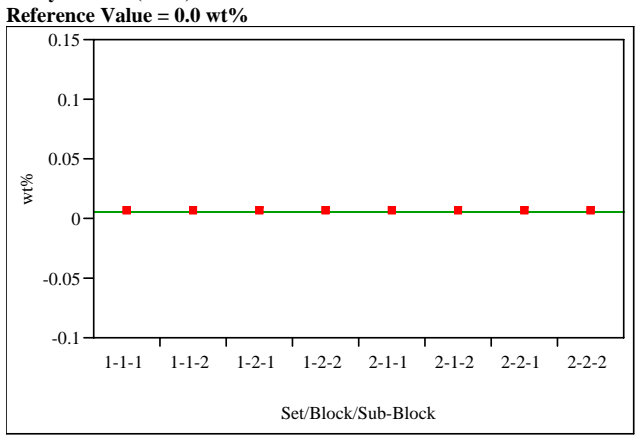

Oneway Anova

Rsquare

Adj Rsquare

Root Mean Square Error

Mean of Response
Observations (or Sum Wgts)

Analysis of Variance

Source DF Sum of Squares Mean Square F Ratio Prob > F

Set/Block/Sub-Block

$\begin{array}{ll}\text { Error } & 16 \\ \text { C. Total } & 23\end{array}$

\begin{tabular}{|c|c|c|c|c|c|}
\hline evel & $\begin{array}{l}\text { sor One } \\
\text { Number }\end{array}$ & $\begin{array}{l}\text { Anovi } \\
\text { Mean }\end{array}$ & & Lower 95\% & Upper 95\% \\
\hline$-1-1$ & & 0.005712 & 0 & 0.00571 & 0.00571 \\
\hline$-1-2$ & & 0.005712 & 0 & 0.00571 & 0.00571 \\
\hline-1 & & 0.005712 & 0 & 0.00571 & 0.00571 \\
\hline-2 & & 0.005712 & 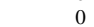 & 0.00571 & 0.005 \\
\hline & & 0.005712 & 0 & 00571 & 0.00571 \\
\hline & 3 & 0.005712 & 0 & $\begin{array}{l}0.00571 \\
0\end{array}$ & 0.00571 \\
\hline & 3 & 0.005712 & 0 & 0.00571 & 0.00571 \\
\hline $2-2-2$ & 3 & 0.005712 & 0 & 0.00571 & 0.005 \\
\hline
\end{tabular}

Std Error uses a pooled estimate of error variance
Oneway Analysis of wt\% By Set/Block/Sub-Block Glass ID=Batch 1,

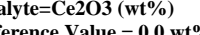

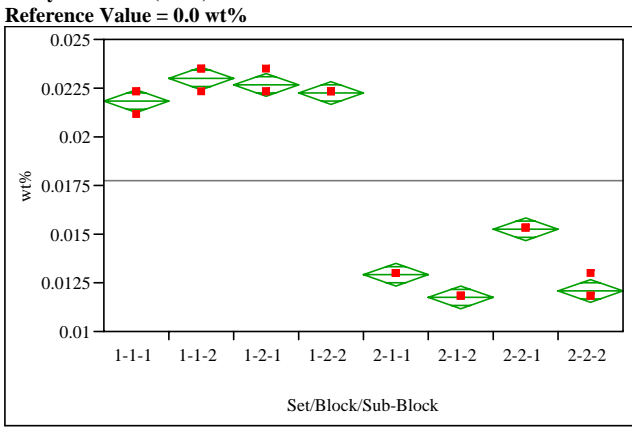

Oneway Anova
Summary of Fit

$\begin{array}{lc}\text { Rsquare } & 0.993537 \\ \text { Adj Rsquare } & 0.99071 \\ \text { Reot } & 0.00478\end{array}$

Mean of Response 0.017716

Observations (or Sum Wgts) $\quad 24$

Analysis of Variance

Source $\quad$ DF Sum of Squares Mean Square F Ratio Prob $>$ F

Set/Block/S
Error

Crror

$\begin{array}{ll}16 & 0.00000366 \\ 23 & 0.00056610\end{array}$

Means for Oneway Anov

Level Number Mean Std Error Lower 95\% Upper 95\%

$\begin{array}{lllllll}1-1-1 & & 3 & 0.021864 & 0.00028 & 0.02128 & 0.02245 \\ 1-1-2 & 3 & 0.023036 & 0.00028 & 0.02245 & 0.02362\end{array}$

$\begin{array}{lllllll}1-1-1 & 3 & 0.021864 & 0.00028 & 0.02128 & 0.02245 \\ 1-2-1 & & 3 & 0.02336 & 0.00028 & 0.02245 & 0.02362\end{array}$

$\begin{array}{llllll}1-2-2 & 3 & 0.022255 & 0.00028 & 0.02167 & 0.02284\end{array}$

$\begin{array}{lllllll}2-1-1 & 3 & 0.012884 & 0.00028 & 0.01230 & 0.01347\end{array}$

$\begin{array}{llllll}2-1-2 & 3 & 0.011713 & 0.00028 & 0.01113 & 0.01230\end{array}$

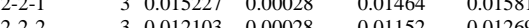

Std Error uses a pooled estimate of error variance
Oneway Analysis of wt\% By Set/Block/Sub-Block Glass ID=Batch 1, nalyte $=\mathrm{Cr} 2 \mathrm{O} 3(\mathrm{wt} \%)$

Reference Value $=0.107 \mathrm{wt} \%$

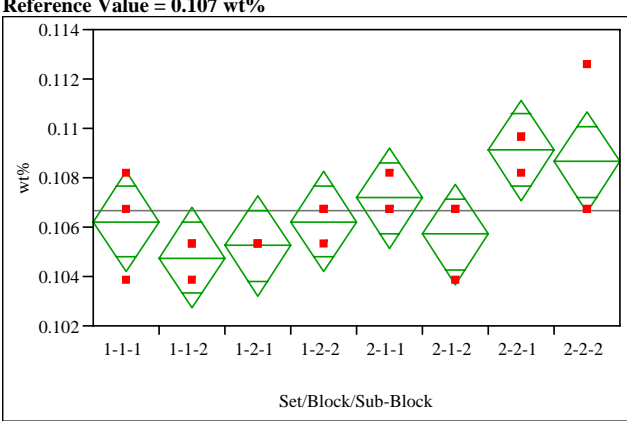

Oneway Anova

ummary of Fit

$\begin{array}{ll}\text { Rsquare } & 0.540315 \\ \text { Adj Rsquare } & 0.339203 \\ \text { Root Mean Square Error } & 0.001661\end{array}$

Mean of Response $\quad 0.106636$

Observations (or Sum Wgts)

Analysis of Varianc

Source DF Sum of Squares Mean Square F Ratio Prob $>$ F

$\begin{array}{llllll}\text { Set/Block/Sub-Block } & 7 & 0.00005189 & 7.4134 \mathrm{e}-6 & 2.6866 & 0.048\end{array}$

Error $16 \quad 0.0000405$

Means for Oneway Anova

Level Number Mean Std Error Lower 95\% Upper 95\%

$\begin{array}{lllllll}1-1-1 & & 3 & 0.106210 & 0.00096 & 0.10418 & 0.10824\end{array}$

$\begin{array}{llllll}1-1 & 3 & 0.104748 & 0.00096 & 0.10271 & 0.10678 \\ 1-2-1 & 3 & 0.105235 & 0.00096 & 0.10320 & 0.10727\end{array}$

$\begin{array}{llllll}1-2-2 & 3 & 0.105235 & 0.00096 & 0.10271 & 0.10678 \\ 1-2-2 & 3 & 0.106210 & 0.00096 & 0.10418 & 0.10824\end{array}$

$\begin{array}{lllllll}2-1-1 & 3 & 0.107184 & 0.00096 & 0.10515 & 0.10922\end{array}$

$\begin{array}{lllll}-3 & 0.105722 & 0.00096 & 0.10369 & 0.10776\end{array}$

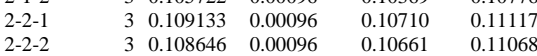

Std Error uses a pooled estimate of error variance 


\section{Exhibit A3. PSAL Measurements by Analytical Block for Samples of the}

\section{Standard Glasses Prepared Using the LM Method}

Oneway Analysis of wt $\%$ By Set/Block/Sub-Block Glass ID=Batch 1 ,

Reference Value $=0.399 \mathrm{wt} \%$

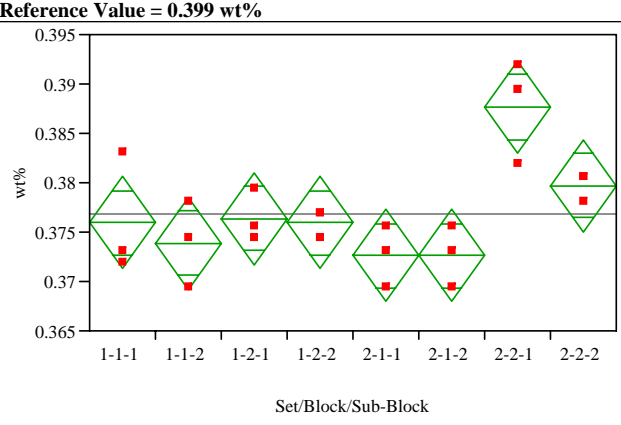

Oneway Anova
Summary of Fit

$\begin{array}{ll}\text { Rsquare } & 0.689797 \\ \text { Adj Rsquare } & 0.554084 \\ \text { Root Mean Square Error } & 0.000799 \\ \text { Mean of Response } & 0.376844\end{array}$

Observations (or Sum Wgts)

Analysis of Variance

$\begin{array}{lrrrrr}\text { Source } & \text { DF } & \text { Sum of Squares } & \text { Mean Square } & \text { F Ratio } & \text { Prob }>\text { F } \\ \text { Set/Block/Sub-Block } & 7 & 0.00051339 & 0.000073 & 5.0827 & 0.0034\end{array}$

\begin{tabular}{lrr} 
& & \\
Error & 16 & 0.00051339 \\
\hline
\end{tabular}

Error

$16 \quad 0.00023087$ 0.000014

$\begin{array}{lrrrrr}\text { Means for Oneway Anova } & & & \\ \text { Level } & \text { Number } & \text { Mean } & \text { Std Error } & \text { Lower 95\% } & \text { Upper 95\% } \\ 1-1-1 & 3 & 0.375957 & 0.00219 & 0.37131 & 0.38061 \\ 1-1-2 & 3 & 0.373871 & 0.00219 & 0.36922 & 0.37852 \\ 1-2-1 & 3 & 0.376375 & 0.00219 & 0.37173 & 0.38102 \\ 1-2-2 & 3 & 0.375957 & 0.00219 & 0.37131 & 0.38061 \\ 2-1-1 & 3 & 0.372619 & 0.00219 & 0.36797 & 0.37727 \\ 2-1-2 & 3 & 0.372619 & 0.00219 & 0.36797 & 0.37772 \\ 2-2-1 & 3 & 0.387641 & 0.00219 & 0.38299 & 0.39229 \\ 2-2-2 & 3 & 0.379713 & 0.00219 & 0.37506 & 0.38436\end{array}$

Std Error uses a pooled estimate of error variance
Oneway Analysis of wt\% By Set/Block/Sub-Block Glass ID=Batch 1,

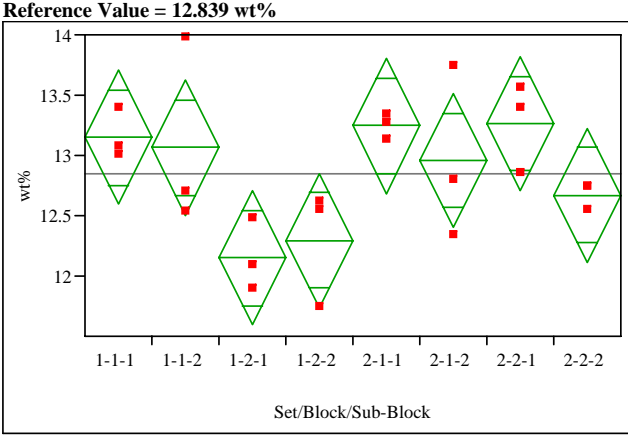

Oneway Anova

Rsquare

0.341571

Rool Mean Square Error $\quad 0.454572$

Observations (or Sum Wgs) 12.84883

Analysis of Variance Source Sum of Squares Mean Square F Ratio Prob >

$\begin{array}{lllllll}\text { Set/Block/Sub-Block } & 7 & 3.9119474 & 0.558850 & 2.7045 & 0.0471\end{array}$

$\begin{array}{llll}\text { Error } & 16 & 3.3061699 & 0.206636 \\ \text { C. Total } & 23 & 7.2181173 & \end{array}$

Means for Oneway Anova

Level Number Mean Std Error Lower 95\% Upper 95\%

$\begin{array}{lrrrrr}1-1-1 & 3 & 13.1485 & 0.26245 & 12.592 & 13.705\end{array}$

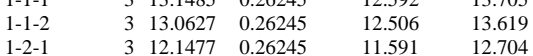

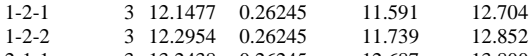

$\begin{array}{llllll}2-1-1 & 3 & 13.2438 & 0.26245 & 11.687 & 13.800 \\ 2-1-2 & 3 & 12.9578 & 0.26245 & 12.401 & 13.514\end{array}$

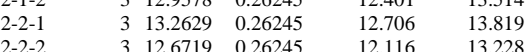

Std Error uses a pooled estimate of error variance
Oneway Analysis of wt\% By Set/Block/Sub-Block Glass ID=Batch 1, Analyte $=\mathrm{K} 2 \mathrm{O}(\mathrm{wt} \%)$

Reference Value $=3.327 \mathrm{wt} \%$

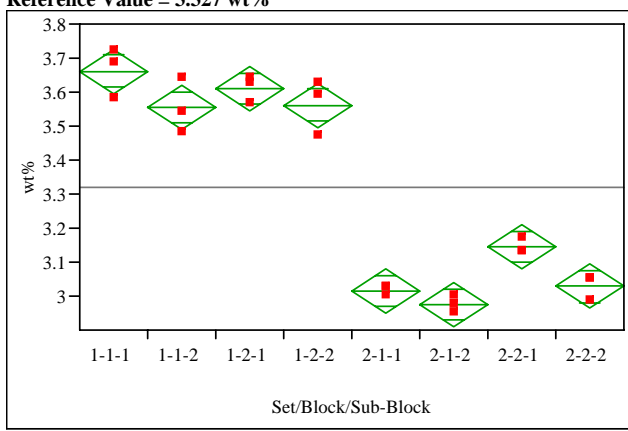

Oneway Anova

mary of Fit

Adj Rsquare $\quad 0.977123$

Root Mean Square Error $\quad 0.05308$ $\begin{array}{lr}\text { Mean of Response } & 3.318673 \\ \text { Observations (or Sum Wgts) } & 24\end{array}$

Analysis of Variance

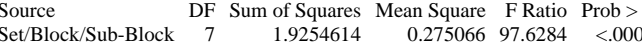
$\begin{array}{llll}\text { Error } & 16 & 0.0450796 & 0.002817 \\ \text { C. Total } & 23 & 1.9705411 & \end{array}$

Means for Oneway Anova

Level Number Mean Std Error Lower 95\% Upper 95\%

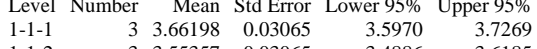

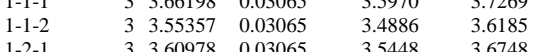

$\begin{array}{lllllll}1-2-1 & & 3 & 3.60978 & 0.03065 & 3.5448 & 3.6748 \\ 1-2-2 & & 3 & 3.56160 & 0.03065 & 3.4966 & 3.6266\end{array}$

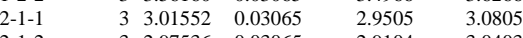

$\begin{array}{lllllll}2-1-2 & 3 & 2.97536 & 0.03065 & 2.9104 & 3.0403 \\ 2-2-1 & & 3 & 3.14401 & 0.03065 & 3.0790 & 3.2090\end{array}$

$\begin{array}{llllll}2-2-2 & 3 & 3.02756 & 0.03065 & 2.9626 & 3.2090 \\ \end{array}$

Std Error uses a pooled estimate of error variance 


\section{Exhibit A3. PSAL Measurements by Analytical Block for Samples of the}

\section{Standard Glasses Prepared Using the LM Method}

Oneway Analysis of wt\% By Set/Block/Sub-Block Glass ID=Batch 1, (wt\%)

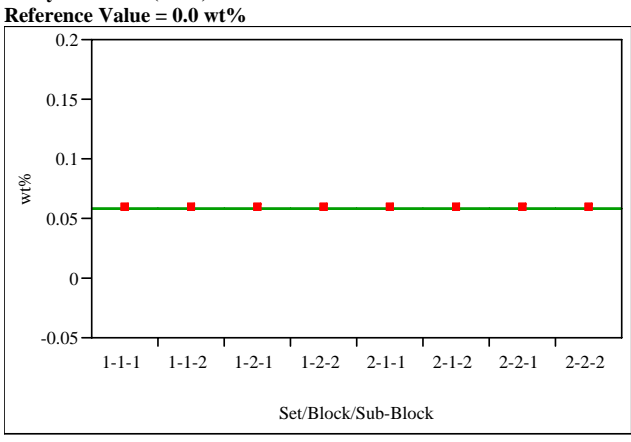

Oneway Anova

Rsquare
Adj Rsquare

Root Mean Square Erro

$\begin{array}{ll}\text { Mean of Response } & 0.05864 \\ \text { Observations (or Sum Wgts) }\end{array}$

Analysis of Variance

Source DF Sum of Squares Mean Square F Ratio Prob > F

$\begin{array}{lrrr}\text { Set/Block/Sub-Block } & 7 & 4.6222 \mathrm{e}-33 & 6.603 \mathrm{e}-34 \\ \text { Error } & 16 & 0 & \end{array}$

Error

$16-4.6222 \mathrm{e}-33$

$\begin{array}{lrlrrr}\text { Means for Oneway Anova } & & & \\ \text { Leevel Number } & \text { Mean } & \text { Std Error } & \text { Lower 95\% } & \text { Upper 95\% } \\ 1-1-1 & 3 & 0.058640 & 0 & 0.05864 & 0.05864 \\ 1-1-2 & 3 & 0.058640 & 0 & 0.05864 & 0.05864 \\ 1-2-1 & 3 & 0.058640 & 0 & 0.05864 & 0.05864 \\ 1-2-2 & 3 & 0.058640 & 0 & 0.05864 & 0.05864 \\ 2-1-1 & 3 & 0.058640 & 0 & 0.05864 & 0.05864 \\ 2-1-2 & 3 & 0.058640 & 0 & 0.05864 & 0.05864 \\ 2-2-1 & 3 & 0.058640 & 0 & 0.05864 & 0.05684 \\ 2-2-2 & 3 & 0.058640 & 0 & 0.05864 & 0.05864\end{array}$

Std Error uses a pooled estimate of error variance
Oneway Analysis of wt\% By Set/Block/Sub-Block Glass ID=Batch 1, gO ( $w$ t\%)

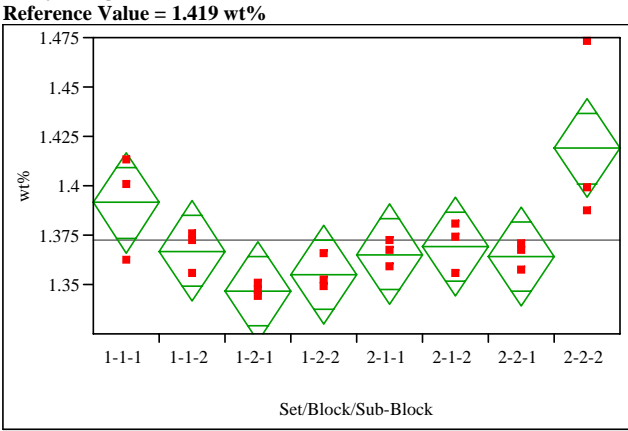

Oneway Anova

$\begin{array}{lr}\text { Rsquare } & 0.620051 \\ \text { Adj Rsquare } & 0.453824 \\ \text { Root Mean Square Error } & 0.020501 \\ \text { Mean of Response } & 1.372174 \\ \text { Observations (or Sum Wgts) } & \end{array}$

Observations (or Sun

Analysis of Variance

Source DF Sum of Squares Mean Square F Ratio Prob $>$ F

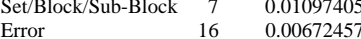

$\begin{array}{rrrr}0.001568 & 3.7301 & 0.0139\end{array}$

$\begin{array}{lll}\text { Error } & 16 & 0.0067245 \\ \text { C. Tolal } & 23 & 0.1769862\end{array}$

Means for Oneway Anova

Level Number Mean Std Error Lower 95\% Upper 95\%

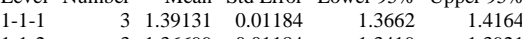

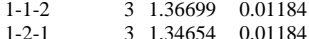

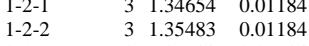

$\begin{array}{llll}2-1-1 & 3 & 1.36533 & 0.01184\end{array}$

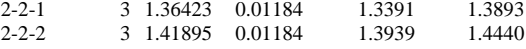

Std Error uses a pooled estimate of error variance
Oneway Analysis of wt\% By Set/Block/Sub-Block Glass ID=Batch 1, $\mathrm{te}=\mathrm{MnO}(\mathrm{wt} \%)$

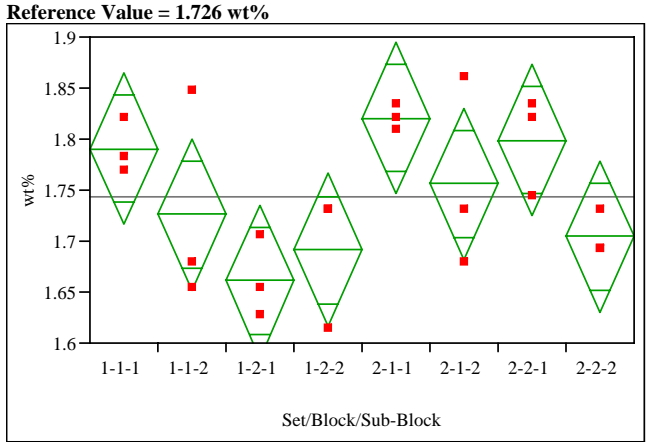

Oneway Anova

ary of Fit

Adj Rsquare $\quad 0.535143$

Root Mean Square Error $\quad 0.060792$ $\begin{array}{lr}\text { Mean of Response } & 1.743658 \\ \text { Observations (or Sum Wgts) } & 24\end{array}$

Analysis of Variance
Source

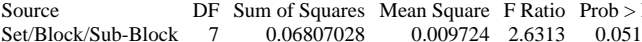
$\begin{array}{llll}\text { Error } & 16 & 0.05912994 & 0.003696 \\ \text { C. Total } & 23 & 0.12720022 & \end{array}$

Means for Oneway Anova

Level Number Mean Std Error Lower 95\% Upper 95\% $\begin{array}{lllllll}1-1-1 & & 3 & 1.79046 & 0.03510 & 1.7161 & 1.8649 \\ 1-1-2 & & 3 & 1.7250 & 0.03510 & 1.615 & 1.7003\end{array}$

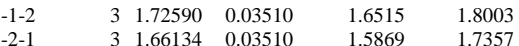

$\begin{array}{llllll}1-2-2 & 3 & 1.69147 & 0.03510 & 1.6171 & 1.7659\end{array}$

$\begin{array}{lllllll}2-1-1 & & 3 & 1.82059 & 0.03510 & 1.7462 & 1.8950 \\ 2-1-2 & & 3 & 1.75603 & 0.03510 & 1.6816 & 1.8504\end{array}$

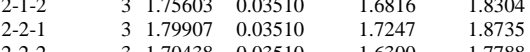

Std Error uses a pooled estimate of error variance 


\section{Exhibit A3. PSAL Measurements by Analytical Block for Samples of the}

\section{Standard Glasses Prepared Using the LM Method}

Oneway Analysis of wt\% By Set/Block/Sub-Block Glass ID=Batch 1, (wt\%)

Reference Value $=9.003 \mathrm{wt} \%$

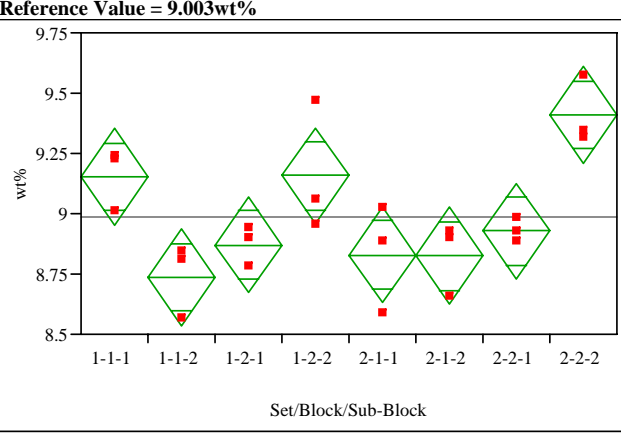

Oneway Anova

Rsquare

0.598827

Root Mean Square Error $\quad 0.163089$

Mean of Response 8.988352

Analysis of Variance

Source DF Sum of Squares Mean Square F Ratio Prob > F

$\begin{array}{llllll}\text { Set/Block/Sub-Block } & 7 & 1.0993403 & 0.157049 & 5.9046 & 0.0016\end{array}$

C. Total

$16 \quad 0.4255658$

0.026598

Level Number Mean Std Error Lower 95\% Upper 95\%

$\begin{array}{llllll}1-1-1 & 3 & 9.15292 & 0.09416 & 8.9533 & 9.3525\end{array}$

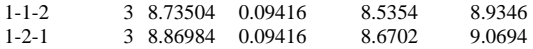

$\begin{array}{lll}3 & 9.15741 & 0.09416\end{array}$

$\begin{array}{llll}2-1-2 & 3 & 8.82491 & 0.09416\end{array}$

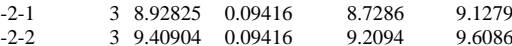

Std Error uses a pooled estimate of error variance
Oneway Analysis of wt\% By Set/Block/Sub-Block Glass ID=Batch 1, Analyte=NiO Reference Value $=0.751 \mathrm{wt} \%$

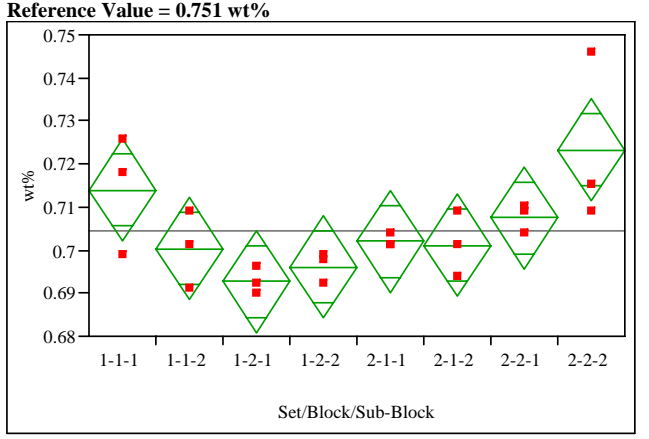

Oneway Anova

Rsquare

$\begin{array}{ll}0.58035 \\ \text { Adj Rsquare } & 0.396754\end{array}$

Root Mean Square Error $\quad 0.009695$

Mean of Response $\quad 0.704594$

Analysis of Variance

Source DF Sum of Squares Mean Square F Ratio Prob > F

$\begin{array}{lllll} & & 0.000297 & 3.1610 & 0.0268\end{array}$

C. Total

$16 \quad 0.0015037$ 0.000094

Means for Oneway Anova

Level Number Mean Std Error Lower 95\% Upper 95\%

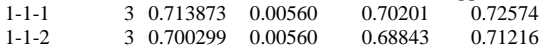

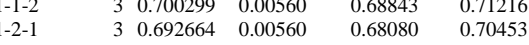

$\begin{array}{llll}1-2-1 & 3 & 0.696058 & 0.00560 \\ 1-2-2 & 3 & 0.70096 & 0.0056\end{array}$

$\begin{array}{llll}1-2-2 & 3 & 0.69058 & 0.00560 \\ 2-1-2 & 3 & 0.701966 & 0.00560 \\ 2-2-1 & 3 & 0.70748 & 0.00560\end{array}$

$\begin{array}{lll}0.707510 & 0.00560 \\ 3 & 0.723204 & 0.00560\end{array}$

0.71301

\begin{tabular}{lll}
0.00560 & 0.69564 & 0.71938 \\
\hline & 0.71134 & 0.73507
\end{tabular}

Std Error uses a pooled estimate of error variance
Oneway Analysis of wt\% By Set/Block/Sub-Block Glass ID=Batch 1 , Analyte $=$ PbO ( $(\mathrm{wt} \%)$
Reference Value $=0.0 \mathrm{w} \%$

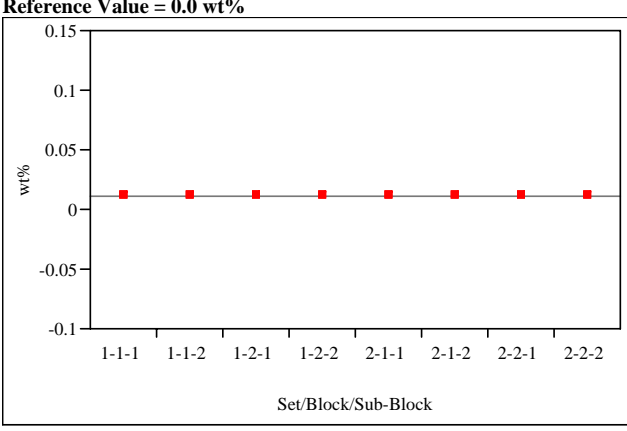

Oneway Anova

Rsquare

Adj Rsquare $\quad 5.3125$

Root Mean Square Erro
Mean of Response

Mean of Response
Observations (or Sum Wgts)

Analysis of Variance

Source DF Sum of Squares Mean Square F Ratio Prob $>F$

$\begin{array}{lrrrrr}\text { Set/Block/Sub-Block } & 7 & 2.8889 \mathrm{e}-34 & 4.127 \mathrm{e}-35 & -3.0476 & 0.0000 \\ \text { Error } & 16 & -2.167 \mathrm{e}-34 & -1.35 \mathrm{e}-35 & & \end{array}$

ova

Level Number Mean Std Error Lower 95\% Upper 95\%

$\begin{array}{lrr}1-1-1 & 3 & 0.010772 \\ 1-1-2 & 3 & 0.010772 \\ 1-2-1 & 3 & 0.01072\end{array}$

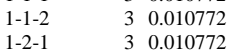

$\begin{array}{lll}1-2-1 & 3 & 0.010772 \\ 1-2-2 & 3 & 0.010772\end{array}$

$\begin{array}{lll}2-1-1 & 3 & 0.010772 \\ 2-1-2 & 3 & 0.010772\end{array}$

$2-2-1$

$2-2-2 \quad 3 \quad 0.010772$

Std Error uses a pooled estimate of error variance 


\section{Exhibit A3. PSAL Measurements by Analytical Block for Samples of the}

\section{Standard Glasses Prepared Using the LM Method}

Oneway Analysis of wt\% By Set/Block/Sub-Block Glass ID=Batch 1,

Analyte $=$ SiO2 (wt $\%)$
Reference Value $=50.22$ wt

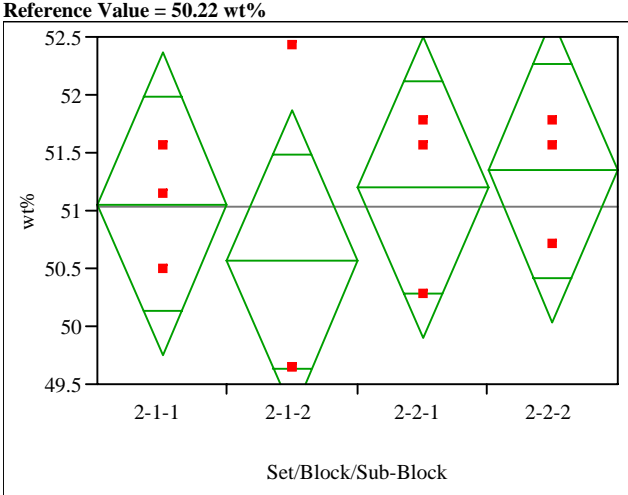

Missing Rows

Oneway Anova

Rsquare

Adj Rsquare $\quad \begin{array}{ll}0.120035 \\ -0.20995\end{array}$

Root Mean Square Error $\quad 0.98035$

Mean of Response
Observations (or Sum Wgts)

Analysis of Variance

Source DF Sum of Squares Mean Square F Ratio Prob $>$ F

$\begin{array}{llllll}\text { Set/Block/Sub-Block } & 3 & 1.0488052 & 0.349602 & 0.3638 & 0.7812\end{array}$

Error

7.6886955 0.961087

Means for Oneway Anova

Level Number Mean Std Error Lower 95\% Upper 95\%

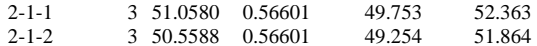

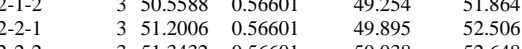

Std Error uses a pooled estimate of error variance
Oneway Analysis of wt\% By Set/Block/Sub-Block Glass ID=Batch 1, nalyte $=\mathrm{SO} 4(\mathrm{wt} \%)$

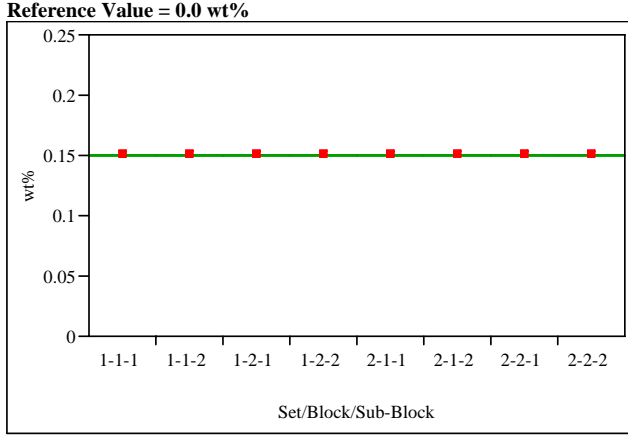

Oneway Anova

\section{Rsquare}

Aj Rsquare

Observations (or Sum Wgts)

0
0.149795
24

Analysis of Variance

Source DF Sum of Squares Mean Square F Ratio Prob $>F$

C. Total

16
23

Means for Oneway Anova

Level Number Mean Std Error Lower 95\% Upper 95\%

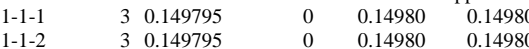

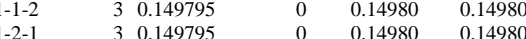

$\begin{array}{lllllll}1-2-2 & 3 & 0.149795 & 0 & 0.14980 & 0.14980 \\ 2-14980 & 0.14980\end{array}$

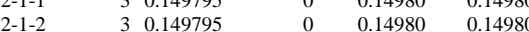

$\begin{array}{llllll}2-2-1 & 3 & 0.149795 & 0 & 0.14980 & 0.14980\end{array}$

$\begin{array}{llllll}2-2-2 & 3 & 0.149795 & 0 & 0.14980 & 0.14980\end{array}$

Std Error uses a pooled estimate of error variance
Oneway Analysis of wt\% By Set/Block/Sub-Block Glass ID=Batch 1, Analyte=TiO2 (wt $\%)$

Reference Value $=\mathbf{0 . 6 7 7} \mathbf{w t} \%$

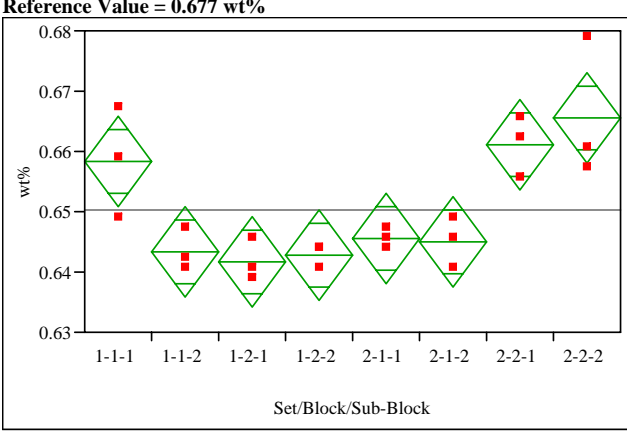

Oneway Anova

$\begin{array}{ll}\text { Rsquare } & 0.766477 \\ \text { Adj Rsquare } & 0.664311\end{array}$

Root Mean Square Error $\quad 0.006072$

Mean of Response $\quad 0.65038$

Analysis of Variance

$\begin{array}{lrrrrr}\text { Source } & \text { DF } & & & & \\ \text { Set/Block/Sub-Block } & 7 & 0.00193596 & 0.000277 & 7.5022 & 0.0004\end{array}$

$\begin{array}{llll}\text { Error } & 16 & 0.00058983 & 0.000037 \\ \text { C. Total } & 23 & 0.00252580 & \end{array}$

Means for Oneway Anova

Level Number Mean Std Error Lower 95\% Upper 95\%

$\begin{array}{lllllll}1-1-1 & 3 & 0.658304 & 0.00351 & 0.65087 & 0.66574\end{array}$

$\begin{array}{llllll}1-1-2 & 3 & 0.643292 & 0.00351 & 0.63586 & 0.65072\end{array}$

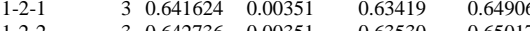

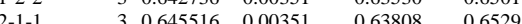

$\begin{array}{lllllll}2-1-2 & 3 & 0.644960 & 0.00351 & 0.63753 & 0.65239\end{array}$

$\begin{array}{lllllll}2-2-1 & 3 & 0.661084 & 0.00351 & 0.65365 & 0.66852\end{array}$

$\begin{array}{llllll}2-2-2 & 3 & 0.665532 & 0.00351 & 0.65810 & 0.67296\end{array}$

Std Error uses a pooled estimate of error variance 


\section{Exhibit A3. PSAL Measurements by Analytical Block for Samples of the}

\section{Standard Glasses Prepared Using the LM Method}

Oneway Analysis of wt\% By Set/Block/Sub-Block Glass ID=Batch 1, Analyte $=\mathrm{U} 308$ (wt\%)

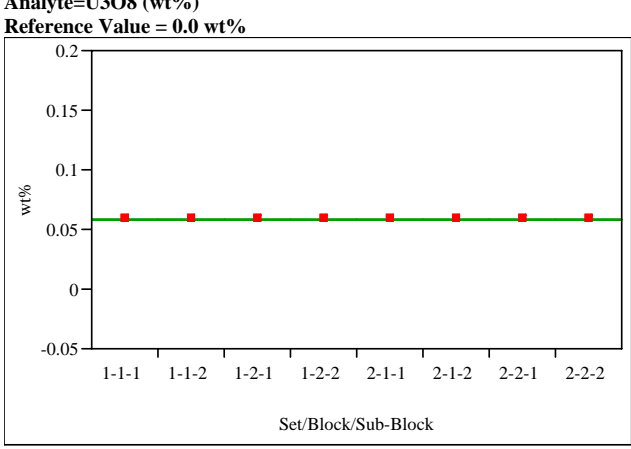

Oneway Anova

Rsquare

Adj Rsquare

Root Mean Square Erro

$\begin{array}{ll}\text { Mean of Response } & 0.05896 \\ \text { Observations (or Sum Wgts) }\end{array}$

Analysis of Variance

Source DF Sum of Squares Mean Square F Ratio Prob > F

$\begin{array}{lrrr}\text { Set/Block/Sub-Block } & 7 & 4.6222 \mathrm{e}-33 & 6.603 \mathrm{e}-34 \\ \text { Error } & 16 & 0 & 0\end{array}$

Error
C. Total

$16-4.6222-33$

\begin{tabular}{|c|c|c|c|c|}
\hline & & & & \\
\hline Number & Mean & Std Error & Lower 95\% & Upper 95\% \\
\hline & 30.058960 & 0 & 0.05896 & \\
\hline & B 0.058960 & 0 & 0.05896 & \\
\hline & $\begin{array}{l}3.058960 \\
0.050\end{array}$ & 0 & 0.05896 & 0.05896 \\
\hline & $\begin{array}{l}3 \\
0.058960\end{array}$ & 0 & 0.05896 & 0.05896 \\
\hline & $\begin{array}{l}3 \\
0.058960\end{array}$ & 0 & 0.05896 & 0.05896 \\
\hline & $\begin{array}{l}30.058960 \\
\end{array}$ & 0 & 0.05896 & 0.0589 \\
\hline & 30.058960 & 0 & 0.05896 & 0.05896 \\
\hline & 30.058960 & 0 & 0.05896 & 0.05896 \\
\hline
\end{tabular}

Std Error uses a pooled estimate of error variance
Oneway Analysis of wt\% By Set/Block/Sub-Block Glass ID=Batch 1, Analyte $=\mathrm{ZnO}(\mathrm{wt} \%)$

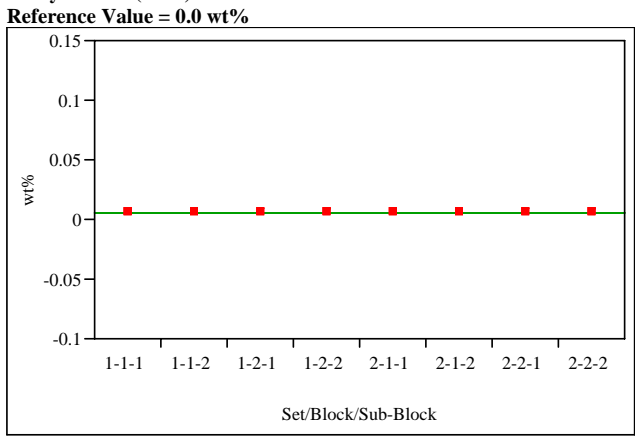

\section{Oneway Anova}

Rsquare

Adj Rsquare

Root Mean Square Erro

$\begin{array}{lr}\text { Mean of Response } & 0.006224 \\ \text { Observations (or Sum Wgts) } & 24\end{array}$

Analysis of Variance

Source DF Sum of Squares Mean Square F Ratio Prob > F

Set/Block/Sub-Block
Error

7
23

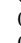

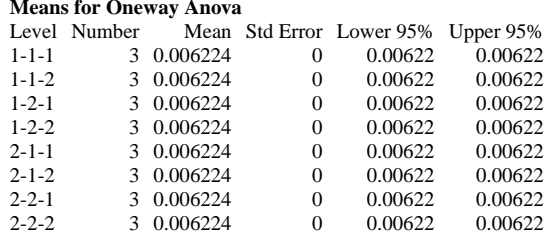

Std Error uses a pooled estimate of error variance
Oneway Analysis of wt\% By Set/Block/Sub-Block Glass ID=Batch 1, Analyte $=\mathrm{ZrO} 2(\mathrm{wt} \%)$

Reference Value $=0.098 \mathrm{wt} \%$

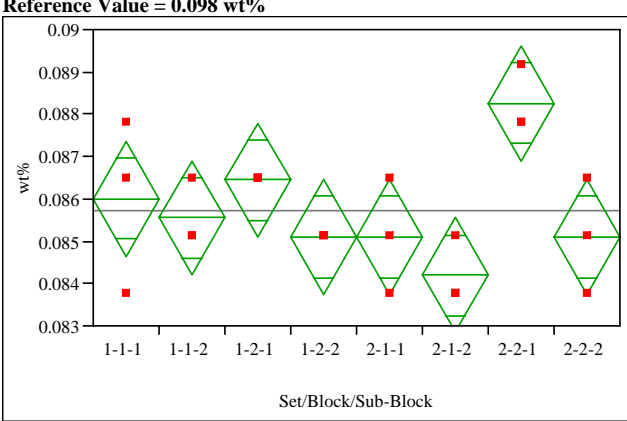

Oneway Anova

$\begin{array}{lr} & \\ \text { Rsquare } & 0.61848 \\ \text { Adj Rsquare } & 0.451565 \\ \text { Root Mean Square Error } & 0.001103 \\ \text { Mean of Response } & 0.08572 \\ \text { Observations (or Sum Wgts) } & 24\end{array}$

Analysis of Varianc

Source DF Sum of Squares Mean Square F Ratio Prob >

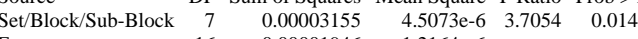

Error $\quad 16 \quad 0.00001946$ $1.2164 \mathrm{e}-6$

Means for Oneway Anova

Level Number Mean Std Error Lower 95\% Upper 95\%

$\begin{array}{lllllll}1-1-1 & 3 & 0.086001 & 0.00064 & 0.08465 & 0.08735\end{array}$

$\begin{array}{lllllll}1-1-2 & 3 & 0.085551 & 0.00064 & 0.08420 & 0.08690 \\ 1-2-1 & 3 & 0.006451 & 0.00064 & 0.08510 & 0.08780\end{array}$

$\begin{array}{lllllll}1-2-2 & & 3 & 0.085100 & 0.00064 & 0.08375 & 0.08645 \\ 2-1-2 & & 3 & 0.085100 & 0.0064 & 0.0835 & 0.0845\end{array}$

$\begin{array}{lllllll}2-1-1 & & 3 & 0.085100 & 0.00064 & 0.08375 & 0.08645\end{array}$

$\begin{array}{lllllll}2-1-2 & 3 & 0.084200 & 0.00064 & 0.08285 & 0.08555 \\ 2-2-1 & & 3 & 0.088252 & 0.00064 & 0.08690 & 0.08960\end{array}$

$\begin{array}{lllllll}2-2-1 & & 3 & 0.088252 & 0.00064 & 0.08690 & 0.08960 \\ 2-2-2 & & 3 & 0.085100 & 0.00064 & 0.08375 & 0.08645\end{array}$

Std Error uses a pooled estimate of error variance 


\section{Exhibit A3. PSAL Measurements by Analytical Block for Samples of the}

\section{Standard Glasses Prepared Using the LM Method}

Oneway Analysis of wt\% By Set/Block/Sub-Block Glass ID=U std, Analyte $=\mathrm{Al} 2 \mathrm{O} 3(\mathrm{wt} \%)$

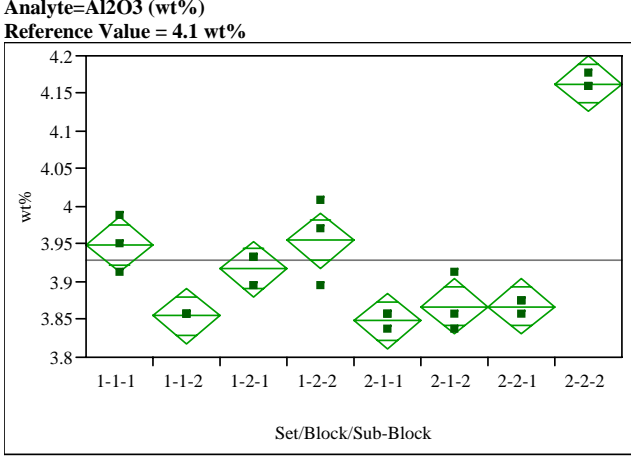

Oneway Anova

Rsquare
Adj Rsquar

0.940883

Root Mean S

Mean of Response

.029876

$\begin{array}{lrrrrr}\begin{array}{l}\text { Analysis of Variance } \\ \text { Source }\end{array} & \text { DF } & \text { Sum of Squares } & \text { Mean Square } & \text { F Ration } & \text { Prob }>\text { F } \\ \text { Set/Block/Sub-Block } & 7 & 0.22728851 & 0.032470 & 36.3786 & <.0001\end{array}$

Error

$\begin{array}{rr}7 & 0.2272885 \\ 16 & 0.01428084\end{array}$

0.000893

Means for Oneway Anova

$\begin{array}{lrrrrr}\text { Level } & \text { Number } & \text { Mean } & \text { Std Error } & \text { Lower 95\% } & \text { Upper 95\% } \\ 1-1-1 & 3 & 3.94906 & 0.01725 & 3.9125 & 3.9856 \\ 1-1-2 & 3 & 3.85458 & 0.01725 & 3.8180 & 3.8911 \\ 1-2-1 & 3 & 3.91756 & 0.01725 & 3.8810 & 3.9541 \\ 1-2-2 & 3 & 3.95535 & 0.01725 & 3.9188 & 3.9919 \\ 2-1-1 & 3 & 3.84828 & 0.01725 & 3.8117 & 3.8848 \\ 2-1-2 & 3 & 3.86718 & 0.01725 & 3.8306 & 3.9037 \\ 2-2-1 & 3 & 3.86718 & 0.01725 & 3.8306 & 3.9037 \\ 2-2-2 & 3 & 4.16320 & 0.01725 & 4.1266 & 4.1998\end{array}$

Std Error uses a pooled estimate of error variance
Oneway Analysis of wt $\%$ By Set/Block/Sub-Block Glass ID=U std Analyte $=\mathbf{B a O}(\mathbf{w t} \%)$

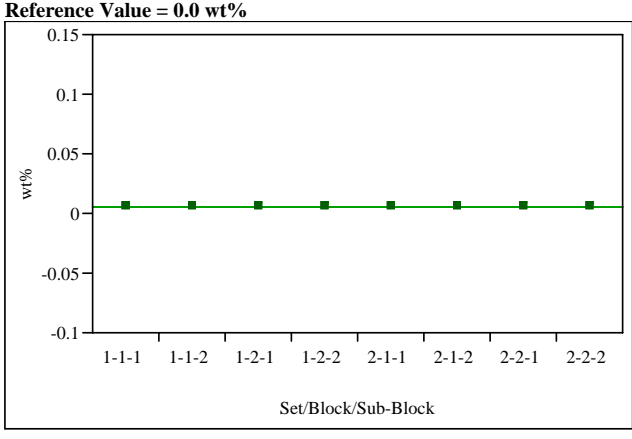

\section{Oneway Anova}

Rsquare

Adj Rsquare

Root Mean Square Erro

$\begin{array}{lr}\text { Mean of Response } & 0.005583 \\ \text { Observations (or Sum Wgts) } & 24\end{array}$

Analysis of Variance

Source DF Sum of Squares Mean Square F Ratio Prob > F

Set/Block/Sub-Block
Error

Error

7
16
23

Means for Oneway Anova

Level Number Mean Std Error Lower 95\% Upper 95\%

$\begin{array}{lrrrrr}\text { Level } & \text { Number } & \text { Mean } & \text { Std Error } & \text { Lower 95\% } & \text { Upper 95\% } \\ 1-1-1 & 3 & 0.005583 & 0 & 0.00558 & 0.00558 \\ 1-1-2 & 3 & 0.005583 & 0 & 0.00558 & 0.00558 \\ 1-2-1 & 3 & 0.005853 & 0 & 0.00558 & 0.0058\end{array}$

$0.00558 \quad 0.00558$

$\begin{array}{lll}1-2-2 & 3 & 0.005583 \\ 2-1-1 & 3 & 0.00583\end{array}$

$\begin{array}{ll}3 & 0.005583 \\ 3 & 0.005583 \\ 3 & 0.005583\end{array}$

$\begin{array}{ll}3 & 0.005583 \\ 3 & 0.005583\end{array}$

$\begin{array}{ll}0.00558 & 0.00558 \\ 0.00558 & 0.00558 \\ 0.00588 & 0.0058\end{array}$

Std Error uses a pooled estimate of error variance
Oneway Analysis of wt \% By Set/Block/Sub-Block Glass ID=U std, Analyte $=\mathbf{C a O}(\mathrm{wt} \%)$

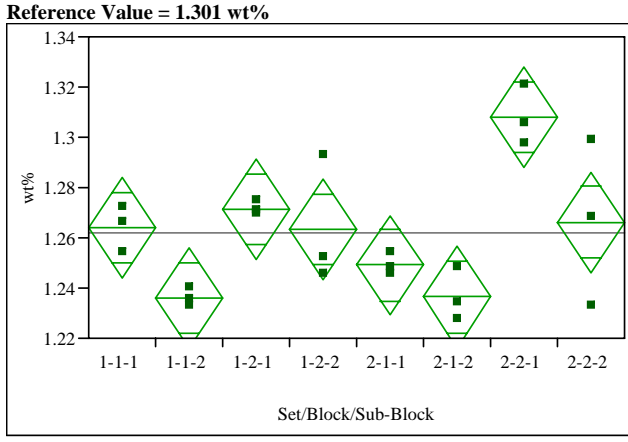

Oneway Anova

$\begin{array}{ll}\text { Rsquare } & 0.723338 \\ \text { Adj Rsquare } & 0.602298\end{array}$

Root Mean Square Error $\quad 0.01631$

$\begin{array}{lr}\text { Mean of Response } & 1.261787 \\ \text { Observations (or Sum Wgts) } & 24\end{array}$

Analysis of Variance

Source DF Sum of Squares Mean Square F Ratio Prob > F

$\begin{array}{lllllll}\text { Set/Block/Sub-Block } & 7 & 0.01112783 & 0.001590 & 5.9760 & 0.0015\end{array}$

C. Total

$\begin{array}{rr}7 & 0.01112783 \\ 16 & 0.00425617\end{array}$

0.000266

Level Number Mean Std Error Lower 95\% Upper 95\%

$\begin{array}{llllll}1-1-1 & 3 & 1.26394 & 0.00942 & 1.2440 & 1.2839\end{array}$

$\begin{array}{llll}1-1-2 & 3 & 1.23596 & 0.00942\end{array}$

$\begin{array}{llll}1-2-1 & 3 & 1.27141 & 0.00942 \\ 1-2-2 & 3 & 1.26348 & 0.00942\end{array}$

$\begin{array}{llll}1-2-1 & 3 & 1.27141 & 0.00942 \\ 2-1-1 & 3 & 1.26348 & 0.00942\end{array}$

$\begin{array}{lllll}2-1-2 & 3 & 1.23643 & 0.00942\end{array}$

$\begin{array}{ll}1.2435 & 1.2834 \\ 1.2291 & 1.2690\end{array}$

$\begin{array}{lllll}3 & 1.26628 & 0.00942 & 1.2463 & 1.2862\end{array}$

Std Error uses a pooled estimate of error variance 


\section{Exhibit A3. PSAL Measurements by Analytical Block for Samples of the}

\section{Standard Glasses Prepared Using the LM Method}

Oneway Analysis of wt $\%$ By Set/Block/Sub-Block Glass ID=U std, nalyte $=\mathbf{C d O}(\mathrm{wt} \%)$

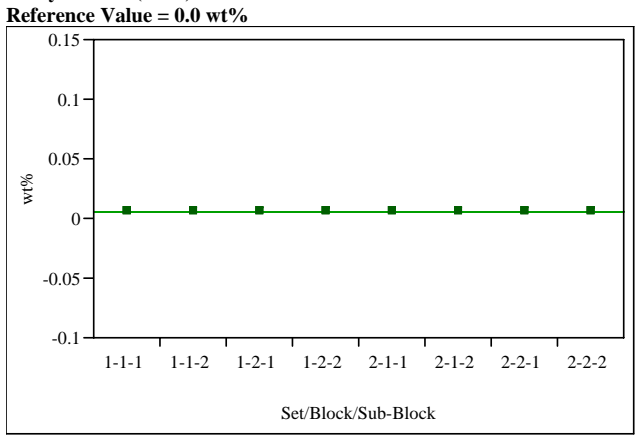

Oneway Anova

Rsquare

Adj Rsquare

Root Mean Square Error

$\begin{array}{lr}\text { Mean of Response } & 0.005712 \\ \text { Observations (or Sum Wgts) } & 24\end{array}$

Analysis of Variance

Source DF Sum of Squares Mean Square F Ratio Prob > F

Set/Block/Sub-Block
Error

Error

7
6

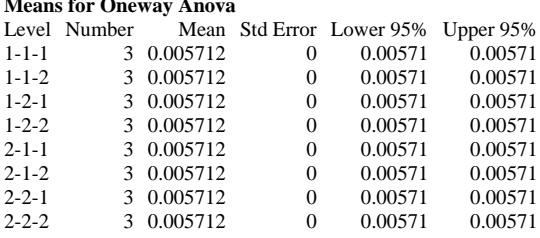

Std Error uses a pooled estimate of error variance
Oneway Analysis of wt\% By Set/Block/Sub-Block Glass ID=U std, Analyte $=\mathrm{Ce} 2 \mathrm{O3}(\mathrm{wt} \%)$

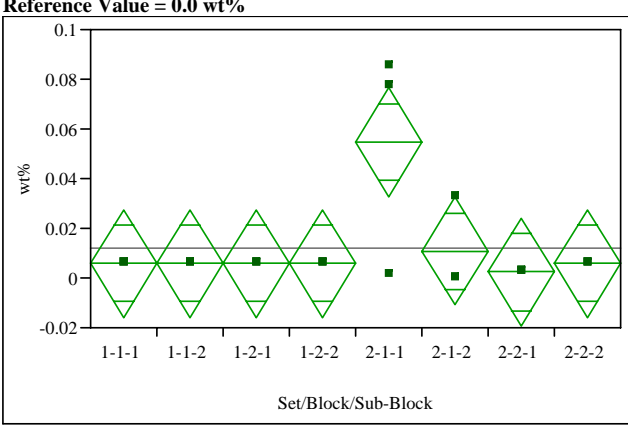

\section{Oneway Anova}

Rsquare

Root Mean Square Error $\quad 0.017752$

Mean of Response $\quad{ }^{0.012152}$

Analysis of Variance

$\begin{array}{lrrrrr}\text { Source } & \text { DF } & \text { Sum of Squares } & \text { Mean Square } & \text { F Ratio } & \text { Prob }>\text { F } \\ \text { Set/Block/Sub-Block } & 7 & 0.00630860 & 0.000901 & 2.8597 & 0.0387\end{array}$ Set/Blorks

Error

$\begin{array}{ll}16 & 0.00504235 \\ 23 & 0.01135095\end{array}$ 0.000315

$\begin{array}{rrrrrr}\text { Level } & \text { Number } & \text { Mean } & \text { Std Error } & \text { Lower 95\% } & \text { Upper 95\% } \\ 1-1-1 & 3 & 0.005857 & 0.01025 & -0.0159 & 0.02758\end{array}$

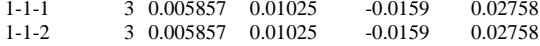

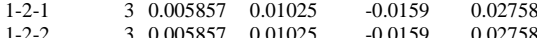

$\begin{array}{llllll}2-1-1 & 3 & 0.054661 & 0.01025 & 0.0329 & 0.027639\end{array}$

$\begin{array}{lllll}3 & 0.010932 & 0.01025 & -0.0108 & 0.0326\end{array}$

$\begin{array}{lllll}3 & 0.002343 & 0.01025 & -0.0194 & 0.02407\end{array}$

$\begin{array}{llllll}2-2-2 & 3 & 0.005857 & 0.01025 & -0.0159 & 0.02758\end{array}$

Std Error uses a pooled estimate of error variance
Oneway Analysis of wt\% By Set/Block/Sub-Block Glass ID=U std, Analyte $=\mathrm{Cr} 2 \mathrm{O} 3(\mathrm{wt} \%)$

Reference Value $=0.0 \mathrm{wt} \%$

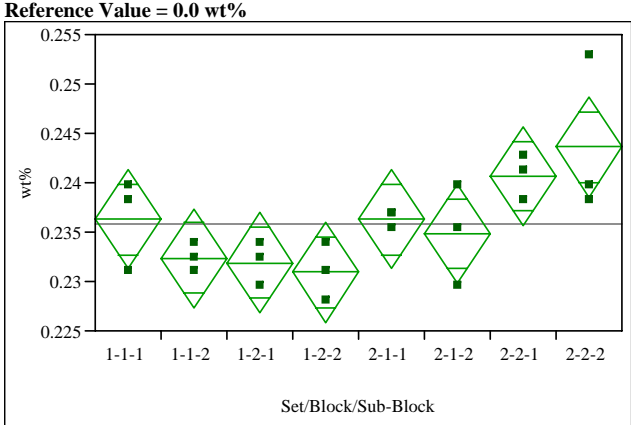

Oneway Anova

Rsquare

$\begin{array}{ll}0.603306 \\ \text { Adj Rsquare } & 0.429752\end{array}$

Aor Mean Square Error 0.004102

Observations (or Sum Wgts)

Analysis of Variance

Source DF Sum of Squares Mean Square F Ratio Prob > F $\begin{array}{lllll}\text { Set/Block/Sub-Block } & 70.00040936 & 0.000058 & 3.4762 & 0.018\end{array}$ C. $16 \quad 0.0002617$

Means for Oneway Anova

Level Number Mean Std Error Lower 95\% Upper 95\%

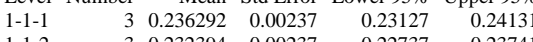

$\begin{array}{lllllll}1-1-2 & 3 & 0.232394 & 0.00237 & 0.22737 & 0.2374 \\ 1-2-1 & & 3 & 0.231907 & 0.00237 & 0.22689 & 0.23493\end{array}$

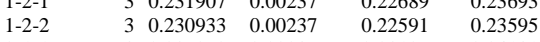

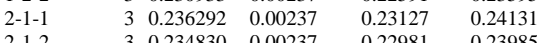

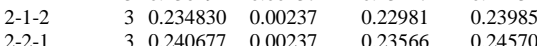

$\begin{array}{lllllll}2-2-1 & & 3 & 0.240677 & 0.00237 & 0.23566 & 0.24570 \\ 2-2-2 & & 3 & 0.243600 & 0.00237 & 0.23858 & 0.24862\end{array}$

Std Error uses a pooled estimate of error variance 


\section{Exhibit A3. PSAL Measurements by Analytical Block for Samples of the}

\section{Standard Glasses Prepared Using the LM Method}

Oneway Analysis of wt $\%$ By Set/Block/Sub-Block Glass ID=U std, nalyte $=\mathrm{CuO}(\mathrm{wt} \%)$

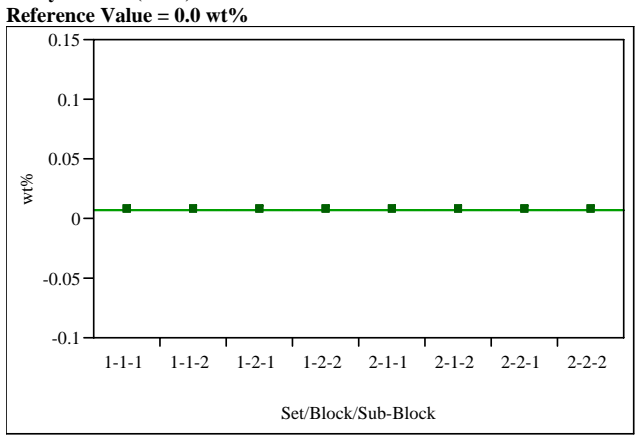

Oneway Anova

Rsquare

Adj Rsquare

0.006259
Mean of Response

Source of Variance DF Sum of Squares Mean Square F Ratio Prob > F

Set/Block/Sub-Block

$\begin{array}{ll}\text { Error } & 16 \\ \text { C. Total } & 23\end{array}$

\begin{tabular}{|c|c|c|c|c|}
\hline$s$ sor Une & eway fuov & & & \\
\hline Number & Mean & Std & Lower 95\% & Upper 95\% \\
\hline & 30.006259 & 0 & 0.00626 & 0.00 \\
\hline & 0.006259 & 0 & 0.00626 & 0.00 \\
\hline & 0.006259 & 0 & 0.00626 & 0.006 \\
\hline & $\begin{array}{ll}30.006259\end{array}$ & 0 & 0.00626 & 0.006 \\
\hline & 30.006259 & 0 & 0.00626 & 0.00626 \\
\hline & 30.006259 & 0 & 0.00626 & 0.00626 \\
\hline & 0.006259 & 0 & 0.00626 & 0.00626 \\
\hline & $\begin{array}{ll}3 & 0.006259\end{array}$ & 0 & 0.00626 & 0.00626 \\
\hline
\end{tabular}

Std Error uses a pooled estimate of error variance
Oneway Analysis of wt\% By Set/Block/Sub-Block Glass ID=U std, Analyte=Fe2O3 (wt\%)

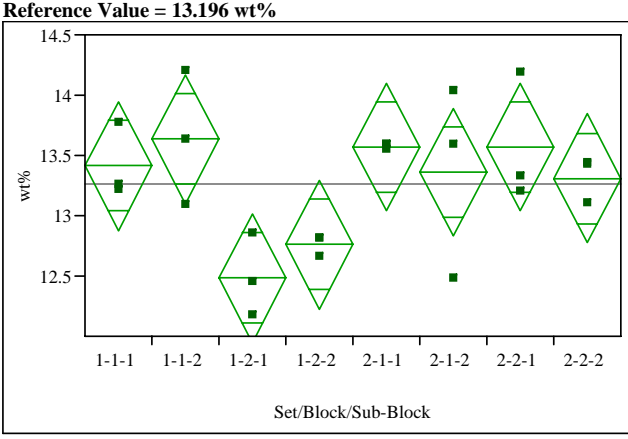

Oneway Anova

Rsquare
Adj Rsquar

0.433365

Observations (or S 13.26166

Analysis of Variance DF Sum of Squares Mean Square F Ratio Prob > F

$\begin{array}{lllllll}\text { Set/Block/Sub-Block } & 7 & 3.6480360 & 0.521148 & 2.7749 & 0.043\end{array}$

$16 \quad 3.004878$

0.187805

Means for Oneway Anova

$\begin{array}{lrrrrr}\text { Level } & \text { Number } & \text { Mean } & \text { Std Error } & \text { Lower 95\% } & \text { Upper 95\% } \\ 1-1-1 & 3 & 13.4106 & 0.25020 & 12.880 & 13.941 \\ 1-1-2 & 3 & 13.6346 & 0.25020 & 13.104 & 14.165 \\ 1-2-1 & 3 & 12.4860 & 0.25020 & 11.956 & 13.016 \\ 1-2-2 & 3 & 12.7577 & 0.25020 & 12.227 & 13.288 \\ 2-1-1 & 3 & 13.5679 & 0.25020 & 13.037 & 14.098 \\ 2-1-2 & 3 & 13.3582 & 0.25020 & 12.828 & 13.889 \\ 2-2-1 & 3 & 13.5679 & 0.25020 & 13.037 & 14.098 \\ 2-2-2 & 3 & 13.3105 & 0.25020 & 12.780 & 13.841\end{array}$

Std Error uses a pooled estimate of error variance
Oneway Analysis of wt\% By Set/Block/Sub-Block Glass ID=U std, Analyte $=\mathrm{K} 2 \mathrm{O}(\mathrm{wt} \%)$

Reference Value $=2.999 \mathrm{wt} \%$

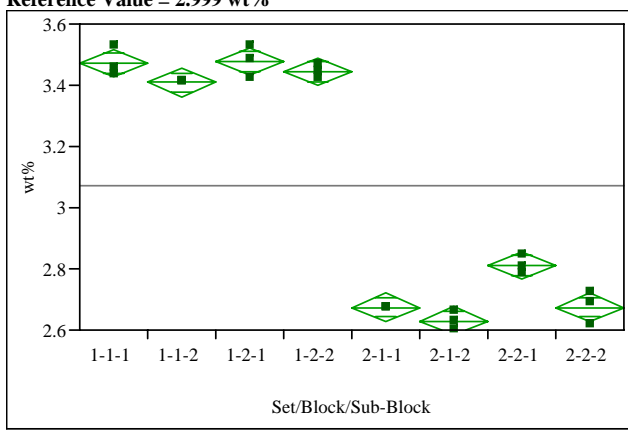

Oneway Anova

mary of $\mathrm{Fi}$

Rsquare

Root Mean Square Error $\quad 0.036965$

$\begin{array}{lr}\text { Mean of Response } & 3.07424 \\ \text { Observations (or Sum Wgts) } & 24\end{array}$

Analysis of Variance
Source DF Sum of Squares Mean Square F Ratio Prob $>$ F

Source Set/Block/Sub-Block DF Sum of Squares Mean Square F Ratio Prob > F

$\begin{array}{llll}\text { Error } & 16 & 0.0218627 & 0.001366 \\ \text { C. Total } & 23 & 3.4961807 & \end{array}$

Means for Oneway Anova

$\begin{array}{lrrrrr}\text { Means for Oneway Anova } & & & \\ \text { Level } & \text { Number } & \text { Mean } & \text { Std Error } & \text { Lower 95\% } & \text { Upper 95\% } \\ 1-1-1 & 3 & 3.47326 & 0.02134 & 3.4280 & 3.5185\end{array}$

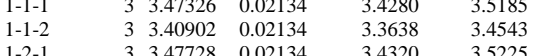

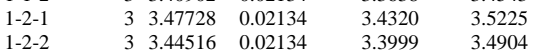

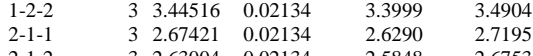

$\begin{array}{llllll}2-1-2 & 3 & 2.63004 & 0.02134 & 2.5848 & 2.6753 \\ 2-2-1 & 3 & 2.81073 & 0.02134 & 2.7655 & 2.8560\end{array}$

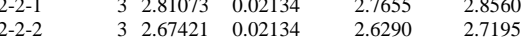

Std Error uses a pooled estimate of error variance 


\section{Exhibit A3. PSAL Measurements by Analytical Block for Samples of the}

\section{Standard Glasses Prepared Using the LM Method}

Oneway Analysis of wt\% By Set/Block/Sub-Block Glass ID=U std, Analyte $=\mathrm{La} 2 \mathrm{O} 3(\mathrm{wt} \%)$

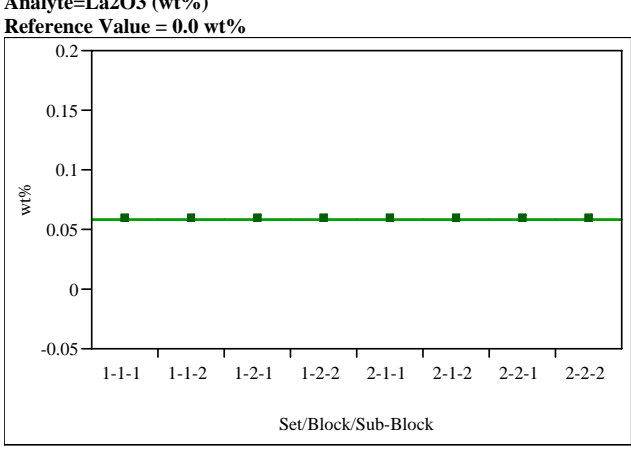

Oneway Anova

Rsquare

Adj Rsquare

Root Mean Square Error

Mean of Response $\quad 0.05864$

Analysis of Variance

Source DF Sum of Squares Mean Square F Ratio Prob > F

$\begin{array}{lrrr}\text { Set/Block/Sub-Block } & 7 & 4.6222 \mathrm{e}-33 & 6.603 \mathrm{e}-34 \\ \text { Error } & 16 & 0 & \end{array}$

Error
C. Total

$16-4.6222 \mathrm{e}-33$

\begin{tabular}{|c|c|c|c|c|}
\hline & & & & \\
\hline Number & r Mean & Std Error & Lower 95\% & $\begin{array}{r}\text { Upper } 95 \% \\
0.05864\end{array}$ \\
\hline & $\begin{array}{l}30.058640 \\
3\end{array}$ & 0 & $\begin{array}{l}0.05864 \\
0.05864\end{array}$ & $\begin{array}{l}0.05864 \\
0.05864\end{array}$ \\
\hline 3 & $\begin{array}{l}30.058640 \\
\end{array}$ & 0 & 0.05864 & 0.05864 \\
\hline & 0.058640 & 0 & 0.05864 & 0.05864 \\
\hline & $\begin{array}{l}3 \\
0.058640\end{array}$ & 0 & 0.05864 & 0.05864 \\
\hline & 30.058640 & 0 & 0.05864 & 0.05864 \\
\hline & 0.058640 & 0 & 0.05864 & 0.058 \\
\hline & 058640 & 0 & 0.05864 & 0.058 \\
\hline
\end{tabular}

Std Error uses a pooled estimate of error variance
Oneway Analysis of wt\% By Set/Block/Sub-Block Glass ID=U std, Analyte $=\mathrm{MgO}(\mathrm{wt} \%)$

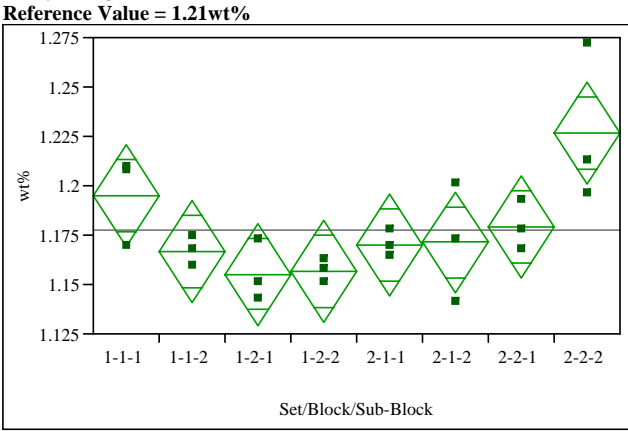

Oneway Anova

$\begin{array}{lr}\text { Rsquare } & 0.619616 \\ \text { Adj Rsquare } & 0.453197 \\ \text { Root Mean Square Error } & 0.021028 \\ \text { Mean of Response } & 1.177669 \\ \text { Observations (or Sum Wgts) } & 24\end{array}$

Analysis of Variance

$\begin{array}{lrrrrrr}\text { Source } & \text { DF } & \text { Sum of Squares } & \text { Mean Square } & \text { F Ratio } & \text { Prob }>\text { F } \\ \text { Set/Block/Sub-Block } & 7 & 0.01152416 & 0.001646 & 3.7232 & 0.0140\end{array}$

Error $\quad 16 \quad 0.00707473$

$\begin{array}{lll}\text { Error } & 16 & 0.00707473 \\ \text { C. Total } & 23 & 0.01859889\end{array}$

\begin{tabular}{|c|c|c|c|c|c|}
\hline \multirow{2}{*}{\multicolumn{6}{|c|}{ Means for Onev }} \\
\hline & Number & an & & $\%$ & Upper 95\% \\
\hline 1-1-1 & 3 & 1.19508 & 0.01214 & 1.1693 & $1.22 \mathrm{Z}$ \\
\hline 1-1-2 & 3 & 1.16689 & 0.01214 & 1.1412 & $1.1 \mathrm{C}$ \\
\hline $2-1$ & 3 & 1.15528 & 0.01214 & 1.1295 & 1.1810 \\
\hline$-2-2$ & 3 & 1.15694 & 0.01214 & 1.1312 & 1.1827 \\
\hline$-1-1$ & 3 & 1.17021 & 0.01214 & 1.1445 & 1.1959 \\
\hline $2-1-2$ & 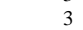 & 1.17131 & 0.01214 & 1.1456 & 1.19 \\
\hline $2-2-1$ & 3 & 1.17905 & 0.01214 & 1.1533 & 1.2048 \\
\hline $2-2-2$ & & 1.22659 & 0.01214 & 1.2009 & 1.2523 \\
\hline
\end{tabular}

Std Error uses a pooled estimate of error variance
Oneway Analysis of wt \% By Set/Block/Sub-Block Glass ID=U std, Analyte $=\mathrm{MnO}(\mathrm{wt} \%)$ Reference Value $=2.892 \mathrm{wt} \%$

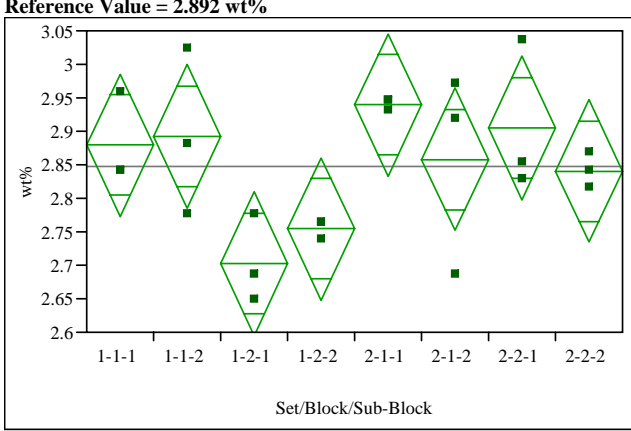

Oneway Anova

Rsquare

Root Mean Square Error $\quad 0.087016$

Mean of Response 2.846558

Analysis of Variance

Source DF Sum of Squares Mean Square F Ratio Prob $>$ F

$\begin{array}{llllll}\text { Set/Block/Sub-Block } & 7 & 0.13359114 & 0.019084 & 2.5204 & 0.0596\end{array}$

$\begin{array}{lll}\text { Error } & 16 & 0.1211496\end{array}$

0.007572

Means for Oneway Anova

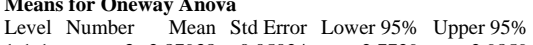

$\begin{array}{llrrrr}1-1-1 & 3 & 2.87938 & 0.05024 & 2.7729 & 29859\end{array}$

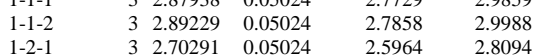

$\begin{array}{lllllll}1-2-1 & & 3 & 2.70291 & 0.05024 & 2.5964 & 2.8094 \\ 1-2-2 & & 3 & 2.75456 & 0.05024 & 2.6481 & 2.8611\end{array}$

$\begin{array}{llllll}2-1-1 & 3 & 2.93963 & 0.05024 & 2.8331 & 3.0461 \\ 2-1-2 & 3 & 2.85786 & 0.05024 & 2.7514 & \\ 2-2-1 & & 3 & 2.9644\end{array}$

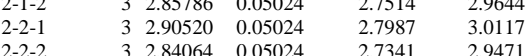

Std Error uses a pooled estimate of error variance 


\section{Exhibit A3. PSAL Measurements by Analytical Block for Samples of the}

\section{Standard Glasses Prepared Using the LM Method}

Oneway Analysis of wt\% By Set/Block/Sub-Block Glass ID=U std,

Reference Value $=11.795 \mathrm{wt} \%$

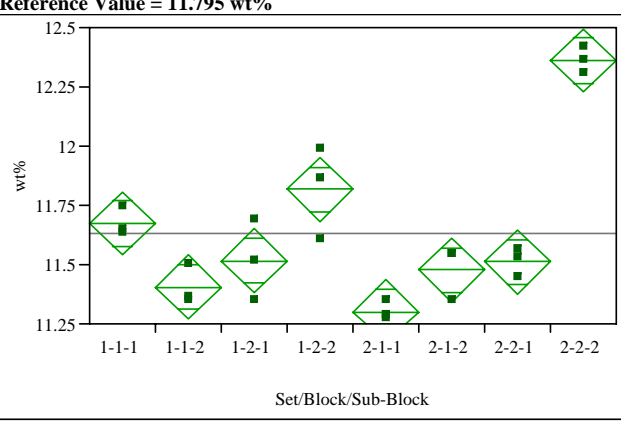

Oneway Anova

$\begin{array}{lr}\text { Rsquare } & 0.92418 \\ \text { Adj Rsquare } & 0.891009 \\ \text { Root Mean Square Error } & 0.10965 \\ \text { Mean of Response } & 11.63268 \\ \text { Observations (or Sum Wgts) } & \end{array}$

Observations (or S

Analysis of Variance

$\begin{array}{lrrrrr}\text { Source } & \text { DF } & \text { Sum of Squares } & \text { Mean Square } & \text { F Ratio } & \text { Prob }>\text { F } \\ \text { Set/Block/Sub-Block } & 7 & 2.3448440 & 0.334978 & 27.8610 & <.0001\end{array}$

\begin{tabular}{lll} 
Error & \\
C. & 16 & 0.3448440 \\
\hline
\end{tabular}

Error

2.5372147

\begin{tabular}{|c|c|c|c|c|c|}
\hline Level & Number & Mean & Std Error & Lower 95\% & Upper 95\% \\
\hline 1-1-1 & & 11.6737 & 0.06331 & 11.539 & 11.808 \\
\hline$-1-2$ & & 11.4041 & 0.06331 & 11.270 & 11.538 \\
\hline $2-1$ & 3 & 11.5164 & 0.06331 & 11.382 & 11.651 \\
\hline$-2-2$ & 3 & 11.8175 & 0.06331 & 11.683 & 11.952 \\
\hline & 3 & 11.3007 & 0.06331 & 11.167 & 11.435 \\
\hline & 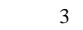 & 11.4760 & 0.06331 & 11.342 & 11.610 \\
\hline & 3 & 11.5119 & 0.06331 & 11.378 & 11.646 \\
\hline $2-2-2$ & 3 & 12.3612 & 0.06331 & 12.227 & 12.495 \\
\hline
\end{tabular}

Std Error uses a pooled estimate of error variance
Oneway Analysis of wt\% By Set/Block/Sub-Block Glass ID=U std, Analyte= $\mathrm{NiO}(\mathrm{wt} \%)$

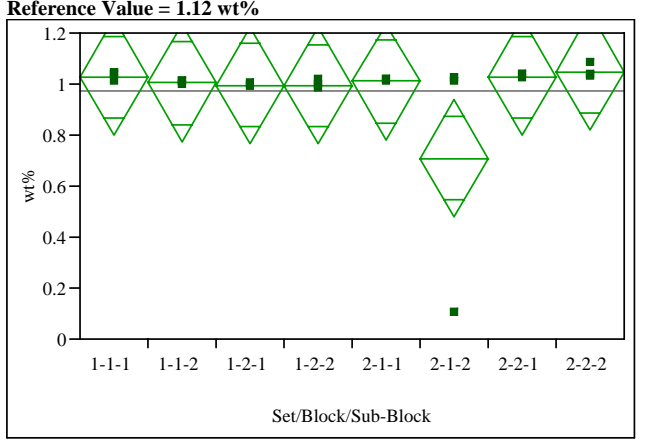

Oneway Anova

ary of Fit

Rsquare

Adj Rsquare $\quad 0.309587$

Root Mean Square Error $\quad 0.187382$

$\begin{array}{lr}\text { Mean of Response } & 0.976644 \\ \text { Observations (or Sum Wgts) } & 24\end{array}$

Analysis of Variance
Source

Source
Set/Block/Sub-Block DF Sum of Squares Mean Square F Ratio Prob $>$

$\begin{array}{llll}\text { Error } & 16 & 0.56179016 & 0.035112 \\ \text { C. Total } & 23 & 0.81370217 & \end{array}$

Means for Oneway Anova

Level Number Mean Std Error Lower 95\% Upper 95\%

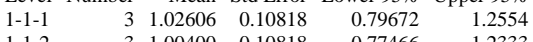

$\begin{array}{lllllll}1-1-2 & & 3 & 1.00400 & 0.10818 & 0.77466 & 1.2333 \\ 1-2-1 & & 3 & 0.99552 & 0.10818 & 0.76618 & 1.2249\end{array}$

$\begin{array}{llll}1-2-2 & 3 & 0.99425 & 0.10818\end{array}$

$\begin{array}{llll}2-1-1 & 3 & 1.01037 & 0.10818 \\ 2-1-2 & 3 & 0.70921 & 0.0818\end{array}$

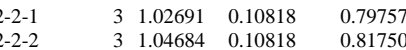

or variance
Oneway Analysis of wt\% By Set/Block/Sub-Block Glass ID=U std, nnalyte $=\mathbf{P b O}(\mathbf{w t} \%)$

Reference Value $=0.0 \mathrm{wt} \%$

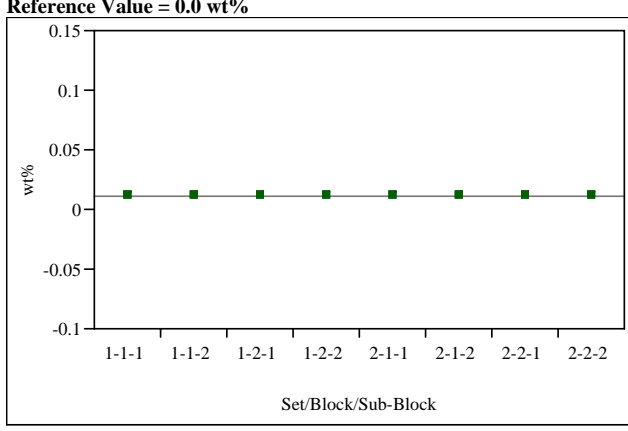

Oneway Anova

Rsquare

Adj Rsquare $\quad 5.3125$

Mean of Response

Mean of Response
Observations (or Sum Wgts)

Analysis of Variance

Source DF Sum of Squares Mean Square F Ratio Prob $>$ F

$\begin{array}{lrrrrr}\text { Set/Block/Sub-Block } & 7 & 2.8889 \mathrm{e}-34 & 4.127 \mathrm{e}-35 & -3.0476 & 0.0000 \\ \text { Error } & 16 & -2.167 \mathrm{e}-34 & -1.35 \mathrm{e}-35 & & \end{array}$

$\begin{array}{ll}16 & -2.167 \mathrm{e}-34 \\ 23 & 7.2222 \mathrm{e}-35\end{array}$

Std Error Lower 95\% Upper 95\%

Means for Oneway Anova

$\begin{array}{rrrr}\text { Level } & \text { Number } & \text { Mean } \\ 1-1-1 & 3 & 0.010772 \\ 1-1-2 & 3 & 0.01072\end{array}$

$\begin{array}{lll}1-1-2 & 3 & 0.010772 \\ 1-2-1 & 3 & 0.010772\end{array}$

$\begin{array}{lll}1-2-1 & 3 & 0.010772 \\ 1-2-2 & 3 & 0.010772\end{array}$

$\begin{array}{lll}2-1-1 & 3 & 0.010772 \\ 2-1-2 & 3 & 0.010772\end{array}$

$2-2-1$

$2-2-2 \quad 3 \quad 0.010772$

Std Error uses a pooled estimate of error variance 


\section{Exhibit A3. PSAL Measurements by Analytical Block for Samples of the}

\section{Standard Glasses Prepared Using the LM Method}

Oneway Analysis of wt\% By Set/Block/Sub-Block Glass ID=U std,

Analyte $=\mathrm{SiO} 2(\mathrm{wt} \%)$

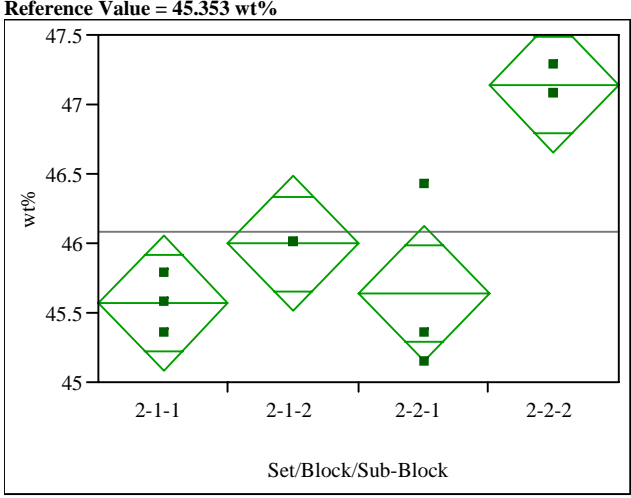

Missing Rows

12

Oneway Anova

$\begin{array}{ll}\text { Rsquare } & 0.816152 \\ \text { Adj Rsquare } & 0.747209\end{array}$

Root Mean Square Error $\quad 0.365355$

Mean of Response 46.0840

Analysis of Variance
Source

$\begin{array}{lrrrrr}\text { Set/Block/Sub-Block } & 3 & 4.7405995 & 1.58020 & 11.8381 & 0.0026\end{array}$

Error

1.0678744

0.13348

Means for Oneway Anova

Level Number Mean Std Error Lower 95\% Upper 95\%

$\begin{array}{llllll}2-1-1 & 3 & 45.5671 & 0.21094 & 45.081 & 46.054\end{array}$

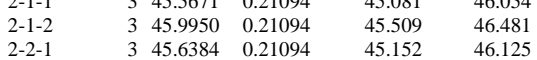

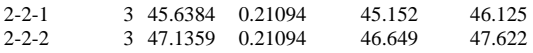

Std Error uses a pooled estimate of error variance
Oneway Analysis of wt\% By Set/Block/Sub-Block Glass ID=U std, Analyte $=\mathrm{SO} 4(\mathrm{wt} \%)$

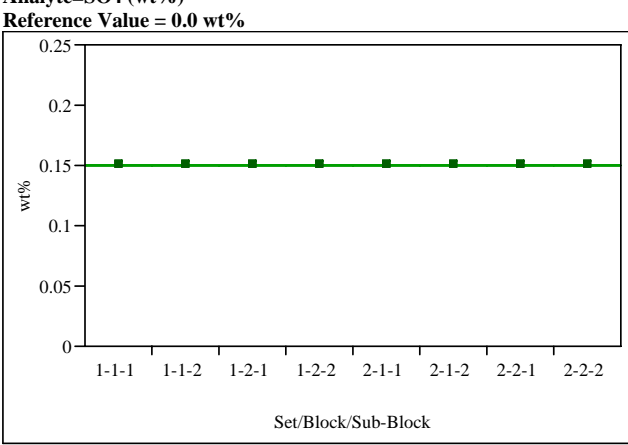

Oneway Anova

Adj Rsquare

Root Mean Square Erro

Mean of Respons

0.149795

Analysis of Variance

Source DF Sum of Squares Mean Square F Ratio Prob > F

Set/Block/Sub-Block

Error
C. Total

16
23

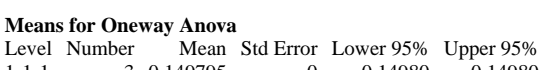

$\begin{array}{lrrrrr}1-1-1 & 3 & 0.149795 & 0 & 0.14980 & 0.14980 \\ 1-1-2 & 3 & 0.14995 & 0 & 0.1490 & 0.14980\end{array}$

$\begin{array}{llll}1-1-1 & & 3 & 0.149795 \\ 1-2-149795 & & 3 & 0.19795\end{array}$

$\begin{array}{lll}1-2-1 & 3 & 0.149795 \\ 1-2-2 & 3 & 0.149795\end{array}$

$\begin{array}{lll}2-1-1 & 3 & 0.149795 \\ & 3 & 0.14795\end{array}$

$2-2-1-30.140 .149795$

30.149795

$\begin{array}{lll}0 & 0.14980 & 0.14980 \\ 0 & 0.14980 & 0.14980 \\ 0 & 0.14980 & 0.14980 \\ 0 & 0.14980 & 0.14980 \\ 0 & 0.14980 & 0.14980 \\ 0 & 0.14980 & 0.14980 \\ 0 & 0.14980 & 0.14980 \\ 0 & 0.14980 & 0.14980\end{array}$

Std Error uses a pooled estimate of error variance
Oneway Analysis of wt \% By Set/Block/Sub-Block Glass ID=U std, Analyte $=$ TiO2 (wt $\%)$

Reference Value $=1.049 \mathrm{wt} \%$

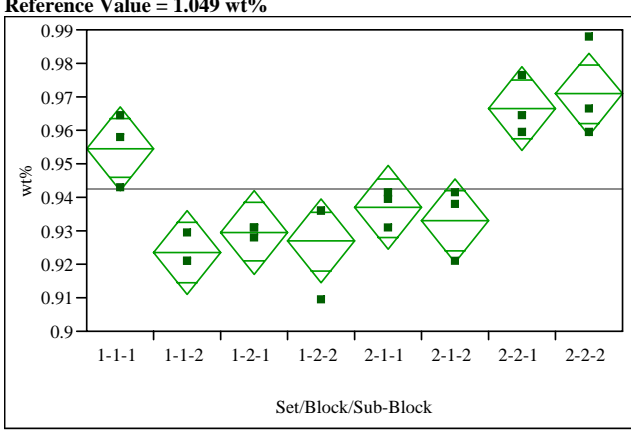

Oneway Anova

Rsquare $\quad 0.814172$

0.732873
Adj Rsquare

$\begin{array}{ll}\text { Root Mean Square Error } & 0.010152 \\ \text { Mean of Response } & 0.942698\end{array}$

Observations (or Sum Wgts)

Analysis of Variance
Source

$\begin{array}{llllll}\text { Set/Block/Sub-Block } & 7 & 0.00722451 & 0.001032 & 10.0145 & <.0001\end{array}$

$\begin{array}{llll}\text { Error } & 16 & 0.00164893 & 0.000103 \\ \text { C. Total } & 23 & 0.00887344 & \end{array}$

Means for Oneway Anova

Level Number Mean Std Error Lower 95\% Upper 95\%

$\begin{array}{lllllll}1-1-1 & 3 & 0.954652 & 0.00586 & 0.94223 & 0.96708\end{array}$

$\begin{array}{llllll}1-1-2 & 3 & 0.923516 & 0.00586 & 0.91109 & 0.93594\end{array}$

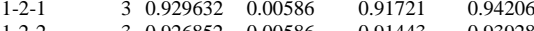

$\begin{array}{llllll}1-2-2 & 3 & 0.926852 & 0.00586 & 0.91443 & 0.93928 \\ 2-1-1 & 3 & 0.93680 & 0.00586 & 0.9243 & 0.9429\end{array}$

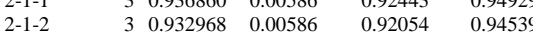

$\begin{array}{llllll}2-2-1 & 3 & 0.966328 & 0.00586 & 0.95390 & 0.97875\end{array}$

$\begin{array}{llllll}2-2-2 & 3 & 0.970776 & 0.00586 & 0.95835 & 0.98320\end{array}$

Std Error uses a pooled estimate of error variance 


\section{Exhibit A3. PSAL Measurements by Analytical Block for Samples of the}

\section{Standard Glasses Prepared Using the LM Method}

Oneway Analysis of wt $\%$ By Set/Block/Sub-Block Glass ID=U std, Analyte=U308 (wt \%)

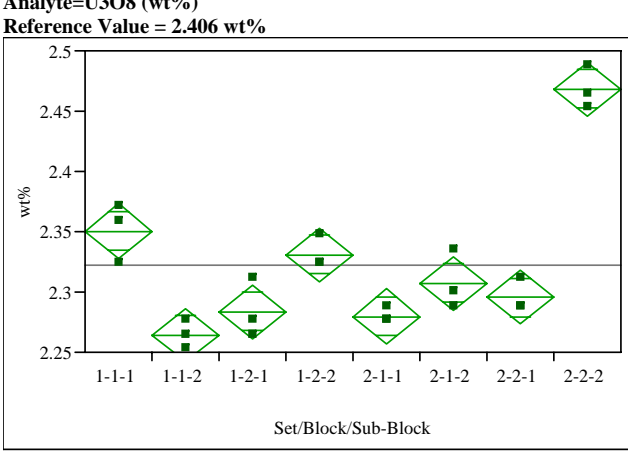

Oneway Anova

Rsquare
Adj Rsquar

0.943384
0.918614

0.918614

Mean of Response

0.018331

Observations (or Sum Wgts)

$\begin{array}{lrrrrr}\text { Analysis of Variance } & & & & & \\ \text { Source } & \text { DF } & \text { Sum of Squares } & \text { Mean Square } & \text { F Ratio } & \text { Prob }>\text { F } \\ \text { Set/Block/Sub-Block } & 7 & 0.08958957 & 0.012799 & 38.0862 & <.0001 \\ \text { Error } & 16 & 0.00537665 & 0.000336 & & \\ \text { C. Total } & 23 & 0.09496622 & & & \end{array}$

Means for Oneway Anova

Level Number Mean Std Error Lower 95\% Upper 95\%

$\begin{array}{lllllll}1-1-1 & & 3 & 2.35054 & 0.01058 & 2.3281 & 2.3730 \\ 1-1-2 & & 3 & 2.26406 & 0.01058 & 2.2416 & 2.2865\end{array}$

$\begin{array}{llll}1-2-1 & 3 & 2.28372 & 0.01058\end{array}$

$\begin{array}{llll}3 & 2.33089 & 0.01058 \\ 3 & 2.27979 & 0.01058\end{array}$

$\begin{array}{llll}2-1-2 & 3 & 2.30730 & 0.01058\end{array}$

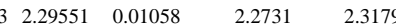

Std Error uses a pooled estimate of error variance
Oneway Analysis of wt\% By Set/Block/Sub-Block Glass ID=U std, Analyte $=\mathbf{Z n O}(\mathrm{wt} \%)$

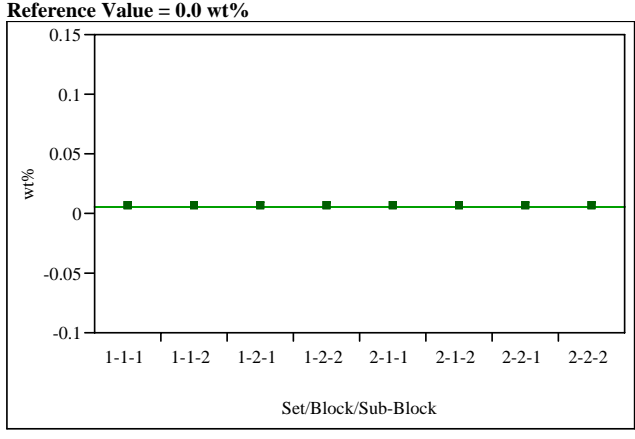

\section{Oneway Anova}

Rsquare

Adj Rsquare

Root Mean Square Erro

$\begin{array}{lr}\text { Mean of Response } & 0.006224 \\ \text { Observations (or Sum Wgts) } & 24\end{array}$

Analysis of Variance

Source DF Sum of Squares Mean Square F Ratio Prob > F

Set/Block/Sub-Block
Error

Error

7
16
23

Means for Oneway Anova

Level Number Mean Std Error Lower 95\% Upper 95\%

$\begin{array}{lrrrrr}\text { Level } & \text { Number } & \text { Mean } & \text { Std Error } & \text { Lower 95\% } & \text { Upper 95\% } \\ 1-1-1 & 3 & 0.006224 & 0 & 0.00622 & 0.00622 \\ 1-1-2 & 3 & 0.006224 & 0 & 0.00622 & 0.00622\end{array}$

30.006224

$\begin{array}{lll}3 & 0.006224 \\ 3 & 0.006224\end{array}$

$\begin{array}{ll}3 & 0.006224 \\ 3 & 0.006224\end{array}$

$\begin{array}{lll}2-2-1 & 3 & 0.006224 \\ 2-2-2 & 3 & 0.006224\end{array}$

$\begin{array}{lll}0.00622 & 0.00622 \\ 0.00622 & 0.00622\end{array}$

$\begin{array}{llll}0 & 0.00622 & 0.00622 \\ 0 & 0.00622\end{array}$

0.00622

Std Error uses a pooled estimate of error variance
Oneway Analysis of wt\% By Set/Block/Sub-Block Glass ID=U std, Analyte=ZrO2 (wt\%)

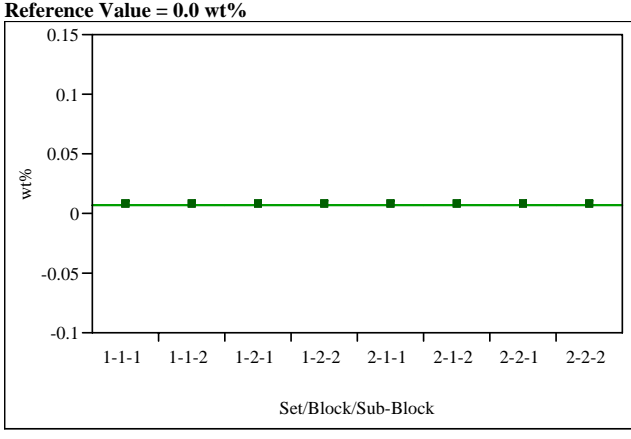

Oneway Anova

Rsquare

Adj Rsquare

$\begin{array}{lr}\text { Mean of Response } & 0.006754 \\ \text { Observations (or Sum Wgts) } & 24\end{array}$

Analysis of Variance

Source DF Sum of Squares Mean Square F Ratio Prob > F

Set/Block/Sub-Block

$\begin{array}{ll}\text { Error } & 16 \\ \text { C. Total } & 23\end{array}$

Means for Oneway Anova

Level Number Mean Std Error Lower 95\% Upper 95\%

$\begin{array}{llllll}1-1-1 & 3 & 0.006754 & 0 & 0.00675 & 0.00675 \\ 1-1-2 & 3 & 0.006754 & 0 & 0.0075 & 0.00675\end{array}$

$\begin{array}{llllll}1-1-2 & 3 & 0.006754 & 0 & 0.00675 & 0.00675 \\ 1-2-1 & 3 & 0.006754 & 0 & 0.00675 & 0.00655\end{array}$

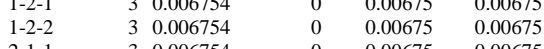

$\begin{array}{llllll}2-1-2 & 3 & 0.006754 & 0 & 0.00675 & 0.00675\end{array}$

$\begin{array}{lll}0 & 0.00675 & 0.00675 \\ 0 & 0.00675 & 0.00675\end{array}$

$\begin{array}{llllll}2-2-2 & 3 & 0.006754 & 0 & 0.00675 & 0.00675\end{array}$

Std Error uses a pooled estimate of error variance 


\section{Exhibit A4: PSAL Measurements by Analytical Block for Samples of the Standard Glasses Prepared Using the PF Method}

Oneway Analysis of wt\% By Set/Block/Sub-Block Glass ID=Batch 1, Analyte $=$ B2O3 ( $w$ t $\%)$

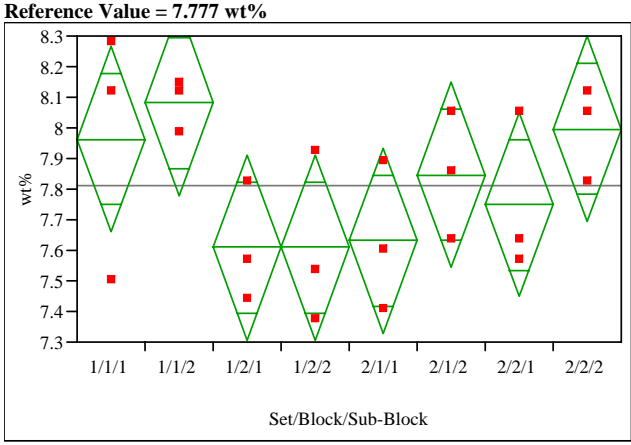

Oneway Anova

$\begin{array}{ll}\text { Rsquare } & 0.434883 \\ \text { Adj Rsquare } & 0.187644 \\ \text { Root Mean Square Error } & 0.246538\end{array}$

$\begin{array}{lr}\text { Root Mean Square Error } & 0.246538 \\ \text { Mean of Response } & 7.810941 \\ \text { Observations (or Sum Wgts) } & 24\end{array}$

Analysis of Varianc

Source DF Sum of Squares Mean Square F Ratio Prob > F

$\begin{array}{lrllll}\text { Set/Block/Sub-Block } & 7 & 0.7483792 & 0.106911 & 1.7590 & 0.1653\end{array}$

C. Total

1.7208747

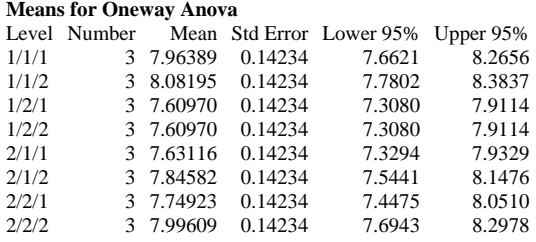

Std Error uses a pooled estimate of error variance
Oneway Analysis of wt\% By Set/Block/Sub-Block Glass ID=Batch 1, Analyte $=\mathrm{Li2O}$ (wt \%)

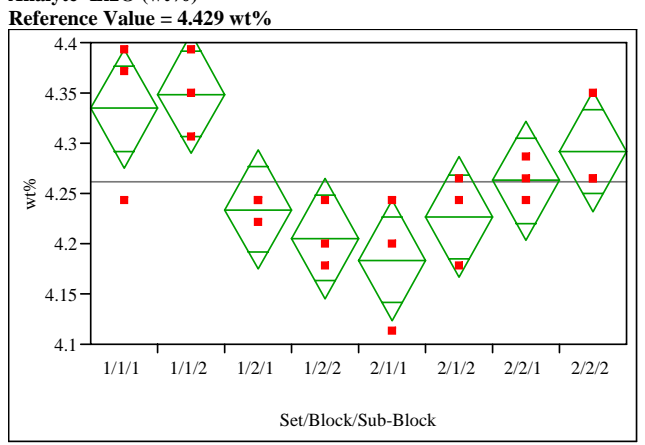

Oneway Anova
Summary of Fit

$\begin{array}{lr}\text { Rsquare } & 0.663705 \\ \text { Adj Rsquare } & 0.516576 \\ \text { Root Mean Square Error } & 0.048738 \\ \text { Mean of Response } & 4.260948 \\ \text { Observations (or Sum Wgts) } & 24\end{array}$

Analysis of Variance

DF Sum of Squares Mean Square F Ratio Prob $>F$

$\begin{array}{lllrlll}\text { Set/Block/Sub-Block } & 7 & 0.07500940 & 0.010716 & 4.5110 & 0.0060\end{array}$

C. Total

$\begin{array}{ll}16 & 0.03800682 \\ 23 & 0.11301622\end{array}$

Means for Oneway Anova

Level Number Mean Std Error Lower 95\% Upper 95\%

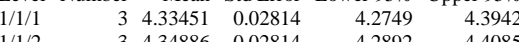

$\begin{array}{lllllll}1 / 1 / 2 & & 3 & 4.34886 & 0.02814 & 4.2892 & 4.4085 \\ 1 / 2 / 1 & & 3 & 4.23404 & 0.02814 & 4.1744 & 4.2937\end{array}$

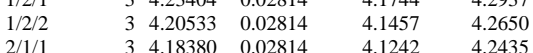

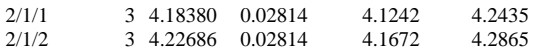

$\begin{array}{llllll}2 / 2 / 1 & 3 & 4.26274 & 0.02814 & 4.2031 & 4.3224\end{array}$

Std Error uses a pooled estimate of error variance
Oneway Analysis of wt\% By Set/Block/Sub-Block Glass ID=Batch 1

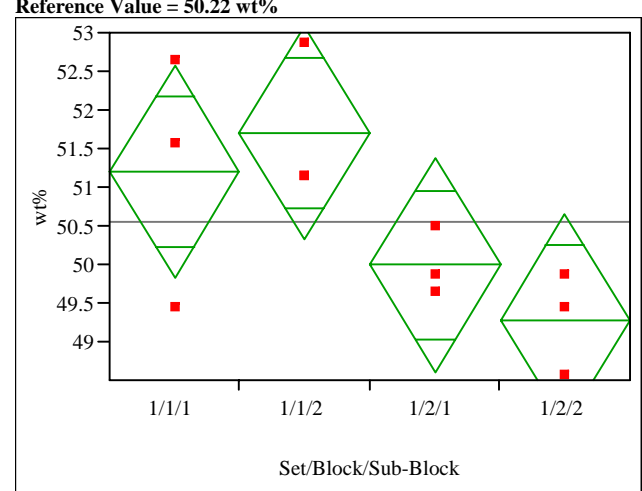

Oneway Anova
Summary of Fit

Rsquare

0.564118

Root Mean Square Error $\quad 0.400662$

Observations (or Sum Wgts) 50.54096

Analysis of Variance

Source DF Sum of Squares Mean Square F Ratio Prob $>$ F $\begin{array}{lrrrrr}\text { Set/Block/Sub-Block } & 3 & 11.056314 & 3.68544 & 3.4512 & 0.0716\end{array}$ C. Total $\begin{array}{rr}8 & 8.54299 \\ 11 & 1959930\end{array}$

Means for Oneway Anova

Level Number Mean Std Error Lower 95\% Upper 95\%

$\begin{array}{llllll}1 / 1 / 1 & 3 & 51.2006 & 0.59662 & 49.825 & 52.576 \\ 11 / 1 / 2 & & 3 & 51.6998 & 0.59662 & 50.324\end{array}$

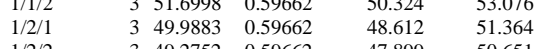

Std Error uses a pooled estimate of error variance 


\section{Exhibit A4: PSAL Measurements by Analytical Block for Samples of the Standard Glasses Prepared Using the PF Method}

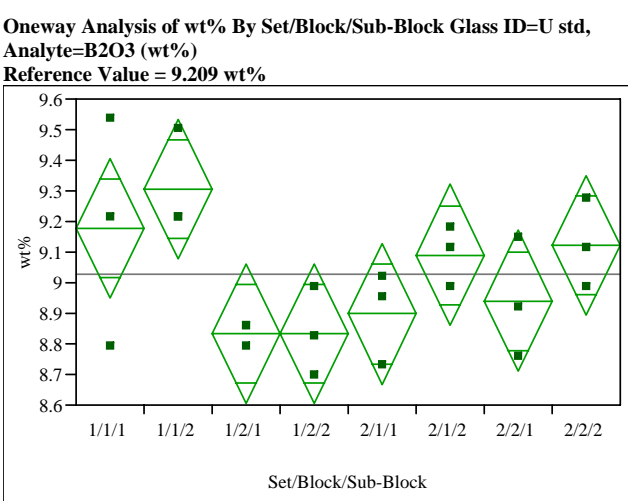

\section{Oneway Anova}

$\begin{array}{lr}\text { Rsquare } & 0.532451 \\ \text { Adj Rsquare } & 0.327898 \\ \text { Root Mean Square Error } & 0.187059 \\ \text { Mean of Response } & 9.025111 \\ \text { Observations (or Sum Wgts) } & 24\end{array}$

Analysis of Varianc

Source DF Sum of Squares Mean Square F Ratio Prob > F

$\begin{array}{lllllll}\text { Set/Block/Sub-Block } & 7 & 0.6375738 & 0.091082 & 2.6030 & 0.0536\end{array}$

Error

1.1974326

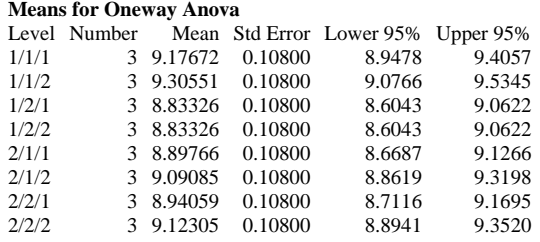

Std Error uses a pooled estimate of error variance
Oneway Analysis of $w t \%$ By Set/Block/Sub-Block Glass ID=U std Oneway Analysis of $w$ Analyte $=\mathrm{Li} 2 \mathrm{O}(\mathrm{wt} \%)$

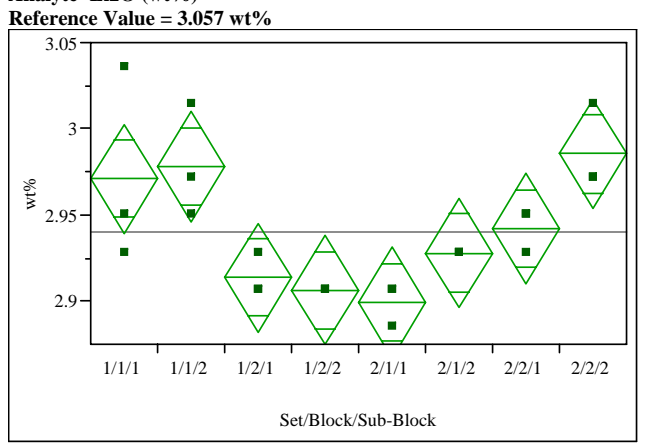

\section{Oneway Anova
Summary of Fit}

$\begin{array}{lr}\text { Rsquare } & 0.692308 \\ \text { Adj Rsquare } & 0.557692 \\ \text { Root Mean Square Error } & 0.025999 \\ \text { Mean of Response } & 2.940503 \\ \text { Observations (or Sum Wgts) } & 24\end{array}$

Analysis of Variance

DF Sum of Squares Mean Square F Ratio Prob >

$\begin{array}{lllllll}\text { Set/Block/Sub-Block } & 7 & 0.02433364 & 0.003476 & 5.1429 & 0.0032\end{array}$

C. Total

$23 \quad 0.03514859$

Means for Oneway Anova

Level Number Mean Std Error Lower 95\% Upper 95\%

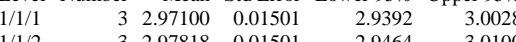

$\begin{array}{llllll}1 / 1 / 2 & 3 & 2.97818 & 0.01501 & 2.93964 & 3.0100 \\ 1 / 2 / 1 & 3 & 2.91359 & 0.01501 & 2.8818 & 2.9454\end{array}$

$\begin{array}{lllllll}1 / 2 / 2 & 3 & 2.90642 & 0.01501 & 2.8746 & 2.9382\end{array}$

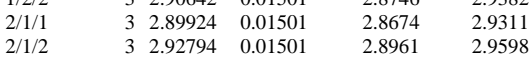

\begin{tabular}{llllll}
$2 / 2 / 1$ & 3 & 2.92794 & 0.01501 & 2.8674 & 2.931 \\
\hline & 3 & 2.94230 & 0.01501 & 2.9105 & 2.9598 \\
2 & & & 2.974
\end{tabular}

Std Error uses a pooled estimate of error variance
Oneway Analysis of $w t \%$ By Set/Block/Sub-Block Glass ID=U std, Analyte $=$ SiO2 $(w \%)$

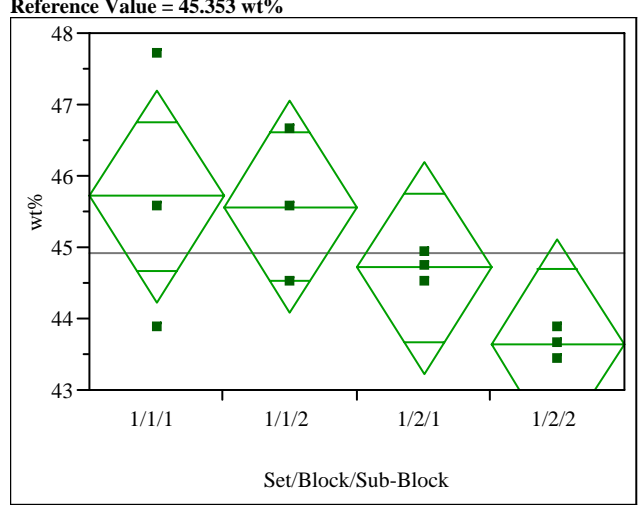

Oneway Anova

Rsquare

Root Mean Square Error $\quad 1.113327$

$\begin{array}{lr}\text { Mean of Response } & 44.90747 \\ \text { Observations (or Sum Wgts) } & 12\end{array}$

Analysis of Variance
Source DF Sum of Squares Mean Square F Ratio Prob $>$ F $\begin{array}{lrrrrr}\text { Source } & \text { DF } & & & & \\ \text { Set/Block/Sub-Block of Squares } & 3 & 8.157798 & 2.71927 & 2.1938 & 0.1665\end{array}$ $\begin{array}{lrrr}\text { Error } & 8 & 9.915976 & 1.23950 \\ \text { C. Total } & 11 & 18.073774 & \end{array}$

Means for Oneway Anova

Level Number Mean Std Error Lower 95\% Upper 95\%

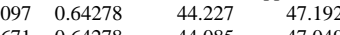

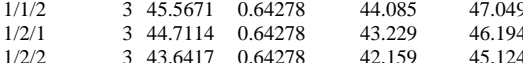

$\begin{array}{llllll}1 / 2 / 2 & 3 & 43.6417 & 0.64278 & 42.159 & 45.124\end{array}$

Std Error uses a pooled estimate of error variance 
Exhibit A5. Oxide Measurements by Solution ID for Samples Prepared Using the LM Method

Set $=1$

Variability Chart for Al2O3 (wt\%)

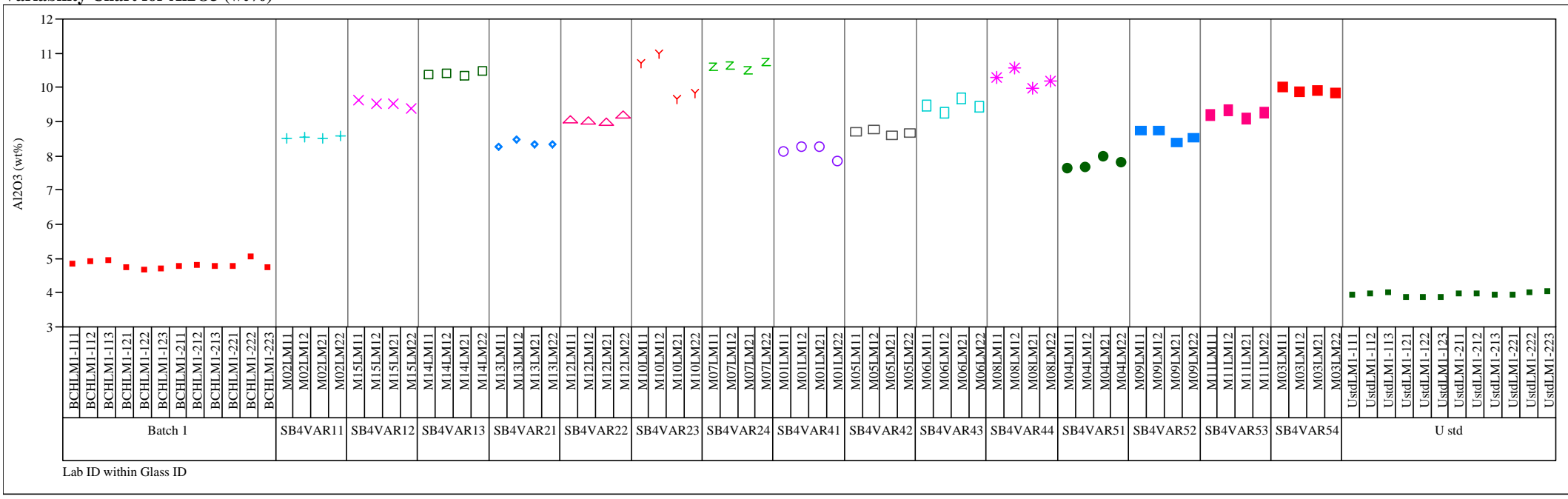

Set $=1$

Variability Chart for BaO (wt \%)

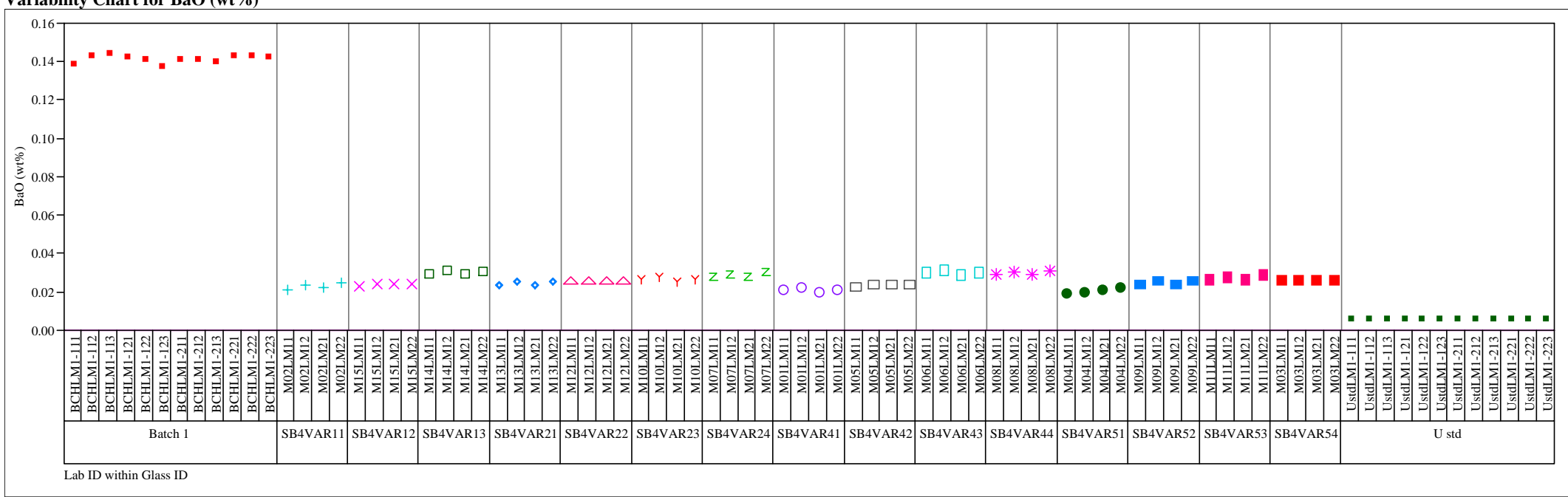


Exhibit A5. Oxide Measurements by Solution ID for Samples Prepared Using the LM Method

Set $=1$

Variability Chart for $\mathrm{CaO}$ (wt \%)

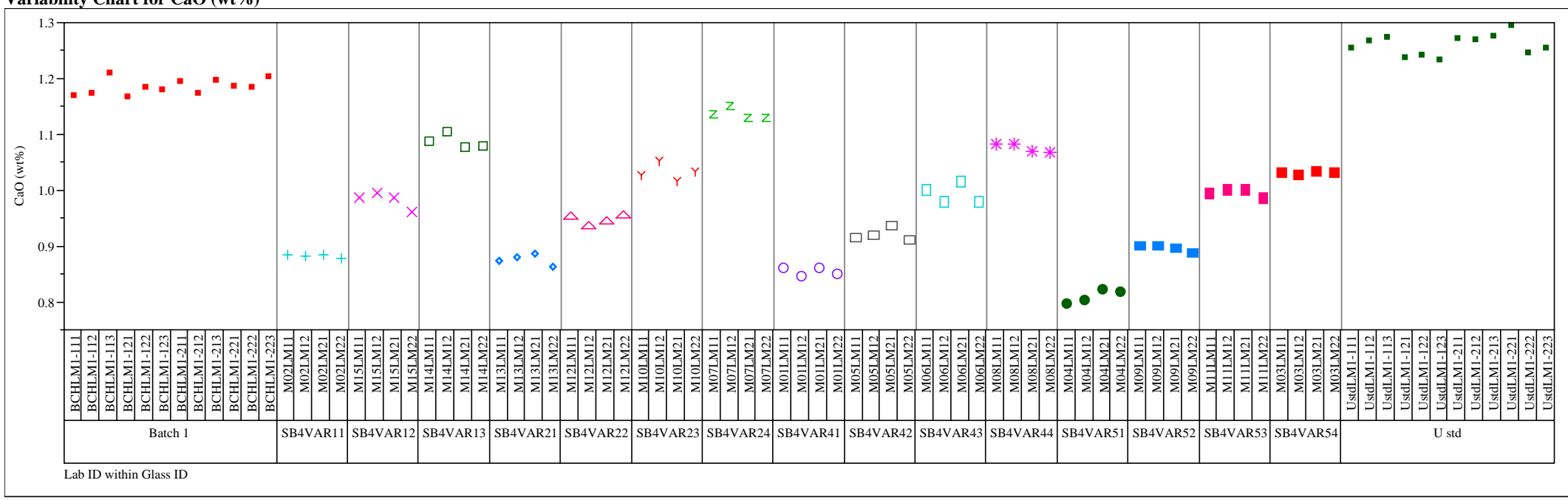

Set $=1$

Variability Chart for CdO (wt \%)

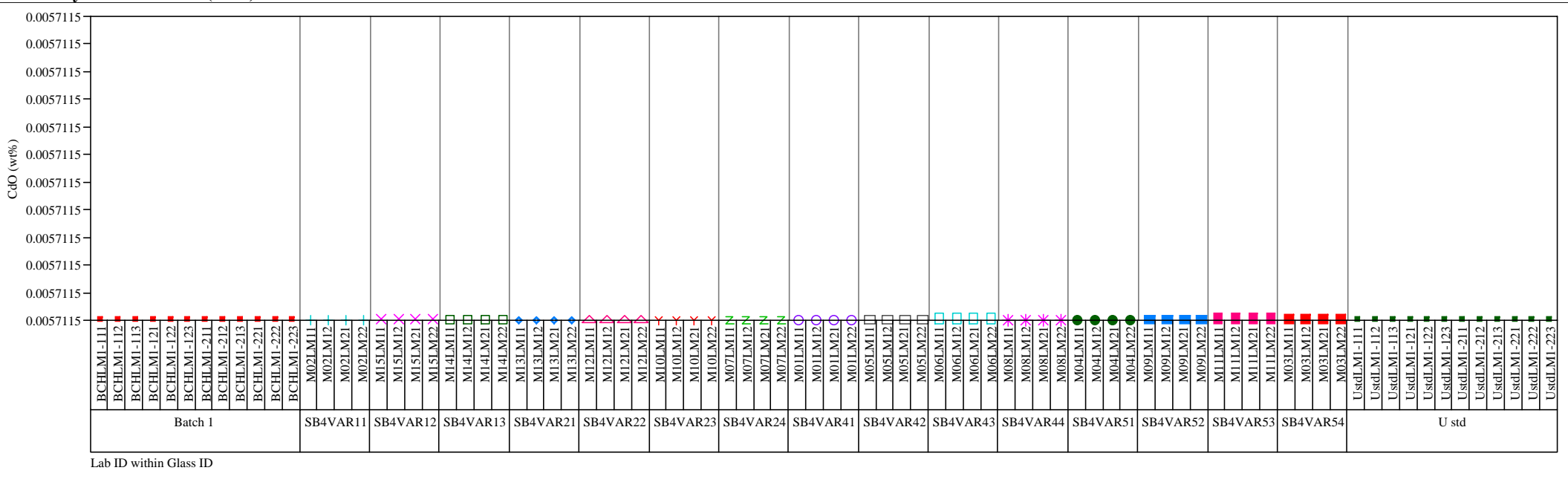




\section{Exhibit A5. Oxide Measurements by Solution ID for Samples Prepared Using the LM Method}

Set $=1$

Variability Chart for Ce2O3 (wt \%)

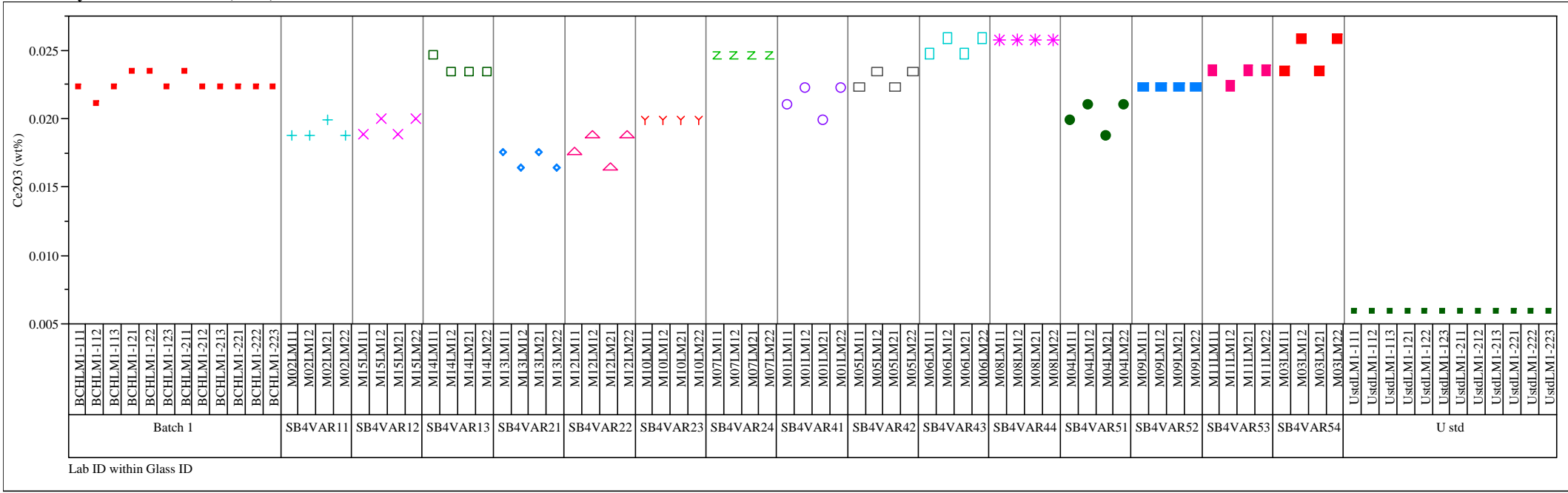

Set $=1$

Variability Chart for Cr2O3 (wt\%)

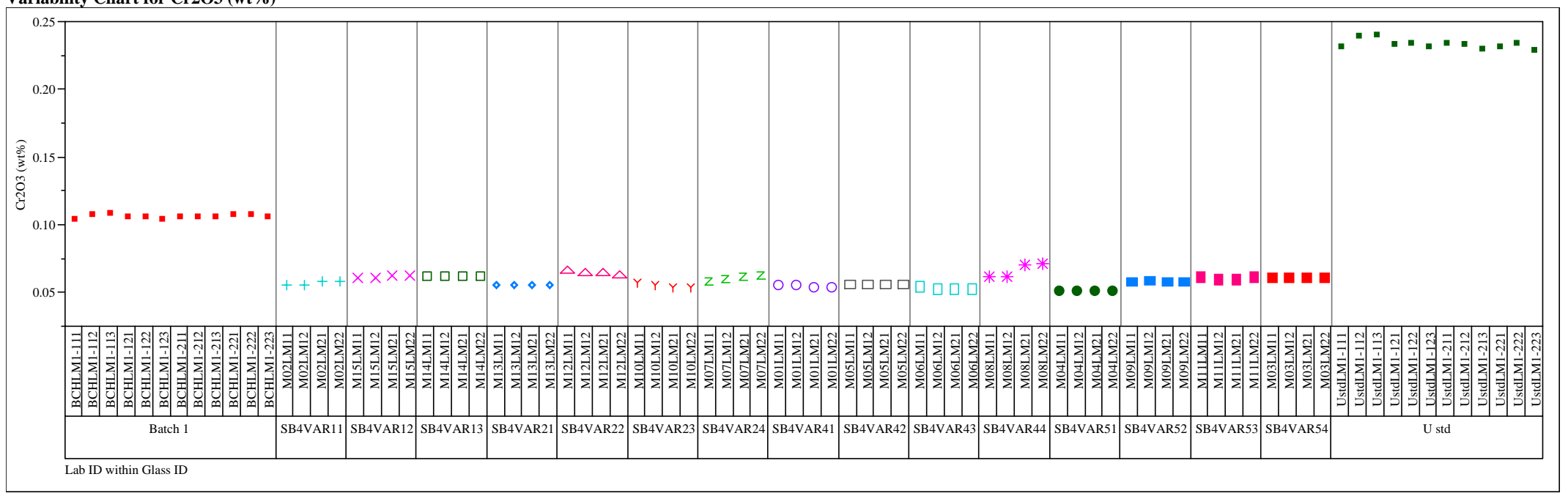


Exhibit A5. Oxide Measurements by Solution ID for Samples Prepared Using the LM Method

Set $=1$

Variability Chart for $\mathrm{CuO}$ (wt \%)

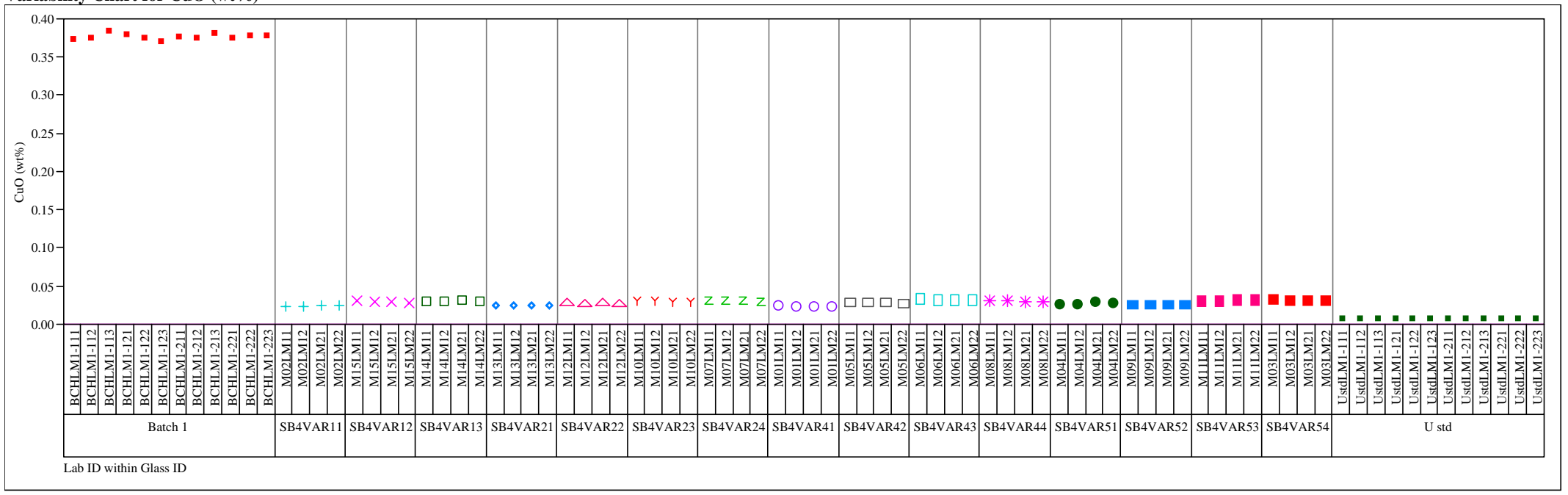

Set $=1$

Variability Chart for Fe2O3 (wt\%)

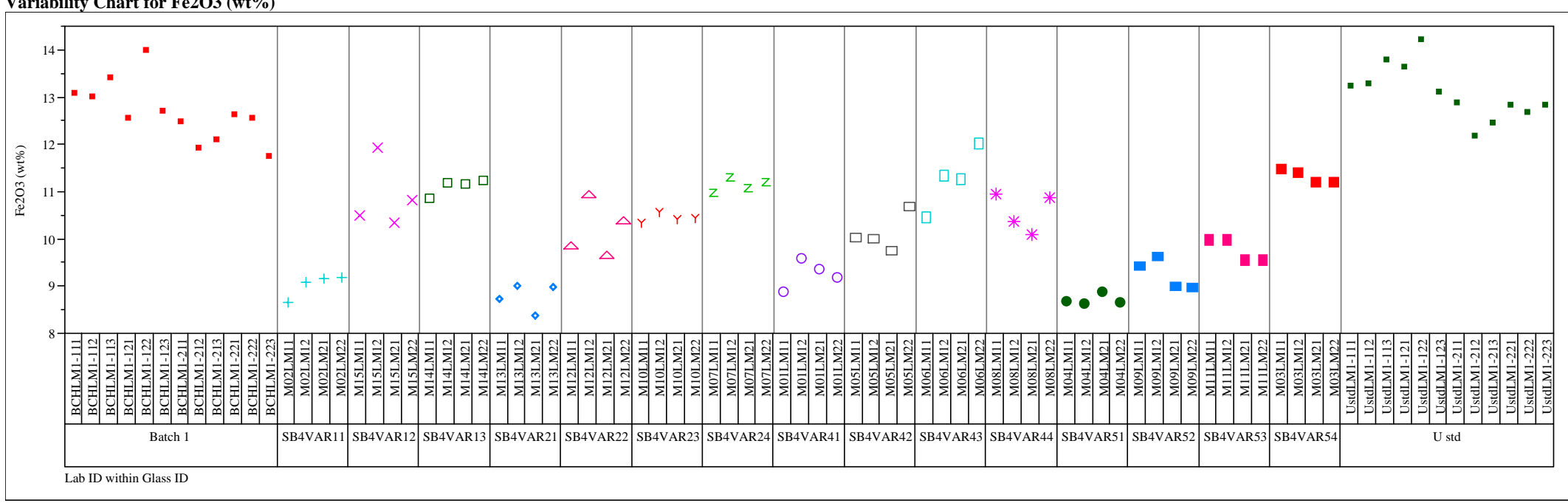


Exhibit A5. Oxide Measurements by Solution ID for Samples Prepared Using the LM Method

Set $=1$

Variability Chart for K2O (wt\%)

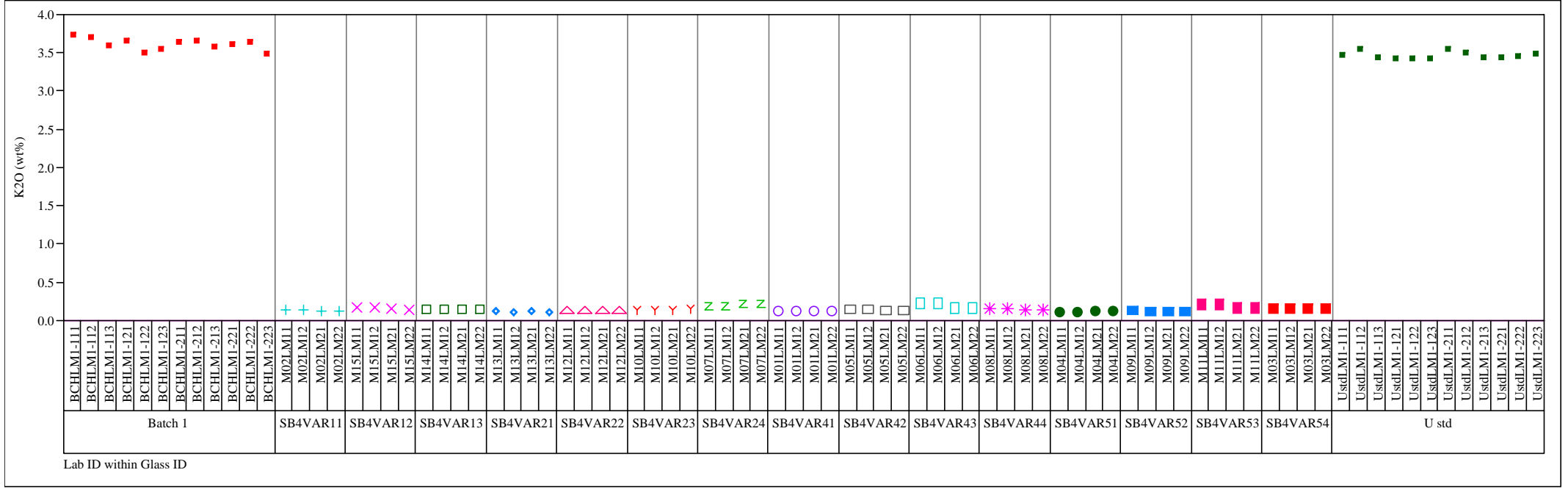

Set $=1$

Variability Chart for La2O3 (wt\%)

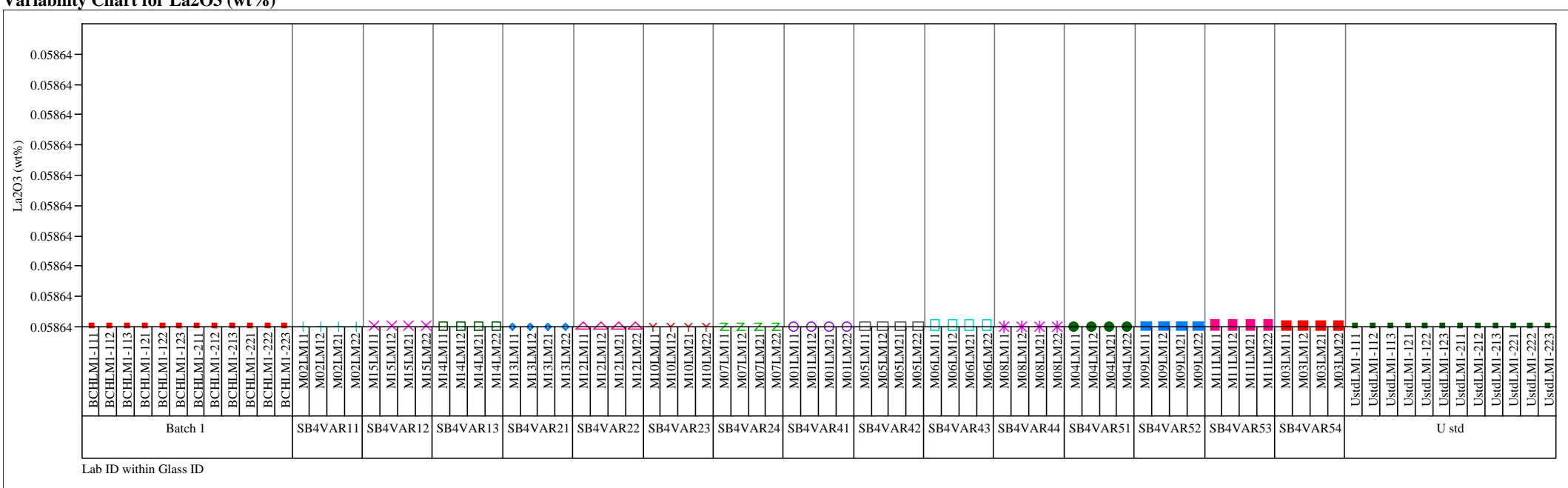




\section{Exhibit A5. Oxide Measurements by Solution ID for Samples Prepared Using the LM Method}

Set $=1$

Variability Chart for MgO (wt\%)

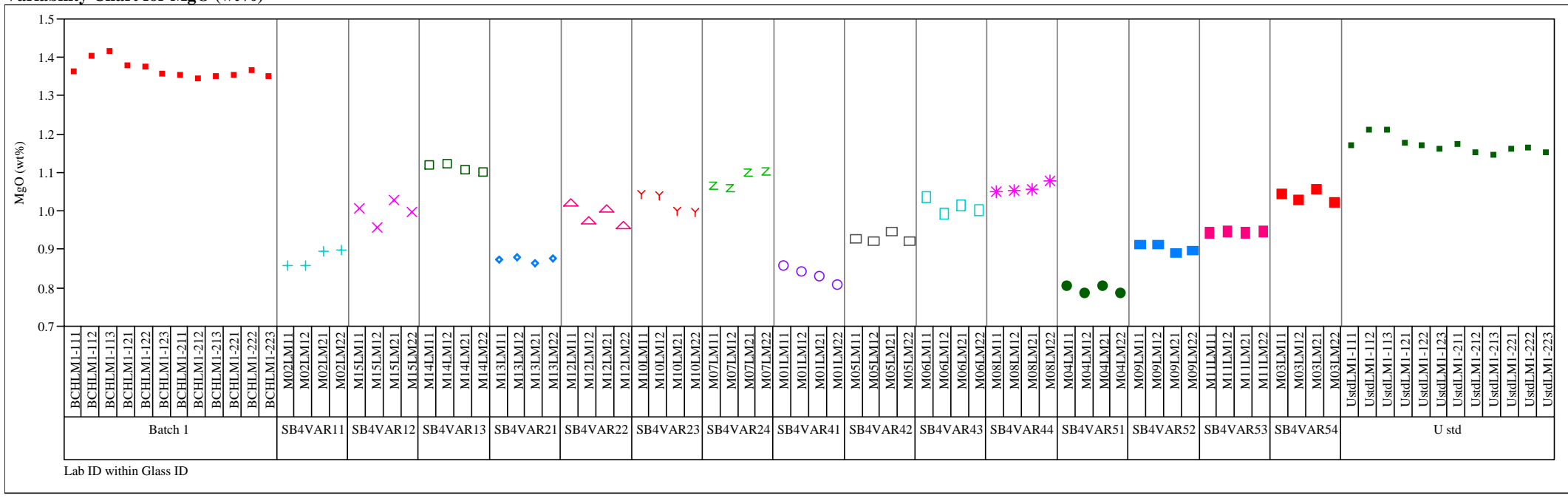

Set $=1$

Variability Chart for MnO (wt\%)

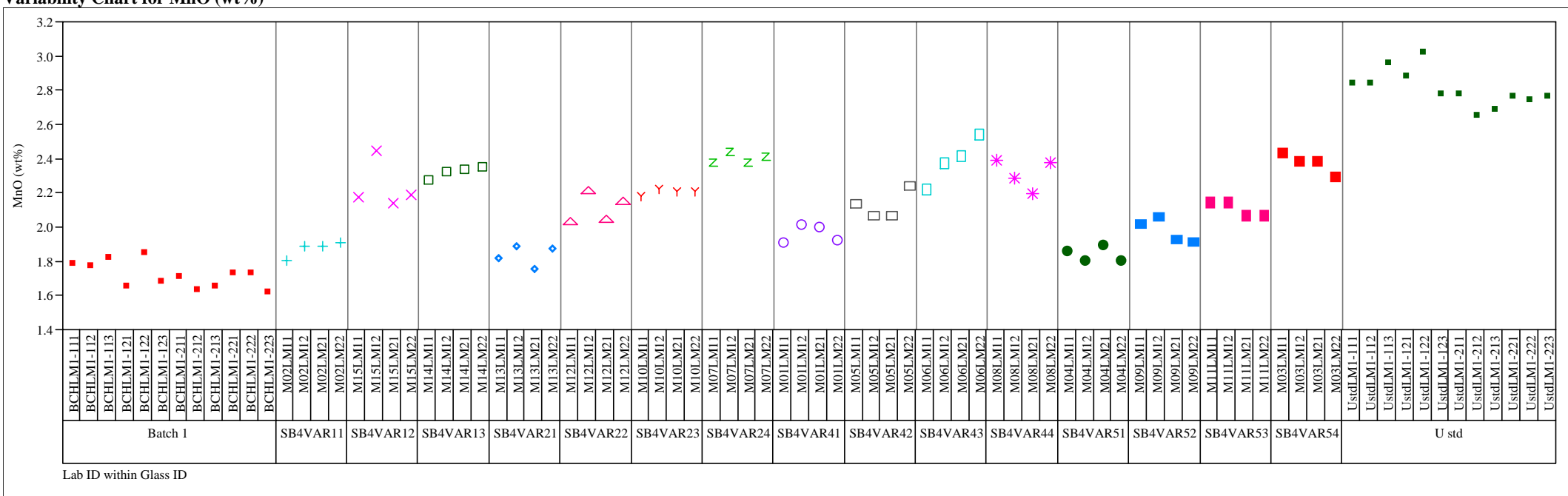




\section{Exhibit A5. Oxide Measurements by Solution ID for Samples Prepared Using the LM Method}

Set $=1$

Variability Chart for Na2O (wt \%)

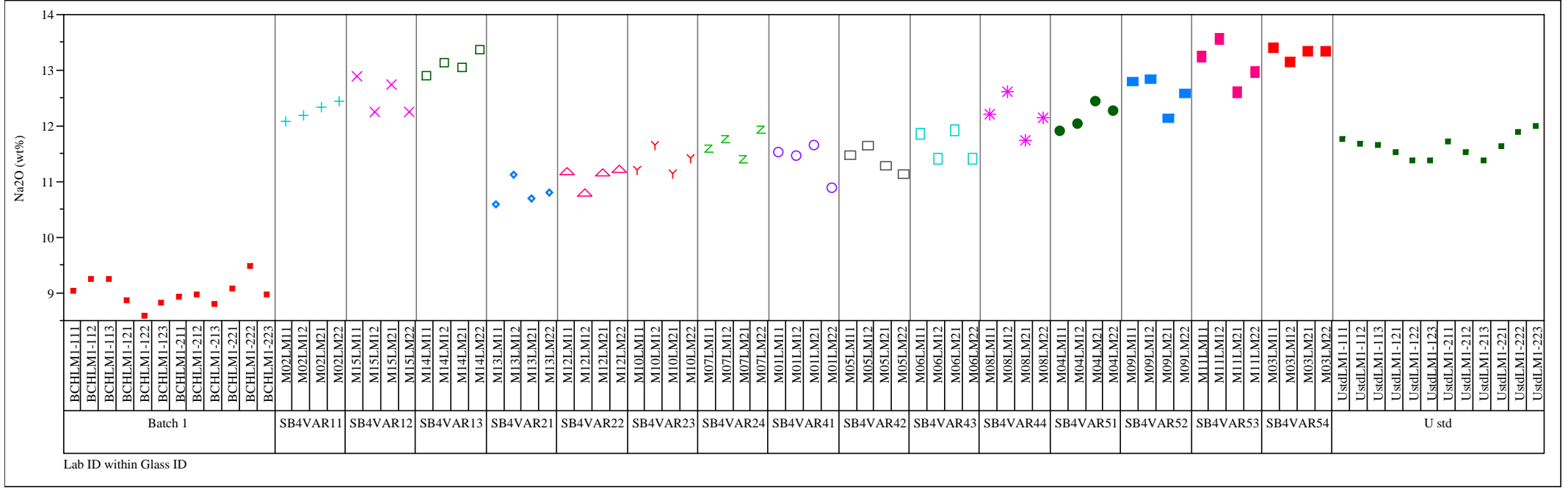

Set $=1$

Variability Chart for NiO (wt \%)

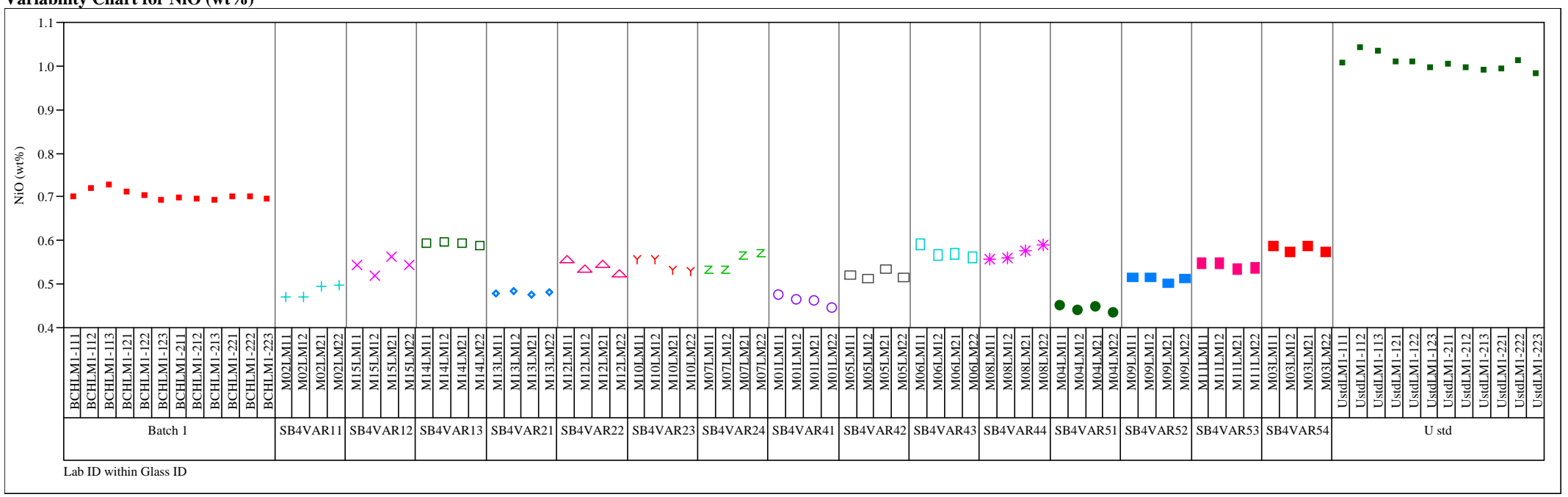


Exhibit A5. Oxide Measurements by Solution ID for Samples Prepared Using the LM Method

Set $=1$

Variability Chart for PbO (wt \%)

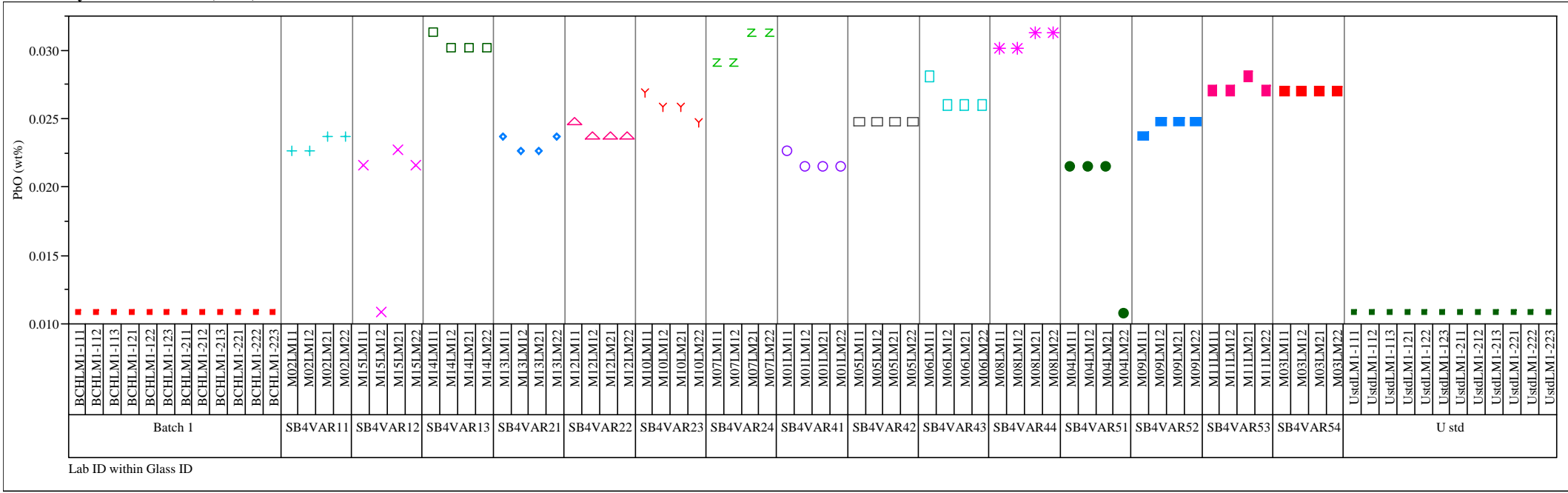

Set $=1$

Variability Chart for SO4 (wt\%)

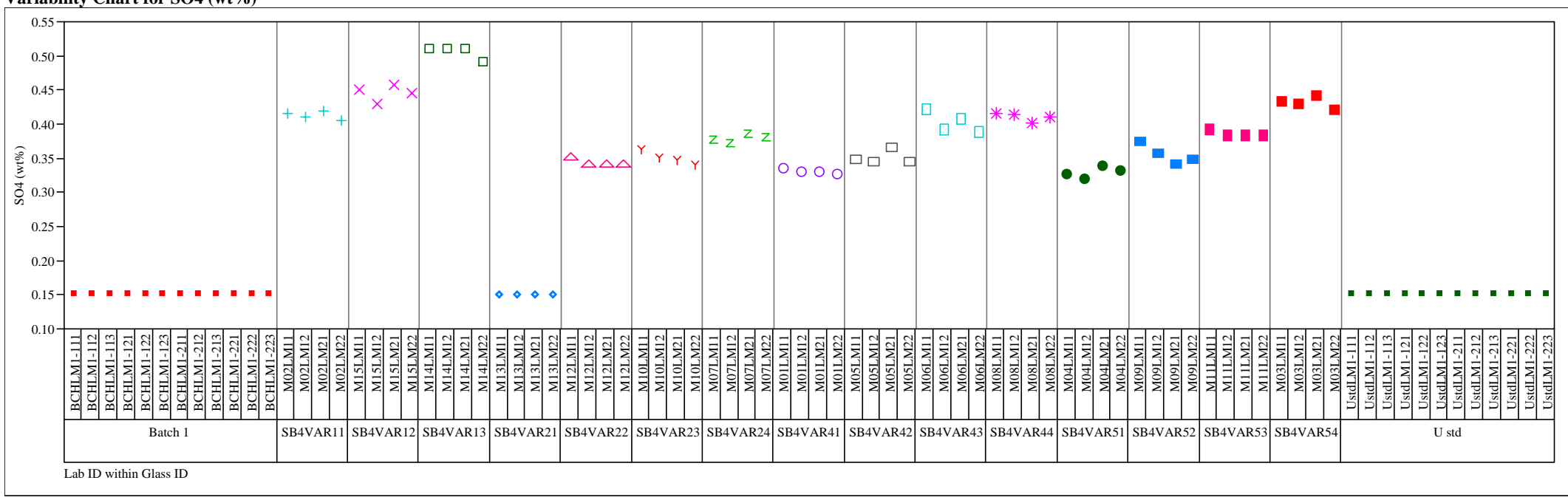


Exhibit A5. Oxide Measurements by Solution ID for Samples Prepared Using the LM Method

Set $=1$

Variability Chart for SiO2 (wt\%)

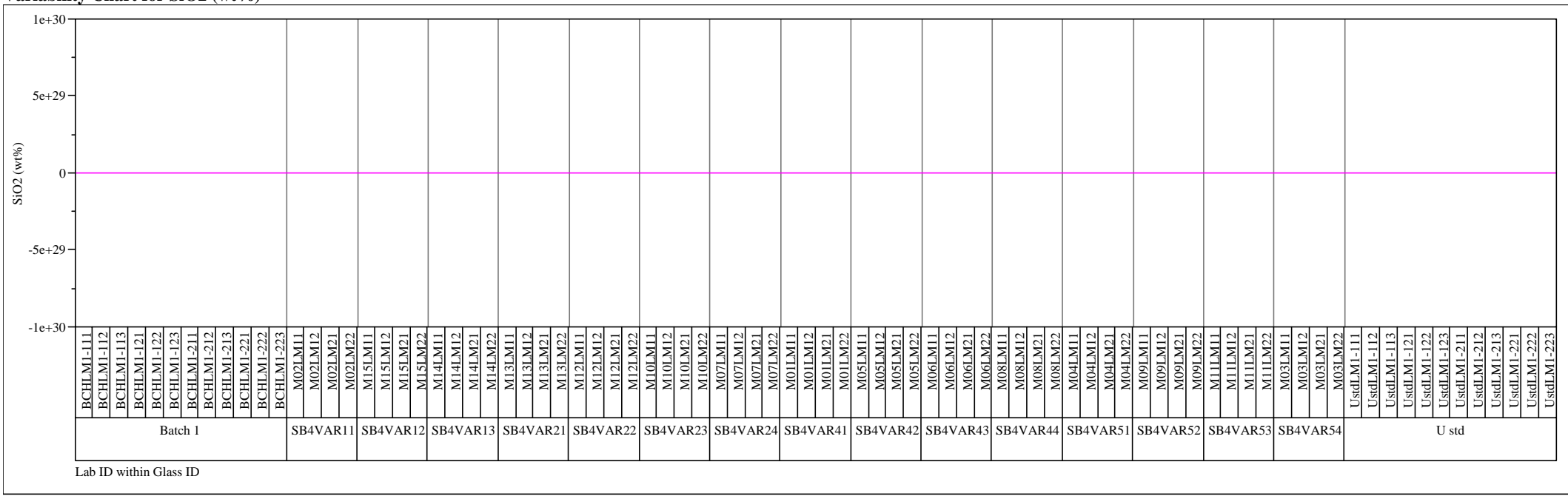

Set $=1$

Variability Chart for TiO2 (wt\%)

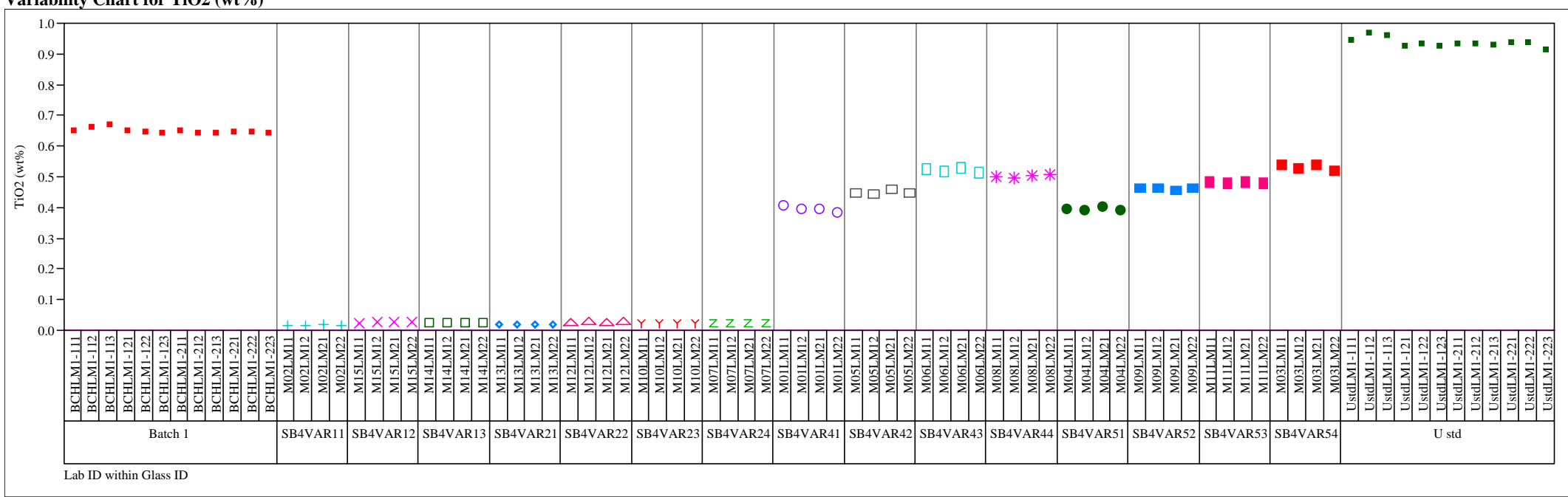




\section{Exhibit A5. Oxide Measurements by Solution ID for Samples Prepared Using the LM Method}

Set $=1$

Variability Chart for U3O8 (wt \%)

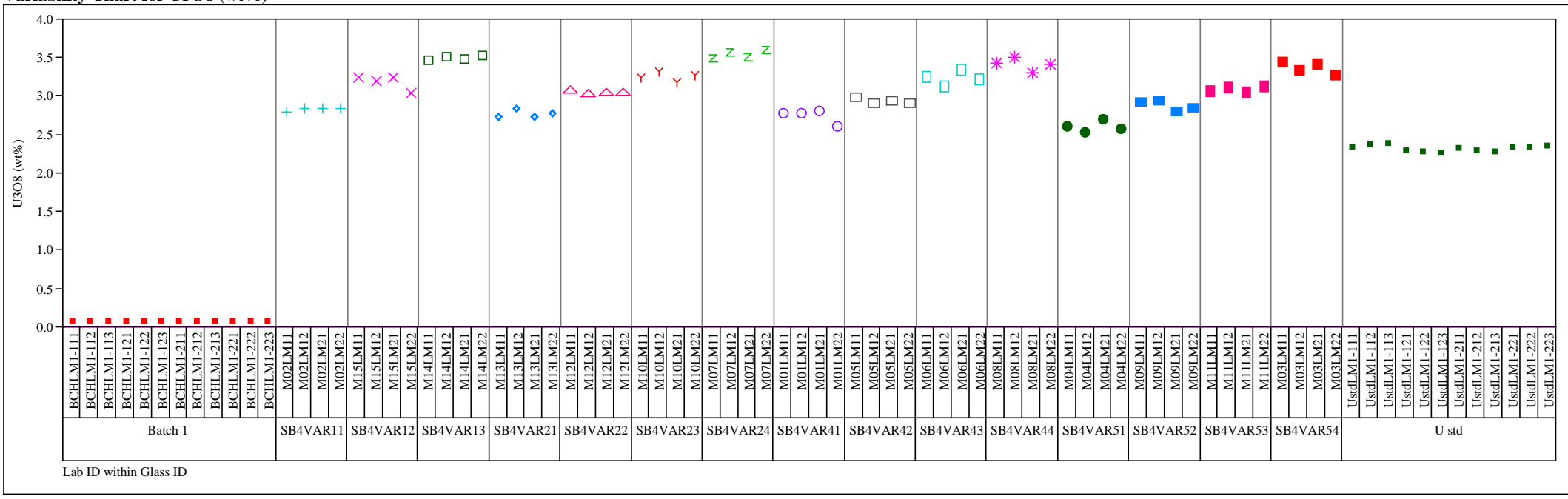

Set $=1$

Variability Chart for $\mathrm{ZnO}$ (wt \%)

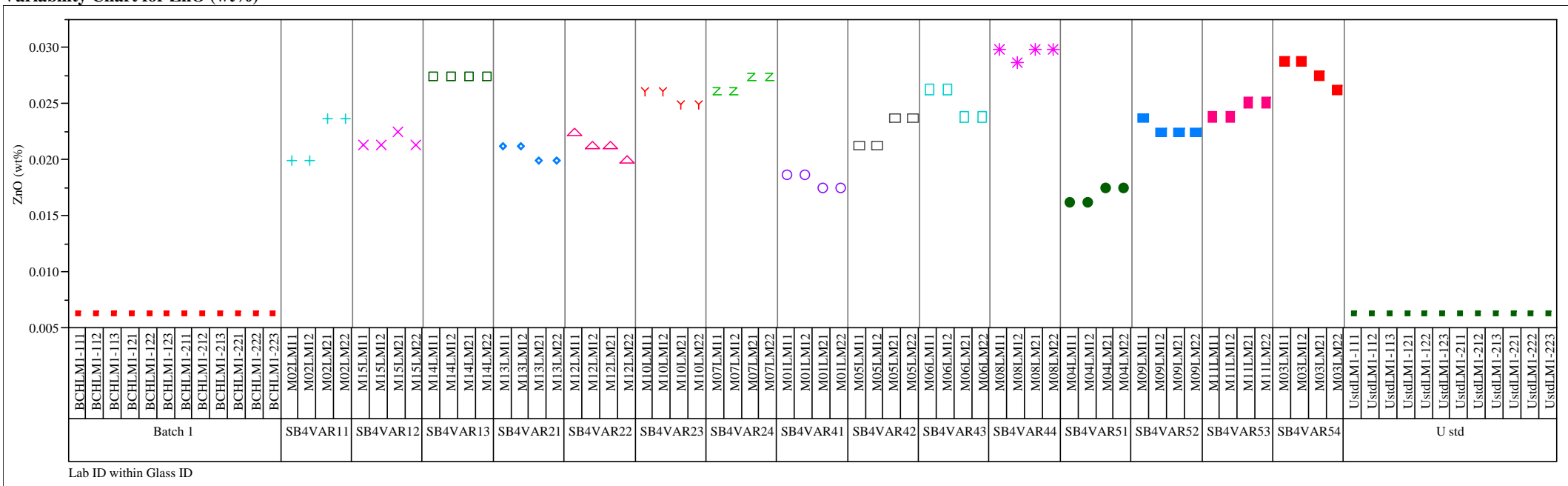




\section{Exhibit A5. Oxide Measurements by Solution ID for Samples Prepared Using the LM Method}

Set $=1$

Variability Chart for $\mathrm{ZrO} 2$ (wt \%)

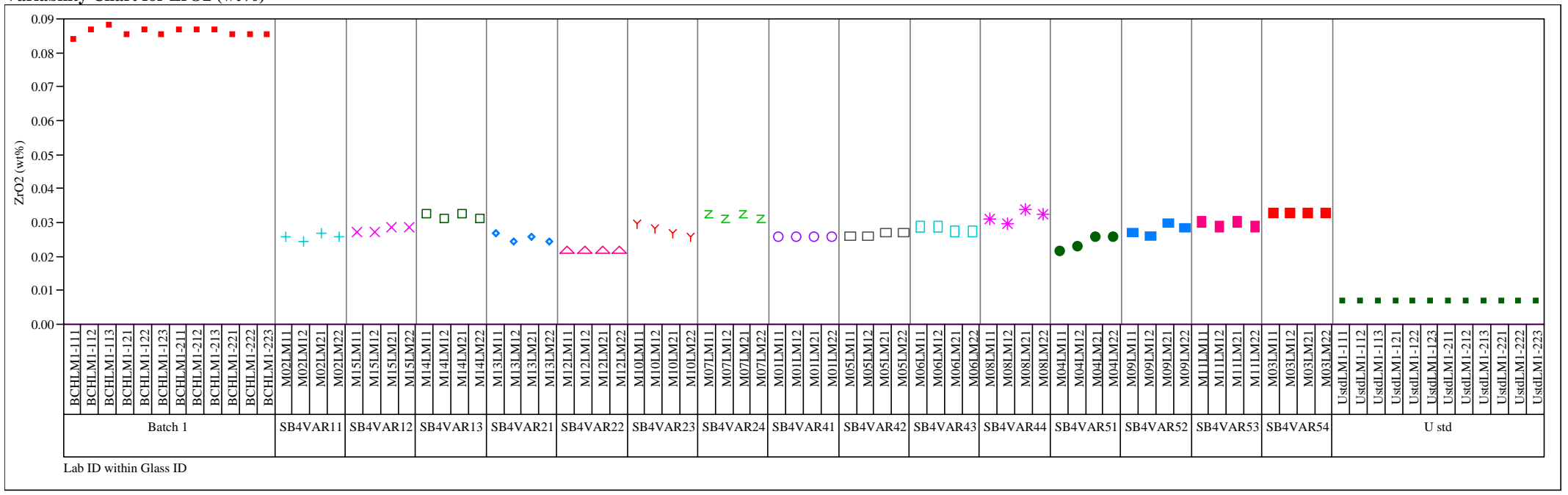

Set $=1$

Variability Chart for Al2O3 bc (wt \%)

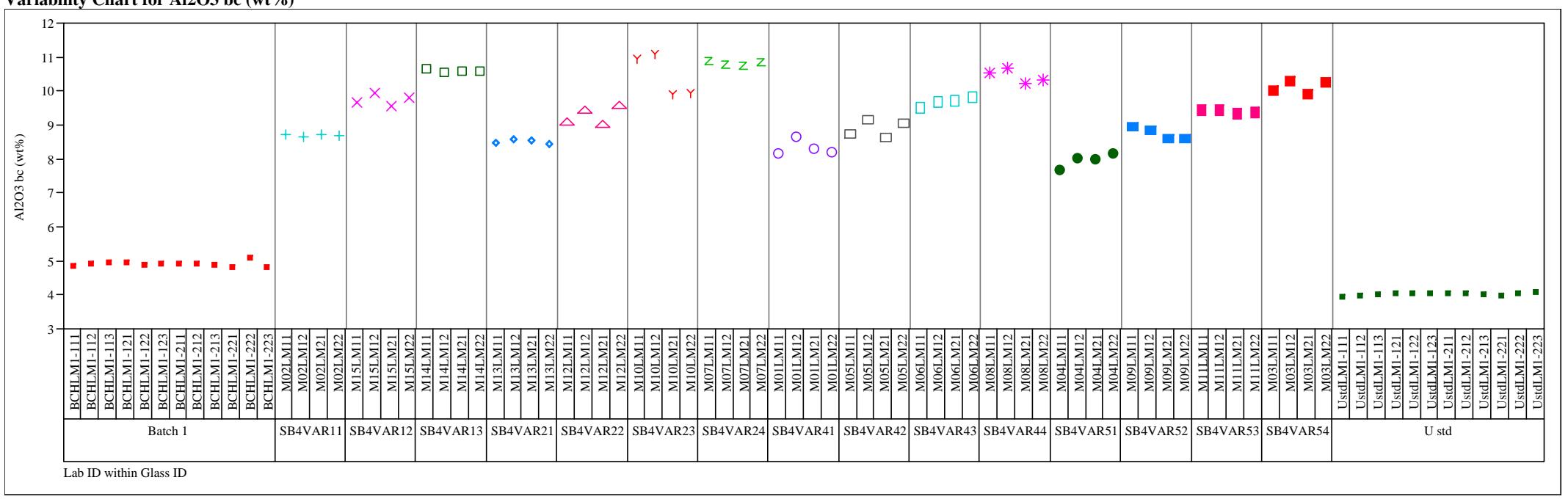


Exhibit A5. Oxide Measurements by Solution ID for Samples Prepared Using the LM Method

Set $=1$

Variability Chart for BaO bc (wt\%)

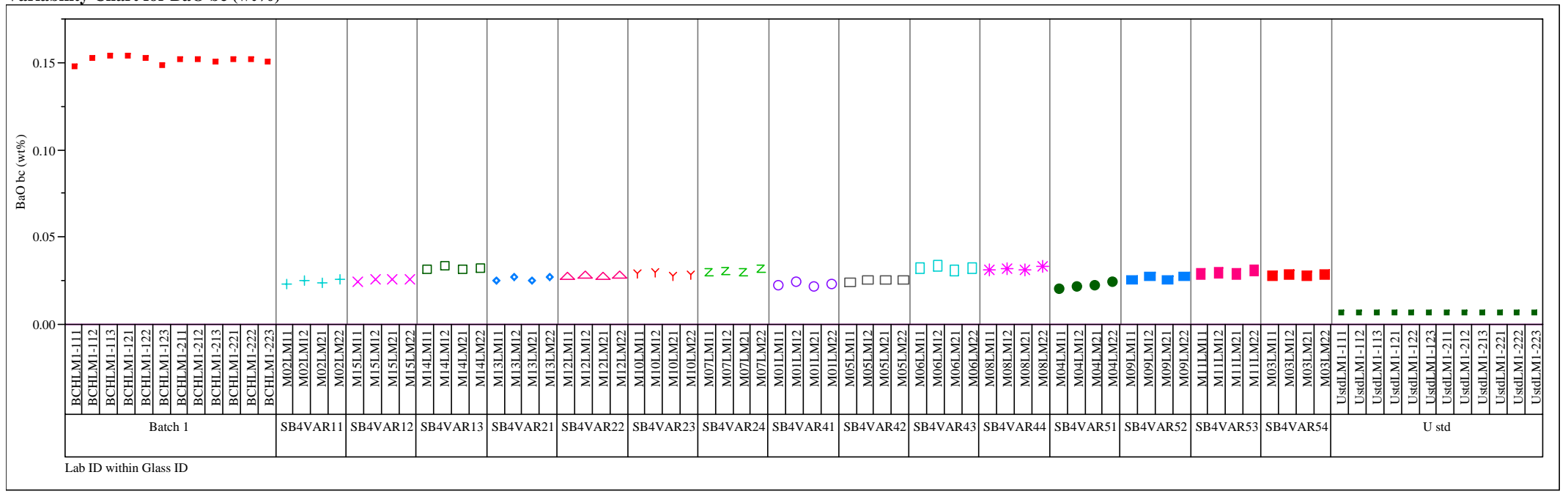

Set $=1$

Variability Chart for CaO bc (wt\%)

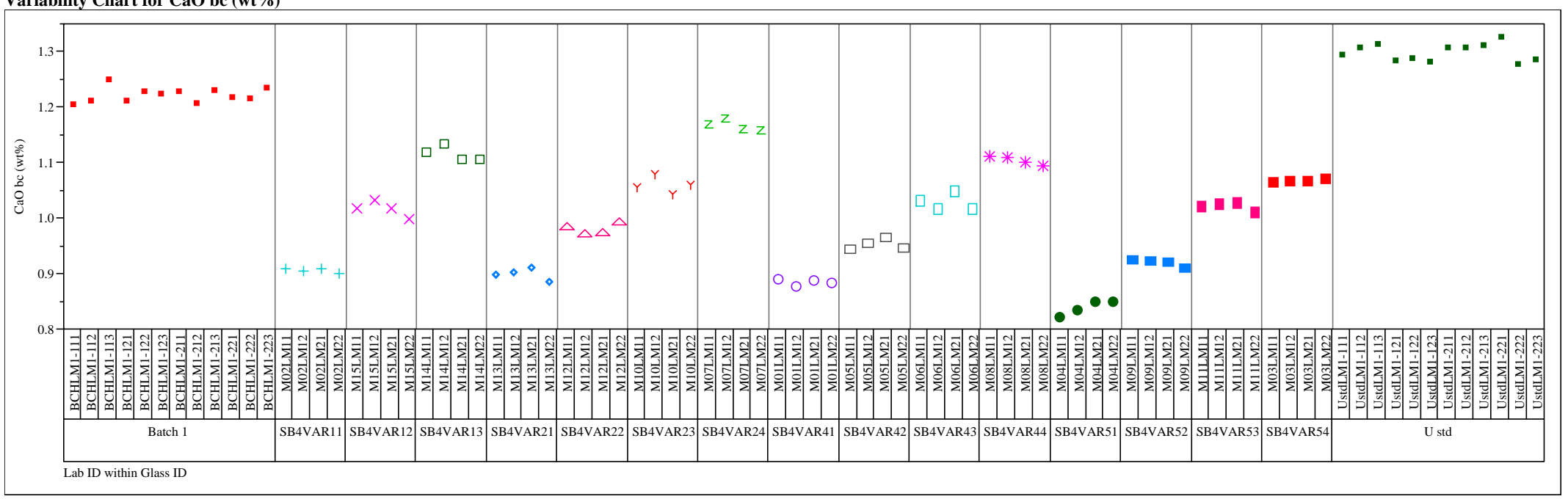


Exhibit A5. Oxide Measurements by Solution ID for Samples Prepared Using the LM Method

Set $=1$

Variability Chart for CdO bc (wt\%)

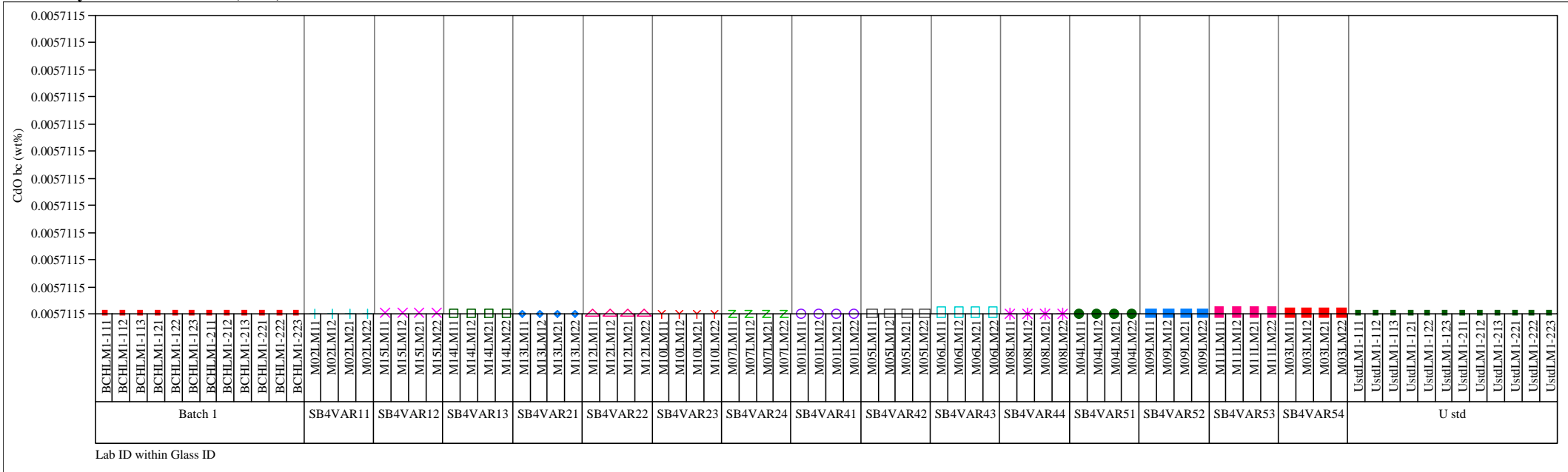

Set $=1$

Variability Chart for Ce2O3 bc (wt \%)

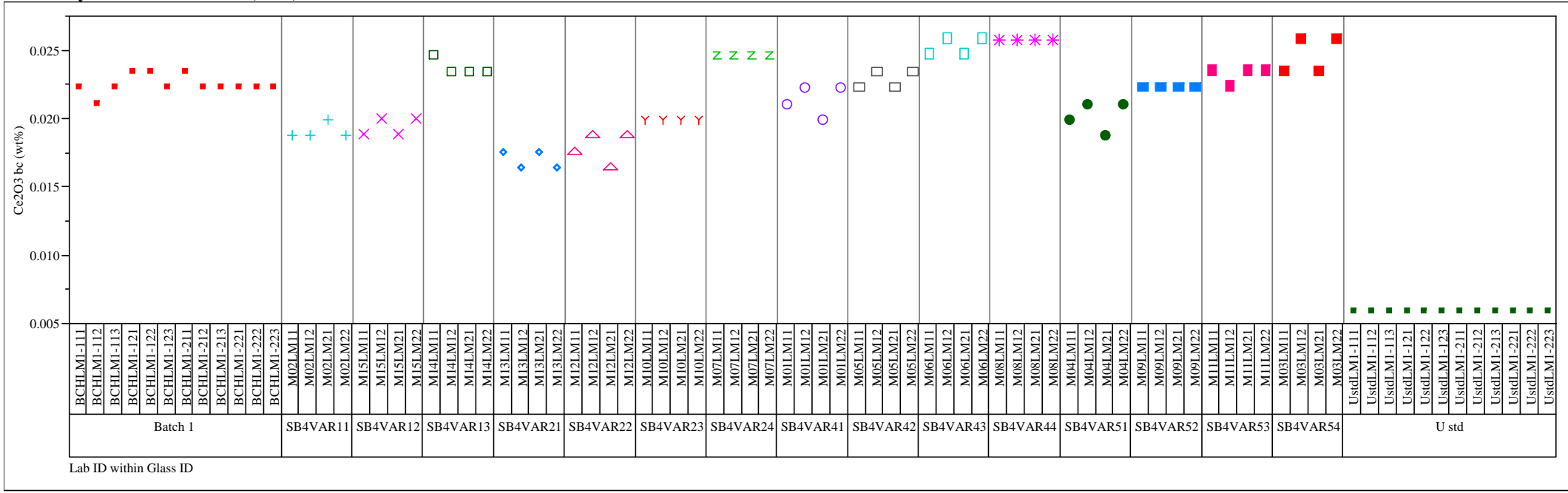




\section{Exhibit A5. Oxide Measurements by Solution ID for Samples Prepared Using the LM Method}

Set $=1$

Variability Chart for Cr2O3 bc (wt \%)

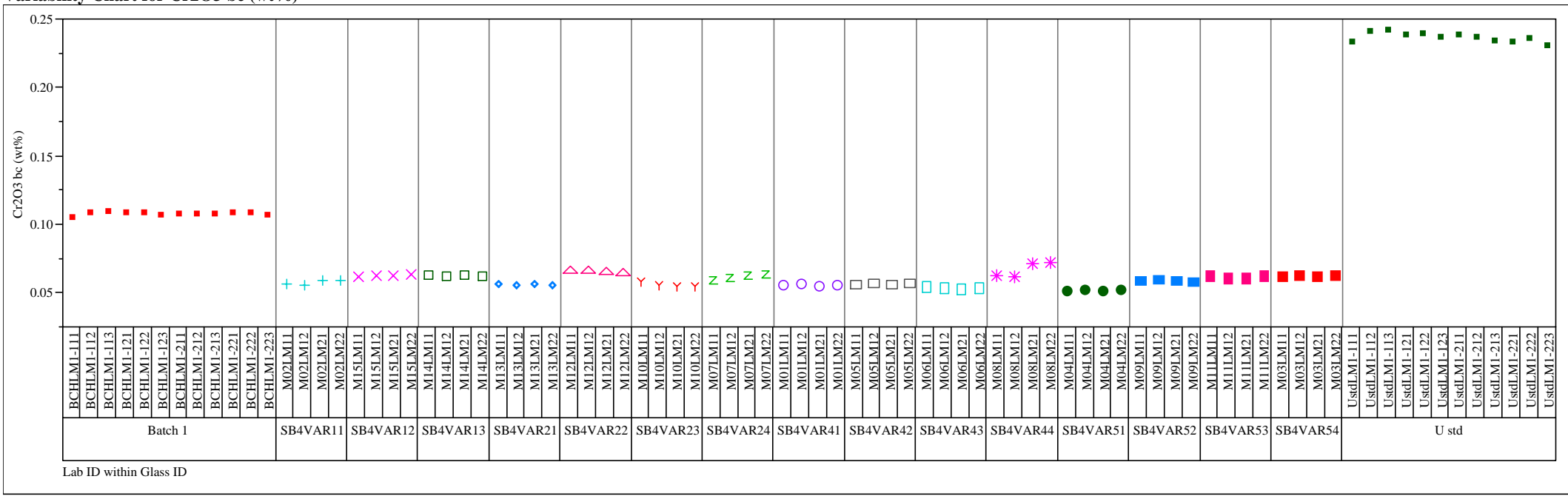

Set $=1$

Variability Chart for $\mathrm{CuO}$ bc (wt \%)

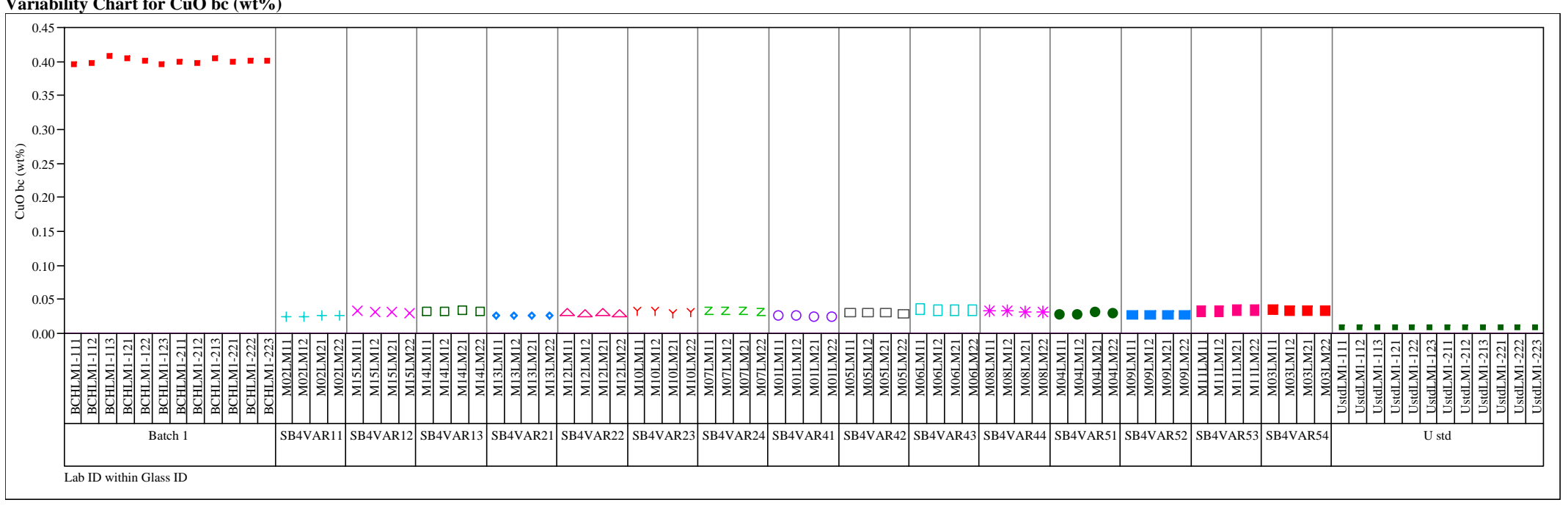


Exhibit A5. Oxide Measurements by Solution ID for Samples Prepared Using the LM Method

Set $=1$

Variability Chart for Fe2O3 bc (wt\%)

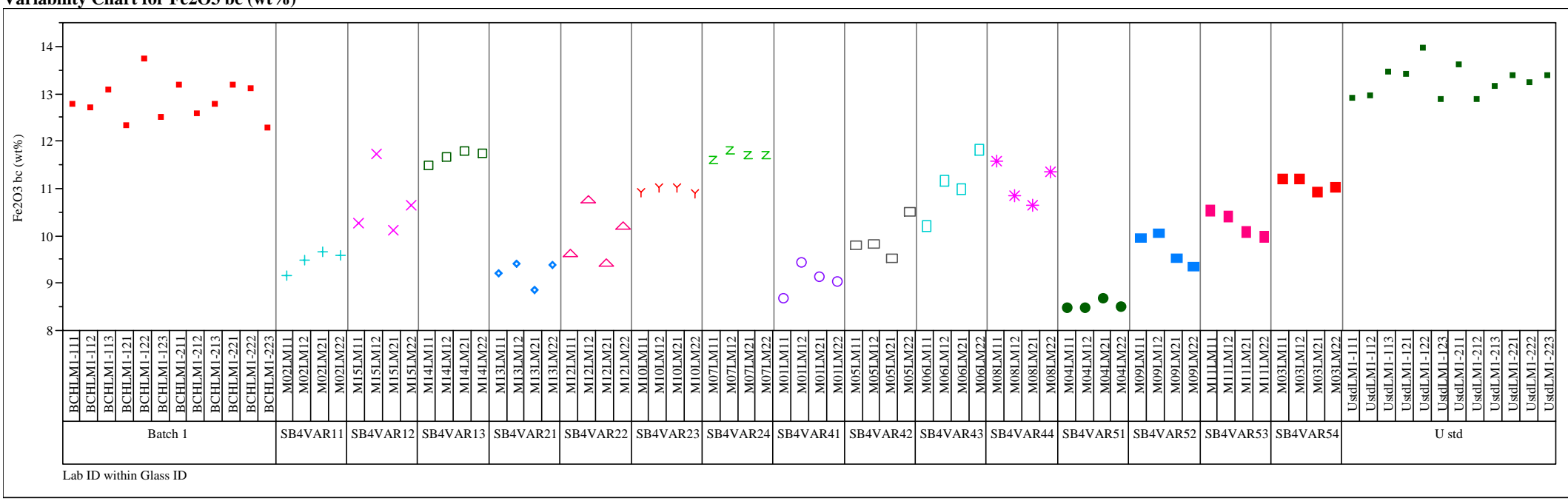

Set $=1$

Variability Chart for K2O bc (wt\%)

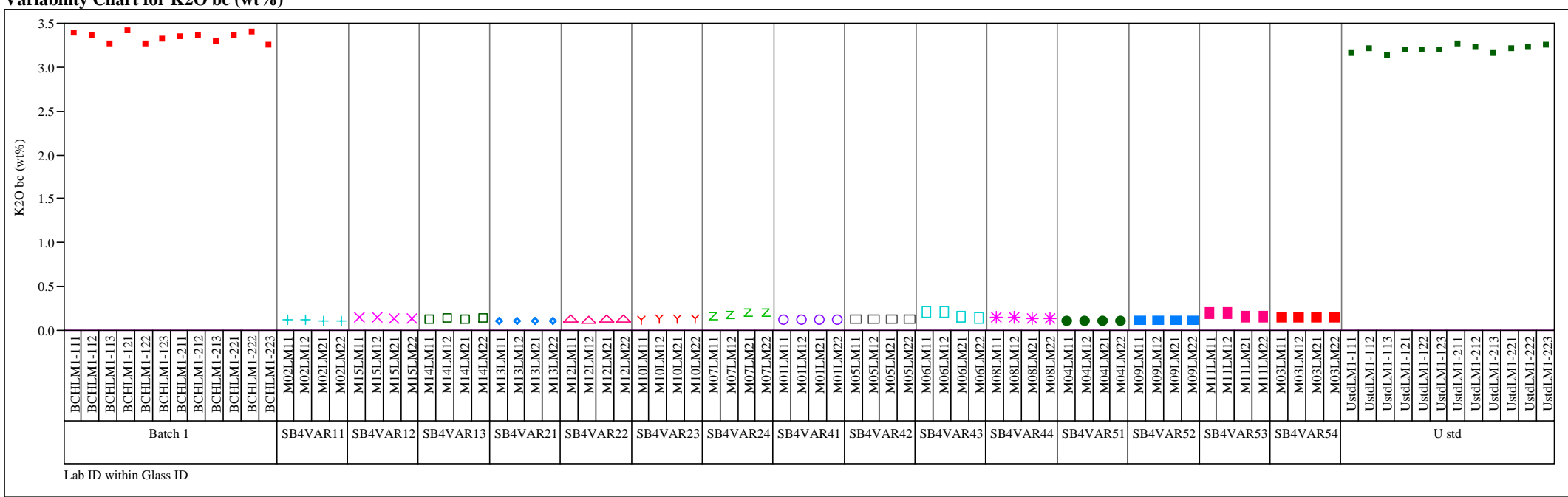




\section{Exhibit A5. Oxide Measurements by Solution ID for Samples Prepared Using the LM Method}

Set $=1$

Variability Chart for La2O3 bc (wt\%)

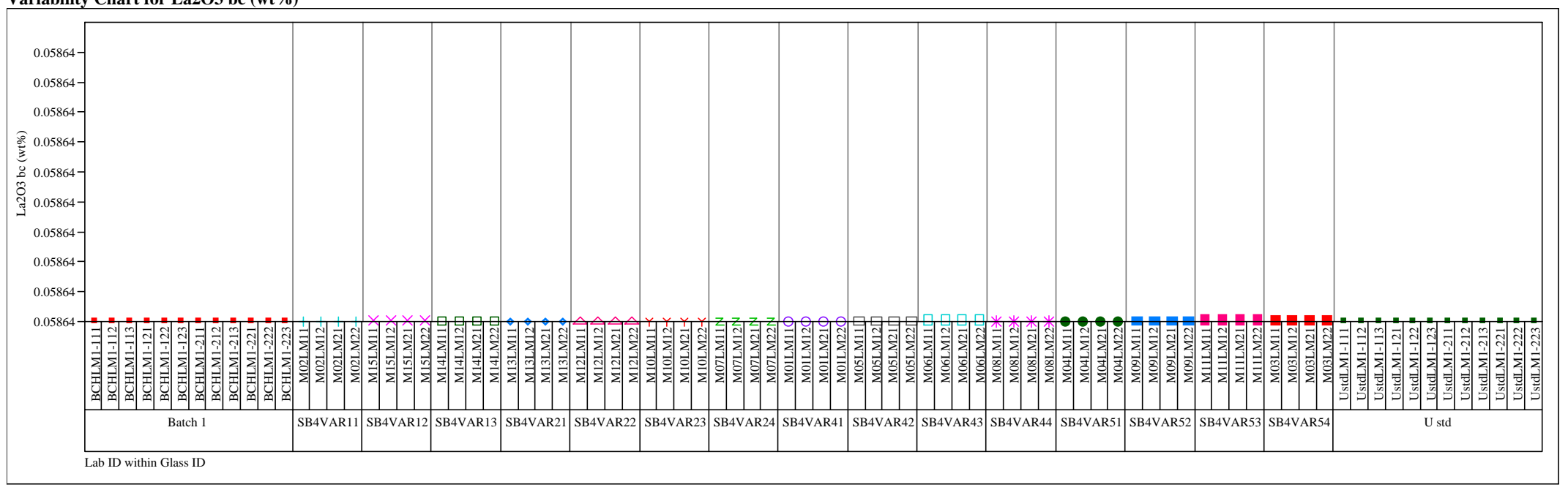

Set=1

Variability Chart for MgO bc (wt \%)

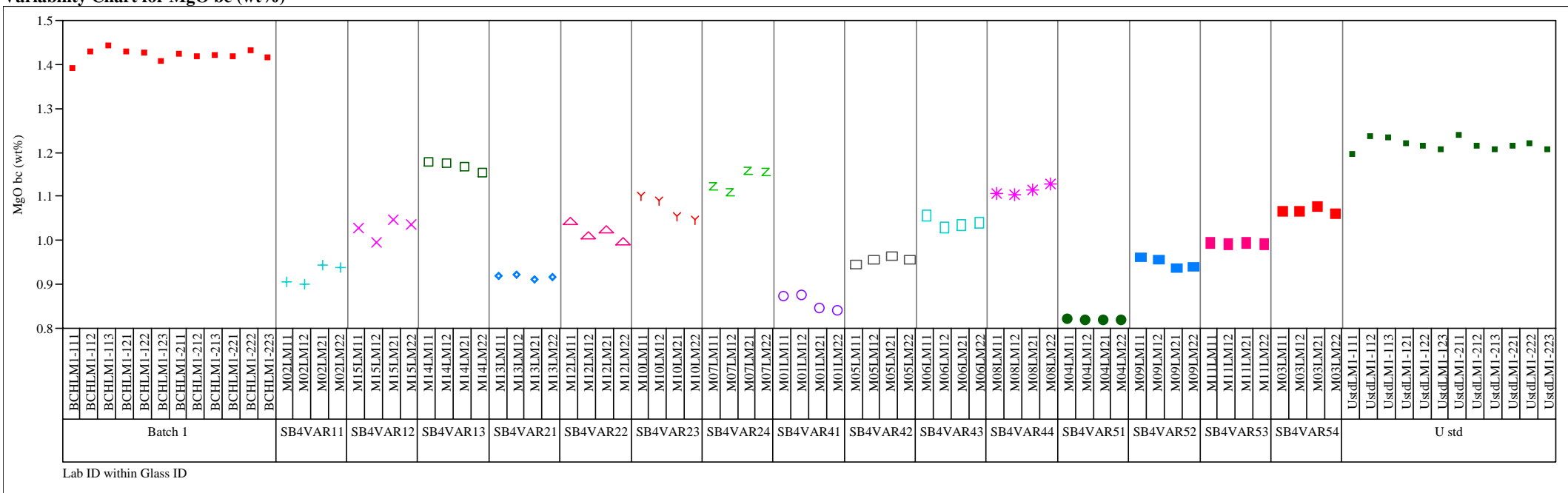




\section{Exhibit A5. Oxide Measurements by Solution ID for Samples Prepared Using the LM Method}

Set $=1$

Variability Chart for MnO bc (wt\%)

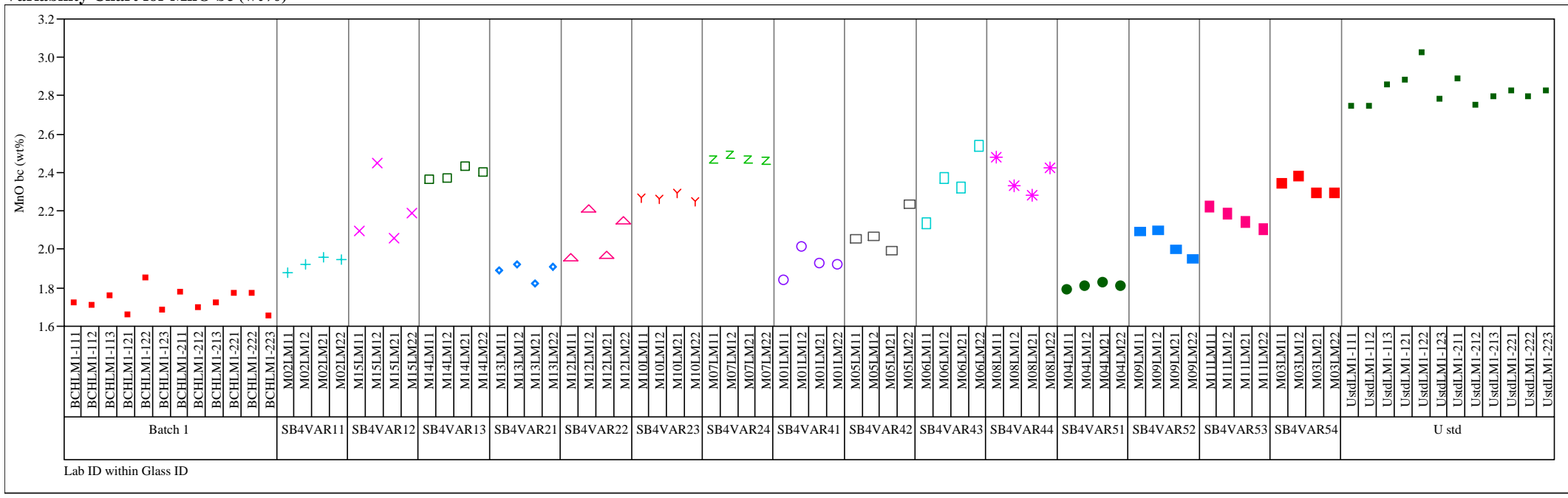

Set $=1$

Variability Chart for Na2O bc (wt\%)

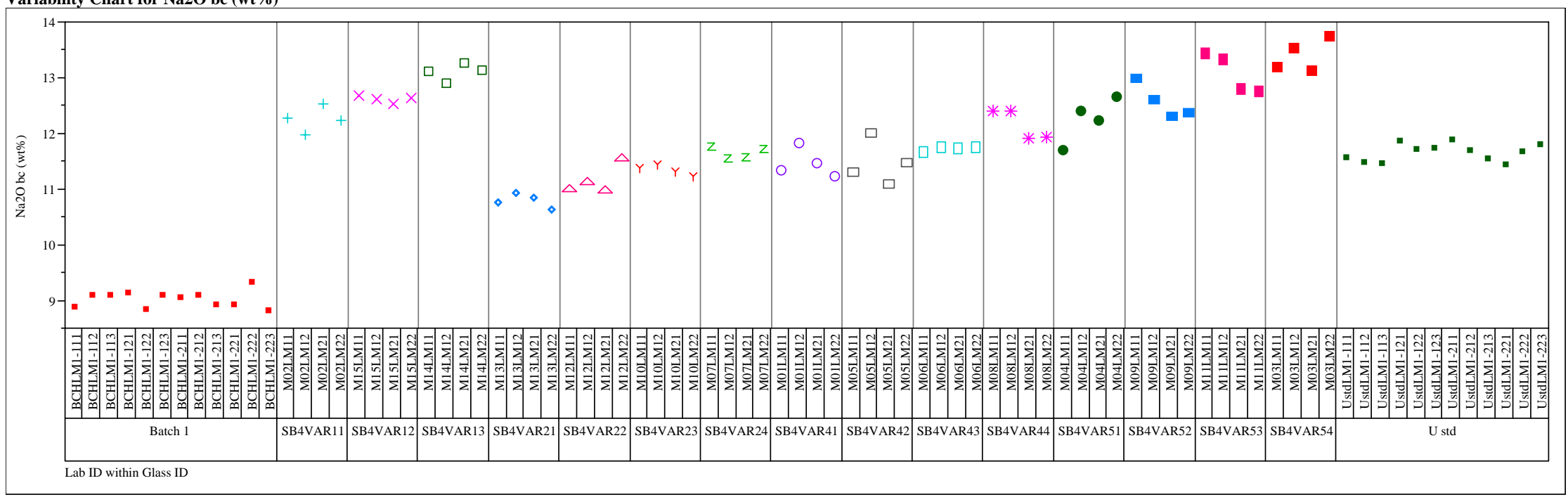




\section{Exhibit A5. Oxide Measurements by Solution ID for Samples Prepared Using the LM Method}

Set $=1$

Variability Chart for NiO bc (wt\%)

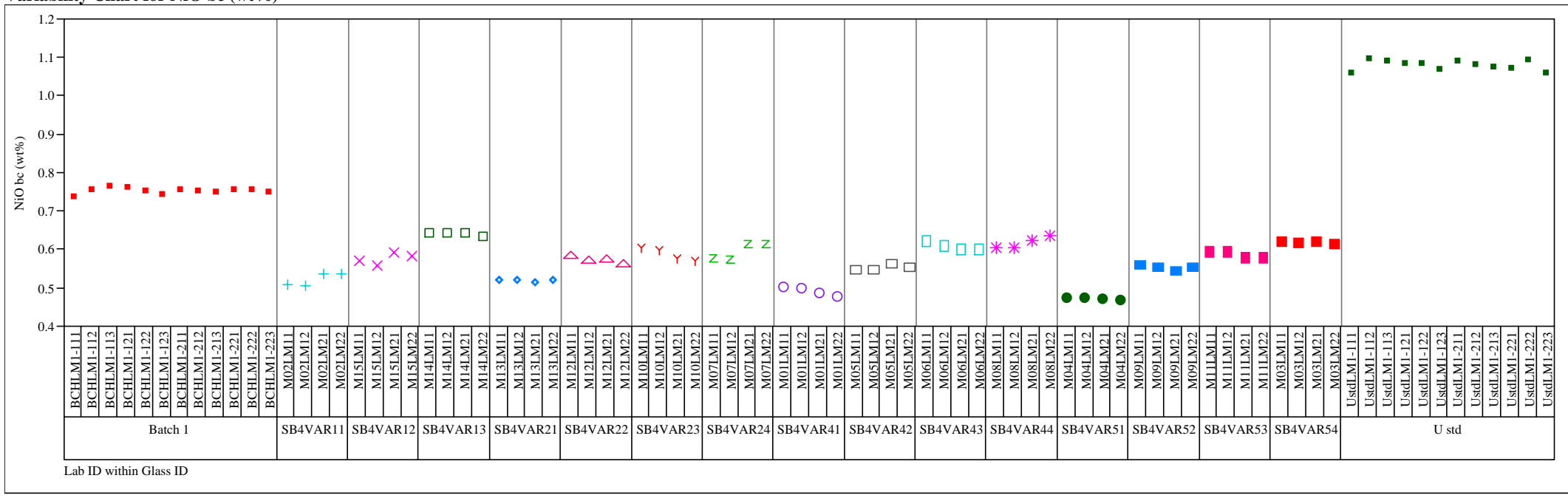

Set $=1$

Variability Chart for PbO bc (wt\%)

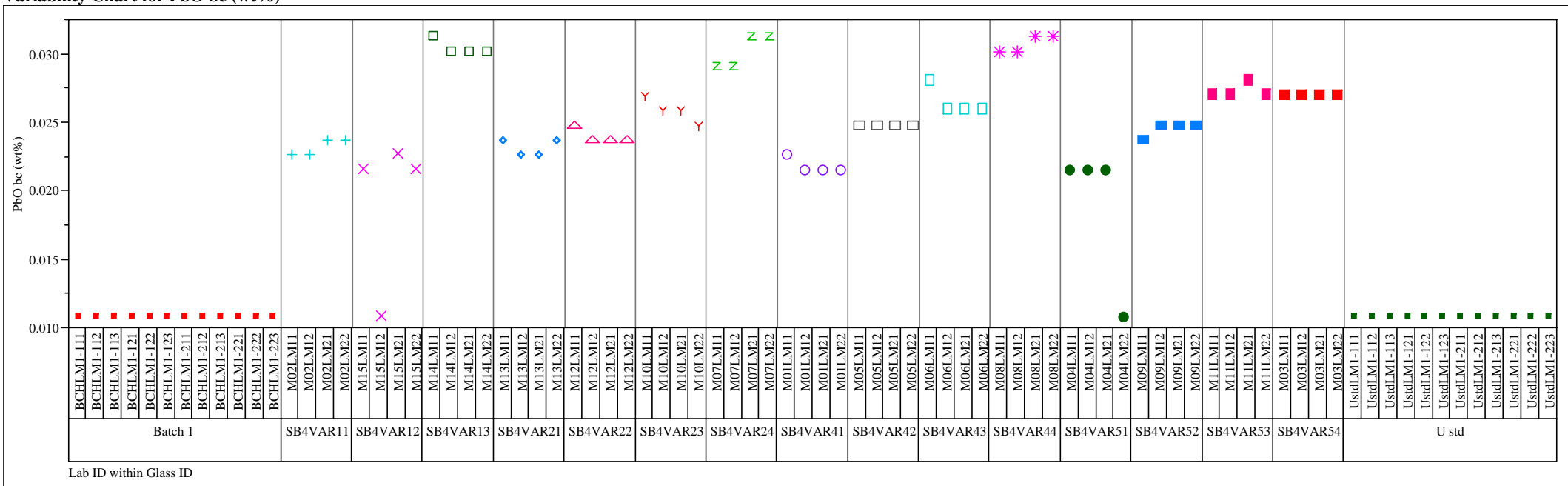


Exhibit A5. Oxide Measurements by Solution ID for Samples Prepared Using the LM Method

Set $=1$

Variability Chart for SO4 bc (wt \%)

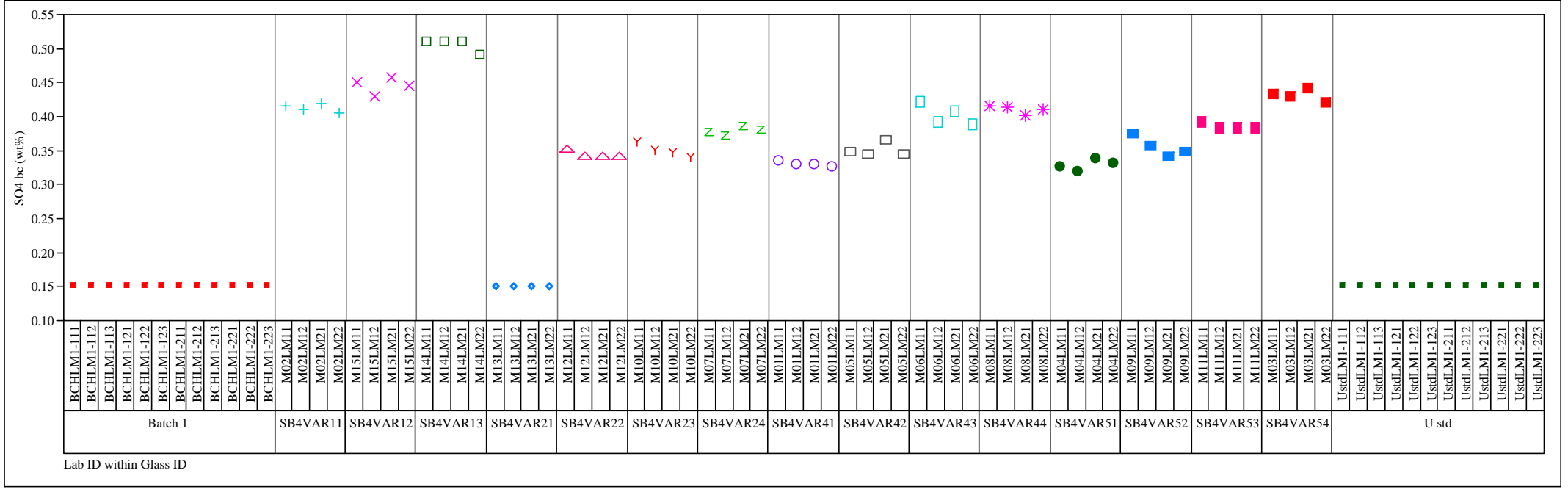

Set $=1$

Variability Chart for SiO2 bc (wt\%)

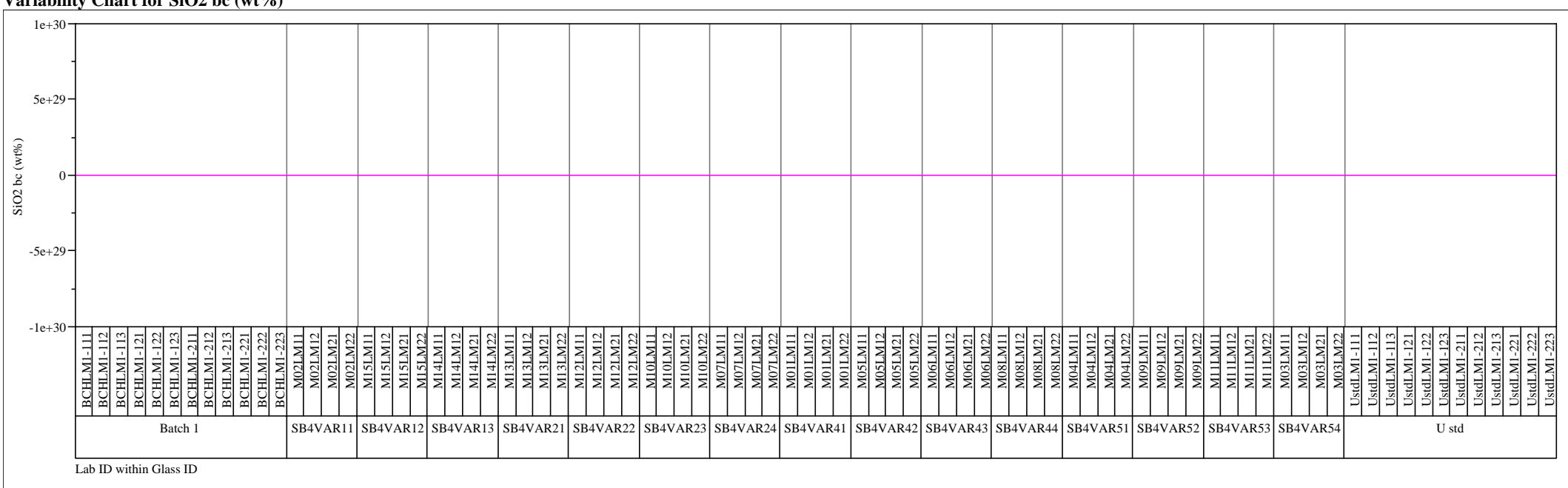


Exhibit A5. Oxide Measurements by Solution ID for Samples Prepared Using the LM Method

Set $=1$

Variability Chart for TiO2 bc (wt \%)

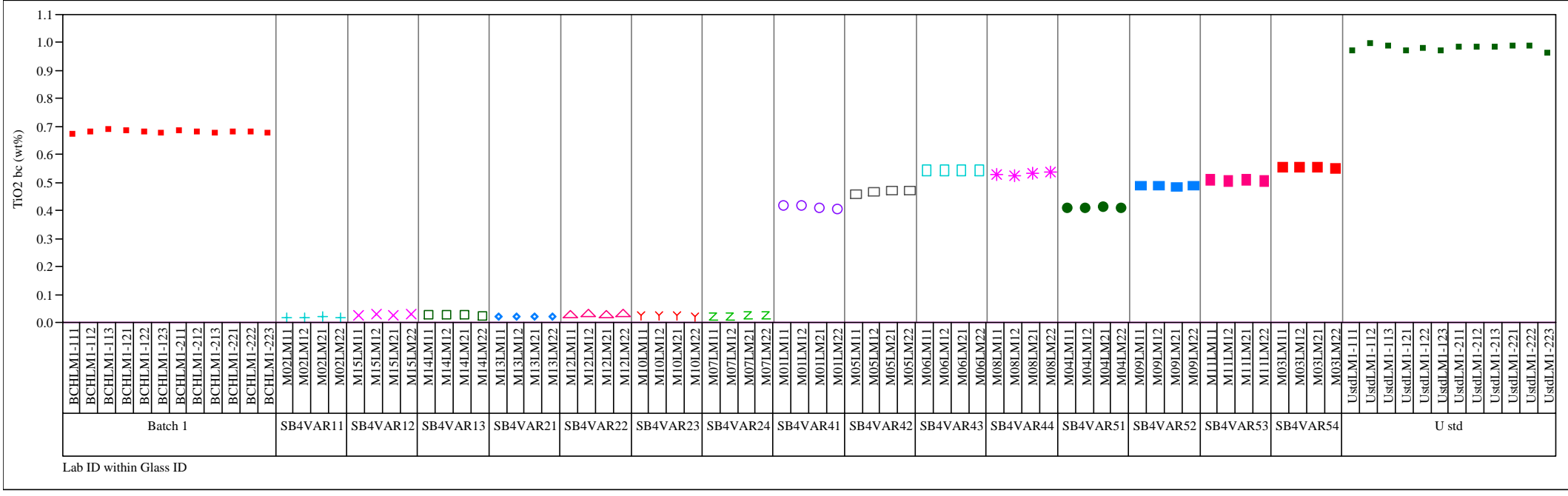

Set $=1$

Variability Chart for U3O8 bc (wt\%)

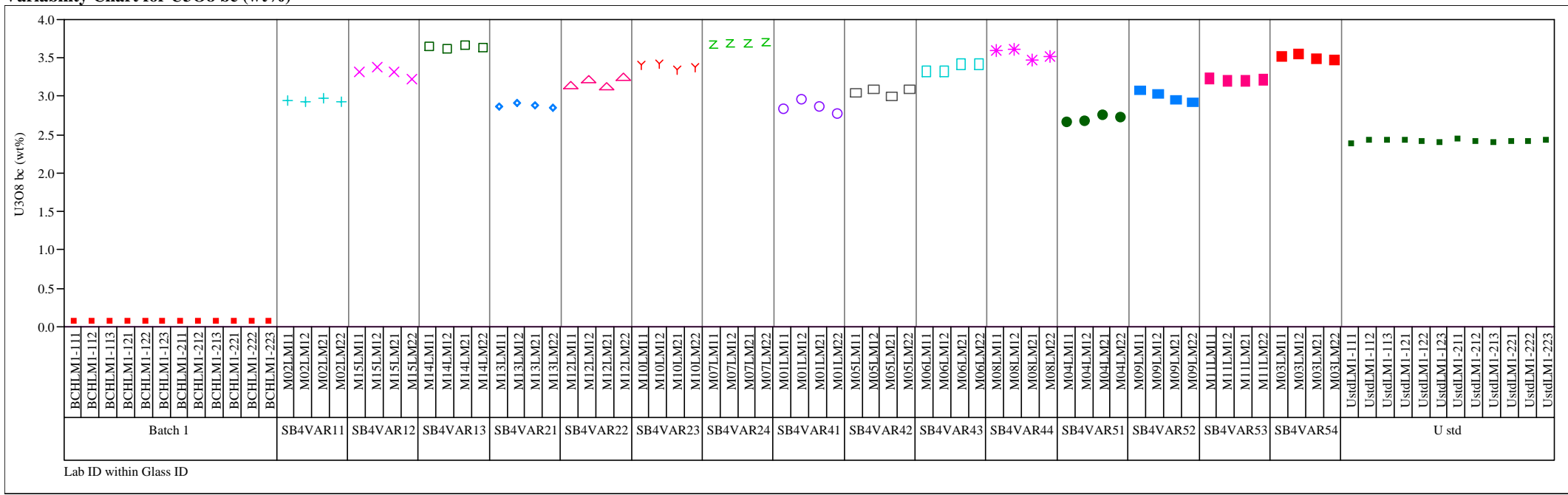


Exhibit A5. Oxide Measurements by Solution ID for Samples Prepared Using the LM Method

Set $=1$

Variability Chart for ZnO bc (wt\%)

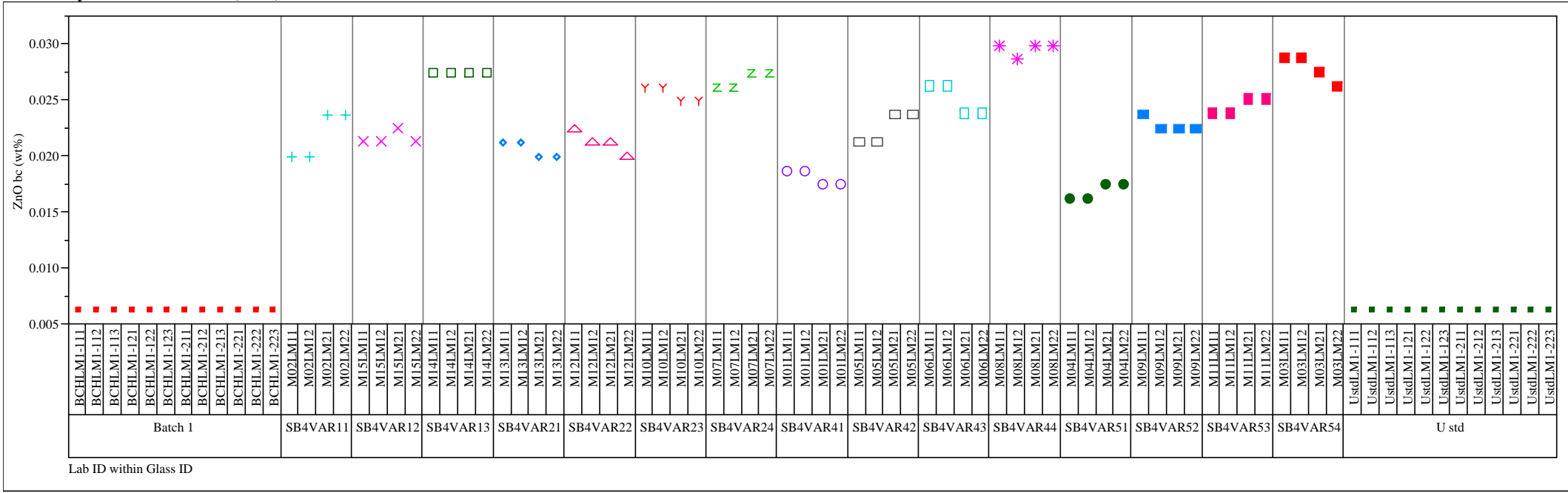

Set $=1$

Variability Chart for $\mathrm{ZrO} 2 \mathrm{bc}(\mathrm{wt} \%)$

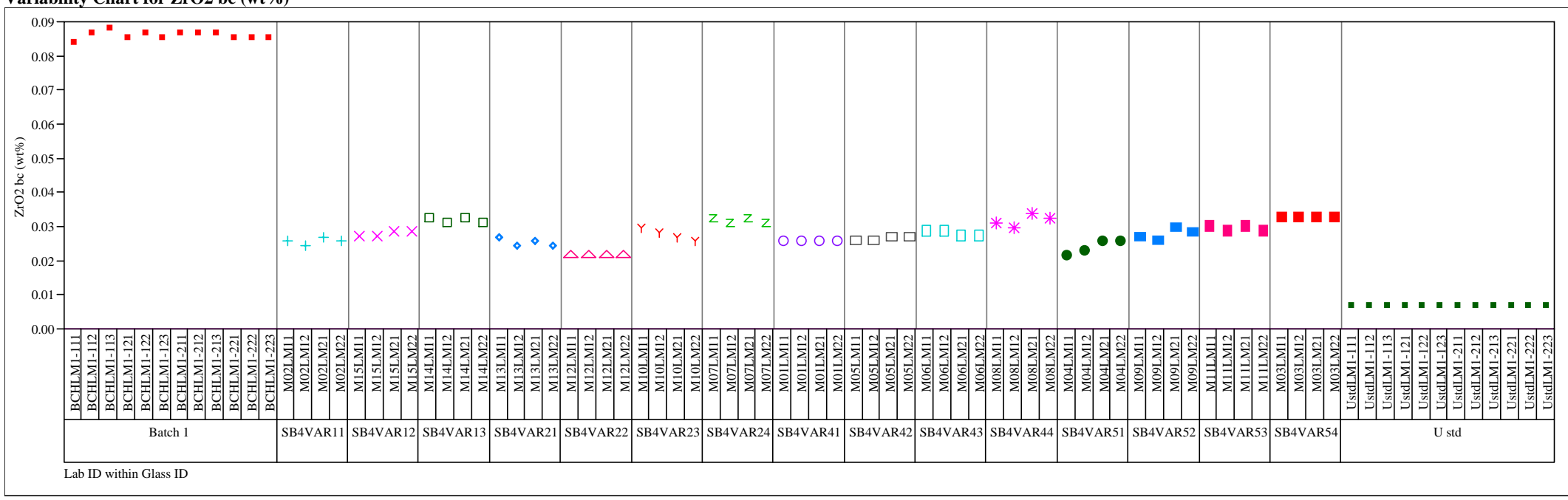




\section{Exhibit A5. Oxide Measurements by Solution ID for Samples Prepared Using the LM Method}

Set $=2$

Variability Chart for Al2O3 (wt \%)

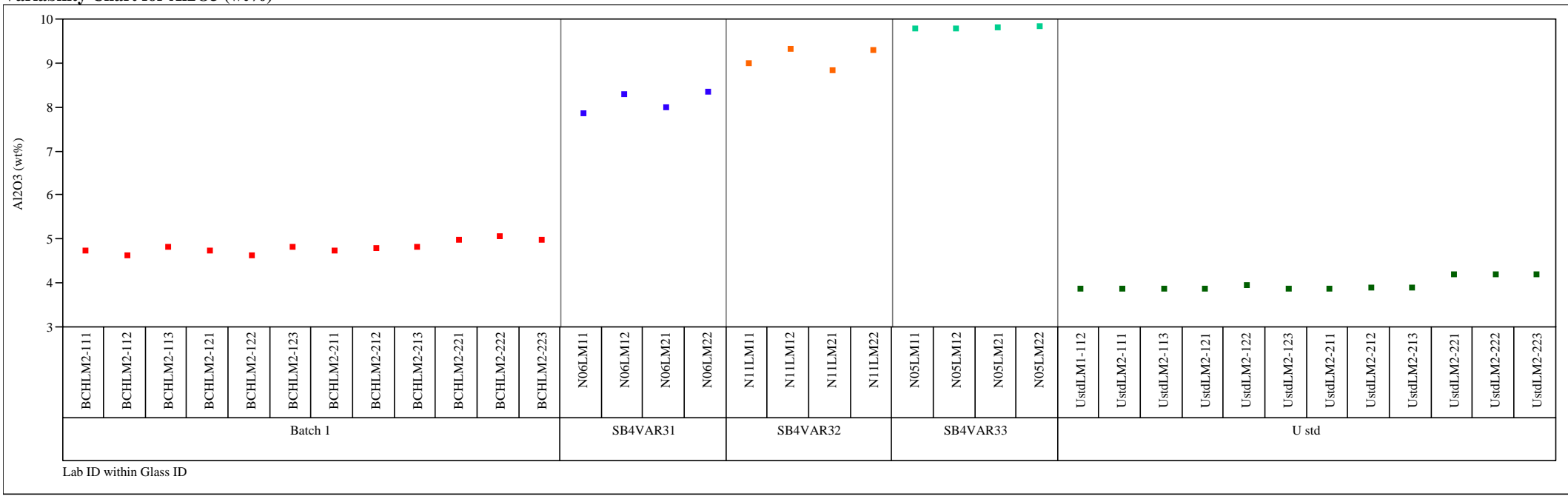

Set $=2$

Variability Chart for BaO (wt \%)

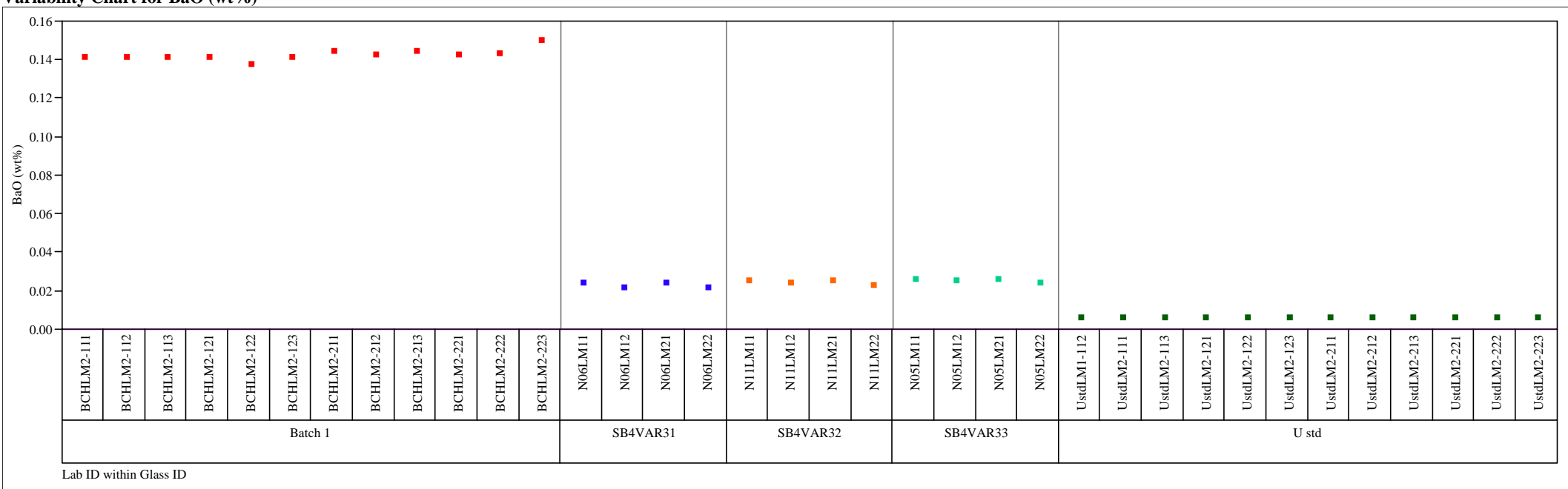


Exhibit A5. Oxide Measurements by Solution ID for Samples Prepared Using the LM Method

Set $=2$

Variability Chart for $\mathrm{CaO}$ (wt\%)

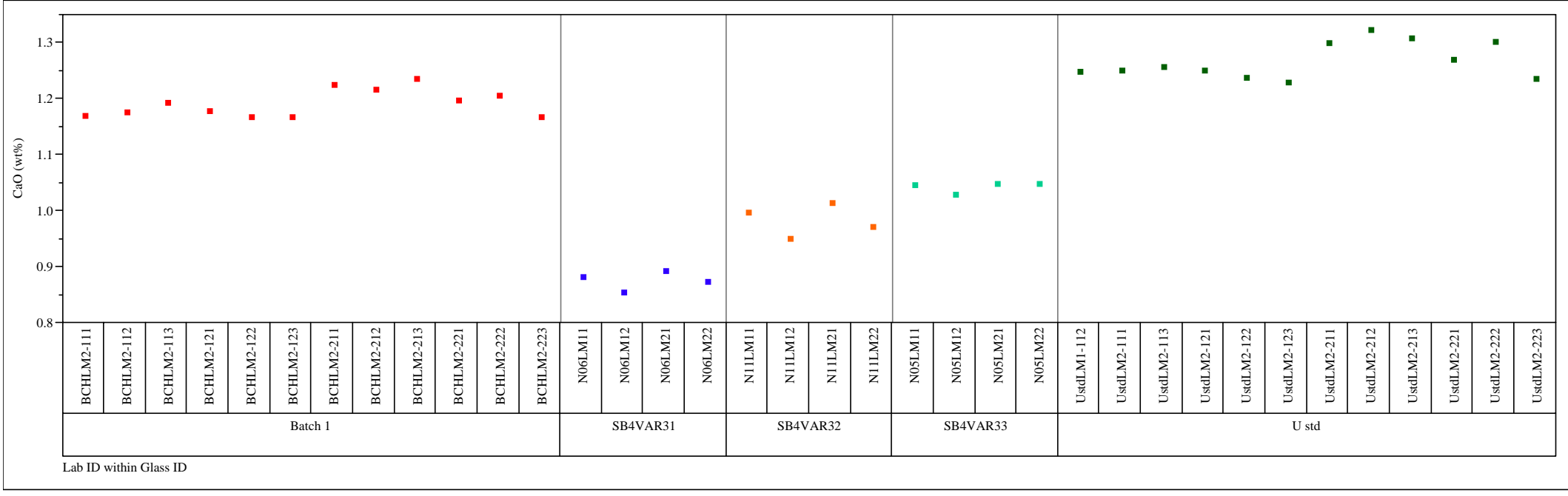

Set $=2$

Variability Chart for CdO (wt\%)

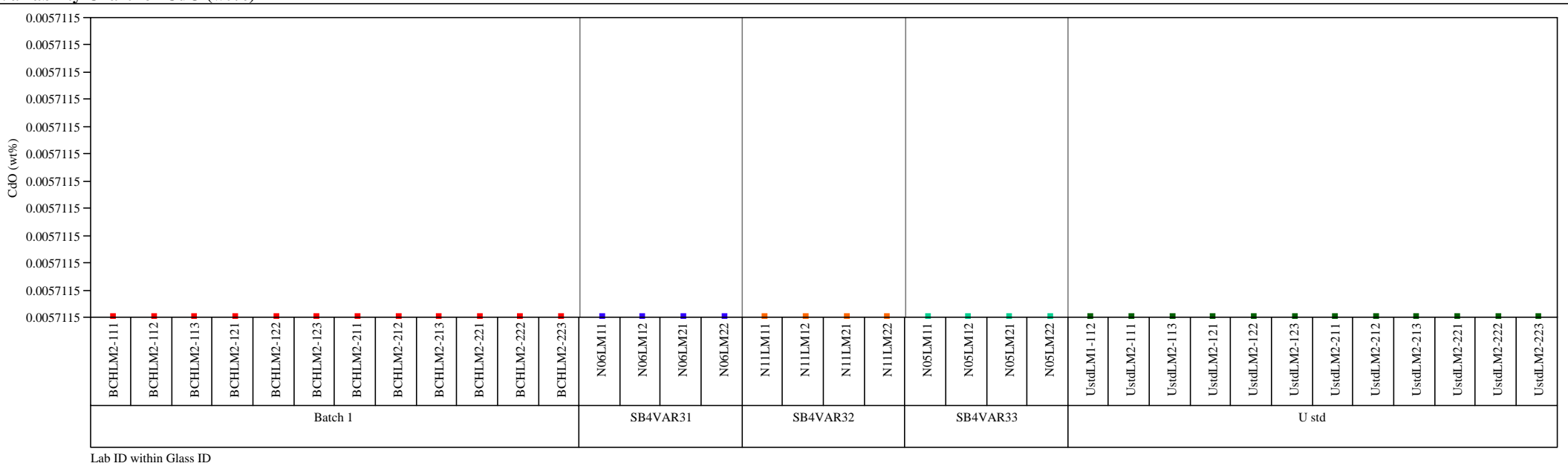




\section{Exhibit A5. Oxide Measurements by Solution ID for Samples Prepared Using the LM Method}

Set $=2$

Variability Chart for Ce2O3 (wt \%)

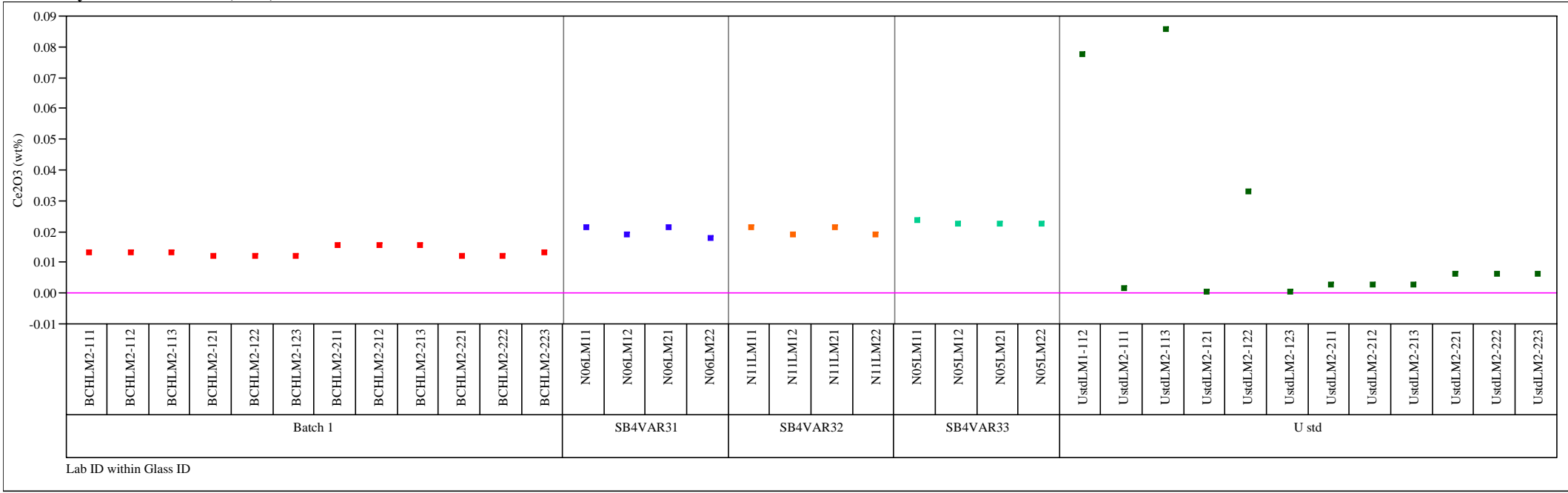

Set $=2$

Variability Chart for Cr2O3 (wt\%)

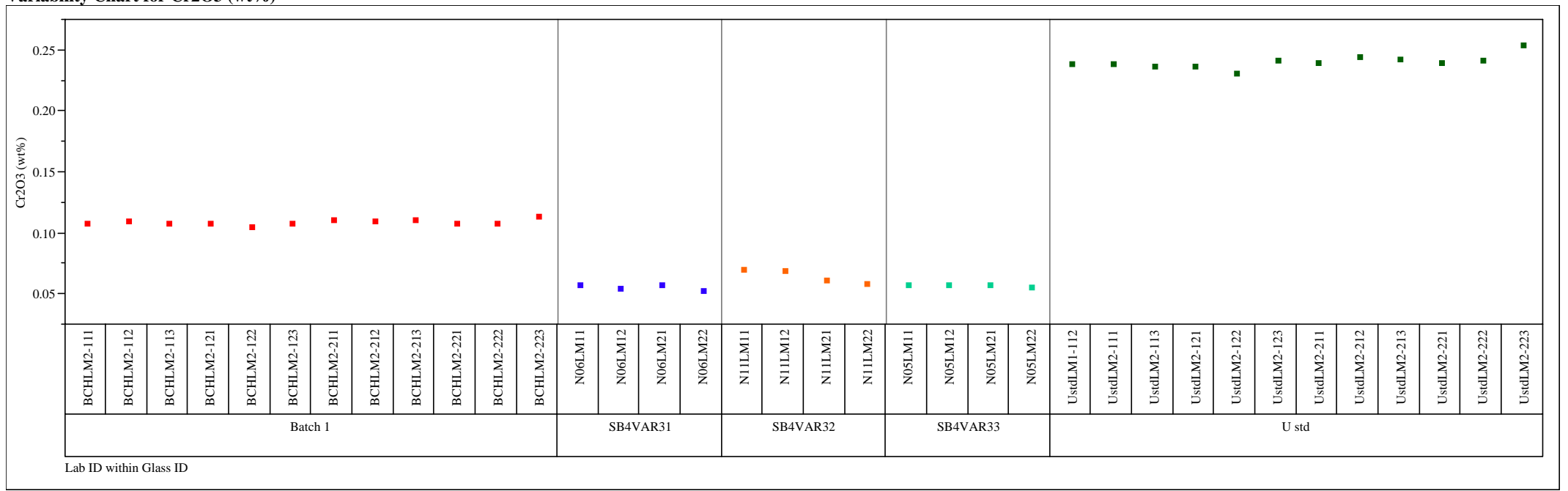


Exhibit A5. Oxide Measurements by Solution ID for Samples Prepared Using the LM Method

Set $=2$

Variability Chart for $\mathrm{CuO}$ (wt\%)

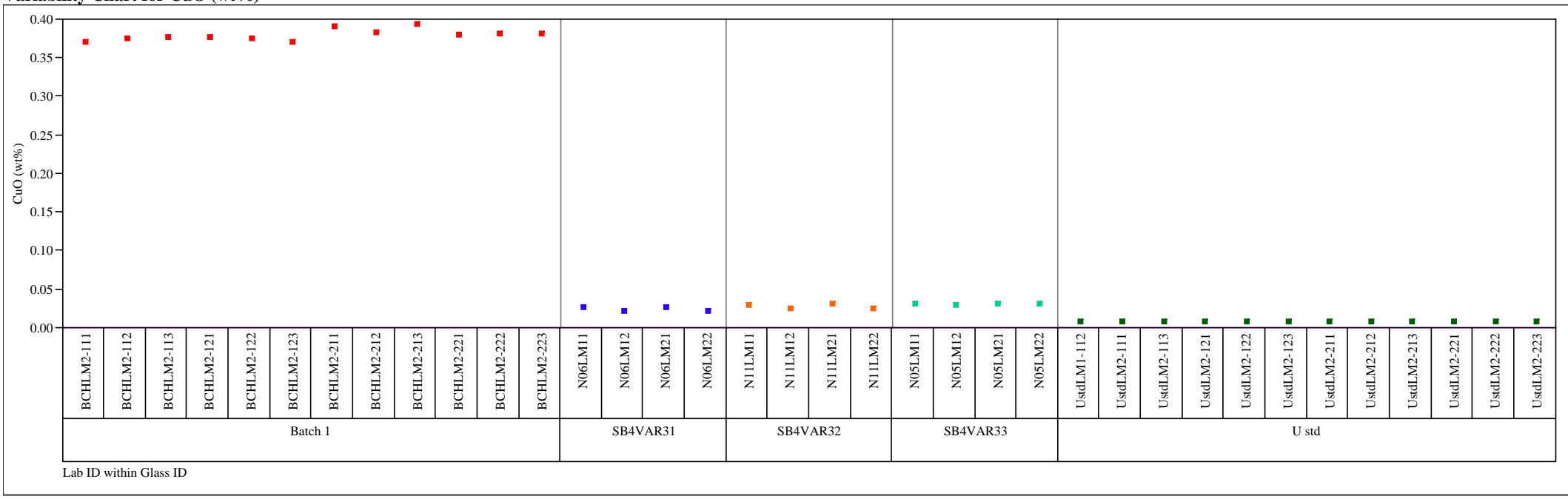

Set $=2$

Variability Chart for Fe2O3 (wt\%)

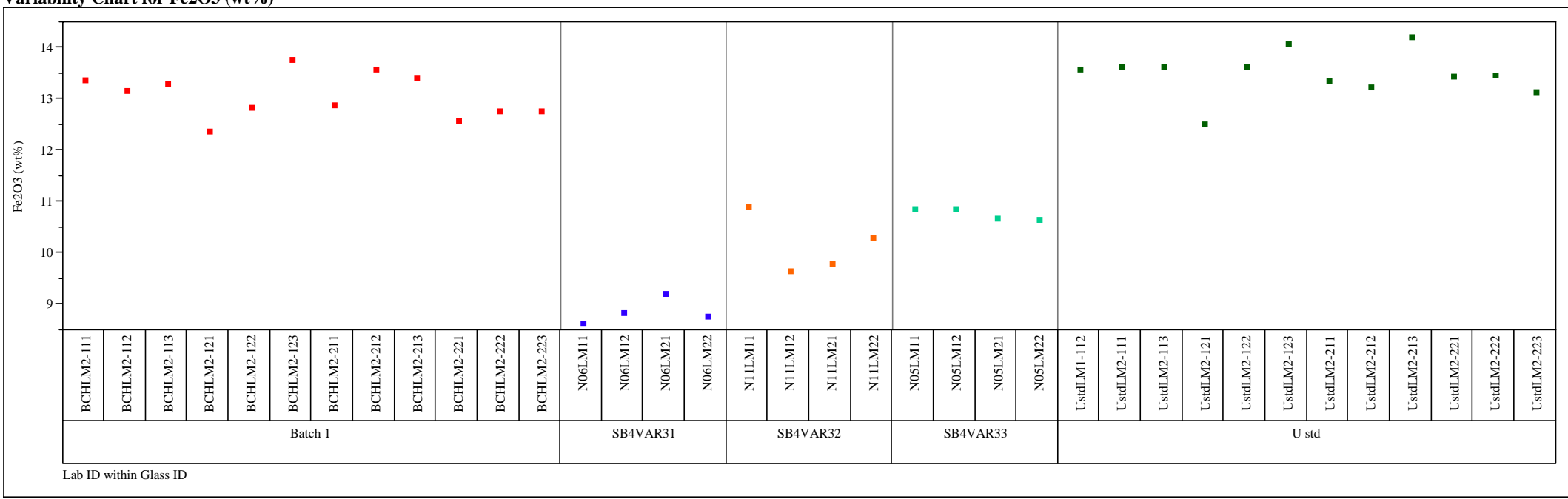




\section{Exhibit A5. Oxide Measurements by Solution ID for Samples Prepared Using the LM Method}

Set $=2$

Variability Chart for K2O (wt \%)

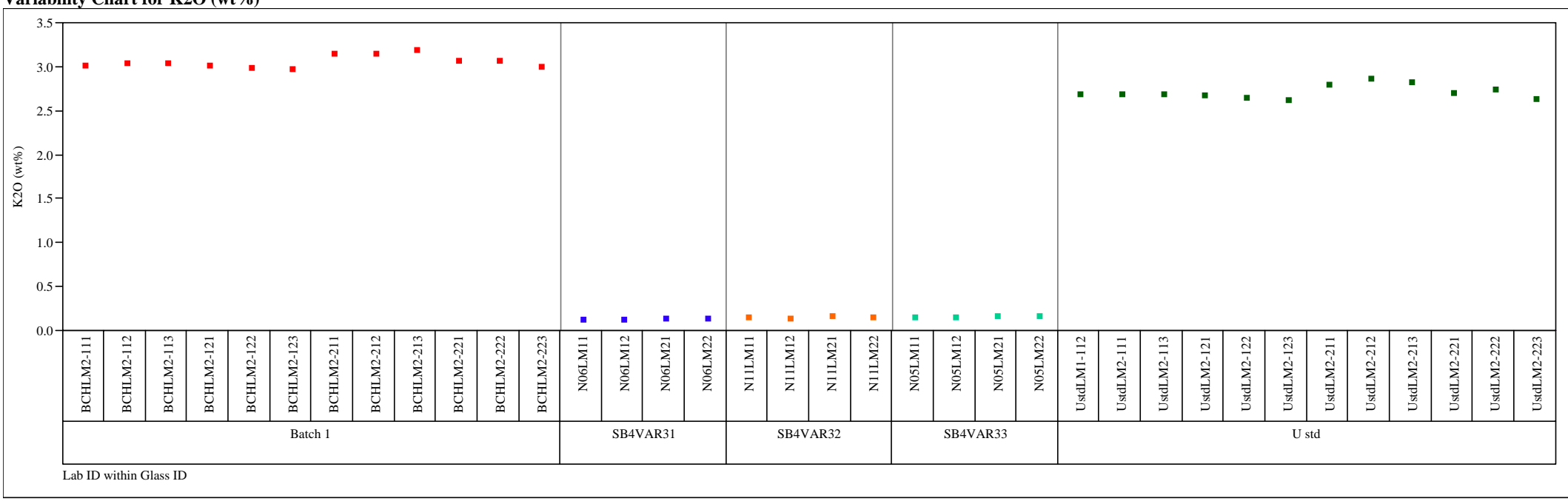

Set $=2$

Variability Chart for La2O3 (wt\%)

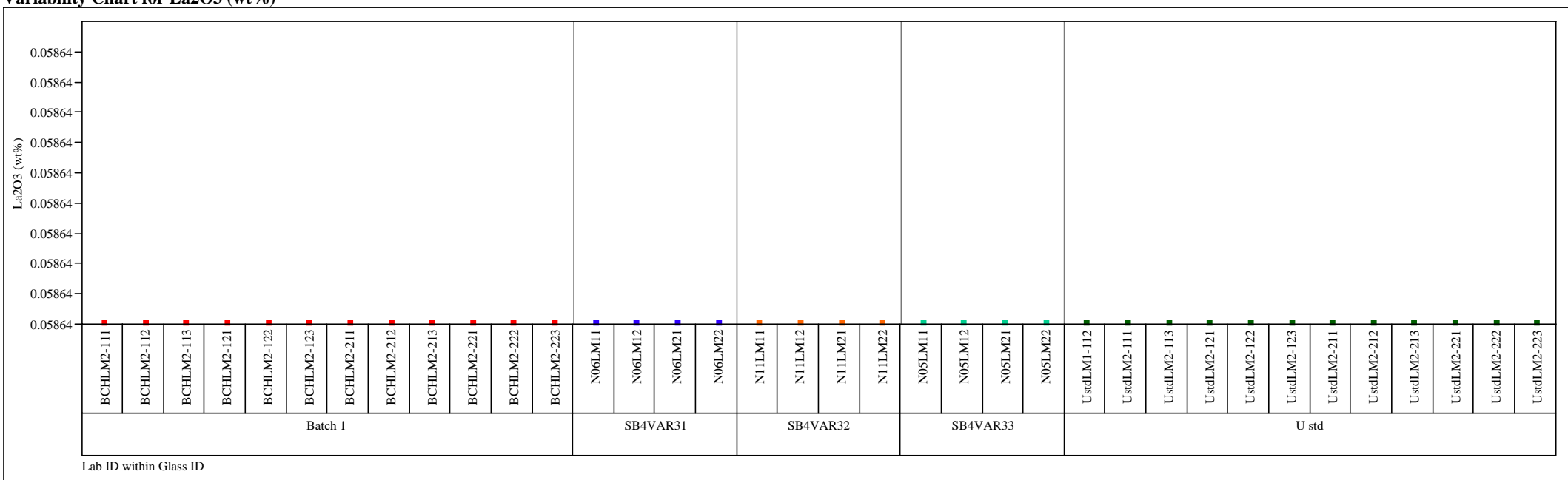


Exhibit A5. Oxide Measurements by Solution ID for Samples Prepared Using the LM Method

Set $=2$

Variability Chart for MgO (wt\%)

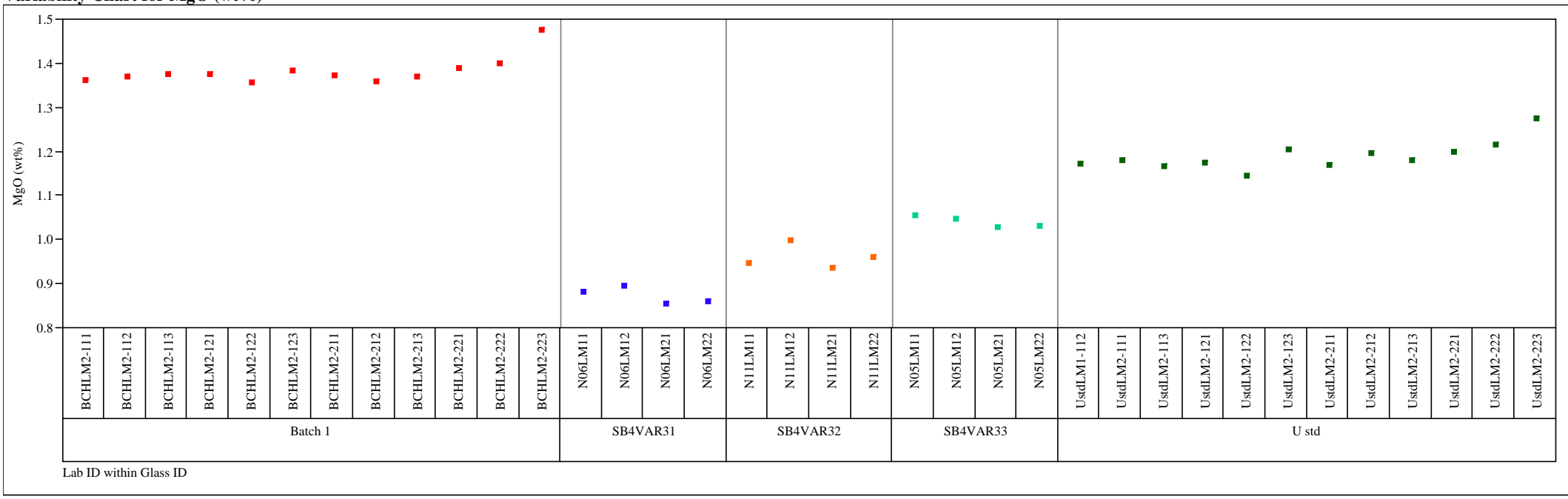

Set $=2$

Variability Chart for MnO (wt\%)

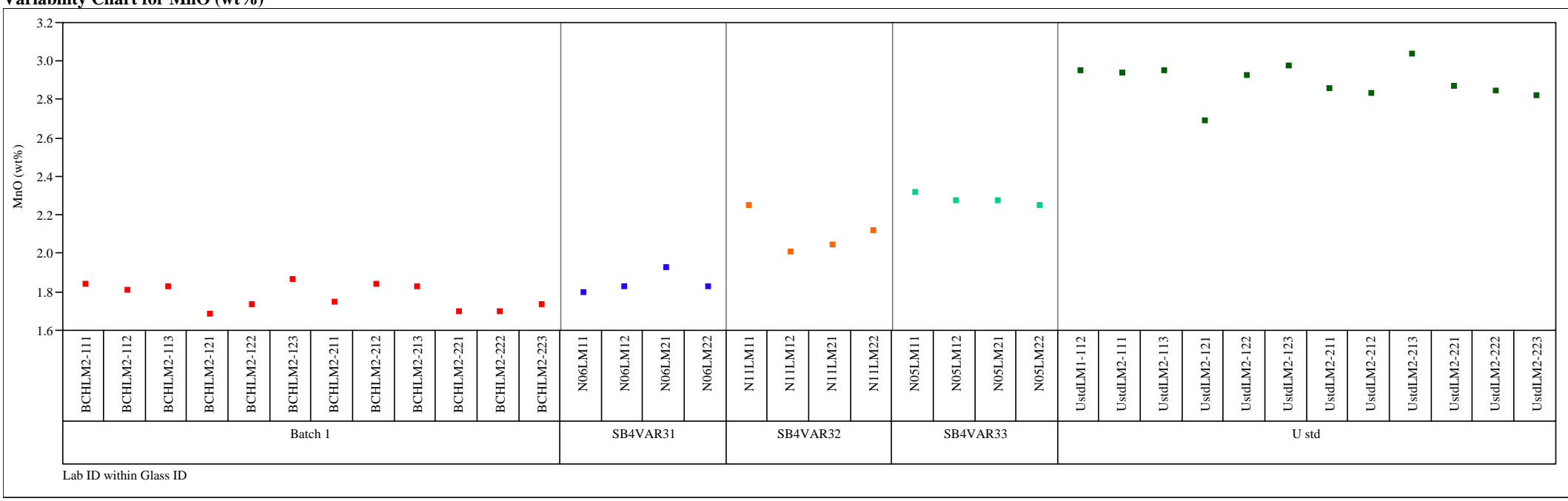




\section{Exhibit A5. Oxide Measurements by Solution ID for Samples Prepared Using the LM Method}

Set $=2$

Variability Chart for $\mathrm{Na2O}$ (wt \%)

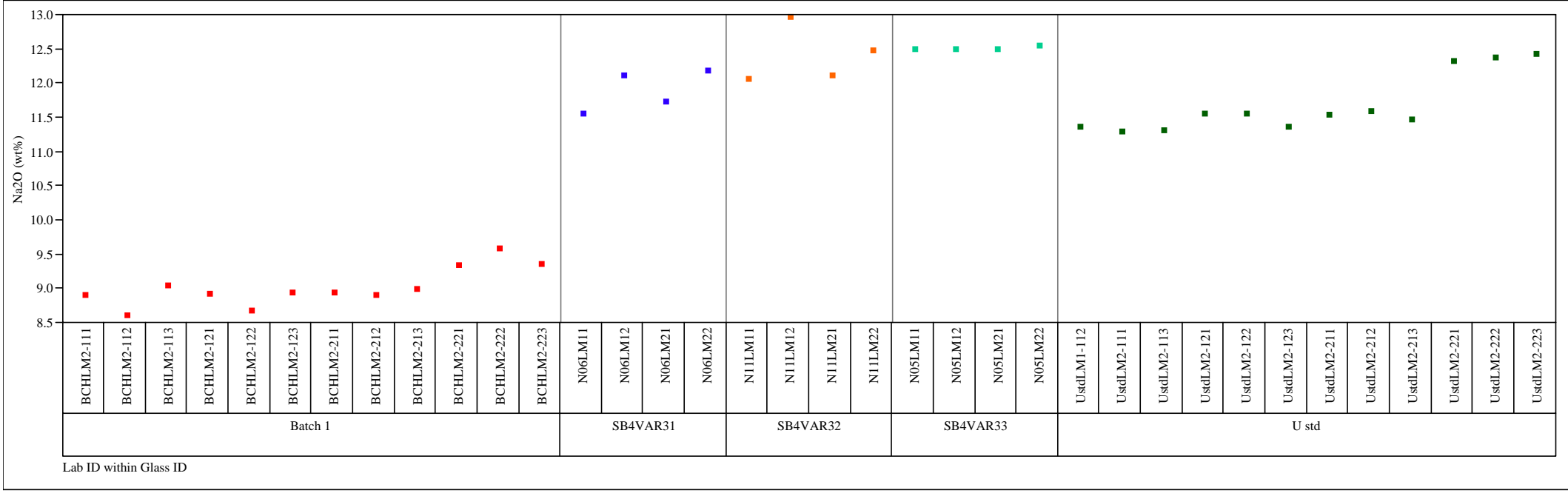

Set=2

Variability Chart for NiO (wt\%)

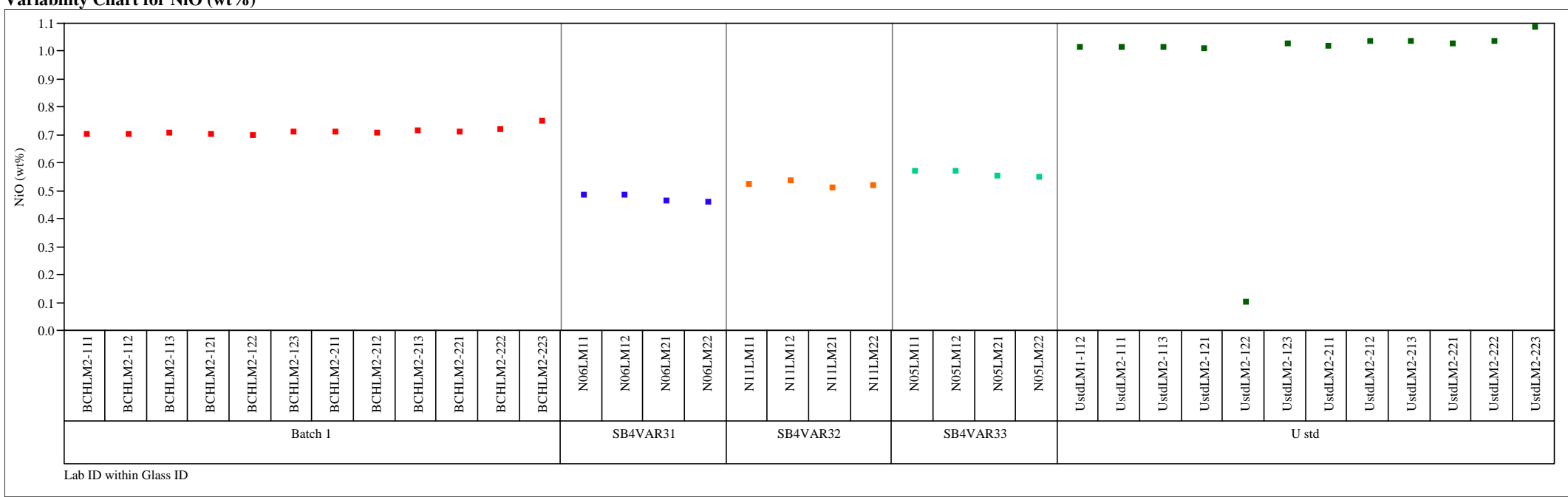




\section{Exhibit A5. Oxide Measurements by Solution ID for Samples Prepared Using the LM Method}

Set $=2$

Variability Chart for PbO (wt \%)

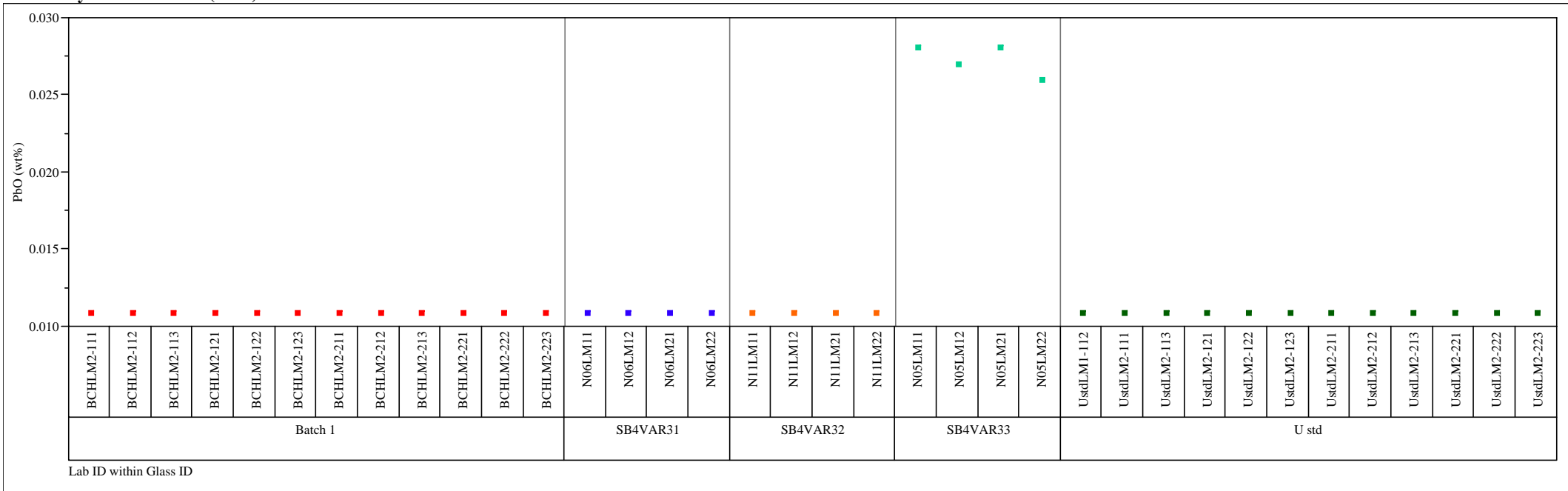

Set $=2$

Variability Chart for SO4 (wt \%)

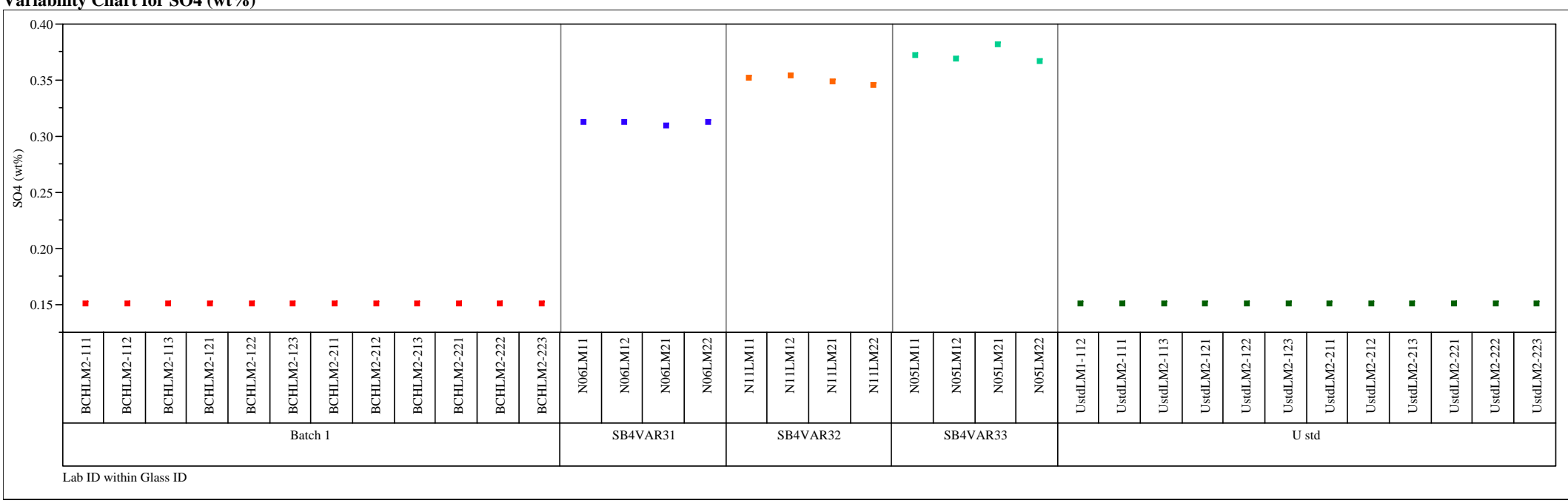


Exhibit A5. Oxide Measurements by Solution ID for Samples Prepared Using the LM Method

Set $=2$

Variability Chart for SiO2 (wt\%)

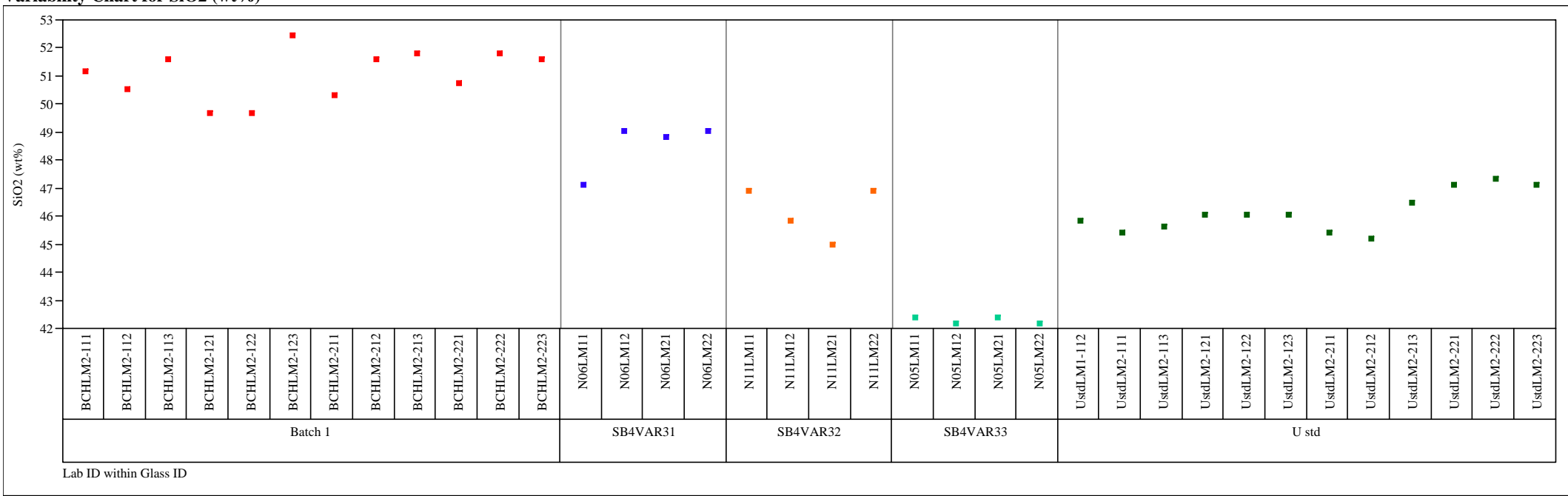

Set $=2$

Variability Chart for TiO2 (wt\%)

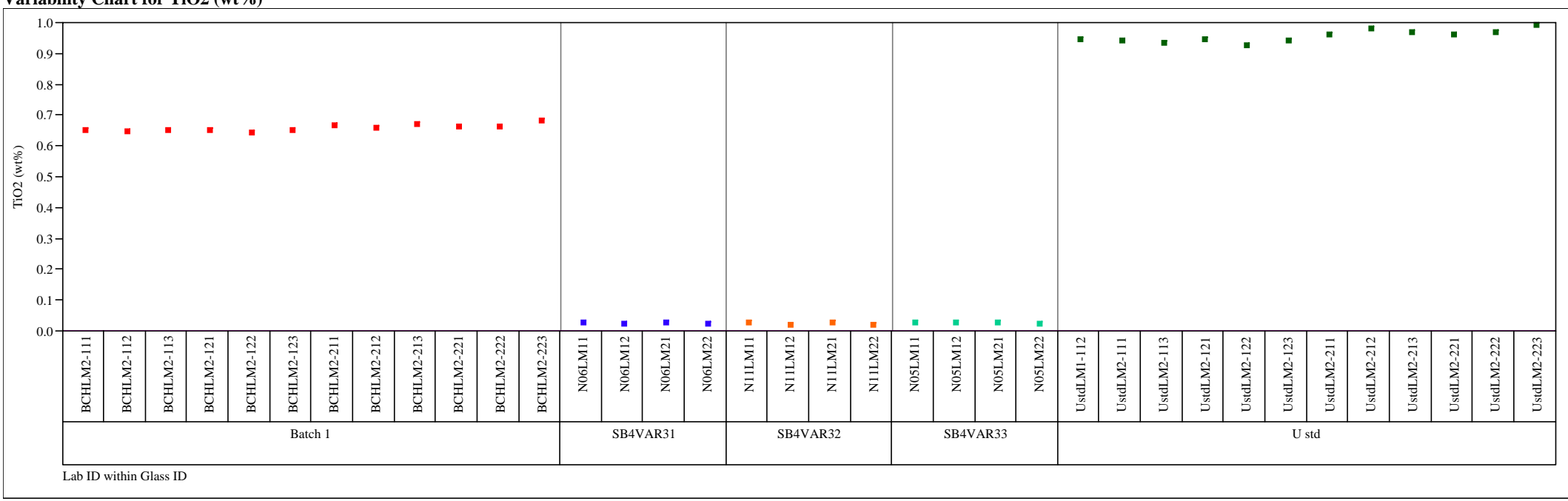




\section{Exhibit A5. Oxide Measurements by Solution ID for Samples Prepared Using the LM Method}

Set $=2$

Variability Chart for U3O8 (wt \%)

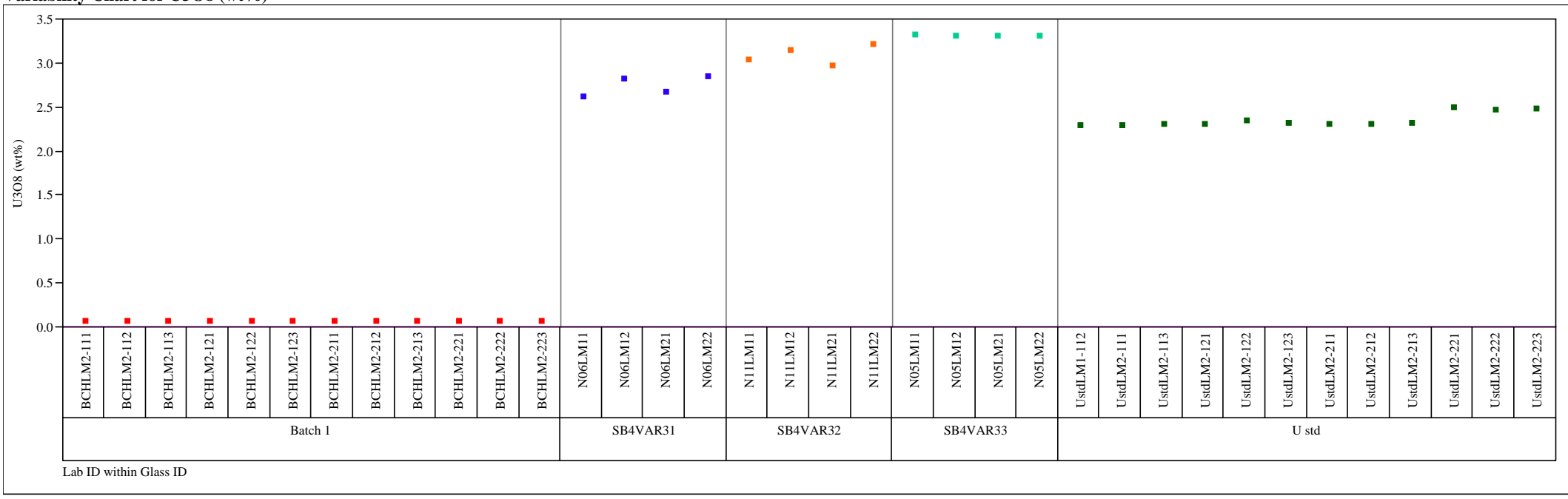

Set $=2$

Variability Chart for ZnO (wt\%)

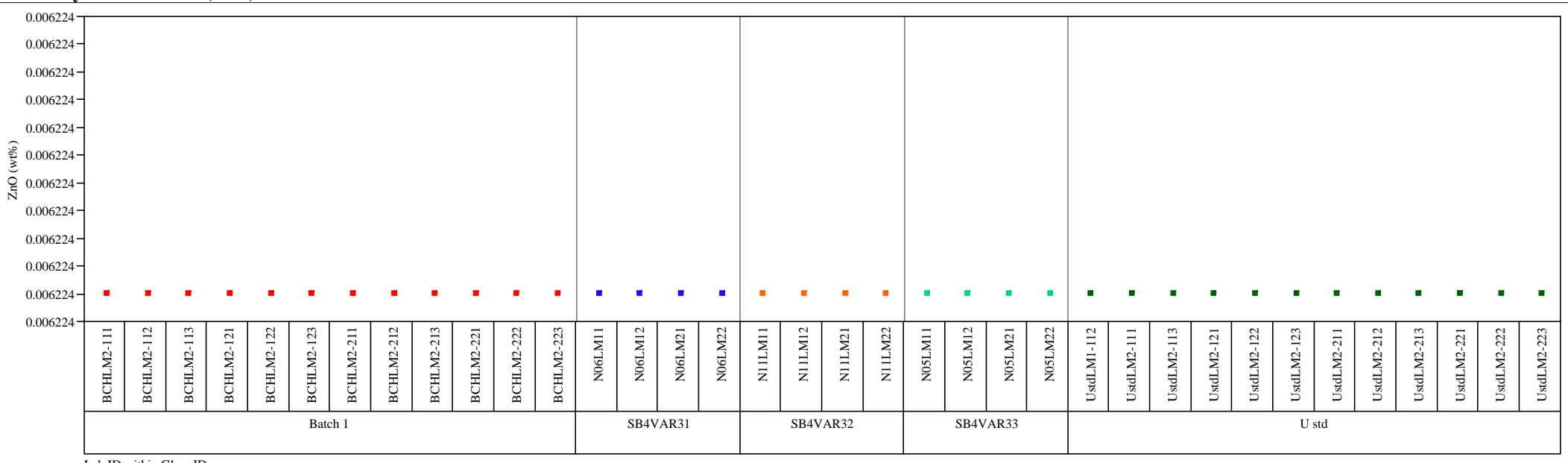




\section{Exhibit A5. Oxide Measurements by Solution ID for Samples Prepared Using the LM Method}

Set $=2$

Variability Chart for $\mathrm{ZrO} 2$ (wt\%)

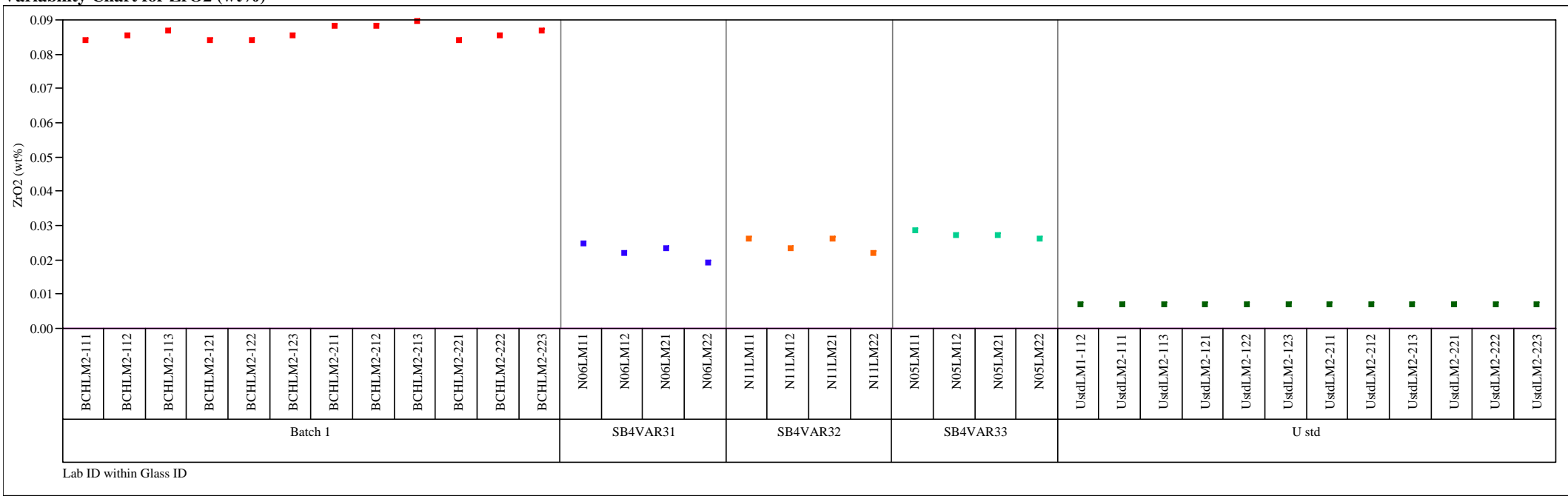

Set $=2$

Variability Chart for Al2O3 bc (wt \%)

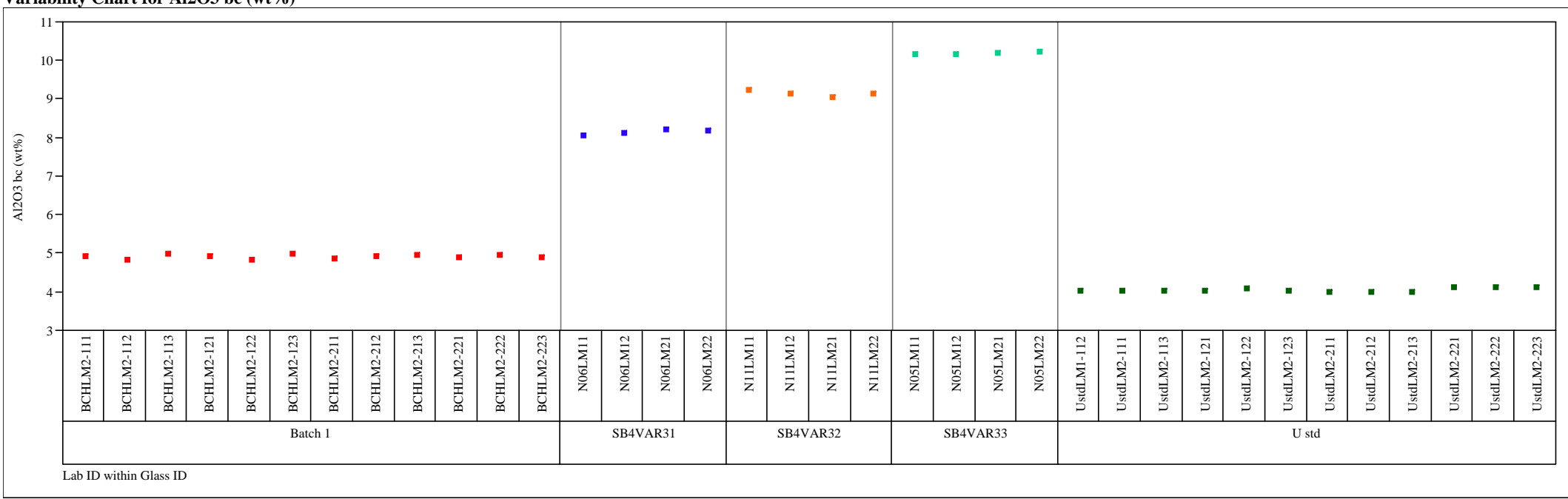




\section{Exhibit A5. Oxide Measurements by Solution ID for Samples Prepared Using the LM Method}

Set $=2$

Variability Chart for BaO bc (wt \%)

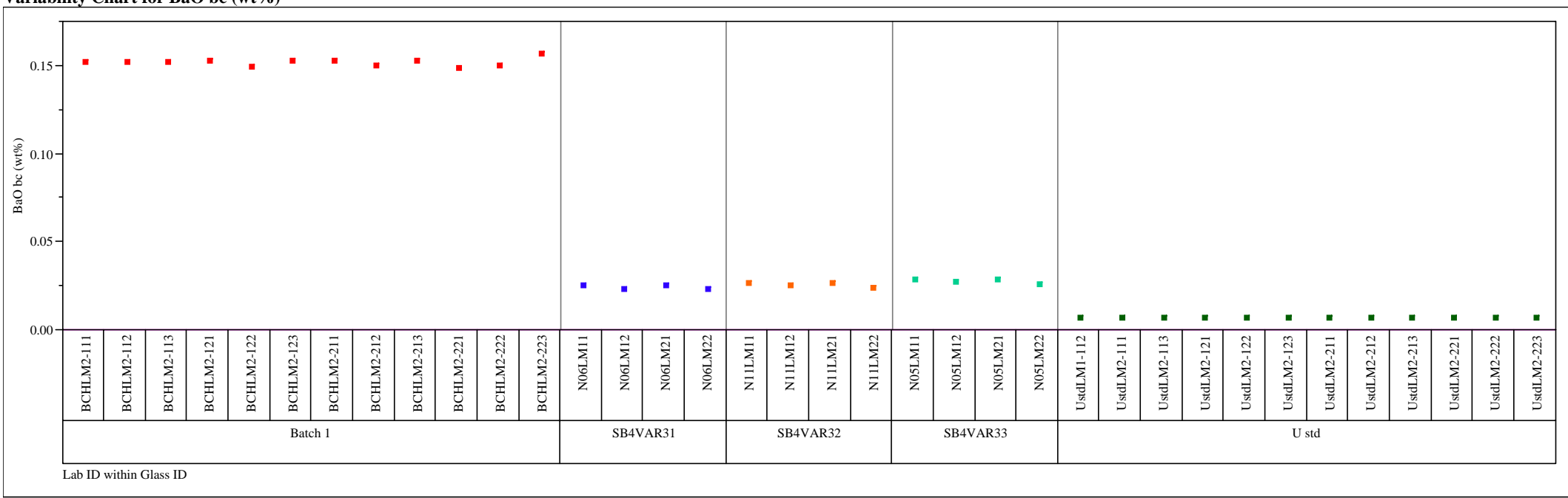

Set $=2$

Variability Chart for CaO bc (wt \%)

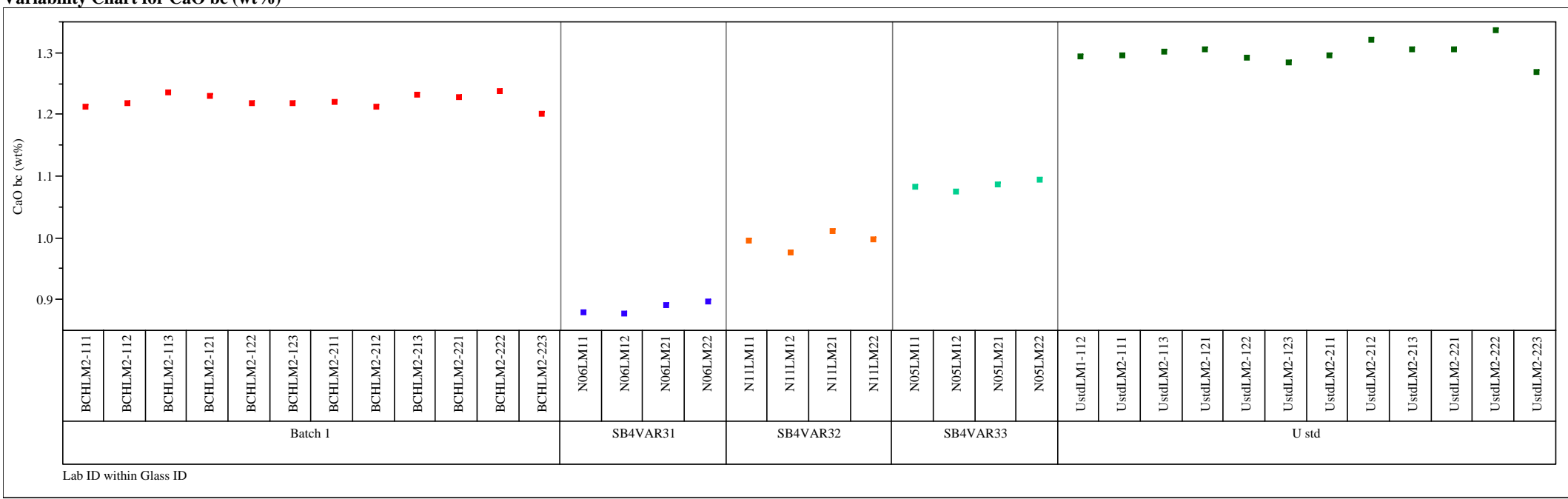




\section{Exhibit A5. Oxide Measurements by Solution ID for Samples Prepared Using the LM Method}

Set $=2$

Variability Chart for CdO bc (wt\%)

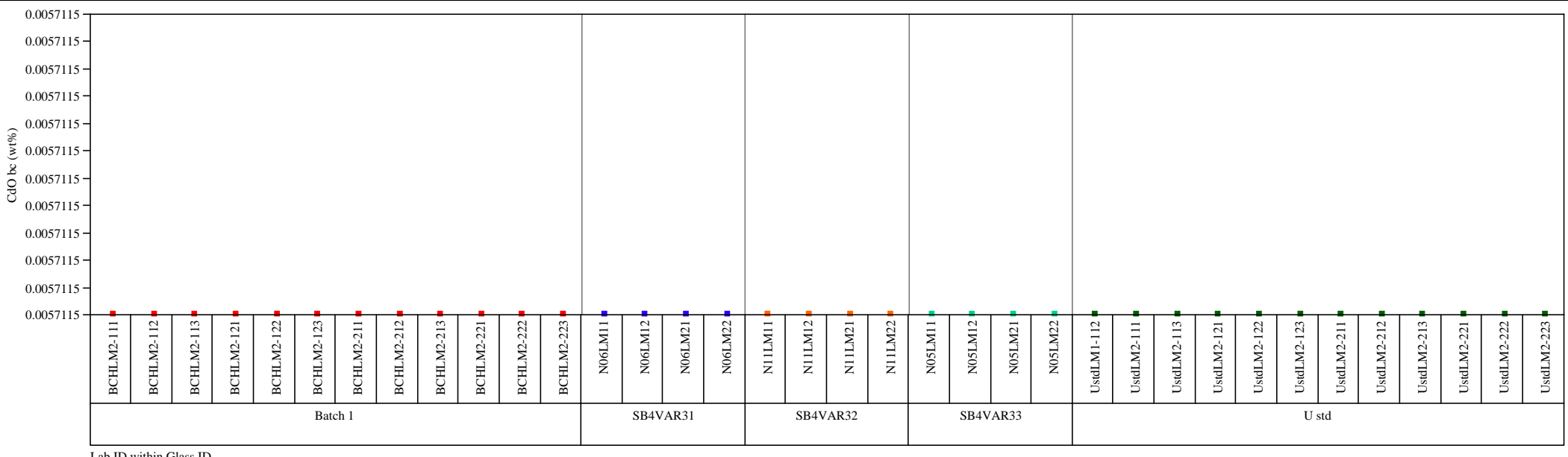

Set $=2$

Variability Chart for Ce2O3 bc (wt\%)

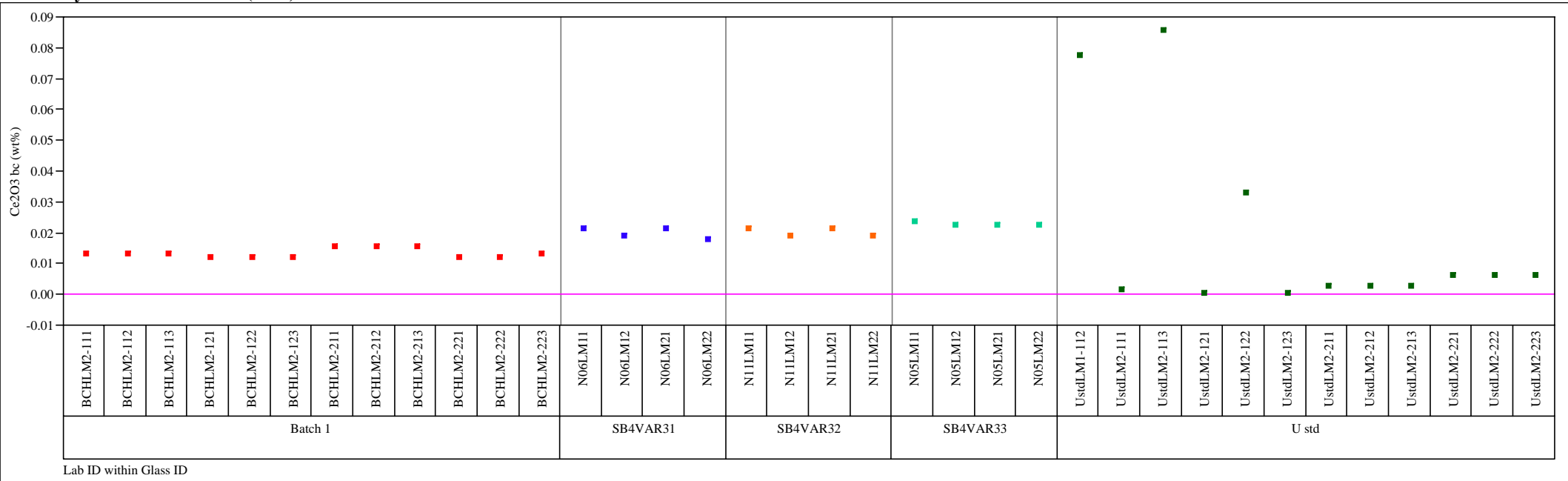




\section{Exhibit A5. Oxide Measurements by Solution ID for Samples Prepared Using the LM Method}

Set $=2$

Variability Chart for Cr2O3 bc (wt \%)

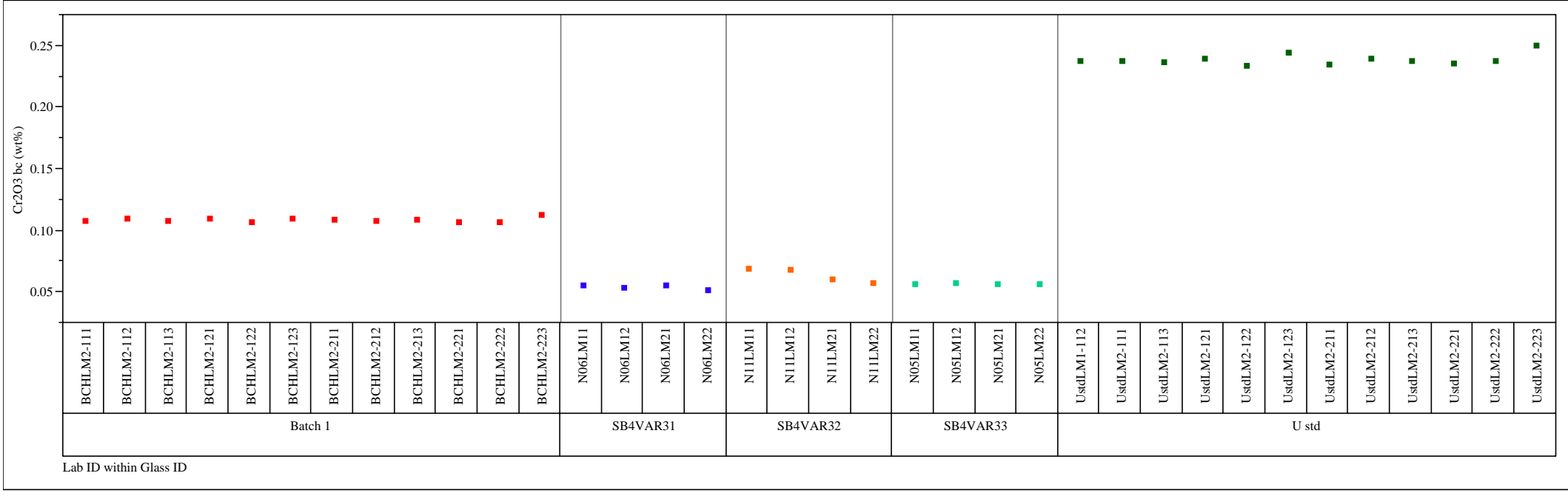

Set $=2$

Variability Chart for CuO bc (wt\%)

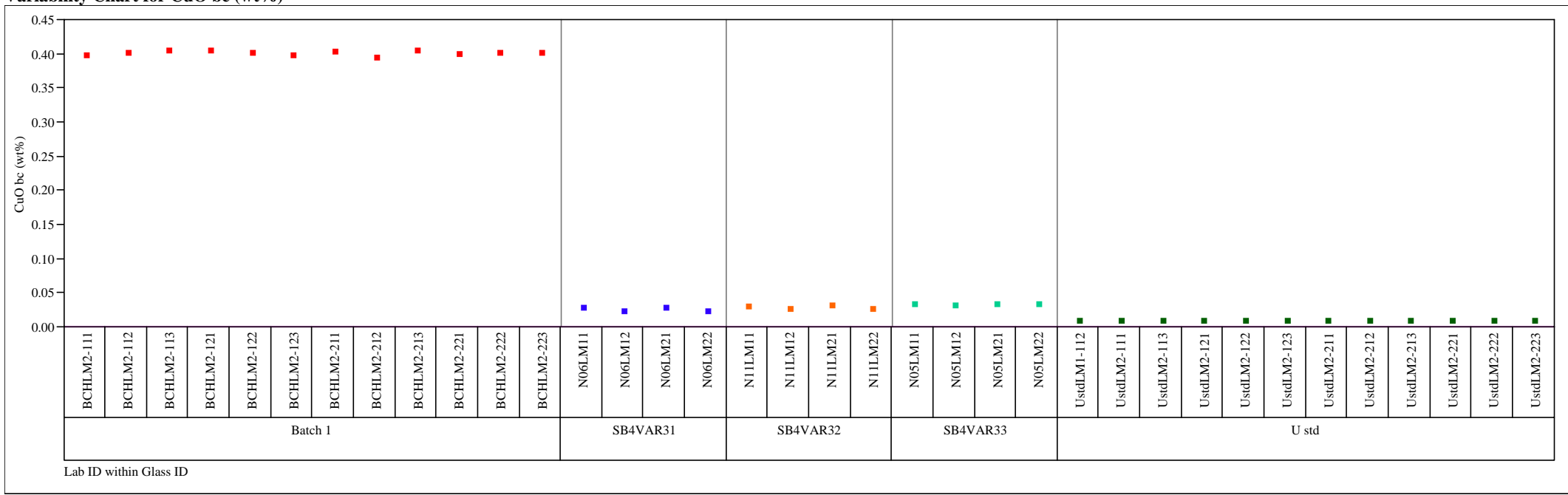




\section{Exhibit A5. Oxide Measurements by Solution ID for Samples Prepared Using the LM Method}

Set $=2$

Variability Chart for Fe2O3 bc (wt \%)

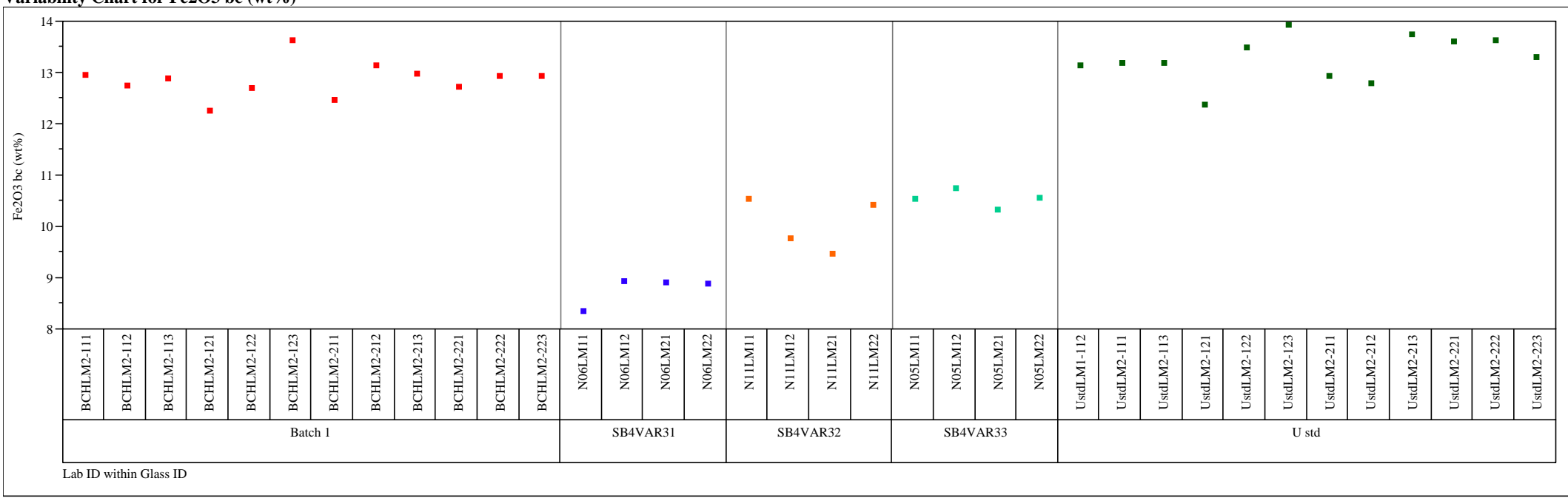

Set $=2$

Variability Chart for K2O bc (wt \%)

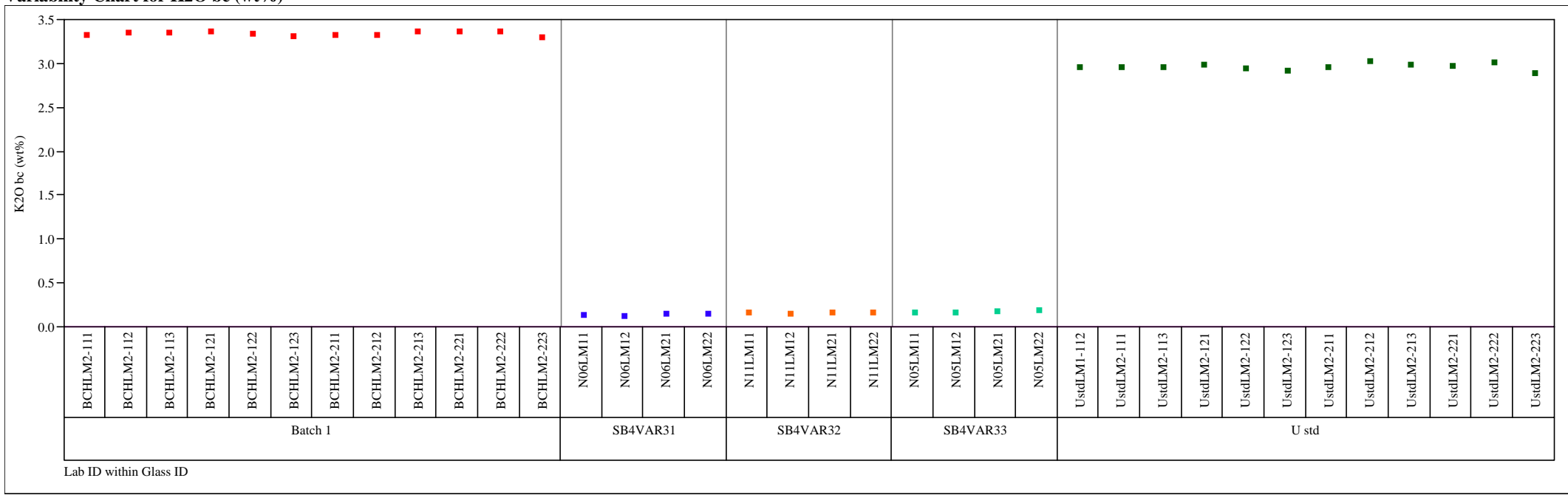




\section{Exhibit A5. Oxide Measurements by Solution ID for Samples Prepared Using the LM Method}

Set $=2$

Variability Chart for La2O3 bc (wt \%)

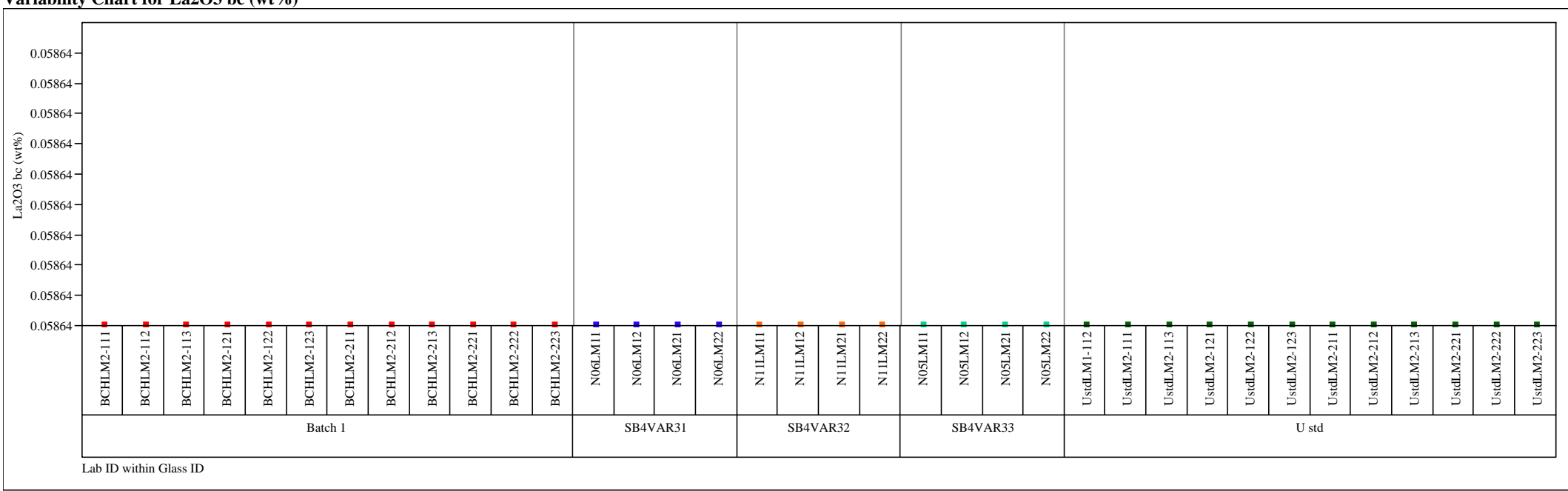

Set=2

Variability Chart for MgO bc (wt \%)

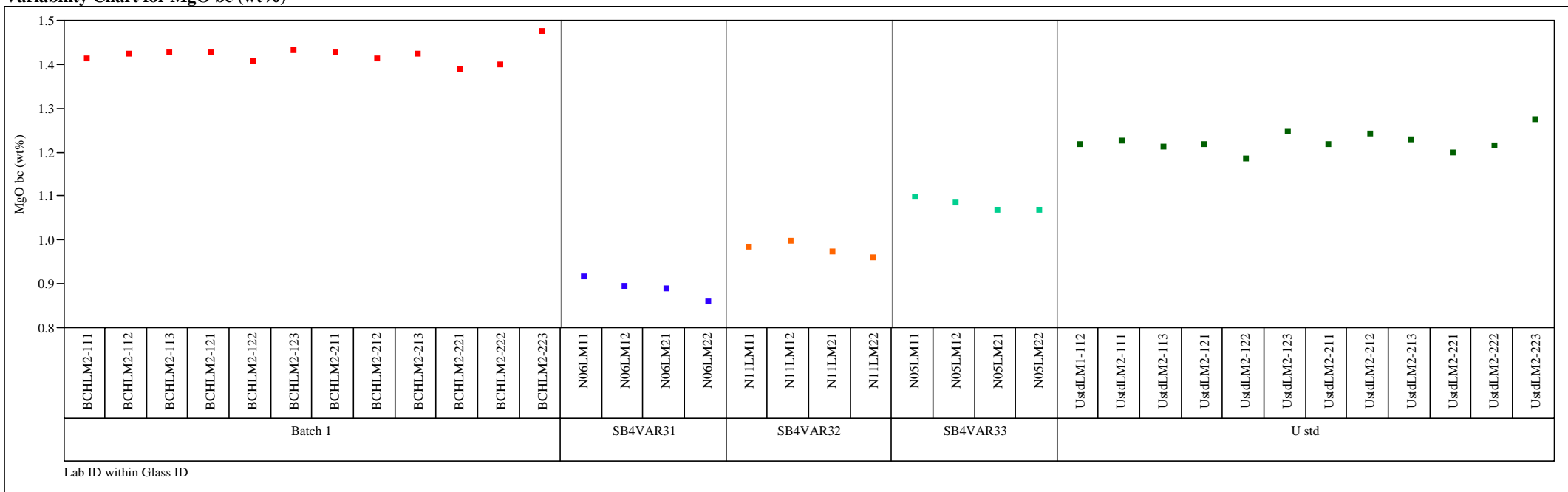


Exhibit A5. Oxide Measurements by Solution ID for Samples Prepared Using the LM Method

Set $=2$

Variability Chart for MnO bc (wt\%)

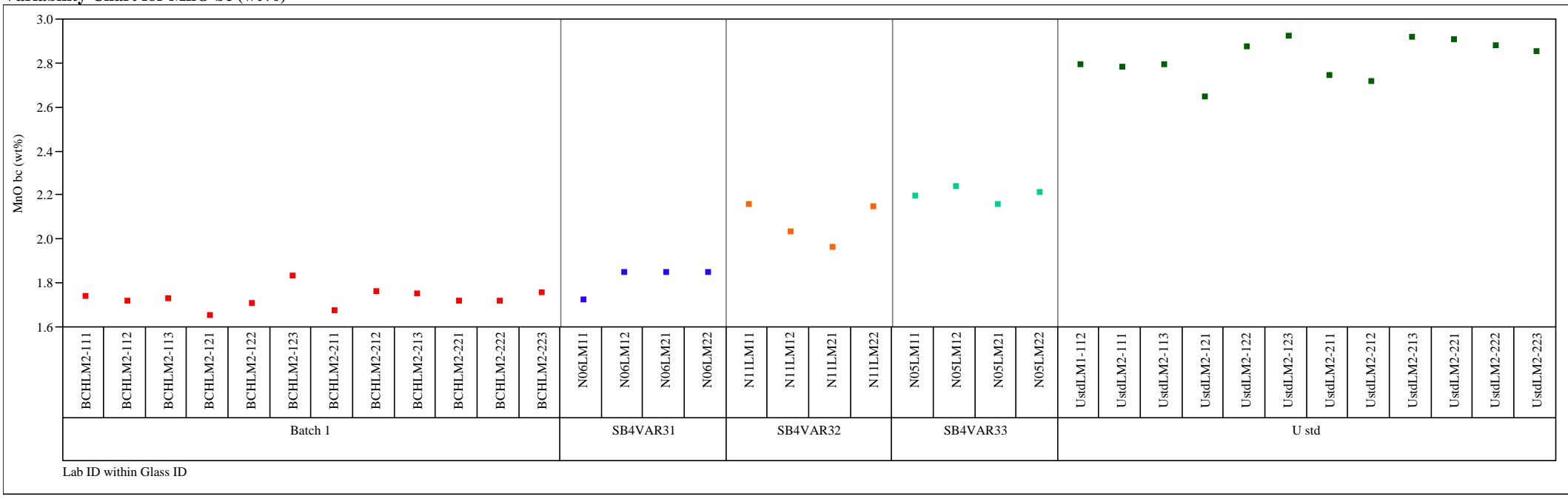

Set $=2$

Variability Chart for Na2O bc (wt\%)

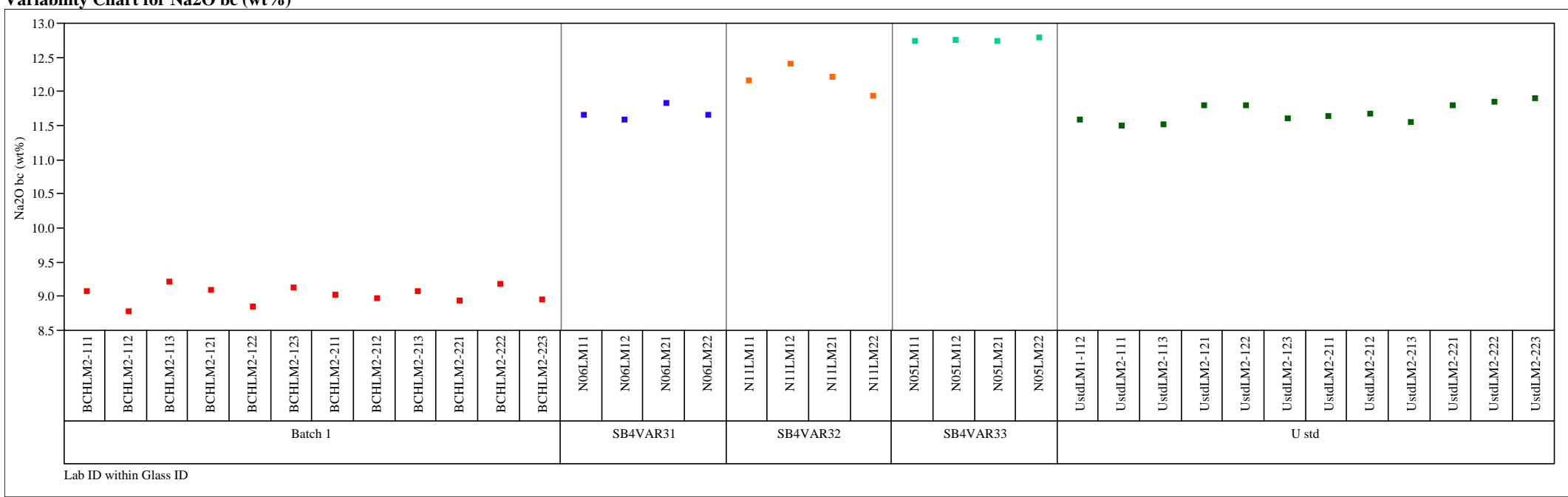


Exhibit A5. Oxide Measurements by Solution ID for Samples Prepared Using the LM Method

Set $=2$

Variability Chart for NiO bc (wt\%)

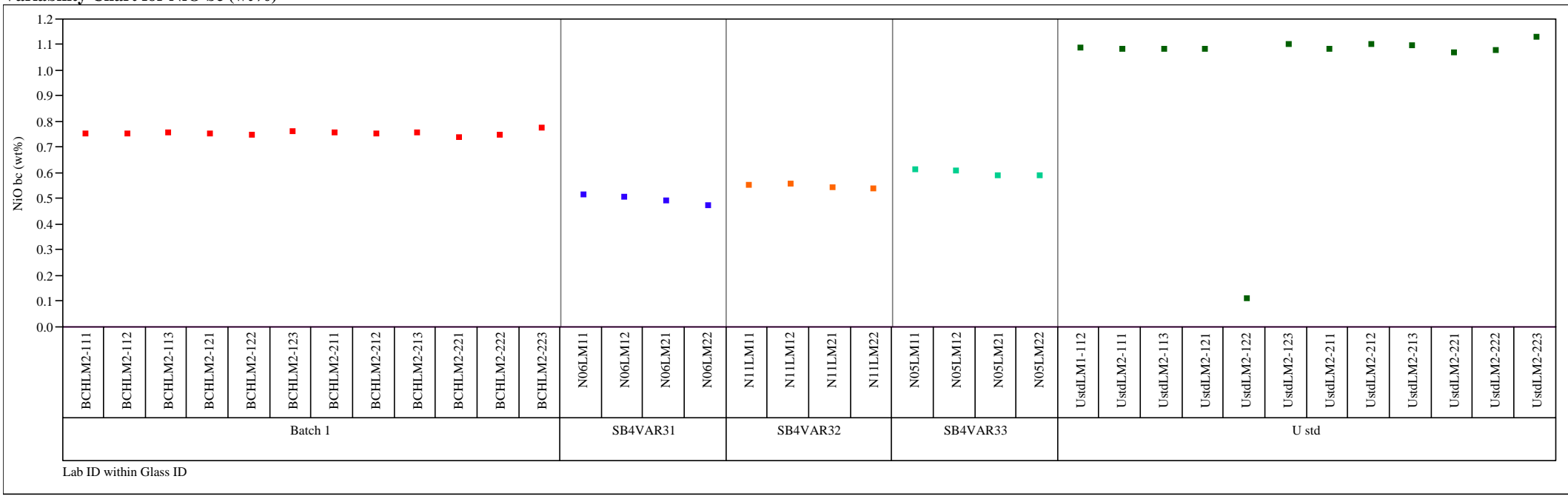

Set $=2$

Variability Chart for PbO bc (wt\%)

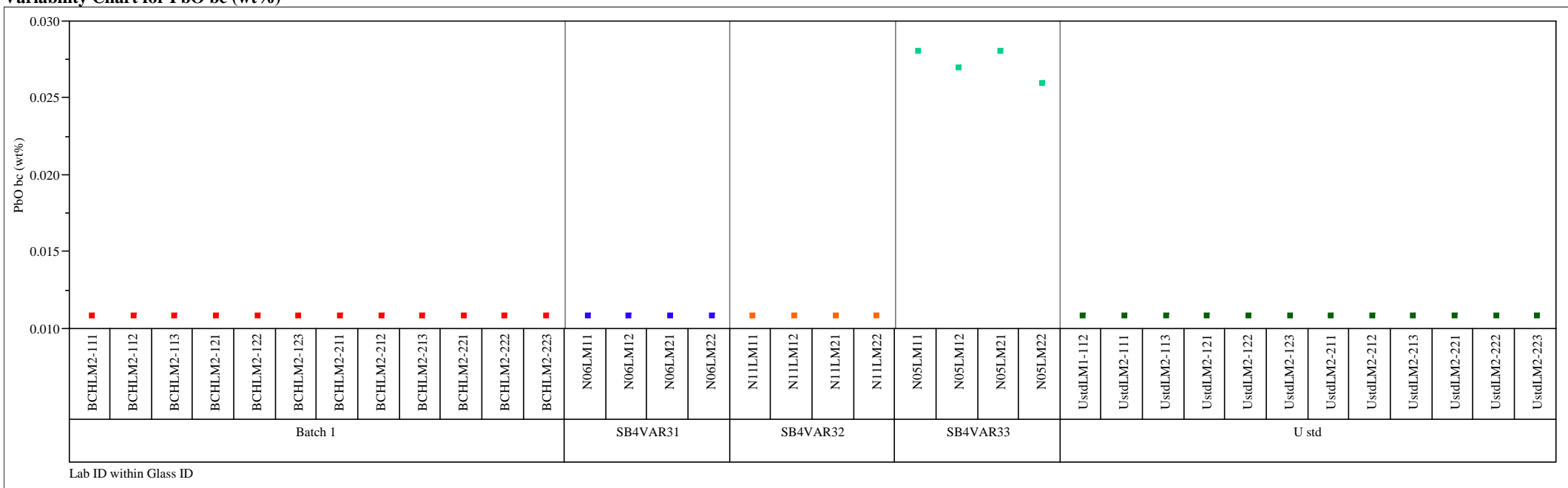




\section{Exhibit A5. Oxide Measurements by Solution ID for Samples Prepared Using the LM Method}

Set $=2$

Variability Chart for SO4 bc (wt \%)

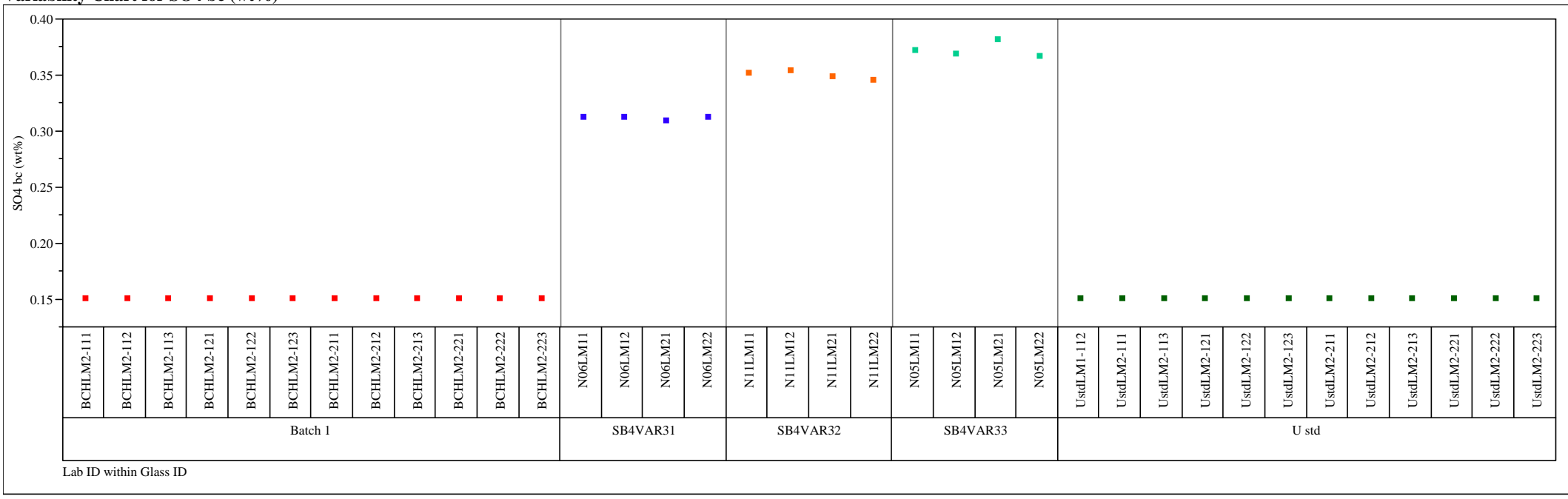

Set $=2$

Variability Chart for SiO2 bc (wt\%)

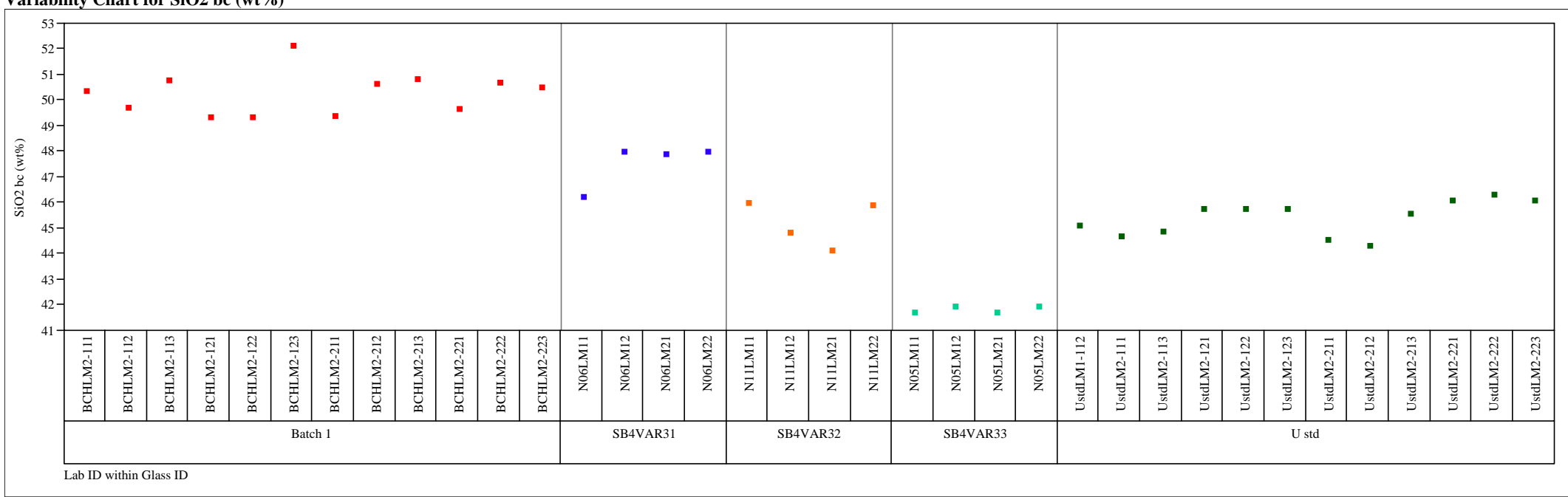




\section{Exhibit A5. Oxide Measurements by Solution ID for Samples Prepared Using the LM Method}

Set $=2$

Variability Chart for TiO2 bc (wt \%)

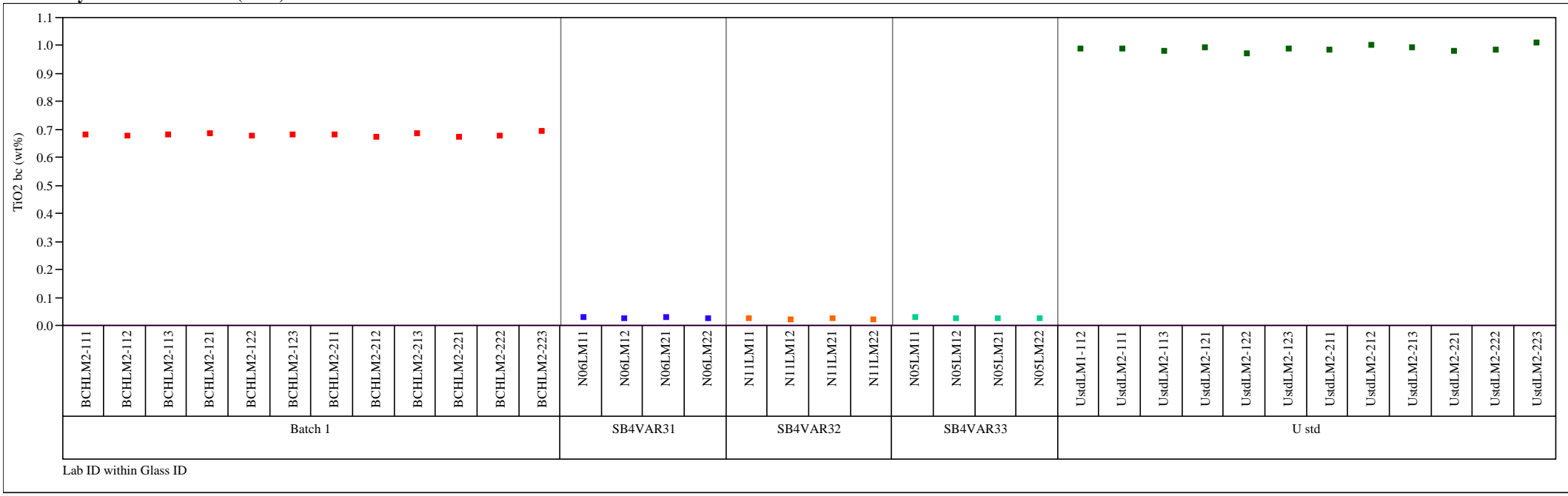

Set $=2$

Variability Chart for U3O8 bc (wt \%)

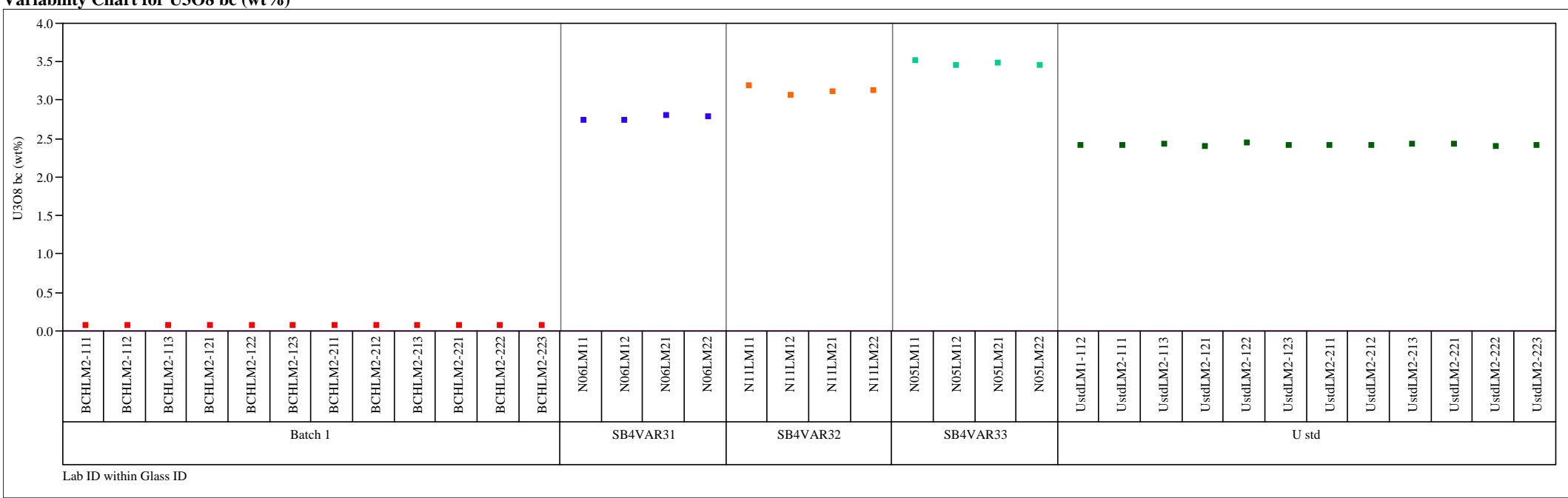




\section{Exhibit A5. Oxide Measurements by Solution ID for Samples Prepared Using the LM Method}

Set $=2$

Variability Chart for $\mathrm{ZnO}$ bc (wt \%)

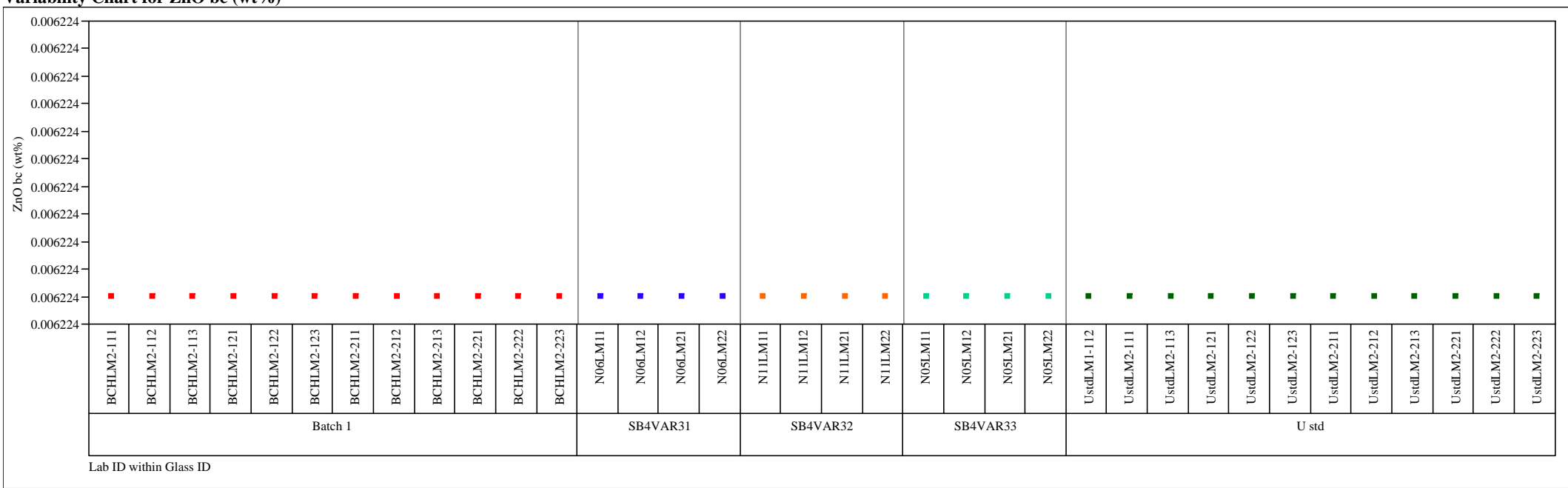

Set $=2$

Variability Chart for ZrO2 bc (wt\%)

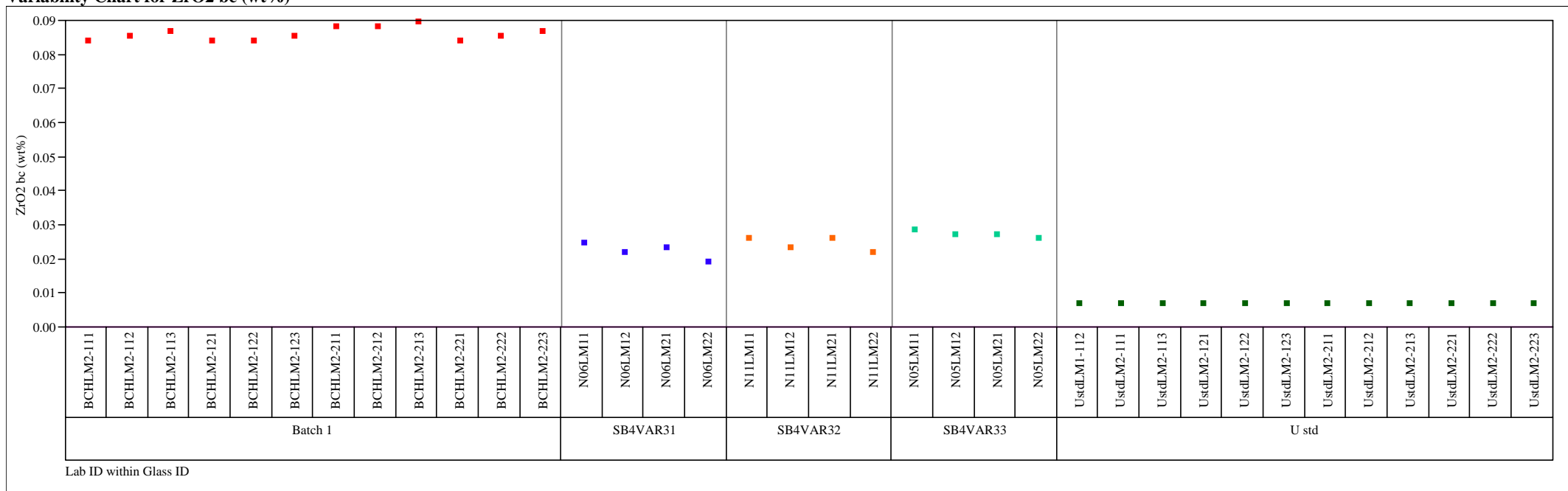




\section{Exhibit A6. Average Oxide Measurements by Solution ID for Samples Prepared Using the PF Method}

Set $=1$

Variability Chart for B2O3 (wt \%)

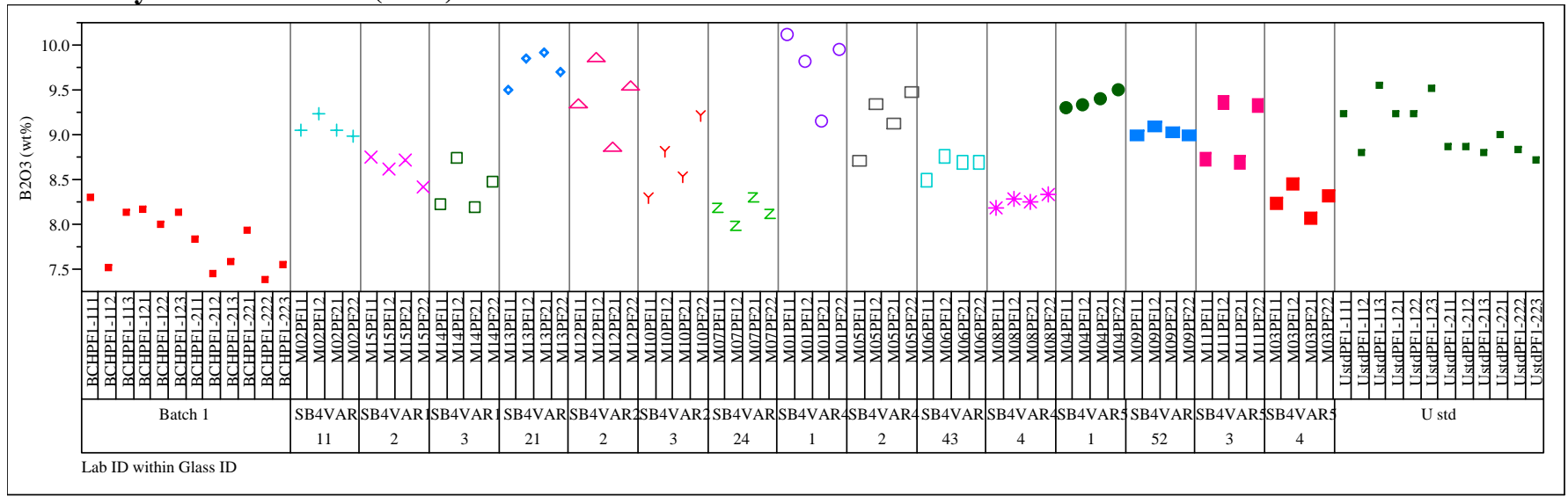

Set=1

Variability Chart for Li2O (wt\%)

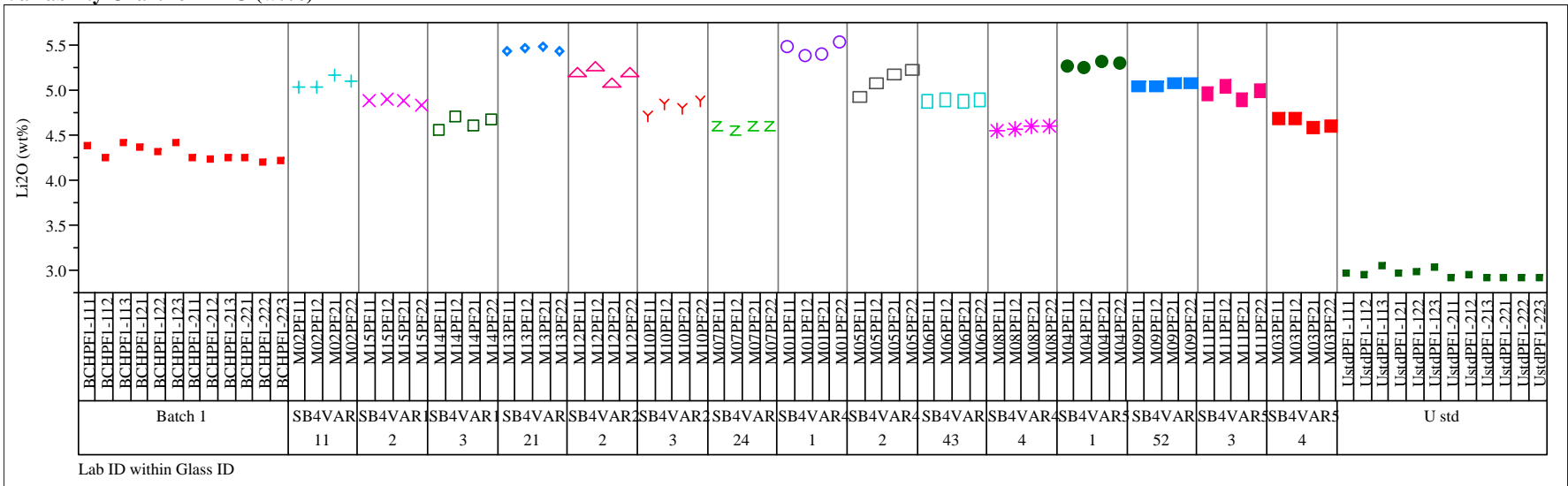




\section{Exhibit A6. Average Oxide Measurements by Solution ID for Samples Prepared Using the PF Method}

Set=1

Variability Chart for SiO2 (wt \%)

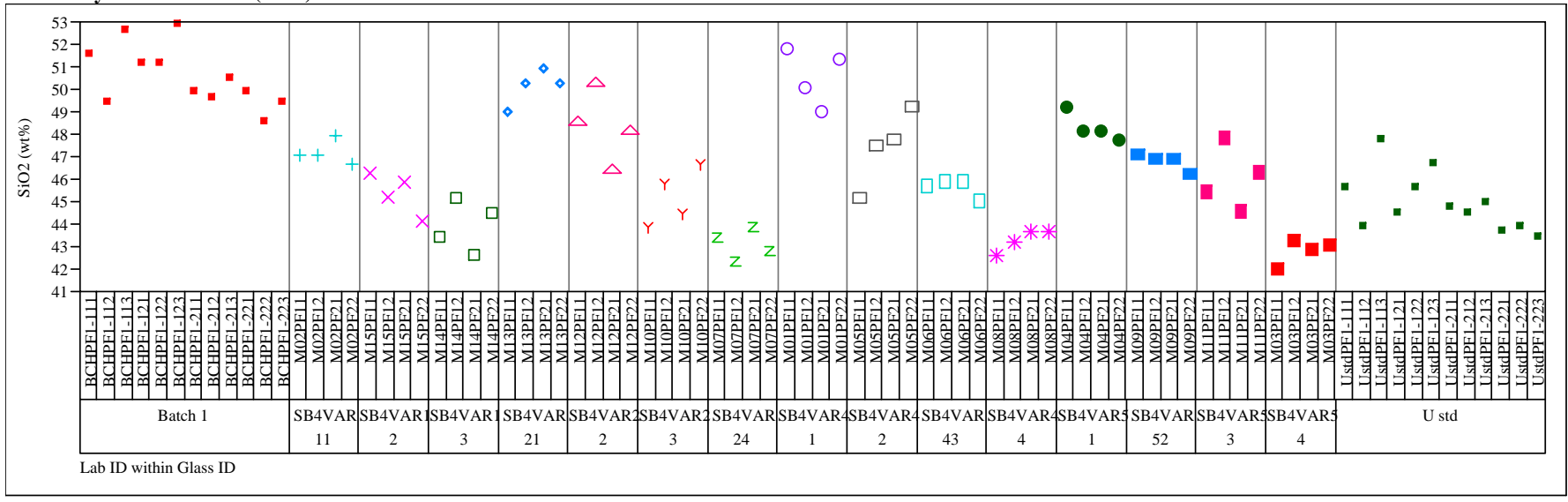

Set $=1$

Variability Chart for B2O3 bc (wt\%)

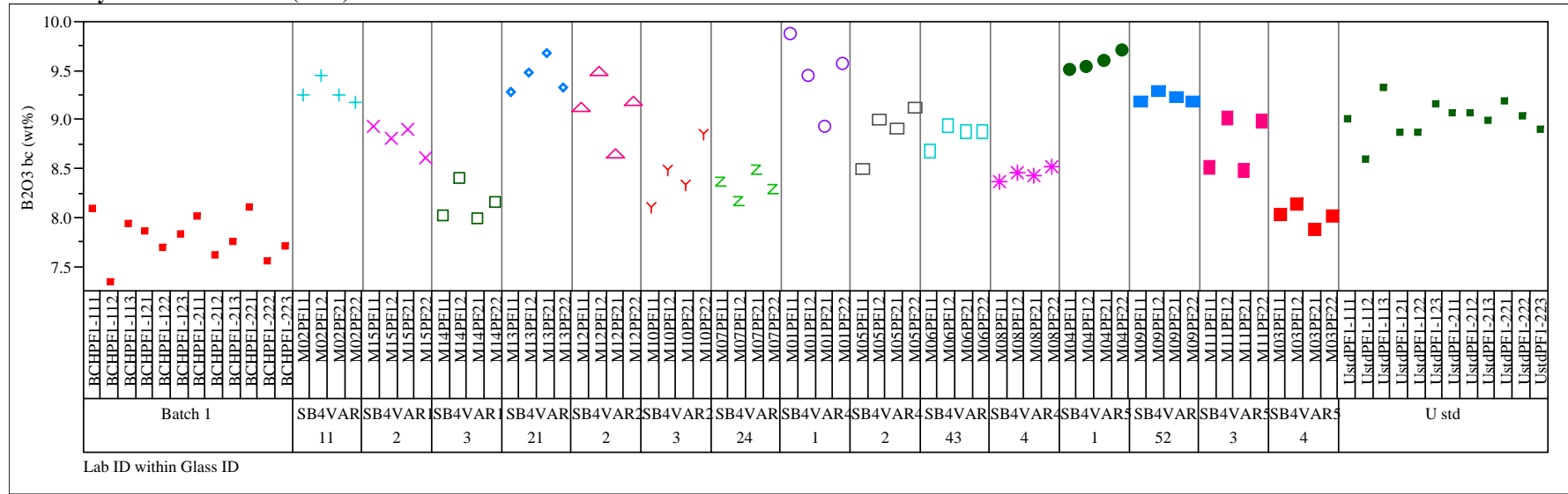




\section{Exhibit A6. Average Oxide Measurements by Solution ID for Samples Prepared Using the PF Method}

Set $=1$

Variability Chart for Li2O bc (wt \%)

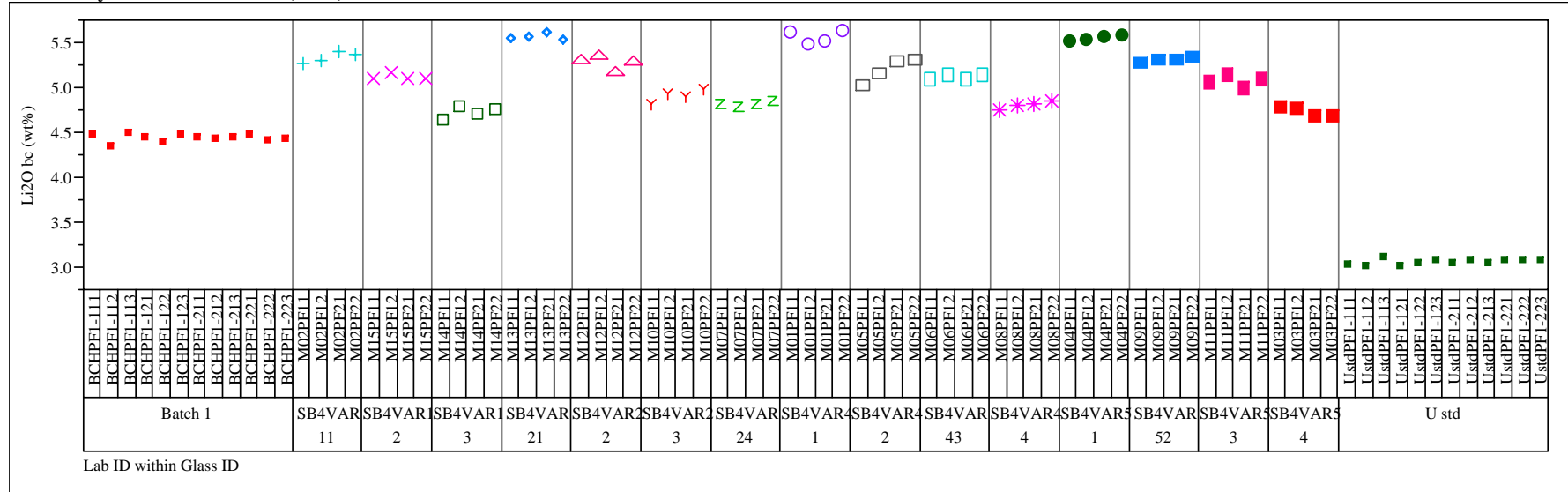

Set $=1$

Variability Chart for SiO2 bc (wt\%)

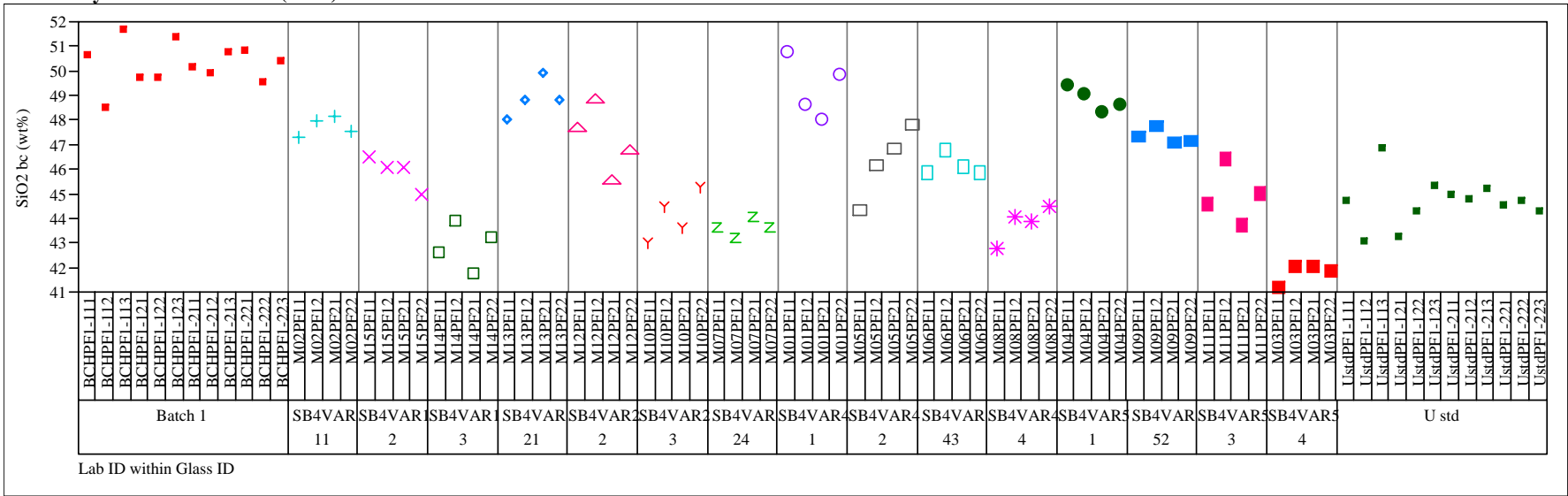




\section{Exhibit A6. Average Oxide Measurements by Solution ID for Samples Prepared Using the PF Method}

Set=2

Variability Chart for B2O3 (wt\%)

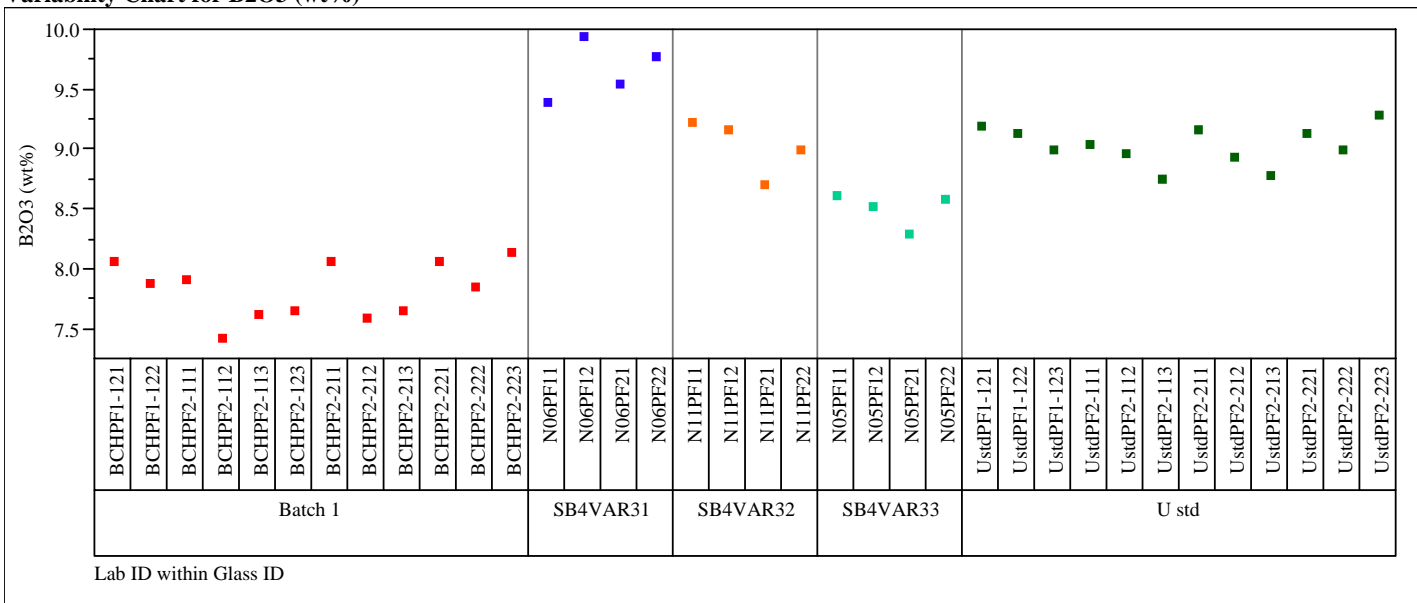

Set $=2$

Variability Chart for Li2O (wt\%)

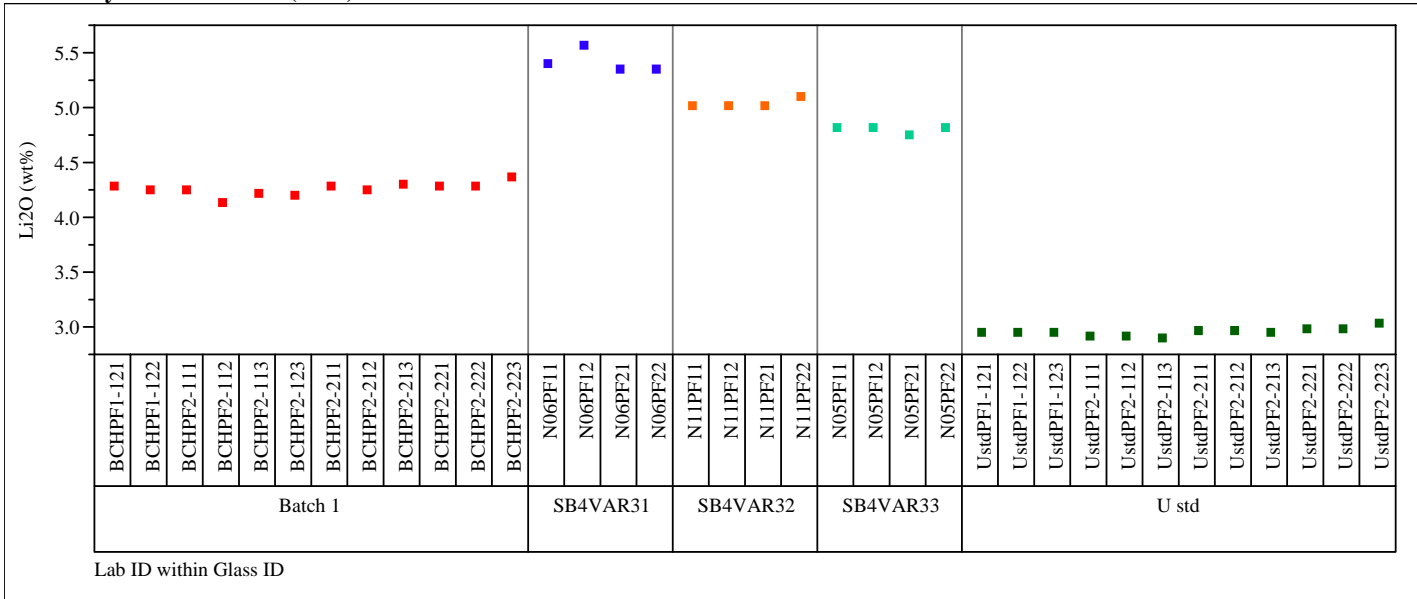




\section{Exhibit A6. Average Oxide Measurements by Solution ID for Samples Prepared Using the PF Method}

Set=2

Variability Chart for SiO2 (wt\%)

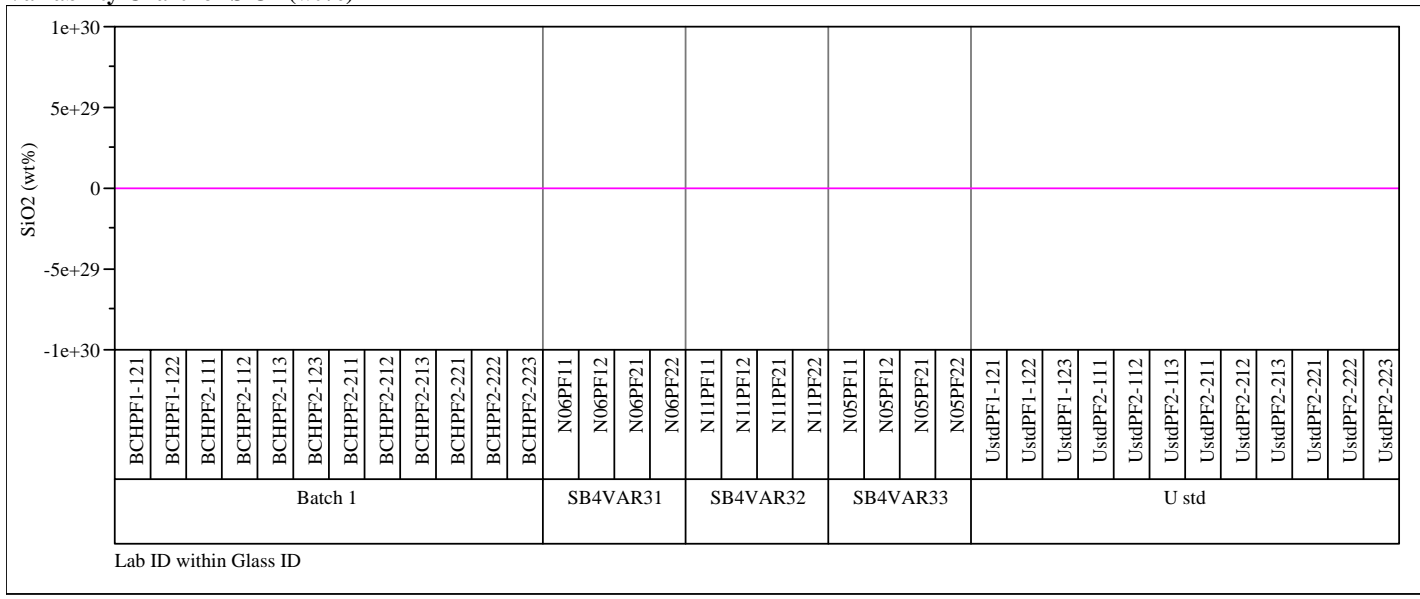

Set=2

Variability Chart for B2O3 bc (wt\%)

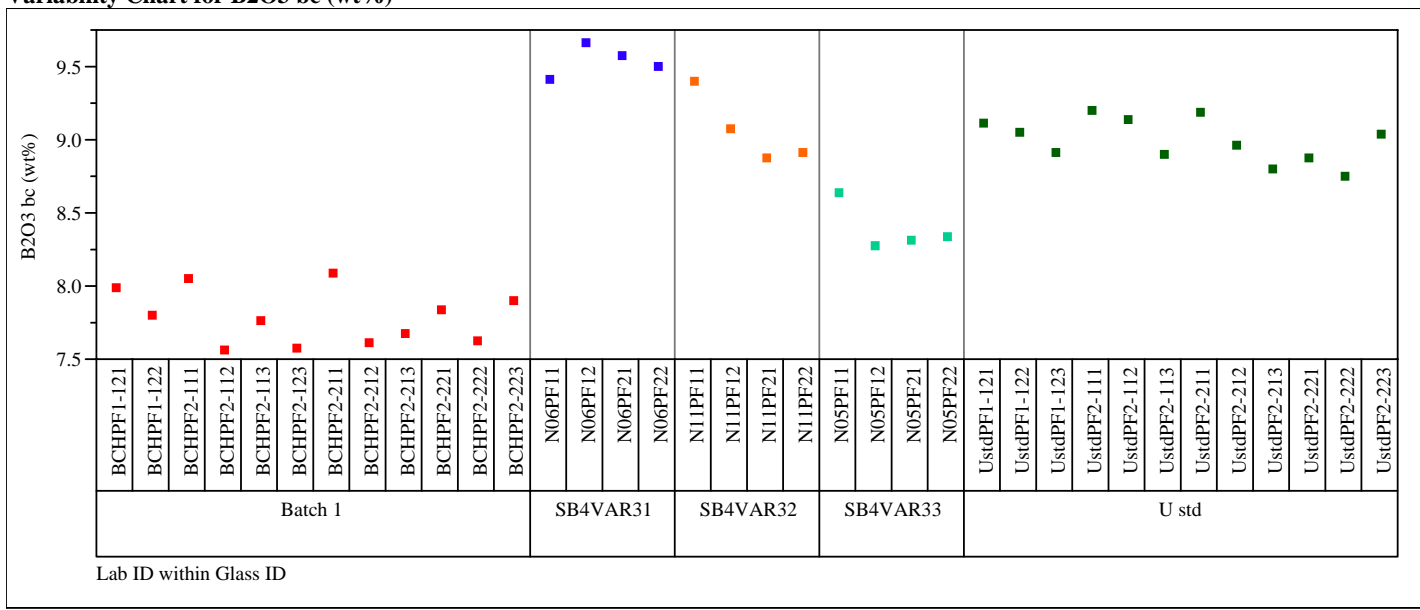




\section{Exhibit A6. Average Oxide Measurements by Solution ID for Samples Prepared Using the PF Method}

Set=2

Variability Chart for Li2O bc (wt\%)

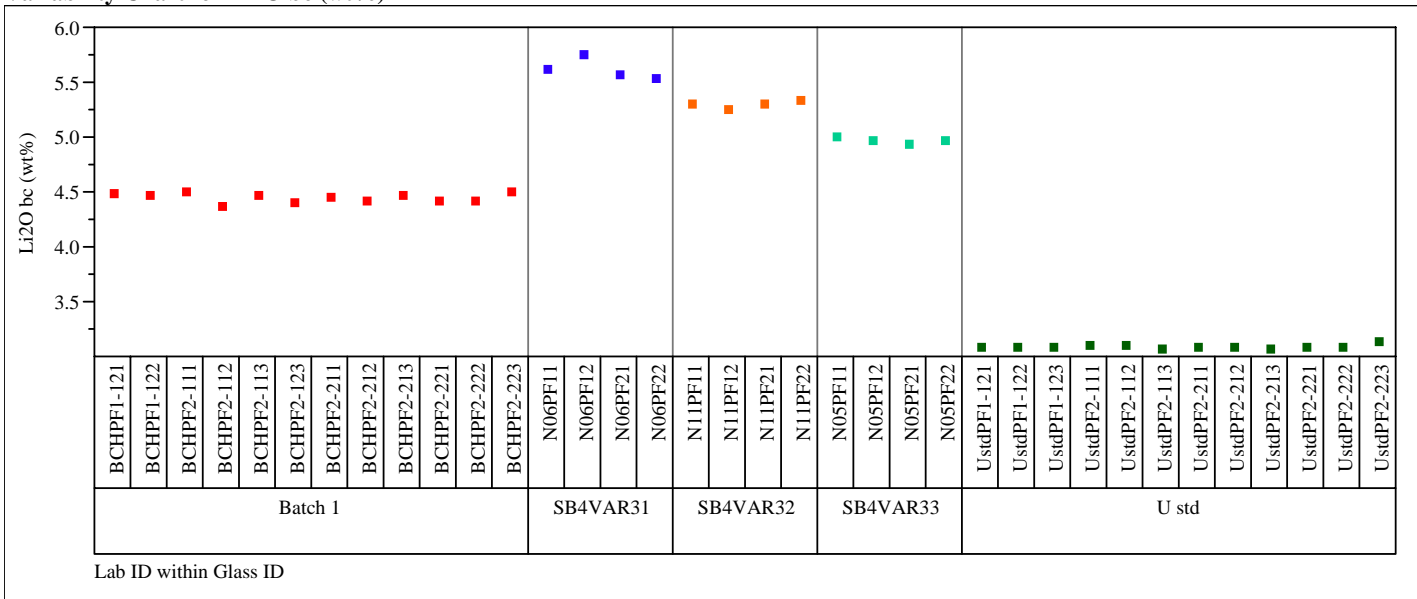

Set $=2$

Variability Chart for SiO2 bc (wt \%)

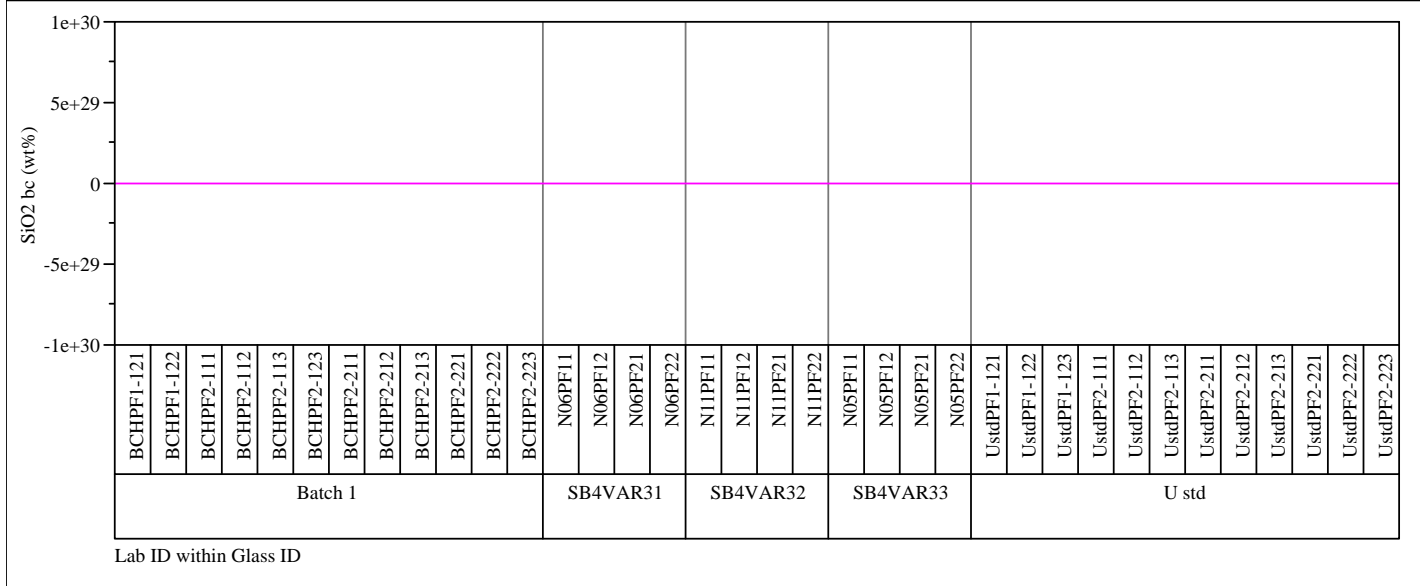




\section{Exhibit A7. Average Measured and Bias-Corrected (bc) Versus Targeted} Compositions by Glass \# by Oxide

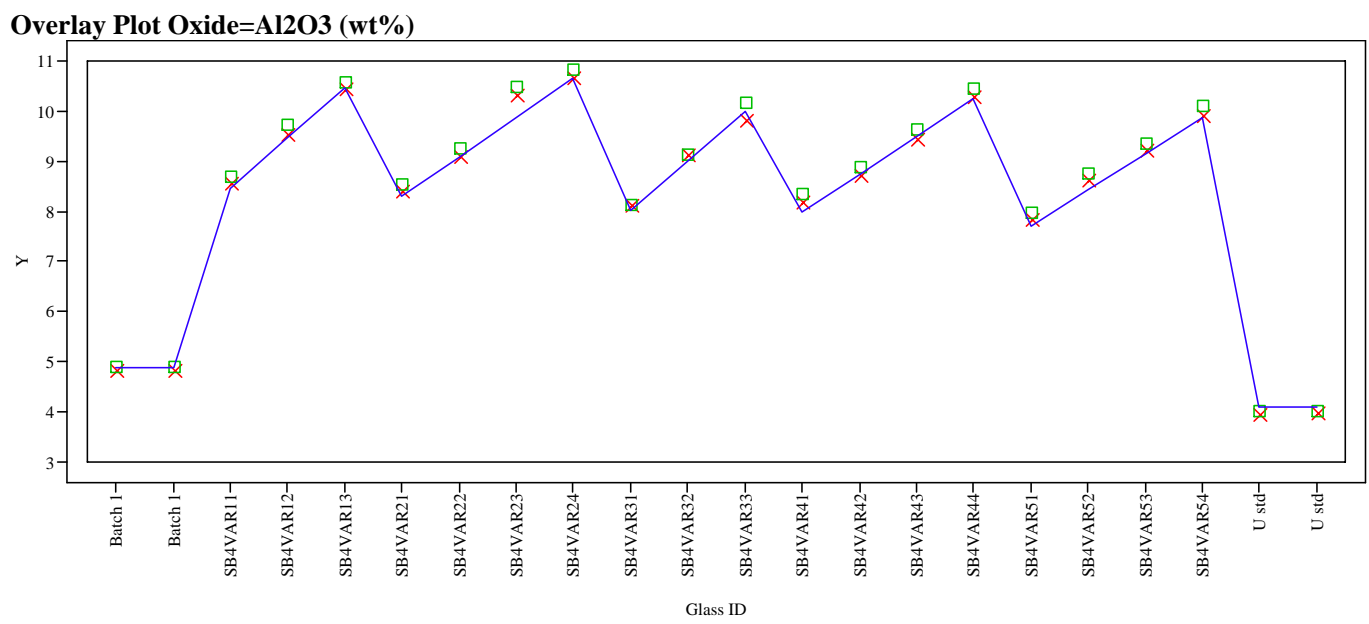

Overlay Plot Oxide=B2O3 (wt \%)

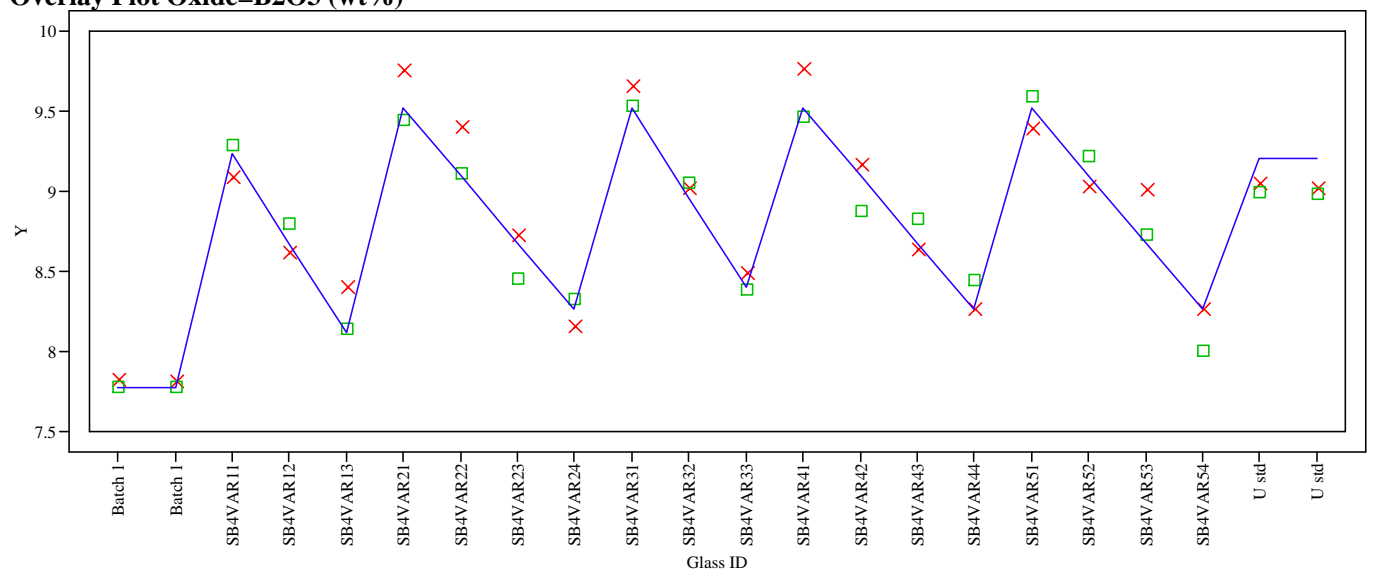

Overlay Plot Oxide=BaO (wt\%)

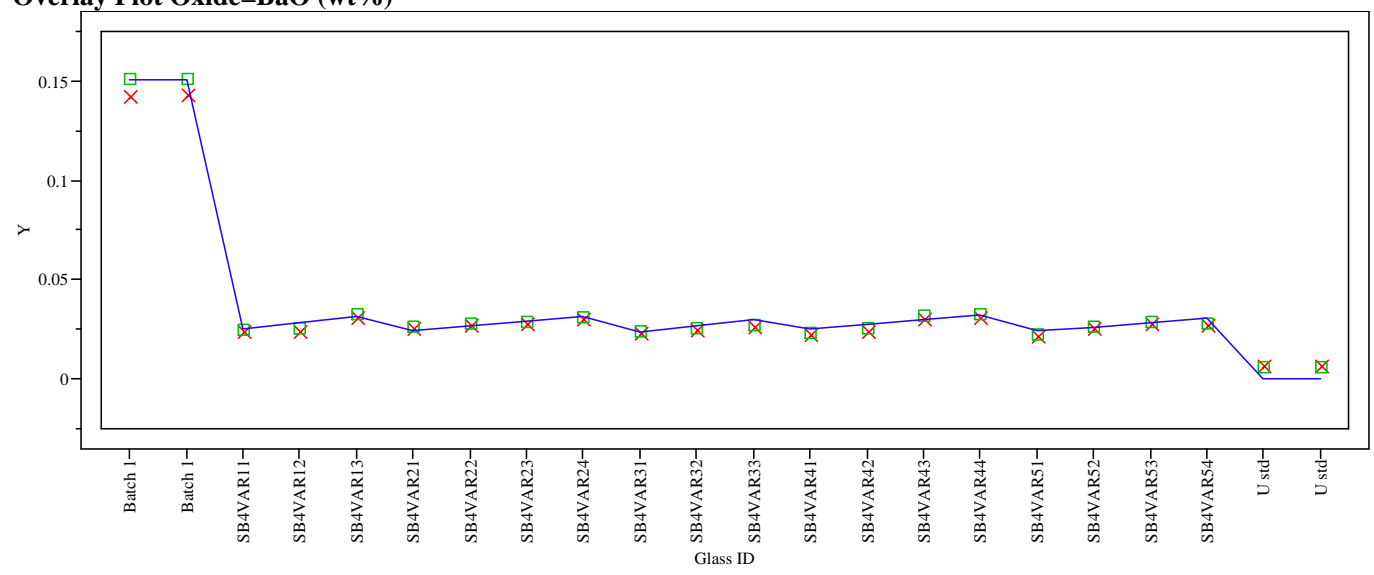

Y $\times$ Measured

⿴囗 Measured bc - Targeted 


\section{Exhibit A7. Average Measured and Bias-Corrected (bc) Versus Targeted} Compositions by Glass \# by Oxide
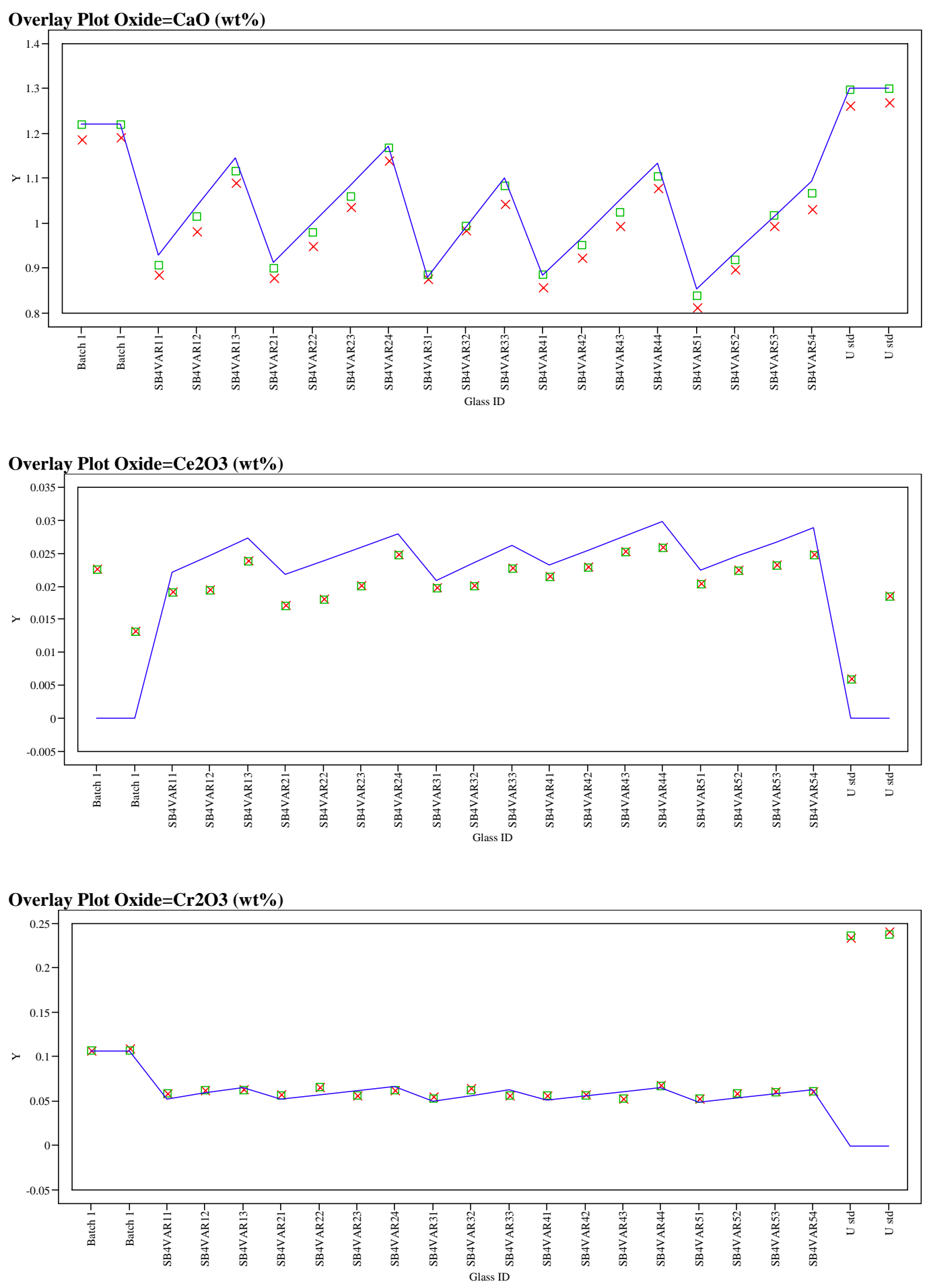

Y $\times$ Measured

a Measured bc - Targeted 


\section{Exhibit A7. Average Measured and Bias-Corrected (bc) Versus Targeted} Compositions by Glass \# by Oxide
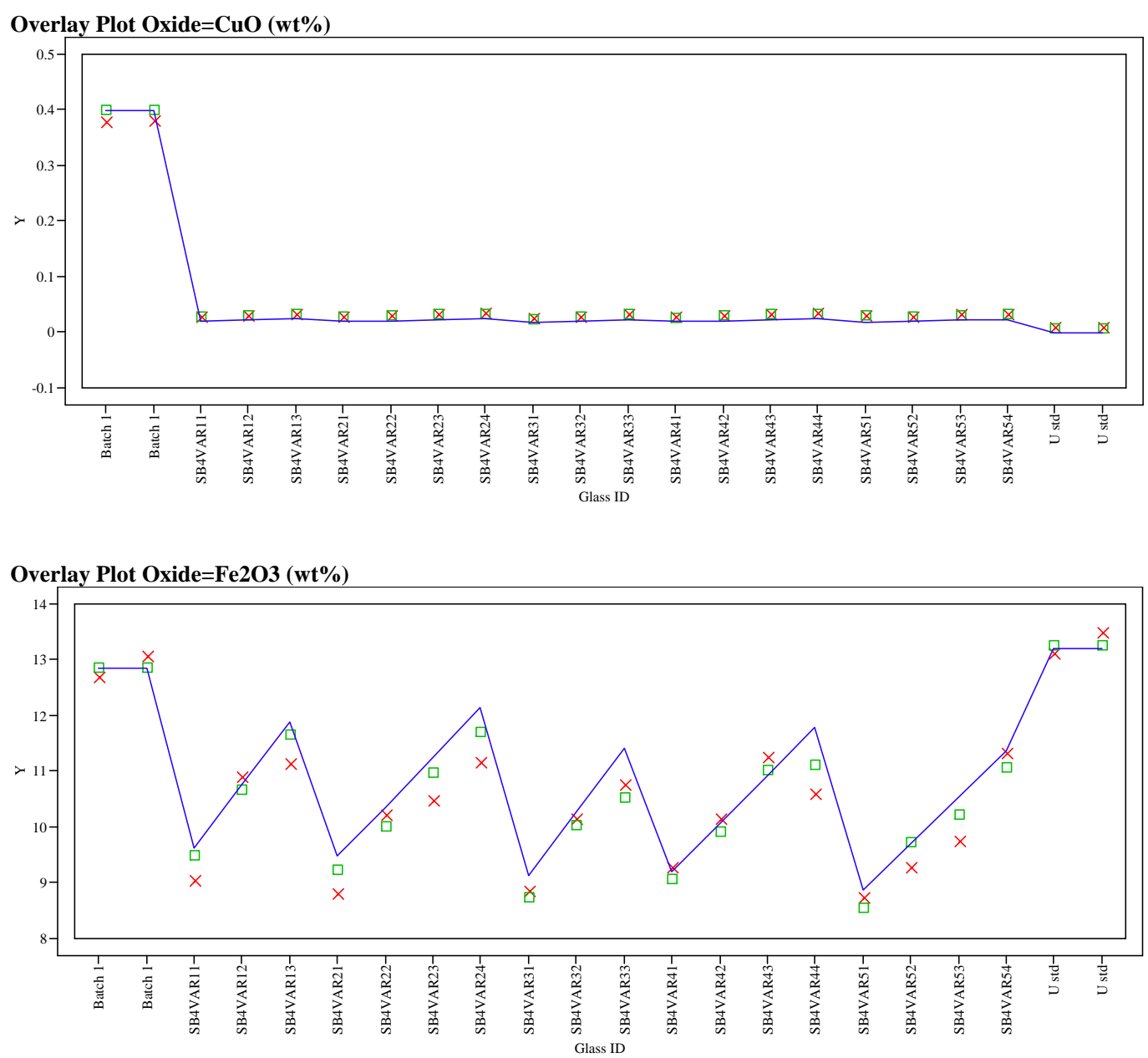

Overlay Plot Oxide=K2O (wt \%)

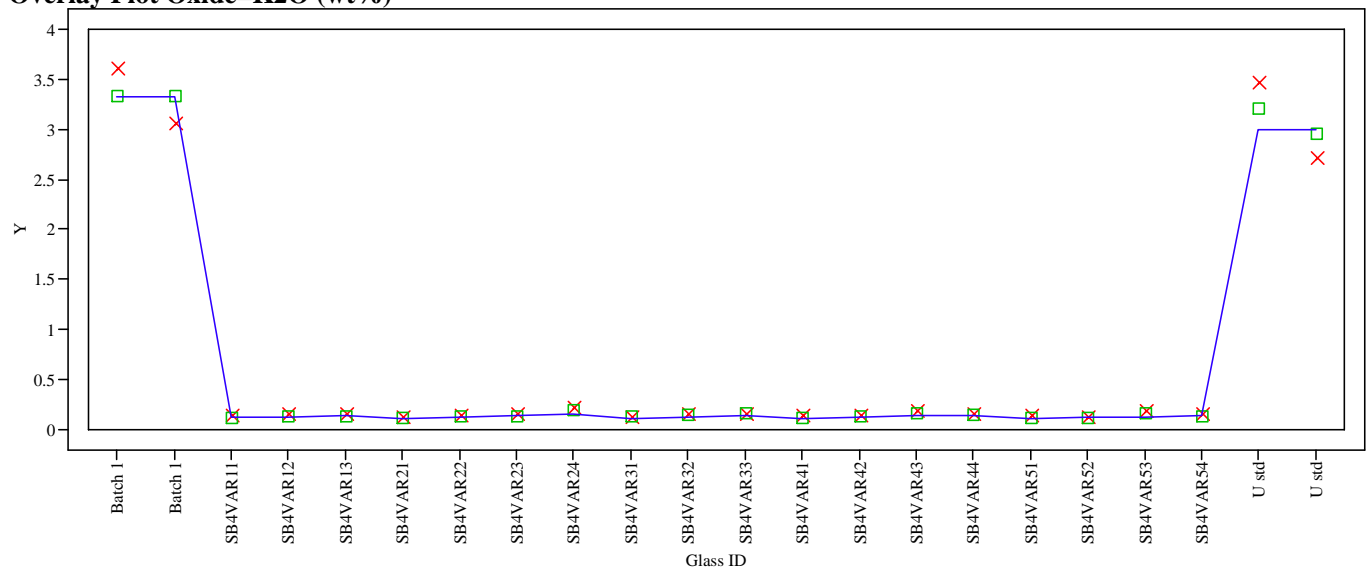

Y $\times$ Measured

⿴囗M Measured bc — Targeted 


\section{Exhibit A7. Average Measured and Bias-Corrected (bc) Versus Targeted} Compositions by Glass \# by Oxide

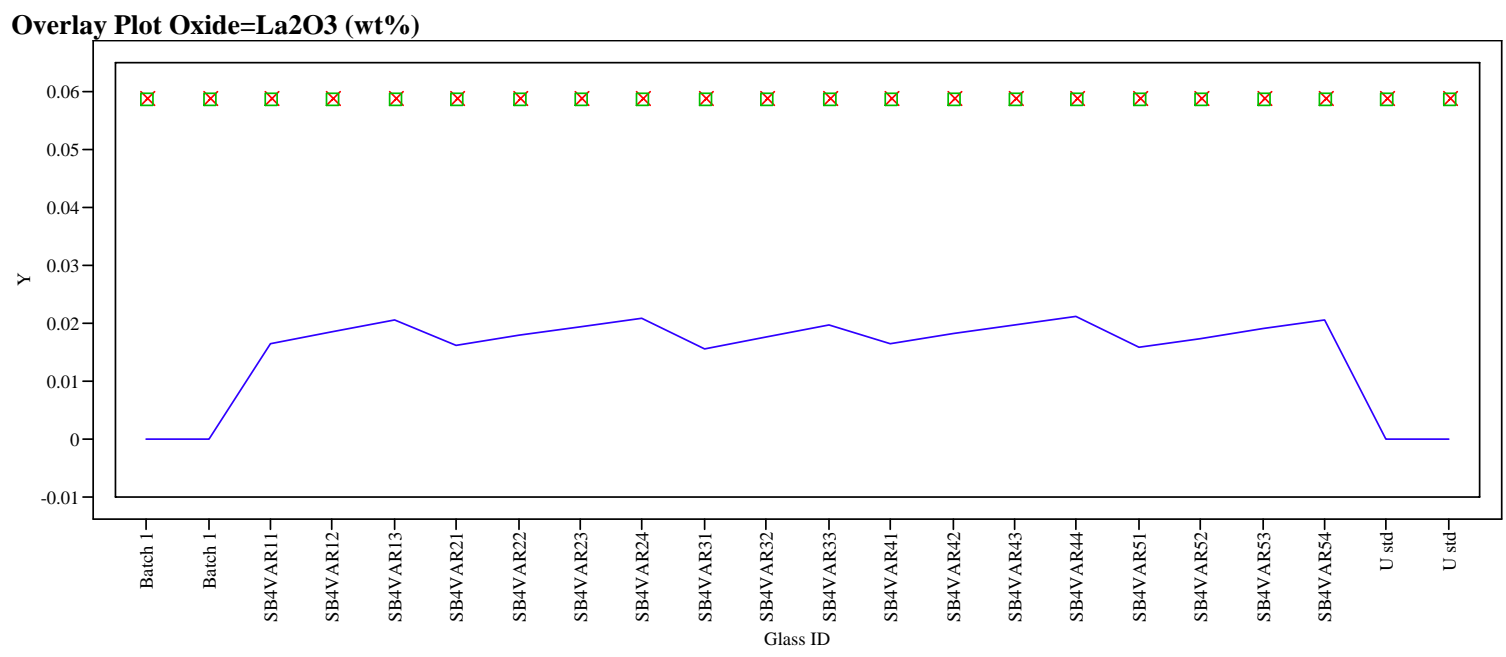

Overlay Plot Oxide=Li2O (wt \%)
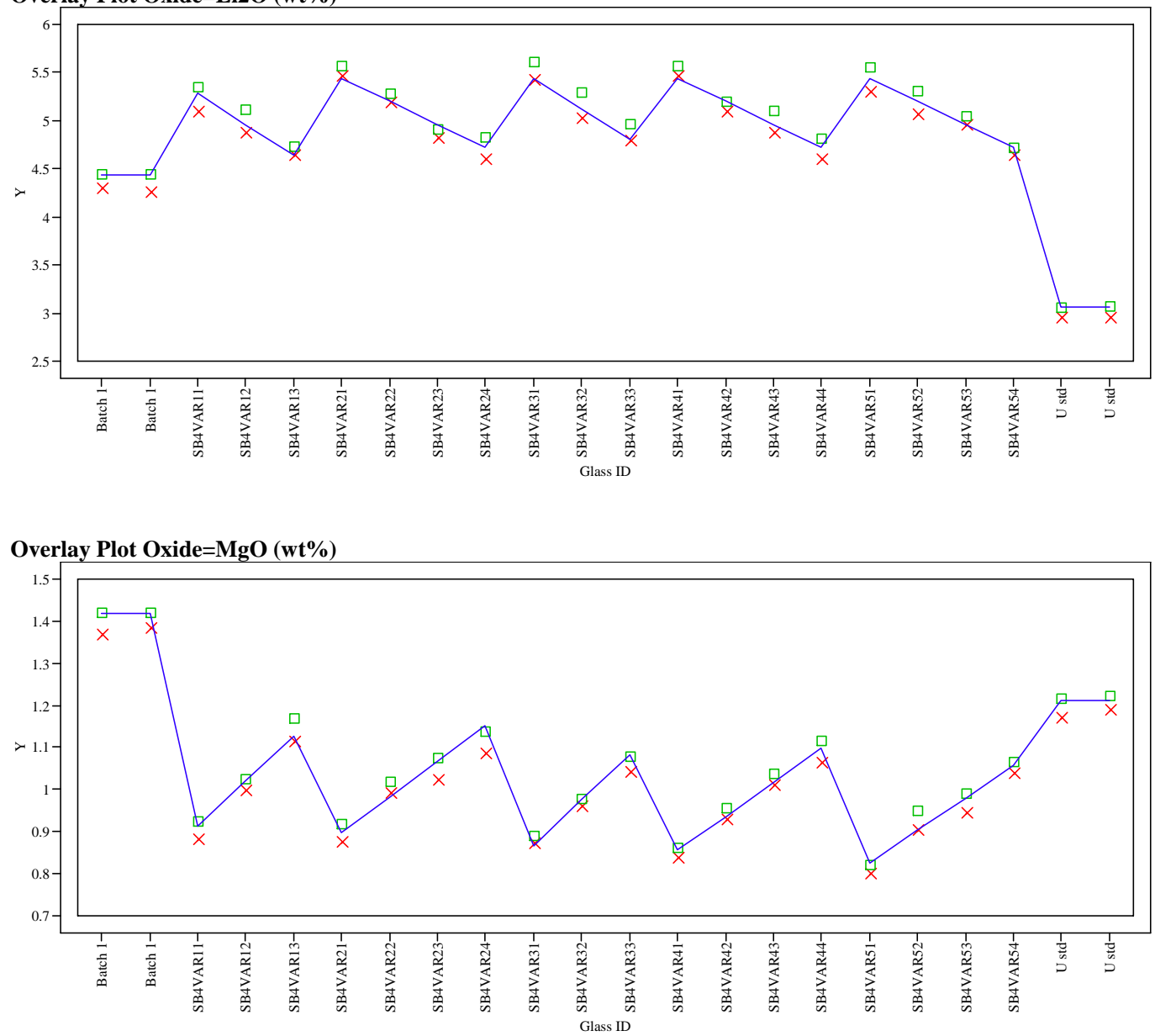

Y $\times$ Measured

■ Measured bc — Targeted 


\section{Exhibit A7. Average Measured and Bias-Corrected (bc) Versus Targeted} Compositions by Glass \# by Oxide
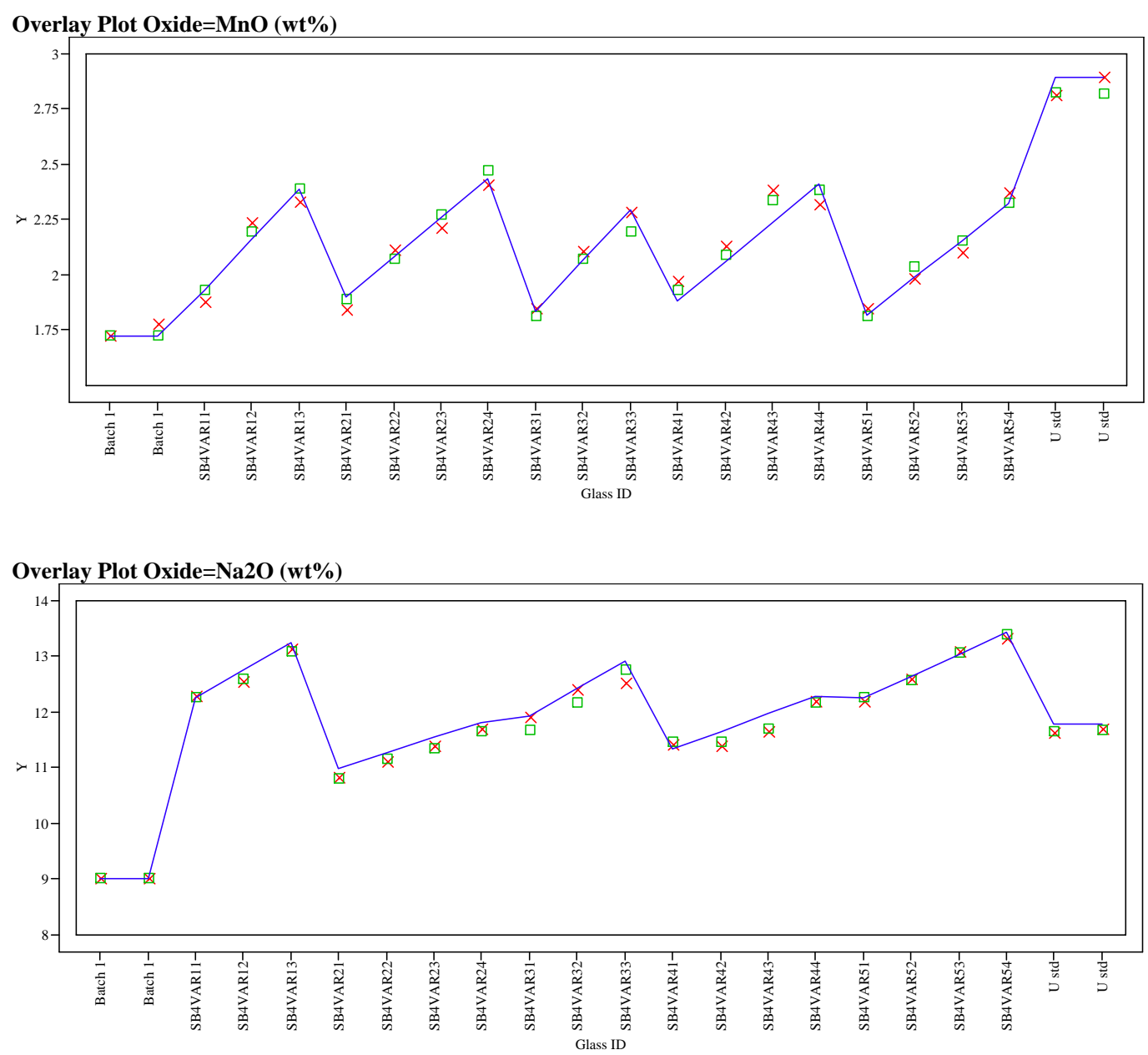

\section{Overlay Plot Oxide=NiO (wt \%)}

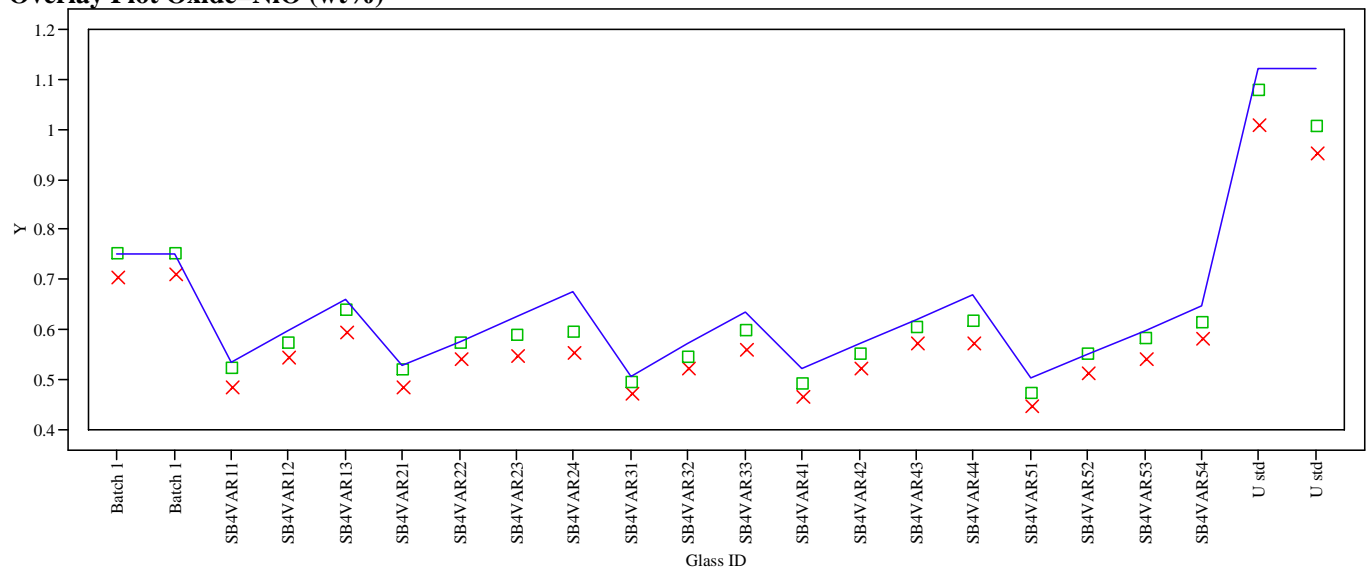

Y $\times$ Measured $\square$ Measured bc - Targeted 


\section{Exhibit A7. Average Measured and Bias-Corrected (bc) Versus Targeted} Compositions by Glass \# by Oxide

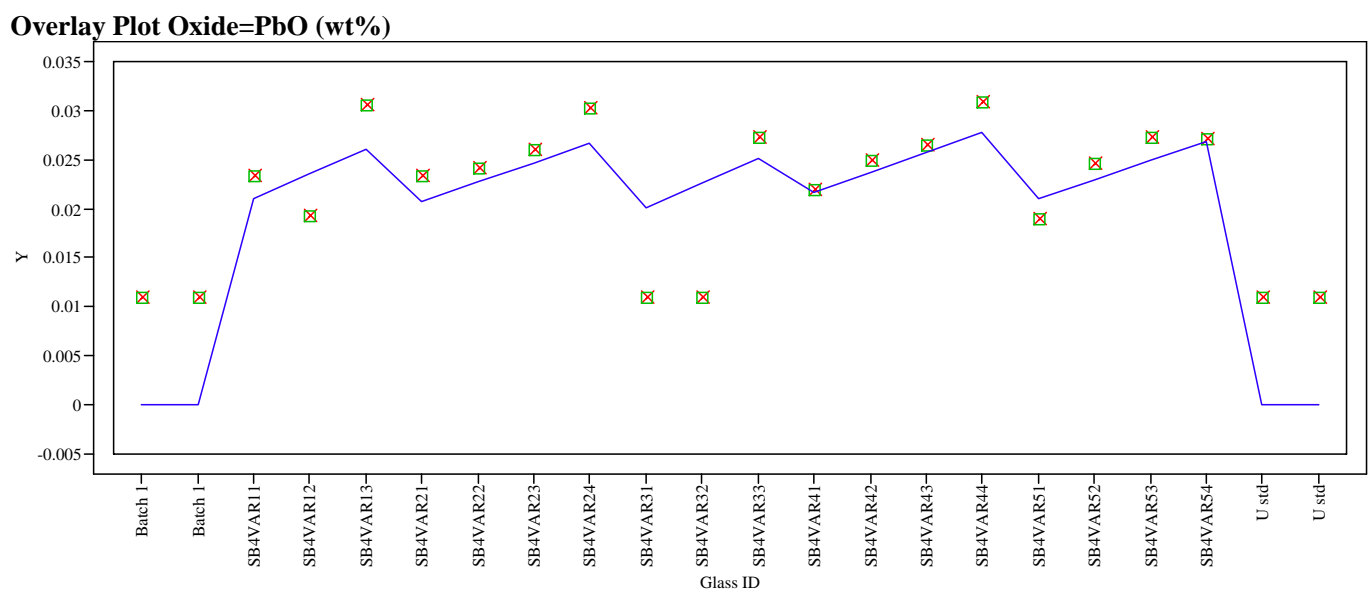

Overlay Plot Oxide=SiO2 (wt \%)

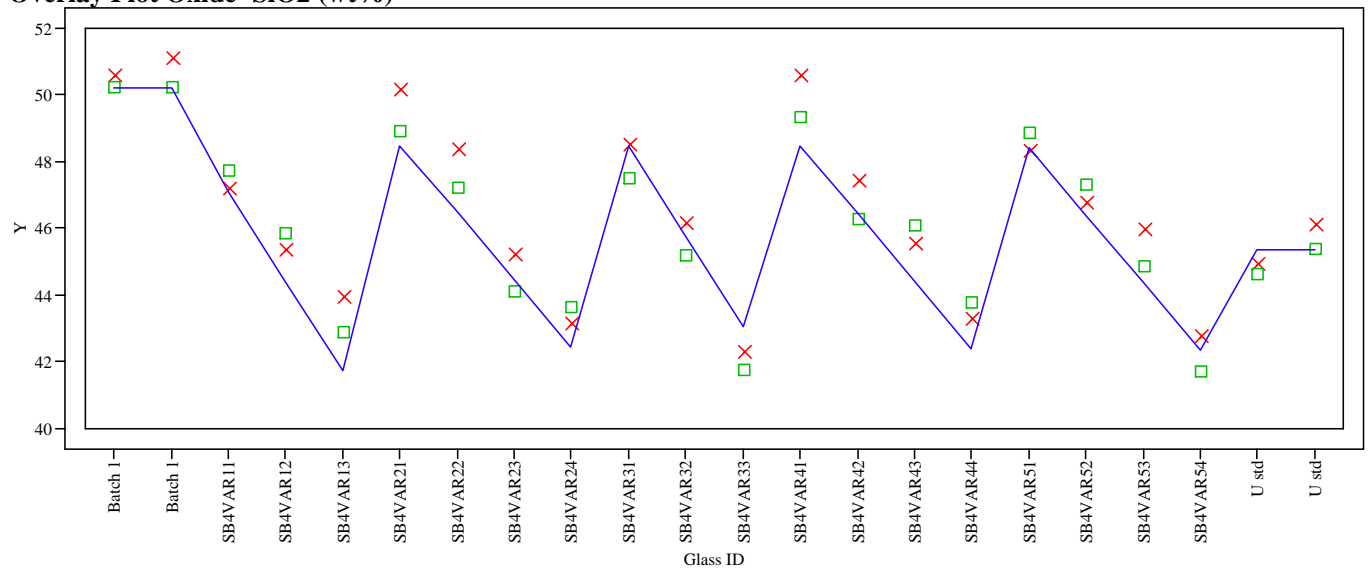

Overlay Plot Oxide=SO4 (wt \%)

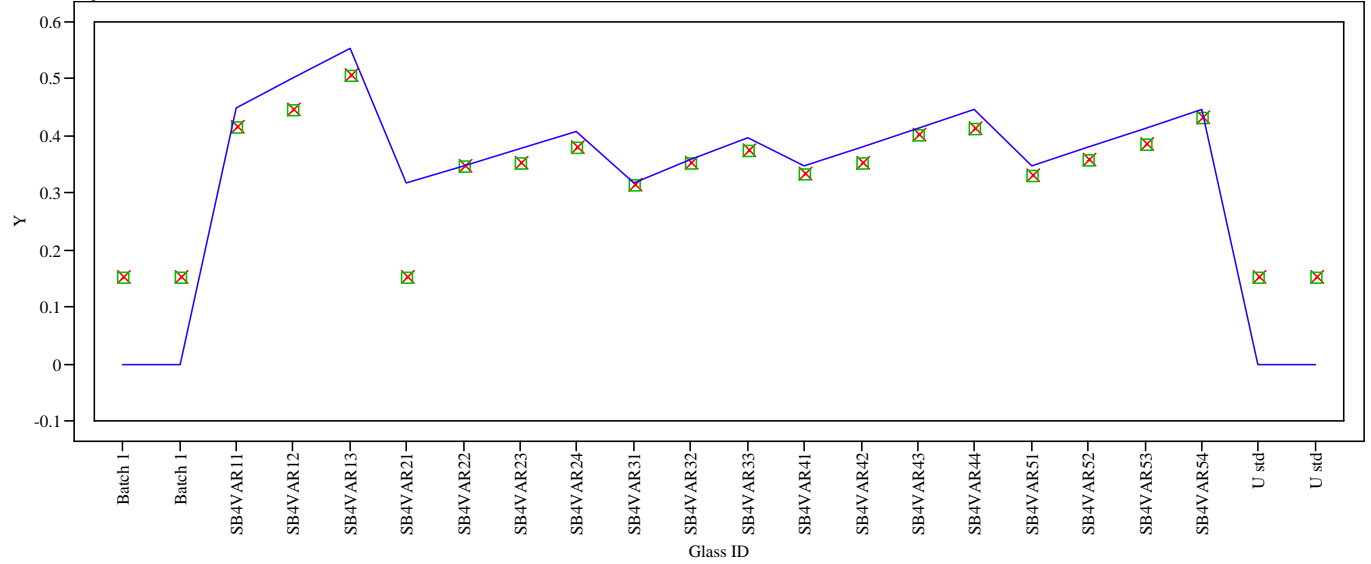

Y X Measured $\square$ Measured bc - Targeted 


\section{Exhibit A7. Average Measured and Bias-Corrected (bc) Versus Targeted} Compositions by Glass \# by Oxide
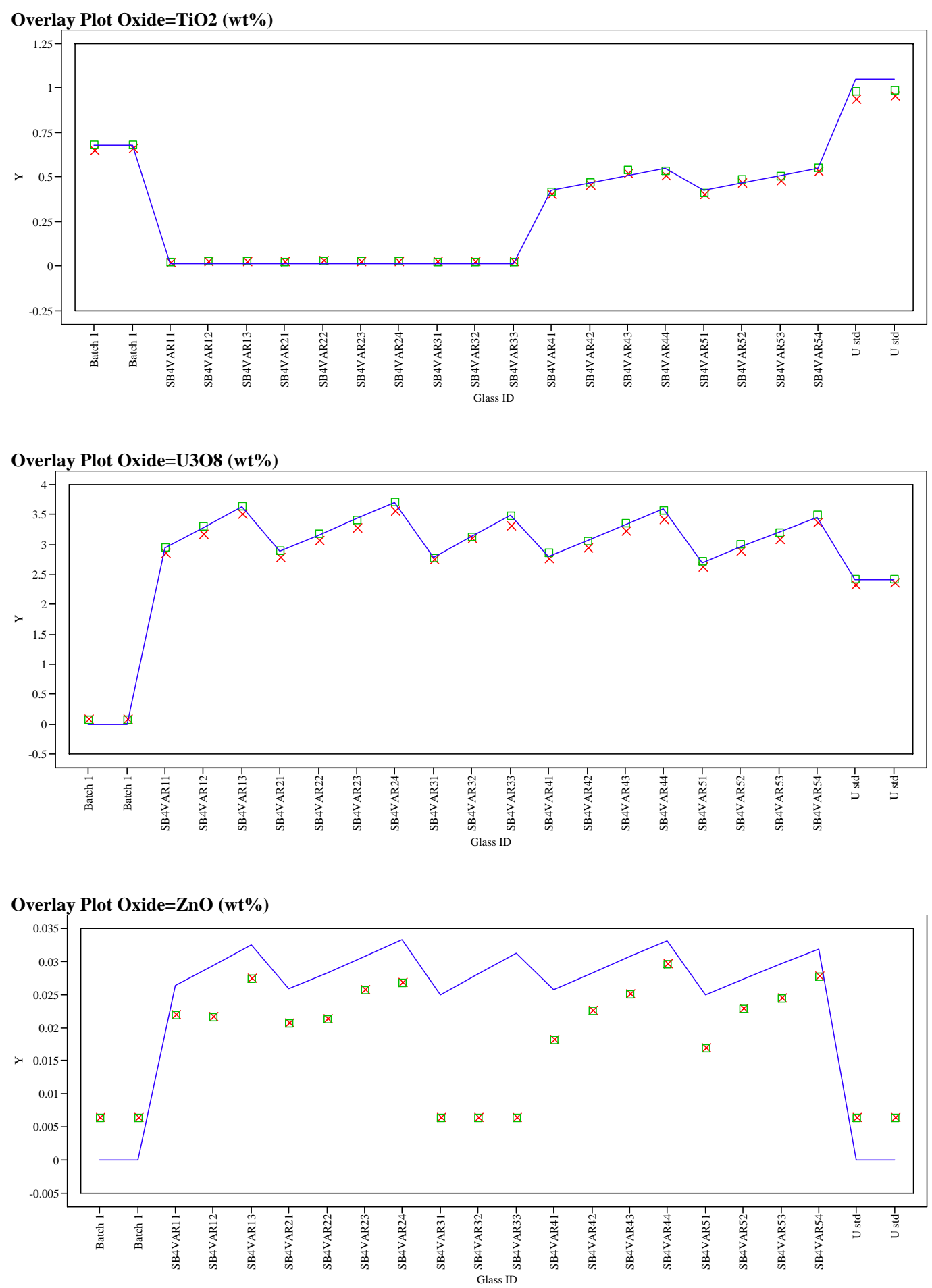

Y $\times$ Measured

口 Measured bc - Targeted 


\section{Exhibit A7. Average Measured and Bias-Corrected (bc) Versus Targeted}

\section{Compositions by Glass \# by Oxide}

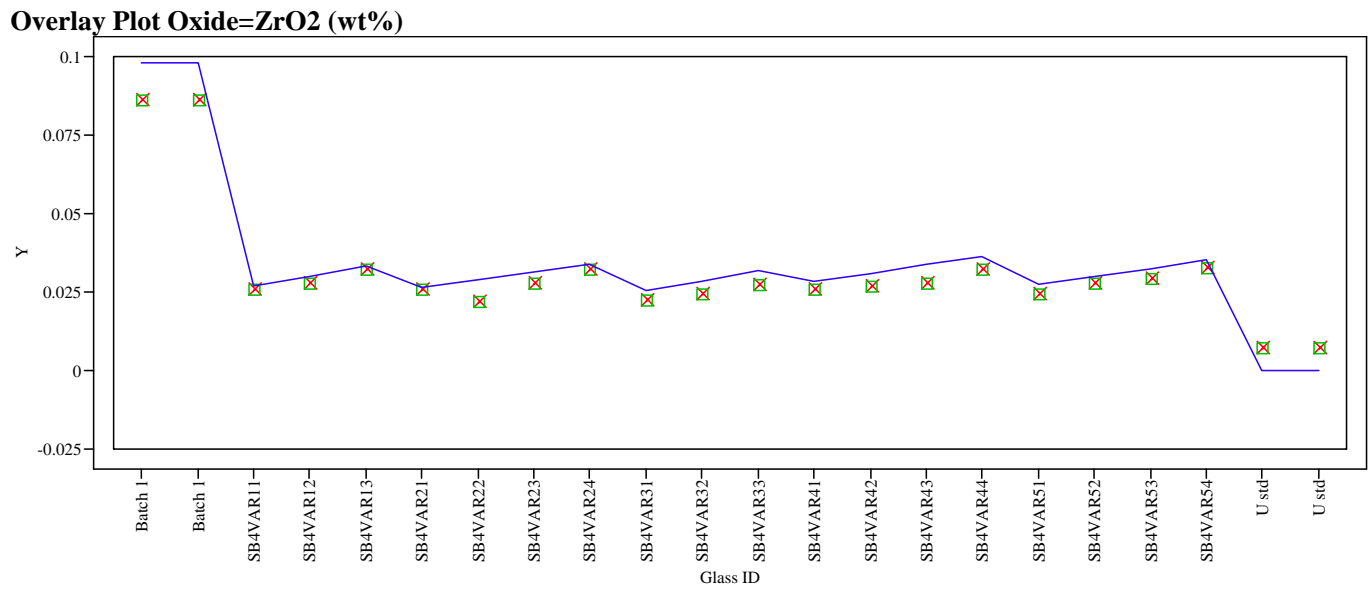

Overlay Plot Oxide=Sum of Oxides (wt\%)

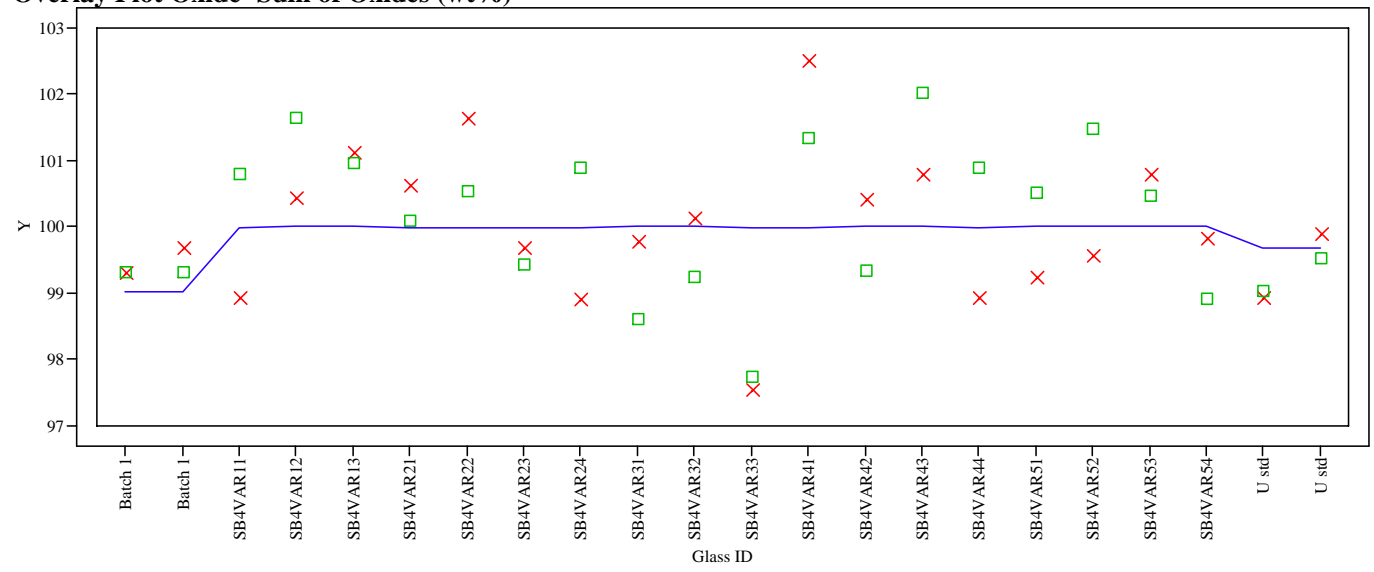

$\mathrm{Y} \times$ Measured $\square$ Measured bc —Targeted 


\section{APPENDIX B}

TABLES AND EXHIBITS SUPPORTING THE ANALYSIS OF THE PCT RESULTS FOR THE SB4-DECANT VARIABILITY STUDY GLASSES 
WSRC-STI-2008-00149

Revision 0

This page intentionally left blank. 


\section{Table B1. PSAL Measurements of the PCT Solutions for the Study Glasses As- Received (ar) and After Appropriate Adjustments (in ppm)}

\begin{tabular}{|c|c|c|c|c|c|c|c|c|c|c|c|c|}
\hline Set & $\begin{array}{l}\text { Glass ID } \\
\text { (w HT) }\end{array}$ & Block & Seq & Lab ID & $\mathrm{B}$ ar & $\mathrm{Li}$ ar & $\mathrm{Na}$ ar & Si ar & B (ppm) & Li (ppm) & $\mathrm{Na}(\mathrm{ppm})$ & Si (ppm) \\
\hline $\mathrm{q}$ & Soln Std & 1 & 1 & STD-11-1 & 20.6 & 9.81 & 79.9 & 49.1 & 20.600 & 9.810 & 79.900 & 49.100 \\
\hline $\mathrm{q}$ & SB4VAR21ccc & 1 & 2 & Q49 & 12.1 & 11 & 29.1 & 61.3 & 20.167 & 18.334 & 48.501 & 102.169 \\
\hline $\mathrm{q}$ & SB4VAR54ccc & 1 & 3 & Q47 & 16.1 & 13.1 & 54.2 & 61.6 & 26.834 & 21.834 & 90.335 & 102.669 \\
\hline $\mathrm{q}$ & EA & 1 & 4 & Q67 & 34.3 & 10.6 & 91.8 & 50.4 & 571.668 & 176.667 & 1530.003 & 840.002 \\
\hline $\mathrm{q}$ & SB4VAR22 & 1 & 5 & Q30 & 12.3 & 10.8 & 31.8 & 58.2 & 20.500 & 18.000 & 53.001 & 97.002 \\
\hline $\mathrm{q}$ & SB4VAR44 & 1 & 6 & Q25 & 12.1 & 10.2 & 40.2 & 55.2 & 20.167 & 17.000 & 67.001 & 92.002 \\
\hline $\mathrm{q}$ & SB4VAR21 & 1 & 7 & Q69 & 11.9 & 10.9 & 30.2 & 59.4 & 19.834 & 18.167 & 50.334 & 99.002 \\
\hline $\mathrm{q}$ & SB4VAR42ccc & 1 & 8 & Q45 & 11.9 & 10.8 & 34.3 & 58.6 & 19.834 & 18.000 & 57.168 & 97.669 \\
\hline $\mathrm{q}$ & SB4VAR23 & 1 & 9 & Q14 & 11.8 & 10.5 & 35.2 & 54.7 & 19.667 & 17.500 & 58.668 & 91.168 \\
\hline $\mathrm{q}$ & SB4VAR41 & 1 & 10 & Q59 & 12.3 & 11.2 & 33.9 & 61 & 20.500 & 18.667 & 56.501 & 101.669 \\
\hline $\mathrm{q}$ & SB4VAR51 & $\overline{1}$ & 11 & Q53 & 11.9 & 10.6 & 36.7 & 58.8 & 19.834 & 17.667 & 61.168 & 98.002 \\
\hline $\mathrm{q}$ & ARM-1 & 1 & 12 & Q24 & 12.3 & 9.44 & 25.3 & 39.3 & 20.500 & 15.734 & 42.168 & 65.501 \\
\hline $\mathrm{q}$ & SB4VAR22ccc & 1 & 13 & Q79 & 11.6 & 10.6 & 32.9 & 57.8 & 19.334 & 17.667 & 54.834 & 96.335 \\
\hline $\mathrm{q}$ & blank & 1 & 14 & Q23 & $<1.00$ & $<1.00$ & $<1.00$ & $<0.100$ & 0.833 & 0.833 & 0.833 & 0.083 \\
\hline $\mathrm{q}$ & Soln Std & 1 & 15 & STD-11-2 & 19.7 & 9.94 & 81.5 & 48.8 & 19.700 & 9.940 & 81.500 & 48.800 \\
\hline $\mathrm{q}$ & SB4VAR42 & 1 & 16 & Q42 & 12.2 & 10.8 & 35.7 & 59 & 20.334 & 18.000 & 59.501 & 98.335 \\
\hline $\mathrm{q}$ & SB4VAR24 & 1 & 17 & Q52 & 12.5 & 10.7 & 39.3 & 55.3 & 20.834 & 17.834 & 65.501 & 92.169 \\
\hline $\mathrm{q}$ & SB4VAR52 & 1 & 18 & Q19 & 12.6 & 10.9 & 41.2 & 60.3 & 21.000 & 18.167 & 68.668 & 100.502 \\
\hline $\mathrm{q}$ & SB4VAR44ccC & 1 & 19 & Q01 & 13.7 & 11.8 & 43.1 & 56.7 & 22.834 & 19.667 & 71.835 & 94.502 \\
\hline $\mathrm{q}$ & SB4VAR24ccC & 1 & 20 & Q71 & 28.2 & 22.3 & 60.6 & 72.4 & 47.001 & 37.167 & 101.002 & 120.669 \\
\hline $\mathrm{q}$ & SB4VAR41ccc & 1 & 21 & Q48 & 13.1 & 12 & 34.3 & 65.4 & 21.834 & 20.000 & 57.168 & 109.002 \\
\hline $\mathrm{q}$ & SB4VAR53ccc & 1 & 22 & Q61 & 12.3 & 10.8 & 43.9 & 58.1 & 20.500 & 18.000 & 73.168 & 96.835 \\
\hline $\mathrm{q}$ & SB4VAR53 & 1 & 23 & Q51 & 12.6 & 10.8 & 46.8 & 59.1 & 21.000 & 18.000 & 78.002 & 98.502 \\
\hline $\mathrm{q}$ & SB4VAR52ccc & 1 & 24 & Q13 & 12.4 & 11 & 41 & 60.9 & 20.667 & 18.334 & 68.335 & 101.502 \\
\hline$q$ & SB4VAR43 & 1 & 25 & $\mathrm{Q} 80$ & 11.8 & 10.6 & 38.3 & 57.1 & 19.667 & 17.667 & 63.835 & 95.169 \\
\hline $\mathrm{q}$ & SB4VAR43ccc & 1 & 26 & Q77 & 11.5 & 10.4 & 36.8 & 55.4 & 19.167 & 17.334 & 61.335 & 92.335 \\
\hline $\mathrm{q}$ & SB4VAR51ccc & 1 & 27 & Q60 & 12.6 & 11.5 & 37.4 & 63.5 & 21.000 & 19.167 & 62.335 & 105.835 \\
\hline $\mathrm{q}$ & SB4VAR54 & 1 & 28 & Q76 & 12.3 & 10.3 & 49.2 & 55.8 & 20.500 & 17.167 & 82.002 & 93.002 \\
\hline $\mathrm{q}$ & SB4VAR23ccc & 1 & 29 & Q18 & 12.3 & 10.9 & 36.6 & 57 & 20.500 & 18.167 & 61.001 & 95.002 \\
\hline $\mathrm{q}$ & Soln Std & 1 & 30 & STD-11-3 & 20.3 & 10.1 & 82.2 & 50.2 & 20.300 & 10.100 & 82.200 & 50.200 \\
\hline $\mathrm{q}$ & Soln Std & 2 & 1 & STD-12-1 & 20.7 & 9.74 & 78.2 & 50.3 & 20.700 & 9.740 & 78.200 & 50.300 \\
\hline $\mathrm{q}$ & ARM-1 & 2 & 2 & Q28 & 11.9 & 8.7 & 23.1 & 38.2 & 19.834 & 14.500 & 38.501 & 63.668 \\
\hline $\mathrm{q}$ & SB4VAR23ccc & 2 & 3 & Q12 & 12.3 & 10.5 & 34 & 57.7 & 20.500 & 17.500 & 56.668 & 96.169 \\
\hline $\mathrm{q}$ & SB4VAR52 & 2 & 4 & Q03 & 12.5 & 10.7 & 40.8 & 61 & 20.834 & 17.834 & 68.001 & 101.669 \\
\hline $\mathrm{q}$ & SB4VAR21ccc & 2 & 5 & Q75 & 11.7 & 10.7 & 30.7 & 61.1 & 19.500 & 17.834 & 51.168 & 101.835 \\
\hline $\mathrm{q}$ & SB4VAR54 & 2 & 6 & Q08 & 12.6 & 10.4 & 49 & 58.5 & 21.000 & 17.334 & 81.668 & 97.502 \\
\hline$q$ & SB4VAR24 & 2 & 7 & Q09 & 12.1 & 10.4 & 38 & 56.1 & 20.167 & 17.334 & 63.335 & 93.502 \\
\hline $\mathrm{q}$ & SB4VAR23 & 2 & 8 & Q74 & 11.8 & 10.6 & 35.4 & 57.3 & 19.667 & 17.667 & 59.001 & 95.502 \\
\hline $\mathrm{q}$ & SB4VAR44ccC & 2 & 9 & Q63 & 13.4 & 11.9 & 43 & 58.1 & 22.334 & 19.834 & 71.668 & 96.835 \\
\hline $\mathrm{q}$ & SB4VAR53 & 2 & 10 & Q07 & 12.1 & 10.5 & 45.8 & 59.9 & 20.167 & 17.500 & 76.335 & 99.835 \\
\hline $\mathrm{q}$ & SB4VAR44 & 2 & 11 & Q29 & 11 & 9.64 & 38.4 & 53.8 & 18.334 & 16.067 & 64.001 & 89.668 \\
\hline $\mathrm{q}$ & SB4VAR24cCC & 2 & 12 & Q55 & 27.6 & 21.8 & 59.1 & 73.2 & 46.001 & 36.334 & 98.502 & 122.002 \\
\hline $\mathrm{q}$ & SB4VAR43ccc & 2 & 13 & Q41 & 11.2 & 10 & 35.5 & 56.8 & 18.667 & 16.667 & 59.168 & 94.669 \\
\hline $\mathrm{q}$ & SB4VAR21 & 2 & 14 & Q32 & 12 & 11.3 & 31.2 & 62.6 & 20.000 & 18.834 & 52.001 & 104.335 \\
\hline$q$ & Soln Std & 2 & 15 & STD-12-2 & 20 & 9.9 & 81.5 & 50.8 & 20.000 & 9.900 & 81.500 & 50.800 \\
\hline $\mathrm{q}$ & SB4VAR52ccc & 2 & 16 & Q02 & 12.7 & 11 & 39.6 & 63.7 & 21.167 & 18.334 & 66.001 & 106.169 \\
\hline $\mathrm{q}$ & SB4VAR42ccc & 2 & 17 & Q26 & 12.4 & 11.2 & 35.5 & 63.1 & 20.667 & 18.667 & 59.168 & 105.169 \\
\hline $\mathrm{q}$ & SB4VAR54ccc & 2 & 18 & Q64 & 16.1 & 13.2 & 53.9 & 63.6 & 26.834 & 22.000 & 89.835 & 106.002 \\
\hline$q$ & SB4VAR22 & 2 & 19 & Q10 & 12.1 & 11 & 32.3 & 61.2 & 20.167 & 18.334 & 53.834 & 102.002 \\
\hline $\mathrm{q}$ & SB4VAR41 & 2 & 20 & Q44 & 12.4 & 11.4 & 32.5 & 64.1 & 20.667 & 19.000 & 54.168 & 106.835 \\
\hline $\mathrm{q}$ & SB4VAR42 & 2 & \begin{tabular}{|l|}
21 \\
\end{tabular} & Q34 & 11.9 & 10.8 & 33.8 & 61.1 & 19.834 & 18.000 & 56.334 & 101.835 \\
\hline$q$ & EA & 2 & 22 & Q31 & 39 & 11.4 & 99.3 & 56.6 & 650.001 & 190.000 & 1655.003 & 943.335 \\
\hline $\mathrm{q}$ & SB4VAR51 & 2 & 23 & Q06 & 12.9 & 11.2 & 36.1 & 65.3 & 21.500 & 18.667 & 60.168 & 108.836 \\
\hline $\mathrm{q}$ & SB4VAR43 & 2 & 24 & Q70 & 12.5 & 10.8 & 37.1 & 61.4 & 20.834 & 18.000 & 61.835 & 102.335 \\
\hline $\mathrm{q}$ & SB4VAR41ccc & 2 & 25 & Q65 & 13 & 11.8 & 32.5 & 67.9 & 21.667 & 19.667 & 54.168 & 113.169 \\
\hline $\mathrm{q}$ & SB4VAR53ccc & 2 & 26 & Q38 & 12.2 & 10.7 & 42.7 & 60.6 & 20.334 & 17.834 & 71.168 & 101.002 \\
\hline $\mathrm{q}$ & SB4VAR22ccc & 2 & 27 & Q15 & 11.5 & 10.6 & 32.7 & 59.7 & 19.167 & 17.667 & 54.501 & 99.502 \\
\hline $\mathrm{q}$ & SB4VAR51ccc & 2 & 28 & Q16 & 12.3 & 11.4 & 36.9 & 64.6 & 20.500 & 19.000 & 61.501 & 107.669 \\
\hline $\mathrm{q}$ & Soln Std & 2 & 29 & STD-12-3 & 20.1 & 9.9 & 80.7 & 50.8 & 20.100 & 9.900 & 80.700 & 50.800 \\
\hline $\mathrm{q}$ & Soln Std & 3 & 1 & STD-13-1 & 20 & 9.75 & 79.3 & 50.5 & 20.000 & 9.750 & 79.300 & 50.500 \\
\hline
\end{tabular}




\section{Table B1. PSAL Measurements of the PCT Solutions for the Study Glasses As- Received (ar) and After Appropriate Adjustments (in ppm)}

\begin{tabular}{|c|c|c|c|c|c|c|c|c|c|c|c|c|}
\hline Set & $\begin{array}{l}\text { Glass ID } \\
\text { (w HT) }\end{array}$ & Block & Seq & Lab ID & $\mathrm{B}$ ar & $\mathrm{Li}$ ar & $\mathrm{Na}$ ar & Si ar & B (ppm) & Li (ppm) & $\mathrm{Na}(\mathrm{ppm})$ & Si (ppm) \\
\hline $\mathrm{q}$ & SB4VAR53 & 3 & 2 & Q04 & 11.2 & 10.1 & 44.8 & 56.6 & 18.667 & 16.834 & 74.668 & 94.335 \\
\hline $\mathrm{q}$ & SB4VAR52ccc & 3 & 3 & Q68 & 11.2 & 10.4 & 38.9 & 59.1 & 18.667 & 17.334 & 64.835 & 98.502 \\
\hline $\mathrm{q}$ & SB4VAR22ccc & 3 & 4 & Q78 & 11.1 & 10.4 & 31.3 & 58.2 & 18.500 & 17.334 & 52.168 & 97.002 \\
\hline $\mathrm{q}$ & EA & 3 & 5 & Q62 & 37.5 & 11.5 & 102 & 55.4 & 625.001 & 191.667 & 1700.003 & 923.335 \\
\hline $\mathrm{q}$ & SB4VAR42 & 3 & 6 & Q58 & 11.2 & 10.5 & 34.2 & 59 & 18.667 & 17.500 & 57.001 & 98.335 \\
\hline $\mathrm{q}$ & SB4VAR53ccc & 3 & 7 & Q57 & 11 & 10 & 41.4 & 57.6 & 18.334 & 16.667 & 69.001 & 96.002 \\
\hline $\mathrm{q}$ & SB4VAR22 & 3 & 8 & Q43 & 11.1 & 10.6 & 30.9 & 58.1 & 18.500 & 17.667 & 51.501 & 96.835 \\
\hline $\mathrm{q}$ & SB4VAR23ccc & 3 & 9 & Q73 & 10.8 & 10.3 & 33.7 & 56.3 & 18.000 & 17.167 & 56.168 & 93.835 \\
\hline $\mathrm{q}$ & SB4VAR42ccc & 3 & 10 & Q17 & 11 & 10.7 & 33.6 & 59.6 & 18.334 & 17.834 & 56.001 & 99.335 \\
\hline $\mathrm{q}$ & SB4VAR54 & 3 & 11 & Q39 & 11.3 & 9.85 & 47.2 & 56.5 & 18.834 & 16.417 & 78.668 & 94.169 \\
\hline $\mathrm{q}$ & SB4VAR51ccc & 3 & 12 & Q33 & 11.2 & 11 & 35.1 & 62.3 & 18.667 & 18.334 & 58.501 & 103.835 \\
\hline $\mathrm{q}$ & SB4VAR43 & 3 & 13 & Q27 & 10.4 & 9.92 & 37.3 & 56.6 & 17.334 & 16.534 & 62.168 & 94.335 \\
\hline $\mathrm{q}$ & SB4VAR51 & 3 & 14 & Q72 & 11.4 & 10.9 & 37.3 & 61.8 & 19.000 & 18.167 & 62.168 & 103.002 \\
\hline $\mathrm{q}$ & Soln Std & 3 & 15 & STD-13-2 & 19.1 & 9.8 & 78.9 & 50.4 & 19.100 & 9.800 & 78.900 & 50.400 \\
\hline $\mathrm{q}$ & SB4VAR43ccc & 3 & 16 & Q36 & 10.7 & 10.1 & 33.8 & 56.6 & 17.834 & 16.834 & 56.334 & 94.335 \\
\hline $\mathrm{q}$ & SB4VAR44ccc & 3 & 17 & Q37 & 12.9 & 11.5 & 40.6 & 57.6 & 21.500 & 19.167 & 67.668 & 96.002 \\
\hline $\mathrm{q}$ & SB4VAR24ccc & 3 & 18 & Q66 & 26.5 & 21.1 & 56.9 & 71.3 & 44.168 & 35.167 & 94.835 & 118.836 \\
\hline $\mathrm{q}$ & SB4VAR54ccc & 3 & 19 & Q56 & 14.9 & 12.6 & 50.4 & 61.1 & 24.834 & 21.000 & 84.002 & 101.835 \\
\hline $\mathrm{q}$ & blank & 3 & 20 & Q20 & $<1.00$ & $<1.00$ & $<1.00$ & 0.142 & 0.833 & 0.833 & 0.833 & 0.237 \\
\hline $\mathrm{q}$ & SB4VAR24 & 3 & 21 & Q40 & 10.9 & 10.1 & 35.6 & 53.9 & 18.167 & 16.834 & 59.335 & 89.835 \\
\hline $\mathrm{q}$ & SB4VAR21ccc & 3 & 22 & Q35 & 10.6 & 10.4 & 27.7 & 59.4 & 17.667 & 17.334 & 46.168 & 99.002 \\
\hline $\mathrm{q}$ & SB4VAR52 & 3 & 23 & Q22 & 11.1 & 10.5 & 38.5 & 59.9 & 18.500 & 17.500 & 64.168 & 99.835 \\
\hline $\mathrm{q}$ & SB4VAR44 & 3 & 24 & Q50 & 10.4 & 9.85 & 38.3 & 53.1 & 17.334 & 16.417 & 63.835 & 88.502 \\
\hline $\mathrm{q}$ & SB4VAR41 & 3 & 25 & Q54 & 10.5 & 10.5 & 30.2 & 58.9 & 17.500 & 17.500 & 50.334 & 98.169 \\
\hline$q$ & ARM-1 & 3 & 26 & $\mathrm{Q} 46$ & 9.5 & 8.24 & 21.2 & 36.1 & 15.834 & 13.734 & 35.334 & 60.168 \\
\hline $\mathrm{q}$ & SB4VAR21 & 3 & 27 & Q05 & 10.7 & 10.7 & 28.1 & 59.7 & 17.834 & 17.834 & 46.834 & 99.502 \\
\hline $\mathrm{q}$ & SB4VAR23 & 3 & 28 & Q11 & 10.5 & 10 & 32.4 & 54 & 17.500 & 16.667 & 54.001 & 90.002 \\
\hline $\mathrm{q}$ & SB4VAR41ccC & 3 & 29 & Q21 & 10.9 & 10.9 & 30.1 & 62 & 18.167 & 18.167 & 50.168 & 103.335 \\
\hline $\mathrm{q}$ & Soln Std & 3 & 30 & STD-13-3 & 19.1 & 10.1 & 83.1 & 50.3 & 19.100 & 10.100 & 83.100 & 50.300 \\
\hline $\mathrm{r}$ & Soln Std & 1 & 1 & STD-21-1 & 20.5 & 9.89 & 79.3 & 50.7 & 20.500 & 9.890 & 79.300 & 50.700 \\
\hline $\mathrm{r}$ & EA & 1 & 2 & R62 & 36.5 & 11 & 98.2 & 53.3 & 608.335 & 183.334 & 1636.670 & 888.335 \\
\hline $\mathrm{r}$ & SB5-17 & 1 & 3 & R55 & 11.9 & 11.3 & 34.1 & 59.4 & 19.834 & 18.834 & 56.834 & 99.002 \\
\hline $\mathrm{r}$ & SB4VAR13ccc & 1 & 4 & R09 & 23.8 & 17.9 & 65.9 & 71.6 & 39.667 & 29.834 & 109.836 & 119.336 \\
\hline $\mathrm{r}$ & SB5-18ccC & 1 & 5 & R38 & 11.7 & 8.53 & 45.8 & 58 & 19.500 & 14.217 & 76.335 & 96.669 \\
\hline $\mathrm{r}$ & SB4VAR33 & 1 & 6 & R77 & 12.5 & 10.3 & 44.9 & 57.5 & 20.834 & 17.167 & 74.835 & 95.835 \\
\hline $\mathrm{r}$ & SB4VAR11ccc & 1 & 7 & R06 & 12.2 & 10.6 & 35.2 & 61.1 & 20.334 & 17.667 & 58.668 & 101.835 \\
\hline$r$ & SB5-14cCC & 1 & 8 & R49 & 11.1 & 10.5 & 60.5 & 68.5 & 18.500 & 17.500 & 100.835 & 114.169 \\
\hline $\mathrm{r}$ & SB5-17ccC & 1 & 9 & R45 & 11.3 & 10.5 & 32 & 58.4 & 18.834 & 17.500 & 53.334 & 97.335 \\
\hline$r$ & SB4VAR33ccc & 1 & 10 & R84 & 13.1 & 10.7 & 43.6 & 59.1 & 21.834 & 17.834 & 72.668 & 98.502 \\
\hline $\mathrm{r}$ & SB5-20ccc & 1 & 11 & R26 & 11.7 & 4.55 & 39 & 49.9 & 19.500 & 7.583 & 65.001 & 83.168 \\
\hline$r$ & SB4VAR32 & 1 & 12 & R52 & 12.3 & 10.4 & 37.7 & 59.9 & 20.500 & 17.334 & 62.835 & 99.835 \\
\hline $\mathrm{r}$ & SB5-16 & 1 & 13 & R68 & 15 & 6.42 & 55.3 & 56.2 & 25.001 & 10.700 & 92.169 & 93.669 \\
\hline $\mathrm{r}$ & ARM-1 & 1 & 14 & R17 & 10.2 & 8.03 & 21.1 & 36 & 17.000 & 13.384 & 35.167 & 60.001 \\
\hline $\mathrm{r}$ & SB5-15 & 1 & 15 & R69 & 13.3 & 8.12 & 58.9 & 59.2 & 22.167 & 13.534 & 98.169 & 98.669 \\
\hline$r$ & Soln Std & 1 & 16 & STD-21-2 & 19.8 & 9.69 & 79.1 & 49.4 & 19.800 & 9.690 & 79.100 & 49.400 \\
\hline $\mathrm{r}$ & SB5-16сcC & 1 & 17 & R61 & 13.8 & 5.94 & 48.2 & 54.4 & 23.000 & 9.900 & 80.335 & 90.668 \\
\hline $\mathrm{r}$ & SB4VAR12 & 1 & 18 & R85 & 12.9 & 10.5 & 41.5 & 59.1 & 21.500 & 17.500 & 69.168 & 98.502 \\
\hline $\mathrm{r}$ & SB5-15ccC & 1 & 19 & R78 & 12.8 & 7.93 & 51.6 & 57.3 & 21.334 & 13.217 & 86.002 & 95.502 \\
\hline$r$ & SB4VAR13 & 1 & 20 & R57 & 13.3 & 10.5 & 48.7 & 56.6 & 22.167 & 17.500 & 81.168 & 94.335 \\
\hline $\mathrm{r}$ & SB5-20 & 1 & 21 & R31 & 11.8 & 4.44 & 41.1 & 48.3 & 19.667 & 7.400 & 68.501 & 80.502 \\
\hline $\mathrm{r}$ & SB4VAR31 & 1 & \begin{tabular}{|l|}
22 \\
\end{tabular} & R30 & 12.4 & 10.9 & 34.3 & 61.2 & 20.667 & 18.167 & 57.168 & 102.002 \\
\hline$r$ & blank & 1 & 23 & R08 & $<1.00$ & $<1.00$ & $<1.00$ & 0.116 & 0.833 & 0.833 & 0.833 & 0.193 \\
\hline$r$ & SB4VAR12ccc & 1 & 24 & R46 & 12.2 & 10.3 & 46.2 & 57.7 & 20.334 & 17.167 & 77.002 & 96.169 \\
\hline $\mathrm{r}$ & SB5-18 & 1 & 25 & R48 & 11.7 & 8.35 & 46.7 & 57.4 & 19.500 & 13.917 & 77.835 & 95.669 \\
\hline $\mathrm{r}$ & SB4VAR11 & 1 & 26 & R01 & 12.7 & 10.7 & 36.9 & 62 & 21.167 & 17.834 & 61.501 & 103.335 \\
\hline $\mathrm{r}$ & SB4VAR31ccc & 1 & 27 & R59 & 12.4 & 11.1 & 34.3 & 62.2 & 20.667 & 18.500 & 57.168 & 103.669 \\
\hline $\mathrm{r}$ & SB5-19 & 1 & 28 & R65 & 12.3 & 5.63 & 51.5 & 52.8 & 20.500 & 9.384 & 85.835 & 88.002 \\
\hline $\mathrm{r}$ & SB4VAR32ccc & 1 & 29 & R02 & 12 & 10.4 & 38.4 & 58.4 & 20.000 & 17.334 & 64.001 & 97.335 \\
\hline $\mathrm{r}$ & SB5-19ссс & 1 & 30 & R37 & 11.9 & 5.66 & 49.7 & 51.8 & 19.834 & 9.434 & 82.835 & 86.335 \\
\hline $\mathrm{r}$ & SB5-14 & 1 & 31 & R53 & 11.1 & 10.2 & 64.4 & 67.8 & 18.500 & 17.000 & 107.335 & 113.002 \\
\hline
\end{tabular}




\section{Table B1. PSAL Measurements of the PCT Solutions for the Study Glasses As- Received (ar) and After Appropriate Adjustments (in ppm)}

\begin{tabular}{|c|c|c|c|c|c|c|c|c|c|c|c|c|}
\hline Set & $\begin{array}{c}\text { Glass ID } \\
\text { (w HT) }\end{array}$ & Block & Seq & Lab ID & B ar & $\mathrm{Li}$ ar & $\mathrm{Na}$ ar & Si ar & B (ppm) & Li (ppm) & $\mathrm{Na}$ (ppm) & Si (ppm) \\
\hline $\mathrm{r}$ & Soln Std & 1 & 32 & STD-21-3 & 20.1 & 9.82 & 79.4 & 49.9 & 20.100 & 9.820 & 79.400 & 49.900 \\
\hline $\mathrm{r}$ & Soln Std & 2 & 1 & STD-22-1 & 20.1 & 9.63 & 78.7 & 50.8 & 20.100 & 9.630 & 78.700 & 50.800 \\
\hline $\mathrm{r}$ & SB5-17сcс & 2 & 2 & R83 & 10.8 & 10.4 & 31.5 & 58.1 & 18.000 & 17.334 & 52.501 & 96.835 \\
\hline $\mathrm{r}$ & SB4VAR11ccC & 2 & 3 & R03 & 11.4 & 10 & 34.1 & 59.3 & 19.000 & 16.667 & 56.834 & 98.835 \\
\hline $\mathrm{r}$ & SB5-15 & 2 & 4 & R20 & 12.3 & 7.6 & 56.8 & 56.8 & 20.500 & 12.667 & 94.669 & 94.669 \\
\hline $\mathrm{r}$ & SB5-14 & 2 & 5 & R54 & 10.1 & 9.53 & 61.3 & 65 & 16.834 & 15.884 & 102.169 & 108.336 \\
\hline $\mathrm{r}$ & SB4VAR11 & 2 & 6 & R13 & 11 & 9.73 & 35.4 & 57.9 & 18.334 & 16.217 & 59.001 & 96.502 \\
\hline $\mathrm{r}$ & SB5-15ccc & 2 & 7 & R80 & 11.4 & 7.42 & 52.8 & 55.1 & 19.000 & 12.367 & 88.002 & 91.835 \\
\hline $\mathrm{r}$ & SB4VAR13ccc & 2 & 8 & R47 & 21.5 & 16.5 & 62.3 & 65.8 & 35.834 & 27.501 & 103.835 & 109.669 \\
\hline $\mathrm{r}$ & SB4VAR12 & 2 & 9 & R82 & 11.7 & 10 & 41.3 & 58.1 & 19.500 & 16.667 & 68.835 & 96.835 \\
\hline $\bar{r}$ & SB5-18 & 2 & 10 & R66 & 10.4 & 7.51 & 44.6 & 54.6 & 17.334 & 12.517 & 74.335 & 91.002 \\
\hline $\mathrm{r}$ & SB4VAR12ccc & 2 & 11 & R32 & 11.7 & 10.1 & 39.7 & 59 & 19.500 & 16.834 & 66.168 & 98.335 \\
\hline $\mathrm{r}$ & SB4VAR13 & 2 & 12 & $\mathrm{R} 40$ & 12.2 & 10 & 48.9 & 56.2 & 20.334 & 16.667 & 81.502 & 93.669 \\
\hline $\mathrm{r}$ & SB4VAR33ccc & 2 & 13 & R74 & 12 & 10.2 & 53.4 & 56.9 & 20.000 & 17.000 & 89.002 & 94.835 \\
\hline $\mathrm{r}$ & SB5-19ccC & 2 & 14 & R50 & 11.2 & 5.38 & 48.9 & 52.3 & 18.667 & 8.967 & 81.502 & 87.168 \\
\hline $\mathrm{r}$ & SB5-17 & 2 & 15 & R27 & 11.1 & 11 & 34.3 & 60.2 & 18.500 & 18.334 & 57.168 & 100.335 \\
\hline $\mathrm{r}$ & Soln Std & 2 & 16 & STD-22-2 & 19.6 & 9.64 & 80.1 & 50.9 & 19.600 & 9.640 & 80.100 & 50.900 \\
\hline $\mathrm{r}$ & SB5-14ccc & 2 & 17 & R81 & 10.4 & 10.1 & 60.5 & 68.1 & 17.334 & 16.834 & 100.835 & 113.502 \\
\hline $\mathrm{r}$ & SB4VAR33 & 2 & 18 & R42 & 12.2 & 10.1 & 44.7 & 58.1 & 20.334 & 16.834 & 74.501 & 96.835 \\
\hline $\mathrm{r}$ & ARM-1 & 2 & 19 & R25 & 9.64 & 7.65 & 20.5 & 35.6 & 16.067 & 12.750 & 34.167 & 59.335 \\
\hline $\mathrm{r}$ & SB5-16сcс & 2 & 20 & R35 & 12.4 & 5.52 & 47.9 & 52.6 & 20.667 & 9.200 & 79.835 & 87.668 \\
\hline $\mathrm{r}$ & SB5-19 & 2 & 21 & R41 & 12.2 & 5.6 & 52.9 & 53.6 & 20.334 & 9.334 & 88.168 & 89.335 \\
\hline $\mathrm{r}$ & SB5-20ccc & 2 & 22 & R19 & 11.1 & 4.34 & 40.6 & 49.6 & 18.500 & 7.233 & 67.668 & 82.668 \\
\hline $\mathrm{r}$ & EA & 2 & 23 & R51 & 36.6 & 10.7 & 98.6 & 53.8 & 610.001 & 178.334 & 1643.337 & 896.668 \\
\hline $\mathrm{r}$ & SB4VAR32 & 2 & 24 & R36 & 11.9 & 10.2 & 38.8 & 60.9 & 19.834 & 17.000 & 64.668 & 101.502 \\
\hline $\mathrm{r}$ & SB5-20 & 2 & 25 & R11 & 11.7 & 4.3 & 41.1 & 51.1 & 19.500 & 7.167 & 68.501 & 85.168 \\
\hline $\mathrm{r}$ & SB4VAR32ccC & 2 & 26 & R34 & 12.5 & 10.5 & 37.8 & 62.7 & 20.834 & 17.500 & 63.001 & 104.502 \\
\hline $\mathrm{r}$ & SB4VAR31 & 2 & 27 & R24 & 12.3 & 10.8 & 34.1 & 63.4 & 20.500 & 18.000 & 56.834 & 105.669 \\
\hline $\mathrm{r}$ & SB5-18ccC & 2 & 28 & R12 & 11.5 & 8.4 & 46.1 & 59.9 & 19.167 & 14.000 & 76.835 & 99.835 \\
\hline $\mathrm{r}$ & SB4VAR31ccc & 2 & 29 & R72 & 11.7 & 10.4 & 34.1 & 62.4 & 19.500 & 17.334 & 56.834 & 104.002 \\
\hline $\mathrm{r}$ & SB5-16 & 2 & 30 & R70 & 14.8 & 6.11 & 55.1 & 57.2 & 24.667 & 10.184 & 91.835 & 95.335 \\
\hline $\mathrm{r}$ & Soln Std & 2 & 31 & STD-22-3 & 21.5 & 10.1 & 81.5 & 54.3 & 21.500 & 10.100 & 81.500 & 54.300 \\
\hline $\mathrm{r}$ & Soln Std & 3 & 1 & STD-23-1 & 20.8 & 9.7 & 79.1 & 49.9 & 20.800 & 9.700 & 79.100 & 49.900 \\
\hline $\mathrm{r}$ & SB4VAR32ccc & 3 & 2 & R73 & 12.5 & 10.7 & 40.2 & 60.4 & 20.834 & 17.834 & 67.001 & 100.669 \\
\hline $\mathrm{r}$ & SB5-17 & 3 & 3 & R60 & 11.1 & 10.1 & 31.8 & 54.8 & 18.500 & 16.834 & 53.001 & 91.335 \\
\hline $\mathrm{r}$ & SB4VAR12ccC & 3 & 4 & R23 & 11.9 & 10 & 40.1 & 56.6 & 19.834 & 16.667 & 66.835 & 94.335 \\
\hline $\mathrm{r}$ & SB4VAR11ccc & 3 & 5 & R15 & 11.8 & 10 & 34.2 & 59.2 & 19.667 & 16.667 & 57.001 & 98.669 \\
\hline $\mathrm{r}$ & SB4VAR31ccc & 3 & 6 & R58 & 11.9 & 10.3 & 32.7 & 60.5 & 19.834 & 17.167 & 54.501 & 100.835 \\
\hline $\mathrm{r}$ & SB5-19ccC & 3 & 7 & R76 & 12.5 & 5.46 & 48.4 & 54.5 & 20.834 & 9.100 & 80.668 & 90.835 \\
\hline $\mathrm{r}$ & SB5-14 & 3 & 8 & R79 & 11.5 & 10 & 63.7 & 70.2 & 19.167 & 16.667 & 106.169 & 117.002 \\
\hline $\mathrm{r}$ & SB4VAR33ccc & 3 & 9 & R63 & 13.7 & 10.8 & 45.1 & 60.6 & 22.834 & 18.000 & 75.168 & 101.002 \\
\hline $\mathrm{r}$ & SB4VAR32 & 3 & 10 & R67 & 12.5 & 10.1 & 37.5 & 59.4 & 20.834 & 16.834 & 62.501 & 99.002 \\
\hline $\mathrm{r}$ & EA & 3 & 11 & R64 & 36.9 & 10.7 & 91.3 & 55 & 615.001 & 178.334 & 1521.670 & 916.669 \\
\hline $\mathrm{r}$ & SB5-20ccC & 3 & 12 & R22 & 12.6 & 4.39 & 40.2 & 51.4 & 21.000 & 7.317 & 67.001 & 85.668 \\
\hline $\mathrm{r}$ & SB4VAR12 & 3 & 13 & R44 & 13.6 & 10.6 & 43.9 & 63.3 & 22.667 & 17.667 & 73.168 & 105.502 \\
\hline $\mathrm{r}$ & SB5-17сcC & 3 & 14 & R07 & 12.6 & 11.2 & 33 & 64.2 & 21.000 & 18.667 & 55.001 & 107.002 \\
\hline $\mathrm{r}$ & SB4VAR13ccc & 3 & 15 & R28 & 25 & 18.1 & 64.6 & 73.7 & 41.668 & 30.167 & 107.669 & 122.836 \\
\hline $\mathrm{r}$ & Soln Std & 3 & 16 & STD-23-2 & 22 & 10.1 & 80 & 54.6 & 22.000 & 10.100 & 80.000 & 54.600 \\
\hline $\mathrm{r}$ & SB4VAR11 & 3 & 17 & R33 & 12.8 & 10.4 & 37.1 & 58.9 & 21.334 & 17.334 & 61.835 & 98.169 \\
\hline $\mathrm{r}$ & SB4VAR31 & 3 & 18 & R04 & 14.2 & 11.2 & 36.4 & 67.2 & 23.667 & 18.667 & 60.668 & 112.002 \\
\hline $\mathrm{r}$ & SB5-19 & 3 & 19 & R86 & 14 & 6 & 56 & 57.2 & 23.334 & 10.000 & 93.335 & 95.335 \\
\hline$r$ & SB4VAR13 & 3 & 20 & R10 & 12.3 & 10.4 & 50.9 & 52.6 & 20.500 & 17.334 & 84.835 & 87.668 \\
\hline $\mathrm{r}$ & SB5-15ccC & 3 & 21 & R16 & 11.4 & 7.68 & 55.1 & 51.2 & 19.000 & 12.800 & 91.835 & 85.335 \\
\hline $\mathrm{r}$ & SB5-14cCC & 3 & 22 & R71 & 9.94 & 10.2 & 62.5 & 60.3 & 16.567 & 17.000 & 104.169 & 100.502 \\
\hline $\mathrm{r}$ & SB5-20 & 3 & 23 & R56 & 11.3 & 4.29 & 42.3 & 46.7 & 18.834 & 7.150 & 70.501 & 77.835 \\
\hline $\mathrm{r}$ & SB5-16 & 3 & 24 & R75 & 13.8 & 6.17 & 58 & 53.3 & 23.000 & 10.284 & 96.669 & 88.835 \\
\hline $\mathrm{r}$ & blank & 3 & 25 & R39 & $<1.00$ & $<1.00$ & $<1.00$ & 0.456 & 0.833 & 0.833 & 0.833 & 0.760 \\
\hline$r$ & SB5-18 & 3 & 26 & R21 & 10.9 & 8.22 & 49.7 & 53.5 & 18.167 & 13.700 & 82.835 & 89.168 \\
\hline $\mathrm{r}$ & SB4VAR33 & 3 & \begin{tabular}{|l|}
27 \\
\end{tabular} & R18 & 12.3 & 10.7 & 48.4 & 57 & 20.500 & 17.834 & 80.668 & 95.002 \\
\hline $\mathrm{r}$ & ARM-1 & 3 & 28 & R29 & 10.1 & 8.25 & 23.2 & 35 & 16.834 & 13.750 & 38.667 & 58.335 \\
\hline
\end{tabular}


Table B1. PSAL Measurements of the PCT Solutions for the Study Glasses AsReceived (ar) and After Appropriate Adjustments (in ppm)

\begin{tabular}{|c|c|c|c|c|c|c|c|c|c|c|c|c||}
\hline \hline Set & $\begin{array}{c}\text { Glass ID } \\
\text { (w HT) }\end{array}$ & Block & Seq & Lab ID & B ar & Li ar & Na ar & Si ar & B (ppm) & Li (ppm) & Na (ppm) & Si (ppm) \\
\hline r & SB5-16ccc & 3 & 29 & R14 & 13.7 & 6.3 & 55.1 & 53.2 & 22.834 & 10.500 & 91.835 & 88.668 \\
\hline r & SB5-18ccc & 3 & 30 & R43 & 10.8 & 8.28 & 48.5 & 54.2 & 18.000 & 13.800 & 80.835 & 90.335 \\
\hline r & SB5-15 & 3 & 31 & R05 & 14.4 & 8.23 & 58.7 & 61.6 & 24.000 & 13.717 & 97.835 & 102.669 \\
\hline r & Soln Std & 3 & 32 & STD-23-3 & 19.7 & 10.2 & 83.1 & 50 & 19.700 & 10.200 & 83.100 & 50.000 \\
\hline
\end{tabular}


Exhibit B1. Laboratory PCT Measurements in Analytical Sequence for Study Glasses with and without Other Results from the Analytical Plans

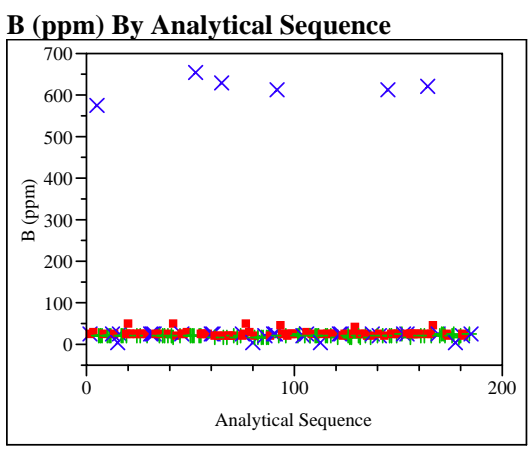

Li (ppm) By Analytical Sequence

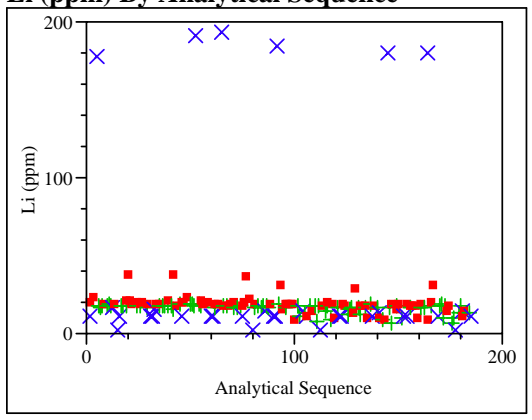

Na (ppm) By Analytical Sequence

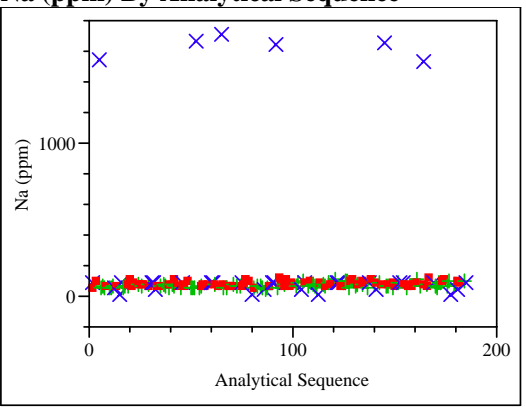

Si (ppm) By Analytical Sequence

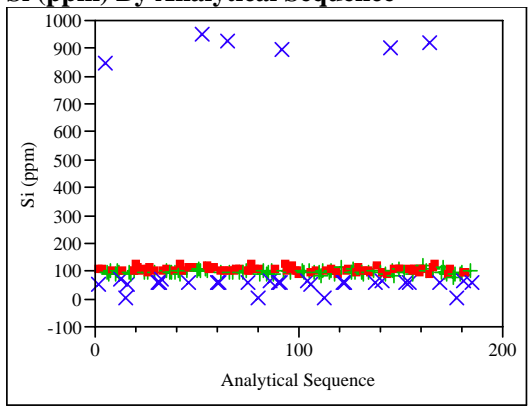

B (ppm) By Analytical Sequence

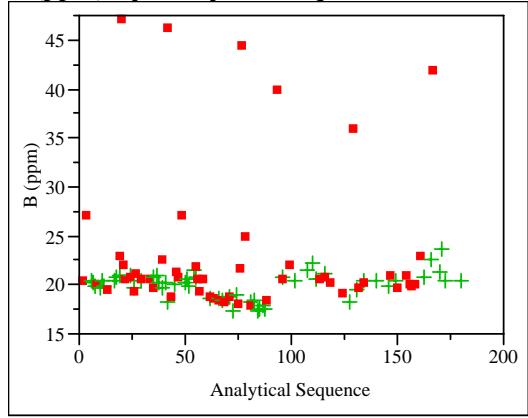

Li (ppm) By Analytical Sequence

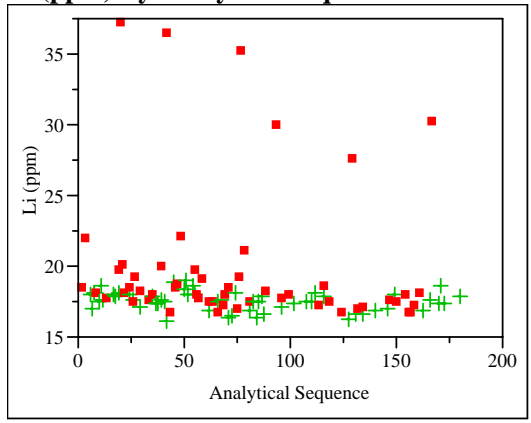

Na (ppm) By Analytical Sequence

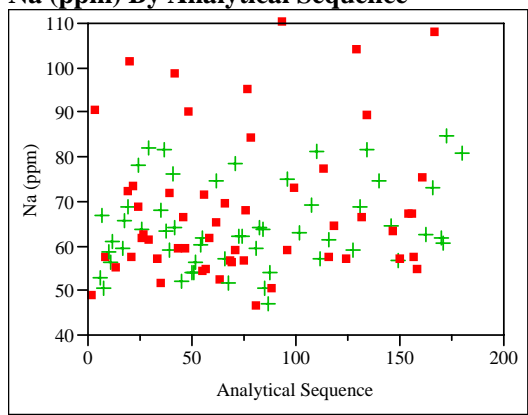

Si (ppm) By Analytical Sequence

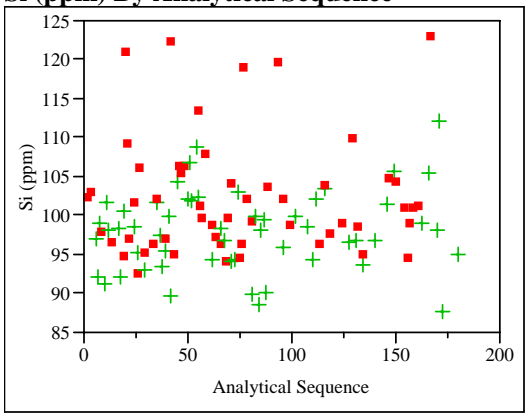




\section{Exhibit B2. Measurements of the Multi-Element Solution Standard by ICP Block}

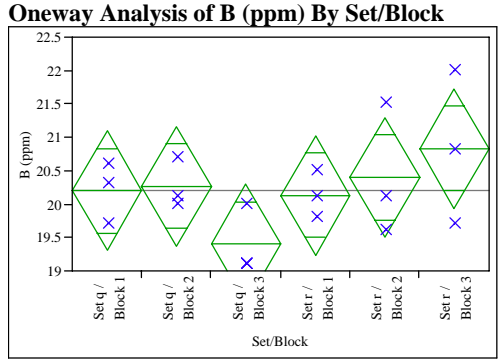

Oneway Anova

Summary of Fit

Rsquare

are Error $\quad 0.711805$

Mean of Response 20.20556

Observations (or Sum Wgts)

18

Analysis of Variance

Source DF Sum of Squares Mean Square F Ratio Prob $>$ F

$\begin{array}{lllll}\text { Set/Block } 5 & 3.2694444 & 0.653889 & 1.2906 & 0.3305\end{array}$

$\begin{array}{llll}\text { Error } & 12 & 6.0800000 & 0.506667\end{array}$

C. Total $17 \quad 9.3494444$

Means for Oneway Anova

Level Number Mean Std Error Lower 95\% Upper 95\%

$\begin{array}{lllll}\text { Set q/Block } 1 & 320.2000 & 0.41096 & 19.305 & 21.095\end{array}$

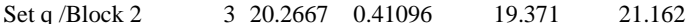

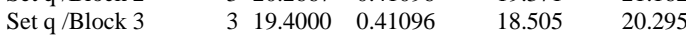

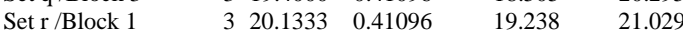

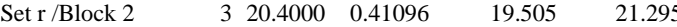

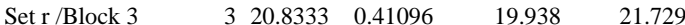

Std Error uses a pooled estimate of error variance

Oneway Analysis of Li (ppm) By Set/Block

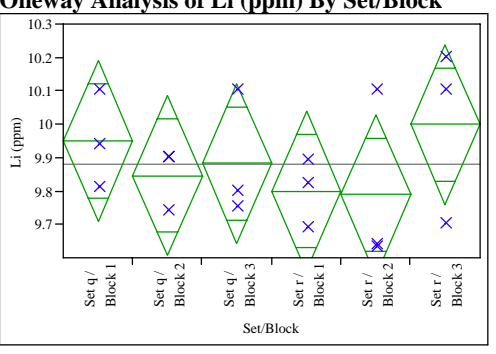

Oneway Anova

Summary of Fit

Rsquare $\quad 0.193758$

Adj Rsquare $\quad-0.14218$

Root Mean Square Error $\quad 0.190555$

Mean of Response $\quad 9.878333$

Observations (or Sum Wgts) 18

Analysis of Variance

Source DF Sum of Squares Mean Square F Ratio Prob $>$ F

$\begin{array}{llllll}\text { Set/Block } & 5 & 0.10471667 & 0.020943 & 0.5768 & 0.7173\end{array}$

$\begin{array}{llll}\text { Error } & 12 & 0.43573333 & 0.036311\end{array}$

C. Total $17 \quad 0.54045000$

Means for Oneway Anova

Level Number Mean Std Error Lower 95\% Upper 95\%

$\begin{array}{llllll}\text { Set q /Block } 1 & 3 & 9.9500 & 0.11002 & 9.7103 & 10.190\end{array}$

$\begin{array}{llllll}\text { Set q /Block } 2 & 3 & 9.8467 & 0.11002 & 9.6070 & 10.086\end{array}$

$\begin{array}{llllll}\text { Set q /Block } 3 & 3 & 9.8833 & 0.11002 & 9.6436 & 10.123\end{array}$

$\begin{array}{llllll}\text { Set } r \text { /Block } 1 & 3 & 9.8000 & 0.11002 & 9.5603 & 10.040\end{array}$

$\begin{array}{llllll}\text { Set } \mathrm{r} / \text { Block } 2 & 3 & 9.7900 & 0.11002 & 9.5503 & 10.030\end{array}$

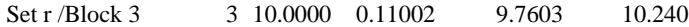

Std Error uses a pooled estimate of error variance
Oneway Analysis of Na (ppm) By Set/Block

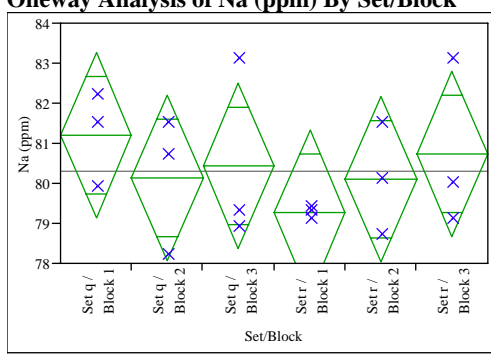

Oneway Anova

Summary of Fit

Rsquare

0.166791

Adj Rsquare

$-0.18038$

Root Mean Square Error $\quad 1.638766$

Mean of Response

80.31111

Observations (or Sum Wgts)

Analysis of Variance

Source DF Sum of Squares Mean Square F Ratio Prob $>$ F

$\begin{array}{lrrrrr}\text { Set/Block } & 5 & 6.451111 & 1.29022 & 0.4804 & 0.7844\end{array}$

$\begin{array}{llll}\text { Error } & 12 & 32.226667 & 2.68556\end{array}$

C. Total $17 \quad 38.677778$

Means for Oneway Anova

Level Number Mean Std Error Lower 95\% Upper 95\%

$\begin{array}{llllll}\text { Set q /Block } 1 & 3 & 81.2000 & 0.94614 & 79.139 & 83.261\end{array}$

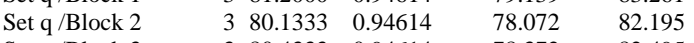

$\begin{array}{llllll}\text { Set q/Block } 3 & 3 & 80.4333 & 0.94614 & 78.372 & 82.495\end{array}$

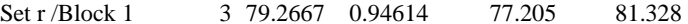

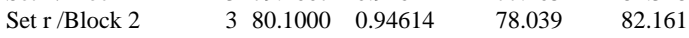

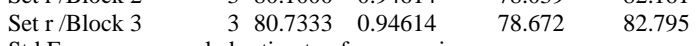

Std Error uses a pooled estimate of error variance

Oneway Analysis of Si (ppm) By Set/Block

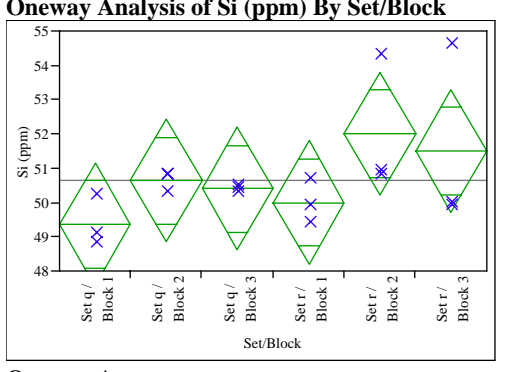

Oneway Anova

Summary of Fit

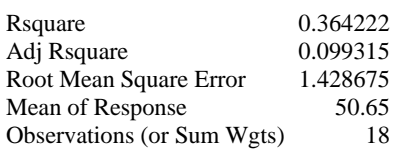

Observations (or Sum Wgts)

Analysis of Variance

Source DF Sum of Squares Mean Square F Ratio Prob $>$ F $\begin{array}{llllll}\text { Set/Block } & 5 & 14.031667 & 2.80633 & 1.3749 & 0.3005\end{array}$

$\begin{array}{llll}\text { Error } & 12 & 24.493333 & 2.04111\end{array}$

C. Total $17 \quad 38.525000$

Means for Oneway Anova

Level Number Mean Std Error Lower 95\% Upper 95\%

$\begin{array}{lllrrr}\text { Set q/Block } 1 & 3 & 49.3667 & 0.82485 & 47.569 & 51.164\end{array}$

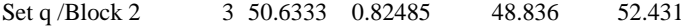

$\begin{array}{lllll}\text { Set q /Block } 3 & 350.4000 & 0.82485 & 48.603 & 52.197\end{array}$

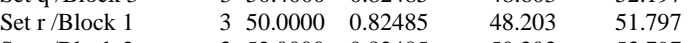

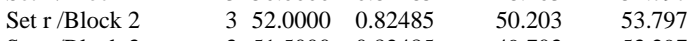

$\begin{array}{llll}\text { Set } r \text { /Block } 3 & 351.5000 & 0.82485 & 49.703\end{array}$

Std Error uses a pooled estimate of error variance 


\section{Exhibit B3. Laboratory PCT Measurements by Glass Identifier for Study Glasses and Standards}

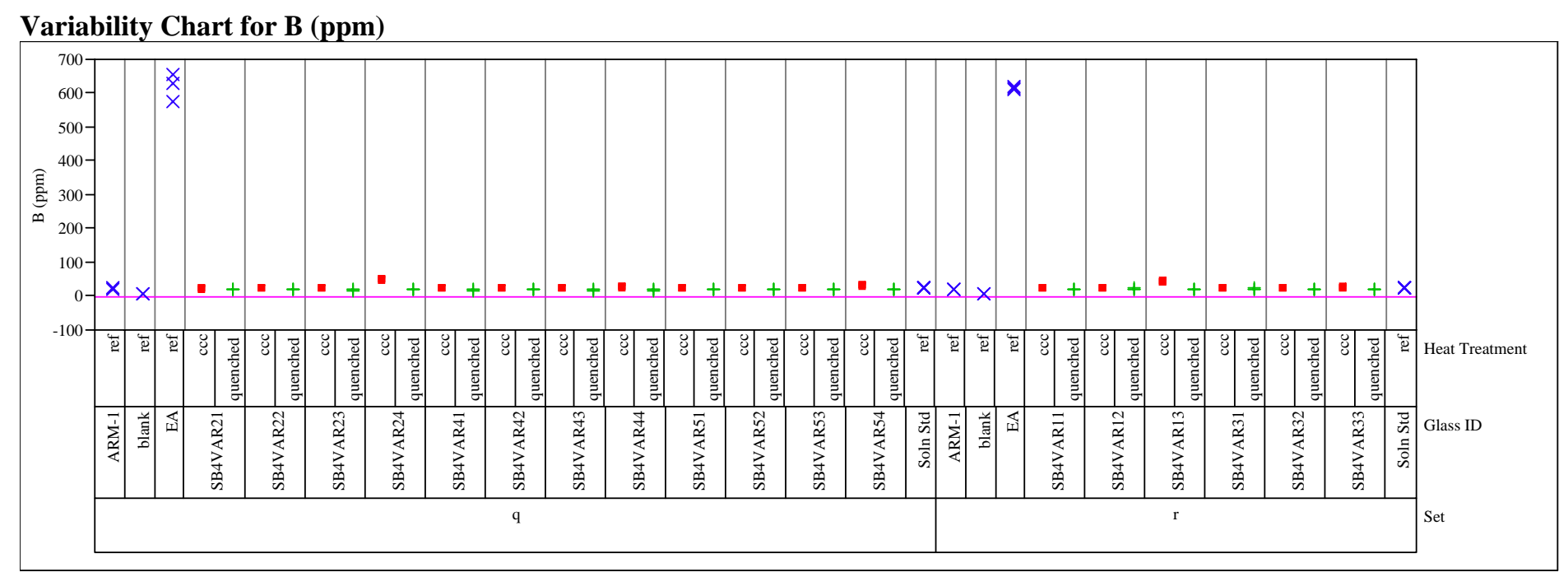

\section{Variability Chart for Li (ppm)}

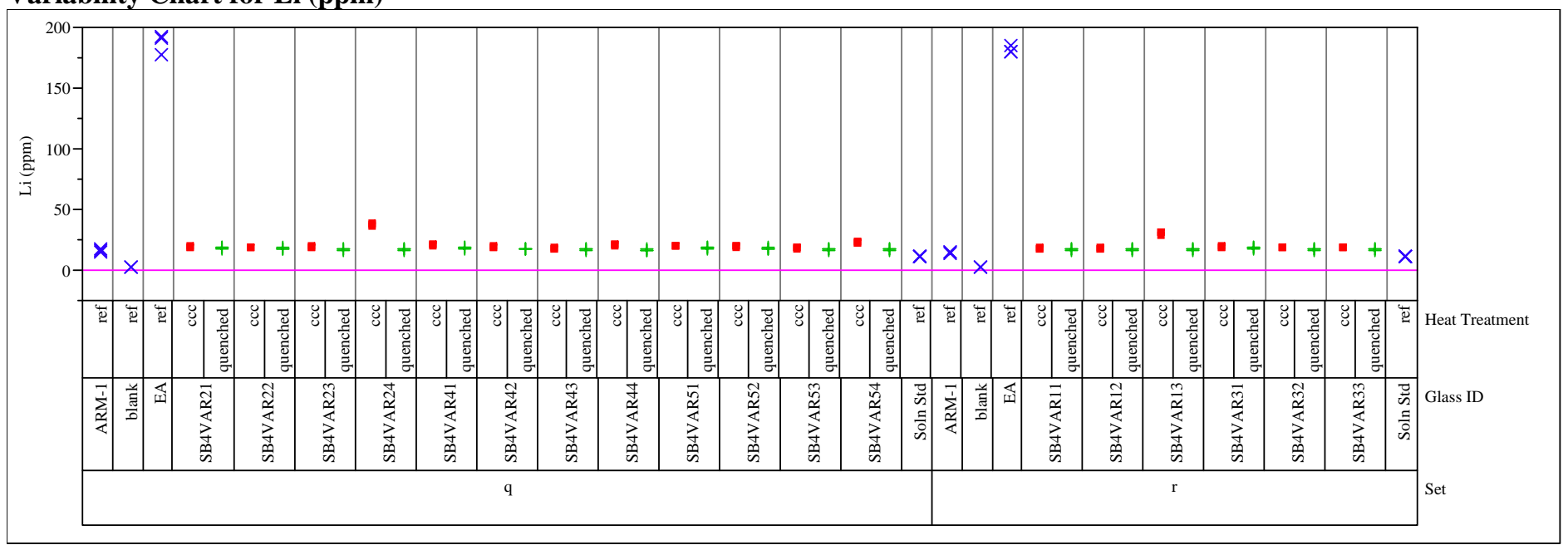




\section{Exhibit B3. Laboratory PCT Measurements by Glass Identifier for Study Glasses and Standards}

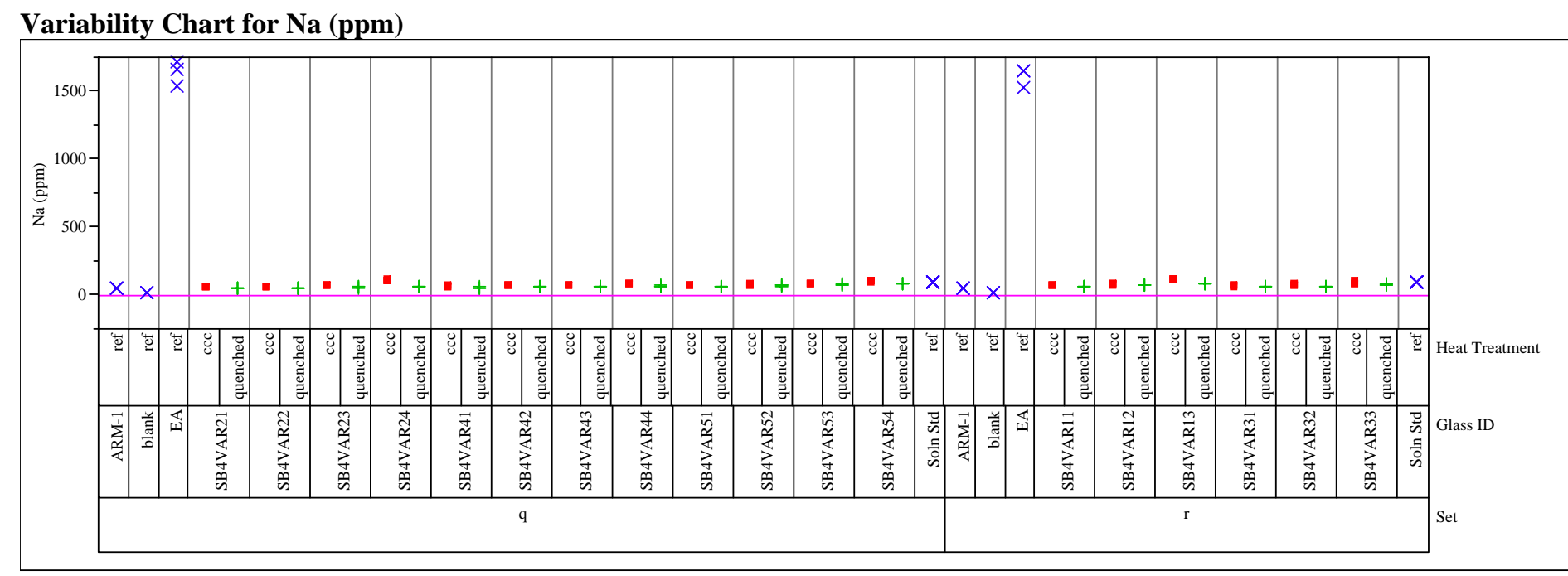

\section{Variability Chart for Si (ppm)}

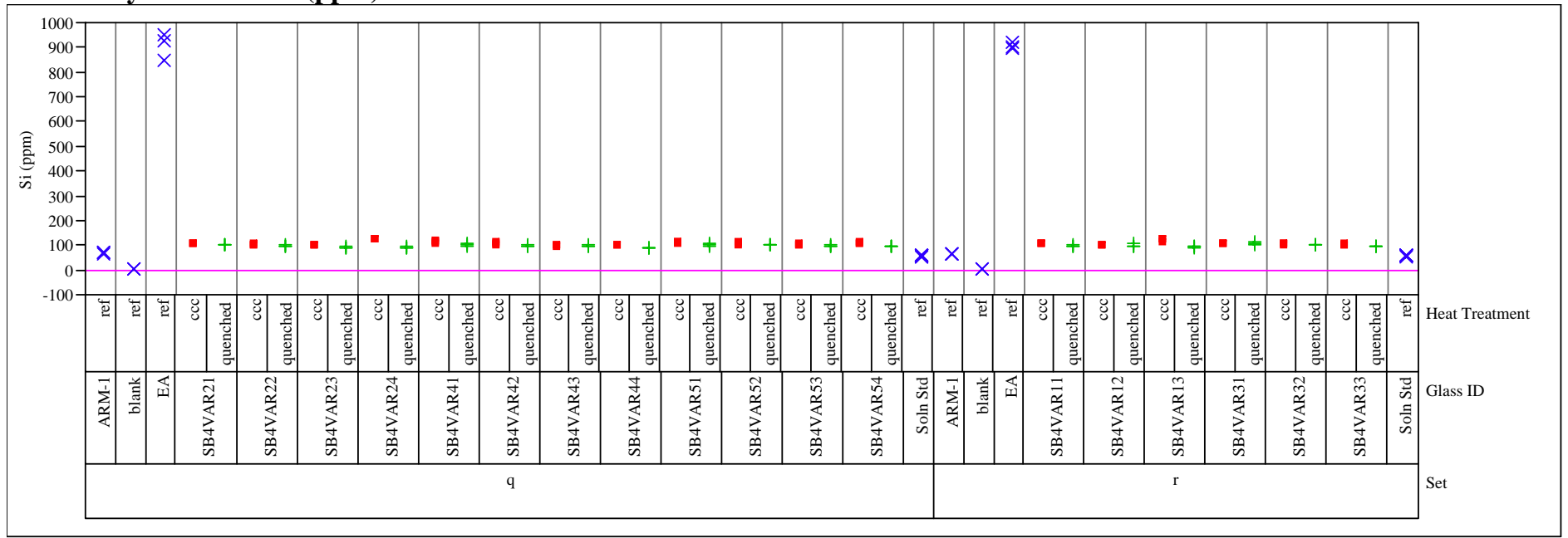


Exhibit B4. Laboratory PCT Measurements by Glass Identifier for Study Glasses

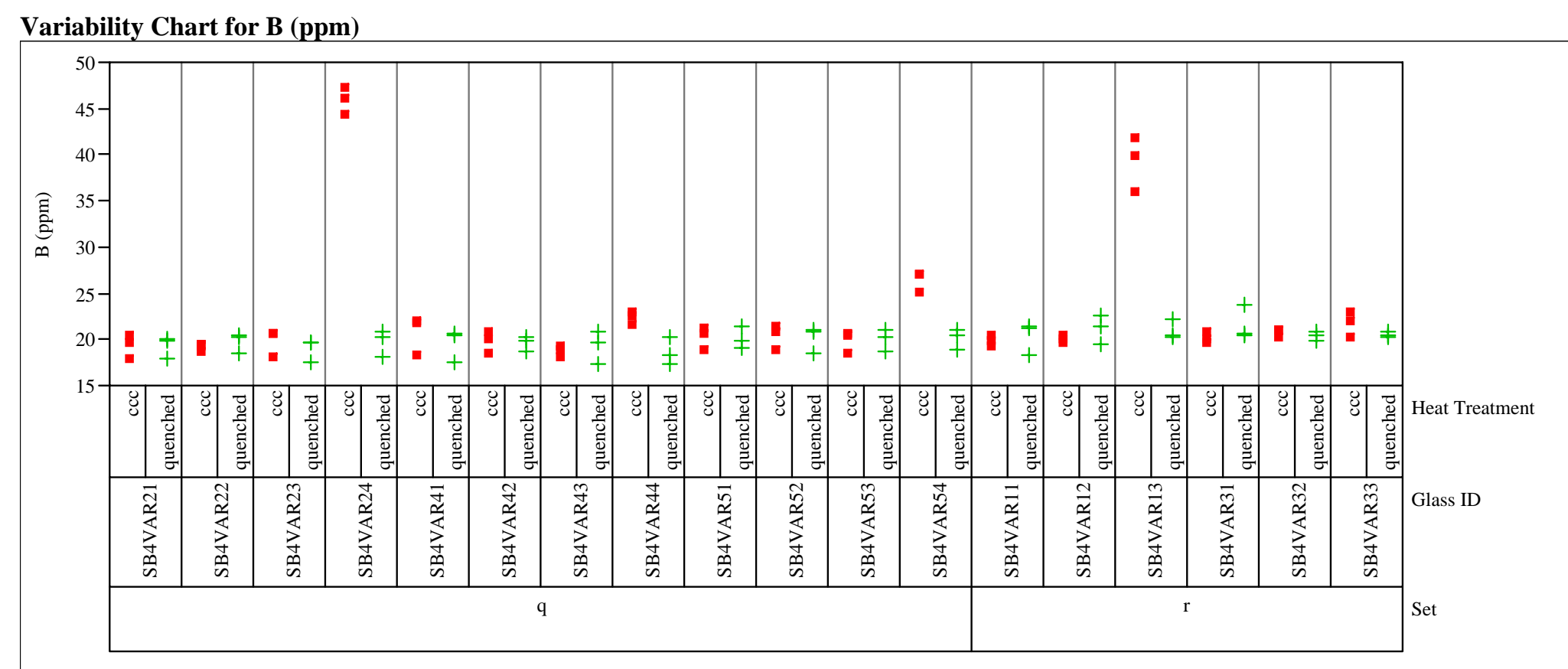


Exhibit B4. Laboratory PCT Measurements by Glass Identifier for Study Glasses

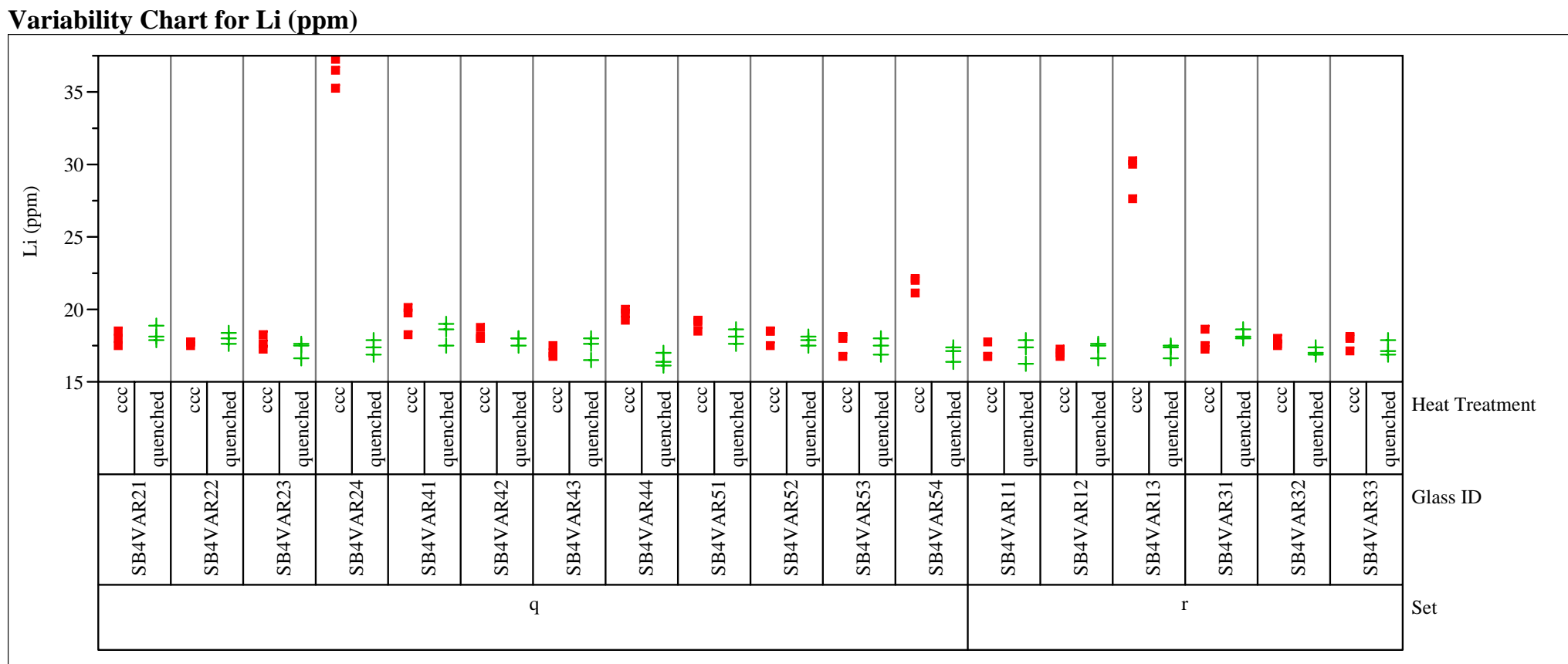


Exhibit B4. Laboratory PCT Measurements by Glass Identifier for Study Glasses

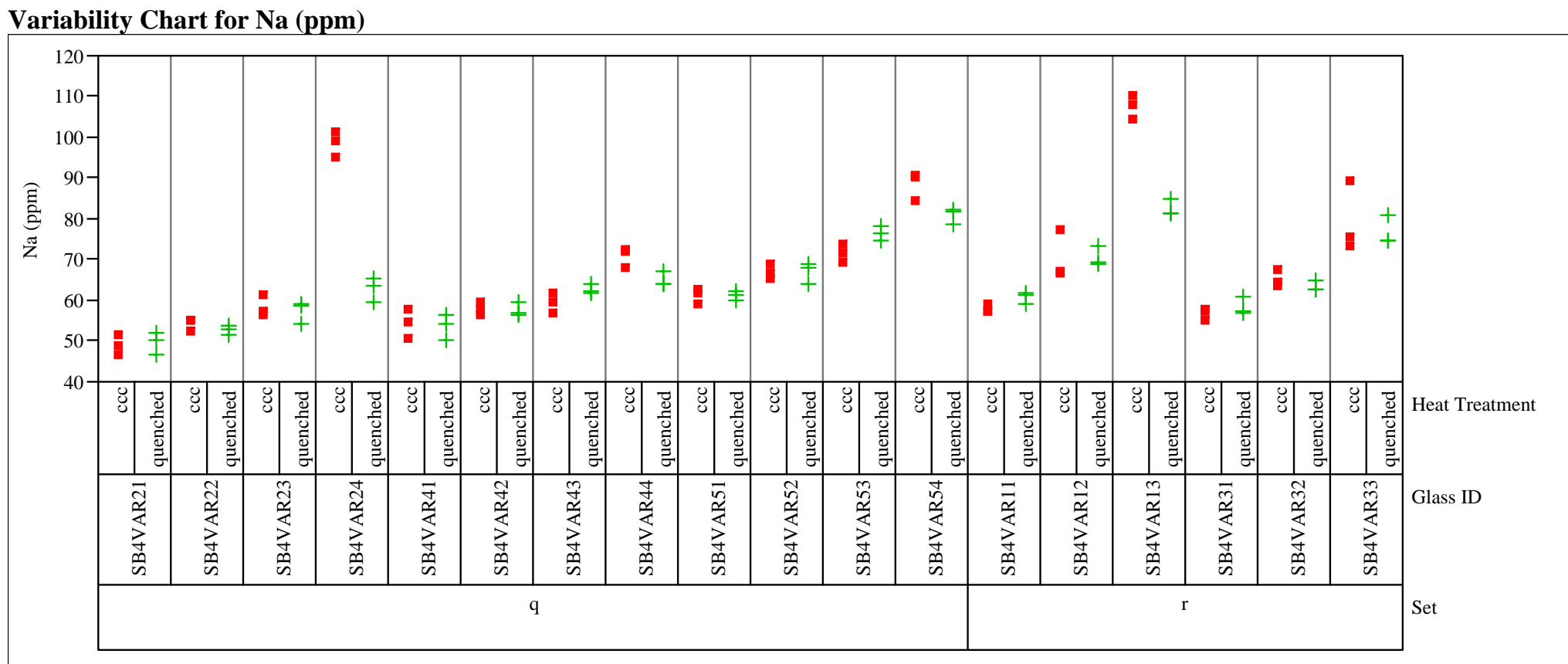


Exhibit B4. Laboratory PCT Measurements by Glass Identifier for Study Glasses

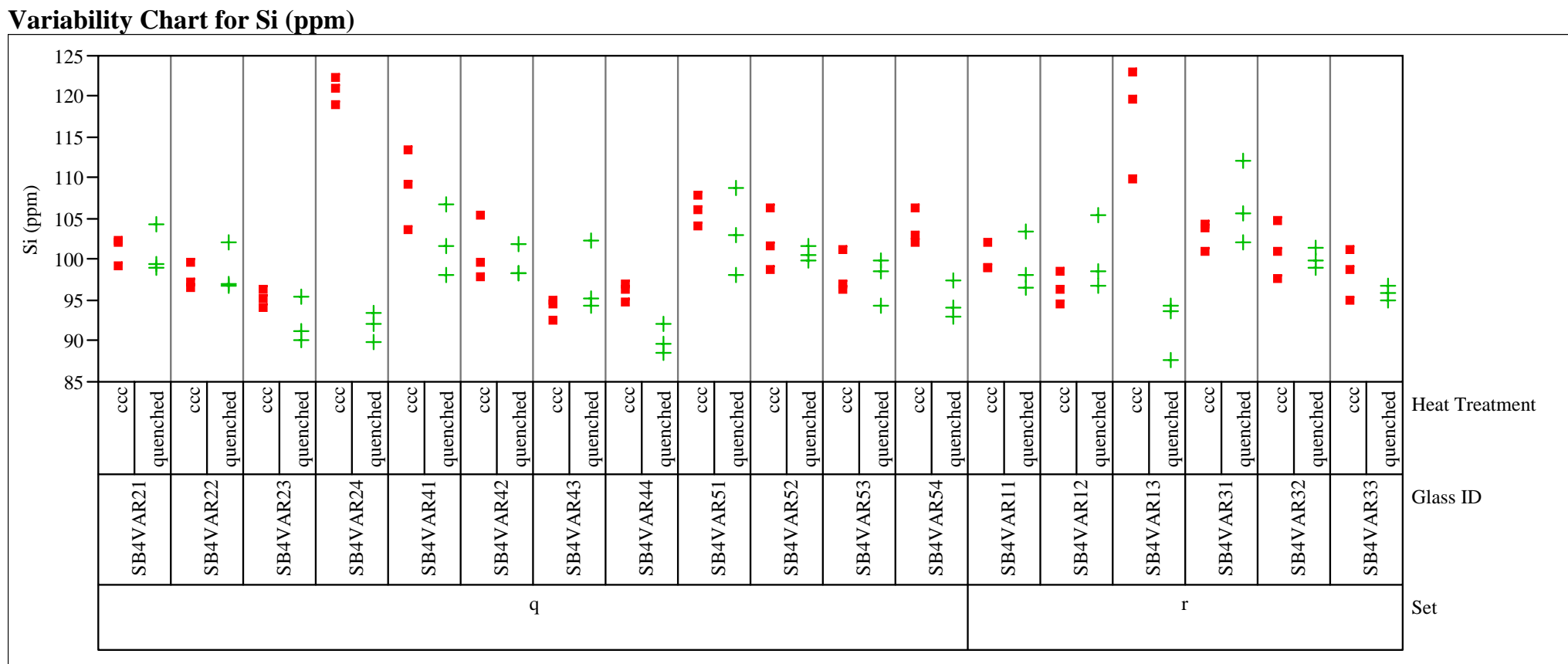




\section{Exhibit B5. Correlations and Scatter Plots of Normalized PCTs Over All Compositional Views and Heat Treatments}

\section{Correlations}

$\log \mathrm{NL}[\mathrm{B}(\mathrm{g} / \mathrm{L})]$

$\log \mathrm{NL}[\mathrm{Li}(\mathrm{g} / \mathrm{L})]$

$\log \mathrm{NL}[\mathrm{Na}(\mathrm{g} / \mathrm{L})]$

$\log \mathrm{NL}[\mathrm{Si}(\mathrm{g} / \mathrm{L})]$

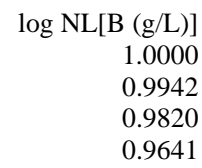

1.0000

0.9942

0.9820

0.9641
$\log \mathrm{NL}[\mathrm{Li}(\mathrm{g} / \mathrm{L})]$
0.9942
1.0000
0.9702
0.9529

$\log \mathrm{NL}[\mathrm{Na}(\mathrm{g} / \mathrm{L})]$

0.9820

$\log \mathrm{NL}[\mathrm{Si}(\mathrm{g} / \mathrm{L})]$

0.9702

0.9776

0.9641
0.9529

0.9776

1.0000

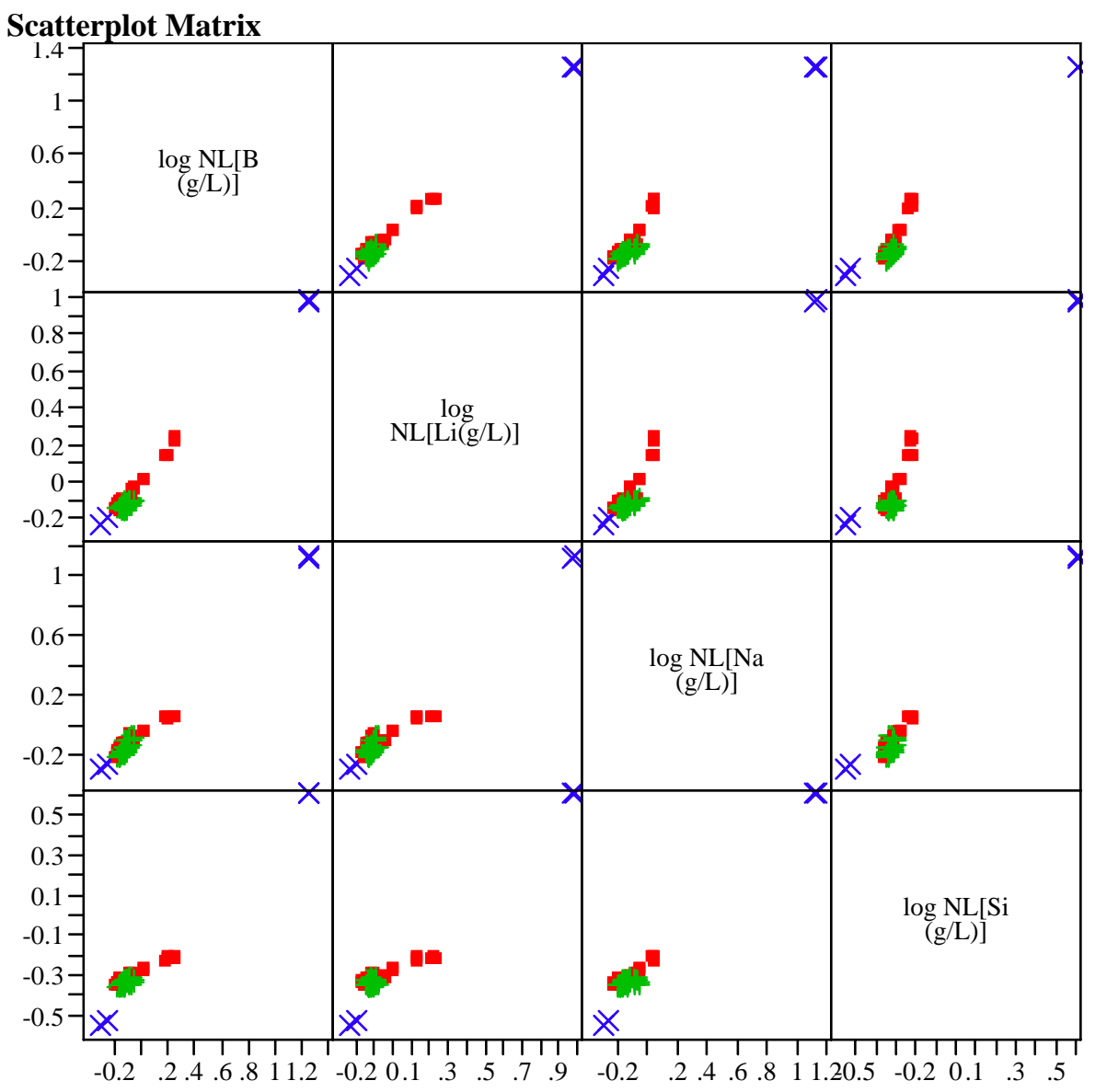


Exhibit B6. Effects of Heat Treatment on PCT $\log (\mathrm{ppm})$-Response of Study Glasses

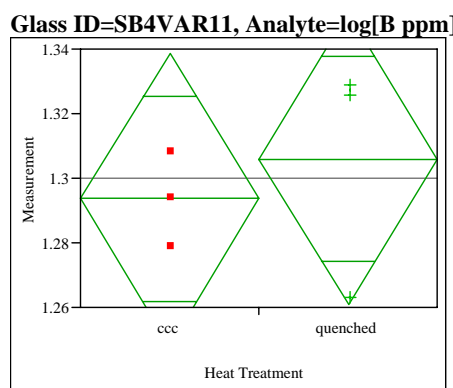

Difference $\quad 0.01242$ t Ratio $\quad 0.539481$ $\begin{array}{lrlr}\text { Std Err Dif } & 0.02302 & \text { DF } & 4 \\ \text { Upper CL Dif } & 0.07634 & \text { Prob }>|t| & 0.6182 \\ \text { Lower CL Dif } & -0.05150 & \text { Prob }>t & 0.3091\end{array}$ $\begin{array}{lll}\text { Confidence } & 0.95 \text { Prob }<\mathrm{t} \quad 0.6909\end{array}$

Glass ID=SB4VAR11, Analyte=log[Li ppm $]$

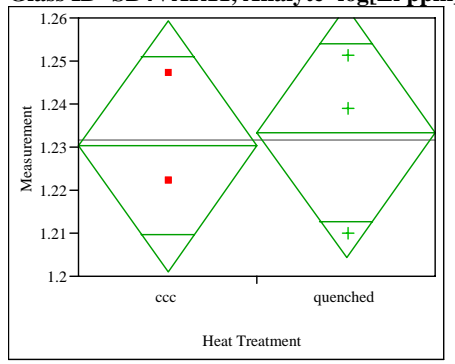

Difference $\quad 0.00307$ t Ratio $\quad 0.206957$ Std Err Dif 0.01486 DF

Upper CL Dif 0.04432 Prob $>|\mathrm{t}| \quad 0.8462$

Lower CL Dif -0.03817 Prob $>t \quad 0.4231$

$\begin{array}{lll}\text { Confidence } & 0.95 \text { Prob }<\mathrm{t} & 0.5769\end{array}$

Glass ID=SB4VAR11, Analyte=log[Na ppm]

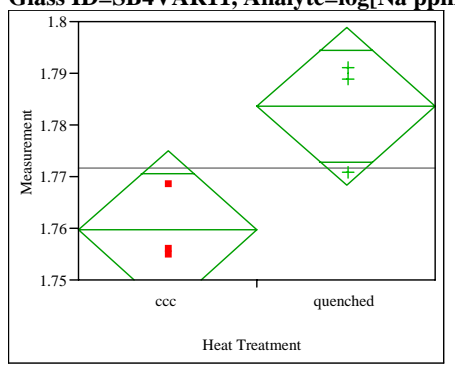

Difference $\quad 0.024027$ t Ratio 3.082351 Std Err Dif 0.007795 DF

Upper CL Dif 0.045669 Prob $>|t| \quad 0.0368$

Lower CL Dif 0.002385 Prob $>t \quad 0.0184$

Confidence $\quad 0.95$ Prob $<t \quad 0.9816$
Glass ID=SB4VAR11, Analyte=log[Si ppm $]$

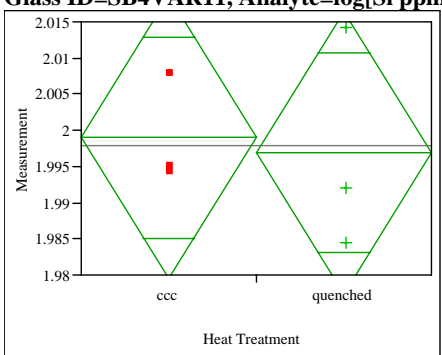

Difference $\quad-0.00208$ t Ratio -0.20821 Std Err Dif $\quad 0.00998$ DF

Upper CL Dif 0.02562 Prob $>|t| \quad 0.8452$

Lower CL Dif - -0.02978 Prob $>t \quad 0.5774$

$\begin{array}{lll}\text { Confidence } & 0.95 \text { Prob }<\mathrm{t} & 0.4226\end{array}$

Glass ID=SB4VAR12, Analyte $=\log [B$ ppm $]$

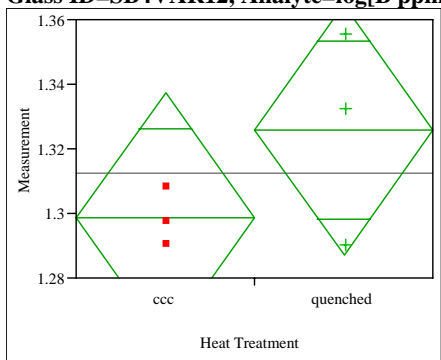

Difference $\quad 0.02741$ t Ratio 1.380256 Std Err Dif $\quad 0.01986$ DF

Upper CL Dif $\quad 0.08254$ Prob $>|t| \quad 0.2396$

Lower CL Dif -0.02772 Prob $>t \quad 0.1198$

Confidence $\quad 0.95$ Prob $<\mathrm{t} \quad 0.8802$

Glass ID=SB4VAR12, Analyte $=\log [\mathrm{Li}$ ppm $]$

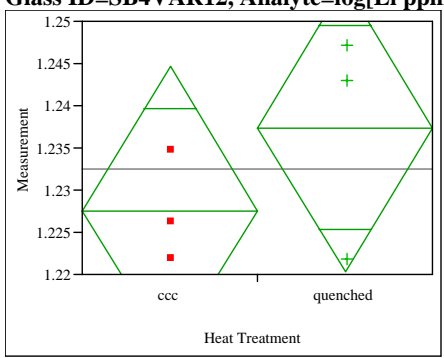

Difference $\quad 0.00978$ t Ratio $\quad 1.124053$ Std Err Dif $\quad 0.00870$ DF Upper CL Dif 0.03393 Prob $>|t| \quad 0.3239$

Lower CL Dif -0.01438 Prob $>\mathrm{t} \quad 0.1619$ Confidence $\quad 0.95$ Prob $<\mathrm{t} \quad 0.8381$

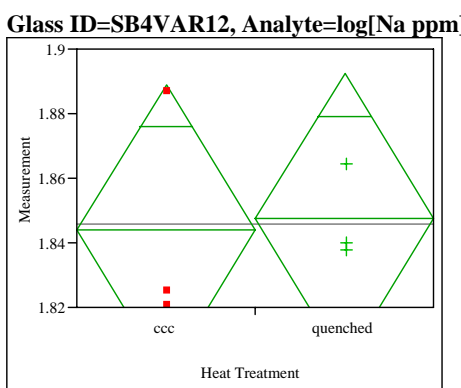

Difference $\quad 0.00330$ t Ratio 0.143887 Std Err Dif 0.02290 DF 4 Upper CL Dif 0.06688 Prob $>|t| \quad 0.8925$ Lower CL Dif -0.06029 Prob $>t \quad 0.4463$ Confidence $\quad 0.95$ Prob $<\mathrm{t} \quad 0.5537$

Glass ID=SB4VAR12, Analyte $=\log [\mathrm{Si}$ ppm $]$

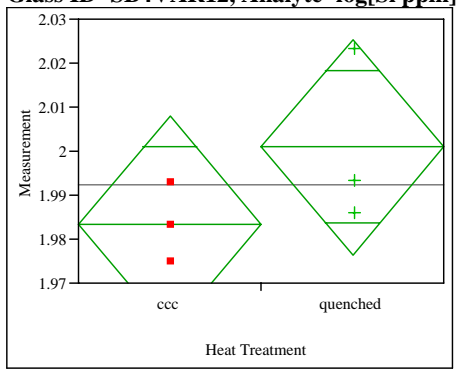

Difference $\quad 0.01744$ t Ratio 1.393767 Std Err Dif $\quad 0.01251$ DF 4 Upper CL Dif 0.05218 Prob $>|t| \quad 0.2358$ Lower CL Dif -0.01730 Prob $>t \quad 0.1179$ Confidence $\quad 0.95$ Prob $<\mathrm{t} \quad 0.8821$

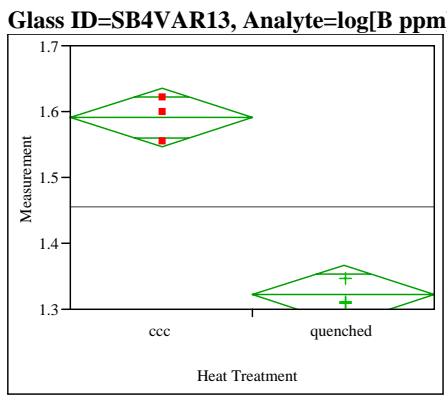

Difference $\quad-0.26895$ t Ratio $\quad-11.854$ Std Err Dif 0.02269 DF 4 Upper CL Dif -0.20595 Prob $>|t| 0.0003$ Lower CL Dif -0.33194 Prob $>$ t 0.9999 $\begin{array}{lll}\text { Confidence } & 0.95 \text { Prob }<\mathrm{t} & 0.0001\end{array}$ 
Exhibit B6. Effects of Heat Treatment on PCT $\log (\mathrm{ppm})-$ Response of Study Glasses

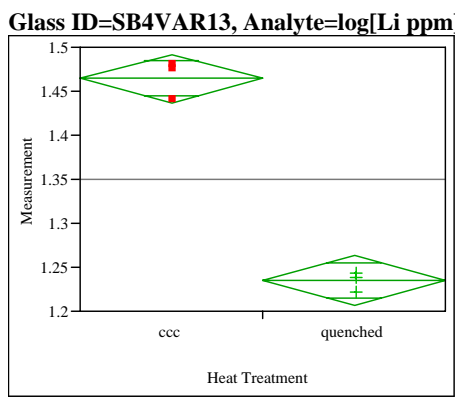

Difference $\quad-0.22993$ t Ratio $\quad-16.1551$ Std Err Dif 0.01423 DF

Upper CL Dif -0.19041 Prob $>|\mathrm{t}|<.0001$

Lower CL Dif -0.26945 Prob $>$ t 1.0000

$\begin{array}{lll}\text { Confidence } & 0.95 \text { Prob }<\mathrm{t} \quad<.0001\end{array}$

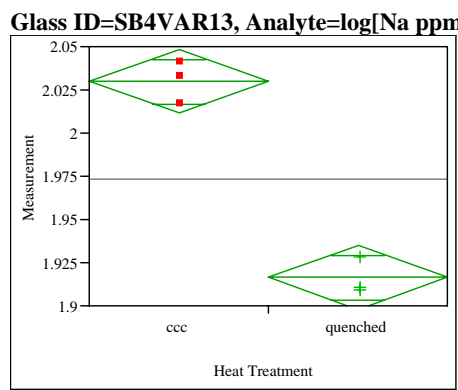

Difference $\quad-0.11335$ t Ratio $\quad-12.0511$ Std Err Dif 0.00941 DF

Upper CL Dif -0.08724 Prob $>|t| \quad 0.0003$ Lower CL Dif -0.13946 Prob $>t \quad 0.9999$

Confidence $\quad 0.95$ Prob $<\mathrm{t} \quad 0.0001$

Glass ID=SB4VAR13, Analyte=log[Si ppm $]$

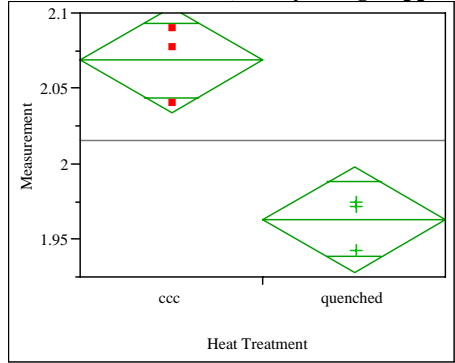

Difference $\quad-0.10569$ t Ratio $\quad-5.89922$ Std Err Dif $\quad 0.01792$ DF 4

Upper CL Dif -0.05595 Prob $>|\mathrm{t}| \quad 0.0041$

Lower CL Dif -0.15543 Prob $>t \quad 0.9979$

$\begin{array}{lrl}\text { Confidence } & 0.95 \text { Prob }<\mathrm{t} & 0.0021\end{array}$
Glass ID=SB4VAR21, Analyte= $=\log [B$ ppm $]$

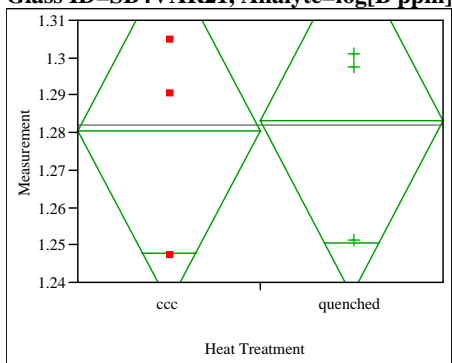

Difference $\quad 0.00261$ t Ratio 0.110914 Std Err Dif $\quad 0.02355$ DF

Upper CL Dif 0.06799 Prob $>|\mathrm{t}| \quad 0.9170$

Lower CL Dif -0.06276 Prob $>\mathrm{t} \quad 0.4585$

$\begin{array}{lll}\text { Confidence } & 0.95 \text { Prob }<\mathrm{t} & 0.5415\end{array}$

Glass ID=SB4VAR21, Analyte $=\log [\mathrm{Li}$ ppm $]$

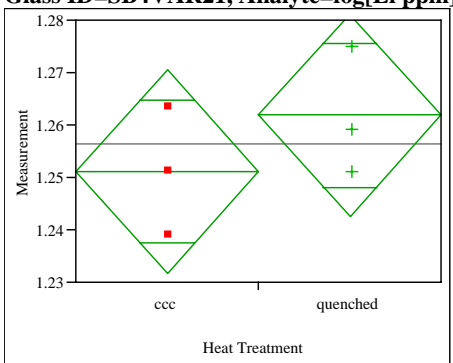

Difference $\quad 0.01069$ t Ratio 1.081002 Std Err Dif $\quad 0.00989$ DF

Upper CL Dif 0.03816 Prob $>|t| \quad 0.3405$ Lower CL Dif -0.01677 Prob $>\mathrm{t} \quad 0.1703$

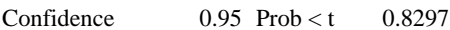

Glass ID=SB4VAR21, Analyte=log[Na ppm]

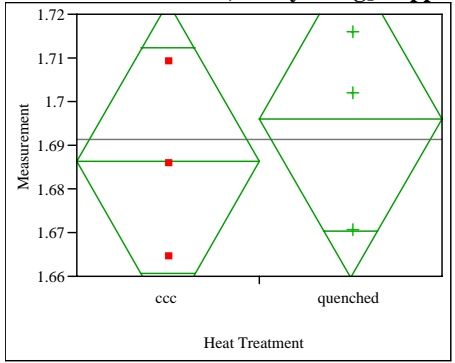

Difference $\quad 0.00979$ t Ratio 0.525625 Std Err Dif $\quad 0.01862$ DF

Upper CL Dif 0.06147 Prob $>|t| \quad 0.6269$ Lower CL Dif -0.04190 Prob $>t \quad 0.3135$ $\begin{array}{lrl}\text { Confidence } & 0.95 \text { Prob }<\mathrm{t} & 0.6865\end{array}$

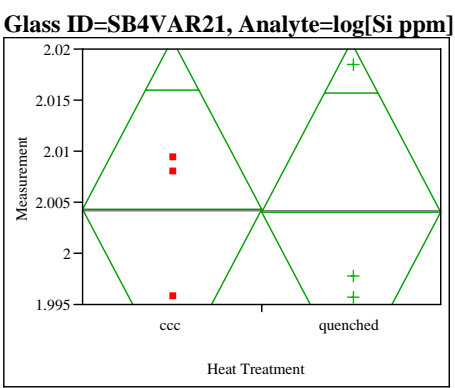

Difference $\quad-0.00032$ t Ratio $\quad-0.03756$ $\begin{array}{llll}\text { Std Err Dif } \quad 0.00846 \text { DF } & 4\end{array}$ Upper CL Dif 0.02316 Prob $>|t| \quad 0.9718$ Lower CL Dif -0.02380 Prob $>t \quad 0.5141$ Confidence $\quad 0.95$ Prob $<\mathrm{t} \quad 0.4859$

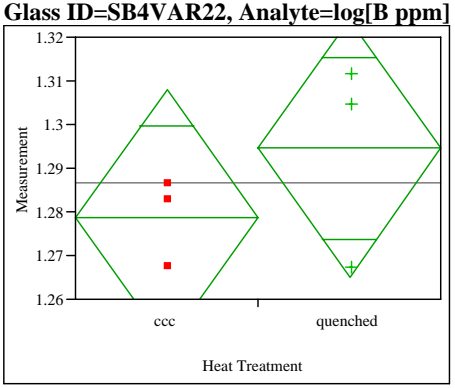

Difference $\quad 0.01584$ t Ratio 1.055237 Std Err Dif $\quad 0.01502$ DF

Upper CL Dif 0.05753 Prob $>|\mathrm{t}| \quad 0.3508$ Lower CL Dif -0.02584 Prob $>\mathrm{t} \quad 0.1754$

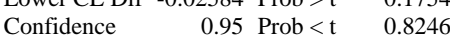

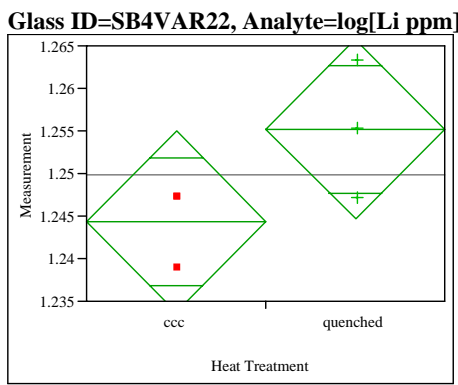

Difference $\quad 0.01083$ t Ratio 2.004426 Std Err Dif 0.00540 DF 4 Upper CL Dif 0.02582 Prob $>|t| 0.1155$ Lower CL Dif -0.00417 Prob $>\mathrm{t} \quad 0.0578$ Confidence $\quad 0.95$ Prob $<\mathrm{t} \quad 0.9422$ 
Exhibit B6. Effects of Heat Treatment on PCT $\log (\mathrm{ppm})-$ Response of Study Glasses

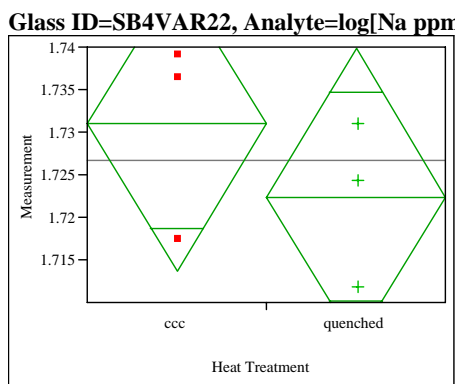

Difference $\quad-0.00857$ t Ratio $\quad-0.96838$ Std Err Dif $\quad 0.00885$ DF Upper CL Dif 0.01599 Prob $>|t| \quad 0.3877$ Lower CL Dif -0.03313 Prob $>\mathrm{t} \quad 0.8062$

$\begin{array}{lll}\text { Confidence } & 0.95 \text { Prob }<\mathrm{t} \quad 0.1938\end{array}$

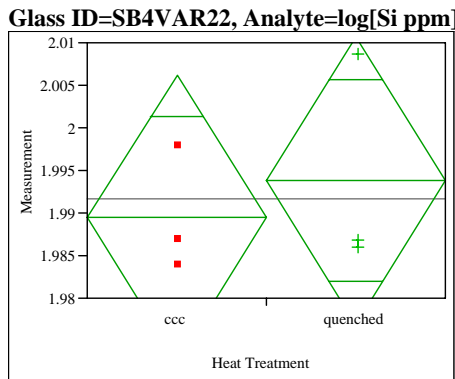

Difference $\quad 0.00434$ t Ratio 0.507958 $\begin{array}{lrlr}\text { Std Err Dif } & 0.00855 & \text { DF } & 4 \\ \text { Upper CL Dif } & 0.02807 & \text { Prob }>|t| & 0.6382\end{array}$ $\begin{array}{rrr}\text { Upper CL Dif } & 0.02807 \text { Prob }>|t| & 0.6382\end{array}$ $\begin{array}{lll}\text { Confidence } & 0.95 \text { Prob }<\mathrm{t} \quad 0.6809\end{array}$

Glass ID=SB4VAR23, Analyte $=\log [B$ ppm $]$

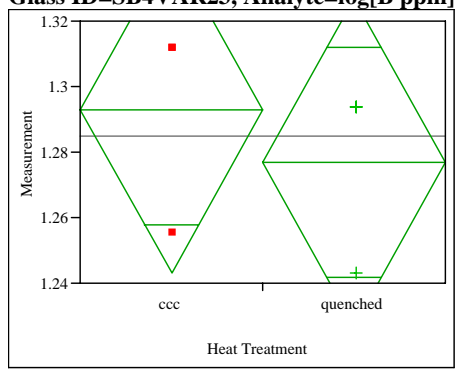

Difference $\quad-0.01609$ t Ratio $\quad-0.63616$ Std Err Dif $\quad 0.02530$ DF

Upper CL Dif 0.05414 Prob $>|t| 0.5593$

Lower CL Dif -0.08633 Prob $>\mathrm{t} \quad 0.7204$

$\begin{array}{lll}\text { Confidence } & 0.95 \text { Prob }<\mathrm{t} & 0.2796\end{array}$

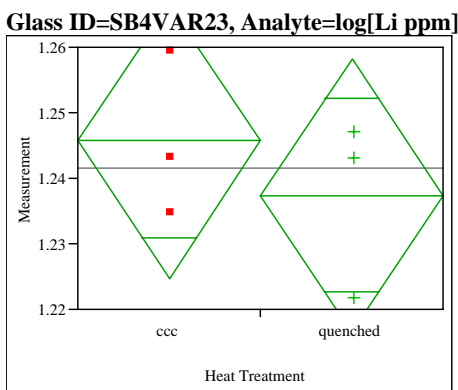

Difference $\quad-0.00832$ t Ratio $\quad-0.78063$ Std Err Dif $\quad 0.01066$ DF

Upper CL Dif 0.02127 Prob $>|\mathrm{t}| \quad 0.4786$

Lower CL Dif -0.03791 Prob $>\mathrm{t} \quad 0.7607$

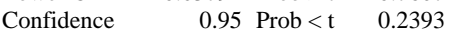

Glass ID=SB4VAR23, Analyte $=\log [\mathrm{Na}$ ppm]

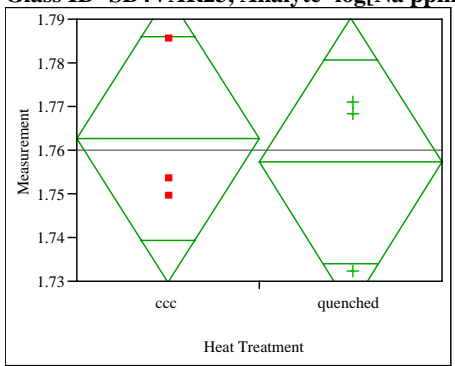

Difference $\quad-0.00550$ t Ratio $\quad-0.32656$ Std Err Dif $\quad 0.01684$ DF

Upper CL Dif 0.04126 Prob $>|t| \quad 0.7604$

Lower CL Dif -0.05226 Prob $>t \quad 0.6198$

Confidence $\quad 0.95$ Prob $<\mathrm{t} \quad 0.3802$

Glass ID=SB4VAR23, Analyte $=\log [S i$ ppm $]$

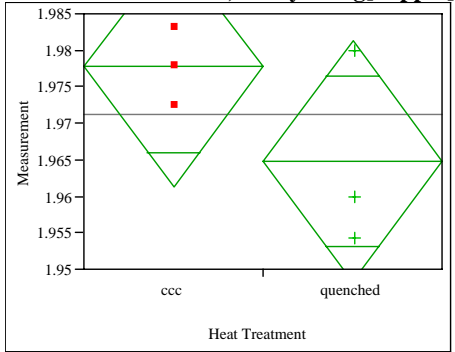

Difference $\quad-0.01301$ t Ratio $\quad-1.54718$ Std Err Dif $\quad 0.00841$ DF

Upper CL Dif 0.01033 Prob $>|t| \quad 0.1967$

Lower CL Dif -0.03635 Prob $>t \quad 0.9016$

Confidence $\quad 0.95$ Prob $<\mathrm{t} \quad 0.0984$

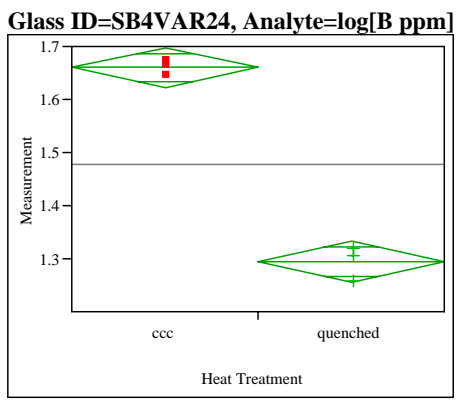

Difference $\quad-0.36576$ t Ratio -18.6495 Std Err Dif 0.01961 DF 4

Upper CL Dif -0.31131 Prob $>|t|<.0001$ Lower CL Dif -0.42021 Prob $>\mathrm{t} \quad 1.0000$

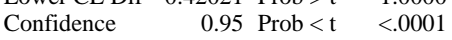

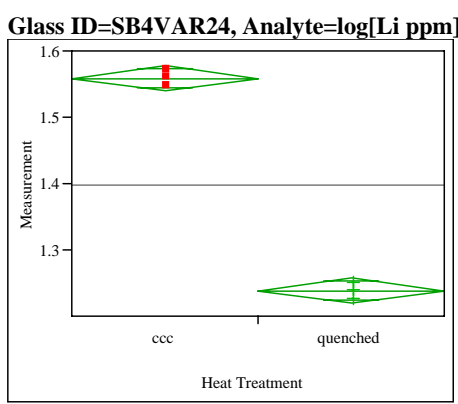

Difference $\quad-0.32010$ t Ratio $\quad-31.8581$ Std Err Dif $\quad 0.01005$ DF 4 Upper CL Dif -0.29220 Prob $>|\mathrm{t}|<.0001$ Lower CL Dif -0.34800 Prob $>t \quad 1.0000$ $\begin{array}{llll} & \text { Confidence } & 0.95 \text { Prob }<\mathrm{t} & <.0001\end{array}$

Glass ID=SB4VAR24, Analyte $=\log [\mathrm{Na}$ ppm $]$

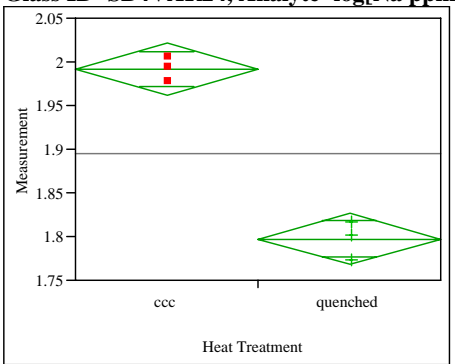

Difference $\quad-0.19452$ t Ratio $\quad-13.0505$ Std Err Dif $\quad 0.01490$ DF 4 Upper CL Dif -0.15313 Prob $>|t| 0.0002$ Lower CL Dif -0.23590 Prob $>t \quad 0.9999$ Confidence $\quad 0.95$ Prob $<\mathrm{t}<.0001$ 
Exhibit B6. Effects of Heat Treatment on PCT $\log (\mathrm{ppm})-$ Response of Study Glasses

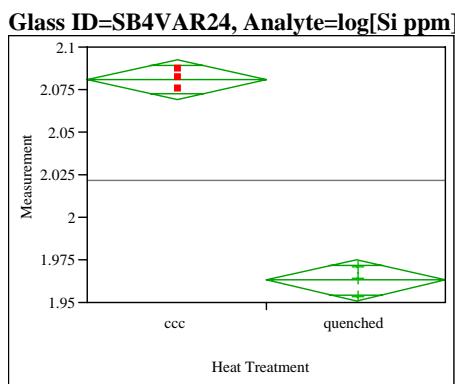

Difference $\quad-0.11802$ t Ratio $\quad-19.4577$ Std Err Dif $\quad 0.00607$ DF Upper CL Dif -0.10118 Prob $>|t|<.0001$ Lower CL Dif -0.13486 Prob $>\mathrm{t} \quad 1.0000$ Confidence $\quad 0.95$ Prob $<\mathrm{t} \quad<.0001$

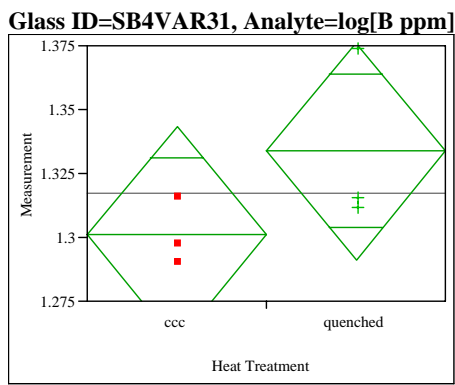

Difference $\quad 0.03282$ t Ratio 1.521106 $\begin{array}{lrlr}\text { Std Err Dif } & 0.02158 & \text { DF } & 4 \\ \text { Upper CL Dif } & 0.09273 & \text { Prob }>|t| & 0.2029 \\ \text { Lower CL Dif } & -0.02709 & \text { Prob }>t & 0.1014\end{array}$ $\begin{array}{lll}\text { Confidence } & 0.95 \text { Prob }<\mathrm{t} \quad 0.8986\end{array}$

Glass ID=SB4VAR31, Analyte=log[Li ppm]

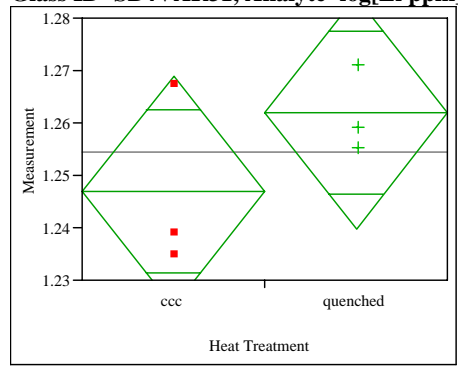

Difference $\quad 0.01496$ t Ratio 1.329729 Std Err Dif 0.01125 DF

Upper CL Dif 0.04619 Prob $>|t| \quad 0.2544$

Lower CL Dif - 0.01627 Prob $>t \quad 0.1272$

$\begin{array}{lll}\text { Confidence } & 0.95 \text { Prob }<\mathrm{t} & 0.8728\end{array}$
Glass ID=SB4VAR31, Analyte=log[Na ppm]

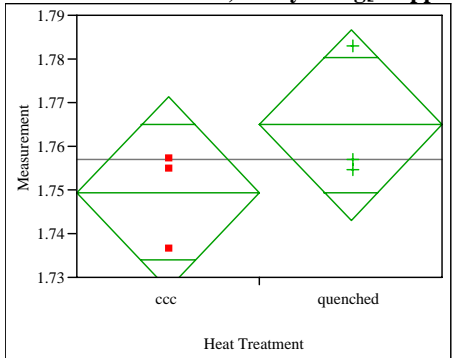

Difference $\quad 0.01552$ t Ratio 1.389704 Std Err Dif $\quad 0.01117$ DF

Upper CL Dif 0.04652 Prob $>|t| \quad 0.2370$

Lower CL Dif -0.01548 Prob $>\mathrm{t} \quad 0.1185$

$\begin{array}{lll}\text { Confidence } & 0.95 \text { Prob }<\mathrm{t} \quad 0.8815\end{array}$

Glass ID=SB4VAR31, Analyte=log[Si ppm]

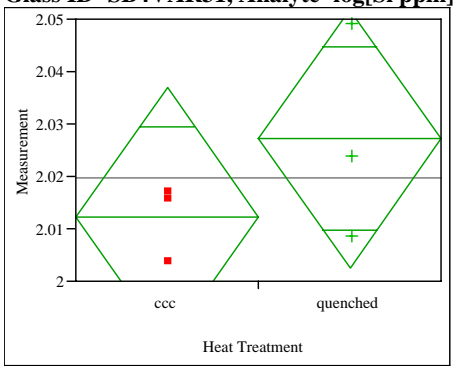

Difference $\quad 0.01516$ t Ratio $\quad 1.204516$ Std Err Dif $\quad 0.01259$ DF

Upper CL Dif 0.05010 Prob $>|\mathrm{t}| \quad 0.2948$ Lower CL Dif -0.01978 Prob $>t \quad 0.1474$

$\begin{array}{lll}\text { Confidence } & 0.95 \text { Prob }<\mathrm{t} & 0.8526\end{array}$

Glass ID=SB4VAR32, Analyte=log[B ppm]

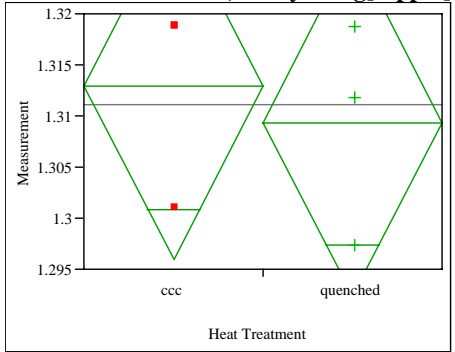

Difference $\quad-0.00355$ t Ratio $\quad-0.41099$ Std Err Dif $\quad 0.00863$ DF

Upper CL Dif 0.02041 Prob $>|t| \quad 0.7021$

Lower CL Dif -0.02750 Prob $>t \quad 0.6489$

$\begin{array}{lll}\text { Confidence } & 0.95 \text { Prob }<t \quad 0.3511\end{array}$
Glass ID=SB4VAR32, Analyte=log[Li ppm $]$

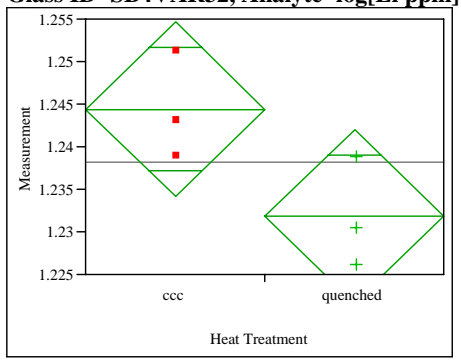

Difference $\quad-0.01255$ t Ratio $\quad-2.41045$ Std Err Dif $\quad 0.00521$ DF 4 Upper CL Dif 0.00191 Prob $>|t| \quad 0.0735$ Lower CL Dif -0.02701 Prob $>\mathrm{t} \quad 0.9632$

Confidence $\quad 0.95$ Prob $<\mathrm{t} \quad 0.0368$

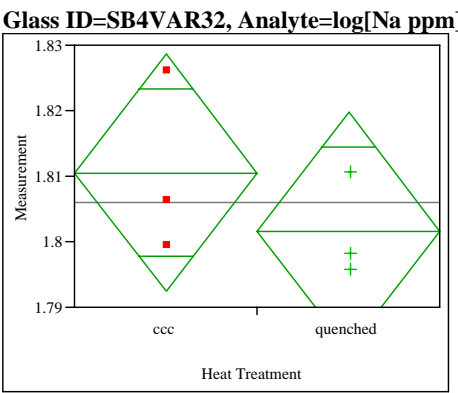

Difference $\quad-0.00895$ t Ratio $\quad-0.96814$ Std Err Dif 0.00924 DF 4 Upper CL Dif 0.01671 Prob $>|t| 0.3878$ Lower CL Dif -0.03461 Prob $>t \quad 0.8061$ $\begin{array}{lll}\text { Confidence } & 0.95 \text { Prob }<\mathrm{t} & 0.1939\end{array}$

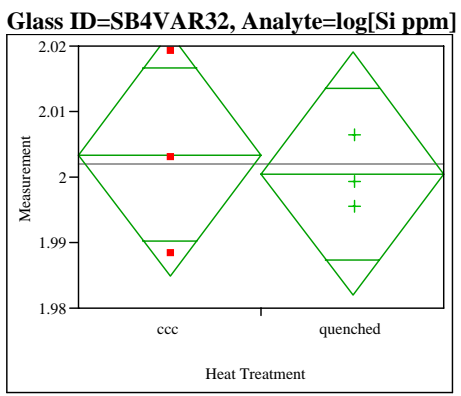

$\begin{array}{lrlr}\text { Difference } & -0.00296 & \text { t Ratio } & -0.31306 \\ \text { Std Err Dif } & 0.00946 \text { DF } & 4 \\ \text { Upper CL Dif } & 0.02331 \text { Prob }>|t| & 0.7699 \\ \text { Lower CL Dif } & -0.02923 \text { Prob }>\text { t } & 0.6151 \\ \text { Confidence } & 0.95 \text { Prob }<\mathrm{t} & 0.3849\end{array}$


Exhibit B6. Effects of Heat Treatment on PCT $\log (\mathrm{ppm})-$ Response of Study Glasses

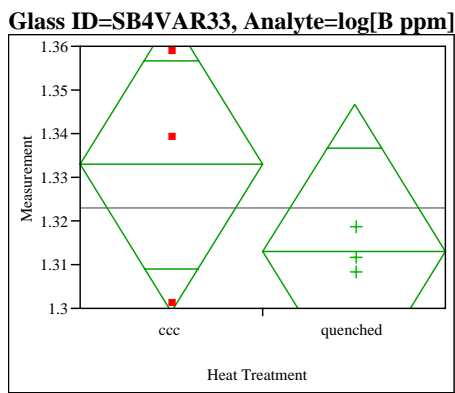

Difference $\quad-0.02000$ t Ratio $\quad-1.1641$ Std Err Dif 0.01718 DF

Upper CL Dif 0.02770 Prob $>|\mathrm{t}| \quad 0.3091$

Lower CL Dif -0.06770 Prob $>t \quad 0.8455$

Confidence $\quad 0.95$ Prob $<\mathrm{t} \quad 0.1545$

Glass ID=SB4VAR33, Analyte= $=\log [\mathrm{Li}$ ppm $]$

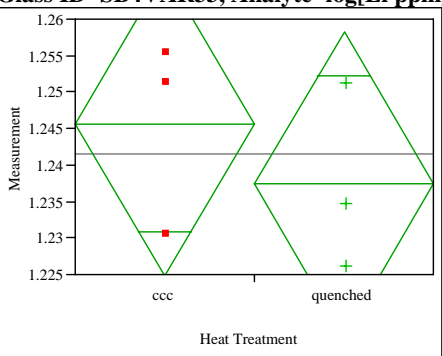

Difference $\quad-0.00829$ t Ratio $\quad-0.77877$ Std Err Dif $\quad 0.01064$ DF

Upper CL Dif 0.02126 Prob $>|t| \quad 0.4796$

Lower CL Dif -0.03784 Prob $>\mathrm{t} \quad 0.7602$

$\begin{array}{lll}\text { Confidence } & 0.95 \text { Prob }<\mathrm{t} & 0.2398\end{array}$

Glass ID=SB4VAR33, Analyte=log[Na ppm]

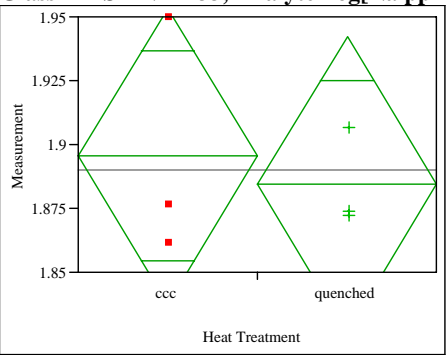

Difference $\quad-0.01127$ t Ratio $\quad-0.38263$ Std Err Dif $\quad 0.02945$ DF 4

Upper CL Dif 0.07050 Prob $>|t| 0.7215$

Lower CL Dif -0.09303 Prob $>\mathrm{t} \quad 0.6393$

Confidence

0.95 Prob $<\mathrm{t} \quad 0.3607$
Glass ID=SB4VAR33, Analyte $=\log [S i$ ppm $]$

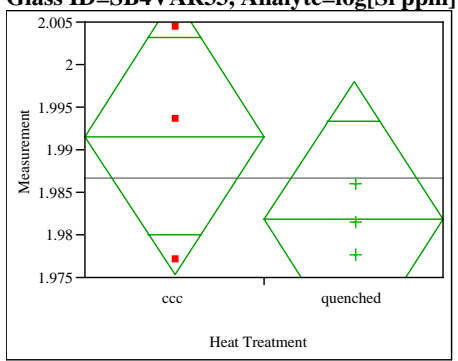

Difference $\quad-0.00982$ t Ratio $\quad-1.18187$ Std Err Dif $\quad 0.00831$ DF

Upper CL Dif 0.01325 Prob $>|t| \quad 0.3027$

Lower CL Dif -0.03288 Prob $>\mathrm{t} \quad 0.8486$

$\begin{array}{lll}\text { Confidence } & 0.95 \text { Prob }<\mathrm{t} \quad 0.1514\end{array}$

Glass ID=SB4VAR41, Analyte=log[B ppm $]$

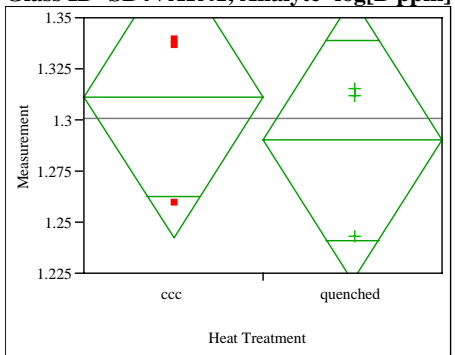

Difference $\quad-0.02138$ t Ratio -0.60875 Std Err Dif $\quad 0.03511$ DF 4 Upper CL Dif 0.07611 Prob $>|t| \quad 0.5756$ Lower CL Dif -0.11886 Prob $>\mathrm{t} \quad 0.7122$

Confidence $\quad 0.95$ Prob $<\mathrm{t} \quad 0.2878$

Glass ID=SB4VAR41, Analyte $=\log [\mathrm{Li}$ ppm $]$

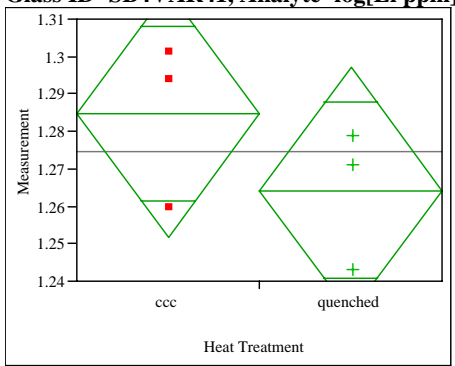

Difference $\quad-0.02039$ t Ratio $\quad-1.211$

Std Err Dif $\quad 0.01684$ DF 4

Upper CL Dif 0.02636 Prob $>|t| 0.2926$

Lower CL Dif -0.06715 Prob $>\mathrm{t} \quad 0.8537$

Confidence $\quad 0.95$ Prob $<\mathrm{t} \quad 0.1463$

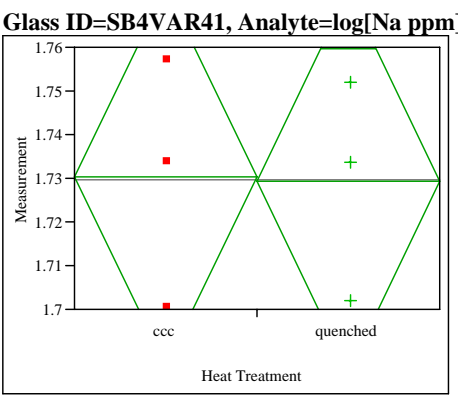

Difference $\quad-0.00122$ t Ratio $\quad-0.05525$ Std Err Dif $\quad 0.02204$ DF 4 Upper CL Dif 0.05999 Prob $>|t| 0.9586$ Lower CL Dif -0.06242 Prob $>\mathrm{t} \quad 0.5207$ $\begin{array}{lll}\text { Confidence } & 0.95 \text { Prob }<\mathrm{t} \quad 0.4793\end{array}$

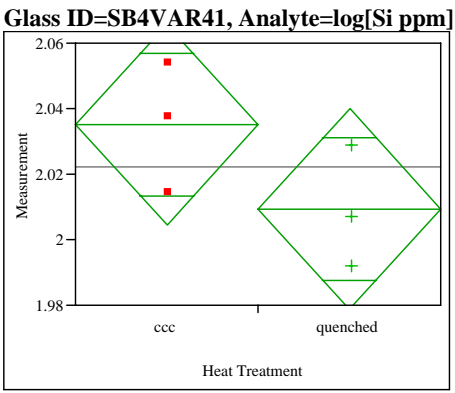

Difference $\quad-0.02585$ t Ratio $\quad-1.65185$ Std Err Dif $\quad 0.01565$ DF 4 Upper CL Dif 0.01760 Prob $>|t| \quad 0.1739$ Lower CL Dif -0.06929 Prob $>\mathrm{t} \quad 0.9130$ Confidence $\quad 0.95$ Prob $<\mathrm{t} \quad 0.0870$

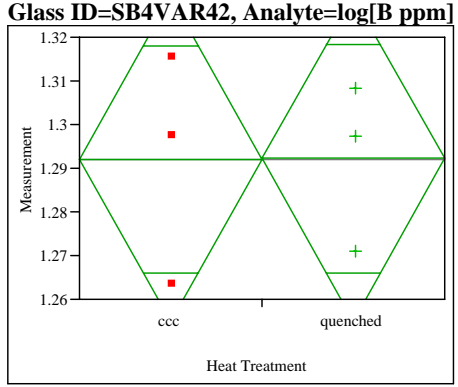

Difference $\quad 0.00025$ t Ratio 0.013515 Std Err Dif 0.01883 DF 4

Upper CL Dif 0.05254 Prob $>|t| 0.9899$ Lower CL Dif -0.05203 Prob $>t \quad 0.4949$ Confidence $\quad 0.95$ Prob $<\mathrm{t} \quad 0.5051$ 
Exhibit B6. Effects of Heat Treatment on PCT $\log (\mathrm{ppm})-$ Response of Study Glasses

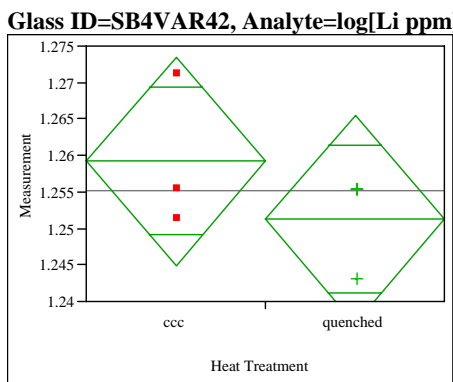

Difference $\quad-0.00800$ t Ratio $\quad-1.09576$ Std Err Dif $\quad 0.00730$ DF Upper CL Dif 0.01226 Prob $>|t| \quad 0.3347$ Lower CL Dif -0.02826 Prob $>\mathrm{t} \quad 0.8326$

Confidence $\quad 0.95$ Prob $<\mathrm{t} \quad 0.1674$

Glass ID=SB4VAR42, Analyte $=\log [\mathrm{Na} p \mathrm{pm}]$

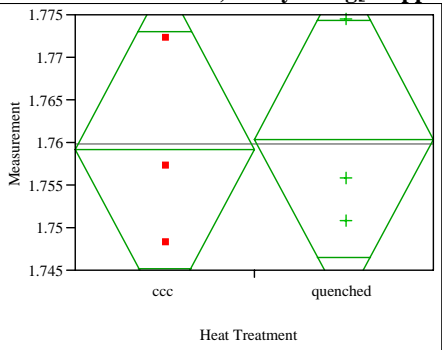

Difference $\quad 0.00125$ t Ratio $\quad 0.124572$ $\begin{array}{lrlr}\text { Std Err Dif } & 0.01003 & \text { DF } & 4 \\ \text { Upper CL Dif } & 0.02910 & \text { Prob }>|t| & 0.9069\end{array}$ Lower CL Dif -0.02660 Prob $>t \quad 0.4534$ Confidence $\quad 0.95$ Prob $<\mathrm{t} \quad 0.5466$

Glass ID=SB4VAR42, Analyte=log[Si ppm

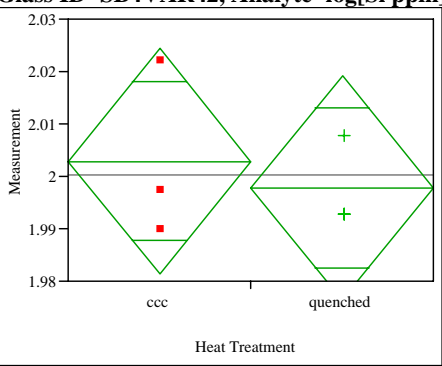

Difference $\quad-0.00514$ t Ratio -0.46923 Std Err Dif 0.01096 DF

Upper CL Dif 0.02529 Prob $>|t| 0.6633$

Lower CL Dif -0.03557 Prob $>\mathrm{t} \quad 0.6683$

$\begin{array}{lll}\text { Confidence } & 0.95 \text { Prob }<\mathrm{t} & 0.3317\end{array}$
Glass ID=SB4VAR43, Analyte=log[B ppm $]$

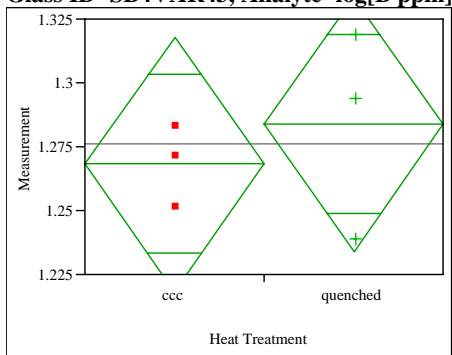

Difference $\quad 0.01551$ t Ratio 0.613009 Std Err Dif $\quad 0.02530$ DF

Upper CL Dif 0.08575 Prob $>|t| \quad 0.5730$ Lower CL Dif -0.05473 Prob $>t \quad 0.2865$

$\begin{array}{lll}\text { Confidence } & 0.95 \text { Prob }<\mathrm{t} & 0.7135\end{array}$

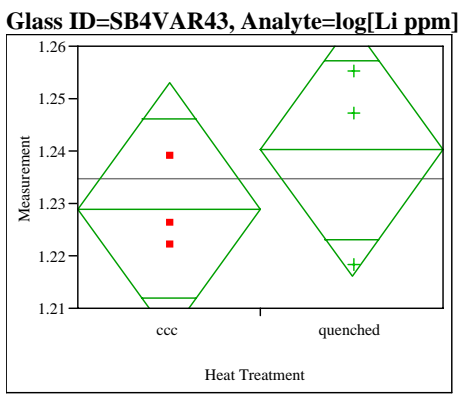

Difference $\quad 0.01130$ t Ratio 0.917546 Std Err Dif $\quad 0.01231$ DF

Upper CL Dif 0.04548 Prob $>|t| 0.4108$ Lower CL Dif -0.02288 Prob $>\mathrm{t} \quad 0.2054$ $\begin{array}{lrl}\text { Confidence } & 0.95 \text { Prob }<\mathrm{t} & 0.7946\end{array}$

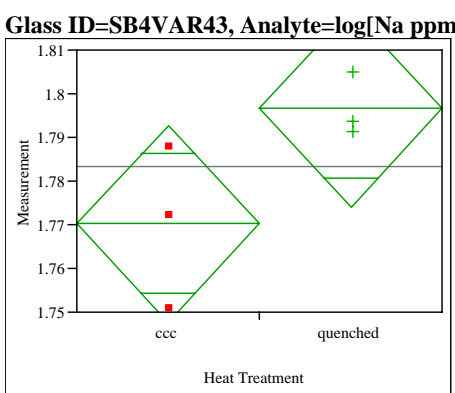

Difference $\quad 0.02643$ t Ratio 2.293333 Std Err Dif $\quad 0.01152$ DF

Upper CL Dif 0.05843 Prob $>|t| \quad 0.0835$

Lower CL Dif -0.00557 Prob $>t \quad 0.0418$

$\begin{array}{lll}\text { Confidence } & 0.95 \text { Prob }<\mathrm{t} \quad 0.9582\end{array}$

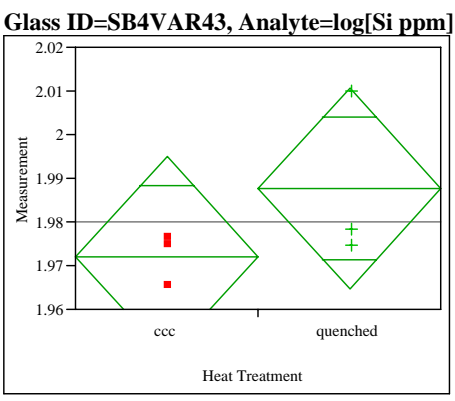

Difference $\quad 0.01565$ t Ratio $\quad 1.337219$ Std Err Dif $\quad 0.01170$ DF

Upper CL Dif 0.04814 Prob $>|t| \quad 0.2521$

Lower CL Dif -0.01684 Prob $>t \quad 0.1261$

$\begin{array}{lll}\text { Confidence } & 0.95 \text { Prob }<\mathrm{t} & 0.8739\end{array}$

Glass ID=SB4VAR44, Analyte $=\log [B$ ppm $]$

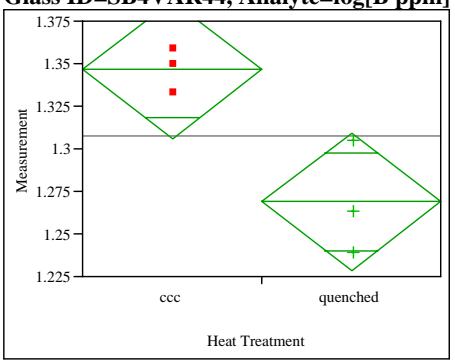

Difference $\quad-0.07773$ t Ratio $\quad-3.76378$ Std Err Dif $\quad 0.02065$ DF 4

Upper CL Dif -0.02039 Prob > $|\mathrm{t}| \quad 0.0197$

Lower CL Dif -0.13508 Prob $>t \quad 0.9901$

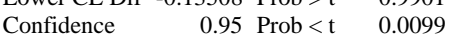

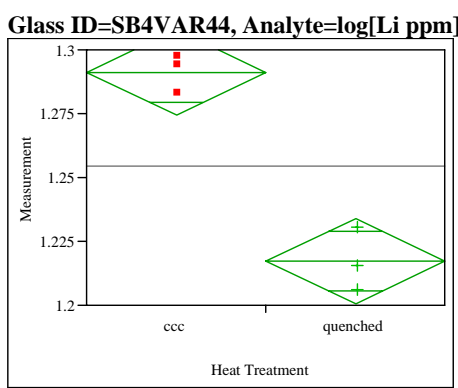

Difference $\quad-0.07400$ t Ratio $\quad-8.78293$ Std Err Dif 0.00843 DF 4

Upper CL Dif -0.05061 Prob $>|t| 0.0009$

Lower CL Dif -0.09740 Prob $>t \quad 0.9995$

Confidence $\quad 0.95$ Prob $<\mathrm{t} \quad 0.0005$ 
Exhibit B6. Effects of Heat Treatment on PCT $\log (\mathrm{ppm})-$ Response of Study Glasses

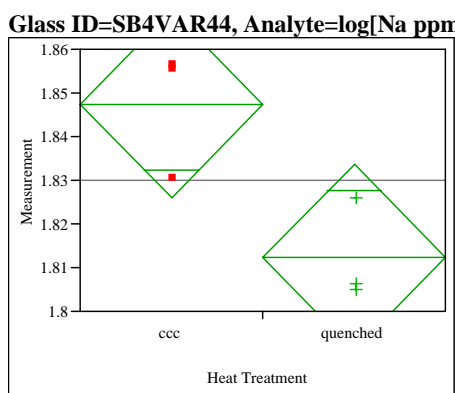

Difference $\quad-0.03491$ t Ratio $\quad-3.20437$ Std Err Dif $\quad 0.01089$ DF

Upper CL Dif -0.00466 Prob > $|\mathrm{t}| 0.0328$

Lower CL Dif -0.06515 Prob $>\mathrm{t} \quad 0.9836$

$\begin{array}{lll}\text { Confidence } & 0.95 \text { Prob }<\mathrm{t} \quad 0.0164\end{array}$

Glass ID=SB4VAR44, Analyte=log[Si ppm $]$

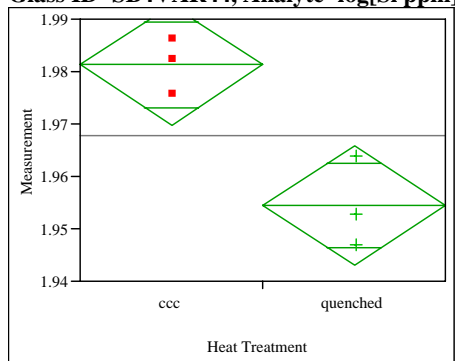

Difference $\quad-0.02679$ t Ratio $\quad-4.58805$ Std Err Dif $\quad 0.00584$ DF

Upper CL Dif -0.01058 Prob $>|t| \quad 0.0101$

Lower CL Dif -0.04300 Prob $>t \quad 0.9949$

Confidence $\quad 0.95$ Prob $<t \quad 0.0051$

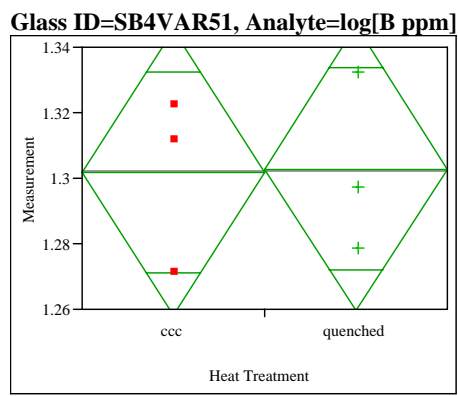

Difference 0.00118 t Ratio 0.053367 Std Err Dif 0.02216 DF 4

Upper CL Dif 0.06271 Prob $>|t| \quad 0.9600$ Lower CL Dif -0.06034 Prob $>t \quad 0.4800$ $\begin{array}{lrl}\text { Confidence } & 0.95 \text { Prob }<\mathrm{t} & 0.5200\end{array}$

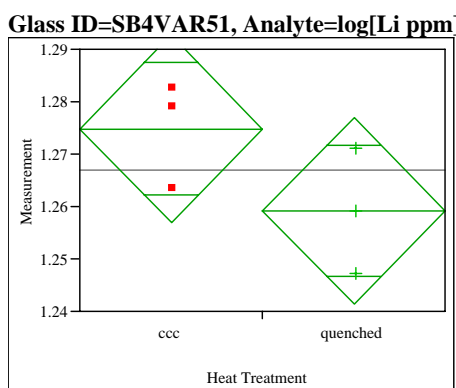

Difference $\quad-0.01568$ t Ratio -1.72624 Std Err Dif 0.00908 DF

Upper CL Dif 0.00954 Prob $>|t| \quad 0.1594$

Lower CL Dif -0.04090 Prob $>t \quad 0.9203$

$\begin{array}{lll}\text { Confidence } & 0.95 \text { Prob }<\mathrm{t} & 0.0797\end{array}$

Glass ID=SB4VAR51, Analyte $=\log [\mathrm{Na}$ ppm]

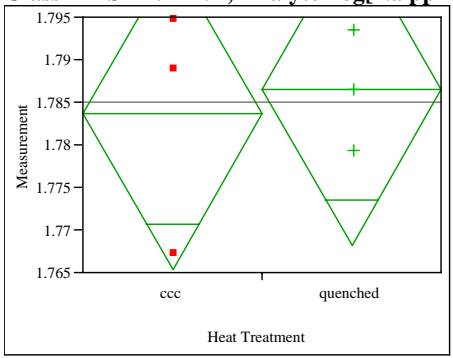

Difference $\quad 0.00289$ t Ratio 0.309871 Std Err Dif $\quad 0.00933$ DF

Upper CL Dif 0.02881 Prob $>|\mathrm{t}| \quad 0.7721$

Lower CL Dif -0.02302 Prob $>t \quad 0.3861$

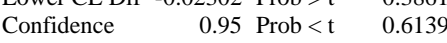

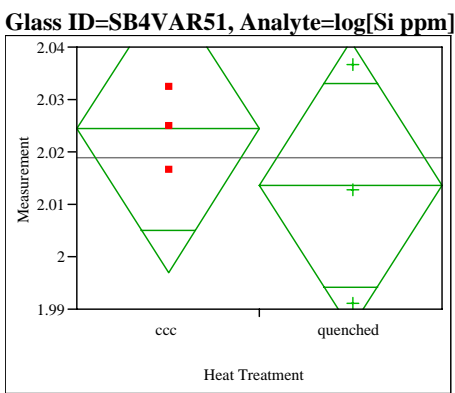

Difference $\quad-0.01074$ t Ratio $\quad-0.77174$ Std Err Dif $\quad 0.01391$ DF

Upper CL Dif $0.02789 \operatorname{Prob}>|\mathrm{t}| \quad 0.4833$

Lower CL Dif - 0.04937 Prob $>t \quad 0.7583$

Confidence $\quad 0.95$ Prob $<\mathrm{t} \quad 0.2417$

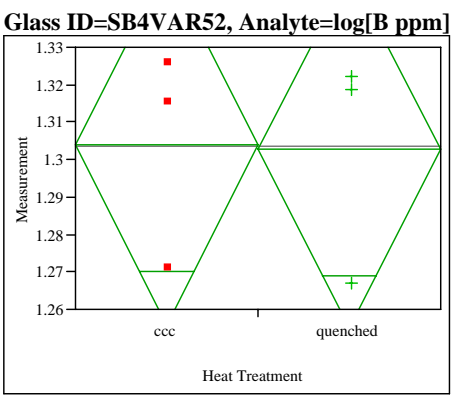

Difference $\quad-0.00128$ t Ratio $\quad-0.05239$ Std Err Dif 0.02443 DF 4 Upper CL Dif 0.06655 Prob $>|t| \quad 0.9607$ Lower CL Dif -0.06911 Prob >t 0.5196 $\begin{array}{lll}\text { Confidence } & 0.95 \text { Prob }<\mathrm{t} \quad 0.4804\end{array}$

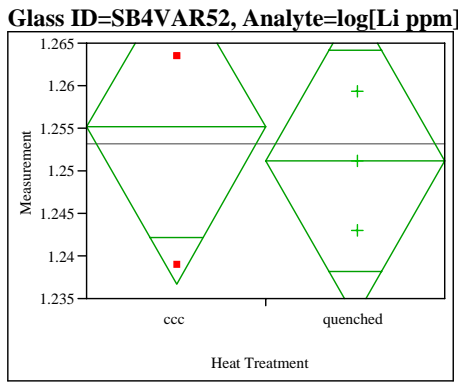

Difference $\quad-0.00394$ t Ratio $\quad-0.42021$ Std Err Dif 0.00938 DF 4 Upper CL Dif 0.02209 Prob $>|t| \quad 0.6959$ Lower CL Dif -0.02997 Prob $>t \quad 0.6520$ $\begin{array}{lll}\text { Confidence } & 0.95 \text { Prob }<\mathrm{t} & 0.3480\end{array}$

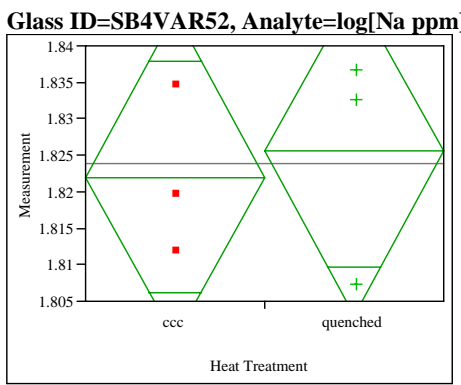

Difference $\quad 0.00353$ t Ratio $\quad 0.310347$ Std Err Dif $\quad 0.01137$ DF Upper CL Dif 0.03511 Prob $>|t| 0.7718$ Lower CL Dif -0.02805 Prob $>\mathrm{t} \quad 0.3859$ $\begin{array}{lll}\text { Confidence } & 0.95 \text { Prob }<\mathrm{t} & 0.6141\end{array}$ 
Exhibit B6. Effects of Heat Treatment on PCT $\log (\mathrm{ppm})-$ Response of Study Glasses

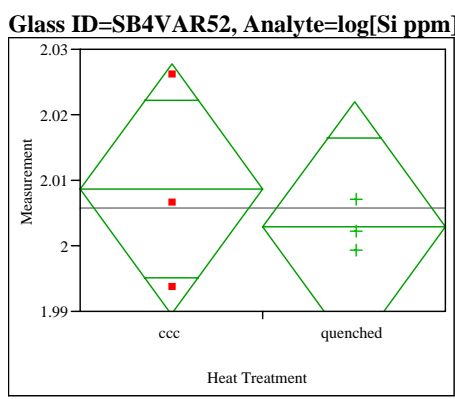

Difference $\quad-0.00576$ t Ratio $\quad-0.59124$ Std Err Dif 0.00974 DF

Upper CL Dif 0.02128 Prob $>|t| 0.5862$

Lower CL Dif -0.03279 Prob $>\mathrm{t} \quad 0.7069$

$\begin{array}{lll}\text { Confidence } & 0.95 \text { Prob }<\mathrm{t} \quad 0.2931\end{array}$

Glass ID=SB4VAR53, Analyte=log[B ppm $]$

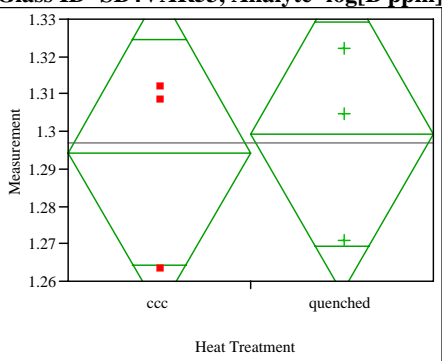

Difference $\quad 0.00491$ t Ratio 0.22653

Std Err Dif $\quad 0.02165$ DF 4

Upper CL Dif 0.06503 Prob $>|t| \quad 0.8319$

Lower CL Dif -0.05522 Prob $>t \quad 0.4159$

Confidence $\quad 0.95$ Prob $<\mathrm{t} \quad 0.5841$

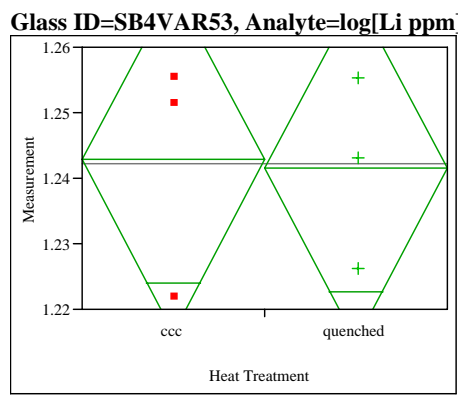

Difference $\quad-0.00129$ t Ratio -0.09567 Std Err Dif 0.01349 DF 4

Upper CL Dif 0.03618 Prob $>|t| 0.9284$

Lower CL Dif -0.03876 Prob $>t \quad 0.5358$

$\begin{array}{lrl}\text { Confidence } & 0.95 \text { Prob }<\mathrm{t} & 0.4642\end{array}$
Glass ID=SB4VAR53, Analyte=log[Na ppm]

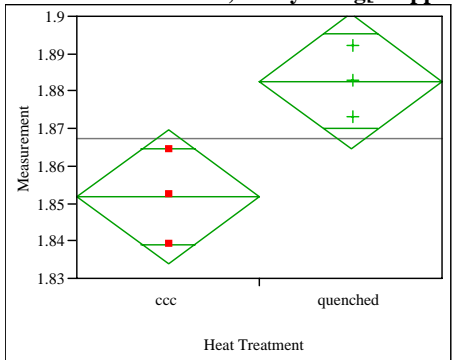

Difference $\quad 0.030832$ t Ratio 3.362631 Std Err Dif $\quad 0.009169$ DF

Upper CL Dif 0.056290 Prob $>|t| 0.0282$

Lower CL Dif 0.005375 Prob $>t \quad 0.0141$

$\begin{array}{lll}\text { Confidence } & 0.95 \text { Prob }<\mathrm{t} \quad 0.9859\end{array}$

Glass ID=SB4VAR53, Analyte $=\log [$ Si ppm $]$

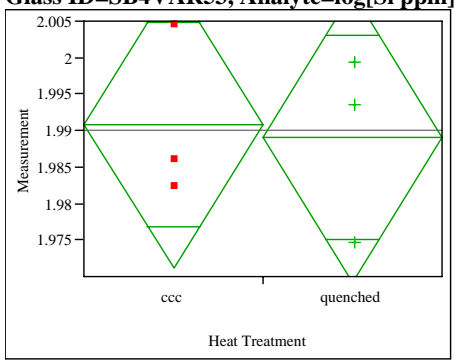

Difference $\quad-0.00175$ t Ratio -0.17338

Std Err Dif $\quad 0.01008$ DF

Upper CL Dif 0.02623 Prob $>|\mathrm{t}| \quad 0.8708$

Lower CL Dif -0.02972 Prob $>\mathrm{t} \quad 0.5646$

$\begin{array}{lrll} & \text { Confidence } & 0.95 \text { Prob }<\mathrm{t} & 0.4354\end{array}$

Glass ID=SB4VAR54, Analyte=log[B ppm $]$

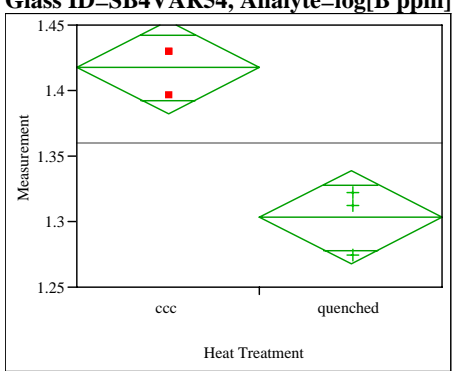

Difference $\quad-0.11449$ t Ratio -6.28923

Std Err Dif $\quad 0.01820$ DF

Upper CL Dif -0.06395 Prob $>|t| \quad 0.0033$

Lower CL Dif -0.16504 Prob $>t \quad 0.9984$

Confidence $\quad 0.95$ Prob $<\mathrm{t} \quad 0.0016$

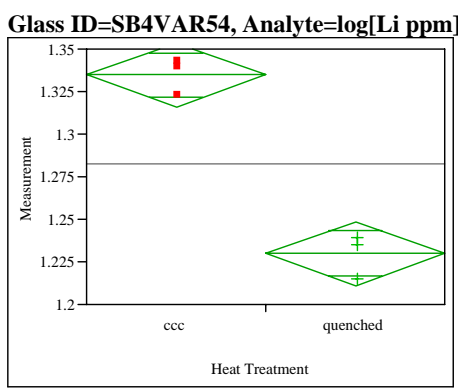

Difference $\quad-0.10497$ t Ratio $\quad-10.9453$ Std Err Dif $\quad 0.00959$ DF 4 Upper CL Dif -0.07834 Prob > |t| 0.0004 Lower CL Dif -0.13160 Prob $>t \quad 0.9998$ Confidence $\quad 0.95$ Prob $<\mathrm{t} \quad 0.0002$

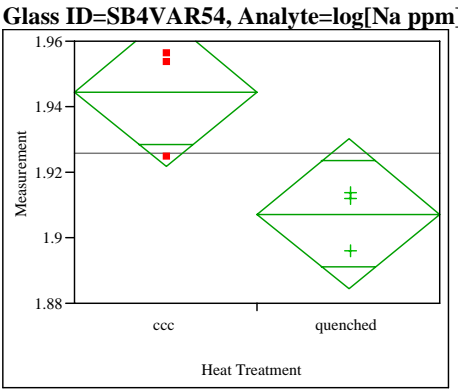

Difference $\quad-0.03731$ t Ratio $\quad-3.20101$ Std Err Dif 0.01165 DF 4 Upper CL Dif -0.00495 Prob > $|\mathrm{t}| \quad 0.0329$ Lower CL Dif -0.06966 Prob $>t \quad 0.9836$ $\begin{array}{lll}\text { Confidence } & 0.95 \text { Prob }<\mathrm{t} & 0.0164\end{array}$

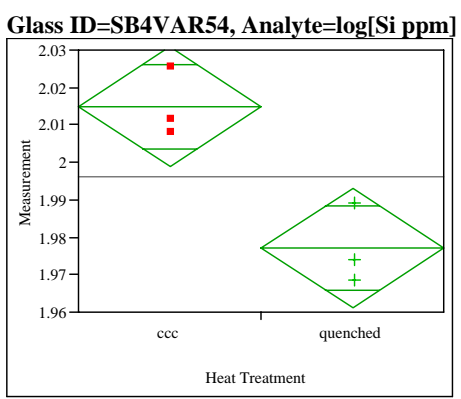

$\begin{array}{lrlr}\text { Difference } & -0.03775 & \text { t Ratio } & -4.64811 \\ \text { Std Err Dif } & 0.00812 & \text { DF } & 4 \\ \text { Upper CL Dif } & -0.01520 & \text { Prob }>|t| & 0.0097 \\ \text { Lower CL Dif } & -0.06029 & \text { Prob }>\text { t } & 0.9952 \\ \text { Confidence } & 0.95 & \text { Prob }<\mathrm{t} & 0.0048\end{array}$




\section{Exhibit B7. Effects of Heat Treatment for Study Glasses by Compositional View}

Sludge Case $=$ Case 1

Variability Chart for $\log$ NL[B (g/L)]

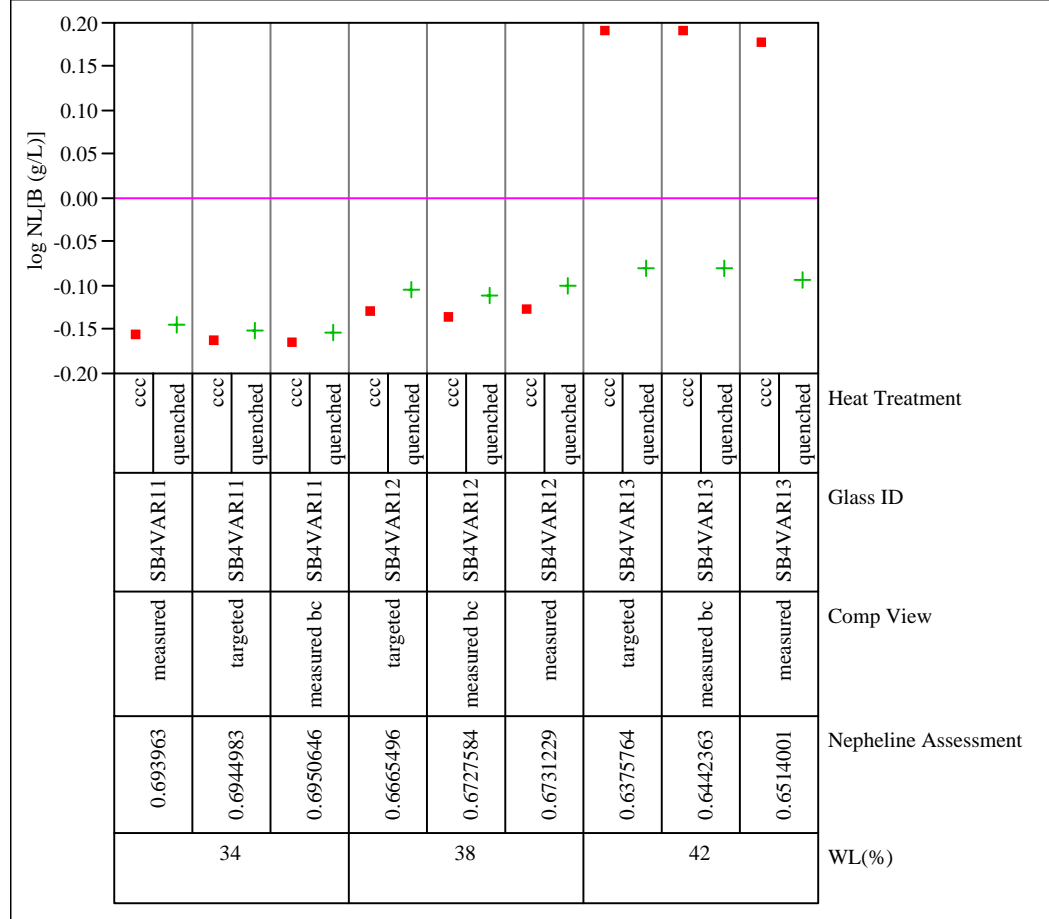

Sludge Case $=$ Case 1

Variability Chart for log NL[Li(g/L)]

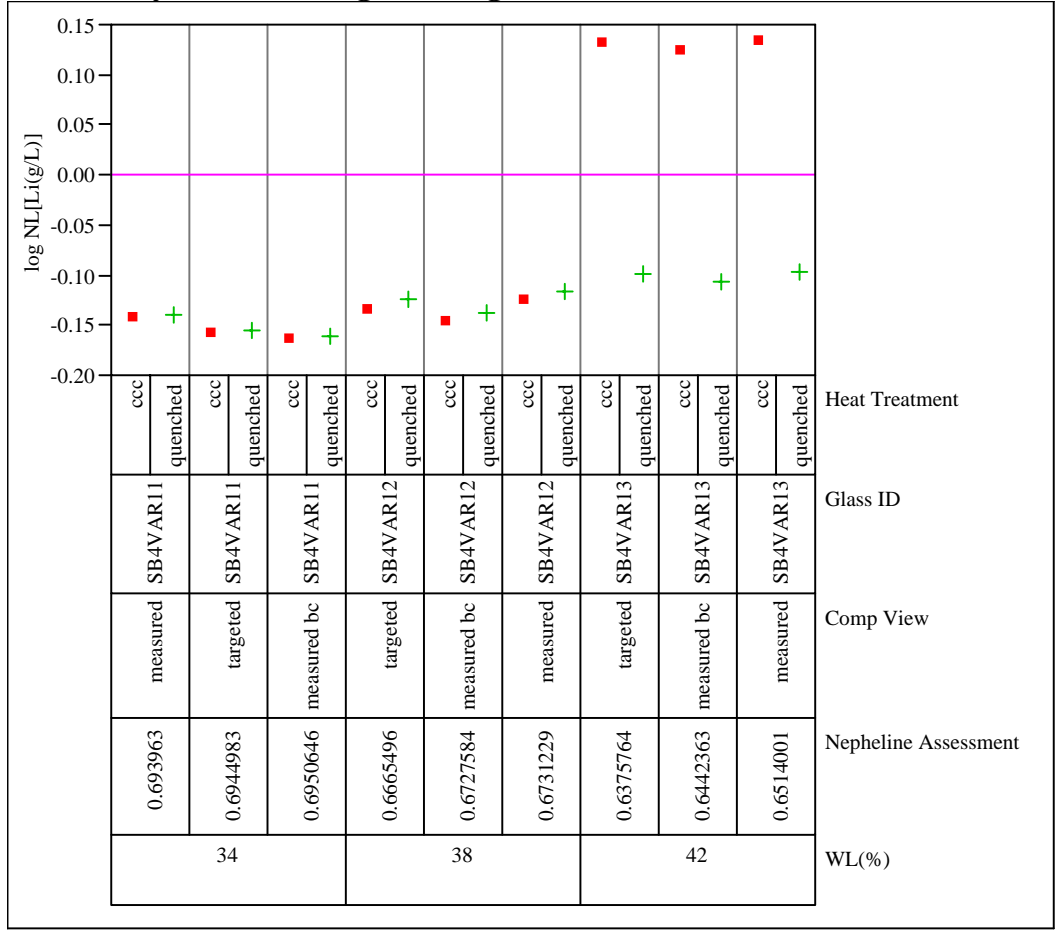




\section{Exhibit B7. Effects of Heat Treatment for Study Glasses by Compositional View}

Sludge Case $=$ Case 1

Variability Chart for $\log \mathrm{NL}[\mathrm{Na}(\mathrm{g} / \mathrm{L})]$

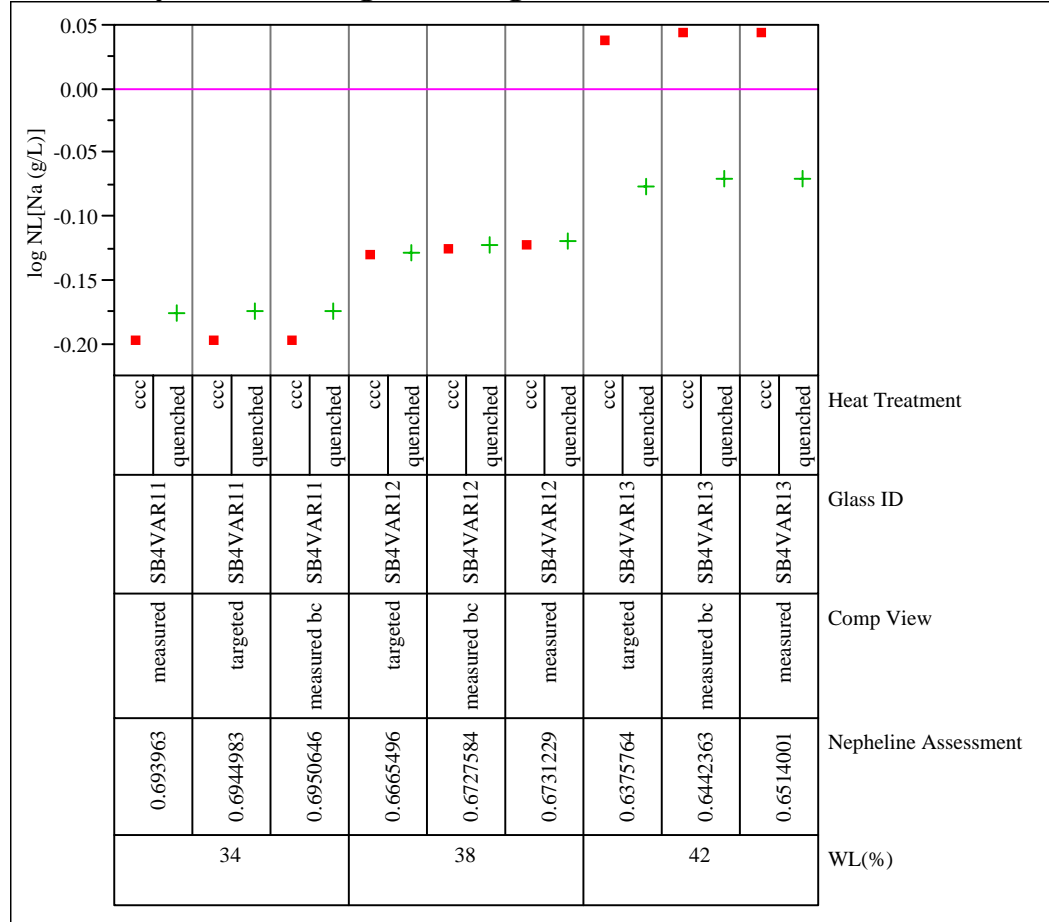

Sludge Case $=$ Case 1

Variability Chart for log NL[Si (g/L)]

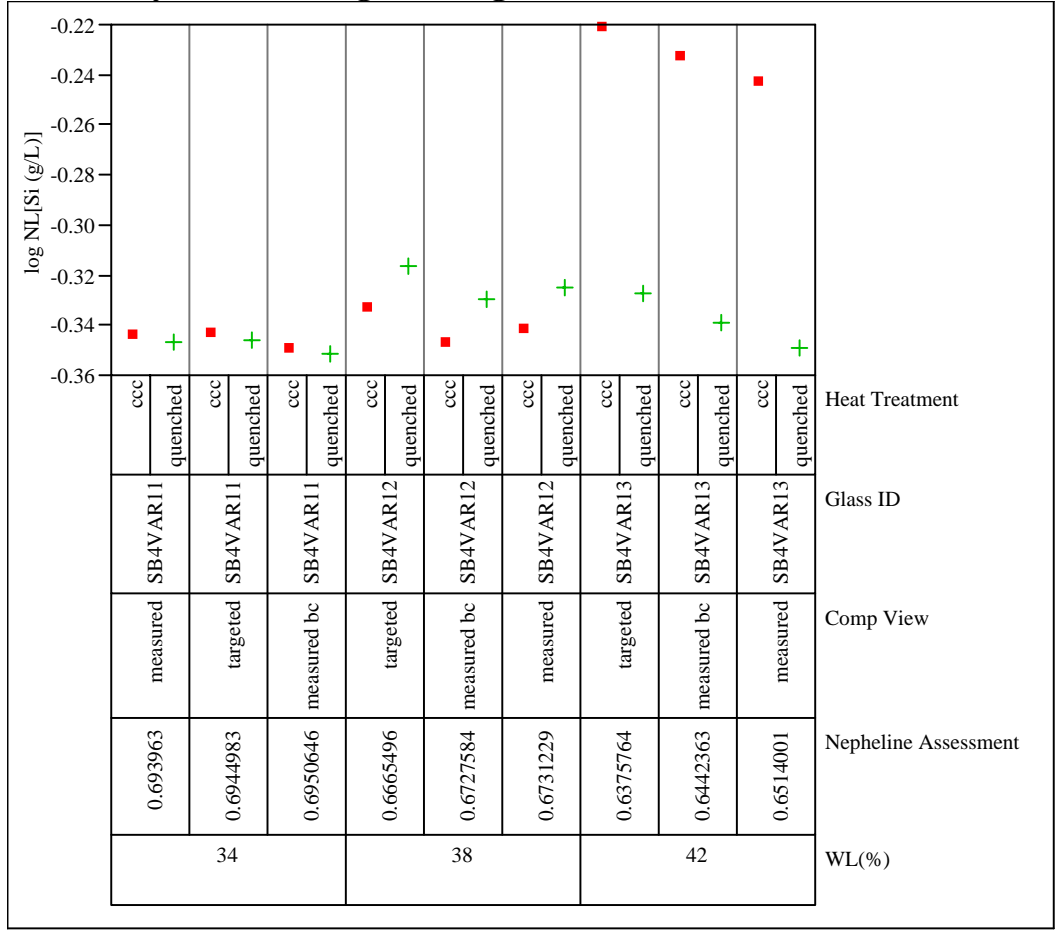




\section{Exhibit B7. Effects of Heat Treatment for Study Glasses by Compositional View}

Sludge Case $=$ Case 2

Variability Chart for $\log \mathrm{NL}[\mathrm{B}(\mathrm{g} / \mathrm{L})]$

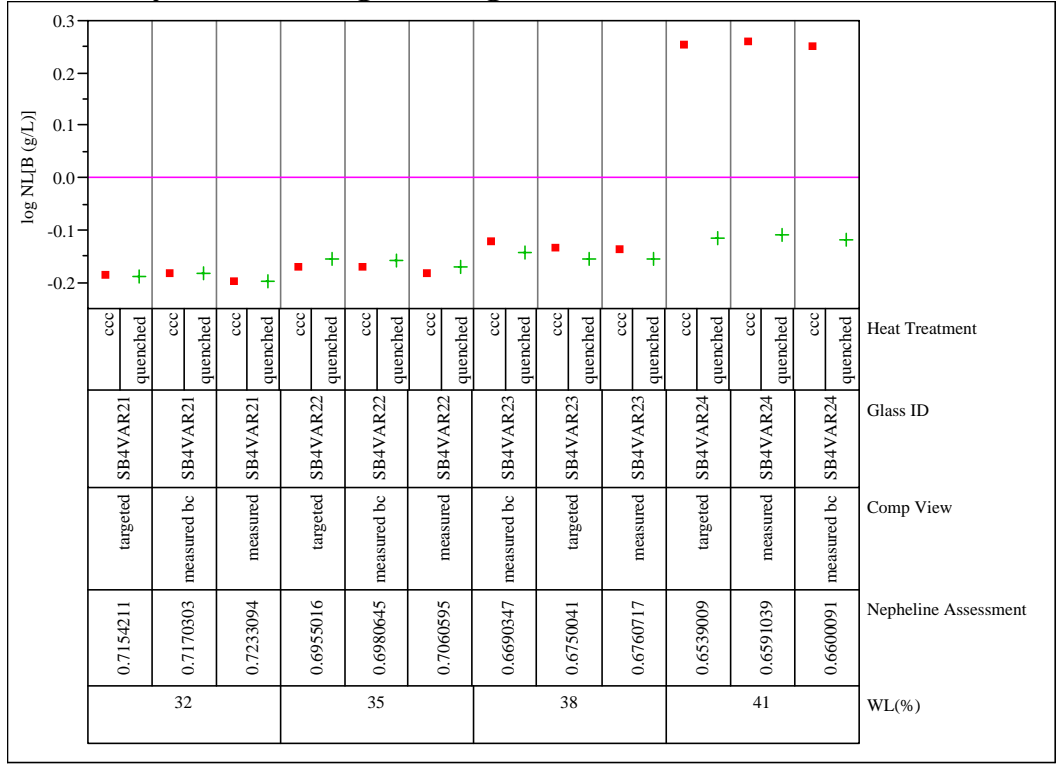

Sludge Case $=$ Case 2

Variability Chart for log NL[Li(g/L)]

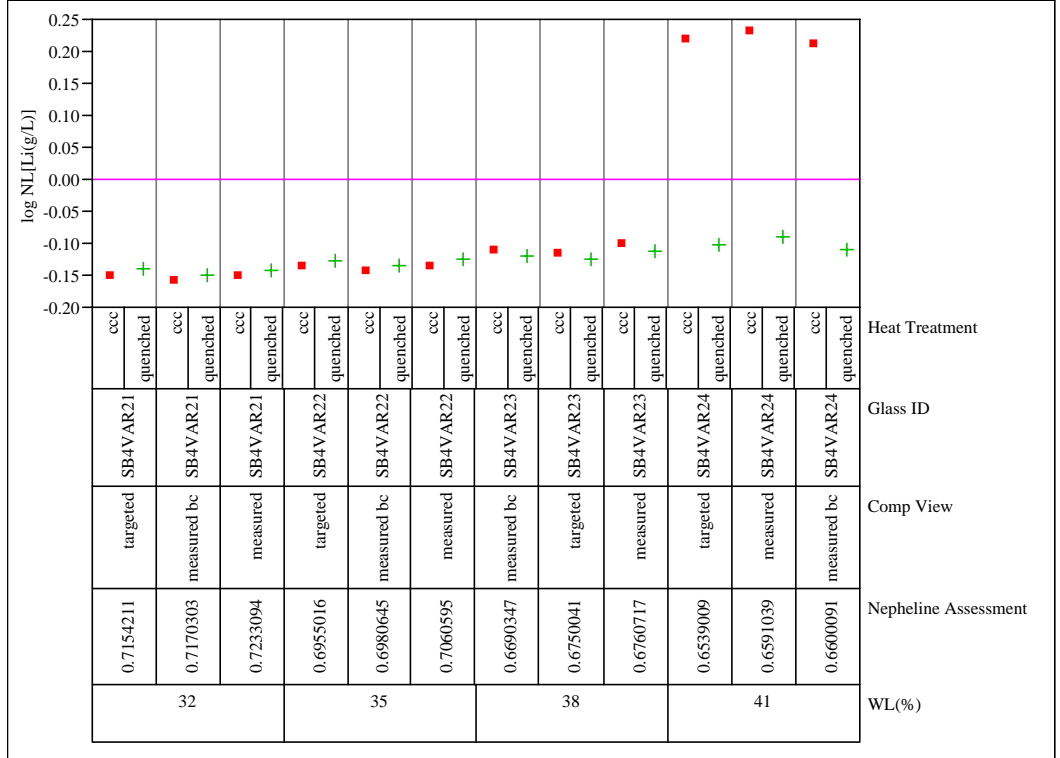




\section{Exhibit B7. Effects of Heat Treatment for Study Glasses by Compositional View}

Sludge Case $=$ Case 2

Variability Chart for $\log \mathrm{NL}[\mathrm{Na}(\mathrm{g} / \mathrm{L})]$

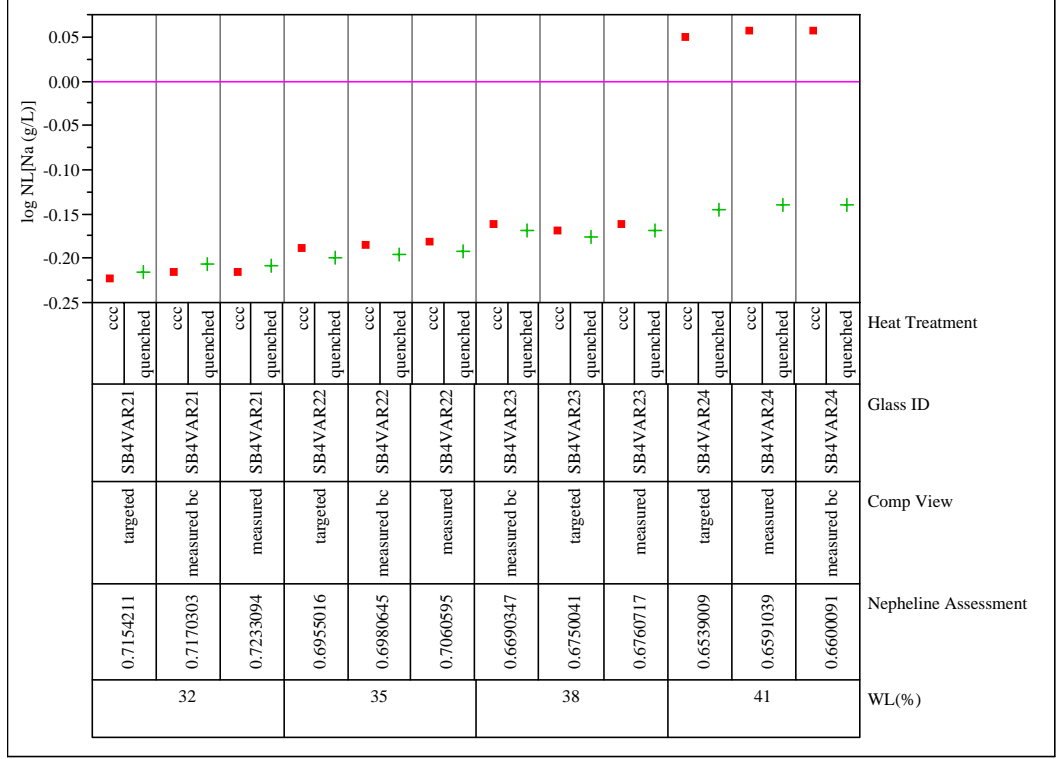

Sludge Case $=$ Case 2

Variability Chart for log NL[Si (g/L)]

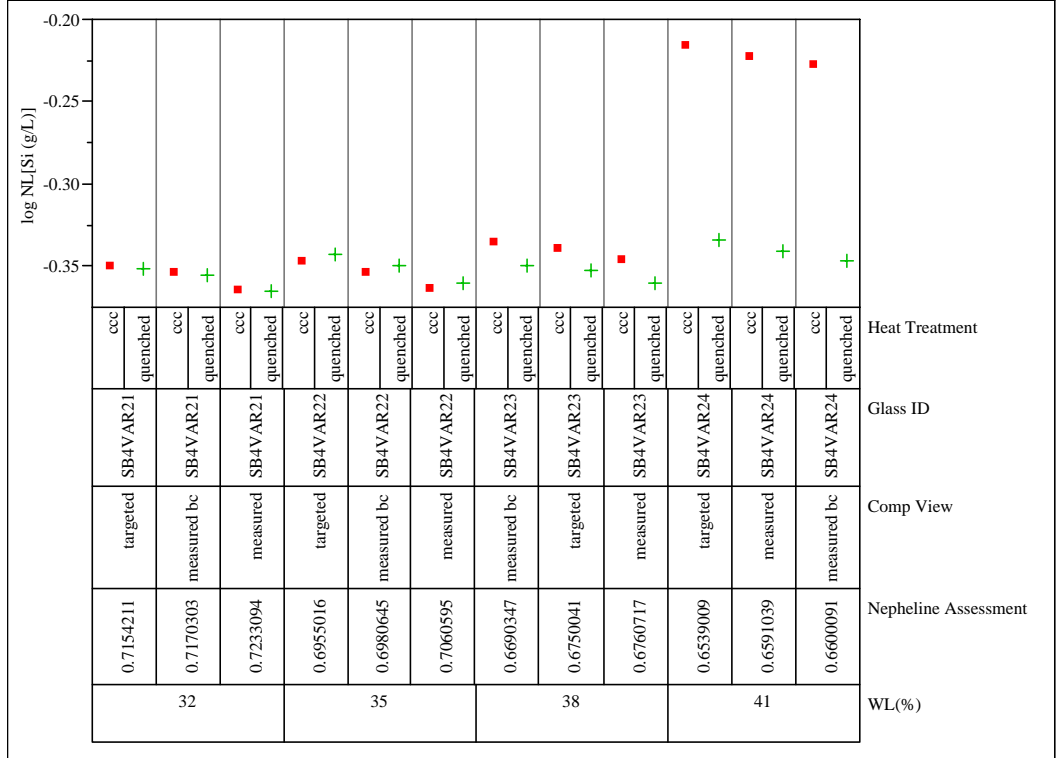




\section{Exhibit B7. Effects of Heat Treatment for Study Glasses by Compositional View}

Sludge Case $=$ Case 3

Variability Chart for $\log$ NL[B (g/L)]

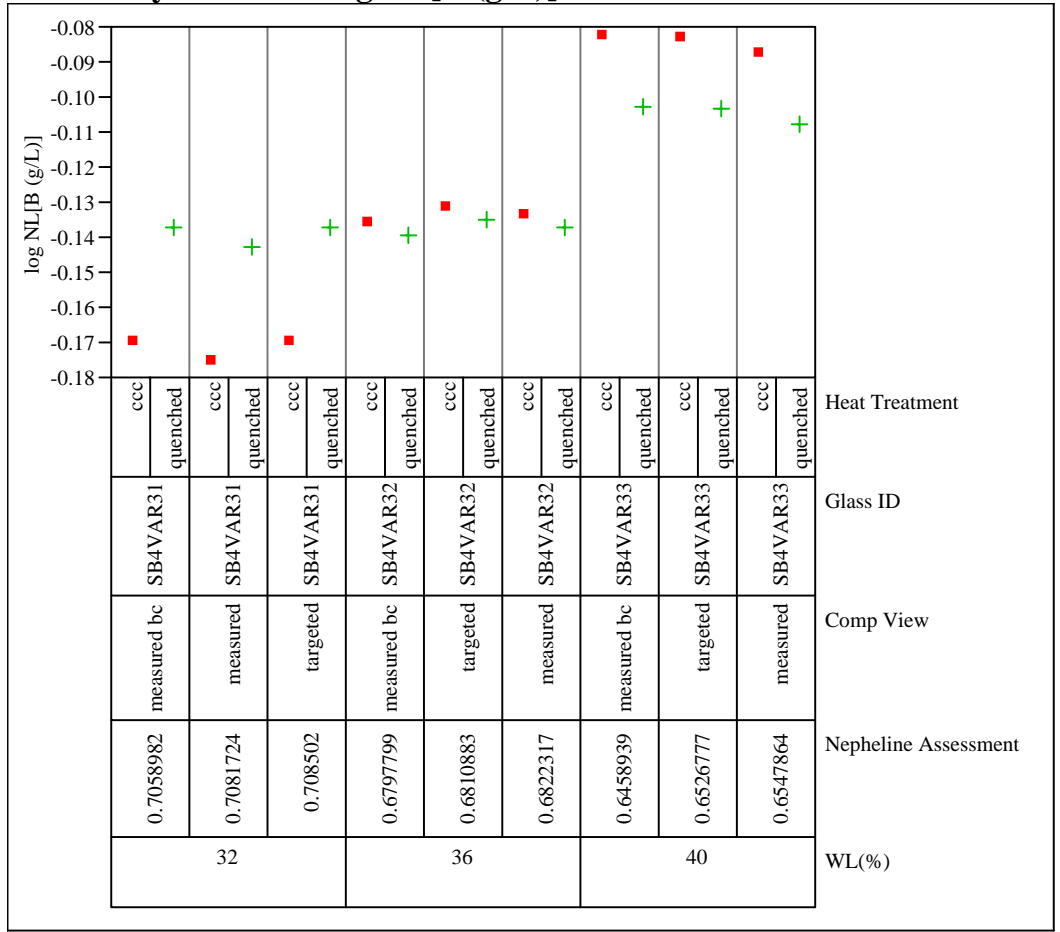

Sludge Case $=$ Case 3

Variability Chart for $\log \mathrm{NL}[\mathrm{Li}(\mathrm{g} / \mathrm{L})]$

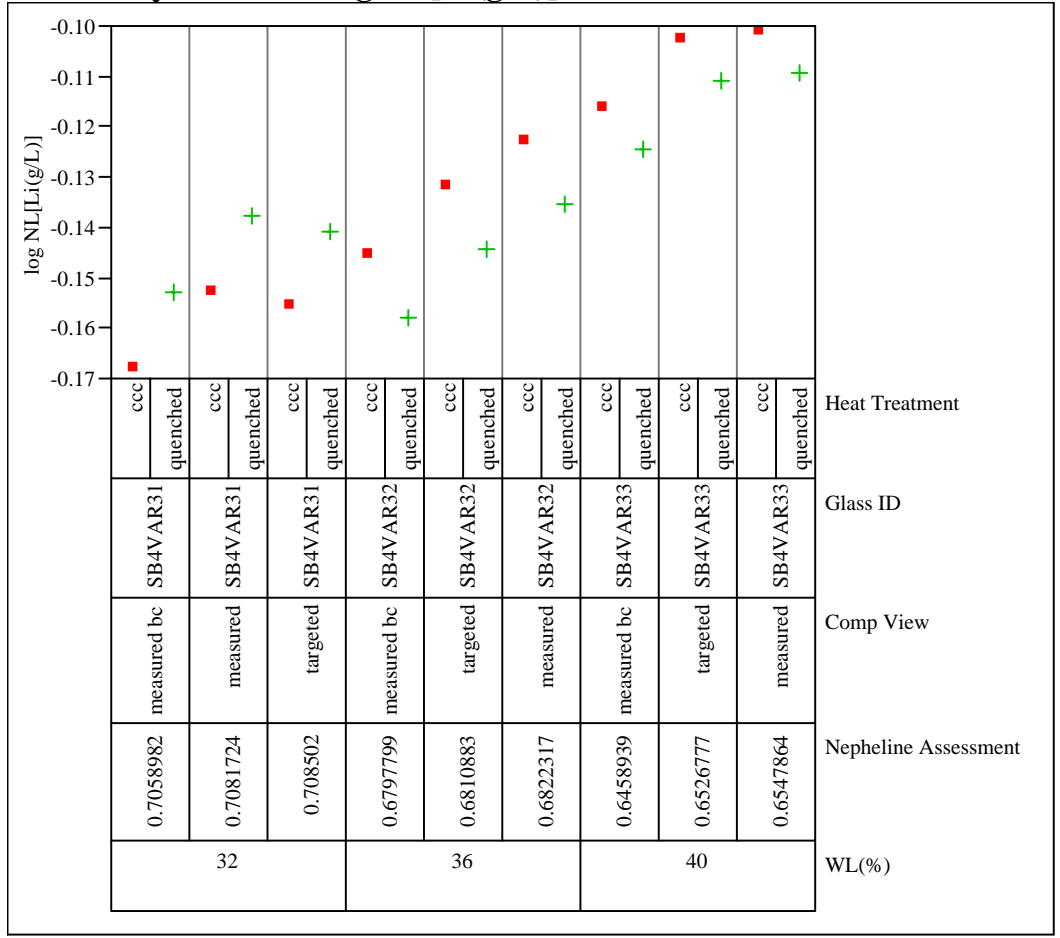




\section{Exhibit B7. Effects of Heat Treatment for Study Glasses by Compositional View}

Sludge Case $=$ Case 3

Variability Chart for $\log$ NL[Na (g/L)]

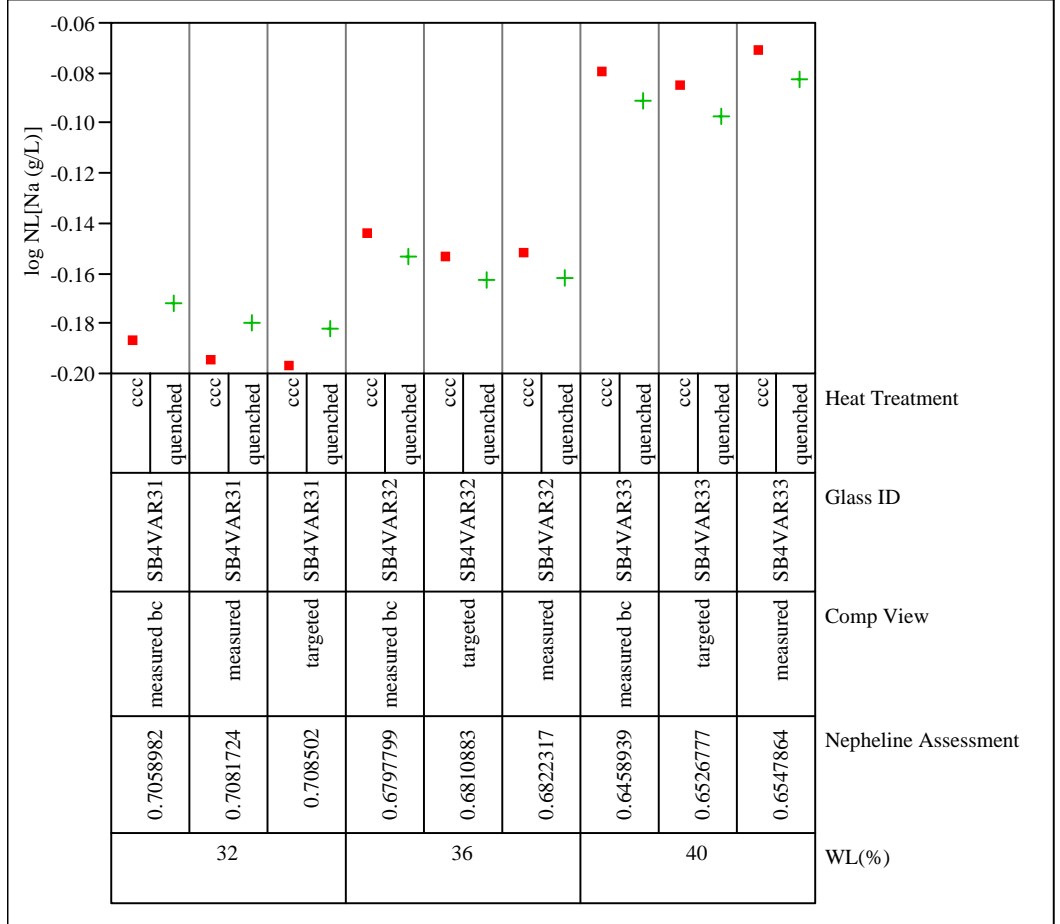

Sludge Case $=$ Case 3

Variability Chart for log NL[Si (g/L)]

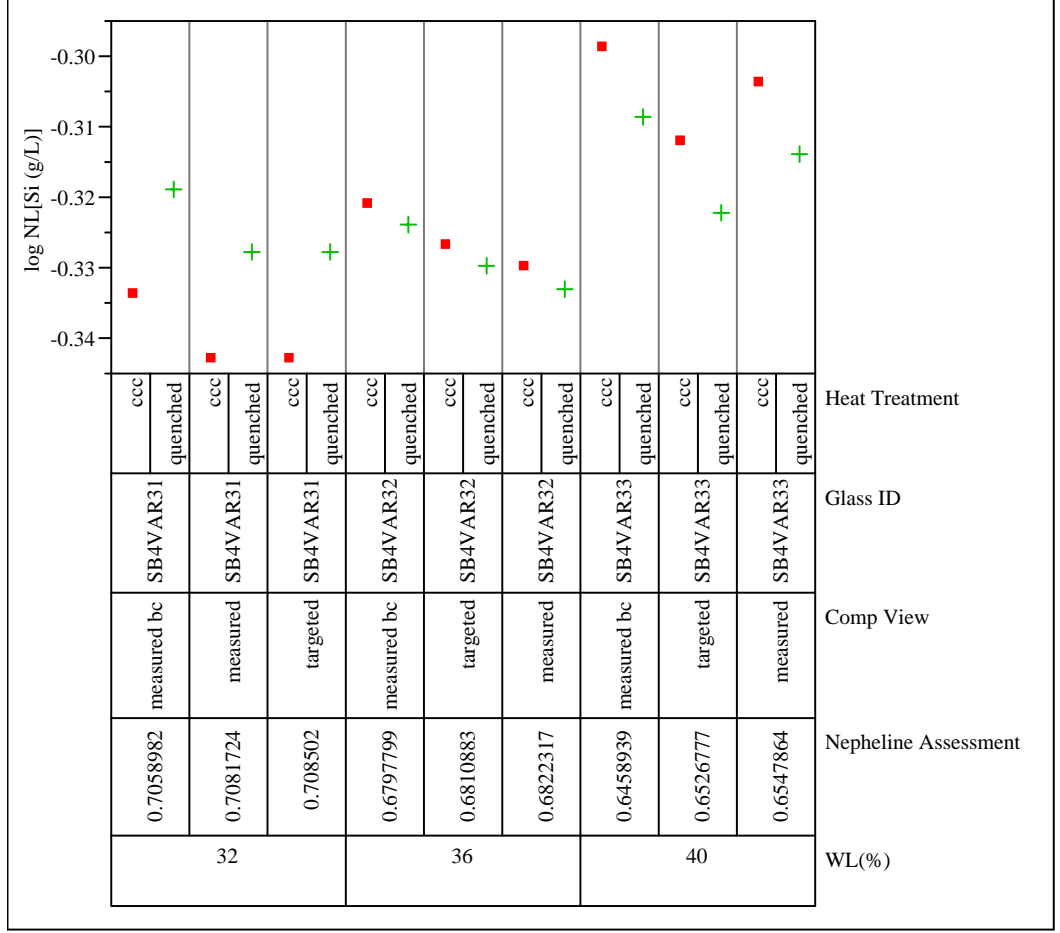




\section{Exhibit B7. Effects of Heat Treatment for Study Glasses by Compositional View}

\section{Sludge Case $=$ Case 4}

Variability Chart for $\log$ NL[B (g/L)]

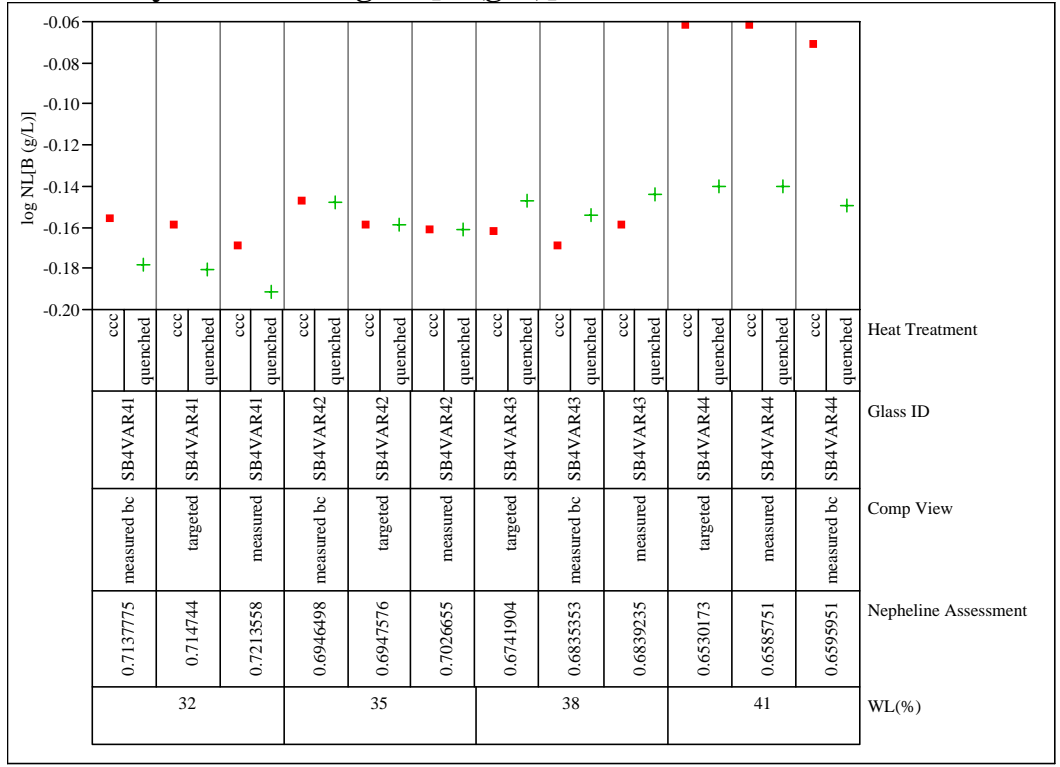

\section{Sludge Case $=$ Case 4}

Variability Chart for $\log \mathrm{NL}[\mathrm{Li}(\mathrm{g} / \mathrm{L})]$

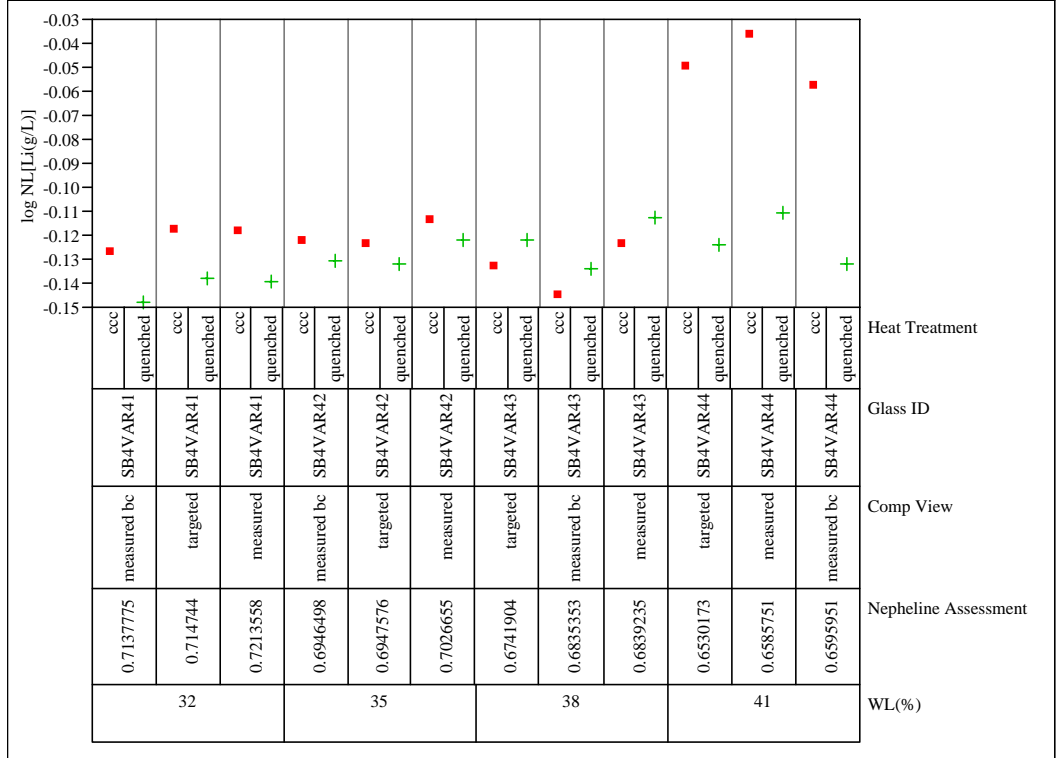




\section{Exhibit B7. Effects of Heat Treatment for Study Glasses by Compositional View}

Sludge Case $=$ Case 4

Variability Chart for log NL[Na (g/L)]

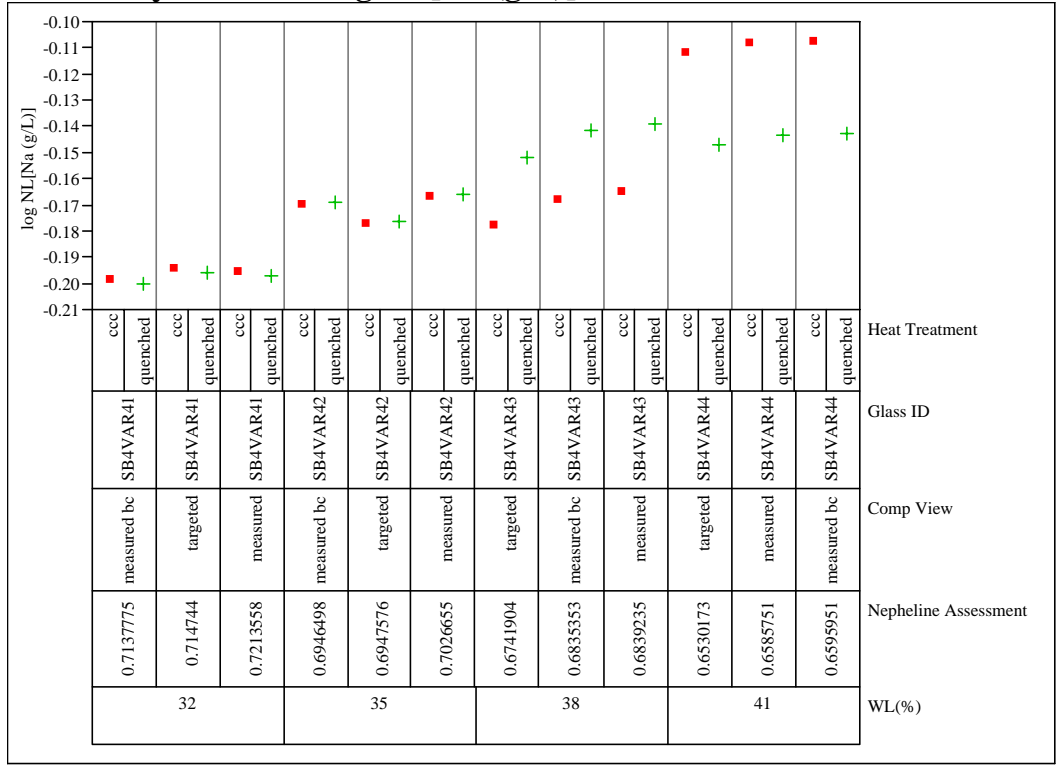

Sludge Case $=$ Case 4

Variability Chart for $\log$ NL[Si (g/L)]

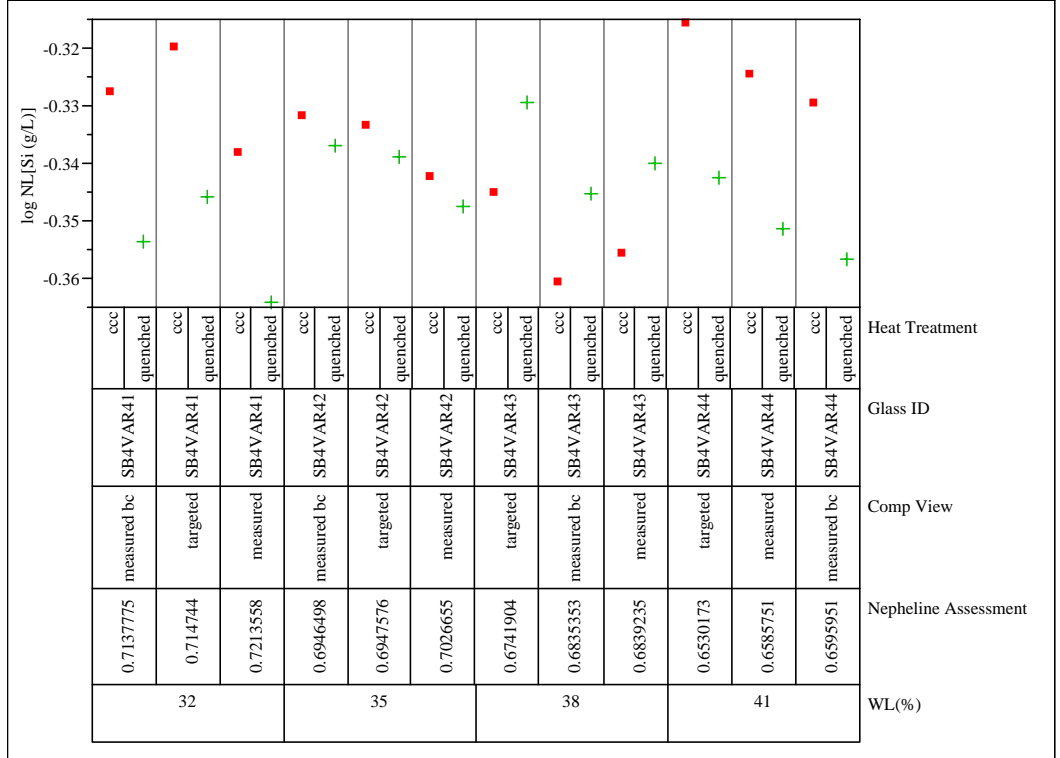




\section{Exhibit B7. Effects of Heat Treatment for Study Glasses by Compositional View}

Sludge Case $=$ Case 5

Variability Chart for $\log$ NL[B (g/L)]

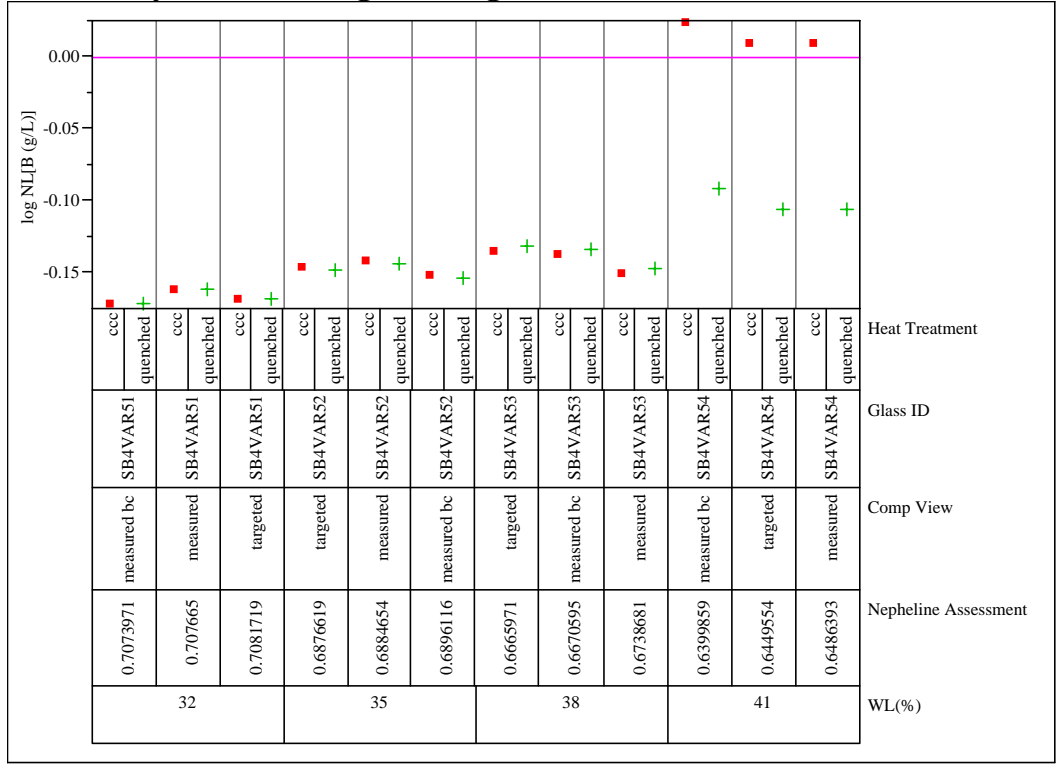

Sludge Case $=$ Case 5

Variability Chart for log NL[Li(g/L)]

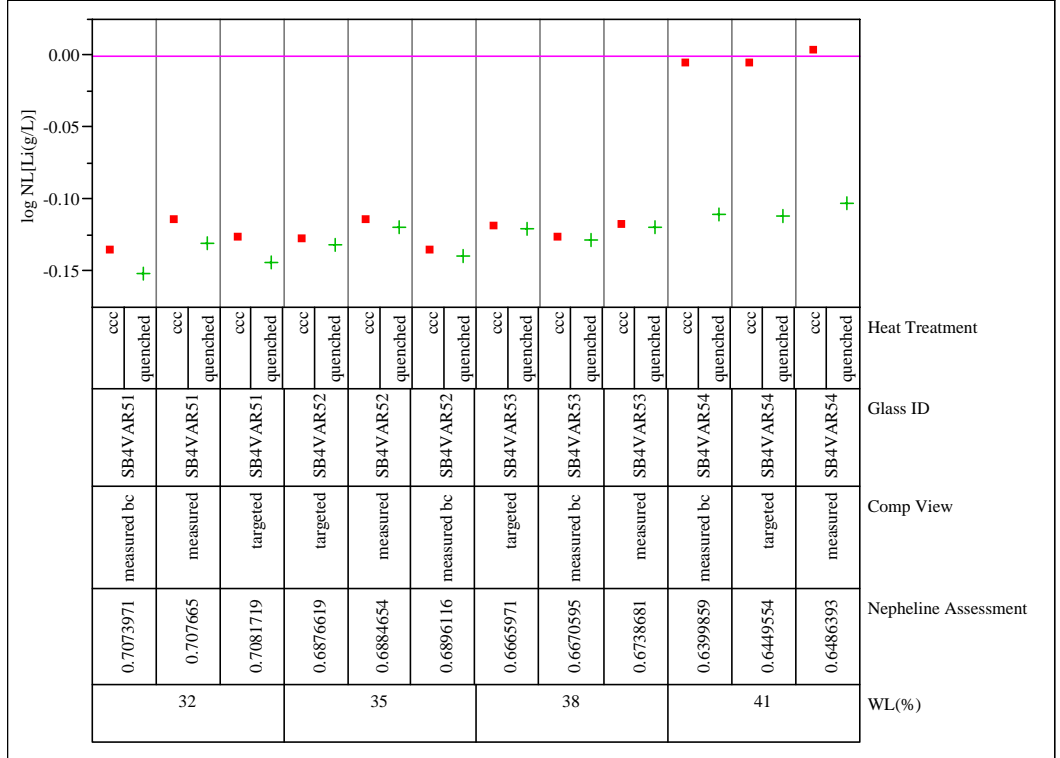




\section{Exhibit B7. Effects of Heat Treatment for Study Glasses by Compositional View}

Sludge Case $=$ Case 5

Variability Chart for $\log$ NL[Na (g/L)]

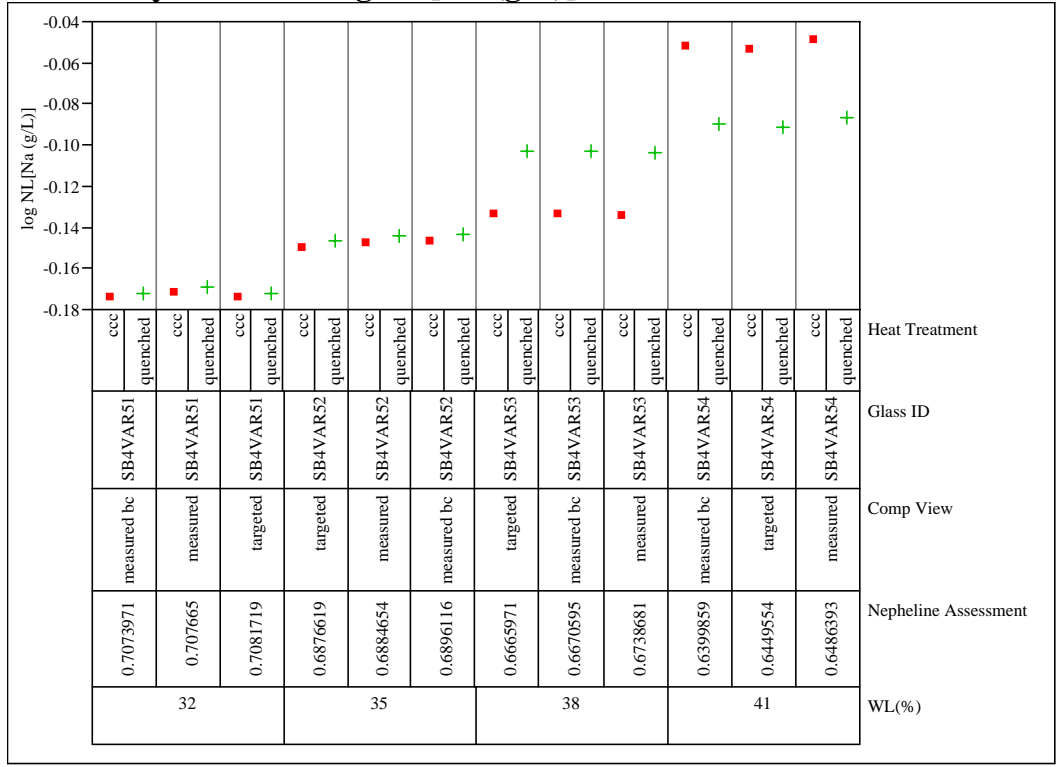

Sludge Case $=$ Case 5

Variability Chart for $\log$ NL[Si (g/L)]

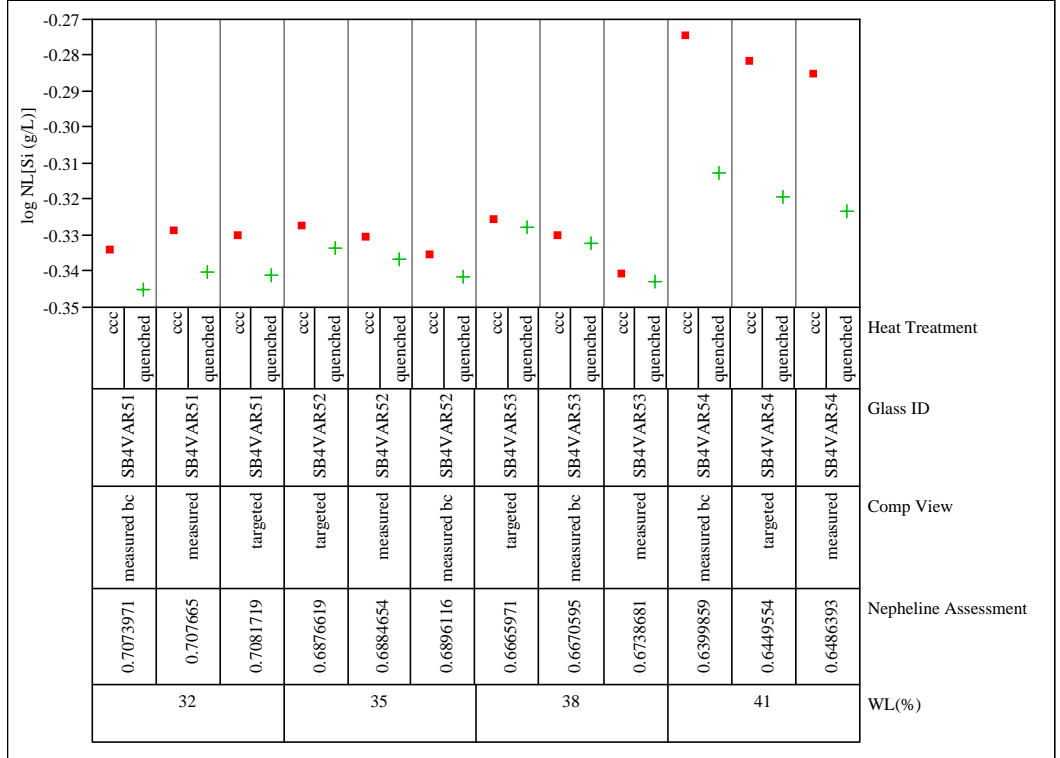




\section{Exhibit B8. del $G p\left(\Delta G_{p}\right)$ Predictions versus Common Logarithm Normalized Leachate $(\log \mathrm{NL}[]$.$) for \mathrm{B}, \mathrm{Li}, \mathrm{Na}$, and $\mathrm{Si}$ \\ Over All Compositional Views and Heat Treatments}

\begin{tabular}{|c|c|c|c|}
\hline \multicolumn{2}{|c|}{ Legend } & Heat Treatment & Composition \\
\hline $\mathbf{z}$ & 1 & ARM & ARM \\
\hline 0 & 2 & $\operatorname{coc}$ & measured \\
\hline$\Delta$ & 3 & $\operatorname{cec}$ & measured bc \\
\hline a & 4 & $\csc$ & targeted \\
\hline$\diamond$ & 5 & EA & EA \\
\hline 0 & 6 & quenched & measured \\
\hline$\Delta$ & 7 & quenched & measured bc \\
\hline a & 8 & quenched & targeted \\
\hline
\end{tabular}
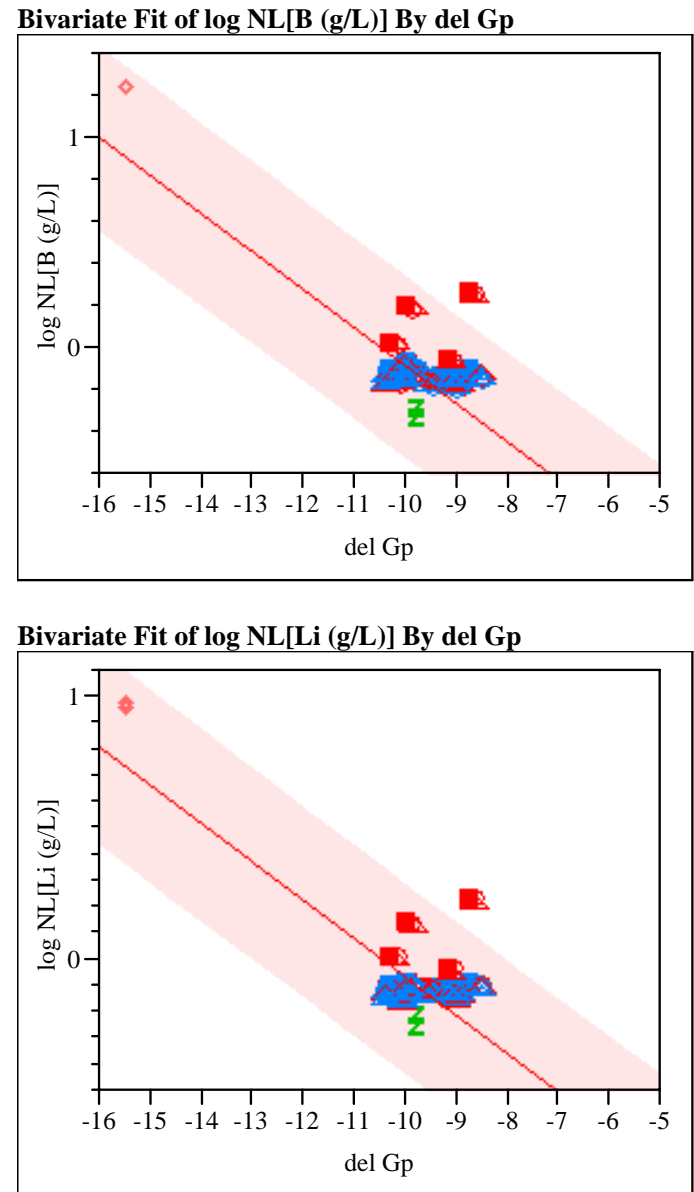

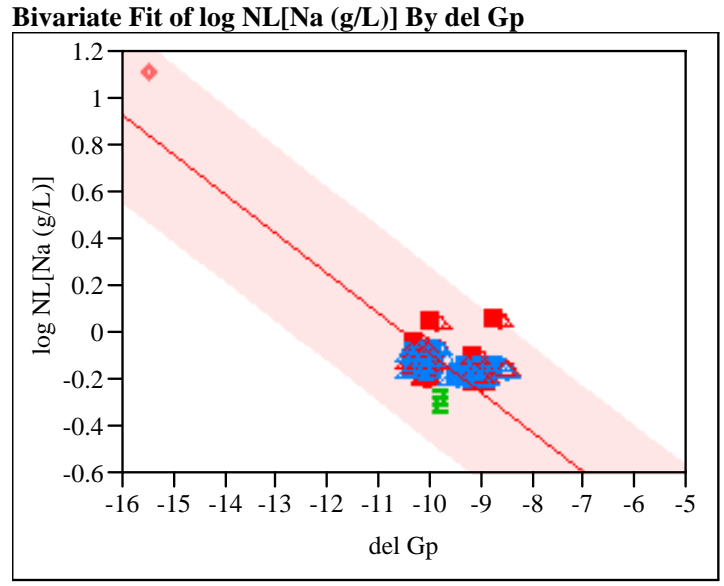

Bivariate Fit of log NL[Si (g/L)] By del Gp

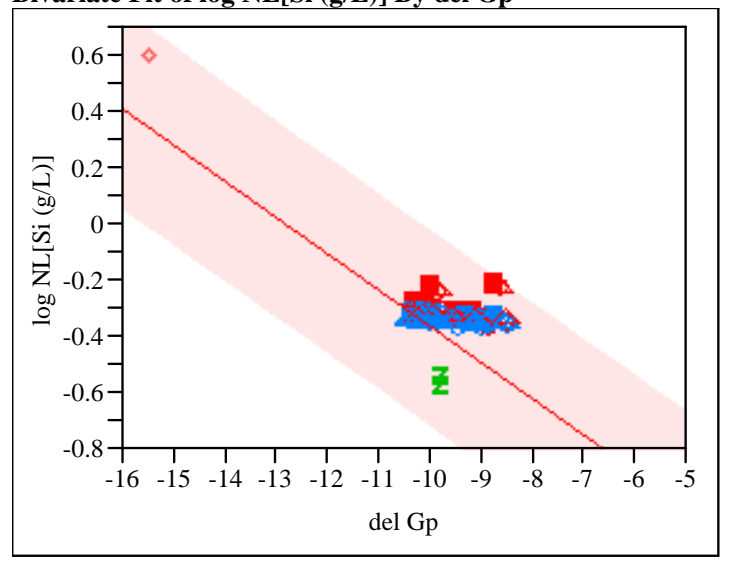




\section{Exhibit B9. del $G p\left(\Delta G_{p}\right)$ Predictions versus Common Logarithm Normalized Leachate $(\log \mathrm{NL}[]$.$) for \mathrm{B}, \mathrm{Li}, \mathrm{Na}$, and $\mathrm{Si}$ Over All Compositional Views for Quenched Glasses}

\begin{tabular}{|c|c|c|c|}
\hline \multicolumn{2}{|c|}{ Legend } & Heat Treatment & Composition \\
\hline $\mathbf{z}$ & 1 & ARM & ARM \\
\hline 0 & 2 & $\operatorname{coc}$ & measured \\
\hline$\Delta$ & 3 & $\operatorname{coc}$ & measured bc \\
\hline $\mathbf{\square}$ & 4 & $\operatorname{coc}$ & targeted \\
\hline$\diamond$ & 5 & EA & EA \\
\hline 0 & 6 & quenched & measured \\
\hline$\triangle$ & 7 & quenched & measured bc \\
\hline E & 8 & quenched & targeted \\
\hline
\end{tabular}

Bivariate Fit of $\log$ NL[B (g/L)] By del Gp

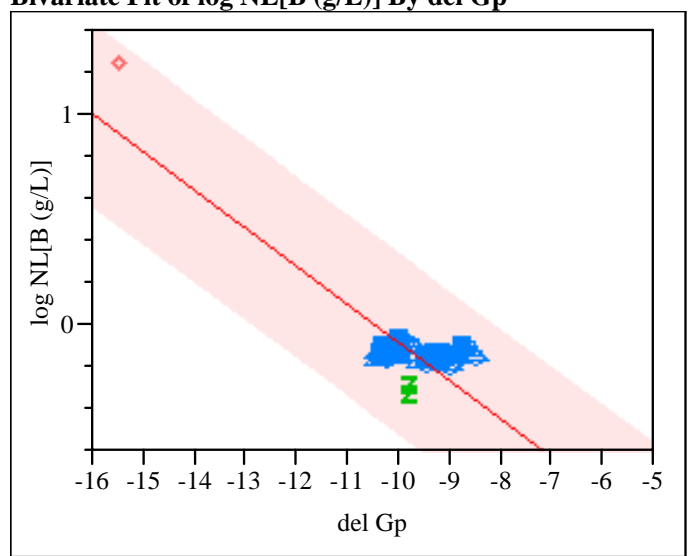

Bivariate Fit of $\log$ NL[Li (g/L)] By del Gp

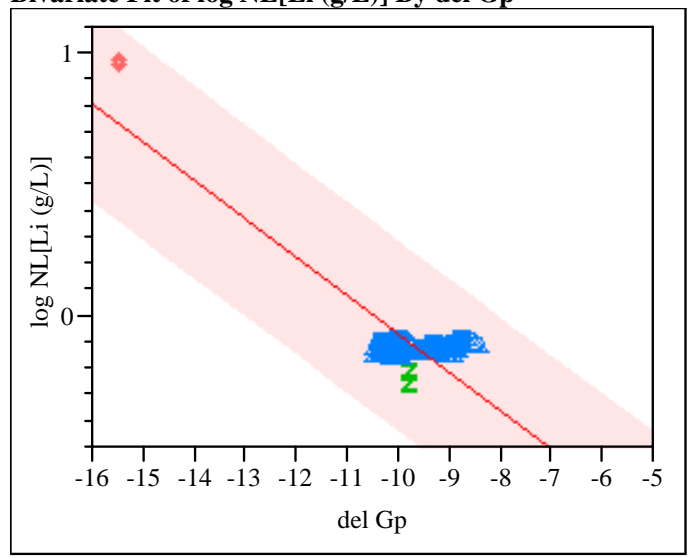

Bivariate Fit of $\log$ NL[Na (g/L)] By del Gp

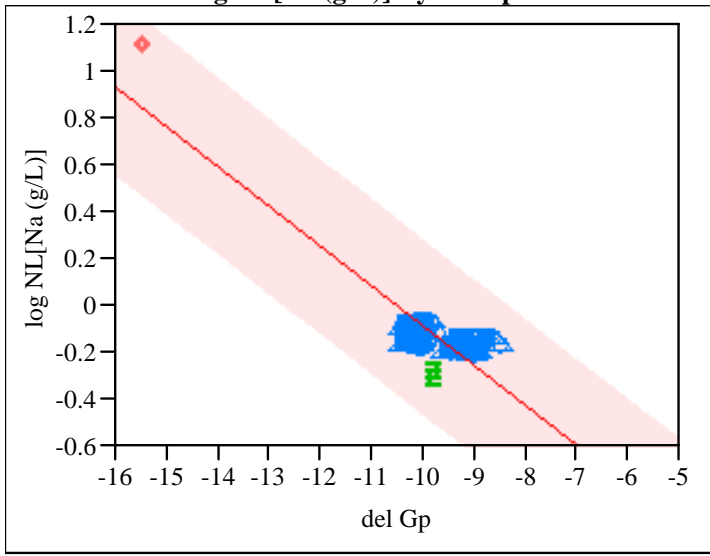

Bivariate Fit of $\log$ NL[Si (g/L)] By del Gp

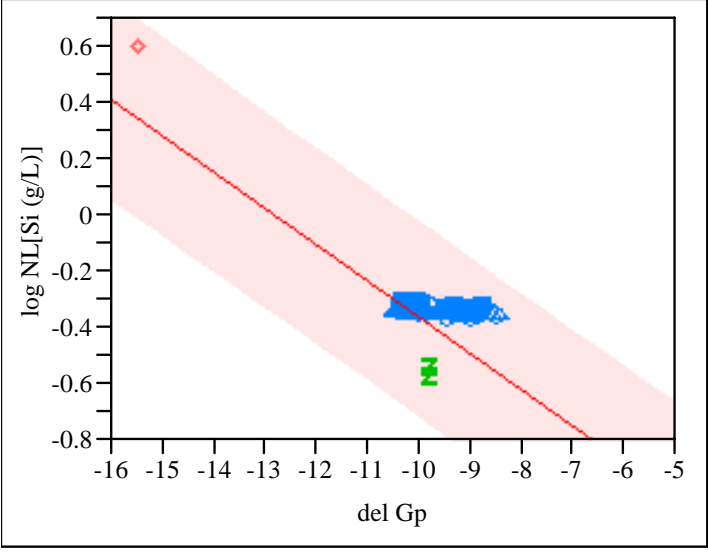


Exhibit B10. del $G p\left(\Delta G_{p}\right)$ Predictions versus Common Logarithm Normalized Leachate $(\log \mathrm{NL}[]$.$) for \mathrm{B}, \mathrm{Li}, \mathrm{Na}$, and $\mathrm{Si}$ Over All Compositional Views for ccc Glasses

\begin{tabular}{|c|c|c|c|}
\hline \multicolumn{2}{|c|}{ Legend } & Heat Treatment & Composition \\
\hline $\mathbf{z}$ & 1 & ARM & ARM \\
\hline$\circ$ & 2 & $\operatorname{ccc}$ & measured \\
\hline$\Delta$ & 3 & $\operatorname{coc}$ & measured bc \\
\hline ! & 4 & $\operatorname{cec}$ & targeted \\
\hline$\diamond$ & 5 & EA & EA \\
\hline 0 & 6 & quenched & measured \\
\hline$\Delta$ & 7 & quenched & measured bc \\
\hline - & 8 & quenched & targeted \\
\hline
\end{tabular}

Bivariate Fit of $\log$ NL[B (g/L)] By del Gp

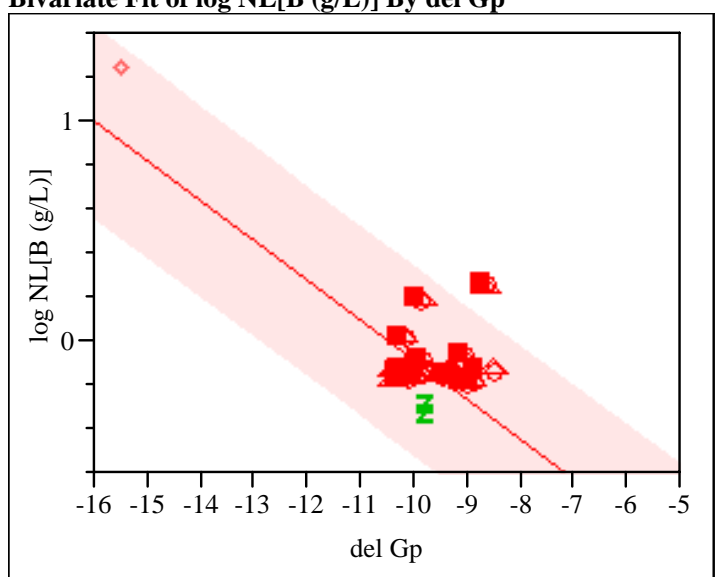

Bivariate Fit of $\log \mathrm{NL}[\mathrm{Li}(\mathrm{g} / \mathrm{L})]$ By del Gp

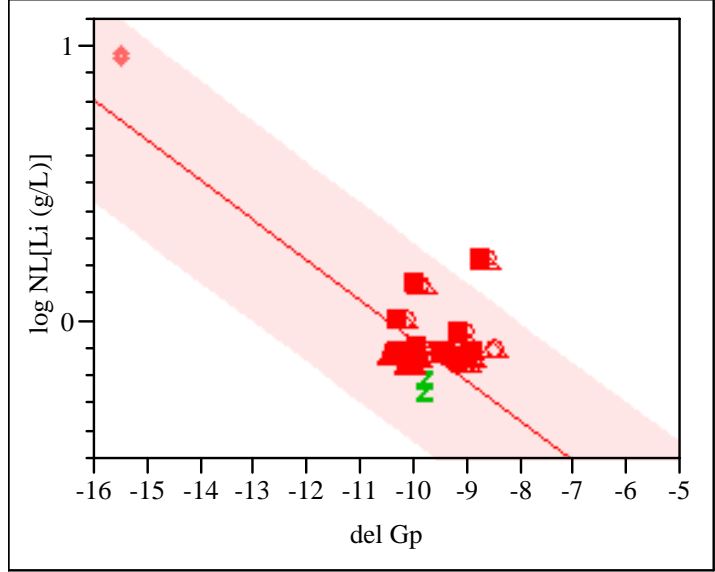

Bivariate Fit of $\log \mathrm{NL}[\mathrm{Na}(\mathrm{g} / \mathrm{L})]$ By del Gp

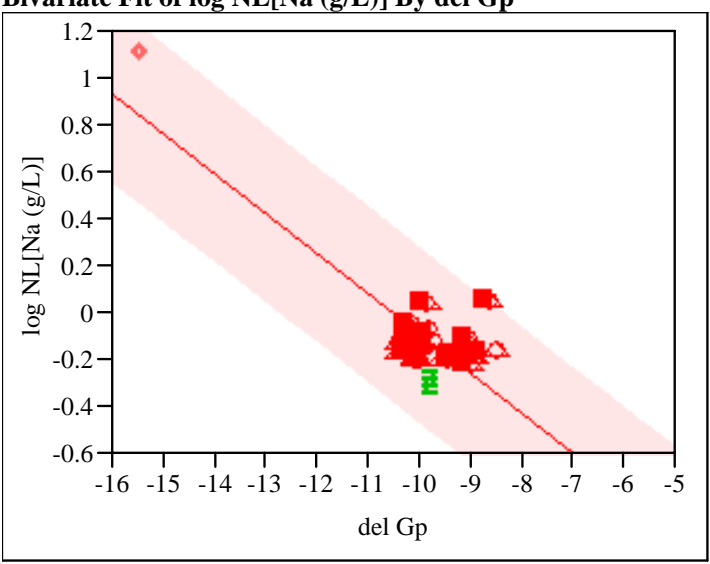

Bivariate Fit of $\log$ NL[Si (g/L)] By del Gp

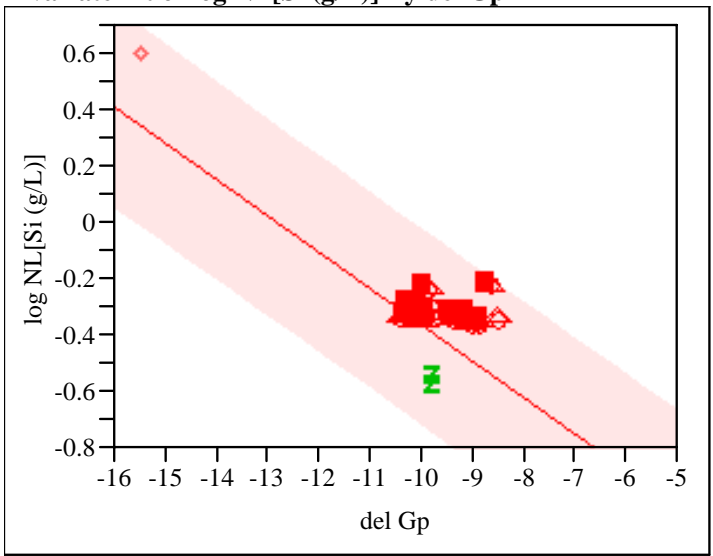




\section{APPENDIX C}

\section{COMPARISON OF SME BATCH COMPOSITIONS TO VARIABILITY STUDY GLASS COMPOSITIONS}




\begin{tabular}{|c|c|c|c|c|}
\hline \multirow{2}{*}{ SME Batch } & $\mathbf{A l}_{2} \mathbf{O}_{3}$ & $\mathrm{Fe}_{2} \mathrm{O}_{3}$ & $\mathrm{Na}_{2} \mathrm{O}$ & $\mathbf{U}_{3} \mathbf{O}_{8}$ \\
\hline & \multicolumn{4}{|c|}{ wt \% } \\
\hline 402 & 6.61 & 12.50 & 12.72 & 3.31 \\
\hline 403 & 7.11 & 9.74 & 11.22 & 2.43 \\
\hline 404 & 7.90 & 9.33 & 11.56 & 2.42 \\
\hline 405 & 8.06 & 8.64 & 10.54 & 2.42 \\
\hline 406 & 8.35 & 9.26 & 11.44 & 2.35 \\
\hline 407 & 7.79 & 8.79 & 11.18 & 2.19 \\
\hline 408 & 7.86 & 8.63 & 11.60 & 2.81 \\
\hline 409 & 8.33 & 9.24 & 11.26 & 2.43 \\
\hline 410 & 7.80 & 8.93 & 11.80 & 2.35 \\
\hline 411 & 8.76 & 9.75 & 10.69 & 2.46 \\
\hline 412 & 8.22 & 9.40 & 11.39 & 2.41 \\
\hline 413 & 7.80 & 8.83 & 11.09 & 2.28 \\
\hline 414 & 7.97 & 9.09 & 10.86 & 2.34 \\
\hline 415 & 8.12 & 9.38 & 9.47 & 2.30 \\
\hline 416 & 8.45 & 9.33 & 10.47 & 2.26 \\
\hline 417 & 8.32 & 9.46 & 10.35 & 2.15 \\
\hline 418 & 8.47 & 9.64 & 10.43 & 2.08 \\
\hline 419 & 8.14 & 8.98 & 11.04 & 2.51 \\
\hline 420 & 7.88 & 9.02 & 11.76 & 2.16 \\
\hline 421 & 8.21 & 9.27 & 10.36 & 2.63 \\
\hline 422 & 7.52 & 8.73 & 10.97 & 2.15 \\
\hline 423 & 7.72 & 8.76 & 10.81 & 2.21 \\
\hline 424 & 7.87 & 8.80 & 11.39 & 2.15 \\
\hline 425 & 8.18 & 9.44 & 12.39 & 2.36 \\
\hline 426 & 7.74 & 8.56 & 11.34 & 2.04 \\
\hline 427 & 8.43 & 8.57 & 11.56 & 1.95 \\
\hline 428 & 8.46 & 8.98 & 12.29 & 2.20 \\
\hline 429 & 8.33 & 8.83 & 11.23 & 2.15 \\
\hline 430 & 8.29 & 9.07 & 11.00 & 3.02 \\
\hline 431 & 8.54 & 9.46 & 11.68 & 3.14 \\
\hline 432 & 7.88 & 8.63 & 11.66 & 2.96 \\
\hline 433 & 8.13 & 9.12 & 11.47 & 2.94 \\
\hline 434 & 7.65 & 8.12 & 12.19 & 2.75 \\
\hline 435 & 7.29 & 7.94 & 11.86 & 2.66 \\
\hline 436 & 8.03 & 8.73 & 11.63 & 2.46 \\
\hline MINIMUM & 6.61 & 7.94 & 9.47 & 1.95 \\
\hline MAXIMUM & 8.76 & 12.50 & 12.72 & 3.31 \\
\hline
\end{tabular}

\begin{tabular}{|c|c|c|c|c|c|}
\hline \multirow{2}{*}{ Glass ID } & \multirow{2}{*}{ WL (\%) } & $\mathrm{Al}_{2} \mathbf{O}_{3}$ & $\mathrm{Fe}_{2} \mathrm{O}_{3}$ & $\mathrm{Na}_{2} \mathrm{O}$ & $\overline{\mathrm{U}_{3} \mathrm{O}_{8}}$ \\
\hline & & \multicolumn{4}{|c|}{ wt $\%$} \\
\hline SB4VAR11 & 34 & 8.46 & 9.62 & 12.26 & 2.94 \\
\hline SB4VAR12 & 38 & 9.46 & 10.75 & 12.76 & 3.28 \\
\hline SB4VAR13 & 42 & 10.46 & 11.88 & 13.26 & 3.63 \\
\hline SB4VAR21 & 32 & 8.31 & 9.47 & 10.98 & 2.89 \\
\hline SB4VAR22 & 35 & 9.09 & 10.36 & 11.26 & 3.16 \\
\hline SB4VAR23 & 38 & 9.86 & 11.25 & 11.54 & 3.43 \\
\hline SB4VAR24 & 41 & 10.64 & 12.14 & 11.82 & 3.70 \\
\hline SB4VAR31 & 32 & 8.00 & 9.13 & 11.93 & 2.79 \\
\hline SB4VAR32 & 36 & 9.01 & 10.27 & 12.42 & 3.13 \\
\hline SB4VAR33 & 40 & 10.01 & 11.41 & 12.92 & 3.48 \\
\hline SB4VAR41 & 32 & 8.00 & 9.20 & 11.34 & 2.80 \\
\hline SB4VAR42 & 35 & 8.75 & 10.06 & 11.66 & 3.06 \\
\hline SB4VAR43 & 38 & 9.50 & 10.92 & 11.97 & 3.32 \\
\hline SB4VAR44 & 41 & 10.25 & 11.79 & 12.28 & 3.59 \\
\hline SB4VAR51 & 32 & 7.71 & 8.87 & 12.25 & 2.70 \\
\hline SB4VAR52 & 35 & 8.43 & 9.70 & 12.65 & 2.95 \\
\hline SB4VAR53 & 38 & 9.15 & 10.53 & 13.04 & 3.21 \\
\hline SB4VAR54 & 41 & 9.88 & 11.37 & 13.44 & 3.46 \\
\hline MINIMUM & & 7.71 & 8.87 & 10.98 & 2.70 \\
\hline MAXIMUM & & 10.64 & 12.14 & 13.44 & 3.70 \\
\hline SB4PS1 & & 7.61 & 8.07 & 12.12 & 2.73 \\
\hline SB4PS2 & & 7.37 & 8.03 & 11.98 & 2.68 \\
\hline SB4PS3 & & 7.92 & 9.00 & 11.99 & 2.75 \\
\hline SB4PS4 & & 8.29 & 9.42 & 12.18 & 2.88 \\
\hline SB4PS5 & & 8.49 & 9.65 & 12.28 & 2.94 \\
\hline MINIMUM & & 7.37 & 8.03 & 11.98 & 2.68 \\
\hline MAXIMUM & & 8.49 & 9.65 & 12.28 & 2.94 \\
\hline
\end{tabular}

Table C1. Comparison of SME batch compositions and variability study glass compositions. Note the variability study glass compositions (SB4VAR11 - SB4VAR54 and SB4PS1 - SB4PS5) are target compositions. The maximum contents of the variability study glasses in bold indicate that these are outside the range of the SME batch compositions. 


\section{Distribution:}

C.J. Bannochie, 773-42A

A.B. Barnes, 999-W

D.R. Best, 786-1A

D.B. Burns, 786-5A

D.A. Crowley, 999-W

B.A. Davis, 704-27S

T.B. Edwards, 999-W

H.H. Elder, 766-H

T.L. Fellinger, 704-26S

K.M. Fox, 999-W

J.M. Gillam, 766-H

J.C. Griffin, 773-A

B.A. Hamm, 766-H

C.C. Herman, 999-W

J.F. Iaukea, 704-30S

J.E. Marra, 773-A

R.T. McNew, 704-27S

T.A. Nance, 773-42A

J.D. Newell, 999-W

J.E. Occhipinti, 704-S

D.K. Peeler, 999-W

F.C. Raszewski, 999-W

J.W. Ray, 704-S

I.A. Reamer, 999-1W

H.B. Shah, 766-H

M.E. Smith, 999-W

M.E. Stone, 999-W

J. Stuberfield, 766-H

M.F. Williams, 999-1W

R.J. Workman, 999-1W

A.L. Youchak, 999-W 